P.

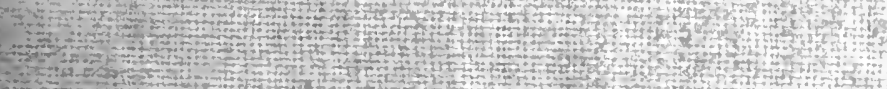

M

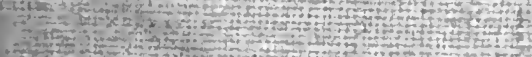

1

C.

My

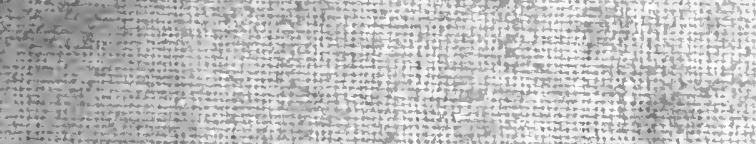

Ty

Now

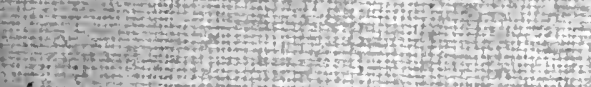

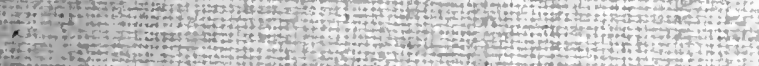

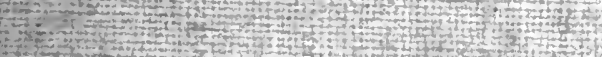

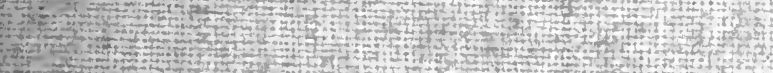

(1.

W.

15y

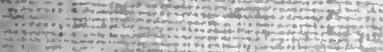

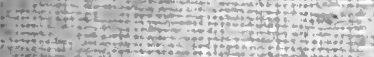

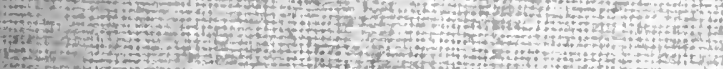

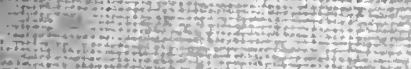

-

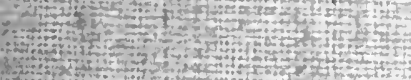

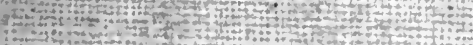

$x^{2}+x^{2}$

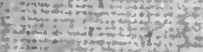

ind is

f.8.

$5=32$

castivis

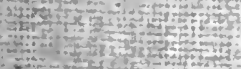

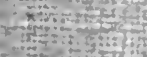

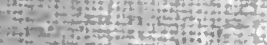

tiv

Unwar Тонอบть

WhBARY

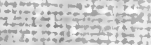

Tith 


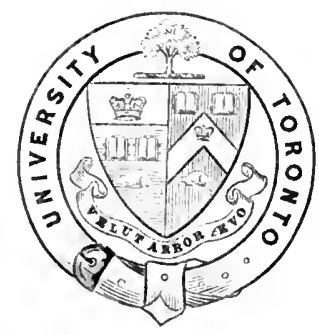

Dresented to

\section{The Iibrary}

of the

\section{Univergity of Toronto}

by

From The Library of

H.T. Gerrans

by Wis wite 
BIRDING LISM:.UG 151923 

it 




$$
74^{6^{3}}
$$

\section{L'A D R I A T I C O.}



A 2432

Studio geografico, storico e politico
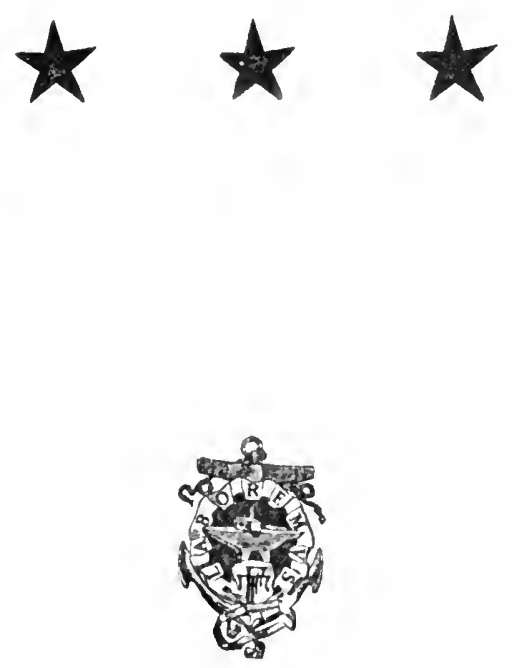

MI L A N O

FRATELLI TREVES, EDITORI

1915

secondo migliaio. 
PROPRIET I LETTERARIA.

$I$ deritti di riproduzione e di traduzione sono riservati per tutti $i$ paesi, compresi la Svezia, la Norvegia e l'Olanda.

Copyright by Fratelli Treves, 1914. 


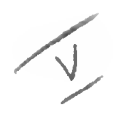

\section{Avvertenza dell' Editore.}

Il libro che si presenta oggi al lettore ha una storia; una di quelle piccole oscure storie di libri che nascono, e che valgono talora a dare ai volumi una doppia vita: quella che spirat dalle loro pagine, e quclla che spira dall'imagine dell'antore.

Imagine ignota. L'anonimo che si nasconde sotto gli asterischi del frontispizio è mo scrittore adriatico, di quella nobilissima terra dalmata che attende in quest'ora la sua sorte suprema, e non può essere rivelato al gran pubblico per ragioni che ognumo comprende. Nell'ora in cui escono queste pagine ch'egli ha scrilte, ma che non ha più riviste nè corrette, l'autore è trascinato sotto le insegne dell'impero austriaco per i campi di Galizia e di Polonia, e noi ne ignoriamo la sorte. La sua ultima parola è quella che ci ha mandata con questo manoscritto nel mese di luglio trascorso, nascondendo fin da allora il suo nome: parola di profezia che pare mirabile a chi ripensi la calma in cui l'Europa si addormiva prima della bufera di agosto. Auguriamoci di riavere dall'autore anonimo tra poco un'altra parola libera, col suo nome e non col suo travestimento, dopo che la realtà abbia sostituito il desiderio e la profezia.

$\mathrm{Ci}$ sia permesso aggiungere un'avvertenza. Come l'editore, esaminando forse svogliatamente le pagine manoscritte d'autore giovane e sconosciuto, fu sorpreso nello scoprire un forte lavoro degno d'essere divulgato; così crediamo accadrà al lettore che, scorrendo da prima questo volume con la curiosità o con la commozione che la sua origine può destare. si accorgerà, mano mano che proceda nella lettura, di trovarsi dinanzi ad un'opera fondamentale non soltanto per Ia storia, per la geografia e per la politica dell' Adriatico, ma anco per la storia, per la geografia, per la politica d'Italia nel mondo.

Novembre 1914 

PARTE PRIMA.

\section{L'ADRIATICO NELLA GEOGRAFIA.}

\section{LADRIATICO E LA PADANIA FORMANO UN BACINO SOLO.}

\section{Perchè l'Allrialico è un mare?}

L'Adriatico i un mare, perehè il massimo fattor prese e distribui la sua terra onde formale le calene di monti, che racchiurlono il suo bacino. Partendo da questo roncello si arriva all'idea che l'Adriatico è lightio delle Nipi.

Lásserzione sembrerà ardita. Prima pero di pronunziare un giudizio contratio si voglia osservare una buona carta geograliea e accompagnare le considerazioni che seguono.

Ai piedi ed in immediala dipendenza dalla cononal delle Mpi si estende la pianmat hombardo-renela-linulana, chiusa ad occidente (con maggior esallezza nella parte del Piemonte dagli Apennini "and oriente dal Carso.

Quesla pianmara è alforrersata abbondimlemente da fiumi, che avendo le loro origini appunto nelle Alpi, sborcano tulti nell'Adriatico. Il londo di questo mare puo pere conseguenza essere considerato come una continuazione abbassata della pianura pardana o delle $x_{p i}$ (un ter\%o gradino ideale.

Lidea della dipendenza dell'Ariatico dalle $\left.M_{p}\right) \mathrm{i}$ apparisce ancorar piò evidente guando si considerino i suoi conlini longitudinali.

Da tulti si ammette che gli Apennini siano man continuazione delle Alpi. Meno diffusal e atecettala is inverese

LiAdriatico. 
l'idea, che anche il Carso non sia alteo che una continuazione di fuetle importantissime catene di montagne.

All'accettazione di questa seconda idea si oppone sopratutto la diversa composizione geologica. Però anche gli Apennini sono lormati geologicamente in maniera diversa dalle Alpi ed anche in questi, come nel Carso, manca una linca esatta di demarcazione tra la corona delle Alpi e le sue prolungazioni apenniniche e carsiche.

Esiste invece una certa analogia geologica tra gli Apennini e il Carso. Per gli Apennini il confine colle Alpi viene da alcuno collocato al Col di Tenda, da allri al passo della Bocchetta e da altri inline a quello dei Giovi, ciò che dimostra appunto la difficoltà di separare queste due catene di monti anche secondo criteri geologici.

Per il Carso manca addirittura una divisione accentuata. L'ultimo tratto alpino riconoscibile come lale per configurazione geologica e imponenza di catena c̀ quello della Carnia. Dopo le Carniche i geografi riconoscono le Alpi Giulie, che vamno dalla ferrovia pontebbana fino ai fiumicelli Idria e Zeyer; ma queste differiscono dalle prime perchè vi mancano non solo alte montagne, ma perfino vere e proprie catene. Dopo di queste incominciano le montagne del Carso, propriamente detto, che si unisce poi al sistema montuoso della penisola balcanica.

Però altipiani calcarei carsici come quello dei Tredici Commni, sopra Verona, dei Sette Commi e l'altro ad est della frattura di Santa Croce, che raggiungono tutti i 1000 metri, incominciano a mezzogiorno delle Alpi Carniche e Giulie, ancora molto più ad occidente nelle Alpi renete e ricompariscono poi a settentrione di Trieste. Il collegamento geologico tra le Alpi e il Carso e poi tra questo e i monti balcanici è quindi ancora più stretto, che quello tra le Alpi e gli Apennini. - La forma del Carso sciolto in molte catene parallele e più basso in confronto all'Apennino, ch'è più elevato e robusto nel suo dorso principale, non è, come si vedrà più avanti, una ragione assoluta per negarne la continuazione geologica dalle Alpi. 
Liopinione differente riguardo al Carso potrebbe avere una causa più che sostanziale, occasionale. Si deve notare infalli, che gli Apennini furono studiati per lo più dalle stesse persone che rivolsero la loro altenzione alle $\mathrm{Al}_{\mathrm{p}}$, persone che se non proprio un interesse, doverano provare almeno piacere nell'affermare la parentela naturate tra i due sistemi di montagne, che dàmo vita alla penisola italica. Il Carso al contrario, pì̀ incerto come confine naturale e che rese per conseguenza valriabili anche $\mathrm{i}$ confini elnografico e politico, è stato studiato finora con altri criteri.

Ma ammesso pure che il Carso soltanto non possil essere considerato geologicamente come ma continuazione delle Alpi, per la geografia è luori di dubbio che Api, Apemnini e Carso racchiudono nettamente un grande bacino in parte asciutto - la pianura lombarclo-reneta-friulana - o in parte coperto dal mare - l'Adriatico.

Irocchio unnano non può abbracciare lestensione di questo bacino che nella rappresentazione artificiale ridotta. All'osservatore allento però non slugge la sua lorma di numero selle: forma che s'incontra spessissino più in piccolo nella natura e che si può vedere lanto nelle valli asciulte o atlraversate solo da fiumi nella ferraferma, fuanto nelle insenature marine dellat costa. in cui appunto il trallo superiore del selte è terrat. mentre lá gamba è mare.

Ma che laa le Mpi, gli Apemini a il Carso, ollere al nesso geogralien suacermulo esiste anche minallar colrispondenza di origine geologica, ma di grande valore per Collegamenti delle I'realpi e delle isole. la geogralia, ì provalo prescindendo dai movimenti sismici che partendo dall'oceidente degli Apemnini abbratediano fuasi sempere lutle le due coste aldrialichej dai molti rimasugli di minori calcne traversali di congimnzione tra le Mpri "gli Apemmini e lra questi e il Carso, che, come frangie o brandelli, adomano i versanti convergenti, ma specialmente quello orientale carsico.

Incominciande da occidente troviamo te colline del

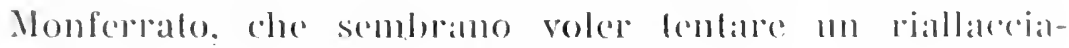
mento fra Apennini ed Mpi a scomanne la divisione trat 
Piemonte e Lombardia. Segnendo it dectivio nalurale deI bacino verso orienle, ei sono le Jrealpi del Trentino, che, staceandosi lumgo l'Alige colle Dolomili e coi monti Lessini, seendono a lorma di sperone fino a Terona, dividono la pianura della Lombardia dal Veneto e contimuando nei monli Berici e nei rolli Euganci, sembrano voler tendere incontro all'Istria. Tra il Vencto e il Friuli la separazione geografica é poco marcala e consiste solamente in un conline idrografiero.

Sullo specehio del mare questi tentalivi di riavicinamenlo tra i due versanti opposti, convergenti, sono ancora più evidenti. La penisola dell'Istria si prolende col suo dorso montuoso principale direttamente verso Ancona, dove gli Apemini fanmo gomito nell'Adriatico. Pola ed Ancona potrebbero dirsi i cardini idcali di quella grande porta, che olle a segnare il confine geografico orienlalle d'Italia sul mare, marea il limite di una, per noi ipotetica, contimuazione d'inferramento della Padania. Questa porta poi congiunge il bacino setlentrionale detI'Adriatico o golfo di Venezia coll'Adrialico di mezzo.

Dalla linea Ravema-Pola l'Adriatico si estende ampio verso sud-est. finchè penisole ed isole datla costa dalmata - Sabbioncello, Curzola, Lesina. Lissa - lo sperone del Gargano colle appendici delle Tremiti, di Pianosa e di Pelagosa dall'Apemino, non lo restringono per la seconda volta, cuass nella sua metà r lamno da controporte ad un nuovo limite geografico, chebbe la sua espressione storica nel cosidetto Mare nostrum e nella Dalmazia renela. Qui Lissa fu detta e ritenuta per molto tempo la chiare o la Malla dell'Adriatico.

Osservando allentamente le due coste in questo tratto scmbra di redere che la cassica dopo Sebenico (Punta Planca) si sia gentimente ritirata per far posto al gomito legli Apennini nel mare e non pregiudicarne lampieza, mat ehe poi per afferione abbia lanciato incontro al frattello Apemnino le sue isole. I gruppi Brazza-Solta-Zirona in alto, Lesina-Lissa-Pomo, Sabbioncello-Curzola nel mezzo, Meleda-Lagosta-Cazziol " Cazza in basso, corrono in direzione duasi nella di est-ovest. Perfino Pelagosa (la 
più grande dei seclici seogli che compongono il gruppo? sta colla sua lunghezza (1400 m.) - mentre la larghezza non ha che poco più di $300 \mathrm{~m}$. - nella direzione da est ad ovest. quasi che volesse piutlosto che sharrare il passaggio, stendere nella sua modestia le braceia per unire le lue coste.

L'ultimo e piò signilicante restringimento è fucllo che la natura hat posto allo sboceo o allentrata delldodrialico fra Otranto e la penisola degli Acoocerami (capo) Linguetla .

La nalura dunque ha segnato non solo i confini per una demareazione regionale della teraterma di guesto immenso arvallamento, ma ne ha diviso anche il mare in tre bacini, aperti e congiunti ncllo slesso lompo, però bene distinti tra loro.

\section{TRE BACINI DELLA ADRIATICO.}

Il grande gollo schtentrionale è caralterizzalo dal pas- Il settentriosaggio che la lerralerma la nel mare. Lal massima parte male. della sua costa da l) uino a Cervia o dall'tsonzo al Savio non i altro dhe an immenso della delle alecpue che seendono dalle Mpi, sempre arescente per alluvione, pantanoso, malsano, stavoperole - meno che in singoli punti alla vila, alla narvigazione, allo sviluppo della civilla. Ad oriente gli rimane lollima costa istriana lino a Pola e ad occidente il litorale emiliano-maredigiano da Riminj ad Ancollal.

Questo gollo per atuse maturali, che non richiedono spiegazione. oggi es semsibilmente rimpiecolito in conlironto al tempi. di cui la storia de ha lamandalo notizia: o basta ricoredare che Mepuileja. Ravemna. Aelria semza nomi-

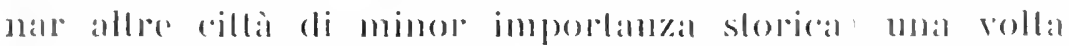

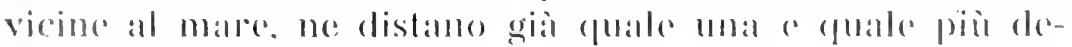
rine di rhilometri. Rimini pure una volla al marre sta-

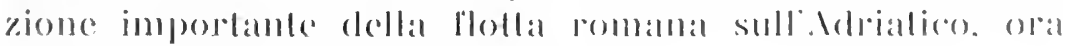

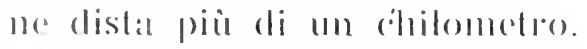

Il Po is stato il fallore principante di questal lental ma 
continua usurpazione della lerar sul mare. Secondo il hechus ${ }^{1}$ ) il Po è il piǹ atlivo di tutti i fimmi lavoratori del bacino del Mediterranco e se continuasse il suo atluale progresso gli basterebhero altri mille anni per formare attraverso P'Adriatico una penisola larga 10 chilometri, che andrebbe a locarre le spiagge opposte dell'Istria.

Ma non volento affaticarsi nell'acuire il cervello per vedere l'epoca in cui potrebbe arverarsi la realizzazione (li queslo calcolo, basterà che il letlore si ponga idealmente su tura vetta delle Mpi del Cardore o della Camia e guardi la pianura e il mare che si stendono ai suoi piedi, perchè si persuada che, come ma volta il mare potrebbe aver quasi lambito gli ullimi contrafforti delle Nlpi, così potrebbe pure venire un giorno in cui $i$ vapori non si novessero piì nel porto di Trieste e che Caporlistria direnisse ma cilladina della piamma lriulana o veneta.

Il medio. Il bacino di mezzo da Ancona (monte Conero) al Gargano (monte Sant'Angelo) e da Pola (capo Promontore) all'isola di Meleda è il più bello e il più l'avorito dalla natura. La costa apenninica $\dot{e}$, è vero, soffocata. I detriti alluvionali che te seendono dal proprio rersinte, indi quelli che provengono dal bacino superiore, principalmente dal Po, e in fine la sabbia che la corrente marina ascendente nell'Adriatico lungo la costa orientale le trasporta nel giro disecndente che la dopo la bipartizione all'altezza di Premuda verso Ancona, ha ostruiscono. Essa è però in ogni caso più salubre della reneta e non manca di piccoli porti.

La parte carsica poi è ricea ad esuberanza di isole, che proleggono la costa in più linee parallele ed è fortmata di avere nella Dalmazia un triangolo di terra, che dalla catena del Velebit (l'antico Bebio: e del Dinara secende digradando al mare e costituisce un terrilorio non spregerole per la vila sociale.

L'inferiore. Il bacino inferiore dal Gargano ad Otranto e da Meledal al capo Linguetta baja thi Vallona) è il meno felice,

1) Makissi, Ceografia (Hoepli), dal quale sono presi questi ed altri dati. 
perchè sconvolgimenti tellurici lo privarono nel corso dei secoli di quelle isole che probabilmente esistettero una volta lungo la costa orientale. Eecettuate le Bocche di Cattaro e di Irrindisi esso manca di buoni porti e le sue coste da ambe le parti sono o magre, o ripide, o paludose.

\section{I GOSTA.}

Se questo studio si limitasse a considerazioni puramente di geografia descrittiva, per costa dell'Adriatico non si potrebbe intendere più di quella striscia di terra, che per alcune diecine di chilometri si estende dalla linea del mare verso i piedi dei monti. Volendosi però in seguito trare delle deduzioni storiche, occorre allargare questo concetto fino a fargli abbracciare tutto il tratto accidentato che va dal mare ai monti, giacchè questo tratto e non la sola striscia costiera è la scena su cui si svolsero gli avrenimenti del progresso umano. Anzi a questo scopo sarà necessario di portare alcune volte il valore storico della costa più in alto ancora, fino guasi alla linea dello spartiacque sulle creste dei monti.

Questo spostanento artiliciale del concelto di costa può passare per l'Adriatico senza difficoltà, dappoichè le pareti che racchiudono il bacino sono ripide dappertutto e il tratto di terra pianeggiante tra i monti e il mare, meno che per la Patania, è relativamente breve tamto sul versante apenninico che sul carsico.

Inlatti per quel tratto dell'Apennino che va da Otranto tispenninica. ad Ancona non è difficile chiamare col nome di costa quasi tutto il versante orientale della montagna. Questo versante preso complessivamente è abbastanza unilorme, tanto che l'italica in questo tratto si può dire sia una costa sola.

I'Apennino proprio ha da cuesta parte per un lungo tratto j fianchi che s'imnalzano bruscamente su dal paese collinoso, ma poi continuano con pendenzal dolece. Esso è earalterizatlo orogralicamente dalla presenza di numerose valli traversali, corte, ma parallele, e disposte con 
bella simmetria, dhe lamno angolo reflo colla rosla. I fiumi hamo per conseguenza per la maggiol parte il più breve corso possibile, sorente con aspello lorrentizio, poichè dállo spartiacefue secudono direltamente al mare. Il terreno eatrareo di questa costa viene corroso e devaslate dalle acque, che lo rendono disgregabile es sinlillratno nel sottostolo. Per (ii) onde salvarsi dagli impeti subitanei delle acyue (" per l'intertanento dei porti, la maggior parte delle ciltà di questa costa non sorge vicino al mare, ma sul pendio del monte a parecedie chibómetri nellinterno.

La Padanica. Anche il trallo di mezzo della costa adrialical al nord di Anconar o particolarmente quello che va da Cattolica a Duino. che nel suo complesso, meno l'estremilà inleriore a mezzogiopno delle lagune di Comacehio, si può dire creato dalte Alpi, é, per quanto dissimite di forma dal precedente, monolono. A causa clella sua unilormila e per semplificazione di termini molto spesso sarà indicalo col nome di Padania lutto questo tratto. che forse dovrebbe essere delto più propriamente Alpino, perchè la Padiania come late cessal presso le foei dell Arlige.

La costa della l'adania, cosidella duncfue, differisce da quella dell Apennino, che corre quasi dirillal da sud-est a nord-ovest, per la piegatura at alreo, ed è caralterizzalat oruncue da lereno alluvionale, basso, piallo e da lagune arequitrinose e malariche. Tale è tulta la striscia che geograllicamente costiluisce la costa. Però questa costa non ¿ altro che il passaggio lento e marcalo o la concuista della lerra prolungalasi dalle falde dei monti nel mare.

Siccome la profondita di questa pianma ì rilevante, non si può estendere a tutlo fuesto terreno il concelto di costa. Però il passaggio geografico della terra nel mare congiunto alle molte linee açuee fluviali della pianura. ( ai molli suoi laghi, alpini che rappresentano un golfo lra terra dietro al bacino det mare, crea, malgrado l'insalubrita e inaceessibilita della linca costiera, un nesso economico della massima imporlanza lral la Parlania e il golfo selfentrionale dell Idriatico.

La Carsica. Con Dnino cessimo e l'uniformità e la monotonia dei 
due tratli di costa italica. Sembrerebbe che la natura, stanca di troppa simmetria e semplicità, abbia roluto ricompensarsi sulla costa carsica col più bizzarro disordinc. Questa è infatti lanto frastagliata, irregolare e piena di anfrattuosita da perdere in alcuni punti apparentemente perfino la sua continuilà. Ne sono causa $i$ monti del Carso, che invece di formare un dorso compalto. alto e distinto, come le Alpi o almeno gli Apennini, corrono in catene disciolte, ora alte, ora basse, a pezzi, ora grandi ed ora piecoli, che incerti nel loro principio alla congiunzione colle Alpi, si disperdono confusamente nella penisola balcanica.

Chi guarda questa costa a Duino dal mare vede disegnarsi subito una cresta che, passando al di sopra di Trieste, come attraverso I'Istria, e dal Monte Maggiore continua sulle isole di Cherso, Lụssin, Premuda, Lunga. Incoronata e Zuri, ove piega verso la terraferma.

Ma questa non è che ma linea esterna o catena secondaria, come secondaria è anche fuella che segue, o mediana, la quale passando per le isole di Veglia, Arbe e Pago, continua nella terraferma della Dalmazia, per acequistare gualche rilevanzal appena alla fine, dopo mal fusione colla linea esterna, nelle montagne del Mossor e del Biocovo.

La catena carsica principale, perehè si collega diretlamente alle Alpi, si eleval con masse alte e compalle e forma - ciò che è più importante - spartiaceque lra il deflusso dei liumi c lorreuli nell' Aelriatico, è fuella che. dopo qualche incertezal al nored dell'Istria, si delinca nellamente colle due monlagne maggiori del Velebit e del Dinara. Quest'ultina montagna linisce arvicinandosi e rimendosi in parte al Biocovo, dhe rappresenta quindi il punto di ricongiungimento delle tre catene carsiche e principali al di qua dello spartiacque. Però a scompiglio della geografia anche questo monte viene troppo presto interrotto dalla valle del Nalrental.

Questo fiume costituisce uno dè piò bizzarri escmpi dei capricei della nalura per conlondere le regole ches-

La valle del Narenta.

sa slessal delte allal geografial. Naser ben addellero in 
terraferma; corre dapprima in direzione da sud-est verso nord-ovest, poi dopo una ripiegatura ritorna, arricinandosi al mare, in direzione di nord-nord-ovest rerso sudsud-est, per sfogarsi alline in direzione di cuasi nord-est sud-orest nell'Adriatico. E nivigabile solo in un piccolo tratto alla foce. Il fiume ì lormato dalle acque di un territorio carsico, montuoso. (listinto, che oggi si chiama Erzegovina, la quaíe per conseguenza senza avere nulla di marino, viene ad appartenere idrograficamente al bacino dell'Adriatico. LErzegovina non ha nulla di comune col mare, perchè una catena accidentata di piceole montagne senza nome speciale, che incominciano subito dalla sponda sinistra del Narenta, formano costa, confine e separazione tra lei e l'Adriatico. Questa catena costiera che corre con pendio ripido, sempre nella direzione di nord-orest sud-est, parallela alla linea dell'Adriatico e degli Apennini. è considerata dai geografi come l'ultimo brano del Carso, che vien fatto finire nell'Orien, alto $1895 \mathrm{~m}$. oggi confine tra la Dalmazia, il Montenegro e l'Erzegovina .

I] Montenegro.

Suddivisioni e tratti.
A questa retta si comnette il nodo montuoso speciale del Montenegro (una transazione tra il Carso e i Balcani) che appartiene pure idrograficamente all'Adriatico, in parte per i fimmicelli che dopo il lago di Scutari sboccano in questo mare col nome di Bojana e in parte in grazia a quella speciale insenatura marina, che sono le Bocche di Caltaro.

Colla punta Menders presso Dulcigno termina la costa montuosa. I monti successivi, che si famno appartenere ormai al sistema balcanico, si sono ritirati (per modo di dire) di una linea più all'interno ed hamno collocato innanzi a sè fino al mare la pianura albanese, con costa bassa, uniforme, paludosa e malsana.

Agli Acrocerauni linisce la costa che si può dire adriatica e incomincia quella del Jonio.

Dopo questa esposizione, un po' più dettagliata, ma necessaria per il buon intendimento di tutte quelle considerazioni, che verrammo srolte in seguito, si rede che sarebbe un errore il voler far coincidere anche da que- 
sta parte il concetto geografico e storico di costal in linea generale collo spartiacque dei monti, che nel Carrso sono così capricciosi da rendere difficile di traceialle la linea di separazione tra l'Adriatico e il Mar Nero. D)a questa parte bisogna invece tistingucere e considerare separatamente i singoli tratti. Sarebbero essi sei.

Il primo sarebbe quello da Duino ai monti dei Cieci. Il valore geografico della costa in questo tralto si limila al versante dei monti visibili dal mare: quello storico invece (litorale di Trieste) deve venir trasportato più in là fino quasi alla linea dello spartiacefue.

Nel seconclo tratto o litorale croato (dal gollo di Finme al fiume Zermagna) ore la cresta del monte Velebil segna nettamente lo sparliacefue geogralico c la separazione storica tra il mare e la terra, i due concelli coincidono. La costa qui si limita in ogni senso al ripirlo pendio dei monti.

La coincidenza di concelti continua nel terzo tralto per i monti Dinara e Biocovo lino al Narenta. colla correzione per la geografia che si acectlino come costa lanto te isole, che il vertice dell'angolo di terra dilmata, che s'insinua nell'interno fino alla congiunzione det Dinara col Velebit.

Il tratlo seguente dalla spondal sinistral del Narenta

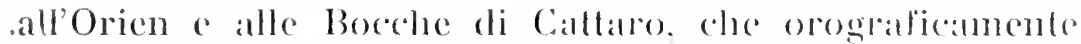
forma l'Erzegovina, poggial sulf Mclriatico solo per legge idrografiea, ma non si puo dire che gli appartenga come -costa, nè per geograllia, nè per storia. La costal geogratlica e storica repubblical di Ragusal i ristrella anche equi al pendio, sempre ripielo, della catenta coterna dei monti.

La stessa rosal si puo dire del quiuto trallo. delle Bocehe di Cattaro col nodo orogralice montenegrino. (doc riversa le sue acepue in parle nel bacino del I)anubio a

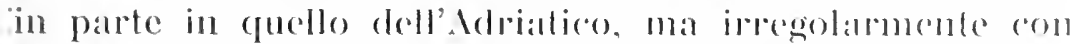
un giro vizioso nella regione della piamura dell Mlbamia.

Per questa pianurar ehe lormal il sesto e ullimo tratlo. si potrebbe ripetere, con qualdere valrinte beninleso, il

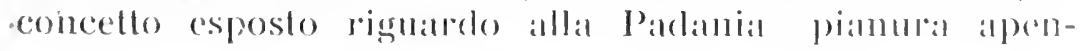


nino-alpina). - Tulte le aceque dei monti seendono allo stesso mare, con corso abbastanza lumgo, trasportano deIriti alluvionali che ingombrano la costa e formano delta bassi, paludosi, malsani. Tulto il bassopiano e il versaute dei monti rappresentano molto bene la costa, che se anche è distinta dalla earsica, perchè è innestata nella penisola balcanica, apparticne indubbiamente per geografia, se anche non veramente per storia, al bacino dell'Adriatico.

Riassumendo i concelti, risultat che la linea costiera in senso lato, rhe nasce in due pumti, uno esterno a Duino e uno interno sul Ionte Re Nanos), dopo il conginngimento presso Fiume, come lungo le ereste dei monti Velebit, Dinara e Biocovo, Laglia la foce del Narenta, prosegue lungo il mare fino allorien, fa un areo per comprendere le Bocche di Catlaro e continua lungo i monti dell' Mbania fino alla baja di Vallona.

Questa sarebbe in senso lato e per brevità di linguaggio la costa carsica.

Malgrado le molte irregolarità esiste dumpue ed è riconoscibile anche per il versante orientale una linea di spartiacque, e con ciò viene tolto ogni valore all'apparente mancanza di collegamento nel contorno generale del bacino adriatico.

\section{LE DUE GOSTE.}

Nel linguaggio comune si odono nominare spesso "le due roste dell'Adriatico».

In profano di buon senso potrebbe obbietlare che veramente non ce n'è che ma sola; il geologo ne dislingue qualtro; il geograto che paragoni l'Adriatico ad un grandissimo fiume, due, e lo storico che voglia distinguere gli arrenimenti, che non furono sempre uguali dall'una a dallatla parte del mare, parimenti due.

Lal distinzione di due coste è determinata quindi più che altro da necessilà pratiche; ma presenta anch'essa 
mal grave diflicoltà, funclla di fissare il punto da cui le duc coste si dipartono.

Il geogratio e lo storico potrebbero accordarsi nel cercare questo punto in qualche segno nalurale al passaggio (lalla costa alpina alla calsica, p. e., al Dnino o al11 confine orientale l'lsonzo, già nominali, o magari all'Aussa. Ma ci sono dei seri scrupoli per tulti duc.

Il geograto sa molto bene che il conline d'Italia sta molto più in là ed anche lo storico non può dissentire da questal opinione.

Prima però di proceclere nelle considerazioni sarà opporluno aprire mal piccolissima parentesi. Nei nostri pases cer lempi che comono il parlare di comlini d'Italia o mo impresa assai scabrosa, perelè si corre pericolo di essere l'raintesi.

Per (iò. onde prevenime eventuali malinlesi e censure,

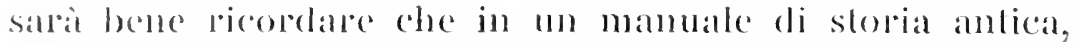
che lesto di sludio nelle semole dell Austria, i conlini d'llalia sono portati al oriente fino al fimmicello Arsa nell'Isliai.

La geogralia insegna però che i limmi, anzichè dividere, mniscono gli abilanti drlle loro sponde. Ln limmicello insignilicante conte l'Mrsa non può inoltre segnare conline lina popoli, e per gli Slati non potreble costibure cle un limile assai primilivo e di breve dorala.

feografial e storia insegnano pintlosto che i monti, specialmente se alli e inlransitalnili, formano i conlini

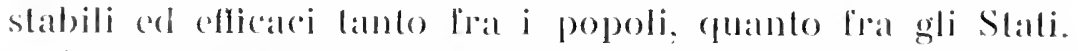

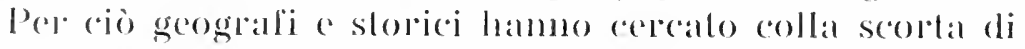
segni malmali esteriori e semza badare eschusivamente agl’inscognamenti sollerranci dellat geologia di lissalre rsilt-

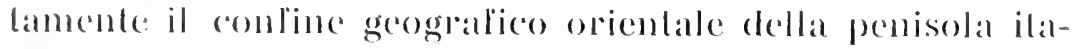
licar. latcenctolo coincidere, molto assemnatamente, collo spartiarefue dei monti. Del resto nelleda di mezor gli Areiduchi d'Austria indioanano i loro paresi alpini al di là dello (iimlie eol nome di comlini italiani.

bopo gli studi di (iiovami Marimelli e le dimostra-

1) (Quello del prof. Cindely di Praga, tradotto dallo Strafforello. 
zioni rigorose del Tamarelli la più logica delimitazione del contine orientale d'Italia sarobbe quella linea che, partendo dal Monte Re (Nanos, passa ad occidente di San Pictro nel Cragno, abbraceia le valli del Recea carsico e del Recina fiumano e raggiunge il mare ả levante di Fiume fra il colle di Tersatto e il porto di Buccari.

Ton tutli però converrebhero nell'idea di far incominciare appena da Portorè quella costa, ch'è detta comunemente l'orientale.

Il titolo di un libro di feelele descrizione di paesi che si specchiano su questo mine, L'Adriatico orientale da Venesia u Corfü, del dottor G. Marcotti. suggerisce tun luogo clue forse sarà accettato dai più: Venezia.

Venezia. Questa città giace inliatti quasi nella metà dell'arco costiero formato dagli Apennini e dalle Alpi tra Ravenna e lo Sdobba: è lo sbocco naturale. lo scalo economico della Padania, lanello di congiunzione tra la terra ed il mare di uno stesso bacino: e fu appunto in grazia della sua posizione geografica la dominatrice dell Adriatico per ollo o nove secoli.

\section{TALORE ECONOMICO E SOCIALE DELLA COSTA.}

L'Apenninica. Tncominciamo di nuovo coll'Apenninica. Esso non è eguale orunque, ma bisogna distinguere a tratli. Distingueremo quindi partendo dalla parte meridionale:

La Puglia. Questo i nella costa occidentale il tratto più farorito dalla natura. perchè insieme colla Capitanata, che le sta a settentrione, da in certo modo agio all uomo di muoversi un poco liberamente. La Puglia va però distinta in due parti: la litoranca, piana, fertile, sebbene un po' arida, popolata, collivata intensivamente. ricea come poche altre zone agricole d'Italia, deusa di abitanti - e accauto a questa una Puglia più interna. alta, priva di risorse. spopolatia. La causa di tale squilibrio è la formazione del tereno; alla costa c'è polpa di terra e ci sono bunni porti, in ognuno dei quali s'è formata per 
conseguenza una cittì. Da Brindisi a Barletta se ne contano otto maggiori, tra cui Bari, che hanno doppia importanza, come porti e come scalo di altri centri agricoli più interni, che stamno in seconda linea.

Viene quindi la Capitanata o provincia di Foggia, delta La Capitanata. nella sua parte meridionale Taroliere di Puglia, per lo più piana, ma con costa bassa, piena di acquitrini, senza buoni porti e ostruita invece da lagune miasmatiche Lesina e Varano. lago Salso e di Salpi). E naturale quindi che sia economicamente inferiore alla prima.

E notevole in essa più che per altro forse per la navigazione il monte Gargano, che orograficamente può essere considerato stante da sè, quantunque un legamento soltile lo allacei all'estrema ondulazione detl'Apemino. $\mathrm{E}$ esso un altipiano, che ha qualche analogia col, Carso ed è senza grandi risorse naturali.

Le due lagune di Lesina e di Varano erano una rolta due seni aperti: ma i cletriti del fimme Fortore. le correnti, l'ondulazione marina vi edificarono lidi e dune quasi segregandoli dal mare. Per ciò se vi si formarono due buoni virai di pesee, la regione c̀ anche un tremenclo fomite di malaria. Per queste ragioni le citla principali sorgono in questo tratto molto all'interno Cerignola, Foggia, San Severo, e questo ì il tratto più infelice della costa Apenninica.

Il litorale successivo, molisano e abbruzzese. che val dal Fortore al Tronto, scbbene non sia nemmeno esso, causa il bruseo innalyamento del paese collinoso dalla sponda del mare, particolarmente adatlo nè allat navigazione, nè allindustria, conta però dei porti discreti Vasto, Ortona, Francavilla, Pescara). Le cilta principali (Chieti, Teramo vi sorgono alquanto all'interno ('alcune nemmeno immediatamente sulle rive, ma in loro prossimità.

Migliore' è il tratto marchigiano dal Tronto a Callolica, perchè il pendio più dolce e la maggion distanzal II Marchigianto. dalla riva alle creste dei monti permette che vi si sviluppino modestamente agricoltura, industria. cillit. Al matre éè il porto, per quella costa noterole; di lurona e

Il litorale molisano e abbruzzese. 
nell'interno ci sono parecehie citlà rimarchevoli Ascoli, Camerino, Macerata, Urbino). In complesso questi due tralli dal Molisano alle Marche hamo caralteristiche abbastanza consimili, per eni in confronto ai meridionali ed a quello che riene al settentrione si possono considerare come aventi un tipo unico.

L'Emiliano. L'emiliano da Rimini alle valli di Comacchio cambia aspetto e va avicinandosi gradalamente al genere lagmare, caratteristico della Padania e della Venezia. La costa non è ancora melmosa, ma i banchi sabbiosi che continuano per un lungo tratto solto il pelo dellacerua, la rendono più adatla alla pesca e ai bagni di marce, che ad un'attivilà dei porti. Una tale costa è veramente disadatta alla grande navigazione, perchè i banchi di sabbia tengono le navi lontane da tera: la mancanza di porti e di isole le priva di un rifugio in caso di burrasea, e la linea dclla terra uniforme loglie l'orientamento pratico. Tutti questi fatti sono purtroppo ostacoli allo stabilirsi di buoni rapporti tra l'uomo e il mare. La zona costicra poi non è vasta da costituire una regione agricola di primo ordine, ma non è nemmeno angusta da imperlire ogni movimento. La terra non d̀ in complesso nè ricca, nè fertile ed è in alcuni punti selvaggia; dà però da vivere se è lavorata. Per ciò eccita gli abitanti alla lolla colla natura e all'attivitì. L'aria marina ed i renti caldi del sud farebbero forse inclinare la popolazione alla mollezza. Però da questo pericolo la salva in parte la posizione geografica, rivolta ad oriente, da cui proviene ma temperatura più bassa causata dall'ombra degli alti monti che la coprono ad occidente. Per questo motivo questa costa ha anche minor pioggia. I venti di sirocero che la porlano dall'Africa nell'Adriatico come nel Mediterraneo, soffiano in direzione da sud verso nord e conducono le nubi ad infrangersi contro l'altro versante dell'Apennino e contro la costa orientale dell'Adriatico. Un tale ambiente geografico ì quanto mai adalto allo sviluppo dei sentimenti estetici. Infatti in esso troviamo centri celcbri, come Bologna, detta la dotta per la sua civiltà (possiede la più antica università del mondo - 1119) e la grassa 
per la sua fertilità: fu la patria del "dolec slil nuovo" e diede i natali a Galvani, a Reni, ai Caraced, cec.; indi Urbino patria del grande Raffaello, Fermignano lì presso del Bramante, Pesaro di Rossini, Recanati del Leopardi, le Alfonsine di Romagna del Monti, Busseto di Giuseppe Verdi, Faenza di Torricelli, Reggio dell'triosto, ece, ece. Il benessere economico di media intensità si palesa socialmente nello sviluppo demografico. Sulla costa apenninica non vi sono empori immensi, ma pullulano dappertulto ciltà e cittadine, linde, formatesi e cresciute per sviluppo naturale e costante nel corso dei secoli, che sono il segnacolo della ciriltà del paese. Citeremo: Bologna. Ferrara (che nel medio evo raggiunse fin 100000 abitanti), Modena, Reggio e Parma e poi lungo la linea ferroviaria Imola, Faenza, Forli, Cesena.

La Padania, che come riva va dal Reno all'Alige o La Padania. dal Savio all'sonzo, ha $u$ valore rhe reramente dovrebbe essere indicato come nullo, nel rimanente in terraferma ha un'importanza di prim'ordine. Per ciò a primo colpo d'ocehio si vede che fuesta è la parte più cospicua del bacino adriatico sollo ogni aspetlo. Ampia, profonda, ricea di lerra fertile e di acepue abbondanti, attraversala in tulli i punti da vie navigabili almeno in parte, densa di popolazione, carica di cillà, piena d’industria e di lavoro, essa costituisce la regione principale d'Italia ed mala delle pianme più importanti d'Enropa.

Occorre però rilevare che la Palania come si prescnta oggi lu nolevolmente migliorala dalla mano dell'uomo, pereluè la matura l'areval latla quasi inospitale. Il tereno per essere fallo di alluvione non solo allodierna riva del mare, ma anche mollo più all'interno, era in antico ingombro di detriti a non frultifero dappertullo; i fiumi correvano acedelentalj fra rive malsiene e confuse, allraversando lratli impaludali e malsami la superfice rimanente ela coperta da boschi intriali. Ai tempi dei Romani lutla la parte marillima della Parlaniat era rivestita da un'immensa pincela, di cui ogggi non sopravive ele il piccolo tratlo di Ravema. La via Acmilia che nel tralto da Bologna per Pandova conducera and 
Aquileja quasi rasente la costa, attraversava un territorio acquitrinoso, boschivo, difficilmente accessibile, e costituiva una strada militare così bene munita dalla natura che gli strateghi averano bisogno solo di poche opere fortificatoric artificiali a grandi distanzc.

Ma la mano dell'nomo in tanti secoli ha abbattuto i boschi, dissodato il terreno, che fu reso fertile dai raggi del sole, ha regolato i fiumi in buona parte, ha creato canali artificiali, fondato città, accumulato ricchezze. Oggi la Padania ha un alto grado di civiltà, ma non ha raggiunto ancora quel limite di perfezionamento economico di cui è certamente capace. Il genio immortale di Leonardo da Vinci concepì negli albori del secolo XVI un piano di canalizzazione, che fu incominciato, ma rimase interrotlo, perchè la potenza economica ed i mezzi tecnici di quell'epoca crano impari alla vastità dell'idea. Quel progetto però vive ancora tra i compiti ereditati dai secoli che seguirono ed attende la realizzazione dalle rinnovate energie italiche. E ognuno che ani l'Italia non può far a meno di angurare che il grandioso e monmmentale lavoro della conginuzione di Tilano col mare sia compiuto quanto prima per it bene del paese e per la gloria della civiltà.

Per il valore economico e sociale della Padania, che sarebbe immensamente maggiore se fosse concepibile al suo sbocco una costa regolare, solida, pianeggiante, favorita da bei porti, è pregiudicato invece dalla presenza di troppi delta, di lagune, di banchi di melma, che rendono la costa inospitale e inaccessibile. Quest'ostacolo, col quale la natura ha danneggiato araramente il benefizio della pianura, è così forte e costante, che gli nomini iardi, a stento e con grandi cure sono riusciti a formare nella maggior laguna lo scalo ed il centro economico di Venezia.

La mancanza di quell'accordo inlimo, di quell'armonia fra terra e mare, che se fossero esistiti, sarebbero stati lecondi dei migliori frutti, non potè d'altro canto impedire che la forza economica della Padania si spandesse e s'imponesse sul mare. E appenachè a Venezia si 
raccolse l'energia materiale e sociale prodoltasi nel suo retroterra fu inevitabile che questa città tentasse di divenire la dominatrice dell'Adriatico.

Tale impulso fu facilitato appunto dal ripiego della navigazione in alto mare, che la mente umana seppe trovare al danno di quella costa inospitale e impraticabile.

La popolazione della Padania è agricola fin là dove la terra tollera di essere lavorata. Ma nella zona costiera, per metà sott'acqua, uniche occupazioni e atlività dell'uomo sono la pesea e la navigazione, da esereitarsi forse più nei mari lontani che nei vicini. Si sa che una costa melmosa e senza porli non attira i naviganti, giacchè dove il mare è insidioso e dove gli sbocchi non hamno nemmeno un segnacolo naturale, i pericoli consigliano di tenersi al largo. Gli stessi abitatori delle lagune poi sono portáti per forza naturale a navigare via dalla costa. L'acqua corrente dei fiumi stacea le loro barche da terra, ma per fortuma non le porta molto lontano. L'Istria piena di otlimi porti e verdeggiante verso il mare è di fronte. Questa penisola sembra destinata dalla natura a completare le risorse naturali ed a rappresentare il giardino d'oltre mare degli abitatori della zona costiera padana. Una volta raggiunta la costa dell'Istria la navigazione ulteriore lungo lat costa dahmata è una cosa che s'impone da sè.

Ma ritornando alla costa e al retroterra della Padania, c'è da notare ch'essi hamno ancora un vantaggio: quello di ma minor siccitì che nel resto dell'Adrialico. Altrove le terie offrono inulibmente le loro arse zolle al contallo delle nubi, che, portate furiosamente dai venti di mezzogiomo, passano sopra e non ne inumidiscono rhe la superlice. Qui invece essendo sbattute contro l'alta ("attena delle $\mathrm{Al}_{\mathrm{p}}$, vi s'infrangono e convertono in pioggia. I monti stessi poi lorniscono alla pianura piogge e temporali. E vero d'altronde che fuando si seatemano bufere (e non sono rare purtroppo) l'atmoslera, stracarical di umidià, più che bene la dammo.

Inline gioverì ricordare che la Padania, cinta da alli monti e relativamente lontana dal nuare, ha mu climal 
continentale. Ciò salva i suoi abitanti dalla mollezza e se 1 distoglie dai godimenti puramente estetici di ma vila contemplativa, li stimola benelicamente all'atlività e li la laboriosi.

La natura chinse la Padania in un recinto di monli e di lagune difficilmente sormontabile, specialmente dall'interno all'esterno. Questa cintura impedì l'espansione territoriale dei popoli di questo bacino tult'all'intorno, ma contribui anche alla fusione di tutti gl'immigrati. Senza ie Alpi i Tedeschi arrebbero allagato anche l'Adriatico, e gl'Italiani devono essere grati a questa montagna perchè se non altro li aiutò a conservarselo.

La segregazione a causa delle Alpi arrebbe potulo essere damosa allo sviluppo della civiltà nella Padania, se l'ampiezza della pianura non fosse stata più che sufficente da permettere un movimento alla civiltà indigena. In queste condizioni l'ancllo di monli e di lagune fu anzi vantaggioso, perchè le assicurò una físionomia propria, incancellabile, e le conlecì una bellezza naturale invidiabile da tulte quelle altre pianure sterminate, ma che per mancanza di confini presentano invece un aspetto di monotonia opprimente.

La Carsica. Come tale sarà inteso in questo capilolo quel tratlo che va da Duino alle Bocche di Cattaro.

Dovendosi passare gradatamente dallosservazione puramente descrittiva a considerazioni organiche, giora ricordare innanzi tutto che la costa carsica oggi è, a cuanto si può constatare, in più punti alquanto abbassata nel mare in confronto dell'epoca romana.

Nel vallone di Risino entro le Bocche di Caltaro (il sinus Rhizonicus dei Romani) (fuando il mare c̀ perfellamente tranquillo e il cielo limpido si vedono ad una prolondilì, relativamente grande, delle grosse linee regolari, biancheggianti, come se fossero mura di costruzioni.

Qualche cosa di simile i pescatori dicono di osservare presso Ragusarecchia nel luogo ore si crede che sia esistila l'antica Epidaurus.

Presso Salona vi sono delle tombe romane per una 
parte sott'acqua. Non essendo ammissibile un seppellimento nell'umidità, è evidente l'abhassamento della terra e la conseguente invasione del mare.

Anche sulla costa dell'Istria presso la Punta Barbarigo fra Rovigno e Fasana nei giorni di bonaccia si intravedono sottacqua i ruderi ragguarderoli della piccola città di Cissa, ricordata da Plinio.

Gli sconvolgimenti sismici, e dietro di loro il mare, hamo inghiottito e rubato molla polpa di terra clalla costa carsica. Oggi non rimangono che l'Istria e la Dalmazia, montuose però in gran parte anche loro, e quel poco di humus che si conservò negli arvallamenti tra le creste delle isole.

Non si dere però diar tulta la colpa di questa rapacità agli dei Vulcano e Nettuno.

It Carso è una composizione montuosa calcarea, poco resistente, che si disgrega anche solto l'azione del sole e delle acque. Per di più vi manca un nucleo compatlo che formi il dorso e il sostegno di tutto il sistema orografico, come nelle Alpi e negli Apennini. Esso si disperde in molte catene longitudinali, parallele, di cui alcune sono rilevanti, ma altre non ragginngono nemmeno l'altezza di vere montagne.

Era quindi, per così dirce, naturale che moli sismici anche di minore entila e senza il valore di avenimenti catastrofici, lacessero, magari inavertitamente, sfrondare, lendersi e staccarsi le minori catene ti m simile sistema, provocando la scomparsa della parte meno resistente, la terra.

Una conseguenza economica immediata ed evidente di una tale conformazione geogralica è la povertà, giacehè la terra lu ognora la fonte principale della ricchezza, e dove essa manea gli uomini possono arere le migliori qualita, ma non quella di render lertili i sassi.

In compenso hamo il mare in casa, per modo di dire.

Lungo questa costa il male penctra ben adelentro in ognuma delle innumerevoli anfrattuositi, tanto de si potrebbe dire che terra e mare sono ancrescinti l'mo nell'altro. 
La pesca e la navigazione furono quindi più che due occupazioni naturali della popolazione, una necessità : solo che se la prima potè e lovelte venir esercitata in patria per il sostentamento della vita, la seconda costringe la massa dei marinai ad esulare altrove per avere m reddito rimunerativo dalla loro atlività e capacità. Giacchè se la costa carsica possiede magnifici golli, baje, rade e porti, queste insenature sono per lo più morte perchè $\mathrm{i}$ monti le soffocano e tagliano dal retroterra.

I porti per essere attivi e divenir la sede di commerei e d'industrie vogliono un retroterra vasto e vallate ampie. L'attività e il progresso tecnico hamno bisogno prima di tutto di un centro naturale, che poi può divenire anche centro economico.

Tutto ciò manca purtroppo lungo la costa carsica, se si eccettui in un certo senso meno letterale il sinus Salonitanus (oggi golfo delle Castella presso Spalato) nel mezzo della Dalmazia.

Se dunque la costa è povera anche là dove, come nell'Istria e nella Dalmazia di mezzo, vi sono e porti e isole e diecine di chilometri di terra digradante, immaginarsi lo squallore di quei tratti dove la costa nuda scende a picco sul mare.

A questo proposito bisogna nolare ancora che in generalc i monti delle catene carsiche esterne lambite dal mare, anzichè discendervi digradando come fa per lo più l'Apennino, si rialzano proprio quando più si avicinano al mare e poi piombano a picco, presentando addirittura la spaccatura d'un fianco. Il pendio anche di molte isole (specialmente di quella denominata Lunga di fronte a Zara, della Brazza, di Lesina, di Meleda) non è rivolto verso occidente al mare, ma verso oricnte alla terra, segno che un tempo dovettero essere state unite col continente.

Questa formazione caratteristica è visibile in grande nel litorale croato. Soltanto alla forma speciale del Capella e del Velebit si deve se corsi d'acqua che hammo origine a pochi chilometri (in linea aerea) dallo spartiaccue ed a poca distanza dal mare vamno a sboccare 
nel Danubio ed appartengono per conseguenza stranamente al bacino immensamente lontano del mar Nero. Così formato ì inoltre il litorale di Trieste, indi quel tratto di costa che diremo dell'Erzegovina dalla penisola di Sabbioncello alla Puntadostro e la costa dalle Bocche di Cattaro avanti fino al capo Menders presso Dulcigno.

Infatti in questi tratti la civilta non ha potuto formare alcuu centro duraturo, perchè lo sviluppo demografico spontaneo è sempre paralizzato là dove una catena di monti si eleva diritta sulla costa. La nascita e la floridezza di Salona nell'antichità, di Ragusa nei tempi di mezzo, di Trieste e di Finme oggigiorno hanno cause speciali e transitorie, che saranno esaminate nella parte storica.

Il valore della costa carsica, già pregiudicato dagli svantaggi ora descritti, è diminuito ancora da un altro difetto della sua conformazione, già accennato, dalla presenza cioè di molte valli longitudinali, ehe le corrono parallele. Gli abitanti di queste valli, anche se esse giacciono poco lontano dal mare, non lo vedono, e, ammenochè la valle non abbia uno sbocco verso la costa alle estremità, per ginngervi devono superare l'ostacolo del monte. Si pongano diverse di fueste valli mua accanto all'altra, com'è appunto il caso in piccolo della Dalmizia e in grande di tulla questa costa, e si comprenderà lacilmente cuanta ¿̀ la resistenza opposta dalla natura alla discesa degli nomini dall'interno al mare e alla penetrazione della civiltà dalla costa allinterno.

Nelle valli strette non si formano centri urbani, ma soltanto villaggi per to più cli scarsa importanza. Valli di tate forma rendendo disagevoli le commnicazioni ostacolano la formazione di un eentro economico diredtivo. Alle valli brevi, streltissime, si deve inoltre lo sminuzzamento della proprietà e la dispersione delle genti in piecolissimi villaggi e minuscole citti.

Queste condizioni topograliche non sono un'eschusiva specialità della catena carsica e s'incontrano tanto netle Api che nell'Apennino. Là però una simile formazione appatiene meno alla regione che noi infendiamo per costa e poi lo svantaggio è compensato dalla vicinamza 
di centri benefici d'irradiazione economica e civile, come la Padania tra le Alpi e gli Apemnini settentrionali e la Toscana, il Lazio, la Campania dall'altro versante apenninico per il rimanente di questo.

La costa carsica invece non ha altro che barricre di monti per diecine di chilometri nell'interno del continente. Essa è abbandonata a sè stessa e non ha altro beneficio che il mare. E si sa che il mare quando tocea un paese felice, lo rende felicissimo, ma se bagna un paese infelice per aridità, non riesce a portargli che un vantaggio assai piccolo. Nella catena carsica poi bisogna rilerare che la sua zona marittima è molto più ristretta che sulla costa occidentale.

Come maggior srantaggio sociale per la costa carsica si deve aggiungere allo sminuzzamento delle valli, il frazionamento del mare. Le penisole e le isole hanno in genere molti minori vincoli naturali con i paesi vicini, che non le regioni continentali.

I dorsi di monte tra le valli e il mare tra le penisole e le isole hamno reso impossibile uno sviluppo omogeneo della vita sociale lụngo questa costa. Benchè il progresso abbia forti muscoli, l'azione di questi ostacoli dev'essere slata profonda e decisiva nel passato e si capisce come anche presentemente la civiltà deva progredire su questa costa più a rilento. che non sull'altra o particolarmente nella Padania.

La costa orientale dell'Adriatico è duncque frazionata, varia, senza un intimo nesso geologico e geografico. Senza dubbio c'è uma relazione causale fra questa particolarità geografica e la mancanza di nesso sociale e nazionale. Anzi forse più a questa causa geografica che all'influenza storica è da ascriversi la presenza di più nazionalità mai fuse. Questo frazionamento economico è aggravato ancora per quella reazione che talvolta le conseguenze esercitano sulla causa, dalla piaga insanabile della discordia e di numerosi partiti locali, formatisi non per sostenere un principio o un'idea, ma perchè capitanati ciascuno da una famiglia o da una persona amante di predominio. La visuale degl'interessi sociali in simili paesi non 
può andare più in là del limite raggiunto dall'occhio, e questo non può spaziare oltre il monte o lo scoglio, ai cui fianchi sta appollaiato il presuntuoso signorotto.

Così le poche e meschine città sulla costa orientale non sono sorte (salvo rare eccezioni) per il commercio, ma sono per lo più la conversione e il rimasuglio di luoghi strategici fortificati nei tempi remoti; e se qualche causa consimile recente non le rianima (come Pola p. c.) conducono vita stentata.

L'enmerazione delle miserie di questa costa non è ancora terminata. Il Carso è ancora più arido e poroso dell'Apennino. Si dice, e ci sono validi motivi per crederlo, che nei tempi antichi anche la costa orientale dell'Adriatico, come tutte le regioni di questo bacino, era ricoperta da boschi piniferi. E un fatto che il pino mariltimo vi alligna benissimo, ma è anche dolorosamente vero che di boschi vi sono assai poche tracee e che sarebbe vano di attendere dalla sola natura la rigenerazione della flora. La generazione avrenne spontanea una volta probabilmente in condizioni climatiche e meteorologiche troppo differenti dalle odierne. Certo è però che il rimboschimento artificiale, di cui tanto si discute per l'Apennino e per il Carso, non è un'impresa da pigmei. Sicchè anche la costa carsica, malgrado che i venti del mare le portino maggior quantitì di pioggia che all'Apennino, ridiviene asciutta subito poco tempo dopo il periodo di umidità, perchè l'acqua non traltenuta dalle piante o seende precipitosamente al mare o scomparisee nei meati calcarei sotterranei.

Ad inaridire la superfice del suolo contribuisee ancora un elemento: l'aria, che su questa cosla soffia dal continente verso il mare.

Il fenomeno dei venti freddi settentrionali che nell'Adrialico molestano solamente la costa orientale s assai facilmente spiegabile dal lato fisico. La catena carsica che nel versante oceidentale discende fino al livello del mare, nel versante orientale dopo un breve abbassamento, continua in altopiano montuoso. L'aria in queste regioni, per sè stesse più nordiche, si mantiene lresca e asciulla; 
quella del mare invece sotto l'azione solare si riscalda e inumidisce. E quando per la differenza di temperatura fra le due regioni confinanti si produce quel riversamento della fredda negli spazi rarelatti della calda, simile a grandi cascate aeriformi, di cui la natura ha bisogno per il ristabilimento del suo equilibrio, gli uomini e la terra sono percossi dalle sferzate gelide di quel vento poco simpatico, ch'è la bora. La violenza di questo vento, che arriva talvolta a superare tulto l'Adriatico fino all'altra costa, è tale da schiantare alberi e macigni nelle gole e da compromettere in certi punti la navigazione. E la bora che rende ancorá più brullo il pendio dei monti e inaridisce colla salsedine del pulviscolo marino ogni traccia di regetazione sui punti più esposti dei versanti interni di molte isole (Pago specialmente). I paesi che stamno dall'altro versante continentale, come la Carniola. la Croazia, la Bosnia, sono verdeggianti.

Nulla di simile può succedere alla costa occidentale, dove l'altro versante degli Apennini dópo di essersi indugiato in qualche vallata laterale, discende anch'esso, e dal lato di mezzogiorno, sino al mare.

La costa orientale, giova ripeterlo ancora una rolta, non ha benefizio maggiore che il mare, col quale s'accompagna tanto bene da offrirgli per la navigazione isole e porti di riparo contro la furia degli elementi e una costa marcatissima, che basterebbe da sola allorientamento del marinaio anche ad occhio nudo.

Pari alla natura del paese, mite e selvaggio nello stesso tempo, si appalesa l'indole dei suoi abitanti. Il contrasto sembra irreconciliabile, eppure il concetto non è esagerato. Gli abitanti della costa orientale hanno l'asprezza della loro terra e la dolcezza del mare e del clima. Sono focosi e violenti, ma si calmano presto; concepiscono grandi cose e non ne compiono che di assai piccole. Sono pigri, ma piuttosto che per indolenza congenita, per scoraggiamento nel vedere che il risultato non compensa i loro sforzi. E chi vuole tentar ia fortuna emigra e per lo più riesce. Non è raro il caso di persone che in patria si sarebbero inaridite e fuori invece 
hammo compiuto opere notevoli, raggiungendo posti cospicui, specialmente nella navigazione o nclle armi, com'è il caso di molti Dalmati e Albanesi. E qui non bisogna pensare solamente al provertio: "nemo propheta in patria». Giacchè non si deve confondere l'allività mal'inara di un paese, di un popolo o di una persona coll'altività dei suoi porti. Questi possono essere morti, ma la capaciti marittima degli abitatori di ma costa come l'orientale dell'Adriatico può svilupparsi anche in porti esteri. Un popolo anche con porti mediocri può arricchirsi sul mare facendo da vettore agli altri popoli, trasportanclo ciò con le proprie navi le loro merci e i viaggiatori. Ed in questo senso principalmente si deve intendere l'allività marinara degli abitanti della costa oricntale adriatica e la loro padronanza sul mare.

Riguardo a questa c’è poco da ággiungere per la geogratia. La costa c̀ bassa, lagliuzzala, paludosa per lo sboceo di molli fimmicelli provenienti dalle alte montagne con pochi porti sicuri (ecectluato Vallona e Durazzo piena di lagune nella parte meridionale, e precechuta in genere da mare immavigabile. Dalla riva non si vede (ap)pertutto il mare, perchè lunghe dune lo nascondono. La costa qua e là è imboscata, ma nella maggior parle dei tratti è damneggiata da lagune e laghetli paludosi. In altri piccoli tratli invece, come a San Giovanni di Medua, si drizza ripida. Da marzo ad aprile e da sellembre a dicembre piove, come del resto in tutto PMArialico, spesso ininterrothamente. Da ciò derivano inondazioni, che mettono sollacefua per parecehi mesi le parti basse della costa. Altra conseguenza delle piogge e delle inondazioni sono quelle pericolose lebbri, dhe desolano il paese nei mesi caldi e costringono gli abilanti a lasciar lo case in pianura e ad emigrare temporancamente nelle parti piu elevate. Inoltre sul mare la bola inluria diuverno: Io siroceo vi è più forte che nell'Adriatico settentrionale e d'estate il maistro rinlorzato dalla lunga corsa genlia le onde lino a pregindicare la navigazione.

Una simile costa 11011 può certamente arere mu valore sociale particolare. Se poi vi si aggiunge che il suo re- 
troterrà è nel genere montuoso quello che si può dare di più aspro, che l'orografia e l'idrografia in questo paese sono tanto capricciosi, che sebbene posseggano una fisonomia caratteristica, non si può dire che esista un'Albania geografica e nazionale nettamente segnata, si comprencle il motivo pel quale il popolo che l'abita potè conservare da oltre 2000 anmi tutte le principali sue caratteristiche di fierezza indomabile e refrattaria agli allettamenti della civiltà occidentale. Ma si spiega anche perchè del resto l'Albania, benchè, come si disse, sia compresa geograficamente nel bacino dell'Adriatico orientale, nel suo complesso e dal lato della civiltà è come se storicamente non appartenesse nemmeno all'Europa. Sperabilmente vi rientrerì nell'avvenire.

Raccordo tra i versanti omonimi.
Il valore economico e sociale di ogni versante montuoso, e per conseguenza anche di tutta la costa che forma oggetto dei nostri studi, dipende inoltre, diremmo in seconda linea, dal valore del versante opposto della stessa catena.

Un versante povero, ma mito ad una regione migliore dall'altra parte, acquista in valore perchè, o poco o molto, riceve sempre dei benefizi dall'essere accompagnato ad uno più ricco; come viceversa un versante che sia mediocre per sè stesso, ma unito ad uno più povero di lui, anzichè un aiuto, soffre da questo un pregiudizio, perchè deve sempre difendersi dalle rapacità della miseria vicina.

Faremo quindi una rapida considerazione riassuntiva sul valore dei tratti principali dei versanti adriatici in rapporto al loro raccordo coi paesi adiacenti dell'altro versante.

L'Apennino è senza dubbi o contrasti più felice nel versante tirrenico che nell'adriatico e ciò è un benefizio per quella parte che guarda sull'Adriatico.

Anche le Alpi hamno discesa più dolce e un'estesa infinitamente più grande di pianure e di terreno dalla parte di settentrione che nel Lombardo-Veneto. Il Rodano, il Reno ed i molti affluenti del Danubio, che hanno la sorgente nelle Alpi e che sono tra i migliori fiumi nell'eco- 
nomia dei popoli, corrono a fecondare le regioni esterne delle Alpi. C'è però una differenza tra questi e il Po. I primi beneficano regioni economicamente indipendenti dalle Alpi. Il Po invece e gli altri fiumi minori del Lombardo-Veneto, quasi fossero i più giovani ed i beniamini, le Alpi se li sono riservati esclusivamente per sè.

A causa dello spessore straordinario della catena alpina le relazioni tra la Padania e le pianure del Rodano, del Reno e del Damubio non poterono essere anticamente viraci. Oggi però che la tecnica dell'uomo riuscì a sulperare anche questa barriera, la Padania, sebbene sia più ristretta della Francia occidentale, della Germania meridionale e della pianura ungherese, risente un benefizio non indifferente, nè trascurabile dal transito di merci svizzere e germaniche, che tendono agli sbocchi del Tirreno e dell'Adriatico. Anche la Padania dunque risente qualche vantaggio dall'altro versante alpino.

Nella catena carsica esiste troppa differenza e troppo squilibrio tra i due versanti per fare un confronto scevro da obbiezioni. Certo è che sono meschini tulti due. Se il versante adriatico è povero, il balcanico non è ricco, perchè i molti fiumi che vi hammo origine sono piccoli, non navigabili e sboccano nell'ultima parte del Danubio. Malgrado ciò per lá sola geografia locale arrebbícro potuto aiutarsi vicenderolmente. Na causa la posizione dei Balcani rispetto at complesso delle regioni europee ed i capricei della storia, il versante balcanico fu infinitamente più infelice dell'adriatico e per ciò agì sempre a detrimento di quello che a noi interessa di studiare.

Anche da questo lato dunque la costa occidentale ì più favorita, perchè i versanti esterni l'avvantaggiarono, portandovi sempre nuovo contributo di beni civili ed economici, pur avendo da attraversare montagne dalle ereste molto alte.

Questo concelto della penetrazione della civilla allraverso le montagne ci conduce ora a fare delle consideratzioni sui valichi e passaggi. 


\section{VALICIII.}

Finora la costa fu considerata nei suoi tratti generali o preferentemente nelle sue parti principali. Occorre però penetrare collo studio anche nelle suddivisioni regionali minori, giacchè queste, ove sono componenti essenziali, servono a spiegare meglio la base geografica dei fatti civili o storici.

Per ottener questo scopo giorerà considerare anche i valichi, che, consistenclo in un abbassamento della cresta che contorna le montagne, segnano oltrechè la transazione da un sistema orografico, gruppo 'o catena all'altro, la strada per cui si mosse il flusso e riflusso umano. I valichi quindi potrebbero dirsi geograficamente confini negativi (in opposizione alle vette che agiscono come separazione positiva) giacchè di fatto congiungono i due versanti. In uno studio sintetico come questo non si potramo beninteso considerare che i principali.

Sull'Apennino.

Per voler incontrare un valico nel senso geografico della parola, vale a dire una strada naturale ben disegnata tra un versante e l'altro, bisognerebbe altendere di arrivare fino alla gola di Popoli, alta solamente $250 \mathrm{~m}$, attraverso alla quale scorre il fimme Pescara e che conginnge il versante orientale degli Abbruzzi col Lazio.

Ciò non per tanto gli scambi umani tra i lue rersanti dell'Apennino meridionale avvennero più al sud, attraverso un terreno difficile, ma che nel suo complesso si presta meglio al passaggio delle genti, attraverso cioè l'altipiano irpino.

Incominciando al mezzogiorno dal golfo di Taranto, l'Apennino lucano, ch'è il più lontano dalla costa orientale, sale gradatamente allontanandosi dal mare fino a raggiungere $m .1836$ e 1425 nelle vette del Volturino c del monte Santa Croce, tra le quali giace la conca di Potenza, per la quale corre anche la ferrovia da Napoli a Taranto. 
Al di là di queste vette e lino al passo di Vinchiaturo, attraversato oggi dalla ferrovia Benevento-Campobasso, si estende l'altipiano irpino, ch'è la zona più bassa di tulto l'Apennino, ma che causa le sue alte e poderose masse montuose, rende disageroli le commicazioni tra un versante e l'altro. Per la sua posizione a cavaliere della penisola l'altipiano irpino domina i due versanti e dovrebbe essere il punto di convegno di numerosissime linee di comunicazione fra la Basilicata, la Puglia, la Campania e gli Abbruzzi. Per di qua passava la grande via Appia, che conginngera Roma a Brindisi e da Durazzo sull'altra sponda proseguiva colla via Aegnatia fino a Bisanzio.

Lungo la cresta degli Abbruzzi, che sono la parte più elevala, più erta, più imponente dell'Apennino, vi sono parecchi altri passaggi e fra (fuesti merita di venir citata la gola Arquata o valle del Tronto $(700 \mathrm{~m}$.); però questi valichi non possono distruggere il fatlo voluto dalla natura, che l'Apemnino abbruzzese abbia da disgiungere per sempre le popolazioni del rersante adriaLico da quella del Lazio.

Importanti sono invece due valichi tra l'Apennino centrale e il settentrionale, cioè il passo di Scheggia (591 m.) e la Bocea Seriola (730 m.), peichè altrarerso di questi l'Adriatico comunica al nord col Tirreno. Per di qua passa ora anche la ferrovia Ancona-Roma e in cuesti paraggi stava la cillà di Tagina, ove i Romani liaccarono l'invasione dei Goti.

Tutti gli altri valichi dell'Apennino seltentrionale fra la Padania e la Toscana non hanno per l'Adriatico (dhe un valore relativo, e lo slesso si può dire degli aviallamenti del resto noteroli tra gli Apennini e le Alpi.

Ognumo conosce al solo nome le vie di comunicat- Tra le Api. zione (oggi ferroviaria) altraverso te Alpi oceidentali, centrali ed orientali, cioce, quelle del Cenisio, del Sempione, del Goltardo, del Brennero e di Pontebha.

Per la costa italica dell'Adriatico acquista importamza appena il passo det Brennero, perehè ralando da esso il fiumicello Eisack, che poi proseguc coll Arlige, si può 
dire che da qui parte una delle principali vie attraversile dai popoli per scendere al mare.

L'importanza di una via non dipende veramente dalla regolarilà o dall'abbassamento di un solo passo, perchè i valichi anche se bassi, ma singoli, in mezzo ad una catena che mantenga alte le sue cime da una parte e dall'altra, non servono che agli scambi tra popolazioni finitime, che sole sono in grado di conoscerli.

I popoli nelle loro grandi trasmigrazioni hanno per lo più evilato simili passaggi angusti, in parte forse perchè meno noti e in parte perchè sono pericolosi, potendo venir lacilmente sbarrati guerrescamente e preparare un'imboscata. Per ciò le orde nmmerose hanno preferito sempre passaggi più aspri, ma composti di parecchi solchi e che siano visiluili all'occhio da lontano. Queste considerazioni servono appunto a spiegare il motivo pel quale malgraclo i molti passi, relativamente non alti, accennati attraverso gli Apennini e le Alpi, i Romani raggiumsero l'Adriatico attraverso l'altipiano irpino, i Germani, dai Cimbri agli Austriaci, immpero in Italia per le strade dell'Adige e la maggior parte degli aitri barbari approfittarono dell'abbassamento aspro, ma visibilissimo, tra le Alpi e il Carso.

Le strade dell'Adige e dei suoi affluenti servirono però anche ai Romani ed agli Italiani per espandersi nell'interno delle vallate alpine. Alcuni scrittori si chiesero se il bacino idrografico dell'Adige sia più accessibile dalle Alpi o dalla Padania. Non è qui il caso di fare un'indagine ed ma discussione geografica. Interrogando brevemente la storia, si vede che vi poterono penetrare tanto. i Germani dal settentrione, che tedeschizzarono l'alto Adige al di qua dello spartiacque puramente geografico, quanto gl'italici che dall'evo medio in poi guadagnano nazionalmente terreno. L'oscillazione del confine etnografico dimostrerebbe quindi clıe la geografia non è sicura in questo punto, se potè subire la correzione di altri fattori imlani.

Per la costa dell'Adriatico ha invece importanza capitale quell'abbassamento, che sta tra le Alpi e il Carso. 
E stato già accennato che geologi e geografi trovano Nel Carso. difficoltà a stabilire la linea di confine tra le Alpi ed il Carso per la ragione che non esiste una demareazione precisa fra l'uno e l'altro di questi sistemi di montagne.

Le Alpi Giulie arrivano fino all'altipiano d'Idria. I] Carso è fatto incominciare dai primi confluenti di sinistra dell'Isonzo; ma una linea di divisione dalla Sava (pianura di Lubiana) al mare non può venir tracciata, perchè non avendo i monti grandi altezze, mancano anche notevoli avvallamenti. L'abbassamento si riscontra invece in questa regione in forma assai grande e vasta dalle sorgenti della Sava a quelle della Culpa, ovverossia dal passo del Predil a Fiume. - Il terreno di transizione è molto lungo ed ha la forma di altipiano, ma i popoli lo utilizzarono egualmente come passaggio. Il termine di valico però non gli si adatterebbe affatto. Volendo qualificarlo bisogna dire ch'è una porta, che la natura creò ampia per proprio comodo, onde restasse libera al passaggio dei venti e degli uomini, ogni volta che un travaso è a lei necessario per ricomporre un equilibrio.

La natura, ch'è stata generosa e benefica verso l'Italia, dandole da tutte le altre parti confini stabili con monti e mari, da questa si mostrò avara, assegnandole un terreno indeciso, aspro, di minimo valore economico. Grande e decisiva è stata da questa parte l'influenza della geografia sulla storia. Mi si conceda ma piccola parentesi storica.

L'indecisione del confine geografico in questo tratto è la causa da cui nacquero contese politiche e nazionali, che non sono risolte ancora, e forse non potranno venir assopite mai. Se la natura avesse messo anche da questa parte un confine netto, come le $\mathrm{Al}_{\mathrm{p}}$ i Carniche, gli Slavi non si sarebbero mai insinuati in territorio geografico italiano, nè i Tedesclıi si sarebbero mai affacciati politicamente sul golfo di Trieste. In buona parte per ragioni geografiche i Romani abbisognarono di lutta la loro potenza per imporsi su queste regioni. L'Istria fu conquistata appena nel secondo secolo a. C. La regione del

\section{L'Adriatico.}


Carso assicme alle Alpi orientali fu poi l'ultima conquista italica dei Romani per opera di Augusto, pochi decenni avanti l'era volgare, colla quale questi dominatori, che avevano già assoggettato l'Africa settentrionale, la Grecia, l'Illiria, la Spagna e di recente anche le Gallie, compirono la formazione nazionale dell'Italia e ne chiusero i confini ad oriente. Allora l'Istria fu da Augusto assegnata alla $\mathrm{X}$ regione italica (Venetia et Histria). Dal nome di questo imperatore le Alpi tra la Sava e l'Isonzo si dicono Giulie.

La mancanza di un solido confine montuoso influì di poi anche sul regresso della romanità. L'Istria, che topograficamente rappresenta l'estremo lembo d'Italia, per essere stata fuori di strada delle trasmigrazioni dei popoli e per la sua forma protesa nel mare fu ognora più italiana del Carso, che confina immediatamente colle Alpi e col Friuli.

L'abbassamento del Carso fu la gran via da cui uscirono le aquile di Roma alla conquista del Danubio; ma appenachè, abbandonate dalla vittoria, si rifugiarono vinte in patria, per la stessa porta vennero inseguite dai nemici trionfanti. Per di là passarono Visigoti, Unni, Ostrogoti, Longobardi, indi Slavi e perfino Turchi. Da allora in poi la porta rimase aperta ed anche oggi gl'Italiani la guardano con trepidazione, presentendo una minaccia.

Forse che la natura, malgrado l'apparenza contraria, costruì quella porta con buona intenzione, per lasciare anche alle energie umane un campo d'attività e per tenerle sempre deste mediante la lotta. Altrimenti i popoli italici, chiusi nei loro confini e snervati da un sole cocente, avrebbero corso il pericolo di infrollirsi nel dolce far niente.

Sarebbe cosa vana negare che il Carso settentrionale appartenga col versante adriatico geograficamente alla regione italica. Geologicamente invece somiglia ai terreni della Carniola e differisce essenzialmente dalle pianure friulane. Siccome inoltre questa regione è più' facilmente raggiungibile dall'esterno, vale a dire dall'altro versante 
carsico, che dall'interno, cioè dalla pianura friulana, essa potè essere contesa etnograficamente e politicamente.

Tutti i popoli in marcia verso le pianure dell'alta Italia, rese ubertose dalla vanga e dal vomere romani, giunti alle alture delle Alpi Giulie o del Carso triestino, devono essersi inebbriati alla vista di tanto bene di Dio in terra, - sul mare, nel cielo. Dalle sommità del monte Re (Nanos) si vede buon tratto del mare Adriatico, coll'Istria da un lato e la pianura friulana dall'altro. La minaccia di un'invasione eccitò sempre gl'Italiani alla difesa e li costrinse a lottare per respingere gl'invasori per lo meno oltre la prima linea dei monti, onde impedire che depredassero le loro messi. Da ciò derivò in queste regioni un perenne stato di lotta, combattuta dagli nomini sull'esempio offerto dalla natura: al di qua terra piana, verde e fruttifera, irrigata d'acque, con sole caldo e clima benefico - al di là la montagna sassosa, arida, infestata dai venti rigidi di bora. Sotto l'influenza della natura, ecco plasmarsi differentemente anche l'indole degli nomini; i pianigiani crescere miti, alle volte indolenti, spesso imprevidenti - i montanari ruvidi, sempre avidi, talora aggressivi: al campo della civiltà stare a ridosso quello della miseria.

Eppure (quiesto duplice pericolo continuo degli elementi e degli uomini forse è benefico, perchè eccita alla vigilanza e ridesta le energie italiche, talvolta assopite.

Lungo i rappresentanti più maestosi della catena carsica, ossia i monti Capella e Velebit, s'incontrano nuovamente dei veri valichi. Servono questi di passaggio alle folate di bora, ai (quadrupedi ed agli nomini singoli, ma più ai primi due che agli ultimi. Non hanno importanza storica.

Notevole è invece l'avvallamento trá il Velebit a il Dinara, perchè da esso hamo origine i tre fiumicelli Z ${ }^{\prime} l^{\circ}-$ magna, Cherca e Cettina (anticamente Tedanio, Tizio e 
Tilluro o Nesto), che ravivano la Dalmazia, cui fanno le funzioni di vene.

Un altro passaggio, gì̀ accennato, esiste tra il Dinara e il Biocovo, e poi viene la valle del Narenta. Questo fiume dovrebbe essere l'arteria principale per congiungere il retroterra colla costa; invece causa il suo corso tortuoso, l'acqua innavigabile, la valle piuttosto stretta, la foce paludosa e malsana, non potè mai venire utilizzato pei commerci.

La stessa cosa si potrebbe ripetere riguardo ai fiumi. dell'Albania.

Tutti questi passaggi insicme hanno importanza dal lato che furono adoperati fin dai popoli dell'antichità per scendere dalle acque del bacino del Danubio alla costa dell'Adriatico. Indi servirono ai Romani per percorrere la strada inversa e furono di poi le vie per le quali i barbari, provocati e allettati dai legionari, rotte le barricre romane irruppero contro Roma. Essi riacquisteranno valore quando, sperabilmente presto, saranno riutilizzati per allacciare mediante linee ferroviarie la costa all'interno e alle regioni del Danubio.

\section{LE REGIONI.}

Come nel fare la conoscenza d'una persona, all'atto della presentazione, non si riceve che un'impressione fuggevole, approssimativa del suo carattere e solo in seguito col trattarla si scopre p. e. che il suo temperamento è focoso perchè la costituzione è pletorica, oppure che.l'animo è fiacco e melanconico perchè il corpo è anemico, così nell'Adriatico e nelle sue coste coll'osservazione accurata e collo studio si viene gradatamente a rilevare che ogni sua particolarità, ogni divisione e caratteristica regionale, e così pure il valore economico, sociale e politico, sono determinati da cause naturali fisse e immutabili.

Nella nostra mente sono infissi fino dall'infanzia nomi di regioni e paesi, conginnti meccanicamente con fatti sto- 
rici. Un esame ragionato scopre facilmente il nesso intimo che passa tra la legge geografica, fissata dalla natura. e l'avvenimento storico, cui contribuirono le azioni degli uomini.

Incominciamo ancora una volta colle Puglie.

La regione che oggi è detta Puglia corrisponde nelle sue linee generali all'antica Apulia. Perchè la storia ab)bia rispettato con tanta fedeltà la compagine di questa regione bisogna che ci sia una ragione molto forte. Infatti quel paese che dai contrafforti orientali dell'Apennino (fiume Fortore) fino al Bradano nel golfo di Taranto, si protende ondulando e digradando nel mare, sebbene per l'osservazione minuta sia composto di più regioni, cioè del cosidetto Tavoliere, un bassopiano, delle Murge collinose, del Gargano quasi carsico e della penisola Salentina, socialmente non può essere ingrandito, nè impiccolito e nemmeno disgiunto, e deve per ciò formare ognora un tutto. I confini di questo complesso sono netti ed immutabili, i mari Adriatico e Jonio, c l'Apennino lucano. Però l'Apennino lucano, che fa da confine col resto del mezzogiorno d'Italia, non è tanto alto e poderoso da costituire una barriera di divisione coll'altro versante tirrenico, come del resto in generale manca in tutto l'Apennino meridionale una catena principale, che agisca da linca di separazione. A motivo di questa configurazione geografica indecisa della parte meridionale d'Italia i due versanti, pur conservando ognuno caratteristiche proprie, ebbero sempre tra loro relazioni scambievoli maggiori che nei rimanenti tratti settentrionali ed anche storicamente subirono per lo più la stessa sorte. La Puglia quindi, sebbene sia tutta bagnata dal mare, Adriatico e Jonio, non vive solo da questa parte, ma trae la sua esistenza accoppiata all'altro versante, il napoletano, formando con esso quel complesso geografico, ch'è appunto delto il mezzogiorno d'Italia.

É strano però che in un complesso così vasto e bagnato da tre mari manchi in tutti i tre versanti, adriatico, ionico e tirrenico, un centro comune e stabile di attrazione. La geografia non l'ha creato e la storia poti 
essere capricciosa. Cause accessorie esterne ne fecero nascere uno secondo le vicende politiche con un'instabilità continua. Durante l'epoca greca antica prevalse la costa ionica: Sibari, Eraclea, 'Taranto; sotto i Romani acquistò maggior valore la sponda tirrenica: Napoli, Stabia, Ercolano, Ponpei, il capo Miseno; sotto i Normanni che vennero dalla parte dell'Arlriatico ebbe maggior importanza la costa pugliese.

La Puglia, facilmente accessibile da due mari e da terra, non tu creata dalla natura per un'indipendenza duratura. E naturale quindi che fosse greca (si pensi alla Magna Graecia), romana o napoletana, secondo la provenienza del dominio.

Il Molise. A settentrione delle Puglie sta il Molise, regione montuosa e frastagliata, ma piuttosto povera e senza caratteristica propria, che geograficamente è soggetta all'altipiano irpino. Fu questo il paese dei Sanniti, forti montanari, che opposero lunga e fiera resistenza alla conquista romana. Però il Molise, come non ha una fisonomia geografica di grande valore, così non ha nemmeno una propria storia. Geograficamente può essere raggiunto dal Napoletano, dal Lazio e anche'dalle Puglie; e in armonia con questa condizione geografica fu congiunto con questi paesi anche politicamente.

Gli Abbruzzi. Negli Abbruzzi la natura ha voluto affermarsi geograficamente con una montagna alta, nuda, aspra, massiccia, le cui creste s'immalzano sorrette da fianchi ripidi e poderosi, intersecati da burroni.

I.'A Apennino abbruzzese, specialmente nel versante adriatico, ebbe fin dall'antichità carattere proprio, notevole per una selvaggia fierezza della natura e degli uomini.

Lo spirito d'indipendenza della popolazione si estrinsccò piuttosto nella difesa, ma dovette inacidire in quanto a manilestazioni affermative, perchè i gruppi montuosi e sporgenti del Gran Sasso e della Majella rendono la zona di terra troppo stretta e troppo povera.

Questa regione, difficilmente accessibile dalle Marche, dal Lazio, dal Napoletano e dal mare, dovette godere in tempi primitivi di una certa indipendenza. Ma la povertà 
gl'impedì di conservarla in tempi più progrediti. Se una catena di montagne molto alta non avesse diviso il versante orientale dal resto d'Italia, esso sarebbe caduto indubbiamente e per sempre sotto la dipendenza del Lazio, che esercita un'influenza d'irradiazione ascensionale sull'altro suo versante occidentale. A causa di cuesta separazione gli Abbruzzi ricevettero la civiltà proveniente dal Tirreno soltanto attraverso il Nolise e lo strello abbassamento Sangro-Volturno (tra la Meta e la montagna del Matese). Fu per di qua appunto che vemnero i suoi dominatori, i Romani ed i Napoletani (reame delle Due Sicilie).

Il territorio delle Marche, dal Tronto allo spartiacque Savio-Tevere, meno aspro e meglio dotato di terra e di risorse naturali degli Abbruzzi è aperto al suo settentrione. Esso può valere quindi nella parte settentrionale come ponte di transizione dalle regioni montuose dell'Apemnino alle piane dell'Emilia e della Padania. Ha però ancora nella sua parte meridionale a causa del terreno collinoso caratteristiche geografiche proprie che lo avvicinano agli Abbruzzi. Forse per ciò quando era occupato dai Galli, coloro che lo abitavano portavano il sopranome di Semones. Paese relativamente piccolo e aperto dalla pianura e dal mare e accessibile dal monte pei valichi di Bocea Seriola e del passo Seheggia fu gallico, greco, latino, bizantino (lá pentapoli) e pontificio. - Il versante settentrionale dell'Apemnino, che va dal punto ora indicato fino alle sorgenti del Reno e del Panaro, è parte dell'odierna Emilia, come era una volta. della Gallia Cispadana; ma queste sono denominazioni generiche e amministrative. Per lo studio della costa basta anche limitarsi a considerare il tratto più vicino al mare, noto col nome di Romagna.

Questa regione appartiene geograficamente e per conseguenza economicamente alla Padania. Difficilmente accessibile da mezzogiorno dall'altro versante dell Apemino e da settentrione per il delta del Po, questo terreno fu oceupato a preferenza da popoli che provenuero dalloccidente, seendendo il corso delle acque o dal mare Adria- 
tico. Non ha fisonomia geografica propria e per ciò anche politicamente ebbe molti dominatori (Romani, Ostrogoti, Longobardi, Franchi, Bizantini, Veneziani, Pontifici ed anche propri, p. e., i Polenta di Ravenna).

Riassumendo dunque si deve dire che tutte le regioni del versante adriatico dell'Apennino, sebbene siano suscettibili di vita civile e abbastanza provvedute di risorse naturali, mancano di quella esuberanza che aiuta a produrre il dominio della sua popolazione. E infatti gli abiiatori della costa adriatica d'Italia, presi singolarmente e come tali, furono popoli dominati.

La Padania. La Padania, che, vista nel suo complesso specialmente idrografico, apparisce una parte integrante del bacino dell'Adriatico, considerata da vicino come pianura mostra delle distinzioni regionali, che meritano di essere considerate particolarmente.

Dal concetto di costa adriatica in senso sociale bisogna a questo punto scindere innanzi tutto la parte occidentale contornata dalle Alpi e dagli Apennini (Piemonte), giacchè questa, sebbene appartenga idrograficamente all'Adriatico, economicamente gravita su Genova e quindi sul Tirreno. Anche la pianura lombarda nel tratto della regione dei laghi fino all'Oglio, essendo attratta dalle Prealpi un po'verso settentrione attorno al suo centro economico naturale, Milano, gravita fino a tanto che non sarà regolata la navigazione mediante canali, piuttosto verso occidente e verso la Liguria. Dal Garda e dal Mincio ha principio e poi coll'Adige pieno sviluppo la tributarietà idrografica della regione veneta al bacino dell'Adriatico. A causa di questo frazionamento nello sbocco economico per la vicinanza del Tirreno la Lombardia e il Veneto non poterono conservare ognora un'unica sorte politica. Però etnograficamente, socialmente ed anche in parte economicamente essa formò sempre un insieme abbastanza omogeneo.

Riserviamoci di trattare separatamente nella parte storica il fenomeno della città di Venezia, come sbocco economico della Padania, e passiamo al Veneto.

Questa pianura (compreso il Friuli) ha un'estensione 
e una ricchezza sufficenti per vivere una vita propria. E circondata tutt'intorno da monti. Di questi però le sole Alpi Carniche per la loro altezza e per l'erto versante fanno da vero confine separatorio. Quelle del Trentino e le Giulie per la direzione delle catene le prime, e per la poca altezza le seconde, sono invece inefficaci a proteggerla. Per ciò non potè conservarsi indipendente che per singoli periodi, quando non fu insidiata da vicini, che la poterono occupare facilmente dalla pianura al Po, dal valico del Brennero o dalla porta del Carso.

Dell'abbassamento del Carso fu fatta già una descri- Il Carso. zione geografica abbastanza dettagliata.

Un terreno di transizione, selvaggio, privo di risorse, non è di solito ricercato da popoli superiori; ma viene occupato come territorio di rifiuto da popoli secondari, condannati per ciò ad un'eterna inferiorità civile.

La penisola d'Istria, marcata geograficamente dai due golfi di Trieste e di Fiume, è divisa in due parti dal 'dorso montuoso, culminante nel Monte Maggiore, che la percorre dalla Montagna dei Cicci alla Punta Nera (al sud di Albona e ad oriente dell'Arsa). Si possono quindi distinguere due Istrie, una, la maggiore, marittima, l'altra, la minore, continentale. Povere e ristrette tutte due, esse gravitano, la marittima, sul bacino settentrionale dell'Adriatico o golfo di Venezia e verso il bassopiano reneto; l'altra, la continentale, verso il Carso. La storia rispettò questa distinzione, dandoci un'Istria marillima, greca, latina, veneta, e un'Istria montuosa, liburnica, illirica, slava.

Il litorale croato col pendio dei monti che scendono a picco sul mare e privo di porti, è il tratto più misero

Il litorale croato. di costa che si specchi nell'Adriatico. Non ha alema importanza econonica, ammenochè non si volesse regalargliene qualche briciola, assegnandogli le isole di Veglia, Arbe e Pago. Socialmente e nella storia non fece parlare di sè che come niolo di pirati (i Liburni, gli Uscocchi).

Se il triangolo della Dalmazia possedesse tutta la terra La Dalmazia. che il mare gl'inghiottì fra le isole, questo paese polrobbe

L'Adriatico. 
dirsi sufficentemente felice e il migliore della costa orientale. Però anche così con'è ridotto, è a miglior partito di alcuni altri, perchè alnıeno può bastare a sè stesso, se anche poveramente.

Per giudicare la Dalmazia non bisogna limitarsi a considerarla nello stato di abbandono in cui è lasciata oggigiorno, ma pensare che al tempo dei Romani era tutta un vero giardino.

Le isole, anclie scarnate, costituirono la risorsa principale, perchè le maggiori agirono da polmoni per respirare e vivere sul mare, e perchè protendendosi fino all'Istria, servirono da ponte di contatto colla civiltà dell'occidente. Chiusa totamente verso settentrione dalla montagna dirupata del Velebit, è aperta verso mezzogiorno tra il Dinara e il Narenta c per ciò ha maggiori relazioni colla Bosnia e coll'Erzegovina, che non colla Croazia.

L'Erzegovina e il Jontenegro

Le regioni dell'Erzegovina e del Montenegro, che si possono accomunare per conformazione geologica e per posizione geografica rispetto al mare, sono le più orride del Carso e forse tra le più desolate délla terra; si possono chiamare i paesi della fame.

lnternati in terraferma e sfavoriti dalla natura anche in quel po' di costa, non possono dar ricetto che a gente povera, indurita ai patimenti e alla miseria. Nè la storia potè correggere questo difetto della geografia. Sarebbe vano sperare che la civiltà vi germogli: è molto se si arriva a penetrare.... dall'aria.

A migliorare queste condizioni non valse nemmeno la costa marittima. Il passaggio dal monte al mare è troppo brusco per permettere un'armonia nelle relazioni. I montanari non sammo e non possono far altro che vivere sui loro monti ed i marittimi sono perduti, lontano dal loro mare. Il passaggio da un ambiente all'altro richiede un lento adattamento e per ciò la popolazione, se anche sia etnograficamente eguale, diviene troppo differente di temperamento e di abitudini. Questa è la causa per cui gli Erzegovesi ed i Montenegrini, per non parlare che dei superstiti, furono divisi e distinti dai Ragusei e dai Bocchesi. 
Considerazioni malinconiche al pari di queste si dovrebbero fare per l'Albania, comprendendori pure anche quel poco di vita marinara, che potè formarsi in qualche piccolo punto al mare.

All'inferiorità del territorio denominato generalmente Albania contribuiscono oltrechè l'asprezza dei monti e la paludosità della pianura, anche la mancanza di un confine geografico, netto e chiuso. Fu già accennato prima, e tutti lo hanno udito o letto nei recenti. rivolgimenti balcanici, che un'Albania geografica veramente non esiste. Ossia esiste, non c'è dubbio, ma non ha consistenza per l'elasticità dei suoi confini. I monti dell'Albania, il lettore l'avrà notato da solo, non hamno nomi noti nella geografia universale. E se anche prendiamo i tre fiumi principali del versante idrografico adriatico dal lago di Scutari a Vallona, ossia Drin, Semeni e Vojuzza e seguiamo il loro corso in linea ascendente per segnare poi lo spartiacque dei monti e dire: tra questo e il mare è l'Albania, redremo che l'impresa non è facile.

Il Drin che nasce dal lago di Ochrida si versa nell'Adriatico con un corso quanto mai capriccioso, simile all'ingrosso a quello del Narenta. Ma il maggior guaio si è che tra il lago di Ochrida e la riva sinistra del Drin per quasi due lerzi della sua lunghezza corre tra il fiume e il mare una catena di monti, che gl'imprime appunto quella linea capricciosa e la quale sareble invece adatta a formare una specie di conline naturale. Il Drin Bianco poi, confluente settentrionale di destra del Drin (che nell'altro tratto è delto Nero) porta idrograficamente in questo fiume e nell'Adriatico un altro bacino, quasi quadrato, che orogralicamente sta disgiunto dal mare.

I duc altri liumi Semeni e Vojuzza hammo un corso relativamente diretto e devono la loro origine in buona parte al monte Grammos, che non silreble un cattivo confine. Ma uno dei due confluenti che dànno vita al Semeni, il Devol, conduce all'Adriatico le acpue dei litghelti, che forse non sono disgimti da cuacllo maggione di Presba, situato al di lì di parecelice catene di monti. 
Il corso del Devol rappresenta dunque una porta sfondata tra le pareti non solide dei confini albanesi.

A rendere ancora più precaria la posizione sociale dell'Albania concorre la sproporzione della sua estensione in lunghezza senza una corrispondente profondità. Da ciò deriva appunto il disgregamento economico, etnografico, sociale dei suoi abitatori e l'animosità degli abitatori che stanno al di là del cosidetto confine, $\mathrm{i}$ quali vedono negli Albanesi un ostacolo alla loro calata al mare. L'Albania ha troppe porte sfondate e per queste tanto gli Albanesi poterono facilmente insinuarsi nei territori dell'altro versante, quanto gli Slavi dai Balcani penetrare in Albania.

L'Albania è povera, e questa povertà oltrechè essere un ostacolo al raggiungimento d'un'indipendenza economica, base di tutti gli altri generi di libertà, spinge i suoi abitatori a cercare ricchezza nei territori adiacenti. Però siccome le regioni adiacenti, senz'essere molto più felici, sono più estese e quindi più popolate numericamente, è da presumersi che in un conflitto riescano vincitori $\mathbf{i}$ più forti in grazia del numero. Infatti gli Albanesi da soli non hamno dominato mai sui loro vicini.

L'ultima e forse non la minore sciagura dell'Albania è quella di essere situata come un cuscinetto al cardine della porta dell'Adriatico e quindi di poter venir implicata e travolta anche nelle vicende di questo mare, rappresentandovi una parte del tutto subordinata a causa della sua piccolezza. E in politica, si sa, come negli abissi del mare, il pesce grande mangia il piccolo.

In quanto all'indole, pure molto studiata e discussa dei suoi abitanti, si comprende che in un paese capriccioso e strano deva essere strana anch'essa. Rude e selvaggia fierezza, amore di libertà, violenza e poca concordia sono sentimenti imposti alla popolazione dalla natura. La molteplicità degli aspetti della loro terra contribuì senza dubbio a dare agli Albanesi vivacità di temperamento e senso artistico. Il popolo albanese ha una intelligenza svegliatissima e certo superiore agli altri che li circondano. Basti dire ch' essi sono i sarti nazionali artistici 
di tutti gli Slavi e dei Greci, loro confinari. Gli Albanesi diedero alla civiltà uomini d'ingegno che divennero insigni, ma fuori della loro patria, come toccò del resto ai più di coloro ch'ebbero la culla sulla sponda orientale adriatica. Ma cumulativamente l'Albania non si elevò mai nella storia, non come Epiro, non come Illiria, non come Turchia. Del resto poco differenti sono le condizioni del bacino idrografico del Narenta e del Montenegro. Solo che in questi paesi i contrasti sono meno stridenti, perchè la loro popolazione non differisce da quella dell'interno balcanico. E se del numeroso popolo degli Illiri sopravvisse soltanto quella parte che oggi chiamianı Albanesi, la causa è forse anche sopratutto geografica: in questo punto la pressione dei sarmati trasmigranti fu esercitata di fianco e l'urto fu più debole, transitorio.

Per questa infelice configurazione geografica l'Albania esercitò nell'Adriatico anche storicamente una parte secondaria in quanto ad attività. Passivamente fu un'altra cosa.

Durazzo è una delle più importanti città adriatiche nella storia dei rapporti tra l'Italia e l'Oriente, il primo acquisto romano oltre l'Adriatico. A Durazzo si svolse l'episodio della lotta fra Cesare e Pompeo che fu il primo segno di quello sfasciamento del troppo colossale impero romano in occidentale e orientale e dello smembramento dell'Adriatico. Durazzo fu poi la mira dei Normanni e degli Angioini. A Durazzo avvenne nei secoli XIII e XIV il cozzo tra Veneziani, Napoletani, Bizantini e Serbi per il predominio nel basso Adriatico. A Durazzo anche di recente collimarono l'antagonismo dei Serbi, degli Austriaci, degl'Italiani. 
Influenza delle regioni sul caratter'e degli abitatori.
Da tutto quanto fu esposto si vede che non le regioni furono trasformate artificialmente dagli uomini, ma che gli uomini dovettero acconciarvisi o bene o male secondo quanto veniva loro offerto dalla natura geografica dei singoli territori.

La geografia quindi forse più che la storia ci porge la chiave per giudicare il valore dei popoli. Se gli abitatori delle Puglie sono attivi marinai, quelli degli Abbruzzi selvaggi pastori, quelli delle Marche e dell'Emilia studiosi e dotti, quelli del Car'so-zotici, quelli dell'Istria e delle isole pescatori e marinai, quelli della Dalmazia continentale infiammabili e discordi, quelli dell'Erzegovina e del Montenegro asciutti e apatici, quelli dell'Albania fieri e indomabili e - presi sommariamente - sani, ricchi, intelligenti quelli delle pianure, pigri e ammalati quelli delle paludi, ostinati, retrogradi, poveri quelli delle montagne, la causa di queste manifestazioni principali del temperamento nazionale, piuttosto che nelle caratteristiche di razza è da ricercarsi nell'ambiente regionale. Il clima, il nutrimento, la natura del suolo, il genere di lotta per l'esistenza, plasmano e modificano la fibra degli uomini, tanto che si potrebbero ancora una volta - come del resto avvenne già nella storia - scambiare i popoli tra le differenti region'j e si vedrebbe che l'indole del paese rimarrebbe la stessa, mentre ne verrebbe coll'andar del tempo modificato il temperamento nazionale. 


\section{CONFRONTO FRA LE DUE COSTE.}

Anche il lettore si sarà ormai domandato: quale dunque delle due coste è la migliore?

Se confrontiamo solamente la zona marittima, specialmente con riguardo all'importanza per la navigazione, si scorge subito l'immensa superiorità dell'orientale sull'occidentale. L'orientale è anche più favorita dalla natura per la sua posizione rivolta a mezzogiorno e per la maggior abbondanza d'accua piovana.

A primo aspetto si dovrebbe duncue optare per l'orientale.

Considerandole invece più attentamente si osserva che gli svantaggi dell'occidentale sono compensati da vantaggi che mancano affatto all'altra.

Innanzi tutto l'occidentale ha più terra e quindi maggior ricchezza e poi, sebbene non sia gran cosa, non è battuta tanto dai venti di bora e di sirocco. Ma la differenza geografica più essenziale e di maggior effetto nel valore sociale si deve cercare nel raccordo dei versanti alla propria catena e nella posizione complessiva di questa rispetto alle altre regioni circostanti.

Due versanti come l'orientale apenninico e l'occidentale carsico, che si guardano e che sono uniti dal mare, devono avere frequenti contatti. Però i versauti di mo stesso monte, per quanto divergenti, non stamno isolati affatto ognumo per sè, ma devono vivere uniti, anche loro malgrado, come due fratelli siamesi ehe si volgessero le spalle.

É stata già rilevata la lormazione geologica differente della catena carsica e dell'apenninica e le divergenze climatiche che ne risultano. Basterebbe questa sola constatazione per lar propendere la bilancia in favore dell'Apemnino. Anche P'Apennino come il Carso hat le sure vette selvagge e i suoi buroni, pendii dirupati, scoscendimenti di terreno, valli parallele e trasversali che ostacolano it 
passaggio da un versante all'altro. Però questo non è il tono dominante come nel Carso. L'Apennino, specialmente nel versante tirrenico, degrada in molte e molte catene collinose, attraversate da fiumi benefici e intersecate da valli ubertose, in cui si formarono molti centri cittadini. Fino al mare c'è un lungo tratto di terreno coltivabile, fertile, per di più armonico, che eccita ogni attività umana, manuale ed intellettuale. La costa tirrenica, anche se poco adatta alla navigazione, ha di fronte le tre grandi isole di Corsica, Sardegna e Sicilia e si può dir padrona sul suo mare. Un confronto efficace dovrebbe veramente venil fatto tra queste due coste, la carsica e la tirrenica, giacchè sono esse che godono di una posizione simmetrica rispetto all'azione solare; e il risultato ridonda allora ad uno schiacciante vantaggio per l'Apennino.

L'Apennino e il Carso discendono ambedue dalle Alpi, ma l'Apennino ne è il figlio prediletto. L'Alpe, la madre, è l'elevamento geologicamente e socialmente più importante d'Europa e la ragione d'essere dell'Italia. La penisola italica, lanciata come un ponte nel mezzo del grande bacino, detto appunto mediterraneo, perchè lambisce ed abbraccia in un grande cerchio economico l'Europa meridionale dalla Spagna al Danubio, tutta l'Africa settentrionale e l'occidente dell'Asia, è la via naturale di comunicazione fra tutti i punti della vasta periferia. L'Italia, protetta dalle Alpi, può essere paragonata ad un molo gigantesco, benefico e indipendente. L'Apennino, il figlio, ha poi il merito di aver fatto del versante tirrenico dell'Italia il giardino del mondo e la seconda culla della civiltà. Il suo versante disteso ad arco e rivolto a mezzogiorno, in posizione in cui i raggi solari gli cadono quasi a perpendicolo, è per l'Europa, come l'India per l'Asia, il Messico colle due penisole di Florida e Yucatan per l'America, il centro da cui devono irradiare e verso il quale devono convergere le energie vitali dell'immenso suo cerchio.

La natura lo suddivise in tre regioni, la Toscana in alto, più fresca, perchè vi germogliassero l'arte e l'intelligenza - il Lazio in mezzo, più caldo, perchè la vo- 
lontà e l'intraprendenzal ne avessero il dominio - e la Campania cocente al sud, perchè la fantasia e l'irrequietezza li mettessero in moto. E da Roma che si mosse il più forte popolo conquistatore e nella Toscana che nacquero i più grandi geni d'Italia e alcuni del mondo: Dante, Leonardo, Michelangelo, Galileo, Machiavelli. Se l'Italia apenninica diveme mat culla inesameibile di civiltà e Roma il Capul mundi non è tul caso o capriccio e nemmeno tullo merito della razza. E il sole che fa crescere il cipresso regolare e serio sui colli toscani, il pino diritlo ed esuberantemente chiomato nella campagna romana, gli aranci molli rosseggianti nel golfo di Napoli ed agita lorse anche le viscere del Vesuvio. Sono i suoi raggi che, scendendo diretli dall'alto, lammo star ritti gl'Italiani e ne inliammano i cervelli. E sempre lui che rinnova la vita in questo paese benedetto, ma pure non tanto ampio da contencre tutta l'esuberanza della sua vitalita. E allora cone da un vaso riboccante il di più si espande all'intorno e, prima che altrove, sull'altro versante dell'Apemino.

Per ciò la costa occidentale dell'Adriatico, meno favorita dell'orientale, acquista dal nesso coll'altro ver'sante dell'Apennino un'imporlanza eceezionale, che la compensa indirellamente della sua posizione secondaria.

La costa orientale dell'Adriatico invece, sebbene sia il versante migliore della catena carsica, per essere stata magramente fornita essa stessa dalla natura, non solo non riuscì a beneficare l'altro, ma ne è addirittura danneggiala. In primo luogo per (quanto sia rivolta al sole, è troppo magra e troppo allungata per divenire terreno fecondo di ma civilta spontanca. Si dira che in compenso ha molte isole e che le isole sono per la costa come klei polmoni per respirat meglio. E vero, ma queste isole, isololti e scogli, sono piecoli, troppi e le stanno a ridosso, lanto che in alcuni punti la soffocano addiriltura. C’è troppe mare e troppo pocal terra e questo squilibrio le toglie molto del suo valore sociale. Un pepeto dhe vive esclusivanente sul malre, vi si afformat sonzal (\{ub)io, mal per divenire conquistalore di terrilori vicini hal bisogno

L'Adriatico. 
di terra alle proprie spalle, come d'un punto d'appoggio, da cui attingere la ricchezza necessaria per sostenere le spese della lotla. Altrimenti sarà un ottimo marinaio, pratico e sagace per muovere le navi e trasportare merci per conto di altri, ma mai un vero conquistatore per proprio conto. Tali sono appunto gli abitanti della costa orientale dell'Adriatico.

L'esempio di due altri popoli che con poca terra (maggiore in ogni caso della costa di cui si tratta) seppero essere anche conquistatori, vale a dire dei Greci e degli Inglesi, non infirma questo criterio, perchè Greci ed Inglesi si trapiantarono prima in territori fertili, dai quali mosséro a sempre maggiori conquiste.

In secondo luogo la costa orientale dell'Adriatico non è del tutto libera nemmeno sul -suo mare, perchè deve tollerare il predominio geografico e sociale della Padania, ch'è esclusivo nel bacino settentrionale e che pesa su tutto il resto per la sua superiorità economica.

Non è per capriccio nè per sola smania di dominio che i popoli della Padania e della costa occidentale si trasportarono sull'orientale. Toccò agli uomini quello che succede alle piante, quando crescono all'ombra, che si piegano dalla parte verso la quale possono raggiungere la luce. Raggiunta nella penisola italica quella maturità che preludia ad un'espansione, i popoli per uscire dall'Adriatico dovettero portarsi sulla costa orientale per ragioni meteorologiche e nautiche. Anche oggigiorno sebbene la navigazione a vapore non tema il mare, lutte le navi, ma particolarmente quelle che ancora adoperano la vela, preferiscono la costa orientale, perchè è la più sicura.

La storia non registra casi di popoli della costa orientale, marinari fino dall'infanzia, che abbiano compiuto atto di conquista duratura sull'occidentale. E ciò è molto sintomatico, e si ha da spiegare non colle attitudini di razza, ma con leggi geografiche.

In terzo luogo la costa orientale dell'Adriatico è infelice perchè oltre ad avere nelle montagne uno spartiacque bizzarro e indeciso, manca di un vero versante 
dall'altra parte ed anche quell'altipiano che lo sostituisce è rivolto a settentrione.

Oltre alle tre catene parallele indicate alla costa, il Carso ne ha un'infinità di altre, che si susseguono in due direzioni. Le une come a gradinate al di sopra di Trieste salgono al settentrione fino a riconfondersi colle Alpi. Le altre ad oriente di Fiume corrono lungo la penisola e vanno a perdersi nei Balcani. Per trovare un po' di pianura bisogna andare da Fiume al corso della Sara o attendere da Trieste fino a Vienna il corso del Danubio. Questi territori, anzi particolarmente l'altipiano montuoso della Croazia e della Bosnia, che ha maggior influenza per la costa, senz'essere regioni gelate, hamo una temperatura marcatamente più fresca, che quelle al mare. Gli nomini vi provano già dei brividi di freddo e la civiltà vi può allignare, ma non germogliare per atto spontaneo, perchè dove la terra non è grassa e il sole non la infuoca non sbocciano fiori così delicati. Molti corsi d'acqua poi che hamno la sorgente non lontano dalla costa la fuggono verso altri bacini e gli nomini li seguono. Manca dunque da questa parte anche un contatto forzato tra i due versanti.

L'infelice e frazionata configurazione geografica della regione carsica com'è causa d'inferiorità economica, determinò anche l'inferioritì sociale. Se l'Apeminica c̀ divisa in regioni, il Carso è rotto a bocconcelli. Ogni valle, ogni monte, ogni corso dacqua delle regioni carsiche ha interessi propri, divergenti da quelli degli altri. Le regioni d'Italia si combatterono, odiarono e sopraffeccio, ma non inbarbarirono mai del tulto e potè venire il giorno in cui interessi superiori fecero tacere le gare e le tendenze particolari per accomunarle tulle in $u$ complesso civile. Vi sono mari e monli che segnano confini inalterabili e più potenti dei campanilismi.

Per le regioni carsiche la geografia non segno allro limite risibile che il mare Adriatico. Dore incomincia veramente il Carso a seltentrione e dove linisce al sud per dar posto ai Balcani? Quali sarebbero i segni malu-

L'Adriatico. 
rali e visibili di divisione tra la Croazia e la Slavonia, tra questa e la Bosnia, tra l'Erzegovina e il Montenegro e così via più in avanti colle regioni del Danubio e dei suoi affluenti? Quali interessi economici comuni potrebbero unire l'Istria marittima alla Croazia, questa alla Dalmazia, il Montenegro all'Albania?

Sarebbe arduo rispondere. La discordia, gran piaga di tutti i popoli balcanici, siano stati gli Illiri, gli Epiroti, i Macedoni, i Greci dell'antichità, i Croati, i Serbi, gli Albanesi odierni, forse non è che una malattia geografica. Come concepire un'unione sociale lungo tutta la costa da Trieste a Vallona, se la natura ne ha rotto in cincue punti la continuità materiale? Quante volte le regioni carsiche balcaniche furono sotto lo stesso dominio e tante altre hanno voluto e vorranno separarsi.

I Romani per trovare un confine naturale da queste parti dovettero spingersi fino al Danubio. Ma anche questo non bastò, perchè era debole, e appena fu rotto l'argine artificiale delle loro legioni la marea barbarica irruppe da valle a valle e li ricacciò fino all'Adriatico, donde appunto erano partiti. Se invece di molte piccole catene ve ne fosse stata una sola del genere delle Alpi è probabile che i Romani non avrebbero raggiunto il Danubio, ma nemmeno i barbari si sarebbero insediati sull'Adriatico.

La presenza di molti bacini nelle regioni balcaniche potè essere utile nei primi momenti di nuove invasioni, perchè ostacolò l'avanzata del nemico, oppure avrebbe potuto giovare nel medio evo, quando soltanto gli Stati piccoli riuscivano a mantenersi in forza e potenza, perchè i territori vasti erano più faciĺmente soggetti a nuove invasioni. Ma un tale frastagliamento ebbe poi lo svantaggio irrimediabile di impedire la fusione fra vincitori e vinti e da rendere quindi impossibile la formazione di grandi Stati basati sulla nazionalità omogenea e su interessi economici consoni. Da ciò fu ritardato anche lo sviluppo delle libertà e della democrazia dei tempi moclerni. Forse che in questa causa geografica è da cercare la ragione prima della composizione polietnica della pe- 
nisola balkanica e della costante presenza di più nazionalità sulla costa orientale dell'Adriatico.

Grande è per conseguenza anche la differenza di civiltà fra i paesi ad est del versante carsico e quelli dell'Apennino. $\mathrm{Fu}$ accennato quale ne potrebbe essere la causa originaria, cioè la posizione geogirafica. Si può ammettere quindi come cosa naturale che la regione italica, una volta raggiunta una superiorità per ragioni intrinseche, l'abbia conservata anche in seguito. Ma l'inferiorità delle regioni carsiche è determinata anche da una causa storica posteriore, che osservata attentamente si risolve in una seconda causa di posizione geografica. Le regioni carsiche e balcaniche, oltrechè esscre aperte a settentrione e in parte ad oriente senza un confine protettore, giacciono nella parte più orientale d'Europa in vicinanza immediata dell'Asia. Ora l'Asia se mandò all'Europa un po' della sua civiltà nei tempi remoti della storia, nei posteriori e particolarmente dall'evo medio in poi, non fece che dammeggiarla. Dall'Asia vemnero in Europa i Persiani, i Mongoli, gli Arabi ed i Turchi. E se non proprio dall'Asia dalle regioni ad essa adiacenti del Volga e dei mari Caspio e Nero mossero gli Unni, i Goti ed altri Germani, gli Slavi, gli Avari. Molte di queste trasmigrazioni, invasioni, conquiste, passarono sopra lc regioni carsiche e le italiche; ma alcune si arrestarono all'Adriatico, come quelle degli Unni, degli Avari, degli Slavi, dei Turchi.

Questo movimento della barbaric da oriente verso occidente, cui corrispose dall'epoca romana quello, anclıe spesso demolitore, della civiltà da occidente verso oriente, transitò nel maggior numero dei casi per l'Adriatico scttentrionale e per la sua costa orientale. Per ciò alcuni storici chiamarono questa costa (anzi particolarmente la Dalmazia) un ponte di passaggio appunto tra l'oriente e l'occidente d'Europa. Privilegio poco invidiabile anche questo, perchè un ponte ch'è troppo calpestato si logora e crolla.

Ad illustrare maggiormente il confronto del valore sociale delle due coste con dati di attualità contropporremo 
il solo numero degli abitanti nelle città e luoghi principali dell'una e dell'altra costa.

Sull'occidentale da Otranto a Ravenna:

Otranto, 2771 - Lecee, 36310 - Brindisi, 28186 - Bari, 103524 Molfetta, 45413 - Barletta, 44233 - Foggia, 76531 - Vasto, 14849 - Ortona, 16881 - Pescara, 9014 - Chieti, 25628 - Teramo, 24538 Ascoli, 30631 - Macerata, 22941 - Ancona, 63145 - Urbino, 18874 - Pesaro 27345 - Rimini, 50862 - Ravenna, 71690.

Sull'orientale da Capodistria a Vallona:

Capodistria, 8\$50 - Pirano, 7600 - Parenzo, 12862 - Rovigno, 12094 - Pisino, 2400 - Pola, 45200 - Fiume, 55000 - Lussino, 7348 - Zara, 13000 - Sebenico, 10000 - Spalato, 25000 - Lesina, 2138 - Curzola, 2068 - Metcovich, 1710 - Cattaro, 5418 - Gravosa-Ragusa, 13200 - Cettigne, 5000 - Antivari, 3000 - Scutari, 35000 - Durazzo, 4000 - Vallona, 5000.

Da questa parte poi in seconda linea a grande contrasto e differenza dall'Apennino non vi sono che borgatelle d'importanza meno che secondaria, eccettuata Mostar, che ha 16000 abitanti.

Riassumendo dunque, la costa occidentale dell'Adrialico fu beneficata dall'altro versante apenninico, è stata protetta dall'Adriatico contro diverse invasioni barbariche e non subì influenze molto nocive dalla costa orientale.

Questa al contrario dovette sottostare ai damni infiniti delle vicende dolorose dell'altro versante e specialmente dei Balcani. Ebbe è vero dei benefizi dall'Adriatico e dall'altra costa; ma siccome questi benefizi le furono imposti, essa nel subire la supremazia delle regioni italiche, si trovò da questo lato in una dipendenza, che mọ-ralmente pregiudicò il valore di quei benefizi.

Se quindi la costa occidentale apparisce diseredata nella zona costiera, perchè è quasi priva di porti e di isole, quel poco di buona costa e la ricchezza d'isole, unico bene, donato dalla natura all'orientale, furono calcolati a troppo caro prezzo.

La costa occidentale adriatica, di poco valore intrin- 
seco, unita agli Apennini e all'Italia conta fra i paesi benedetti d'Europa: l'orientale invece, invidiabile per le ricchezze del suo mare, umita ai Balcani è una povera Cenerentola. La costa italiana dell'Adriatico non perirà mai, perchè la civiltà italica, basata sulla sua unità geografica e nazionale, ch’è indistruttibile, risorgerì sempre. La sponda orientale invece deve sempre trepidare, perchè un eguale' splendore civile dei Balcani è inconcepibile.

\section{FUNZIONE SOCIALE DELL'ADRIATICO.}

Da quanto è stato detto risulta evidente, che l'Adriatico a causa della sua forma allungata colle coste estremamente anguste e magre e della sua naturale utilitì per la navigazione $\grave{e}$ in primo luogo un'importante via di comunicazione. Anzi siccome questa via si prolunga a settentrione coi tentacoli dei fiumi - consolidati nei laghi alpini, dietro ai quali stamo dei valichi, cui corrispondono altre vie acquee di grande valore dall'altro versante alpino - ed a mezzogiorno è aperta tolalmente e si comnette al Jonio, si può dire che è la via ali comunicazione più importante fra il centro d'Europar e l'oriente.

Le vie del mare hamo in generale di fronte a quelle di terra lo svantaggio del risico, perchè sono basale su di un elemento troppo mobile; ciò non per tanto sono preferite economicamente perchè non richierlono nè tracciamenti, nè costruzioni, nè manutenzione. Sono più pericolose, ma sono anche libere e non costano.

L'Adriatico in particolare, favorito da una dolece corrente marina di flusso e riflusso, benclicalo da due venti costanti e distesi, il maistro alpino lresco d'estate e il sirocco africano caldo d'inverno, raramente infestato dalla bora e da burrasche, offre più che altri mari vanlaggi immensi alla navigazione. Ed è naturale fuindi che lulli i popoli lo visitassero. 
Quella ora indicata sarebbe la via di comunicazione in direzione della lunghezza. L'Adriatico si presta però appınto a causa della sua strettezza, in linea secondaria e locale anche come via di comunicazione nella larghezza. Tali scambi si possono considerare in primo luogo, com'è naturale, in senso ristretto tra le due coste e poi in scuso lato tra la penisola italica e il bacino fluviale del Danubio. Abbiamo veduto che non molto lontano dalla costa orientale hanno la sorgente nell'altro versante carsico dei corsi d'acqua, come la Sava coi suoi affluenti di destra Culpa, Unna, Verbas, Bosna e Drina i quali insegnarono ai popoli le vie di comunicazione fra l'Adriatico e il Danubio.

Questa prerogativa dell'Adriatico, ottima dal lato economico, di essere una via di comunicazione facile, lunga ed aperta, si convertì a causa della sproporzione tra la lunghezza e la larghezza in un danno insanabile dal lato sociale. Nel suo bacino cioè non è possibile che cresca e si sviluppi una vita civile unica, indipendente e dominante o che per lo meno formatosi un forte nucleo economico, esso espanda egualmente da ogni lato intorno a sè le sue energie sociali.

La sola Padania avrebbe alcune premesse naturali per l'origine di una civiltà propria, destinata a divenire dominante nell'Adriatico, come l'ampiezza e la fertilità del terreno, le vie acquee pel trasporto di merci pesanti. Na tale sviluppo venne dapprima ritardato nei tempi antichi dall'aggrovigliamento selvaggio del terreno e dall'impraticabilità della zona costiera, indi fu sminuzzato dallo scalo che la natura aperse su Genova nel Tirreno e che assorbe una buona parte del suo commercio.

All'Adriatico rimane solo la parte orientale e meno ricca della Lombardia, il versante apenninico e il Veneto.

Bisogna poi rilevare che la civiltà quando passa dalla terraferma sul mare subisce un arresto leggero, perchè deve assoggettarsi all'adattamento di un nuovo ambiente. Altra sarebbe stata l'espansione della civiltà se l'Adriatico, anzichè un mare, fosse stato una grande pianura. La lontananza dei punti meridionali delle due coste dalla 
Padania fece sì che la civiltà irradiante da questa vi giungesse attraverso il mare affievolita e potesse venir contrastata dalle influenze, che vi esercitarono altri centri situati o fuori dell' Adriatico o al di là del versante interno.

E fuori di dubbio inoltre che la conformazione geologica, arida, franabile, scoscesa del Carso e degli Apennini essendo causa di povertà economica determina anche l'inferiorità civile di queste regioni in confronto a quelle delle Alpi. L'abbondanza di pascoli sulle Alpi, oltrechè concedere quella fonte di ricchezza ch'è un ampio allevamento di bovini, favorisce le industrie ad esso annesse. Sul Carso e sull'Apennino invece non trovano pascolo sufficente che le pecore, le capre, gli asini, i muli, animali che consumano di più il terreno e rendono meno del bue.

Vi sono, è vero, tanto alla costa apenninica che alla carsica, interi distretti - ma non sono grandi - dove la natura esuberante par che inviti con le più soavi lusinghe all'ozio e ai sogni incantati; ma altrove e poco distante la natura cambia ed è tanto aspra da rappresentare qua un vivaio di ruvidi caratteri (la Dalmazia), là un serbatoio di costumi selvatici (gli Abbruzzi), più in là una fucina di energia indomabile, ma purtroppo anclue sterile (Montenegro, Albania).

Appunto per ciò soltanto a Venezia potè formarsi un organismo civile, nato, cresciuto e vissuto su questo mare con forze indigene, il quale riuseì per conseguenza ad affermarsi per nove secoli sulla parte più importante $\mathrm{e}$ più contrastata dell'Adriatico.

Anche in altre parti delle coste adriatiche tentarono di germogliare piccoli organismi civili indipendenti, ma o non nacquero vitali oppure, se vissero, lo doveltero più a felici contingenze passeggere e alla tolleranza degli altri che alla propria energia (come si vedrà meglio nella parte storica).

Non essendo dunque l'Adriatico un bacino adallo per la generazione spontanea di una civilta sua propria, ¿̀ naturale che subisse l'influenza delle civiltì dei paesi 
che lo attorniano e che anche la sua importanza sociale non sia assoluta e diretta, ma derivata e proporzionale allo sviluppo civile dei paesi fra $i$ quali ha da fungere da via di commnicazione. Per ciò l'Adriatico ebbe importanza e floridezza quando la civiltà greca in oriente o la latina nel Mediterraneo brillarono di luce intensa: decadde quando il centro della civiltà d'Europa fu trasportato al nord dai Francesi, dai Tedeschi, dagli AngloSassoni; risorgerà cuando ricacciati i Turchi definitivamente e completamente nell'Asia, anche la penisola balcanica rientrerà a far parte del mondo civile.

Questa polrebbe qualificarsi più particolarmente la funzione internazionale dell'Adriatico. Esso ne ha però anche un'altra più modesta, locale, quella di aiutare gli scambi tra i paesi che si specchiano nel suo mare.

Le costumanze e le idee una volta dell'oriente, oggi dell'Europa, penetrarono da uma regione adriatica all'altra per la via del mare. La legge dei contatti, che agisce prima fra paesi contermini, continua poi ad agire anche tra paesi separati dal mare e con tanto maggior effetto, quanto meglio in posizione geografica, atta agli scambi reciproci. L'Adriatico nella sua maggior larghezza raggiunge appena la distanza di 180 chilometri in media e nello stretto fra Otranto e il capo Linguetta si restringe a soli 72 , si presta eccellentemente ad essere attraversato in tutti i punti c quasi con tutti i tempi.

Dei suoi tre bacini quello settentrionale, se non fosse aperto, potreblue essere equiparato ad un grande lago. I contatti tra la sua sponda semicircolare furono sempre vivi e per ciò anche la vita civile in esso è omogenea ed equamente distesa. In quello di mezzo e nel meridionale tutti i contatti devono avivenire attraverso il mare. Nel primo di questi due prevale per importanza marittima ed economica la costa orientale, nel secondo l'occidentale. La fisionomia locale dell'Adriatico è influenzata quindi per due parti dalla costa occidentale e per una sola dall'orientale. Questo potrebbe essere anche un mezzo d'argomentazione per spiegare la superiorità sociale dell'elemento italiano. 
Dei tre mari che bagnano la penisola italica l'Adrialico è meno italiano del Tirreno, ma più del Jonio. Però l'Adriatico, se anche per l'imperfezione geografica del Carso non può essere esclusivamente italiano, non può prosperare senza la cooperazione della costa occidentale, che, secondaria per la navigazione, è la più importante per la civiltà.

Talc è l'Aclriatico, quale ce lo presenta la geogralia come scienza d'indole sociale.

Andiamo a vedere che cosa ha fiatto di lui la storia. 



\section{L'ADRIATICO NELLA STORIA.}

Per la geografia descrittiva l'Adriatico è un bacino chiuso da tutte le parti, meno che da mezzogiorno (canale di Otranto).

Per la geografia sociale è una grande via di comunicazione.

Per la storia è un croginolo, per il quale passarono c in cui si fuscro quasi tutti i popoli principali d'Europa, dell'Asia occidentale e dell'Africa settentrionale.

Scrivere qualche cosa sulla storia delle coste dell'Adriatico equivarebbe a sfiorare e smuovere per due terzi almeno la storia dell'umanità.

A tanto non arriva l'intenzione modesta di questo studio. Per ciò il lettore vorrà concedere renia, se le vicende storiche dell'Adriatico passeranno in una rapicla rassegna, come in un quadro ad acquaforte, in cui non si vedranno che linee grosse, secoli, o linee medie, decenni, lustri, per i fatti capitali. Poche sfumature completeranno l'abbozzo per dar risalto al nesso che esiste tra i fatti naturali e gli effetti sociali ed economici.

I confini montuosi del bacino adriatico sono alti ed immensi, ma non tanto da impedire all'altivilí instancabile dell'uomo - l'essere piccolo ed irrequicto, ma dominatore della terra - d'insinuarsi allraverso ogni depressione del terreno. Però le vie principali di comunic:azione tra il bacino adriatico e il mondo esterno sono state sempre le seguenti:

Sulle Alpi le valli del Po e dell'Adige per le comuISAdriatico. 
nicazioni coll'Europa occidentale e settentrionale; indi l'abbassamento informe tra le Alpi e il Carso quale via al Danubio medio; sugli Apennini per la penisola italica l'altipiano irpino a mezzogiorno, e il passo di Mandrioli, che avvicina alle sorgenti le valli del Tevere, dell'Arno e del Savio a settentrione; sulla costa orientale la valle del Narenta quale strada tra il mare, la penisola illirica e il Danubio inferiore. Da ultimo il mare stesso pel ca. nale di Otranto quale porta coll'oriente e col Mediterraneo.

\section{POPOLI ANTIGHI.}

F́: opinione dei dotti che gli abitanti più numerosi e più antichi del bacino adriatico a noi noti, ossia gli Illiri, residenti ad est di esso, i Japigi all'ovest in Apulia e Calabria ed i Veneti al nord-est, appartengano a razze di origine strettamente affine tra loro. I tratti rimanenti della pianura del Po e della costa occidentale nell'Italia media erano abitati dagli Umbro-Sabelli, popolo italico, ma, secondo i risultati di studi moderni, di lingua affini ai Greci.

Stando a ciò si dovrebbe indurre che già i primi abitatori a noi meglio noti di questo bacino erano etnicamente misti e che il gruppo principale vi sia venuto da oriente nella massa per la via di terra, probabilmente per la valle del Narenta e per l'abbassaménto del Carso, e che solo un ramo di esso si sia distaccato ed abbia attraversato il mare nel punto più stretto. Questa polietnicità, se esatta, sarebbe un fatto notevole, che confermerebbe immediatamente gl'msegnamenti della geografia. Il passaggio per mare da oriente ad occidente invece potrebbe renir spiegato solamente col cammino materiale dell'umanità dall'Asia all'Europa, perchè in seguito i passaggi di popoli dalla costa orientale all'occidentale furono rari e di minima importanza.

Nessuno di quei popoli primitivi si può dire particolarmente marinaro. Fra gli Illiri, che occuparano tutta 
la costa orientale dell'Adriatico dall'Istria al Drilo (odierno Drin, Drino) che è il confine geografico e storico colla Grecia antica, primeggiarono i Liburni e i Dalmati. Il loro centro però stava al di là dello spartiacque carsico e si crede che Delminium, la capitale dei Dalmati, sia da cercarsi oltre il Dinara nella pianura di Lirno in Bosnia. Solo una parte degl'Illiri, i Liburni, acquistarono domestichezza e padronanza sul mare (l'odierno Quarnero) come pirati e si attirarono per ciò le ire dei Romani. Ma erano tanto esperti, che anche vinti, rimasero apprezzati marinai. Le navi liburniche, rapide ed agili, come si sa, ebbero fama nella flotta romana e si distinsero specialmente nella battaglia d'Azio, ove prestarono segnalati servigi ad Augusto.

Pirati e cattivi furono anche quegli Illiri, che abitavano attorno al sinus Rhizonicus (odierne Bocche di Cattaro), dove si ha probabilmente da cercare la sede della loro terribile regina Teuta (odierna località di Teodo).

I rimanenti popoli, o tribù, in cui si suddividevano gl'Illiri furono dediti a preferenza alla pastorizia. Secondo i racconti di storici greci gli Illiri erano forti, fieri, quasi selvaggi, ma dediti al bere e discordi.

Avendo considerato il valore sociale della configurazione geografica del Carso e dei Balcani, laremo noi le meraviglie perchè gli Illiri crano divisi in molte tribù, non avevano un territorio sieuro, erano disuniti anche militarmente e malgrado tutta la loro fierezza non poterono resistere agli attacchi di altri popoli, meglio organizzali?

Come si vede, del resto, la sola geografia ha fatto sorgere fino dai tempi più remoti sulla costa orientale adriatica fra una stessa razza un piccolo popolo di marinai accanto ad uno numeroso di agricoltori.

Strano popolo quello degli Illiri: così numeroso, così forte e fiero, fu conosciuto non per proprio merito, ma per le aggressioni patite dagli altri e sparì senza lasciar traccia nemmeno della propria civillat.

I segni civili dellepoca preellenica, per fuanto non numerosi, che si rinvengono in tutto il hacino adrialice 
(perfino a Pelagosa) portano invece l'impronta della colLura etrusca. Questo popolo, del quale si hanno le prime tracce nella pianura padana, calatosi dalle bassure del Po nel paese degli Umbri diede all'Italia e ai paesi dell'Adriatico la prima civiltà indigena, relativamente evoiuta. E da notarsi dunque che, sebbene il cammino materiale dell'umanità abbia seguito la via dall'oriente all'occidente, già allora un progresso, che possiamo chiamare civiltà, venne nell'Adriatico per controazione dalla parte italica $\mathrm{e}$ più precisamente dalla Padania e dal versante opposto clell'Apennino settentrionale.

Intanto per la porta del mare di Otranto altri popoli marittimi, come i Pelasgi, i Fenici e poi i Greci vennero su in cerca di commerci e fondarono colonie in tutti i punti migliori delle coste e delle isole dell'Adriatico.

Fin dalla prima alba storica troviamo quindi sulle coste adriatiche una sovrapposizione di razze e per conseguenza popolazione mista, con colonie di navigatori attivi, più evoluti al mare e popoli dediti alla pastorizia, di minor conto, a distanza nell'interno. E ancora a conferma della geografia, dai differenti bisogni economici sorsero attriti fra gli abitatori della costa e quelli dell'interno, che, come si vedrà, degenerarono nella chiamata in soccorso degli stranieri, per l'incapacità in cui si trovavallo i litiganti di soverchiarsi colle proprie forze.

I Galli. Questo popolo dell'antichità, avventuroso, guerriero e coraggioso, che col suo numero scosse e distrusse molti Stati, ma mon fu capace di formarme esso stesso uno durevole e veramente importante, discese nel III secolo a. C. dalla sua prima patria nota, la Gallia, anche nella fertile pianura del Po; ne occupò dapprima la riva sinistra, indi anche la destra (Gallia Cisalpina, divisa alla sua volta in trans e cispadana) e si spinse lungo il versante settentrionale degli Apennini fino all'Adriatico (costa emiliana odierna) nel territorio che da loro prese il nome 
di ager Gallicus (Galli Semmones). Da cui passarono il mare e fondarono colonic sulla costa orientale, fra cui Senna gallica, l'odierna Segna, dirimpetto a Sinigaglia o Senigallia.

Era inevitabile che quando poco dopo passarono gli Apennini, cozzassero coi Romani che venivano espandendosi all'insù. I Galli diedero ai Romani molto filo dia torcere, ma al fine le armi romane, innegabilmente superiori, ebbero la vittoria.

Frattanto altri rami di questi Galli, che erano trasmigrati sul medio Danubio e nell'Illiria, allettati dalle incessanti discordie intestine ond'erano traragliate la Nacedonia e la Grecia, invasero anche questi paesi. Ma non seppero sostenersi nemmeno qui e finirono col disperdersi, senza lasciar notevoli tracce benefiche del loro passaggio.

La parte superiore del bacino adriatico servi loro solamente da via di passaggio ed essi per l'Adriatico non furono altro che causa di nuovi sconrolgimenti. Causa l'irrequietudine nel cambiar sedi e la relativa poca inportanza per l'Adriatico sarebbe superfluo di indagare l'influenza della geografia sul carattere e sulla storia dei Galli.

A questo punto non sarà lorse invece fuori di proposito ricordare che all'epoca del predominio greco, l'Adriatico era un golfo secondario, nido di pirati, e che della penisola illirica non uscirono volontariamente dall'oscurità che la Macedonia e la Tracia (recenti vilajet Lurchi di Salonicco e di Adrianopoli).

Per lortuna dell'Adriatico crescera intanto poco lontano da lui in posizione specialmente farorita dalla natlura un popolo chiamato ai più alti e gloriosi destini.

La struttura geografica della Grecia offre da sola la spiegazione dei pregi e dei diletti del popolo greco. II clima temperato ed il felice accoppiamento della terra col mare, aperto da tutte le parti all'intorno, li spronavano ad una duplice attiviti di vita intellettuale in terra e di espansione commerciale sul mare. La terral, fertile. ma insufficiente a contenere l'esuberanza di popolo, con- 
tribuiva a spingerli ancora più sul mare, che già colla stragrande ricchezza di isole, al di là delle quali stavano fertili continenti, allettava all'espansione marittima.

Equilibrio intellettuale, attività, intraprendenza sono quindi i pregi dei Greci.

Ma il paese di Grecia ha il difetto naturale di mancare di un centro unico, comune, accettabile da tutti e capace quindi di agire come forza di coesione e fulcro di irradiazione. La storia greca ne ricorda per ciò parecchi, Atcne, Sparta, Tebe, Messene ed è auncbbiata dalle. incessanti e improficue lotte intestine per la supremazia.

L'animo discorde, i ripetuti assalti di nemici esterni, chiamati più volte da Greci stessi e la debolezza finale contro l'incalzare dei colpi avversari, non sono dunque anch'essi che conseguenze storiche della struttura e della posizione geografica del paese.

L'espansionc greca avvenne per lo più alla chetichella e a tappe, da isola ad isola, con soste per consolidarsi e riprender fiato. Le conquiste non ebbero sempre come punto di partenza la madrepatria, ma anche le colonie maggiori, una volta consolidatesi, ne intrapresero delle altre, spesso in contrasto e in lotta cogl'intendimenti della madrepatria. Quando arrivavano ad un continente i Greci non si pensavano di conquistarlo tutto, ma si limitavano ad occupare i porti, le penisole, le parti più fertili e lasciavano ai barbari indigeni il possesso indisturbato delle parti meno buone, da loro rifiutate. Essi non cercavano. che il dominio del marc. Si presentavano come mercanti più che come dominatori e per ciò erano per lo più accettati senza resistenza.

La loro espansione potrebbe paragonarsi allo sviluppo della pianta delle fragole, che spinge $\mathrm{i}$ suoi pampini in tutte le direzioni e li appoggia alla superfice del terre120, e dove trova terriccio conveniente immette nuove radici, dalle quali trae i succhi e la forza per proseguire il cammino.

L'Egeo c̀ il mare classico dell'espansione greca. A noi occorre invece rilevare che anche tutto l'Adriatico fu da loro inondato e beneficato per secoli e secoli. 
Seguendo dapprima la prolungazione della loro cosla occuparono la parte meridionale del paese degli Illiri, che da loro prese il nome di Illirico greco (probabilmente l'odierna Albania fino al Drin). Di là si spinsero in su lungo la costa orientale dell'Adriatico fondando colonie e dando il nome, conservatosi fino ad oggi a tutte le penisole ed isole principali della Dalnazia: Molonta, Calamotta (una delle Elafiti), Melitta (Meleda), Korkyra (Curzola), Pharia (Lesina), Bracchya (Brazza), Issa (Lissa) e fondando città Epidamnos (Alessio), Dyrrachion (Durazzo), Antibarion o Antibaris (Antivari), Epidaurum (Ragusavecchia), Epetion (?), Tragurion (Traú, oggi in parte distrulte e in parte esistenti con nomi storpiati. L'odierna Alessio però potrebbe essere più facilmente l'antica Lissos, fondata dai Siracusani, che nella loro auge ai tempi di Dionisio aspirarano al dominio del mare Adriatico.

Dall'altra costa nell'Italia meridionale il numero di colonie e il fiorire della lingua e coltura greca furono tanto grandi che il paese meritò il nome di Magna Graecia. Ancol oggi i nomi delle citta italianc alla costa adriatica lanno chiara origine greea (Brindisi, Monopoli, Bari, Trinitapoli, Rodi, Garganico).

Bisogna poi notare che i Greci arrivarono fino all'ultimo golfo settentrionale dell'Adriatico. Ancona è di indubbia origine greca. Capodistria si chiamava anticamente Iustinopolis. Secondo alcuni dotti i nomi d'Istria e Pola sarebbero anche di origine greca.

Colla comparsa vitloriosa dei Romani lo sjplendore del dominio greco nell'idriatico andò gradatanente decadendo fino a tramontare colla riduzione della Grecia a provincia romana (fine del II sec. a. C.). Ma la lingua e civiltà greche non perirono ancora, perchè assendo superiori a quelle dei Romani, luromo usate da loro stessi per l'ulleriore progresso. Anzi, appena caduto l'impere romano d'occidente e passato il potere a Bisanzio, lolenento greco rivire nell'Adrialico.

Bisanzio possedette per oltre fluattro secoli quasi fulle r hizantin. le due coste dell'Adriatico, è vero, forse più clue per sua 
virtù, per l'avversione innata del mare, che tenne Goti, Longobardi e Franchi lontano dalla costa.

Tracice storiche maggiori di questo dominio sarebbero: la provincia (Thema) Dyrrachion, odierna Albania, indi la Dalmazia, o Thema Dalmatia, sulla costa orientale, che comprendera le cittì municipali romane di Zara, Traú, Spalato, Ragusa e Cattaro in terraferma e di Veglia, Cherso ed Arbe sulle isole, sopravvissute alle devastazioni dei barbari. Sulla costa occidentale, l'esarcato di Ravenna (odierua Romagna) e la Pentapoli (Rimini, Pesaro, Fano, Sinigaglia ed Ancona) da cui dipesero amministrativamente tutta l'Istria, Trieste e perfino Venezia. Infine tra il X-XI sec. d. C. nella bassa Italia al posto della Magna Graecia la provincia Thema Italia colla capitale Bari. Col possesso delle tre regioni principali, Puglia, Romagna e Dalmazia e del punto strategico d'Albania, i Bizantini godettero di fatto il predominio dell'Adriatico.

Ma l'influenza greca decadde realmente quando fu insidiata da due nemici, ad occidente dagl'imperatori di Germania, ad oriente dagli Arabi e poi dai Turchi.

L'esarcato e la Pentapoli tolti da Astolfo, re longobar(lo, andarono perduti definitivamente quando Pipino il Breve li fece cedere al Papa (756 d. C.) e diede così, proprio in una regione dell'Adriatico, origine al potere temporale dei pontefici. Altri imperatori germanici conlinuarono a combattere i Greci Bizantini nell'Italia meridionale, finchè non riuscì loro di darla in feudo ai Normanni, che al principio del secolo XI vi fondarono il reame delle Puglie. A loro si aggiunsero dal IX secolo altri nemici, Venezia e gli Ungheri, che privarono i Greci dell'Istria e della Dalmazia e da ultimo i Turchi, che diedero loro il colpo di grazia. Caduta anche Costantinopoli sotto i colpi della mezzaluna (1453) le colonie greche dell'Adriatico vissero come e quanto poterono. Furono distrutte, assorbite o assimilate ed oggi non esistono più come tali. Ma resiste ancora più che un semplice ricordo di loro in tutto l'Adriatico nei nomi di località, nei tipi, nei costumi, nella lingua dei popoli che 
vi si sovrapposero. E ognuno deve pensare con rammarico e con rispetto a questa potente face di civiltà che illuminò per oltre venti secoli la strada dell'Adriatico. Oggi navi greche lo solcano ancora c la lingua è ancora conosciuta nei porti maggiori, ma troppo umilmente in confronto ai tempi passati. Però siccome la grandezza dei Greci cbbe origini spontanee, e derivò da cause naturali, eterne, forse non ̀̀ vana la speranza, che, cessate le forze che la opprimevano, la luce della civiltà ritorni a germogliare su quella classica terra, unica veramente felice nei Balcani.

Intanto le sciagure della Grecia possono servire almeno di ammaestramento agli altri e insegnare che la civilti da sola, anche se portata all'apice della sua altezza ed intensità, non basta a conservare l'esistenza d'un popolo e perisce se non è accompagnata e sorretta dalla forza. La civiltà tra i suoi immensi vantaggi ha il clifetto di distogliere gli uomini dal lavoro manuale e pesante. Si forma allora uno squilibrio tra l'infinità dei bisognni $\mathrm{e}$ la ricchezza dei popoli civili e la miseria necessariamente ingorda ed invidiosa dei poveri. Avviene allora che i meno civili e talvolta rozzi, ma che dispongono invece di tutta la loro forza, anche se brutale, con un atto barbarico, ma naturalmente spiegabile, si procurano violentemente quello che viene loro conteso in nome di un principio elevato, ma vano praticamente. Quante cose (lolor()se non si apprendono studiando quella cosidetta maestra della vita, ch'è la storia!

I fatti della storia romana sono generalmente tanto noti, che basterà rilevare le ragioni geografiche della loro espansione nell'Adriatico.

La penisola italica ha dato ognora alle genti che l'abitarono due occupazioni e due fonti di ricchezzal l'agricoltura e la marina.

Il sole e la bellezza della terra e dei mari hanno infuso ai suoi abitatori esuberanza d'intelletto e di vitalita, per cui la penisola italica è stata sempre un paese con sovrabbondanza di popolazione attiva e da lei partirono per conseguenza in tutti i tempi correnti migratorie.

IiAdriatico.

I Romani. 
C'è però una differenza essenziale nel genere della loro espansione da quella dei Greci. I Greci, popolo artista, marinaro e appena in terza linea agricoltore, dalle condizioni della loro patria, ricca di mare, povera di terra e priva di un centro comme direttivo, erano indotti a vivere dispersi ed a cercar la ricchezza preferentemente sul mare e sulle coste. I Romani invece, popolo agricoltore, imperioso e in terzo grado marinaro, si sentirono avvinti moralmente al centro matematico della loro città e si avventurarono compatti sul mare, come su una buona via per raggiungere altri continenti, ch'essi occuparono per progetto e per intero fino all'arresto di qualche limite naturale. I Greci colonizzarono col commercio e colla superiorita della loro lingua e coltura: i Romani invece imponendosi con tutti i mezzi concessi dalla forza.

Volendo cercare anche per i Romani un termine di confronto nel regno vegetale, bisognerebbe paragonarli ai cipressi ed ai pini, che crescono rigogliosi e superbi sui colli toscani e nella campagna romana, alberi sempre vivi ed esclusivisti, che non tollerano altre piante accanto a loro, ma che assorbendo tutti i succhi della terra fanno deperire gli altri modesti alberi frondiferi a fogliame caduco.

Dalla fondazione della città occorsero ai Romani quattro secoli per allargare il dominio sul solo Lazio, vincere la potenza etrusca e le invasioni dei Galli a settentrione, e regolare i loro ordinamenti civili interni.

Per quanto vittoriosi erano ancora troppo pochi numericamente per avventurarsi al settentrione degli Apennini. La regione Emilia d'altronde doveva essere allora tutta coperta di boschi e per ciò si presentava come povera e poico allettatrice per un popolo agricolo come i Romani. La configurazione del paese, aperto a mezzogiorno, e lo stato migliore dei campi della bassa Italia, già dissodati dai coloni greci, rese loro più facile ed allettò. l'espansione intanto da questa parte. Questi dovrebbero 
essere i motivi per cui i Romani verso il 400 a. C., anzichè proseguire le concuiste nella Padania, si arrestarono alla linea Macra-Rubicone.

La preparazione lunga fu benefica, perchè li mise in grado di affrontare i Samniti, loro terribili vicini, che abilavano quell'altipiano irpino, descritto geograficamente nella prima parte.

I Sanniti erano un popolo valoroso e superiore ai Romani tanto per munero, quanto per estensione di dominio, ch'essi cercavano naturalmente anche di ampliare: ma mancavano di organizzazione ed erano un po' snervati dalla coltura greca. Venuti a lunga lotta coi Romani soccombettero all'insuperabilità del rigido ordinamento politico di questi.

Le guerre dei Romani coi Samniti durarono mezzo secolo, ebbero, com'è naturale, varia vicenda, ma terminarono colla sottomissione dei Sammiti. I Romani averano ora libera la via all'Adriatico e all'Apulia. Di ciò si preoccuparomo le citlà coloniali greche dell'Italia meridionale e specialmente Taranto. (La Grecia era ormai caduta da mezzo secolo sotto i colpi di Alessandro Magno).

Però le colonie greche oltrechè essere disunite erano ánclıe effeminate e per ciò, secondo quell'uso che potrebbe qualificarsi maliziosamente greco, chiamarono in aiuto l'ambizioso Pirro, re d'Epiro, dall'altra costa. Questa guerra ebbe durata breve da due a tre lustri e terminò coll'assoggettamento di tutte le colonie greche e di altre popolazioni minori. Nel 266 a. C., quindi circa cinque secoli dalla fondazione della città, i Romani crano i dominatori della media e della bassa Italia, ossia, per quanto interessa particolarmente fuesto studio, della parte meridionale della costa occidentale adrialica fino al Rubicone (al nord di Rimini). Anzi questo liume sull'Adriatico e il Macra dallallea parte del Tirreno (al sud del gollo di Spezia) servirono per indicare i confini geogralici d'Italia lino al tempo di Augusto, in cui questi colla soltomissione definitiva della Gallia cisalpina e con una razionale ricostituzione dello Stato romano, portio quelli dell'Italia civile al di là della piantra del loo lino 
alle $A l p i$ al nord ed ai fimmi Varo ad occidente ed Arsia ad oriente.

Ai Romani vittoriosi e sempre più forti doveva star ora a cuore, per ragioni geografiche, il possesso della Sicilia e poi della Sardegna. Così si trovarono di fronte ai Cartaginesi, che li attrassero in Africa e coi quali impegnarono quella lotta gigantesca, che nelle sue conseguenze procacciò ai Romani il dominio del mondo.

Le guerre puniche hanno per l'Adriatico un'importanza relativa e devono venir ricordate come causa indiretta, in quanto che per esse i Romani divennero quello che oggi si suol dire una potenza marittima, e da allora in poi rivolsero le loro cure guerresche non solo all'esercito ma anche alla flotta.

Quali possessori della costa occidentale adriatica essi non potevano vedere di buon occhio le incessanti piraterie dei popoli illirici, annidatisi sulle coste istriane, liburniche (Quarnero) e nel sinus Rhizonicus. Per ciò, dopo che un'ambasciata alla loro regina Teuta a Scodra (Scutari) non sortì alcun effetto, appena terminata la prima guerra punica, senza cercar pretesti, ma per la libertà del commercio e pel bisogno di mantenere, specie nell'Adriatico, il dominio del mare inviarono una squadra di duecento legni, che distrusse la potenza navale degli Illiri e sradicò la pirateria. I Romani colsero da ciò il destro di stabilirsi sulla costa orientale dell'Adriatico, a Corcira (Corfù, piuttosto che Curzola), Epidamno, Alessio o Durazzo ed in altri luoghi dell'Illirico greco, che fu la loro prima conquista su questa costa. - Dyrrachium, nella colonia greca di Epidamnos, è quindi una delle città adriatiche più importanti per la storia dei rapporti dell'Italia coll'oriente, giacchè oltre ad essere il primo acquisto romano oltre l'Adriatico era la città più vicina a Roma, dalla quale per la via Appia da Brindisi non distava che quattro giornate di cammino e una di navigazione. 
Per chi studia le relazioni fra le due coste dell'Adriatico, il passaggio dei Romani sulla costa orientale, per quanto rivesta l'apparenza poco simpatica della prepotenza, è un fatto straordinariamente noterole, perchè dimostra come l'espansione romana avveniva per esuberanza di vita, in nome di una superiorità civile, con una forza conscia e con meta precisa. Essa insegna inoltre che i Romani nell'intraprendere tale atto d'impero non poterono fare a meno di seguire la via naturale, tracciata dalla geografia, lungo la penisola messapica, detta allora Calabria, e di passare quindi l'Adriatico nel suo punto più stretto.

Ciò avveniva alla fine del III secolo a. C. quando la sorte dei Romani era rella parte ascensionale della parabola e alla distanza di meno di un secolo dall'apogeo della sua potenza, se non del dominio, che fu raggiunto verso il 150 a. C.

Posto il piede sulla costa orientale adriatica, i Romani furono distolti dal proseguire la loro mareia subito dopo in parte dalle molestie dei Galli e in parte dalla seconda guerra punica.

Per l'Adriatico interessa più la nuova guerra contro i Galli, giacchè riusciti vittoriosi, i Romani sottomisero in gran parte la regione del Po, piantandovi colonie, e verso il 200 fu ripigliata e continuata la soltomissione tolale dell'alta Italia, rimasta interrotta dalla scenda guerra punica.

Nell'intervallo di cinquant'anni fra la seconda e la terza guerra punica (prima metà del II sec. a. C.) l'attenzione dei Romani fu attratta di nuovo al loro oriente nella penisola illirica, ove non avevano ancora interessi da propugnare, ma dove suscitava loro gelosia l'ingrandimento della Macedonia.

$\mathrm{Si}$ coinvolsero così in tre guerre, nella seconda delle quali i Macedoni guadagnarono alla loro alleanza il $\cdots$ Genzio dell'Illiria.

Conquista romana dell' Illiria. 
L'ainto prestato da Genzio ai Macedoni gli trasse addosso l'immediata vendetta dei Romani. Nel 167 a. C. anche la potenza terrestre degli Illiri fu sconfitta; il loro paese fu sottomesso e diviso in tre repubbliche. I Romani comandavano ora sul mare Adriatico e per conseguenza anche su tutte due le coste, sulle quali non vivevano in quell'epoca seri competitori. Padroni incontrastati di tulti i tre mari che bagnano la loro penisola, prima del Mediterraneo coll'Ârrica, indi del Jonio ed ora anche dell'Adriatico colla penisola illirica, avevano raggiunto l'apogeo della potenza.

Fiaccata la polenza terrestre degli Illiri incominciò la penetrazione e la colonizzazione romana della costa orientale adriatica. Gli Illiri non ristettero ciò non di meno dall'opporre tutta la resistenza di cui erano capaci. La lotta però era impari ed i Romani dovevano fin da principio essere certi della vittoria. Gli Illiri non erano arrivati che al primo stadio di coltura, ossia a quegli albori, che non hanno nemmeno la forza di diradare le tenebre della barbarie. Inoltre per le condizioni geografiche della penisola da loro abitata ed appellata, erano divisi in tante piccole tribù, con nomi ed interessi particolari. Dovevano fatalmente e logicamente soccombere agli assalti dei Romani, che compatti e ben diretti, si trovavano nel fiore della loro organizzazione civile e militare.

L'impresa fu quindi facilitata ai Romani dalla debolezza e dalle discordie della popolazione, ma ostacolata dalla natura del terreno.

Gl'Istri, di cui rimangono ancora numerosi rustici castellieri, opposero ai Romani una seria e lunga resistenza (183-177 a. C.).

Però fra le repubbliche illiriche formatesi dopo la caduta di Genzio primeggiava quella dei Delmati o Dalmati. Contese di dominio tra i Dalmati illirici in terraferma ed i coloni greci delle isole (specialmente di Lissa), che chiamarono anche qui in aiuto i Romani stranicri, porse a questi il destro per comparire nel 156 a. C. con un poderoso esercito ad espugnare Delminium.

Alle vittorie campali romane gli Illiri opposero un mez- 
zo di resistenza non meno efficace: le insurrezioni e le ribellioni, preparate e scoppiate cuando i Romani crano meno attenti o inviluppati in altre imprese. Velleio Patercolo lasciò scritto che i Dalmati si ribellarono più di duecento volte, prima di essere sottoposti all'impero romano.

Per sedare le maggiori di queste ribellioni i Romani dovettero condurre ben nove guerre, che con alterna fortuna si protrassero per oltre un secolo e mezzo (dal 156 a. C. al 12 d. C.).

Da una sopraffazione intestina dei Dalmati a danno dei Liburni i Romani, chiamati in soccorso da questi ultimi, ebbero occasione d'intromettersi mezzo secolo circa a. C. anche nell'Istria montana.

La lentezza dell'avanzata e la breve durata delle vittorie romane e le ripetute guerre coi popoli della penisola illirica si spiega qui, come nella penisola iberica, colla conformazione del terreno. Nel mentre dopo una vittoria campale p. e. sui Galli nelle regioni del Po, i Romani potevano spaziare su ampi tratti di terreno e per tenerli soggetti bastava che vi erigessero singoli grandi presidi militari e colonie agricole, che poi colla superiorità civile ed economica operavano la lenta e pacifica assimilazione dei vinti, nella penisola illirica una vittoria non fruttava il possesso che di vallate. I Romani erano per ciò costretti a piantarvi spessi, piccoli presidi e colonie, di minore efficenza militare e civile, mentre gl'indigeni potevano appiattarsi semi-liberi nelle boscaglie e sui monti delle catene successive e preparare imboscate.

Per ragioni sopratutto geografiche anche la romanizzazione della penisola illirica procedette stentata; però alla fine di queste lotte bisecolari l'Illirico greco, la Dalmazia (compresa l'odierna Bosnia) e l'Istria erano converlite in un vero giardino e godevano di una prosperiti mai veduta prima, nè dopo. La lingua, gli usi, i costumi, la religione dei Romani vi divennero dominanti c cessata l'avversione, anzi cresciuto l'affetto per la romaniti. quei paesi vissero in pace senzar dar da parlare di sè e 
divisero coll'impero romano per. secoli, prima il benessere, poi le sciagure.

I Cimbri. Colle guerre contro gli Illiri i Romani miravano ad assicurarsi il dominio duraturo dclla costa orientale adriatica. A tale scopo occorreva loro di recar pienamente in proprio potere anche i paesi situati a nord e ad est di questa costa, vale a dire di chiudere la porta del Carso. Per ciò avevano già posto il piede gradatamente nell'odierna Carniola e Carinzia. Ma su questa via i Romani si trovarono di fronte inaspettatamente un nuovo e temibile nemico, non indigeno nè in moto di progresso, come finora ovunque, i Cimbri. Erano calati essi nientemeno che dalla Scandinavia e rappresentano il primo accenno di quelle trasmigrazioni dal nord, che dovevano riuscir fatali e dar l'ultimo colpo allo Stato romano.

Da loro i Romani subirono la tremenda sconfitta di Norcia (nel 113 a. C.). - Per fortuna dei Romani i Cimbri, anzichè calare subito in Italia, si mossero verso la Svizzera, la Gallia e la Spagna e solo al ritorno da questi paesi si ripresentarono in Italia per il passo del Brennero e per l'Adige (l'altra porta del Trentino). Ma questa volta furono ricevuti da Mario, che li sconfisse pienamente ai Campi Raudii presso Vercelli (nel 101 a. C.). Mario ebbe in premio il titolo di terzo fondatore della città (dopo Romolo e Camillo).

\section{LA PACE ROMANA DI SETTE SECOLI. PERIODO D'ORO DELL'ADRIATICO.}

Prescindendo dalle lotte di valore secondario e locale per arrotondare le conquiste nella penisola illirica, si può dire che l'Adriatico con tutte le sue coste, dal primo secolo a. C. al quinto d. C., fu un lago romano, sul quale non risuonò che l'eco di avvenimenti interni della storia romana.

Durante questo lungo periodo, per quella legge di na- 
tura contraria all'immutabilità dell'uomo, andarano maturandosi avvenimenti destinati a portarvi mutamenti radicali.

Per comprenderli meglio sarà opportuno di considerare l'aspetto del bacino adriatico durante la pace romana di sette secoli abbondanti, che a ragione si può dire il suo periodo d'oro.

Se metteste davanti agli occhi d'una persona dotata di raziocinio naturale, ma di scarsa coltura, una carta geografica dell'Adriatico, domandando in qual modo può essere avvenuta l'espansione romana dall'Italia sulla costa orientale, è più che probabile che il suo dito segnerebbe da Roma al nord oltre gli Apennini per il Veneto e l'Istria, indi giù, giù, fino alla Grecia.

Invece la storia, seguendo le leggi della geografia in natura anzichè sulla carta, insegna che quest'espansione avvenne in tutt'altro modo. Il primo moto di allargamento dal centro di Roma doveva avvenire in senso circolare e, seguendo la tendenza di raggimngere la corona di monti, spingersi in principio piuttosto a settentrione. Siccome però la barriera dell'Apennino tosco-emiliano e del marchigiano è più alta che quella dell'Apennino napoletano (altipiano irpino) e del hucano, doveva necessariamente succedere un arresto a settentrione e l'espansione proseguire verso il mezzogiomo, seguendo la legge del sole, per cui tutti i popoli si mossero dai loro paesi in regioni più calde. Per non citare che popoli d'Europa vediamo che i Greci si propagarono in tutto il bacino mediteraneo, ma non oltrepassarono etnograficamente la linea dei monti al loro settentrione: che i Germani della Scandinavia e gli Slavi della Sarmazia non si diressero al polo, ma calarono nel mezogiorno d'Europa e nelle tiepide regioni d'Italia e di Grecia.

Anche l'espansione romana, finchè ebbe per oggello l'Italia colle sue grandi isole, le coste d'Alrica, di Iberia, 
d'Illiria ed infine la pianura padana, similmente a quella dei Greci, non seguiva altro che leggi geografiche naturali; ed a questo punto raggiunse l'apice della potenza.

L'espansione romana, inseguendo sempre la sua meta politica particolare, poteva fino a questo punto essere ignara della sua grande missione storica. Formato dai cento popoli d'Italia uno stato unitario, i Romani si videro immanzi agli occhi un nuovo obbiettivo, quello di consolidare definitivamente il loro possesso, e per ciò, abbandonando il sistema segtuito fino allora delle vie naturali più facili e di tendere ai paesi più fertili, si accinsero con maggiore precisione d'intendimenti a conquistare regioni montuose, come il Norico, la Rezia e la Gallia onde murare le tre porte aperte: quella del Carso al di là del sinus tergestinus, quella dell'alta valle dell'Adige a settentrione e cuella del fiume Varo della Gallia Narbonensis ad occidente. La geografia sola li avrebbe arrestati ai piedi di queste vie, troppo in basso. Per raggiungere le vette di confine fu necessario uno sforzo di volontà ed i Romani lo compirono.

Fin qui l'espansione romana fu conquista di popolo: tutti gli altri acquisti territoriali successivi furono fatti, per usare una frase moderna, con intendimenti burocratici o diplomatici, e furono più o meno effimeri.

L'Adriatico fino dal II secolo a. C. fu politicamente romano. Può dirsi che sia stato anche etnograficamente?

Da principio no sicuro, e ci sono motivi per ritenere: che non lo sia stato del tutto nemmeno in seguito.

La sottomissione dell'Illiria terminò appena nel 12 d. C.: ciò indica che esisteva fino allora un elemento etnografico ribelle e capace di reazione. Bisogna poi concedere almeno mezzo secolo per l'assorbimento degli ultimi resti superstiti, ed è da domandarsi ancora se tale assorbimento completo degli Illiri arvenne fino alla caduta dell'impero romano.

Le regioni del Carso (oggi detto Carniolino e Croato) subirono le impronte della civiltà romana; però siccome si tratta di paesi poveri e secondari, montuosi e dal 
clima rigido, è probabile che i Romani non li abbiano colonizzati completamente in modo da togliervi ogni traccia dell'anteriore popolazione. Si sa inoltre che Aquileja era l'ultima grande stazione romana in Italia sulla via Aemilia e che a questa era affidato principalmente il compito di reclutare tutte le milizie semi-barbare, necessaric a completare coll'opera dell'arte lo sbarramento del confine paludoso, naturale.

Anche l'assimilazione dei popoli alla costa occidentale adriatica deve aver costato tempo e fatiche. La storia ci ricorda che nei torbidi che seguirono le riforme dei Gracchi avvenne un'insurrezione della maggior parte dei popoli italici, nella quale i Samniti erano i capi, mentre le città greche, perchè bene trattate, tennero dalla parte delle città latine e dei Romani.

L'elemento greco nell'Adriatico era troppo numeroso ed evoluto per poter venir soppresso d'un tratto. La grandezza romana seppellì la loro importanza politica, ma non la lingua e la civiltà, alle quali anzi i Romani attinsero continuamente ed abbondantemente per progredire. Cesare, quando fu padrone assoluto dell'impero nel 45-44 a. C., concepì il disegno di fondare una grande monarchia mediterranea (l'Adriatico vi sarebbe slato compreso naturalmente) in cui mediante la colonizzazione latina, doveva essere cancellata la differenza delle nazionalità. Solo la greca, che per il suo grande passalo e per la sua letteratura, si raccomandara particolarmente ad un nomo colto come lui, doveva essere risparmiata. Il pugnale che troncò la vita di Cesare, salvò involontariamente l'esistenza a diversi popoli.

Già nel I secolo a. C. le ricchezze ed i vizi, che le accompagnano, fecero un po' vacillare l'organizzazione civile romana. La vitalità era però nella massima esuberanza e questo è il secolo d'oro della letteratura romana. A tale splendore i popoli dell'Adriatico non diedero un contributo noterole, segno forse anche fuesto che non erano completamente romanizali.

In questo e nel successivo secolo d'argento della letteratura (I e II sec. d. C.) avenne tra le due roste del- 
l'Adriatico una corrente di flusso e riflusso per la guale dall'Italia veniva sulla costa orientale la lingua latina, la ricchezza e la civiltà e dall'orientale all'Italia la forza rude, sotto forma di schiavi barbari, da assimilarsi per cementare la potenza dello Stato.

Probabilmente da questo tempo incomincia a datare l'infinità di ville e di stazioni balneari, costruite dai patrizi romani in tutte le insenature delle due coste adriatiche e che ancora oggi non sono interamente scoperte. Le città, fondate o ampliate prima dai Greci, furono ravvivate col commercio e rafforzate militarmente, però senza crescere ad importanza straordinaria. I grandi scali commerciali e strategici erano pochi. La tattica romana, così in guerra come nell'arte, era di costruire poche opere, ma grandiose, perchè fossero più resistenti. I porti romani di prima classe erano a Brindisi, Ravenna, Rimini, Aquileja, Pola, Salona e Durazzo, Iuoghi scelti ottimamente, perchè Brindisi, Pola, Salona e Durazzo erano protetti dalla natura contro assalti dal mare, Ravenna, Rimini ed Aquileja, chiuse da lagune, da maremme e da boscaglie, erano inaccessibili per qualsiasi nemico non pratico dei luoghi. Brindisi e Durazzo chiudevano l'entrata nell'Adriatico; Ravenna e Pola facevano da controporta per il bacino superiore; Pola inoltre dominava la costa liburnica. Aquileja era la chiave che teneva il passaggio tra l'Italia e il Danubio. Salona era una grande base navale di appoggio e di sbocco del commercio della pianura della Pamnonia e del basso Danubio.

Basterebbe la scelta di questi punti sull'Adriatico per far ammirare l'immensa intuizione pratica dei Romani, che non facevano le cose solamente per dire di averle fatte, ma le studiarano con criterio d'utilità e tenendo calcolo delle esigenze e dei vantaggi naturali per farle benc. Brinclisi oltre che un buon porto era lo scalo delle regioni fertili dell'Italia meridionale. Ravenna era lo scalo importantissimo della Padania; Aquileja il punto di congiungimento e di sbocco delle strade dalla Rezia e dal Norico; Pola in regione povera, ma protesa sul mare, era stazione principalmente navale; Salona, una pic- 
cola Roma all'estremità della linea più breve tra l'Adriatico e il Danubio, in posizione favorevole per retroterra, era la sede naturale e migliore per la capilale della vasta provincia d'Illiria; Durazzo era una tappa delle vie Appia ed Aegnatia, ossia della strada più breve tra Roma e la Grecia. Tutti gli altri punti, senza retrolerra e quindi condannati dalla natura ad essere aridi, che poi in condizioni anormali, irregolari o immaturali acquistarono fama e importanza, come Antivari, Rizinium ora Risano nelle Bocche di Cattaro, Epidauro (Ragusa), Jadera (Zara), Tersatica (Tersatto-Fimme) Tergeste (Tricste), Ancona ed anche Bari erano per i Romani luoghi secondari. La geografia e la storia confermano che la scelta dei Romani era ben fatta.

Il possesso della costa orientale adriatica mostrò ai Romani le strade fluviali per arrivare alla fertile Pannonia e al basso Danubio. Per di qua devono essere passati gli eserciti di Trajano (100 d. C.) che sottonisero la Dacia (odierni paesi rumeni), colonizzarono la nuova provincia e latinizzarono il Danubio.

Gli effetti della romanizzazione della costa orientale dell'Adriatico sotto forma di contributo intellettuale alla civiltà latina si fecero sentire appena più tardi. Ed cra naturale. Durante i quattro secoli che stamno allorno all'era di Cristo questa costa fu seminala e fecondata. I frutti dovevano nascere in seguito, e nacquero con Diocleziano nel III secolo d. C., San Girolamo nel IV, Giustiniano nel VI, come l'eco che risponde in ritardo dal fondo della valle ai rumori che furomo prodotti all'altra estremità.

La romanizzazione della costa orientale adriatica lu lenta e difficile, perchè la natura rende i popoli di questa restii ad ogni innovazione. Ma in compenso di poi l'attaccamento all'idea romana fu per la stessa ragione più tenace che altrove. Quando nel 476 (l. C. ebbe line in Roma per opera di Odoacre la diguita imperiale, Giulio Nepote, ultimo inglorioso imperatore romano, si riliri in Dalmazia, ove fu riconosciuto lanto dall'imperalone greco quanto da Odoacre. Proprio sulla sponda orientale 
adriatica e per l'affetto dei Dalmati alla romanità la dignità imperiale potè sopravvivere ancora quattr'anni, almeno di nome, e mandare da (fui l'ultimo bagliore, avanti di spegnersi per sempre.

\section{EVO MEDIO.}

L'Adriatico durante le incursioni dei barbari.
Da quel momento cambia l'aspetto politico dell'Adriatico.

Però già molto tempo prima v'erano stati dei sintomi di mutamenti nazionali, ma in senso contrario agli scopi perseguiti dai Romani.

Era passato appena un secolo forse dalla completa romanizzazione della costa settentrionale ed orientale dell'Adriatico, che già alcuni di quei popoli barbari, che i Romani avevano dovuto combattere fuori del bacino adriatico e dei confini d'Italia per tenerli appunto lontani da queste due regioni, erano riusciti ad infiltrarsi al di qua dei monti che fanno da spartiacque all'Adriatico.

Nel 250 d. C., quando l'impero era sossopra per le continue ribellioni dei soldati, gli Alemanni mettevano impunemente a contribuzione l'alta Italia. Nel 265 d. C. la costa orientale dell'Adriatico, e specialmente Epidauro, fu saccheggiata dai Goti. Nel 270 d. C. l'imperatore Aureliano ebbe da respingere un'incursione dei Marcomanni in Italia. Nel 279 d. C. l'Illiria subì un primo saccheggio per opera di popoli Slavo-Sarmati, che vi rimasero finchè non riuscì all'imperatore Probo di scacciarneli nel 281 d. C.

L'ordinamento civile dei Romani, che avera costituito la base della loro forza, era ormai troppo corrotto e con esso decadera anche la potenza militare. Queste incursioni fino all'Adriatico e alle porte d'Italia erano segno che il grande impero precipitava al tramonto.

Passò ancora un secolo di decadenza per i Romani e di preparazione per i barbari, durante il quale $\mathbf{i}$ rigagnoli d'infiltrazione barbarica s'ingrossarono fino ad assmmere la violenza di una fimmana torrentizia, che, rotte 
le barriere, mon più difese dalle legioni italiche, irruppe per le vie sgombrate dalle legioni, ma in senso inverso, per il Danubio, l'Illiria, l'Adriatico, verso l'Italia e verso Roma, a portare la desolazione là, donde erano partiti la civiltà e l'impero.

Gli storici sogliono porre nel 375 dalla naseita di Cristo il principio della trasmigrazione dei popoli, che sconvolse tutta l'Europa, ma si abbattè colla massima violenza precisamente sull'Adriatico.

La trasmigrazione dei popoli è considerata un fatto storico; però anch'esso porta la sua spiegazione geogralfica. Poichè è avvenuta si comprende che non poteva provenire che dal nord-est d'Europa.

L'occidente d'Europa non è molto vasto ed è ricco naturalmente abbastanza per mantenere l'equilibrio tra la sua potenzialitì economica ed il numero della sua popolazione. Le parti a nord-est dell'Europa e in primo luogo le steppe della Sarmazia sono sterminate per sè stesse, hanno alle loro spalle nell'Asia regioni ancora più sterminate e sono tutte fredde e povere. Non è quindi da meravigliarsi che all'ombra della civiltà greco-romana le popolazioni di quelle steppe siano cresciute a dismisura di numero e che poi in parte per legge di equilibrio della natura, in parte per la legge geografica delle vie acquee, delle pianure e del calore solare, e in parte anche per ragioni morali, perchè provocate dall'espansione romana, siano state attratte come farlalle al lume, verso Roma, donde era venuta loro la luce di tutto quello che non possedevano. Gimnsero nel momento in cui la resistenza romana era ridotta al minimo e la viltoria lu loro.

L'Adriatico, ch'era stato la strada per cui i Romani conobbero e raggimusero il Damubio e l'oriente, servì ora ai barbari per avvicinarsi all'ltalia.

La trasmigrazione dei popoli durò nel suo signilicato storico tre secoli.

A questo studio non apparticne l'indagare le callse, se cioc̀ i barbari furono attratti verso Romal dalle comquiste dei Romani, che li avevano turbati dal foro sonno preistorico, o se le conquiste dei Romani furono provo- 
cale dal bisogno di questi di estendere i possessi territoriali per tener dome o lontane le tribù barbariche, che già avevano urtato minacciosamente i loro confini. Occorre invece notare che colla caduta di Roma scomparisce dall'Adriatico un organismo unico al mondo, che non si rinnovò più nè politicamente, nè socialmente. Del suo tesoro di coltura si arricchirono tutti i popoli posteriori, ma in una forma modificata. La storia dell'Europa e dell'Adriatico nei secoli successivi non può essere parificata a quella dell'epoca romana. Per ciò a causa di questa trasformazione, se siamo dispensati dall'indagare maggiormente $\mathbf{i}$ germi spirituali che promossero l'ingrandimento di Roma, occorrerà invece star attenti al fermento d'idee, che si sviluppò dal miscuglio barbaro-romano, perchè da questo attraverso un lungo filtro si formò la società in cui anche noi viviamo.

Esaminiamo in rapidi tratti i cambiamenti portati all'Adriatico.

In quanto alla decadenza romana bisogna innauzi tutto notare che alla morte di Teodosio (395 d. C.) l'impero fu ripartito per sempre in occidentale ed orientale; che al primo fu assegnata la parte italica dell'Adriatico, compresa la Dalmazia, e il secondo ebbe la costa illirica, e che suo figlio Onorio trasportò la residenza a Ravenna, attirando in seguito su questa città gli eserciti nemici e provocando la caduta di questo grande emporio marittimo di Roma.

Il trasporto della capitale da Roma a Ravenna richiede qualche considerazione. Finora nelle relazioni intime fra Italia e Adriatico, la prima aveva avuto una naturale preminenza. L'elezione di Ravenna a centro dell'impero d'occidente e l'aggregazione ad esso della Dalmazia spostò la preminenza verso l'Adriatico, tanto che ora, formalmente e idealmente, si potrebbe dire che invece di uno Stato d'Italia era stato formato uno Stato dell'Adriatico.

In quanto ai barbari - i primi a comparire, spinti ed incalzati dagli Unni, furono i Visigoti o Goti dell'occidente, che diedero per ciò la vera e forte scossa dello sfacelo al vacillante impero. Sotto il comando del va- 
loroso loro re Alarico essi percorsero dal basso Danubio la penisola illirica e da cui si diressero in Italia, contro la quale, verso il 400 d. C., fecero più spedizioni vittoriose.

A loro tennero dietro mezzo secolo più tardi i feroci Unni, che sotto la condotta di Attila si mossero dal bassopiano ungherese e lungo i fiumi Sava e Drava si diressero dapprima verso le ricche Gallie.

Con uno sforzo supremo i Romani, comandati dal prode generale Ezio, riuscirono ad arrestare la loro marcia, ma poco dopo (452 d. C.) Attila, ridisceso in Italia per la porta del Carso, distrugge Aquileja, devasta tulta l'alta Italia e la parte settentrionale della costa illirica.

La distruzione di questa importante piazzaforte romana, chiave dell'Adriatico e dell'Italia dalla parte di settentrione, ha, per le regioni che vengono qui studiate, un significato particolare: oltre all'aver lasciato sfondata la porta, diede origine alla fondazione di Venezia fra le lagune fin allora inaccessibili.

Pochi lustri più tardi, l'anno 476, segna la fine dell'impero romano d'occidente per opera di Odoacre, capo degli Eruli germanici, e la ritirata di Giulio Nepote in Dalmazia.

Alla romanità tocca ora sull'Adriatico una sorte consimile a quella da lei procurata agli altri popoli vinti.

Dopo la prima sconfitta fatale, che determina la morte di uno Stato, cessa di regola la vita collettiva civile, ma sopravvive una buona parte dell'elemento etnico e talora anche l'idea. L'elemento etnico romano fu abbandonato a sè stesso e non fu più curato da alcumo. Ma l'idea, pur sempre fulgida, dell'impero romano si conservò invece in due siti, a Costantinopoli, rivale momentancamente più fortunata, nell'impero romano orientale o greco-bizantino, e nell'Italia stessa, nella mente dei successivi dominalori barbari, ambiziosi di portare il titolo pomposo d'imperatori romani.

Alla distanza di un altro mezzo secolo dagli Unni l'Italia subi l'invasione degli Ostrogoti o Goti dell'est. V'ennero anch'essi per la Pamnonia e lungo i fiumi Sava e 
Drava e passando per le porte del Carso e del Trentino calarono nell'aita Italia per Aquileja e Verona, condotti dal celebre Teodorico. Teodorico trasportò la sua sede a Ravenna e di là dominò (in modo effimero) su buona parte dell'impero romano, conıpreso l'Adriatico settentrionale. Egli tentò anche, invano per altro, di fondere i suoi Germani colla popolazione indigena d'Italia.

Però i suoi successori caddero in discordie e ciò indusse l'imperatore d'oriente Giustiniano al tentativo di conquistare l'Italia, ciò che gli riuscì dopo una lotta di venti anni. Dal 553 d. C. l'Italia divenne una provincia dell'impero romano d'oriente e tutte le due coste dell'Adriatico appartennero per parecchi secoli all'impero greco di Costantinopoli.

Il dominio bizantino sull'Adriatico raggiunse nel VI secolo un'alta importanza e durò sino al secolo XV. Questa lunga durata perè è da ascriversi più a favorevoli contingenze esterne, alla buona posizione della capitale e in generale alla coltura del popolo, che ad un valore intrinseco dell'amministrazione. Un fasto ingannatore ricopriva la decadenza di una profonda corruzione interna e quando avvemne la caduta, essa fu profonda ed irrimediabile. Bisanzio ritardò, ma non impedì la comparsa di altri popoli vergini e sani, ancora Germanici e poi Slavi.

I iongobardi. Per amministrare i paesi della parte superiore del"Adriatico, i Bizantini collocarono a Ravenna un esarca. Vuolsi che Narsete per vendicarsi di intrighi di corte chiamasse dalla Pannonia i Longobardi. Erano questi un popolo germanico, che nella persona del re Alboino, ebbe un famoso guerricro. Alboino li aveva condotti già negli anni 549 e 560 a saccheggiare l'Illiria, e nel 568 d. C. li guidò per la strada seguita da tutti in Italia, ove conquistarono il paese che da loro prese il nome di Lombardia, elessero a loro capitale Pavia e allargarono i possedimenti anche su parte della costa occidentale adriatica, che, come si vele dunque, contava allora come parte integrante d'Italia.

In questo modo la signoria dei Greci di Bisanzio $\mathrm{ft}_{\mathrm{t}}$ ristretta alle città marittime della costa orientale (Dal- 
mazia bizantina), alla parte meridionale della bassa Italia, a Roma, Genova e alle due isole di Sicilia e Sardegna.

Con ciò cbbe principio quello smembramento dell'Italia che terminò appena mezzo secolo fa e il frazionamento dell'Adriatico che dura ancora.

Colla conquista dei Longobardi finisce la trasmigrazione dei barbari per la costa occidentale: non così invece per l'orientale che, appunto verso quest'epoca (fine del VI secolo), fu tormentata dagli Avari e dagli Slavi.

Così fu rotta quella perfetta comunanza di vicende sloriche, che areva tenuto unite tutte le coste dell'Adriatico per otto secoli.

La comparsa unicamente devastatrice degli Avari nella storia, ha nell'Adriatico una scena secondaria. I disastri da loro apportati anche in questa regione sono però di una entità così grande, che non potrebbero venir passati in silenzio.

Tutte le invasioni, speclizioni e conquiste dei popoli germanici ebbero per conseguenza danneggiamenti d'ogni genere, come uccisioni, depredazioni, devastamenti; ma nessuna fu accompagnata da rovine e stragi così desolanti come quelle che lasciarono sul loro passaggio quei feroci popoli di razza finnica. La comparsa degli Unni alle porte d'Italia aveva segnato la distruzione di Aquileja; quella degli Avari nell'Illiria la distruzione di Salona, che era il secondo grande emporio romano sull'Adriatico. Di queste invasioni ávare fino alla costa orientale dell'Adriatico la storia ne registra particolarmente due: la prima verso il 604 in cui rasarono al suolo quaranta città della Dalmazia (Epidauro, ccc.), c la scconda nel 639 in cui anche seminarono oruncue stragi e devastazione.

Nel. saggio, ma disperato proposito, di frenare le invasioni avariche, opponendo barbari a barbari, l'imperalore d'Oriente Eraclio, che aveva il clominio, più nominale che di fatto, dell'Illiria, chiamò dài Carpazi il popolo slavo dei Croati, cui tennero dietro dalla Galizia i Serbi.

Alcuni di questi Slavi erano già discesi all' Ndrialico

Gli Slav. 
frammisti agli Avari ed avevano partecipato alle loro distruzioni. Questi nuovi Slavi tennero probabilmente la via del medio Danubio, indi dei suoi affluenti Morava e Sava (colla Drina, ecc.) e infine del Narenta.

La potenza degli Avari era già in decadenza. Agli Slavi riuscì di scacciarli dalle regioni dell'Adriatico, ma caddero sotto il dominio avaro nei paesi adiacenti all'Ungheria, ove gli Avari avevano posto le loro sedi attorno al Tibisco.

Di questi Slavi, i Croati si stabilirono nella regione che porta ancora oggi il loro nome e da lì si sparsero un po' alla volta anche nei paesi finitimi della Dalmazia e del Carso istriano. I Serbi occuparono il centro dei Balcani ed ebbero da lottare per ciò coi Bizantini e coi Bulgari. Siccome poi la potenza degli Avari continuò a declinare e il dominio dei Franchi da occidente e quello di Bisanzio dall'oriente giungevano fino a loro affievoliti, riuscirono tutti due Serbi e Croati, nell'anarchia generale, a conquistare ed a conservare per breve tempo i'indipendenza. In questo modo gli Slavi, e particolarmente i Croati, pel fatto di essere stati gli ultimi venuti, rimasero possessori dei paesi ad oriente dell'Adriatico, nel porto occupato una volta dagli Illiri.

Cambiamento dell'Adriatico per la trasmigrazione dei popoli.
L'Adriatico aveva mutato l'aspetto politico e difficilmente si sarebbe più potuto ravvisare in lui il lago romano.

Prima di tutto erano cessati gli scambi tra le due coste ed era scomparso lo splendore della ricchezza patrizia. Distrutti gli empori commerciali, smantellate le città della costa, specialmente orientale, la navigazione decadde e parte passò in mano dei Greci, parte degencrò in pirateria, esercitata per necessità dagli abitanti imporeriti.

Il lettore avrà già osservato che i trambusti della decadenza romana e della trasmigrazione dei popoli furono più forti e più sensibili sulla costa orientale adriatica che sull'occidentale. Le ragioni sono puramente geografiche e facilmente spiegabili.

In primo luogo i popoli trasmigranti erano ignari della 
navigazione. Le strade percorse da loro a preferenza furono o quella del basso Danubio, dei suoi affluenti Morava e Sava colla Drina, Bosna e Verbas, come fu accennato più sopra e che ne portò una parte pel Narenta direttamente all'Adriatico, donde poi ripiegarono a settentrione per raggiungere l'Italia per via di terra; oppure quella del Danubio medio (Pamnonia) e degli affluenti Sava e Drava, che li portava sull'altipiano del Carso. Da qui in mancanza di un solco naturale, evidente, si frazionavano per lo più, calando in parte al sud e proseguendo in Italia per Aquileja oppure proseguendo per la Drava e per la valle del Gail giravano le Alpi Carniche a settentrione e superate le alture di Toblaceo per la Pusteria e la valle dell'Isargo (Eisack) calavano giù lungo l'Adige.

Il primo e più pericoloso urto si riversava quindi per to più sull'Illiria, ossia sulla parte orientale dell'Adriatico. Nessuno però dei popoli barbari vi rimase. Essi provenivano da regioni piane ed erano diretti molto probabilmente alla pianura del Po, descritta come ricea di messi. Le regioni montuose dell'Illiria non si confacevano alle loro intenzioni ed erano buone soltanto per esereitare razzie e procurarsi i mezzi di proseguire la strada.

La lontananza ed i disagi del cammino erano poi tali e tanti che alcuni popoli non ebbero la forzal che di arrivare alle porte d'Italia ed altri vi entrarono, ma con violenza ammortita dalla resistenza delle popolazioni latine della penisola illirica.

Cinque sono le schiatte germaniche, che riuscirono a passare le Alpi e gli Apemnini: i Visigoti con Alarieo, i Vandali con Genserico, gli Eruli con Odoacre, gli Ostrogoti con Teodorico ed i Longobardi con Alboino. Ma questi invasori sono da annoverarsi fra i più miti e declinarono tutti colla morte del capo che ve li avera eondotti. Erano inoltre pochi di numero in paragone a quello dei conquistati e siecome non erano altro (he guerrieri incolti, mentre la popolazione sottomessa conservara i possedimenti rurali e la coltura, si fuscro presto coi 
vinti. Teodorico che a Costantinopoli s'era appropriato Ia coltura greco-romana fece inoltre rolontariamente tutto il possibile per effeltuare la fusione dei suoi Ostrogoti coi Romani e a tale scopo lasciò che l'amministrazione continuasse ad essere regolata alla romana $e$ fosse in gran parte guidata dagli stessi abitanti.

Il clominio dei Longobardi fu per gl'Italiani più oppressivo di quello dei precedenti conquistatori, perclıè il conando militare, l'amministrazione civile e la giustizia crano in mano dei vincitori. Questo peso però durò poco per la l’ajirlissima assimilazione alla popolazione latiua.

In complesso si può dire che gli accennati dominii germanici in Italia ebbero anche dei lati buoni. Essi in primo luogo ristabilirono almeno la giustizia, che prima era purtroppo scomparsa dall'impero romano malgrado le sue famose leggi. In secondo luogo ebbero conseguenze benefiche perchè, dopo la fusione fra vincitori e vinti, lo spixito guerriero dei primi infuse nuova vita alle popolazioni italiche infiacchite dai vizi della troppa ricchezza.

Invece i più feroci demolitori, senz'ombra di bene, che furono gli Unni e gli Avari, non bersagliarono che la costa orientale e si arrestarono quasi alle porte d'Italia. A loro temmero dietro gli Slavi che, sebbene possano essere anche annoverati tra i barbari miti, furono ad ogni modo dammosi per il solo fatto del loro stato di coltura interiore ancora a quello dei Germani, e sono quindi da considerarsi come un flagello, dal quale l'Italia andò esente.

Anche i popoli germanici e particolarmente i Goti e i Vandali, tanto nell'Italia padana che nell'apenninica, saccheggiarono e devastarono; ma lasciarono almeno sussisterc alcune ciltà, in cui poi si ricoverarono essi stessi.

I Goti per escmpio sotto Vitige distrussero e damneggiarono Fano, Pesaro, Ancona, Faenza, Ravenna, ma alfine s'insediarono in quest'ultima città e la eressero anzi a loro capitale. Anche i Yisigoti, condotti da Totila, misero a ferro e a fuoco l'Apennino anche dalla parte 
adriatica fino agli Abbruzzi e distrussero parecehie citià, ma proprio a Tagina nel Piceno (552 d. C.) furono sconfitti definilivamente da Belisario. Belisario si diede quindi a riparare subito ai loro danni e ricostruì direrse città tra cui Pesaro.

In questo studio poi che riguarda particolarmente le due coste dell'Adriatico, va ricordato che i popoli germanici, dopo di aver attraversato le regioni adriatiche dell'alta Italia, erano attratti a Roma e per ciò passavano sull'altro versante degli Apemnini. In complesso si può dire che la costa adriatica dell'Apennino da Ancona alle Puglie ebbe molto meno da soffrire in causa delio trasmigrazioni. Essa sofferse alçuanto solo più tardi per le incursioni dei Saraceni, dei Normanni, dei Turchi.

Al contrario la costa orientale fu saccheggiata, devastata e demolita col primo e maggior impeto in tutta la sua estensione non solo da tutti quei barbari germanici che poi penctrarono in Italia, ma in più, conte si clisse, dagli Unni, Avari e Slavi. Per gli Unni ed Avari si potrebbe anticipare il detto usato più tardi a proposito dei Turchi: che dove era passato lo zoccolo dei loro cavalli non cresceva più nemmeno un filo d'erba. Da tutto ciò si deduce quanto l'Italia fu favorita anche in questo incontro dalla natura geografica, giacchè senza le Alpi, gli Apennini e l'Adriatico essa sarebbe uscita daille trasmigrazioni dei popoli assai più malconcia.

Una delle più terribili irruzioni nell'thiria è quella fatta nel 409 d. C. da turbe di Alani, Goti, Sarmati, Quadi, Unni, Vandali e Marcomanni. Lo stesso San Girolamo, dalmata, uno dei più eminenti dottori tatini della Chiesa, fa un quadro lagrimerole dei damni e delle stragi, che cuesti barbari commisero nei parsi dell'llliria durante. il corso di venti anni. "In una parola, conchude, tutto è pieno di gemiti. e di strida ed altro dappertulto non si vede se non un orrido aspetto di morte, andandes in rovina l'impero romano». E in un allro, passo angiunge che i paesi dell'Illiria fino all'Aclriatico rlane rimasti incolti, senza abitalori, senza bestie c divenuli ixischi e spinai. 
Perchè il lettore si formi un'idea delle stragi degli Avari, basterà tratteggiare alcune scene della distruzione di Salona (anno 615).

Narra Tommaso, arcidiacono di Spalato nel XIII secolo, che all'avvicinarsi del nemico tutti i cittadini si precipitarono verso il porto per ricoverarsi sulle navi. Ma nel trambusto molti caddero in mare e vi annegarono. Intanto però, prima ancora che vi si fossero imbarcati, gli Avari, cui erano commisti molti Slavi, irruppero nella città e la saccheggiarono, distruggendo tutto quello che v'era rimasto. La città stessa coi suoi magnifici palazzi e chiese fu incendiata, demolita e convertita in cenere e polvere. Gli abitanti furono in parte uccisi, in parte fatti schiavi. I superstiti per aver salva la vita dovettero ricoverarsi sulle isole, donde ritornarono un po' alla volta a rivedere le rovine della loro città, quando i terribili barbari se n'erano andati.

La fiorente latinità dell'Illiria fu ridotta in questo tempo alle mura, in parte smantellate, di otto città della costa: Tersatica (Tersatto, ora Fiume), Senia (Segna), Jadera (Zara), Tragurium (Traú), Aspalatha (Spalato, formatasi entro le mura del famoso palazzo di Diocleziano), Stagnum (Stagno), Ragusa e Decatera (Cattaro), nonchè di quattro città sulle isole omonime: Veglia, Cherso, Lussino ed Arbe. Tale era anzi lo stridore del contrasto fra queste città, fari superstiti di una civiltà superiore, e il buio della parte rimanente del paese, che continuarono a formare un corpo amministrativo speciale distinto dal retroterra slavo. Furono queste città che salvarono dal naufragio anche il nome di Dalmazia.

D'ora in poi l'importanza storica della costa orientale adriatica divenne inferiore a quella dell'occidentale. Ciò non per tanto l'orientale s'impone maggiormente all'attenzione degli studiosi per i grandi cambiamenti ivi avvenuti e per il fatto strabiliante ch'essa si trova ancora oggi alla distanza di tredici secoli in uno stato di abbandono press'a poco simile a cuello in cui la lasciarono gli Avari. 
Non tutti saranno forse disposti a sentire rimpianto per il tramonto della romanità dalla costa orientale del- della latinità l'Adriatico.

Qualcuno, con ragione, penserà che venne anche per sulla costa orientale dell'Adriatico. i despoti romani l'ora del redde rationem e che Roma e Salona in fondo avevano meritato la loro sorte.

Roma, quantunque nel 410 avesse racchiuso piì di un milione di abitanti, si arrese ad Alarico, re dei Tisigoti, senza memorabile resistenza.

Salona, la cui vita dopo Diocleziano sembrava una copia di Roma sull'altra sponda ed era grande cruanlo mezza Bisanzio, secondo le narrazioni di Costantino Porfirogenito ( $\mathrm{X}$ sec.) e di Tommaso arcidiacono (XIII sec.), era minata dalla corruzione dei costumi, dalle lotte intestine fra aristocratici e plebei, dalla discordia, dall'indisciplina e dalla rivoluzione del cristianesimo.

L'ora della giustizia viene per tutti. Nessmo però deve dimenticare che, se i Romani abbatterono molto di ciò che appartenera ai loro nemici, riedificarono meglio e le loro opere deslano sempre meraviglia ed invidia. I barbari invece non lasciarono dietro il loro passaggio che rovina e desolazione.

L'Adriatico avrebbe avuto senza dubbio sorte migliore se l'elemento latino anche sulla costa orientale fosse sopravrissuto più numeroso alla trasmigrazione dei popoli. La sua rapida ed estrema riduzione da questa parte, paragonabile quasi ad una scomparsa, provoca per lo meno stupore, se si consideri la lunga e tenace resistenzal opposta alla romanizzazione da tul popolo di gran lunga più debole, quale furono gli Illiri.

La ragione di questo fatto è da ricercarsi in cause sociali e geografiche, però lanto intimamente connesse, da polersi asserire che le geografiche furono dominanti.

Gli Illiri poterono opporre lunga resistenza ai Romani, oltrechè per la conlomazione del terreno, già accenuala, pel molivo che essencto un popolo primilivo ogni nonics era gnerriero. I loro nemici Romani (a differcuza dei barbari più tardi che passavano solamente) (loulo pui venuti per restarvi. L'aversione degli Hlliri averal quimbl 
campo di rinnovarsi e scbbene essi siano stati sottomessi definitivamente verso il principio dell'era volgare, si può ammettere che i Romani sulla costa orientale ebbero per qualche secolo ancora da calcolare con un elemento infido, che sarebbe scomparso totalmente appena in seguito per quelle molteplici cause secolari che determinarono la romanizzazione di altri popoli. Ma la costa illirica ebbe per i Romani ed anche per sè stessa il danno geografico di esscre troppo vicina all'oriente d'Europa e di mancare di un valido confine naturale. Tutte le infiltrazioni di nuovi popoli avvennero da questa parte. Non era forse ancora bene compiuta l'assimilazione degli Illiri, che, prescináendo dallo stragrande numero di schiavi esotici, già nella seconda metà del III secolo d. C. la storia ricorda pericolose incursioni di barbari Germanici e Sarmati dal Danubio. L'imperatore Probo (276-282) non seppe rimediarvi meglio che imitando quanto aveva fatto un secolo prima Marco Aurelio, accoglierli cioè in gran numero nelle legioni romane, allo scopo anche di riassodare la disciplina ormai rilasciata. Mezzo secolo più tardi Costantino terminata una guerra sul Danubio dovette ripetere l'errore di accogliere più di 30000 Vandali e Sarmati, parte nelle province finitime e parte nell'esercito. Per ragioni sociali, ma specialmente geografíche, la superiorità della compagine etnografica romana 1 iu scossa da questa parte fin dal suo principio.

Quando poi alla fine del IV secolo l'urto violento e feroce della trasmigrazione si diresse precisamente all'Adriatico orientale, i Romani, già indeboliti da tante cause, $110 n$ furono in grado di affrontare i barbari nella prima parte del loro cammino per ragioni della loro tattica di guerra. I paesi carsici adiacenti alla costa orientale non si prestano che alla guerriglia. La storia non ricorda alcula grande battaglia in queste regioni. Ora la guerriglia è la tattica adottata sempre dai popoli inferiori in paese montuoso per arrestare d'avanzata nemica e fu usata anclie dagli Illiri con successo. I popoli numericamente e militarmente forti cercano invece il successo in poche, o meglio in ma sola battaglia campale in pianura. 
Per ciò i Romani attesero gli Unni non sulle Alpi, ma nelle Gallie ai Campi Catalauni (451 d. C.) e, sebbene numericamente di gran lunga inferiori, riuscirono colla tattica e col valore a sconfiggerli ed a salvare la civiltà.

Una guerriglia nei paesi carsici i Romani non sarebbero stati più in grado di sostenerla. Le loro milizie in primo luogo composte in gran parte di stranicri, non vi erano adatte e poi non erano addestrate che alla tatlica in grande. In secondo luogo a quello scopo sarebbe occorsa la cooperazione della popolazione indigena, ma su questa i Romani non potevano far calcolo. I Romani, corrotti dalle troppe ricchezze, da molto tempo aborrivano le armi. La custodia delle loro persone e dei loro averi era affidata agli schiavi ed ai mercenari, che, maltrattati, al primo scompiglio avranno fraternizzalo piuttosto cogl'invasori. I barbari poi venivano e scomparivano: piombavano come un fulmine per rubare ed uccidere e tiravano innanzi. Se i Romani non potevano resisterc al primo urto, non era nemmeno il caso di pensare ad una pazicnte riscossa. E stato ricordato che popolazioni numerosissime, come quelle di Roma e Salona, si arresero ignominiosamente. Figurarsi quale sarì stata la paura dei cittadini romani, imbelli o viziosi, raceolti nelle cittadine e nelle vallate del Carso adriatico. Non rimaneva loro altro scampo che lentare di aver salva almeno la vita colla fuga. Erano troppo raffinatamente civilizzati per affrontare la furia feroce dei harbari. E poi bisogna domandarsi se mal tallica artificiale di guerra avrebbe potuto arrestare un disastro elementare, quale fu quello della trasmigrazione dei barbari, che aranzavano non come guerrieri addestrati, ma come una violenta fiumana torrentizia, che schianta e sradica nel sto passaggio alberi secolari c perlino macigni.

Per questi motivi l'Illiria, cui lioma per la sua vicinanza aveva dischiuso $u n$ avvenire di floridezza, pari almeno a quello della Spagna e della Gallia, al causa della sua infelice posizione geografica chbe una sorfo di poco migliore a quella delle province romane nedl'Africa. 
A rendere ancora maggiore il disastro della romanità nella metà orientale dell'Adriatico contribuì una causa puramente morale, la religione cristiana.

Superate le persecuzioni e le incertezze dell'arianesimo e di altri scismi, fonti anche queste di guasti materiali, il cristianesimo prese il sopravvento già al principio del IV secolo con Costantino. Però per quanto emanasse la sua forza da Roma, lo spirito che lo animava era cambiato. Il cristianesimo anzi colle sue tendenze di distruzione del paganesimo e col suo carattere cosmopolita abbattè molte tracce della grandezza romana, non conservando che solo in parte la lingua latina. Così p. e. si deve all'opera germanicamente cristianizzatrice di San Severino nel Norico (Mediterraneo), ossia nei paesi alpini e carsici a settentrione del golfo di Trieste, se sparirono cla fui le ultime tracce di civiltà romana già alla fine del V' secolo d. C.

Malgrado tutti questi rovesci l'elemento latino si conservò, se anche decimato, sulla costa marina dell'Adrialico. Anzi l'Istria, per essere fuori di strada dalle trasmigrazioni dei popoli sprovvisti di marineria, era rimasta lino a questo punto, più che altre terre italiane, esente clalle invasioni devastatrici.

\section{DALLA CADUTA DELLA ROMANITÁ (VII SEG.) ALLA COMPARSA DI VENEZLA (X SEG.)}

Le condizioni ora descritte arrivano fino al VII secolo. Però già dalla fine del IV colla definitiva spartizione dellimpero romano in orientale ed occidentale dopo la morte di Teodosio vien meno sull'Adriatico l'inl'tucnza direttrice unica e l'idea sovrana dell'impero di Roma. In luogo di questa pesò durante il V e VI sacolo la parola di Costantinopoli, però più formalmente che effellivamentc. Da quest'epoca in poi l'Adriatico e le sue coste furono divise in tante zone d'influenza e divennero il teallo di lotte e contese per la supremazia. Invano si 
cercherebbe nella geografia locale la spiegazione originaria dei molti cambiamenti. Per parecchi secoli, fino a tutto il XIV, la violenza, il capriccio, gl'intrighi, fecero dell'Adriatico un calcidoscopio politico. Sembra che i latini, come popolo, siano stati talmente abituali durante i secoli di decadenza dell'impero romano a disinteressarsi della cosa pubblica ed a lasciarsi governare passivamente, che nemmeno pensarono a prendere nelle loro mani le redini della politica.

La storia dell'Adriatico nei secoli futuri è intricatissima e per esporla brevemente bisognerà limitarsi a tracciare una linea rotta a zig-zag, con pochi accenni illustrativi onde spiegare la preminenza dell'uno o dell'altro dei molti suoi competitori. Ne uscirà un quadro arruffato, come certi dipinti ultra-moderni, ostico al lettore per la sua nervosità e poco gradito anche al compilatore, costrelto a trar linee diritte là dove tutto concorre a sminuzzarle. Perì siccome i fatti che si spiegarono dal VII secolo in poi ebbero la loro preparazione, se non esattamente l'origine, nei secoli V e VI, gioverà schiarire questo lavorio di preparazione, onde offrire la base per un migliore intendimento degli avvenimenti successivi.

Bisanzio aveva la supremazia su tulto il mare Adria- Gli eradi politico e sulle sue due coste, ma il suo possesso era effet- tici di Roma tivamente limitato alle ciltà greche della costa oecidentale virn secolo. e alle citta greco-latine della costa e delle isole dal lato orientale. Il centro amministralivo dei possedimenti bizantini nell'Adriatico era nell'esarcato di Ravenna. Il governo di Bisanzio vi rappresentava la depravazione di una grande coltura decadente.

La pianura lombarda era in mano di dominatori germanici, che vi portarono nuovo spirito guerresco e prepararono il suo passaggio nella sfera d'inlluenza degli Stati germanici del centro d'Europa. In questo modo statva per essere inlranto politicamente ([uell'assctto nazionale e civile dell'Italia, portata dai Romani ai confini geografici del Varo, delle Mlpi e dell'Arsia e cementale da Augusto colla fissazione delle dieci regioni italiche,

A Roma la comunita ecelesiastica cristiana assurgerar 
ad un'inportanza capitale. Il suo capo-pastore, prima vescovo, poi patriarca e dal IV secolo Papa, come successore del principe degli Apostoli, andava arrogandosi anche influenza politica e si preparava a far rivivere sotto una nuova forma più spiritualizzata l'idea dominatrice dell'impero romano.

Il papato, pur aiutando a distruggere tutte le opere romane in quanto rivestivano forme pagane, salvò la lingua e la coltura latina, purificata e trasformata dal concetto morale cristiano. Con Gregorio I Magno (590-604) i papi si posero a capo del progresso intellettuale dell'Europa occidentale e non mancarono di esercitare influenza sulle vicende dell'Adriatico colla religione e colla politica.

Rimarrebbero da ricordare ancora i paesi carsici dell'antico Illirio. Queste regioni, condannate alla miseria dalla geografia, furono colpite ora anche dalla storia. Dopo tante vicende furono allagate dal VI al VII secolo dagli Sloveni, dai Croati e dai Serbi, che sono purtroppo le ultime anche fra le schiatte slave. Questi popoli, privi affatto di coltura, non raggiunsero per qualche secolo alcuna importanza. Gli Sloveni caddero fin da bel principio sotto l'influenza tedesca; i Serbi dovettero riconoscere subito la supremazia greca di Bisanzio; i Croati, che si trovarono nella zona morta fra le due sfere d'azione franca e bizantina, conservarono una semi-indipendenza, finchè non furono insidiati anch'essi dai loro potenti vicini.

Albori Nell'osservare la storia dell'Adriatico non bisogna perdi vita nuova dall'VIII dere la visione della storia generale d'Europa e tenere presente per conseguenza che nell'età di mezzo il perno degli avvenimenti stette nell'occidente presso i popoli germanici.

L'Adriatico, clıe quando il mezzogiorno d'Europa aveva il predominio incontrastalo colla civiltà greco-romana, a causa della sua forma (li strada laterale, aveva dovuto pure contentarsi di una parte secondaria e subordinata, or'a che il predominio passò all'occidente d'Europa, cadde totalmente nellombra. Per quattro secoli (VII-X) il 
suo bacino può essere paragonato ad uma spiaggia sulla quale vennero a morire da più circoli esterni le onde di avvenimenti della storia altrui. Appena colla fine del $\mathrm{X}$ secolo l'attività marittima-commerciale di Venezia vicne a toglierlo un po' dalle tenebre in cui l'aveva pionbato la caduta dell'impero romano ed a dargli il benefizio di una modesta attività sua propria. D'ora in poi le vicende locali delle due coste sono distinte e differenti, perchè se anche la civiltà continuò a venire dall'occidente, l'oriente, più popolato che per l'addietro, uscì anche dall'ombra e si fece sentire di tratto in tratto dando segni di vita.

Anche in quest'epoca crepuscolare i fatti più salienti riguardano la metà occidentale del bacino adriatico. Nella lotta per la supremazia in Italia fra i Longobardi e il Papa, questi ricorse ai Carolingi.

Il popolo e la dinastia regnante dei Franchi ebbero la fortuna di arrivare ad insediarsi nella Gallia, ch'era per i Germani la miglior regione, perchè fertile, già dissodata dal lavoro dei coloni romani e cinta da confini naturali immutabili. Questa circostanza geografica più che storica era atta ad aprire loro un avenire più propizio che alle altre schiatte.

I Carolingi naturalmente approfittarono di quell'occasione per allargare la loro influenza. Però le spese della contesa furono pagate... dai Greci Bizantini, che perdettero definitivamente l'esarcato di Ravenna e la Pentapoli, donati da Pipino al Papa (756 (l. C.).

Pipino naturalmente credette di far bene aiulando il più debole per vincere momentaneamente il più forte; però se avesse potuto prevedere che col gettare le fondamenta del futuro Stato della Chiesa avrebbe di poi inviluppato l'impero, cui egli tendeva, in ma folta di tre lunghi secoli col papato e che in questa l'impero tedesco avrebbe logorato la sua lorza migliore, certanente non sarebbe ricorso ad una vittoria così facile.

L'alleanza fra Pipino e la Chiesa cbbe grandi conseguenze: prima l'imnalzanento dei Carolingi sul trono franco col grande impero di Carlomagno; quindi l'indeboli- 
mento della potenza bizantina nell'Adriatico, e infine l'intromissione della Germania nelle faccende interne dell'alta Italia e dell'Adriatico settentrionale per i secoli venturi.

A questo punto si afferma nella storia d'Europa ed anche dell'Adriatico una divisione di poteri bene marcata, disegnatasi già dopo la caduta di Roma e la trasmigrazione dei barbari, fra il papato e gl'imperatori germanici. Prima, durante l'esistenza di Roma, non c'era nel mondo che un solo potere sovrano, assoluto, cui tutti covevano inchinarsi, quello di Roma stessa. C'era anche allora una lotta, ma era oscura ed informe, tra la civiltà romana e la barbarie di tutto ciò che stava all'infuori di questa. Riesce facile studiare la storia generalc di quell'epoca, appunto perchè è semplice.

Col tramonto e colla caduta della romanità questo potere si sgretola e fraziona territorialmente fra Roma e Costantinopoli e si scinde materialmente nei suoi due componenti, potere religioso e potere civile. La religione cristiana in principio separata, anzi in lotta acerba collo Stato, si consolida a Roma nei Papi ed a Costantinopoli nei patriarchi scismatici. Onde ne nasce un'altra contesa politico-religiosa, le periferie dei cui cerchi d'influenza s'incontrarono appunto sull'Adriatico. Ma questa lotta non clura a lungo perchè Costantinopoli impallidisce. Roma invece risorge sotto nuova forma col papato, che per sostenersi cerca di afferrare anche il potere civile. Ma non ci riesce perchè questo colla forza militare e politica era esulato lontano, nelle mani degl'imperatori tedeschi.

La divisione di questi due principi, necessariamente in antagonismo e per ciò più spesso in lotta che in amicizia, ̀̀ il filo secreto degli avvenimenti del medio evo. Non essendovi che due forze non riesce difficile di studiare nemmeno questo periodo.

Coll'evo moderno però subentra un cambiamento: al- 
l'impallidire del potere religioso corrisponde coniemporaneamente la comparsa di un nuovo competitore, a disputare la supremazia dell'alto Adriatico, il re di Francia, rappresentante di una parte della latinità in contrapposto al germanesimo degl'imperatori tedeschi. Ne succede una complicazione di fatti ed avvenimenti, in cui tultavia ancora si possono distinguere le fila. Ben presto però per la creazione di nuove grandi potenze, prima in Europa e poi nel mondo in generale, sorge la cosidetta politica e allora nasee quel guazzabuglio di antagonismi e quella matassa d'intrighi, in cui noi contemporanci a stento ci raccapezziamo.

Il connubio della civilà romana coll'energia intatta germanjea e l'alleanza del Papa coi Carolingi innalzarono il ramo germanico dei Franchi ad insperato e rapido splendore, ch'ebbe il massimo esponente nel genio personale di Carlomagno, uno dei più fortunati fondatori di Stati che la storia ricordi.

Dell'espansione franco-carolingica al nostro studio interessa solamente di rilevare, che Carlomagno alla fine dell'VIII sccolo s'impadronì della Lombardia, fondò anche su territorio italiano delle marche (quella Trevigiana, del Friuli e della Carinzia), e mercè un trattato coi Bizantini riuscì ad incorporare all'impero franco l'Istria, la Liburnia e la Dalnazia settentrionale interna (detta allora veramente Croazia), mentre la Venezia e la Dalmazia marittima (ciltà alla costa) rimasero all'intpero bizantino.

Il dominio carolingico all'infuori della Lombardia, la cui amministrazione fu ordinata alla franca, si limitava ad una supremazia d'indole politica.

Colla comparsa nella storia d'un'amministrazione politica germanica lanno capolino "le marche». - Ne pullularono diverse anche ai confini settentrionali e orientali d'Italia, e sembrano mat novilit. Non sono invere altor 
che imitazioni delle colonie, di cui diedero esempio i Romani e, prima di loro, i Greci. I margravi o marchesi germanici non facevano ora in Italia altra funzione in sostanza che quella esercitata secoli prima al di là delle 'Alpi dai proconsoli romani. Ne citeremo le principali: marca del Friuli, di Aquileja, di Verona, di Treviso, d'Istria, immediatamente sull'Adriatico. Perfino nell'Italia di mezzo ne sorsero alcune, quella di Ancona e quella di Camerino.

Nella formazione di quelle marche e nell'acquisto dei nuovi dominii i Franchi presero per base e furono favoriti dall'affinità di razza con una parte della nuova popolazione infiltratasi prima e dopo la caduta dell'impero romano ai confini settentrionali ed orientali d'Italia.

Sappiamo infatti che i Cimbri; sconfitti da Mario presso Vercelli (101 a. C.), fuggirono sulle Alpi per cercare un asilo in quelle gole inaccessibili. Più tardi Teodorico, che nel 493 d. C. pose la sua residenza anzichè a Roma a Ravenna, aveva fatto di Verona un centro di germanesimo, come lo attestano i canti popolari eroici, che celebrano la sua gloria sotto il nome di Dietrich von Bern. Da ciò si deduce che la valle dell'Adige, ossia la porta settentrionale d'Italia, era rimasta occupata ed otturata dai Germani. Ma i Tedeschi comandarono in questo e nei secoli successivi anche molto più al sud. Si sa p. e. che nel 1093 o 1094 Ancona era sottoposta ad un ministeriale dell'impero germanico, di nome Werner, e si chiamava per ciò marca Guarneri. A lui seguirono marchesi tedeschi per tutto il secolo XII, finchè i Pontefici non affacciarono pretese in quei paesi.

La porta orientale d'Italia o strada del Carso era stata la prima a perdere le tracce di questo benefizio. Popolazione slava vi si era infiltrata fin dal VI secolo e a capo di essa stavano ora principotti ed ecclesiastici germanici, che s'erano spinti fino nella pianura friulana e nell'Istria.

Erano questi el i Germani dell'Adige che parteggiavano per i Franchi, mentre la popolazione romanizzante della Venezia c della Dalmazia teneva per i Bizantini. 
Carlomagno ebbe anche le simpatie dei Croati; e lo si spiega coll'antagonismo che si manifestò subito tra questi e le città latine della costa, protette dai Bizantini.

Che Carlomagno nella sua genialità, coltura ed energia abbia ricostituito l'idea dell'impero romano, si spiega col desiderio di fondere e rianimare la suprema dignità di Roma colla giovane forza germanica. In questo nuovo impero l'Adriatico entrò come parte del tutto secondaria. Questa inclusione dell'Adriatico nell'impero di Carlomagno fu però per il nostro mare apportatrice di gravi conseguenze, che durarono palesi per molti secoli e continuano ancor oggi sotto larvate forme. Vale quindi la pena di considerare un .po' lo spirito ånimatore dei fatti.

Due erano i mezzi dei quali Carlomagno intendeva servirsi per cementare il suo colossale castello: la coltura romana e la riunione nello sceltro imperiale anche della reggenza della Chiesa. La sola sua influenza personale riuscì momentaneamente in un'impresa che non poteva essere duratura per l'incompatibilità degli elementi chiamati a comporla. Il primo a ribellarsi fu, naturalmente per quei tempi, lo spirito dell'indipendenza religiosa, ed è per questo principalmente che l'impero e il papato vennero in breve alle prese per il predominio e che l'intero mondo cristiano prese parte a questa gran lotta, il cui campo principale fu nell'alta Italia e nell'Adriatico settentrionale.

La coltura romana fu un buon cemento finchè i Germani ne avevano un assoluto bisogno. Ma allorehè questi incominciarono ad elaborare una civiltà propria romanogermanica, e che nei paesi romanici della Francia e dell'Italia rinacque la vita, sollo la vernice delle lotte per la supremazia politica, religiosa e culturale, si disegnarono le competizioni nazionali, che portarono un cambiamento nel concetto del sacro romano impero medievale. Piu avanti, al momento opportuno, verranno rilevate le conseguenze di quest'evoluzione per l'Adriatico.

La potenza franca decadde, com'era facile prevedersi, rapidamente sotto i successori di Carlomagno. Ma la direzione degli avvenimenti sull'Adriatico non fu ripresa, 
come potrebbe sembrare, dai Bizantini, perchè anch'essi crano seriamente minacciati ed in pericolo per gli assalti degli Arabi, bensì dal Papa.

I Pontefici. Nicolò I (858-867) si attribuì risolutamente la suprema autorità arbitraria sopra la terra ed ebbe la mira di riunire l'impero occidentale al papato.

Però la supremazia che il Pontefice esercitò anche sull'Adriatico durante quasi un secolo era una supremazia. di nuovo genere. Essa mirava sopratutto al predominio spirituale. Non era più la supremazia nazionale e politica dell'impero romano, nè un dominio esclusivamente politico-amministrativo, come quello dei Bizantini e dei Franchi. Per ciò in questa specie di anarchia politica anche le deboli aspirazioni particolari degli abitanti delle coste adriatiche poterono acquistare forza e farsi strada fino a raggiungere la soglia della coscienza storica. Erano questi i Veneziani, i Croati ed i Narentani, popolazioni della costa marittima, che si divisero o meglio contescro per alquanto tempo il dominio dell'Adriatico.

L'indole di questo studio di mettere succintamente in rilievo la storia particolare dell'Adriatico e lo scopo di dar risalto ai fatti salienti, tanto geografici e storici, delle due costc mediante frequenti opportuni confronti induce ad apprezzamenti riassuntivi, che permettendo di sopprimere le aride esposizioni di avvenimenti, convertono la lettura da oggettiva in soggettiva. L'intenzione, ottima in teoria, di osservare la più stretta oggettività, vale in pratica solo fino ad un certo punto. Dal momento poi in cui i fatti considerati si ripercuotono colle loro consegucnze, come un'eco lontana, fino ai nostri giorni e sono matcria di controversic ancora aperte, ogni tentativo di raggiungere la perfezione dell'oggettività per salvarsi da possibili recriminazioni sarebbe vano. Voglia il lettore quindi non dimenticare questo volontario esame di coscienza.

Venezia. Nella storia di Venezia - che noi non tratteggeremo che nelle sue lince sommarie, perchè è più nota - va rilevata fin dalla sua origine questa circostanza singolare: che la città, sorta quasi per caso in un luogo 
prima evitato da tutti, si trovò ben presto in condizioni geografiche così favorevoli per la navigazione e per l'economia nazionale, da divenire lo scalo di una buona parte della Padania, l'emporio commerciale di congiunzione tra l'occidente e l'oriente d'Europa, la dominatrice dell'Adriatico per otto secoli, conservando durante tutte le molteplici sue vicende un tipo speciale in tutto, anzi unico al mondo.

La città fondata, come fu accemnato, nella metà del $\mathrm{V}$ secolo, crebbe lentamente e relativamente all'oscuro sotto il dominio bizantino, esercitato dall'esarca di Ravenna, che la difese contro i tentativi di conquista dei Longobardi fino al secolo VIII. Da quest'epoca compariscono a capo della sua amministrazione i Dogi, che, gradatamente, sopportando ancora la supremazia bizantina, vammo acquistando sempre maggior influenza fino a farla scomparire di fatto nel $X$ secolo. La potenza marittima di Venezia data precisamente dalla fine dell'VIII secolo, dopochè l'imperatore Giovamni Comneno nel 726 le concesse il dominio sulle acque dell'Adriatico da Duino alle foci del Po.

La storia dei Croati, più oscura, avrì bisogno di maggiori dettagli.

Invitati, come fu ricordato, dall'imperatore bizantino Eraclio contro gli Avari irruppero verso il 620 nella Dalmazia (intesa nel senso di allora, non odicrno) cacciarono via difatti gli Avari e si divisero la provincia, che era rimasta quasi spopolata, specialmente nella terraferma. Essi occuparono le regioni dal confine geografico italiano fino al nodo montuoso del Montenegro nel tratto dalla costa fino circa la catena dinarica ad oriente. It resto alle loro spalle ed a mezzogiono fu occupato dai Serbi. Questi due popoli affini, allora più che oggi, si trovarono quindi ad essere quasi i successori geografici degli Illiri.

In qual modo sia avvenuta questa occupazione non ci fu tramandato. Probabilmente essa avenne alla chetichella, come di una terra abbandonata. All'inrasione degli Slavi successe sulla costa orientale arliatica un perio- 
do di sgomento e di prostrazione generali, che durò un secolo e mezzo, durante il quale nella storia non c'è ricordo della Dalmazia. Appena nel 791 d. C. quando Carlomagno si accinse a debellare gli Avari che dal medio Danubio, ove avevano posto da ultimo le loro sedi, molestavano i Franchi e gli Slavi, si sa che quelli della Slavonia odierna si rivolsero al potente imperatore per aiuto. Carlomagno vinse gli Avari, ma sottomise anche i Croati.

Da notizie posteriori si apprende che i Croati diedero. ai paesi da loro occupati una primitiva amministrazione politica. Nella relativa divisione in distretti riapparisce però subito la conformazione geografica. Si distinse cioè un paese croato settentrionale, che comprendeva il bassopiano tra la Drava, il Danubio e la Sava (una volta Pannonia, più tardi Slavonia) ed un paese croato meridionale, suddiviso alla sua volta in Licca (antica Liburnia), Croazia bianca (Croatiam albam vocabit) dal fiume Zermagna al Cettina, e in Croazia rossa (Croatiam rubeam) dal Cettina fino all'estremità meridionale, che secondo alcuni sarebbe arrivata fino a Durazzo, ma che in realtà non raggiunse probabilmente che la Bojana.

I Serbi dal canto loro diedero il nome a due regioni, la Bosnia (venezianamente Bossina) e la Rascia (Resia), detta oggigiorno Vecchia Serbia, regione tra i fiumi Drin, Vardar, Morava e Lim.

Tolte le differenze orografiche e le divisioni interne del Velebit, del Dinara e della Montagna Nera (forse chiamata così ancora dai Greci e magari dagli Illiri) la regione abbastanza vasta, toccata ora agli Slavi, CroatoSerbi, manca come per gli Illiri di confini montuosi benefici. L'indecisione e la povertà geografica produssero l'indecisione c la povertà etnografica. Queste due cause congiunte collo squilibrio nazionale e culturale colla costa detta ancora dalmatica, furono l'origine principale delle sciagure e dell'inferiorità futura di questo popolo.

Durante la dominazione carolingia, che abbracciò $j$ paesi orientali alpini e la metà settentrionale della costa orientale coi fiumi Drava e Sava fino al Danubio e che fu del resto assai limitata, si presenta nell'agone storico. 
come nucleo croato principale la Croazia bianca (più tardi Dalmazia veneta) con a capo dei Conti, detti nella loro lingua Knezi. - Il fatto politico della prevalenza del distretto della Croazia bianca, si spiega precisamente con ragioni geografiche. $\mathrm{Fu}$ già dimostrato infatti che lungo tutta la costa orientale la regione triangolare tra il. Velebit, il Dinara e la costa (fiumi Zermagna, Cherca, Cettina) è la migliore geograficamente. Per questa causa naturale, congiunta all'altra culturale della maggior presenza in questo tratto di elemento latino, ebbe origine proprio in questo tratto quell'embrione di Stato croato nazionale semi-indipendente, di cui si ode oggigiorno parlar spesso a proposito del trialismo degli jugoslavi in Austria.

Le condizioni politiche suaccennate durarono lungo tutto il IX secolo. Al principio del $\mathrm{X}$ uno di questi conti nazionali, di none Tomislavo, dopo di aver sostenuto coll'aiuto di Bisanzio - ormai in gelosia e in antagonismo con Venezia - che gli cedette le città latine, e lo nominò suo goveruatore (stratega), guerre fortunate contro i Magiari ed i Bulgari e di aver quindi ridolto sotto il proprio potere tutti i paesi croati e serbi, portando il dominio croato a confini mai laggimti prima, nè dopo, s'ineoronò Re dei Croati. Di lui sappiamo solamente che governò dal 903 al 928 e morì poco dopo l'incoronitzione. A lui succedettero i suoi figli che regnarono fino al 1074. Ma alla morte del primo suo successore, avenuta nel 945, il regno si divise, apparentemente per discordie e lotte fratricide di pretendenti, in sostanza per l'immaturità politica del popolo e decadde tanto da subire da lì a mezzo secolo preciso una sconfilta decisiva da parte dei Veneziani.

Nelle lotte che Venezia sostenne lino dal secolo VIII I Nareutani. contro gli abitatori della costa orientale adriatica figurano da questa parte ollre ai Croati anche i Narentani. Siccome tanto gli uni che gli altri non oltrepassarono gli albori di vita civile, le storie, specialmente di quell'epora. non hanno tramandato abbondanti notizie; per cio non sappiamo con esaltezat in quali ripporti politici ed etno- 
grafici si trovavano questi due alleati nel contendere l'espansione di Venezia.

E probabile che negli acquitrini del Narenta abbiano trovato rifugio in tulli i tempi gli elementi refrattari alle imposizioni esterne e straniere e quindi tanto gli Illiri, nemici dei Romani, quanto Romani profughi davanti ai barbari ed indi perfino barbari pagani per salvarsi dalle persecuzioni del cristianesimo.

is probabile che a questo miscuglio si siano aggiunti da ultimo anche degli Slavi (Croati) ed è pure verosimile che i Narentani siano da annoverarsi tra i macstri degli Slavi nell'arte nautica durante l'eclissi storica dei secoli VII e VIII, giacchè i Croati quando si presentarono sulla costa adriatica non avevano altre caratteristiche che quelle di popoli montanari e pastori.

Dei Narentani basterì dire che la storia non ha per Ioro altro titolo che quello di pirati. Un po' pirati saranno stati in principio anche $\mathrm{i}$ Veneziani. Però questi in grazia di una coltura, di un retroterra ricco e di un centro cittadino, poterono evolversi rapidamente a stalo civile. I Narentani al contrario, cui mancava tutto ciò, non poterono superare lo stato barbarico e benchè siano stati forti e temuti, perirono per consunzione naturale. Esercitarono terribili scorrerie su tutto l'Adriatico e per tre secoli (VIII, IX e X) contesero a Venezia, ancora giovane, il dominio di questo mare, ma vinti dal doge Pietro Orseolo II (994-998) non diedero più segno di vita. La loro potenza si potrebbe considerare come un tentativo abortito per legge geografica, di Stato indipendente sulla costa orientale adriatica.

Ma quasi che le condizioni dell'Adriatico non fossero già abbastanza misere, a renderle ancora più dilaniate compariscono sulla sua scena verso la fine del IX secolo altri due attori, fatalmente dammosi, i Saraceni per mare e gli Ungheri o Magiari per terra.

I Saraceni. I Saraceni non sono altro che i pirati di quegli Arabi, che, usciti nel VII secolo dalla loro oscurità, fondarono a spese dell'impero bizantino un dominio mondiale nell'oriente maomettano ed una coltura propria, l'arabica, 
la quale, basata su un fondamento greco, si contrappose all'occidente cristiano.

Gli Arabi non fecero spedizioni di conquista nell'Adriatico che nei distretti costieri della bassa Italia. Distrussero Molfetta nel 988, presero Bari c Brindisi e ne fecero porti di rifugio; si fermarono anche nel Gargano e ne fecero il loro nido principale. Però anche più tardi i pirati degli Stati barbareschi da loro formati sulle coste dell'Alrica (in Egitto, a Tunisi, a Tripoli e nella Mauritania), in Asia minore, a Creta, in Sicilia, si spinsero fin dentro nell'Adriatico e particolarmente sulla costa orientale e sulle isole, perchè più atte alla navigazione. Le loro scorrerie e depredazioni durarono per parecchi sccoli (fino a tulto il XVI) e furono per ciò combattuti da tutti, ma particolarmente dai Veneziani.

La loro presenza nell'Adriatico ebbe conseguenze dannosissime per la navigazione, giacchè le popolazioni delle coste e delle isole, mancando di efficace protezione, e non avendo altra salvezza che nella fuga, disertarono i porti e si rifugiarono al di là delle creste montuose in posizioni non visibili dal mare, ove diedero origine soltanto a miseri villaggi. E appena poco più di un secolo che le insenature minori delle coste e delle isole vanno ripopolandosi.

I Magiari, popolo di ceppo altaico o fimnico e affini Gli tngheri. agli Unni e agli Avari, cacciati verso la metà del IX secolo dai Bulgari via dal basso Danubio, trovarono nuove sedi nel corso del medio Danubio. Da qui incominciarono a devastare i teritori circostanti e si spinsero al principio del $\mathrm{X}$ secolo nell'alta Ilalia e su tutta la costa orientale dell'Adriatico fino a Durazzo. Cessarono di essere un flagello dopo che lurono vinti e civilizzati dai Tedeschi. Ciò non per tanto ricomparirono, male salutati, anche in epoche posteriori. Fu questa ma nuova ingrala preferenza della sorte per la costa orientale.

In una rassegna rapida degli avvenimenti storici di una regione secondaria, paragonabile ad nna corsa in Antraonismo fra il l'apita e. Imper. ferrovia attraverso un paese non bene conosciuto, non si può fermare l'altenzione sui lalti minori, ma bisognal 
limitarsi ad afferrare ora l'uno ed ora l'altro di quei segni e sintomi, che hamno marcato l'attività umana nel cammino del tempo.

Per mantenere il filo materiale degli avvenimenti nel corso del tempo e quello logico nelle relazioni fra causa e conseguenza occorre rilevare che nell'altalena delle lotte tra il papato e l'impero per la supremazia in Europa, il secolo $\mathrm{X}$ segna un rialzo dell'influenza imperiale ed un corrispondente ribasso di quella del Papa.

La tendenza del Papa era di far valere la sua supremazia morale e spirituale sugli Stati per mezzo della sottomissione dei loro capi. A tale scopo la politica papale moveva coll'astuzia o intralciava coll'intrigo i fili delle azioni dei popoli anche sul vicino Adriatico e particolarmente sulla sua costa orientale, ove aveva da combattere l'influenza religiosa e politica di Costantinopoli. I Papi colla nomina dei vescovi, cogli ordini religiosi, colla concessione di benefizi e privilegi, colle investiture di principi e di sovrani erano gli arbitri ed i regolatori della vita politica dell'Adriatico.

Gl'imperatori di Germania al contrario cercavano di consolidare la loro supremazia colla forza materiale e coll'ingrandire la potenza politico-amministrativa dello Stato.

\section{QUATTRO SECOLI (XI-XIV) DI LOT'TE PER L'ADRIATICO}

\section{TRA VENEZIANI, SLAVI ED UNGHERI.}

La decadenza dell'imfluenza papale nel $\mathrm{X}$ secolo cbbe per l'Adriatico già alla fine di esso due importanti conseguenze: l'espansione di Venezia, finora tenuta in scacco da Roma e il tentalivo degl'imperatori di Germania di unire l'Italia in un solo impero colla Germania.

Nel 982 Ottone II cercò di por fine alla signoria greca ed araba nella bassa Italia e nel 983 Ottone III fece guerra alle città marittime della costa orientale per espellere i Bizantini dall'Adriatico. 
Venezia, che aveva incominciato ad uscire dall'ombra sвcon хт. al principio dell'VIII secolo e, forse inconsciamente, si Fra Veneziani apprestava a divenire l'erede dei Romani sul nare Adriae Slavi. tico, sostenne quasi un secolo e mezzo di lotte contro i pirati narentani e gli altri Slavi, che le impedivano l'espansione marittima sulla costa orientale. $E$ verso la fine del $\mathrm{X}$ secolo i Veneziani si apprestarono ad una intrapresa decisiva per la supremazia dell'Adriatico.

Dopo una spedizione guerresca durata quattro anni (994-998) il prode doge Pietro II Orseolo riuscì a vincere la resistenza dei Narentani e dei Croati. In questa lunga campagna non vi fu una battaglia principale decisiva, ma solo qua e là scaramucce. Appoggiati politicamente dai Bizantini e accolli fraternamente dagli abitanti latini della costa, i Veneziani col doge Orseolo occuparono una dietro l'altra Ossero, Zara, Traú, Spalato, Curzola, Lagosta, Lissa, e il doge potè allora ritornare a Venezia cinto del titolo non vano di duca della Dalmazia. Data da allora la tradizionale festa veneziana del Redentore, come simbolo dello sposalizio di Venezia col mare.

Fino a quest'epoca il campo d'azione di Venezia è limilato al mare Adriatico. Come conseguenza di queste vittoric vedremo Venezia nel secolo venturo reggersi a repubblica aristocratica indipendente.

Prima di procedere col racconto degli avvenimenti storici sarí opportuno di sostare un istante per considerare la causa e l'origine delle lotte tra Veneziani e Slavi sull'Adriatico avanti il 1000 d. C.

Chi dei due andò pel primo ad immischiarsi nelle faccende dell'altro e a chi per conseguenza toc'a la colpa nella contesa?

Da parte veneziana sappiamo che i Narcntani colle loro scorreric turbavano il commercio di Venezia; e siccome questa città, anzichè subirle, intendeva di espandersi sul mare e di far navigare liberamente le sue gatere lungo la costa orientale, fu inevitabile un urto. Venezia vinse gli avversari, in casa loro, si noli benc, per superiorità civile, politica c militare. 
Da parte dei Narentani mancano notizie dirette. Però i loro cpigoni, gli scrittori croati odicrni, attingendo alle fonti altrui e commentandole in loro favore, attribuiscono senz'altro ai Vencziani la colpa di essere venuti a turbare l'esistenza e lo sviluppo degli abitatori della costa orientale e li gratificano per conseguenza degli epiteti di ingordi e di astuti mestatori.

Sarebbe ora difficile ed anche ozioso il voler indagare chi fece il primo passo illecito nel campo nautico dell'altro.

É però un falto imnegabile, che Venezia dominò sulla costa orientale adriatica; e per ciò possiamo ammettere senza paura di ledere nè "la carità del natio loco», nè la sensibilità dell'onore, che i Veneziani, essendo i più forti, furono anche i prepotentí. In sostanza essi, non fecero altro che ripetere verso gli Slavi (Narentani e Croati) quello che i Romani avevano fatto verso gli Illiri: con o senza pretesto, è indifferente, essi li attaccarono militarmente per raggiungere il loro scopo economico e civile, e li vinsero. $\mathrm{Si}$ potrebbe forse applicare anche a questo fatto la speciosa spiegazione che i Veneziani agivano in nome di una civiltà superiore. Se però non dimentichiamo la geografia, vedremo che molto probabilmente Romani e Veneziani non fecero altro che obbedire a quella legge naturale, che creò la superiorità complessiva della costa occidentale sull'orientale. E il dominio di Venezia lo dimostra anzi ancora meglio che quello di Roma.

Venezia, superata la resistenza degli abitatori non latini della costa oricntale, continuò a lottare con successo contro i Bizantini e ad estendere i suoi possedimenti anche fuori dell'Adriatico.

Per l'aiuto prestato ai Bizantini contro le incursioni dei Normanni (provenienti dalla bassa Italia) l'imperatore Alessio affidò al doge Vitale Falieri (1084-1096) il dominio non solo sulla Dalmazia e sull'Istria greca, ma anche privilegi commerciali nell'impero bizantino e un quartiere a Pera per la colonia veneziana. 
Nel secolo XI gli avvenimenti continuano a seguire un corso naturale sulla via delineatasi nel precedente, però con maggior rilievo locale per l'Adriatico e colia differenza che l'influenza del Papa risale.

La Lombardia continuò ad essere come al solito il pomo della discordia fra l'impero e il papato e il molivo di discese in Italia degl'imperatori germanici.

I Croati ebbero ancora uno sprazzo di luce soito il Breve storia re Crescimiro, detlo da alcuni più tardi il Grande, discendente di Tomislavo, ma figlio di una dogaressa veneziana (1058-1073), il quale senza portare il suo Stato neppur da lontano all'estensione e alla potenza del primo re Tomislavo, riottenne in nome di Bisanzio il dominio (veramente sarebbe più esatto di dire il governo) sulle città latine della costa $\mathrm{c}$ assunse il titolo di re di Dalmazia.

Alla sua morte si rinnovarono le ambizioni personali, le discordie e le lotte intestine, che, dannose sempre, divemnero fatali, quando nel 1090 si estinse del tulto la dinastia nazionale croata.

Dopo un decennio di gare politiclie e di incertezze, in mancanza di persone adatte tra le famiglie nazionali più in vista, non si sa bene per quale concorso di circostanze, se cioè per opere guerresche o per calcoli politici e a quali patti, la corona col consenso della maggior parte dell'elemento croato (non del latino che dal 1074 si trovava di nuovo solto la supremazia titolare dei Veneziani) fu cinta nel 1102 d. C. da Colomano, re d'Ungheria, e la Croazia passò solto quella dipenłenza dei Magiari, dalla quale ancora oggigiorno tenta inutimente di svincolarsi. 
Da quest'esposizione, Iunga forse per lo studio presente, ma molto succinta per sè stessa della storia della semi-indipendenza croata, si vede che questo Stato, elevato arditamente, ma prematuramente, dall'intelligenza di due governanti, decadde colla loro scomparsa a causa dell'immaturità politica del popolo, ossia della mancanza di un corrispondente grado di civiltà. La coltura era allora ristretta alle città latine della Dalmazia, ormai veneta, che si reggevano a liberi municipi, avverse, meno in pochi periodi, alla signoria slava.

Una prova di questa sorda avversione e della vitalità dell'elemento latino viene offerta dalla lotta, apparentemente religiosa, ma essenzialmente nazionale e civile, per la lingua liturgica. Gli Slavi cioè volevano usare una propria lingua, un po' antica, il cosidetto glagolito (che forma ancora oggetto di dispute religiose-nazionali colla curia di Roma) ed i latini, appoggiati da Roma, esigevano che si continuasse nella loro, ch'era divenuta la lingua del caltolicismo. La contesa ebbe il punto culminante in un concilio turbolento tenuto a Spalato nel 925 (epoca, si noti bene, in cui viveva e governava il re Tomislavo). Eppurc la vittoria rimase al latino spalleggiato dall'influenza dei Pontefici.

Nell'effetto i re Croati non avevano nemmeno una sede stabile ed esercitavano le loro attribuzioni di sovranitả vagando nei coenacula (alberghi) di Nona, Zaravecchia, Sebenico, al mare, Knin (alle sorgenti del Cherca) nell'interno. Talvolta andavano ad abitare come ospiti a Spalato $o$ in altre città latine, alle quali naturalmente dovevano usare deferenza e rispetto e assicurare o concedere moltissimi privilegi.

In mancanza di cause note che indichino il motivo della sottomissione dei Croati ai Magiari si potrebbe ricorrere per spiegazione alla geografia.

Infatti dal Iato geografico la dipendenza, manifestatasi abbastanza per tempo, dei Croati dai Magiari si spiega senza difficoltà.

Croati e Magiari si trovarono di fronte sulla linea geografica della Drava e del Danubio, avanzando $i$ primi 
dalle regioni magre dei monti carsici lungo i fiumi Drava e Sava, i secondi dalla ricca pianura del Danubio e del Tibisco. I paesi del versante carsico orientale, Croazia, Bosnia, Erzegovina, Vecchia Serbia, non esercitarono mai una pressione decisa in nessun senso, non verso l'Adriatico, non verso il Danubio. Una pressione di qualsivoglia genere, se vi fu, provenne sempre dalle regioni sellentrionali a quelle, Ungheria (bassopiano pannonico), Serbia (bassopiano del Danubio). Questo fatto spicgabile ormai facilmente colla natura geografica prova all'evidenza che la regione tra lo spartiacque car'sico dell'Adriatico ed i bassipiani del Danubio medio coi suoi affluenti, può essere libera solamente allora che nessumo si curi di dominarla, ma che la natura l'ha creata tale da essere piuttosto soggetta ai popoli che le stanno a settentrione, senza speranza di poter divenire dominatrice a causa della sua montuosa povertà. Anche prescindendo dunque dalle differenze di razza e di temperamento (i Magiari sono imperiosi, i Croati docili) per ragioni puramente geografiche ai Croati sarebbe mancata l'energia economica d'imporsi a tutto il bassopiano pannonico e per ciò furono i Magiari che attraverso i Croati si aprirono il varco all'Adriatico.

L'effimero lustro dello Stato croato tra il X e l'XI sccolo non è l'unico esempio di questo genere che la storia, in ossequio alla geografia, abbia fatto nascere sulla costa orientale adriatica. Il passato, rispetto al secolo XI, ci addita l'Epiro all'epoca del suo re Pirro (III sec. a. C.) e la storia posteriore ci mostreri nel XIII un regno (Despotia) indipendente di Durazzo per opera di un Angelo, bastardo bizantino Commeno; il ducalo di Durazzo degli Angioini di Napoli (Carlo di Durazzo) nel XIV; un regnó di Bosnia, creato nel XIV, dal bano Trartko; una Serbia all'epoca di Dusciano nel XIV; un'Albania di Scanderbeg nel XV e di Ali di Tepelen, pascia di Giamnina, nel XVIII. Giudicando le cose cogli occhi di un mortale contemporaneo, non sarebbe forse del tulto errato di includere nell'elenco anche l'Albania e il r' gno del Montenegro, almeno come sono ora. 
I Magiari dell'Adriatico.
Lo svolto dall'XI al XII sccolo (anni 1091-1102) segna per l'Adriatico un nuovo fatto importante, la calata alla sua costa dei Magiari.

Questo popolo selvaggio aveva incominciato nel $\mathrm{X}$ secolo dopo le sconfitte inflittegli dai Tedeschi ad abituarsi ad una vila meno nomade, ma la barbarie ed il paganesimo non lo avevano ancora abbandonato del tutto verso il mille. Per sradicare appunto il paganesimo il Papa donò nel 1001 la corona regale a Stefano detto di poi il Santo (995-1038), il quale riorganizzò lo Stato secondo i dettami dell'occidente e del cristianesimo. Così i Magiari si misero sulla via delle riforme, della civiltà e delle conquiste. Nel 1088 sottomisero la Croazia settentrionale e nel 1102 re Colomano (1095-1114) incominciò una guerra coi Veneziani per giungere al possesso di qualche luogo alla costa adriatica. Infatti nel 1111 riuscirono a togliere ai Veneziani la Dalmazia insulare (isole del Quarnero) e più tardi anche le città alla costa verso mezzogiorno. Per il fatto di essere i possessori della Croazia e nel bisogno di un alleato politico, i Magiari si schierarono quali paladini dello slavismo contro la latinità. La loro ingerenza nelle cose dell'Adriatico, durata tre secoli, diede molto da fare ai Veneziani, che per combatterla dovettero ricorrere a tutti gli espedienti della politica e della forza. La lotta, lunga per sè stessa, è complicata per le molte, talvolta anche minime, vicende e per la molteplicità degl'interessi in contrasto.

L'Adriatico occidentale.

Nemmeno la costa occidentale andò esente da mutamenti.

Dopo la decadenza del dominio longobardico e franco e durante le deboli e intermittenti inframettenze degl'imperatori di Germania nella media e bassa Italia, alla costa adriatica tra il possesso pontificio e l'Apulia bizantina, ciò̀ dalla punta d'Ancona al Gargano, s'erano formati avanti il mille due piccoli organismi statali, spesso indipendenti, il ducato di Spoleto colla Marca di Camerino e il ducato di Benevento. Tutti due avevano la loro ragione d'esistenza e base geografica nell'Apennino congiunto al versante adriatico e precisamente il primo nel- 
l'Umbria e il secondo nell'altipiano irpino. Siccome però tanto l'Umbria quanto l'Irpinia gravitano geograficamente piuttosto verso il Tirreno, il momentaneo loro connubio col versante adriatico non diede luogo a grandi organismi statali e andò rotto ben presto. Il ducato di Spoleto colle marche di Ancona e Camerino passò in polere dei Pontefici e servì loro a congiungere Roma colla Romagna; il resto della costa e Benevento, occupati dai Normanni, concorsero ad ingrandire il futuro reame di Napoli e Sicilia.

Questo popolo di audaci avventuricri e pirati scandi- I Normani. navi aveva spinto alcune delle sue schicre nel IX secolo d. C. fin dentro nel Mediterraneo.

Visitarono colle loro scorrerie tutte le coste di questo mare, segnatamente la Spagna, le isole Baleari, l'Africa, la Grecia ed anche l'Anatolia; ma poi scelsero l'Italia, rendendo indirettamente omaggio alla suprema bellezza, fertilità e civiltà della penisola.

Al principio dell'XI secolo si stabilirono nella bassa Italia, tanto dalla costa adriatica (monte Gargano) quanto dalla tirrenica.

I Bizantini per difendere nell'Apulia l'importante possesso della Magna Graecia contro gl'imperatori' Ottoni averano stretto alleanza cogli Arabi (Saraceni) ed ora si servirono di questa per difendersi anche contro gli assalti dei Normami. Ma questi s'intromisero subito molto abilmente e coraggiosamente nelle contese locali, vinsero dapprima i Saraceni, poi posero fine anche alla signoria greca sostituendosi a questa nell'Apulia. Indi destreggiandosi fra gli antagonismi degl'imperatori di Germania e dei Papi, ognumo dei quali avrebbe volentieri esteso la sua signoria sulla bassa Italia, riuscirono colla forza e coll'astuzia a stabilirsi durevolmente nel paese.

Della venuta dei Normami approfittarono gl'imperatori di Germania per estendere la loro influenza solto forma di concessione della provincia in feudo ai nuovi conquistatori. I Normamni alla loro volta approfittarono di questa favorevole contingenza per ampliare le loro conquiste in Italia. Fecero anche delle incursioni cer- 
tamente non del tutto beneliche sull'altra costa adriatica, ed otteunero anche un effimero dominio in Albania (Carlo, duca di Durazzo, 1333) e transitori diritti di protezione sulla repubblica di Ragusa. - In Italia appoggiandosi di poi ai Pontefici fondarono quel reame delle Puglie, che poi, convertitosi in regno delle Due Sicilie e di Napoli, durò fino alla costituzione del regno d'Italia.

Paragone fra i Narentani e i Normanni.
A questo punto non sarà ancora una volta fuori di proposito istituire un conffronto tra le incursioni dei Normanni stranieri nell'Apulia e da qui sulla sponda opposta, che finirono colla fondazione di un reame notevole, ch'ebbe vita per ben sette secoli, e quelle dei Narentani, popolazione indigena, che perirono non lasciando dietro di sè che un brutto ricordo di pirateria.

Le difierenze causali, che produssero effetti così opposti, sarebbero di tre specie: individuale, politica e geografica.

Dal lato individuale non sarà difficile di ammettere anche senza ampie dimostrazioni che i Normanni devono essere stati più forti, più energici, in una parola più bravi dei Narentani. Una prova evidente ci è fornita dall'opera che gli uni e gli altri segnarono nella storia. Nel mentre dei Narentani ci manca una traccia diretta, tanto che ci è difficile determinare perfino la loro identità, dei Nor'manni restano ancora oggi monumenti artistici, tutt'altro che spregeroli, come castelli, chiese, in tutti i luoghi principali della costa occirlentale da Brindisi a Chieti. Sappiamo anzi che quest'ultima città raggiunse sotto di loro una notevole floridezza.

Anche le condizioni politiche, aggrovigliate, ma ardenti, in Italia, in opposizione a quelle più semplici, ma fiacche negli antichi paesi illirici, divenuti slavi, devono aver contribuito all'esito differente delle imprese. La causa però meno evidente, ma più efficace, dev'essere stata anche in questo caso la geografia. I Narentani, sicuri nel loro nido accuitrinoso, poterano razziare impunementé. Ma tolto questo vantaggio mancavano loro quegli altri elementi naturali indispensabili ad un'eroluzione sulla via 
della civiltà. Poveri, in lotta con un retroter'ra più povero ancora, senza agricoltura o industria di sorta, non potevano essere e rimanere altro che pirati.

I Normanni invece trovarono un paese civile, fertile e poterono colla loro intraprendenza metterlo facilmente in valore. Notevole è pure la facilità, già spiegala geograficamente, colla quale passarono dalla costa adriatica (Puglia) alla tirrenica (Napoletano) trasportando seco anche il centro della loro attivitì.

Il tratto rimanente di costa adriatica tra Ie Puglie e I Pontefici. Venezia apparteneva già in quest'epoca allo Stato ponlificio. Bisogna quindi osservare se vi siano stati dei cambiamenti anche da questa parte.

Il papato, caduto molto in basso per la corruzione dell'ambiente romano e rialzato di tanto in tanto per gli sforzi individuali degl'imperatori tedeschi, risorse rapidamente quando il seggio pontificio fu coperto dal toscano Gregorio VII. Sotto la di lui influenza personale il papato gimnse a tanta potenza spirituale e indipendenza dal polere laico, che l'imperatore Arrigo II nel 1077 dovette fare la storica ammenda di Canossa (castello nel Modenese). Nella lotla tra l'imperalore e il Papa quest'ultimo si servi dell'ainto materiale dei Normanni e così l'immigrazione di questi francesi in Italia aiulò gsli sforzi nazionali dei Papi a respingere il germanesimo dall'Italia.

In questo tempo la supremazia feudale del Papa pesava su tutti i paesi dell'Adriatico, e giovò a sostencre sulla costa orientale l'elemento latino delle cilta contro gli attacchi politico-nazionali dei Croati, spalleggiati dagli Ungheri.

Ma arl accrescere ancora più l'importanza dei Pontelici sopraggiunse ora nella storia dell'Adriatico quel lallo rumoroso, che sono le crociate.

Siccome le crociate hanno aperto un nuovo capitolo Considerazioni non solo nella storia degli avvenimenti, ma anche in guello riassuntive. della civiltì, sarà bene di riassumere brevemente lo stato della coltura nei secoli V-XI d. C.

Abbiamo vedulo che i latini sull'Arialico erano i rap- 
presentanti di una coltura, evoluta, ma infrollita e corrotta. Ai bisogni della vita facevano provvedere dagli schiavi, s'erano disabituati dal maneggiare le armi e non pensavano che a godere con tutte le raffinatezze dello spirito greco-orientale. Quando per ciò la trasmigrazione dei popoli travolse questo loro edificio tarlato e gli schiavi defezionarono dalle milizie e dalle case, essi si trovarono senza difesa, senza sostegno, sospesi, diremo così, nell'aria e precipitarono a terra in balia dei barbari, superiori momentaneamente e relativamente a loro soltanto nella rudezza delle armi. Per tre secoli questi barbari fecero il comodo loro e alfine si stabilirono dove piacrue ad ognuno. Secondo le tradizioni della loro patria d'origine e della loro vita nomade, la maggior parte di questi barbari vincitori, divenuti signorotti dispotici per quel cosidetto diritto che veniva loro conferito dalla for$z a$, andarono ad appollaiarsi a preferenza sui punti elevati, ove costruirono castelli e vissero esercitando o la caccia o la guerra per abitudine atavica. La vera coltura romana s'era concentrata e conservata nelle città, cui i principi stranieri dovettero necessariamente concedere vita libera sotto diversi aspétti, e nei conventi, eretti dalla nuova religione di Cristo. Di tutto il resto della popolazione, che oggi si direbbe campagnola, poco si curavano; non erano più schiavi, perchè il cristianesimo aveva abolito la schiavitù, ma secondo un'altra parola dal significato non molto più roseo, erano servi della gleba.

Per la necessità di un contatto a scopo di condominio, principi ed ecclesiastici si avvicinarono. Da ciò derivò l'ingentilimento dei primi, però anche l'esclusivismo della coltura, che in questo periodo e in parecchi dei secoli successivi rimase un privilegio delle classi aristocratiche, dal quale il popolo era assolutamente escluso. Con ciò però era fatto anche il primo passo alla fusione nazionale, che avvenne gradatamente, dove più, dove meno presto, ma fu compiuta durante le crociate.

Di questa fusione romano-barbarica rimane un monumento imperituro nelle lingue.

Nell'italiano non e'è ur'abbondante infusione stranie- 
ra se non nei termini guerreschi, che incominciando dalla stessa parola "guerra» sono germanici.

Al contrario il tedesco, il magiaro, i dialetti slavi parlati all'Adriatico, l'albanese, sono pieni - a parte i tentativi moderni di purismo letterario - di parole latine per indicare oggetti del vivere civile e sentimenti dell'animo.

\section{LE CROCIATE.}

Finora l'Adriatico per la depressione subentrata alla secozo xir. costa occidentale dopo la rovina romana e per il sommo dei popoli senza civiltà che s'erano insediati alla costa orientale aveva passato cinque secoli di letargo. Aveva incominciato a svegliarsi nell'XI, sempre dalla parte occidentale, per opera dei Veneziani e dei Normanni. Le crociate col XII secolo lo fecero rientrare nella scena della storia.

Per la storia universale le crociate sono una grandiosa e rumorosa lotta di tutte le forze collegate dell'occidente cristiano contro l'oriente islamitico; - per la civiltì un progresso, perchè dal contatı, per quanto violento, di popoli diversi ed opposti, crebbero le cognizioni degli uni e degli altri; - per la psicologia uno scoppio di patologia religiosa collettiva; - per l'Adriatico una grande risorsa, giacchè come strada naturale tra i due mondi in lotta, doveva approfittarne sopratulto nei commerei.

L'Adriatico è per l'Europa quello che una via è per una città. Quanto maggiore è il passaggio della gente, tanto maggiore è il numero dei negozi; sono i compratori che stimolano i venditori, non viceversa.

Nelle vie frequentate non e'è posto per le fabbriche: queste stanno meglio nei sobborghi. E l'Adrialico per la sua conformazione geogralica, tolta la Padimia, ch'c̀ 111 buon campo di produzione, non ha sulle sue coste che stazioni di vendita. Le città sono le bolteghe.

All'epoca romana l'Adriatico, più che un mercato, cra 
ull gran lago signorile, di lusso, in cui preponderavano le ville e le stazioni balneari.

Per opera delle crociate esso divenne nel medio evo un variopinto bazar di mercanzie. Vedremo subito chi crano i venditori ed i compratori.

Delle selte crociale principali ch'ebbero luogo nel corso di due secoli (XII e XIII) tre interessano particolarmente l'Adriatico: la prima, la quarta e la quinta.

Nella prima (1096) i crociati francesi seguirono la via terrestre dell'Adriatico lungo ambedue le coste. Divisisi a Lione, una parte, condotta da Raimondo di Tolosa, passò alla riva sinistra del Po per Milano fino ad Aquileja e quindi lungo la costa della Dalmazia raggiunse Durazzo, dove s'incontrò coll'altra parte proveniente dall'Italia. Questo secondo gruppo condotto da Roberto di Fiandra e Ugo di Vermandois per Torino, Genova, Firenze, Roma, attraverso Benevento aveva fatto capo a Bari, donde per mare si fece trasportare a Durazzo.

Nella quarta (1202-1204) il movimento invece seguì per mare ed esclusivamente per la via dell'Adriatico, con immenso vantaggio di questo, ma specialmente di Venezia. L'esercito crociato si unì a Venezia. La comparsa dello spodestato principe bizantino Alessio e l'acuta perspicacia del doge Enrico Dandolo, vecchio cieco di novant'anni, resero i Veneziani arbitri della situazione. Essi imposero ai crociati, come compenso di una spedizione a Costantinopoli, una diversione contro le città della Dalmazia, tolte dagli Ungheri. Nell'amo 1198 tutta la Dalmazia continentale e marittima era ricaduta sotto il dominio degli Ungari-Croati. A Venezia premeva grandemente di riguadagnare la posizione perduta sulla costa orientale. E questo un punto culminante nella storia di Venezia e di Zara, quale capitale della Dalmazia e città più importante della costa dall'età di mezzo in poi. I Veneziani per ción col crociali l'assediarono, I crociati l'as- 
salirono dalla parte di terra, i Veneziani guidati dal doge Enrico Dandolo per mare. Zara resistera. Allora i Veneziani, sbarcate anche le loro milizie, vincitori poi vinti, per ben due volte affrontarono la preponderante potenza terrestre degli Ungari-Croati. Dopo quattordici giorni d'assedio Zara fu presa d'assalto e distrutta in gran parte.

Nella quinta crociata Federico II, la cui altenzione ed attività erano tulte occupate nella bassa Italia, salpò dal porto di Brindisi (1228). Siccome frattanto gli Ungari avevano rioccupato la Dalmazia (nel 1226 Colomano, fratello di Bela IV, re d'Ungheria, è nominato duca della Dalmazia) Venezia riesce ad utilizzare i guerrieri della quinta crociata per ritogliere Zara agli Ungari-Croati e vendicar l'onta subita da Enrico Dandolo.

In grazia di queste spedizioni in Palestina Venezia riacquistò coll'aiuto dei crociati le coste dell'Adriatico orientale (oltre ad altri possedimenti fuori di questo mare).

La partecipazione dei Veneziani e delle loro navi non mancò certamente nemmeno nelle altre crociate; ma ciò che procurò a Venezia grandi guadagni fu il Lrasporto regolare dei pellegrini, che a migliaia si recavano due volte all'anno in Palestina e dovevano pagare il nolo del tragitto.

Cosi i Veneziani divennero non solo i primi navigatori, ma anche i principali commercianti ed esploratori (Marco Polo) e la loro ciltà divenne la più llorida dicil'Adriatico.

Le navi di molte altre ciltà dell'Adriatico settentrionale esercitarono il commercio solto bandicra veneziana.

C'erano poi ancora altri tralli di costa, politicanente liberi. Tra questi devono venir ricordati principalmente quelli dello Stato pontificio nella Romagna dalla loce del Po ad Ancona e del reame delle Puglic - divenuto poi regno di Napoli e delle Due Sicilie secondo i cambiamenti di none più che di sostanza - sulla costa oc:cidentale; sull'orientale la ciltà repubblica di Ragusál. $\hat{\mathrm{E}}$ venuto il momento di dire qualche cosa anche su quest'ultima città. 
Ragusa. Ragusa deve la sua esistenza, come Venezia, alle distruzioni degli Avari.

La data della sua fondazione viene posta nel 656 ed è molto probabile clıe a darle vita abbiano contribuito fuggiaschi di Epidauro, di Salona e di altre città latine. Certo è però che i suoi fondatori erano di sangue romano. A questi si aggimsero nella seconda metà del IX socolo immigrati, pure latini, fuggiaschi dalla costa d'Albania e appena alla fine di questo secolo (XII) incominciarono le prime infiltrazioni di Slavi (Serbi) dal retroterra. Però la lingua italica era dominante fino all'XI secolo.

Dalla fondazione per parte di un elemento già civilizzato, dallo squilibrio tra l'elemento cittadino e l'agricolo, derivarono a Ragusa la signorilità, la costituzione aristocratica colla divisione in patrizi e plebei e la preferenza della lingua e coltura italiche, durate per tutto l'avvenire fino ai secoli recenti.

Ragusa sofferse in principio per parte dei pirati Narentani e Saraceni. Ma poi i suoi cittadini, approfittando dell'esperienza nautica e della superiorità civile in confronto agli abitatori del circondario, seppero estendere un po' alla volta la loro navigazione e il loro commercio. Essi, approfittando delle lotte degli altri e rispettando molto astutamente i più forti o mettendosi sotto la protezione del più potente, secondo i casi, si destreggiarono nelle più violente burrasche politiche. In questo modo il fine senso diplomatico dell'aristocrazia ragusea riuscì a condurre avanti la semi-indipendenza del minuscolo Stato - unica forma di libertà possibile - per quasi un millennio, fino alla fatale epoca napoleonica del principio del XIX secolo.

Nel principio della sua storia, avanti la comparsa di Venezia e durante la supremazia nominale di Bisanzio nei secoli VIII e IX, Ragusa non ebbe da premunirsi che 
dalla parte di terra e verso corrisponsione di un contributo si assicurò la tranquillità da parte dei signorotti slavi del suo retroterra. Questo retroterra è costituito dall'odierna Erzegovina, che allora si chiamava Zachumia e Travun. Quando s'accorse che i Veneziani incominciavano a divenirle pericolosi si appoggiò a Bisanzio ed ottenne la protezione dei Greci. Quando poi dopo la quarla crociata (principio del XIII sec.) Venezia divenne dominante sull'Adriatico, - ed è questo il momento nel quale l'abbiamo fatta comparire sulla soglia di questa esposizione storica - accettò, pur di conservare la propria indipendenza amministrativa, la protezione politica anche di Venezia e la tollerò per un secolo e mezzo. In quest'epoca Ragusa, oltre alla città, comprendeva un piccolo territorio, la vallata longitudinale parallela alla costa fino alla Puntadostro all' entrata nelle Bocehe di Cattaro.

Considerando poi la posizione topograllica di Ragusa si deve dire che la geografia locale, come fu estranca alla sua fondazione, così non contribuì nemmeno alla sua floridezza. Ragusa prosperò sul mare, ma solo lacendo da mezzo di trasporto delle merci d'altri paesi e per conto di altri, e quindi la ricchezza dei Ragusei fu una grandeza effimera. Una floridezza che si lorma colle ricchezze accumulate lontano cessa appena viene a mancare la causa remola e difficilmente risorge, perchè le fa diletto la for\%a intrinseca, costituita sempre per i porti marittimi dalla ricchezza del retroterra. La storia di Ragusa è paragonabile a cuella di Amalfi e Pisa. Se per conseguenza Ragusa potè segnare nella storia tredici secoli di libertì el un'invidiabile opulenza, to si deve attribuire sopratulto al raro senso politico dei suoi cittadini.

Nell'enmmerazione dei venditori, tra i popoli che con- Useita dei Bitribuivano agli scambi dell'Adriatico, bisogna ricoldare zantini dall'Aancora per breve tempo i Greci bizantini, i quali vi esercitavano anche trasporti e commerci. Anzi in questeporal avenne da parte dei Bizantini un fiallo notevole fer l'Adriatico. 
Cadulo transitoriamente l'impero bizantino appunto in seguito alla quarta crociata $u$ congiunto della casa imperiale, Angelo bastardo Comneno, fondò a Durazzo nell'Epiro un regno indipendente, ma di breve durata. Fu questo un barlume d'indipendenza albanese e l'ultima tappa del cammino regressivo dei Bizantini dall'Adriatico. I loro possedimenti sulle due coste di questo mare non avevano solidità. Essi, come i loro predecessori dell'epoca classica, non occupavano che le città e per ciò appena i popoli della rimanente terra raggiungevano forza e coscienza, le colonie bizantine dovevano gradatamente cadere. I Carolingi, i Papalini, i Veneziani, i Normanni, gli Ungheri, i Ragusei li privarono un po' alla volta dal nor:l verso sud. I Turchi si preparavano già a dar loro l'ultimo colpo.

s:

I compratori erano in prima linea i cavalieri crociati che venivano da paesi più o meno vicini, dalla Francia, dall'Alemagna, dall'Inghilterra, secondariamente dalla Spagna, dall'Ungheria, dalla Bocmia e dalle altre regioni italiane.

Il commercio di Venezia si estendeva da una parte fino all'India e dall'altra fino al Belgio e al mare del Nord. A differenza quindi dell'epoca romana, nella quale il commercio transitava l'Adriatico nella sua larghezza da una costa all'altra per congiungere Roma al Danubio, all'epoca di Venezia la lunghezza dell'Adriatico era la via seguita per lo più dalle navi mercantili dell' evo medio.

In linea locale ci sono da ricordare ancora le fiere o mercati annuali, che traendo la loro origine da argomenti religiosi, chiamavano gente e compratori da tutti i luoghi vicini di terra e di mare. Le principali, che si conservarono per tutta l'età di mezzo ed anche più tardi, erano quelle del Santuario di Loreto e di San Nicola a Bari. 
L'insuccesso delle crociate nel loro scopo principale, Conseguenze quello di liberare i luoghi, detti santi, dalle mani degli flelle crociate. infedeli, dimostra che la lotta della eiviltà europea in nome del cristianesimo contro la civiltà orientale, rappresentata dall'islamismo, cr'a stata ingaggiata male. Anzi, nel mentre prima l'islamismo era limitato all'Asia e all'Africa, la provocazione delle crociate lo attrasse su suolo europeo, ove doveva molto presto far tremare la cristianità fino nel centro d'Europa.

Anche per questo fatto le cause sono molte. In consonanza però al tema assunto per questo lavoro, sarì citata una sola, ancora e sempre, la geografia. La cristianità volle combattere l'islanismo troppo da lontano.

Gl'infedeli, com'erano chiamati allora quelli che oggi sono detti da tutti i Turchi, erano gimnti al Mediterraneo orientale e alle porte d'Europa come in casa loro. L'entusiasmo religioso e guerresco avera mosso i principi c cavalieri delloceidente dalla Francia, dall'Alenktgna, dall' Inghilterra. I piè vicini relativamente erano quelli d'Ungheria e d'Italia. Ma anche fra questi e la Palestina intercedeva un lungo tratlo di terra, la penisola illirica o greca, e un mare vasto l'Egeo. Ora per ragsioni appunto religiose le popolazioni scismatiche della penisola balcanica, che incominciavano subito dopo il versante carsico della costa orientale atliatica, non polevano simpatizzare soverchiamente con un'impresa promossa dai Pontefici di Roma a proprio lustro. Non è da meravigliarsi quindi se i cavalieri di Francia e di Lamagna anche per questi soli fatti giungevano in Anatolia estenuati e se i loro trionfi furono eflimeri.

Malgrads quest'insuccesso l'impresa delle crociate segna nella storia un arvenimento di primo ordine, perchè dal tramonto della romanita mondiale questo è il prino risorgimento di tutte le energie dell'Emopa. Una storia, e per alcuni popoli tullalleo che disprezzabile, conte i 
Franchi e i 'Tedeschi, l'hanno anche i secoli intermedi; però con tutto lo splendore locale essi non possono essere considerati che come tempi di preparazione. La vera rielevazione morale, civile e guerresca dell'Europa dopo la corruzione della romanità e l'infusione di nuove energie portate dalle invasioni barbariche incomincia appena colla terza crociata (fine del XII secolo). Finora l'Europa aveva tatto progressi notevoli quasi esclusivamente negli ordinamenti guerreschi e per ciò quando, infiammata da ardore religioso, si trovò di fronte ad un'altra civiltà come l'islamitica, basata pur essa sul valore militare e sul fervore della religione, non potè superarla nel primo momento. Appena quando l'Europa in conseguenza appunto delle crociate sviluppò anche altre sue energie civili potè ottenere la vittoria sulle forze inevolute guerresco-religiose dell'oriente. Ma a ciò le occorsero otto secoli, la cooperazione dei popoli cristiani scismatici della penisola illirica, prima sempre erroneamente trascurati, l'ardore del sentimento nazionale, sostituitosi opportunamente al fervore della fede religiosa, la febbre dei guadagni commerciali e l'ingordigia dello sfruttamento industriale dei paesi soggetti ai Turchi. E con tutto ciò l'islamismo, vinto e curvato, non è ancora domo del tutto.

Per l'Europa in generale le crociate ebbero la conseguenza di far conoscere i popoli tra di loro e di promuovere i commerci. Da ciò derivò dapprima un cambiamento nel tenor di vita e poi anche nel corso delle idee. Si svilupparono lo spirito cavalleresco, la poesia, lo studio delle lingue e delle scienze. Si fecero strada nuove idee di libertà e dai castelli prima chiusi ed armati si formarono nuove città. Per comprendere il valore dello sviluppo preso dagli studi dopo le crociate basti ricordare che appena da quest'epoca data la conoscenza dei numeri arabici. In conseguenza di questa cognizione si svilupparono la malematica e l'algebra. I commercianti italiani trovarono e diffusero nuovi metodi di computisteria. Trace di questo risveglio esistono ancora nei termini commerciali italiani usati nelle lingue straniere.

Oltre però a quegli indicati, che sono gli effetti delle 
crociate per l'Europa, per l'Italia e per l'Adriatico, ec n'è per l'Adriatico uno particolare. Questo mare, che per un paio di secoli era stato la linea di divisione tra il cristianesimo di Roma e quello di Costantinopoli, divennc: ora colla sua costa orientale il confine fra cristianità ed islamismo. Da ciò gliene derivarono danni civili sensibili, ma in compenso qualche piccolo vantaggio economico per essere divenuto la via di contatto nei nuovi scambi commerciali. Nella rivoluzione che ne derivò per le sue condizioni morali e materiali si sviluppò accanto all'ineremento commerciale e nautico, che fu maggiore che altrove, un altro fenomeno: l'evoluzione della lingua.

Era naturale che in Italia, la culla del latino tanto pel paganesimo che per la cristianità, questa lingua resistesse alla corruzione e si conservasse quindi più a lungo che altrove nella sua purezza negli ambienti cosidetti aulici. Però la commistione etnografica e l'abbandono in cui era stato lasciato il popolo avevano fatto nascere una lingua corrotta, volgare, che diede segni di sè già dopo il 1000 .

I movimenti delle crociate, già dopo la terza, diedero origine a componimenti poetici e di prosa, scritti in una nuova lingua, che allora poteva apparire dialetto volgare, ma che era invece il seme della lingua italiana. Certo incominciando dalla fine del XII secolo, ma forse anche prima, trovatori provenzali diedero vita a questo dialetto nelle piceole corti dell'alta Italia (Padania) dove esso era compreso. Contemporaneamente nella bassa Italia si fa sentire un altro dialetto nella scuola siciliana. Anche a Bologna si forma una speciale scuola poetica, detta da Dante "del dolce stil nuovo".

Il rimanente dell'evoluzione è merito della Toscana. Ma per accennare in quanta misura l'Adriatico vi abbia contribuito, vengono ricordati i nomi dei compositori nei dialetti volgari, che provenivano da luoghi del bacino adriatico: Sordello da Mantova, Bartolomeo Zorzi, Mareo Polo, Giacomo Pugliese, tra Bonvesin da Riva, Giacomino da Verona, Ruggerone di Puglia, Pier delle Vigne (che: cantava per le vic di Barletta), ece. 
Similmente poi a quanto avveniva nella parte occidentale dell'Adriatico, sull'altra costa sorgeva il volgare dalmatico. Era questo un dialetto neo-latino, che andava formandosi nelle cillà delle isole e della costa dall'Istria in giù, per corruzione spontanea del latino, giacchè allora le relazioni colla costa occidentale erano assai intermittenti. Esso fu parlato dal tramonto dell'egemonia romana fino ai secoli XII, XIII e XIV e scomparve lentamente dal 1420 in poi assorbito dal veneziano e dall'italiano. Con questa parlata ha molta somiglianza il dialetto vegliotto che sopravvisse fino ai tempi recenti. Un vecchio, morto a Veglia nel 1898, fu l'ultima persona che ricordasse di aver parlato il dalmatico antico dei suoi padri.

Stato Ponti-

Per l'Adriatico l'epoca delle' crociate va notata non solo per il rifiorire dei commerci, che fecero sorgere a nuova vila le città della costa, ma per la mancanza di un potere sovrano riconosciuto.

Chi dirigera la politica era il Papa, la cui importanza era cresciuta cnormemente per il rinato fervore religioso. Dopo la decarlenza dell'impero si vide nel papato la potenza unificatrice e direttrice della politica europea. II rispetto verso i Ponteíici era salito nelle genti a tal punto, che il papato esercitava per tacita sottomissione altrui realmente la signoria temporale, anche quando non era refto da uma persona energica. Pontefici illuminati averano rilevato espressamente il carattere spirituale della loro somma potestá, ma di fatto nella curia romana l'imperium si mescolò e confuse sempre più col sacerdotium. $\mathrm{E}$ così non solamente il papato assunse sempre più il carattere di un potere temporale, ma l'intera Chiesa cattolica accuistò potenza materiale. E siccome la formazione di organismi statali poderosi, specialmente in Italia, non sarebbe stata conforme agl'interessi del minuscolo Stalo pontilicio, la politica dei Papi favoriva tutti i piccoli antagonismi nazionali e le ambizioni locali delle varie ciltà in Italia e sull'Adriatico.

Ercoso xiI. Il medio evo in generale, ma specialmente l'epoca successiva alle crociate, è il periodo del massimo fiore del 
feudalismo, del frazionamento di poteri, di lolte e contese tra signorotti. E non era possibile altrimenti.

$\mathrm{Al}$ naufragio della romanità erano rimaste nella Padania e alle due sponde del mare Adriatico quali superstiti molte ciltà romane (che non tulte erano slate distrutte dai barbari) come segni miliari, colonne o fari, della civiltà sommersa. In ognuma di queste o s'era insediato un principe straniero, oppure una delle più intraprendenti famiglie indigene aveva assunto la direzione e preso il potere. Avanti le crociate partirono da queste cilta guerre, angherie ed assalti, ma tutto questo era prodotto più da indomabilità di temperamento, quasi per passatempo. Quando poi la collura degli studi e le esperienze dei lunghi viaggi per le crociate insegnarono ai principi il valore della ricchezza materiale congiunta al sapere della mente, Lutti quei principi e signori d'um tratto vollero elevarsi, espandersi, arricchirsi. Ora siccome l'ingrandimento di uno non poteva seguire che a scapito dell'allo, ne successero nuove contese, lotle e guerere, piò gravi ancora, determinate da smania di rapina e di con(puista. La cavalleria, sviluppalasi appunto in seguito alle crociate, diede nuovo incentivo allo spirito avventuriero. E così ebbero origine altre imprese, che non si possono qualilicare altrimenti che capriceiose. Siccome inline questi particolarismi erano stali lavoriti in principio tanto dagl'imperatori, che nei principi vedevano i guerrieri pronti ad accorrere ad ogni chiamata, quanto dai Pontelici, che nell'altrui debolezza vedevano crescere la propria autorità, era maturale che ruesto stato di cose non potesse scomparire tanto presto e dovesse anzi durare finchè un potere encrgico non avesse agito da livellatore.

Intanto lo sviluppo della collura negli stali sociali medi, il crescere dei commerei e delle industrie e mal comprensibile reazione contro lo smisurato arbilrio deghl'inperatori germanici, avevano fallo naseere nelle cilli romaniche il desiderio di arere un'amministrazione aulonoma. Sorsero e si svilupparono così i numicipi di lipo italico, che si formarono non solo nell'alta e media Ita- 
lia, ma anche nelle regioni dell'Adriatico. Qui però l'autonomia municipale, oltrechè scopo di amministrazione, aveva anche un sostrato nazionale, perchè le città alla costa orientale erano latine in antagonismo colla popolazione slava delle campagne e alla costa occidentale non erano ancora scomparsi l'elemento ed il sentimento greco.

E naturale quindi che a questo periodo di particolarismi corrisponda il massimo frazionamento e instabilità dei poteri.

Alla costa occidentale esisteva, come fu detto, il reame delle Puglie, che dalla metà del XII secolo (1130), dopochè Ruggero II conquistò anche la Calabria e la Sicilia, prese il nome di regno delle Due Sicilie.

Rispetto al scnso geografico di questo regno va notato che in principio del dominio normanno il lato più importante della bassa Italia era la costa adriatica (Bari), ma che poi il centro della vita fu trasportato nel Tirreno (Napoli, Amalfi, Salerno).

Quando verso la fine del XII secolo (1194) - dunque dopo un secolo e mezzo di dominazione - si estinse la dinastia dei re normanni, parte per diritto di matrimonio, parte colla violenza subentrarono i tedeschi Hohenstaufen. La casa sveva degli Hohenstaufen non durò nella bassa Italia nemmeno mezzo secolo.

Il principio del loro dominio fu luttuoso. Arrigo VI per assodare la signoria acquistata usò la massima durezza. Fece ardere, impiccare ed impalare gli avversari e trasportare con cavalli da soma i tesori della bassa Italia nei castelli degli Hohenstaufen in Germania. La parte meridionale della costa occidentale adriatica sofferse nel medio evo devastazioni simili a quelle patite dal resto nella trasmigrazione delle genti.

I successori di Arrigo VI e particolarmente Manfredi ripararono alle stragi. Col fascino della sua bella mente e del gentile aspelto Manfredi acquistò tanto favore ed aderenti anche nell'Italia media ed alta, che nessun principe italiano poteva più compelergli. L'idea ghibellina celebrava col suo mezzo un trionfo ed a lui sembrava arridere già l'ambito titolo di Rex Italiae; ma l'intro- 
missione dei Papi, che non volevano più saperne degli Hohenstaufen, perchè ligi all'influenza degl'imperatori, fece venire come equilibrio contro gl'imperatori tedeschi i] francese Carlo d'Anjou.

Questa è la seconda volta a breve scadenza clie nella storia delle lotte tra Pontefici e Imperatori germanici per la signoria sulla bassa Italia l'aiuto francese invocato dal Papa arresta o danneggia l'influenza tedesca, senza però realizzare le tendenze dei Pontefici invocatori.

Di queste tre signorie, normanna, sveva ed angioina, la migliore fu la prima. Tutte tre furono però accompagnate da gucrre, distruzioni, che danneggiarono non poco il paese.

Da tutte queste vicende, in complesso più dolorose che liete, si vede che l'influenza del clima e della mollezza greea, che avevano reso deboli i popoli della bassa Italia di fronte alla conquista romana, continuava ostinatamente ancora.

Lo Stato pontificio aveva subìto varie restrizioni e Lo Stato della spogliazioni ogni volta che gli imperatori di Germania o altri principi in Italia averano poluto commellere rappresaglie o usurpazioni sul patrimonio temporale del Pontefice.

In quest'epoca le donazioni di Pipino (che interessano particolarmente la costa dell'Adriatico) erano passate in gran parte in altre mani.

Ma l'altalena delle vicende umane si rialzò per il papato sotto Innocenzo III, che fu uno dei più valenti Pontefici, di cui la storia si vanti (1198-1216). - Innocenzo rivolle tutti quei possessi, cui credeva di aver diritto e approfittando delle lotte per il trono tedesco oltenne dall'imperatore un documento, in cui erano confermate tulte le pretese dei Papi alle donazioni carolinge e per giunta anche Spoleto ed Ancona. Allo Stato pontificio furono assegnati: il cosidetto Palrimonium Sancli Pelri, l'esarcato di Ravenna, la Pentapoli, la marca d'Ancona, il ducato di Spoleto, la contea di Bertinoro el i beni di Matilde di Toscana.

Questo documento ebbe la massina inportanza, mollo L'Adriatico. 
più che l'influcnza papale in Italia e su tutte le due parti dell'Adriatico aveva raggiunto l'apice sotto Innocenzo III.

Di tutto ciò se ne saranno accorti senza dubbio i contemporanei, però soltanto i posteri hanno potuto sentenziare, che quel documento formò il fondamento decisivo per l'estensione ulteriore dello Stato della Chiesa. L'attenzione dei contemporanei è di solito colpita maggiormente dai fatti rumorosi, che sono o l'epilogo di lunghe evoluzioni conosciute oppure scoppi effimeri di forze, che percorrendo una troppo rapida parabola ascendente, sono destinate a cadere presto e poco lontano dal punto di partenza. Quei fatti invece che potrebbero costituire il principio o le tappe di grandi avvenimenti sono silenziosi e trascorrono ignorati. Essi per ciò non vengono scoperti che dai posteri ed anche questo solo quand'ebbero la fortuna di essere coronati da un grande edificio storico.

I Municipi italiani. Albori del trecento.
Una prova di quanto fu detto ora, ci viene offerta dall'alta Italia e specialmente dalla Lombardia.

L'impero romano-germanico, fondato dai Carolingi, aveva ricevuto un'impronta prevalentemente tedesca ancora sotto gl'imperatori Ottoni, della casa sassone (X secolo). Dopo tale cambiamento d'indirizzo politico, la coltura delI'Alemagna illanguidì e già dall'XI secolo fu superata dall'Italia e dalla Francia, che ripresero il primato. Specialmente in Italia si facevano sentire nella coltura e nella vita politica gli albori di quel rinascimento che, spiegatosi nel XIII secolo, doveva affermarsi potentemente nel XV.

Consci di tale risveglio i municipi italiani dell'alta Italia e specialmente quello di Milano cercarono di rendersi indipendenti amministrativamente dalla supremazia degli imperatori di Germania. Si venne così nella seconda metà del XII secolo a quell'epica lotta tra Federico Barbarossa e le città lombarde, in cui si distinsero Arnaldo da Brescia, la lega lombarda ed i vittoriosi di Legnano. La lega porta il nome delle città lombarde, ma era formata da tutti i municipi dell'alta Italia. Vi partecipò anche quello di Trieste. Fra tutte le città primeggia natu- 
ralmente Milano per quella legge geografica che fece di lei il cuore della media Padania e che la risollevò dopo ogni sciagura. A Milano s'impernia per conseguenza anche nel medio evo la lotta contro gl'imperatori tedeschi.

Le guerre rivestirono un carattere nazionale, ebbero rumori strepitosi ed entusiastici, ed essendo terminate colla vittoria dei Lombardi avranno fatto concepire nei contemporanei italiani chi sa quali speranze per l'avvenire. Invece dissidi intestini, causati da mancanza d'un ideale comune, fecero sgretolare dopo alquanti secoli la loro floridezza, libertà e indipendenza, nel mentre il potere temporale dei Papi, che perseguiva uno scopo unico, benchè fosse basato su un documento di carta, riuscì a consolidarsi ed a durare molto di più.

Ma, ritornando all'esposizione di falti, occorre rilevare tutto il valore della pace conchiusa tra Federico Barbarossa e le città lombarde (a Costanza nel 1183). In essa l'imperatore rinunziò all'esercizio della maggior parte dei suoi diritti di un tributo annuo. Ma la clausola più importante era quella con cui l'imperatore riconobbe l'indipendenza dei comuni nella giustizia e nell'amministrazione. Con ciò i comuni ottennero una posizione semi-indipendente e i loro consoli eletti liberamente e investiti soltanto dall'imperatore potevano escrcitare l'autorità suprema.

$\mathrm{Fu}$ questo un grande trionfo della borghesia, che entrò a far parte dell'organismo sociale con diritti eguali a quelli della nobiltà e del clero. La conseguenza di questa vittoria dei municipi fu che la Lombardia, finchè durò la dinastia sveva degli Hohenstaufen, continuò ad apparire di nome come dipendente dall'impero, ma estintasi quella nel 1268, tutto il Milanese fu solamente governato per oltre un secolo, esaltamente sino alla fine del XIV, da vicari imperiali.

Anche Venezia, in progresso per il generale risveglio dell'Italia, per la propria attività e per le lavoreroli cirSecolo Xiv. Venezia. costanze create dal movimento delle crociate, contemporaneamente allo sviluppo dei suoi commerci, cercò di 
estendere, a scopo principalmente commerciale, la sua signoria. Un'espansione di Venezia sui territori circostanti di terraferma era ostacolata dai Papi c dagli imperatori.di Germania. Alle sue spalle nelle regioni dell'Adige c'era il vescovato di Trento, ad oriente il patriarcato di Aquileja, a mezzogiorno oltre il Po le Romagne (contea di Comacchio, esarcato di Ravenna, ecc.) tutto feudi ecclesiastici, più in dentro le città lombarde capitanate da Milano, che lottavano anche contro nemici più potenti di Venezia.

Pel ciò s'era rivolta alla costa orientale adriatica, ove l'impresa si presentava molto più facile $\mathrm{e}$, come si è veduto, era anche riuscita.

Approfittando delle crociate Venezia s'era spinta anzi molto più in là nel Levante, in mari e regioni che non interessano direttamente il nostro argomento, ma dei quali si deve far cenno, perchè a causa di loro Venezia venne dal XIII al XIV secolo in una lunga lotta con Genova. Questa lotta durò centotrent'anni e finì col trionfo di Venezia dopo la vittoria navale di Chioggia (1379).

Durante questa lotta però Venezia ebbe a subire per parte dei Genovesi due disastri navali proprio sull'Adriatico, che meritano di venir ricordati, perchè da loro si possono trarre degli utili ammaestramenti per il presente.

Nel 1298 i Veneziani si scontrarono coi Genovesi nelle acque di Curzola. L'ammiraglio genovese Lamba Doria si pose sopra vento e con polvere di calce viva accecò gli equipaggi veneziani del Dandolo. L'esito dello scontro fu una segnalata vittoria dei Genoresi sui Veneziani, in casa si può dire di questi, riportata a merito dell'astuzia del condottiere genovese. In questa battaglia una galera veneziana era comandata da Marco Polo (il grande autore del Milione) che una tradizione, forse confusa, vorrobbe fare nativo di Curzola.

Nel 1379 i Veneziani incalzati dai Genovesi dovettero accettare battaglia nelle acque di Fasana presso Pola. Nella celebre battaglia del 9 maggio di quell'anno Vettor Pisani, il generale veneto, uccise Luciano Doria, l'ammi- 
raglio genovese, ma, attratto da Ambrogio Doria al largo, fu sconfitto e pagò l'errore col perdere quasi tutta l'armata. I Genovesi si presentarono ora ad assediare la Serenissima nella sua laguna. Venezia, ridotta alle strette, passò all'offensiva c nella battaglia di Chioggia del decembre 1379 il doge Andrea Contarini cogli ultimi avanzi della sua flotta infligge al vincitore una tremenda sconfitta, con cui terminò anche la guerra più che secolare. La pace fu conchiusa a Torino nell'agosto del 1381. Non dunque il trovarsi in casa o in territorio nemico, ma il coraggio e il valore determinarono anche allora la vittoria.

Però nel secolo XIV i Veneziani ebbero nell'Adriatico stesso una vicina pericolosa nell'Ungheria.

Per un caso fortuito e per il rialzo, già accennato, dell'influcnza pontificia avrenne in questo secolo sulla parte orientale dell'Adriatico un cambiamento politico, che ebbe conseguenze lunghe, se anche non durature. Bisogna notare innanzi tutto che ancora sotto Innocenzo III l'Ungheria e la Croazia averano riconosciuto l'alta sovranità del Papa. L'Ungheria era considerata allora quale feudo papale apostolicus e da questa definizione derivò il titolo portato successivamente dai re ungheresi ed ancora oggi dall'imperatore d'Austria di "apostolico re d'Ungheria". - Quando nel 1301 si estinse la dimastia nazionale magiara degli Arpadi, il trono d'Ungheria passò coll'aiuto del pontefice Bonifacio VIII a Carlo Roberto di Napoli della casa d'Anjou. Il suo successoro Luigi il Grande (1342-1382) estese il suo potere, più personale che altro, sulle stirpi slave della penisola balcanica e sull'Adriatico, e riacquistò nel 1358 la Dalmazia, che già nei secoli precedenti era stata oggetlo di contese coi Veneziani.

In questa lotta le città dalmate slavano ora per gli Ungheri, ora per Venezia, secondo che in ogni singola aveva il sopravvento il partito favorevole ai prini o ai secondi. Le simpatie eramo determinate in parte da ratgioni nazionali. L'elemento era qua e lì misto: i latini tenevano per Venezia, gli Slavi per gli Ungheresi, coi 
quali avrebbero comandato i Croati. Però il dominio ungarico sulle città al mare dev'essere stato sgradito, se le popolazioni latine aggiunsero nella recitazione delle litanic un apposito versetto: Et $a b$ ira Hungariorum libera nos domine. - Non sarà difficile spiegare tale stato d'animo qualora si pensi che i Magiari non avevano ancora perduta la loro innata durezza. Malgrado tutti gli sforzi di re illuminati la civiltà, nè in questo nè nei secoli successivi, non riuscì a penetrare in Ungheria nella massa della gente alta e bassa, giacchè, per citare un esempio, il voivoda di Transilvania, Stefano Bathory, e molti altri personaggi consimili, ancora all'epoca del re Mattia Corvino (XV sec.), il cui regno è celebrato come l'età dell'oro della nazione ungherese, non sapevano nè leggere nè scrivere.

Nella lotta tra Ungheri e Veneziani parteciparono ai danni di questi ultimi i pirati di Almissa ed i conti di Bribir (luoghi siti in Dalmazia) istigati o per lo meno autorizzati a ciò dai primi, nonchè Tvartko di Bosnia. Ma di questi nuovi fattori si dirà qualche cosa in dettaglio un po' più avanti.

La perdita della Dalmazia non fu per i Veneziani che transitoria; giacchè colla decadenza della potenza ungarica dopo l'estinzione della casa d'Angiò, essi ritornarono in possesso del dominio perduto. Nel 1409 il re Ladislao vendette Zara e tutti i suoi diritti sulla Dalmazia ai Veneziani per 100000 ducati e al 30 giugno di quest'anno Zara e la Dalmazia tutta solennizzarono con grandiosi spettacoli il loro nuovo passaggio sotto la Serenissima, colla quale rimasero unite d'ora in poi ed in pace per quasi quattro secoli.

In Stato Pon- Abbiamo lasciato lo Stato pontificio sotto lo splendore tificio. d'Innocenzo III e rinvigorito per la conferma delle sue rivendicazioni temporali. La rovina degli Hohenstaufen a Benevento (1265) e Tagliacozzo (1268) suggellò il potere temporale dei Papi.

Colla conferma di quel documento per parte dell'imperatore Rodolfo d'Asburgo (1275) che nel 1279 rinunziò anche a tutta la Romagna, lo Stato della Chiesa era 
completamente libero dall'impero e lo Stato più grande dell'Italia media.

Ma l'autorità dei F'ontefici non cra ancora assicurata all'interno a causa delle famiglie aristocratiche, che signoreggiavano nelle varie città e non volevano sottomettersi al Pontefice che a parole. Quando poi i Papi al principio del secolo XIV (1305) trasportarono la loro sede ad Avignone, la loro potenza nello Stato della Chicsa fu portata all'orlo della rovina. In tali frangenti sorse inaspettatamente Cola di Rienzo come salvatore di Roma e restauratore della repubblica romana.

Le sue imprese fantastiche accaddero lontano dall'Adriatico. Siccome però fra queste c'era auche l'intenzione di porre Roma a capo di una confederazione italiana, l'eventualità della realizzazione di questo piano, che avrebbe portato gravi conseguenze sull'Adriatico, fa sì che meriti farne un cenno fuggevole. D'altronde l'idea di ristabilire peliticamente un'Italia geografica, fantastica allora, fu quella che realmente quando fu attuata portò uno dei più grandi cambiamenti subìti dall'Adriatico.

L'Italia dopo la rovina dell'impero romano non era stata un tutto organico che per opera dei Goti e dei Longobardi. Però l'idea che aveva animato questi due organismi non era partita dall'Italia stessa. Certo che il concetto d'un'Italia geografica e nazionale unita $n o n$ poteva essere una novità nella mente di Italiani. Però le condizioni dell'Italia erano tali che nessun cer'vello di buon senso avrebbe potuto pensarvi come ad una cosa facilmente realizzabile. Áppena nelle lotte tra il papato e l'impero per la supremazia durante e dopo le crociate e nelle competizioni italiane dei Guelfi e dei Ghibellini si formò un embrione forse più che l'idea di speranza. E fu allora che la realizzazione di questo concetto fu intravveduta dalla mente eccelsa e divinatrice, ma solitaria, del più grande dei Ghibellini, Dante. Però anche a suo giudizio era imprescindibile l'aiuto di qualcuno e i Ghibellini, come si sa, volevano cercarlo negl'imperatori di Germania.

Fra le idee fantastiche di Rienzi questa sareble stati 
forse la meno avventata. E se anche forse non è la più originale, va ricordata come contrapposto a quella dei Ghibellini. L'idea ghibellina, per (quanto fosse portata da una mente alta e rara come quella di Dante, tramontò presto a causa del suo esotismo. Quella guelfa, favorita dal suo indigenato, resistette più a lungo, ma non potè nemmeno realizzarsi a causa del fatale connubio e contrasto dei due poteri, divino e temporale.

Il ritorno dei Papi da Avignone (1367) salvò lo Stato temporale dalla dissoluzione. La loro influenza morale nel mondo subì però l'altra scossa terribile dello scisma della riforma (scoppiata nel 1378) che fece distogliere l'attenzione dei Pontefici e del mondo ancora più dalle cose d'Italia e la trasportò lunge dall'Adriatico nel centro d'Europa.

Di questa diminuzione della potenza pontificia approfittarono subito gli Angioini di Napoli, che al principio del XV secolo (1408) s'impossessarono (transitoriamente, c̀ vero) di Roma e del suo Stato.

La concezione d'un'Italia unita in un solo dominio italiano germogliò in questo secolo in forma peregrina anclie in due altre menti e precisamente nei primi anni del secolo in quella di Roberto d'Angiò di Napoli e negli ultimi in quella di Gian Galeazzo Visconti, duca di Milano. Anzi se poniamo mente alla posizione di Roma con Rienzi c di Firenze con Dante rispetto all'Italia media, vedremo che l'Angioino di Napoli ed il Visconti di Milano integrano geograficamente l'idea particolarista, eppure tendente da tutti i punti allo stesso scopo.

Gri Angioini La casa d'Angiò, ristretta per colpa del suo dominio
di Napoli. aspro ben presto al solo Napoletano (terraferma della bassa Italia) durò tutto questo e metà del secolo successivo.

Essa ha importanza per l'Adriatico e segnatamente in questo secolo, perchè coll'acquisto anche del trono d'Ungheria, divenne l'arbitra delle sorti dell'Adriatico e riuscì a far passare in seconda linea perfino la Serenissima. Difatti, se poniamo bene attenzione, è proprio in questo secolo di depressione dell'autorità di .Venezia, che gli ar- 
ciduchi d'Austria arrivano all'Adriatico (Duino 1374 e Trieste 1382) come si vedrà fra poco.

La casa d'Angiò diede all'Adriatico principi valenti, tra i quali merita di ricordare Roberto nella bassa Italia e Carlo Roberto (1308-1342) con suo figlio Luigi il Grande (1342-1382) in Ungheria. Sotto di questi due l'Ungheria ebbe un impulso straordinario per l'introduzione del sapere, degli usi, ma anche del lusso italiano.

Roberto di Napoli (1309-1343) fu un principe prudente e d'ingegno, che seppe acquistare una posizione importante in tutta l'Italia. Fu appunto nella sua mente che si formò l'idea di cingere quella corona d'Italia, che invece coperse il capo di Arrigo VII, accolto con giubilo dai Ghibellini e celebrato da Dante.

I loro successori che si macchiarono di delitti e sconvolsero, mettendo in pericolo, i loro troni, influirono anche involontariamente sull'Adriatico per le spedizioni via mare, intraprese più volte dagli Angioini d'Ungheria nella bassa Italia, onde vendicare le loro onte famigliari. $\mathrm{Fu}$ in questi incontri che Brindisi subì una prima distruzione nel 1348 per opera di Luigi d'Ungheria e poi una seconda nel 1383 per opera di Luigi d'Angiò.

Abbiamo veduto che Milano dopo la pace di Costanza Il Milanese. era retta da vicari imperiali.

Fra questi meritano menzione i Visconti, che nel 1311 si fecero dare dall'imperatore Arrigo VII l'investitura di Milano e del paese da esso dipendente. I suoi discendenti furono uomini di grande valore. Lucchino Visconti (13391349) estese la sua signoria sulla Lombardia e procacciò sicurezza al commercio e al vivere civile. Giovanni Visconti comperò nel 1350 Bologna dai Pepoli; Gian Galeazzo conquistò nel 1387 Verona ai Della Scala, e nel 1395 comperò dall'imperatore il titolo di duca. Negli anni seguenti assoggettò Pisa, Siella, Perugia. Il polso della sua mano era tanto sicuro, che anch'egli pensò di fondare un regno d'Italia. Ma la morte (nel 1403) troncò la sua esistenza e i suoi piani. Non si dimentichi però 
che in questo tentativo dei Visconti di formare un grande Stato unitario, rispunta la posizione geografica favorita di Milano.

L'indeciso orientamento del predominio politico nel XIV secolo permise anche ad organismi civili più deboli di vivere e di farsi sentire.

Nell'Adriatico

Fu già accennato alle marche di creazione longobarda settentrionale.

o franca, in quest'epoca scomparse o quasi. A loro somiglianza esistevano ora il vescovato di Trento e il patriarcato di Aquileja, pure or ora ricordati, e le contee di Gorizia e Pisino (più tardi marchesato d'Istria) dei quali avremo da occuparci fra breve.

La particolarità di tutti questi corpi amministrativi, laici ed ecclesiastici. con giurisdizione spirituale o temporale, consiste in ciò che servivano, almeno nei primi secoli dell'evo medio, da feudo d'investitura a notabili tedeschi in territorio italiano. La ragione di tale usurpazione va cercata nella mancanza, già esposta, di un valido confine orientale.

Sc non indipendenti del tutto, certo abbastanza libere nei loro movimenti, furono invece in quest'epoca diverse città municipali del Veneto e della Romagna, ove per la maggior lontananza dall'insidia straniera, famiglie aristocratiche indigene erano riuscite a mettersi a capo delia cosa pubblica ed a portare ‘e città a benessere e talvolta a splendore. Così si elevarono i Malatesta a Rimini, i Della Rovere a Sinigaglia e ad Urbino, i Polenta a Ravenna, gli Ezzelini a Padova, i Pepoli a Bologna, i Della Scala a Verona, i Gonzaga a Mantova, gli Estensi a Modena e a Ferrara, i Farnese a Parma e Piacenza, e così via, - nomi tulti molto noti agl'Italiani ed agli studiosi di tutto il mondo - che meritano di venir ricordati se non altro per il contributo dato da loro direttamente o indirettamente alla coltura, alla gloria dell'armi, aila storia d'Italia.

Ma anche sulla costa opposta, in condizioni e con tendenze più arretrate, si fecero sentire degli enti, che richiedono un accenno, vale a dire i pirati d'Almissa ed i conti di Bribir, ricordati quali avversari di Venezia. 
Almissa è un luogo (ora conta come città) alle foci I pirati almisdel Cettina in Dalmazia. Geograficamente non ha alcun valore, ma le gole di monti che dall'altipiano scendono quasi a precipizio sul mare ed $\mathbf{i}$ picchi di macigni che lo dominano, fanno di esso un nido naturale di falchi e un covo di pirati.

Nei secoli XII, XIII e XIV, quando nè i Veneziani nè gli Ungheresi riuscivano a imporre alle coste dalmate un governo poderoso e sicuro ed a tenere in freno le incursioni dell'interno, da Almissa partivano dei terribili pirati, probabilmente affini ai Narentani, che scorrazzando il litorale fino a Spalato a settentrione, alle isole del centro della Dalmazia e spingendosi perfino sino alle coste della Puglia rendevano malsicuro l'Adriatico. Le scorrerie degli Almissani erano tanto terribili che il popolo di Spalato aveva inserito anch'esso nelle litanie un versetto: A perfidia Almissanorum libera nos domine, e lo statuto di Ragusa proibiva severamente il commercio coi pirati, segnatamente con quelli di Almissa. Essi non cessarono dalla pirateria che quando furono vinti e sottomessi dai Veneziani e riuscirono molesti ancora nel XIV secolo, quando i conti di Bribii (nel latino di allora detti Breberii) estesero la signoria anche su quelle parti.

Bribir è un luogo ancora più insignilicante di Almissa nei paraggi dell'estuario del Cherea, altro nido di falchi e covo di pirati. Durante l'epoca in cui gli Angioini sedevano sul trono d'Ungheria, la famiglia di questi conti slavi ottenne la carica di bano delle regioni al mare (banus maritlimus); e così avveme che uno di essi sullo scorcio del XIII e sul principio del XIV secolo signoreggiò in nome del re d'Ungheria per alcuni decemni sulla maggior parte della Dalmazia, esercitandovi molte angherie. Però anche questo debole tentativo di vita indigena sulla costa orientale, in ogni caso dalle premesse più vitali della pirateria almissana, abortì e i conti di Bribir perirono quando, cessata la potenza dei loro mandanti, Venezia ridivenne la signora della costa dalmala.

Abbenchè il paese dal Celtina press'a poco all'Alla: nia, quindi in un raggio comprendente e Serbia e Monte-

I Conti di Bribir.

II regno di Serbia. 
negro e Bosnia odierni, fosse stato occupato ancora nel VII sccolo dai Serbi, questo popolo barbaro, che appena nel successivo passò al cristianesimo, dovette subire in principio la signoria dei Bizantini e dei Bulgari. Appena dopo il 1000 incominciò a formarsi uno Stato indipendente, che nei suoi tentativi di espansione giunse quasi all'Adriatico (nelle regioni del Montenegro e di Cattaro) ma fu male accolto e venne a contesa colla latinità dell'occidente (Ragusa ed i Pontefici) per le differenze di religione. I Serbi, avendo ricevuto il cristianesimo da Costantinopoli, erano scismatici e in Bosnia si erano allargati i Patareni. Per ciò questo Stato trasse vita stentata e contrastata, fino che il valore personale di un suo re, Stefano Dusciano (Dušan), 1336-1356, il maggiore di tutti i dominatori serbi, che s'impossessò della Macedonia, della Tessalia, della Bulgaria, dell'Albania e dell'Epiro settentrionale, e giunse quindi fino al mare Adriatico, non la portò ad un rapido, insperato, ma purtroppo anche effimero lustro. Sotto i suoi successori immediati l'impero serbo si sfasciò e la grandezza militare serba in parte per debolezza in parte per tradimento trovò ben presto la tomba nella famosa e per lei fatale battaglia di Cossovo (campo dei merli) nel 1389. Se quindi la grandezza dello Stato serbo, per molti aspetti simile a quella esposta un po' più in dettaglio dei Croati, ci apparisce effimera, addirittura meteorica dev'essere qualificata quella, ai più ignota, del regno di Bosnia.

Trartko, re di Bosnia.

Questo paese dopo la sua slavizzazione fu palleggiato ora tra la signoria dei Serbi ed ora dei Croati e con questi stette anche qualche tempo alle dipendenze dei re ungarici (regno di Rama), finchè un bano o governatore di nome Tvartko, verso il 1376, occupò anche l'Erzegovina e tratti della costa adriatica e si proclamò re di Bosnia. Però i giorni del suo governo furono sì tristi, da uscire tardi dalla memoria degl'infelici calpestati. Le contese dei suoi successori pel trono finirono colla chiamata dei Turchi, che col principio del venturo secolo XV dovevano far cambiar aspetto alla penisola detta finora greca. 
Per completare il quadro della costa orientale del- Ragusa. l'Adriatico alla fine del XIV secolo, col quale stava per chiudersi il periodo della preparazione e dei rivolgimenti derivati dalla caduta della romanità, occorre accennare ancora alla minuscola repubblica di Ragusa, già forte navigatrice e commerciante.

Approfittando dell'auge in cui si trovava l'Ungheria essa riuscì verso la metà di questo sccolo a svincolarsi dal dominio di Venezia, ivi stabilito e rappresentato da un conte, come lungo tutte le coste dalmate in occasione della quarta crociata.

L'epoca in cui Ragusa si giovò della signoria protęitrice dell'Ungheria si estende dalla metà di questo al principio del XVI secolo.

Malgrado tutte le traversie anche la costa orientale del- I Mongoli.

l'Adriatico, in grazia al progresso dell'altra, risorgeva. Però siccome il destino ha scritto nel suo libro che l'orientale debba essere la più bersagliata, anche nel XIII secolo le capitò un nuovo flagello, i Tartari o Mongoli, detti nella bocca dei popoli depredati "teste di cane».

Questi nomadi dell'Asia avevano invaso l'Ungheria e la Croazia, facendo tremare anche la metà orientale dell'Europa e dell'Adriatico. Nell'inseguire Bela IV re d'Ungheria (1241) penetrarono fino in Dalmazia e la devastarono, distruggendo tre cittì della parte meridionale, fra cui Cattaro. Ma nel 1243 toccarono una soleme sconfilta sulle alture al di sopra di Fiume.

Strano destino quello della costa orientale adriatica di essere beneficata solo dal mare e dalla sua sorella italica. Tutto ciò che le venne invece dal suo retroterra, sia che si fosse trattato di barbari trasmigranti e predoni, o di gente finitima dell'altro versante, come gli Ungheri coi loro bani, i Serbi, i Bosniaci, non le portò che danno e avversione.

Per completare il quadro del secolo XIV occorre ricorlare ancora un alvenimento.

Questo secolo segna verso la sua fine nella parte setGli Ashurg'esi. (L'A usiria). tentrionale dell'Adriatico un altro fatto, che ai contemporanei sarà apparso molto probabilnente d'importanza 
inferiore all'estensione del dominio ungherese sulla costa dalmata, ma nel quale i posteri scorgono invece il germe di un avvenimento di grande valore per l'avvenire, cioè l'umile arrivo degli Asburghesi al mare, a Duino nel 1374 e a Trieste nel 1382.

Trieste. Qualcuno si domanderà per quale motivo in questo avvenimento figura il nome di una sola città, anzichè di un territorio o di una regione. Perchè Trieste è un triangolo di terra o un seno di mare che economicamente e politicamente può star unito tanto colla pianura friulana ad occidente, quanto col Carso a settentrione, che coll'Istria ad oriente, ma per la geografia locale non può essere confusa con nessuna delle regioni circostanti. C'è nel terreno che la circonda una caratteristica speciale. Per ciò quell'angolo di terra adriatica ebbe ognora una storia individuale, congiunta negli avvenimenti generali, ma distinta nei fatti particolari dal resto della costa.

La storia di Trieste subì la trafila di tutte le città italiane al confine orientale: colonia militare e commerciale romana nel II secolo a. C., indi municipio (republica tergestina), patì indirettamente nella trasmigrazione dei barbari e direttamente per una scorreria dei Longobardi, appartenne ai Bizantini nell'esarcato di Ravenna, indi cascò in mano dei Franchi, che l'aggregarono alla marca del Friuli, istituita da Carlomagno nell'803. Come si sa a capo di ogni marca stava un margravio, o in italiano marchese, cui era subordinato un conte ed a questi un barone e più giù ancora un gastaldo. Ora tutti questi feudatari, marchesi, conti e baroni (che in principio erano anche ecclesiastici, vescovi e patriarchi) come fu già accemuato crano di nazione germanica. Così avvenne che anche i paesi da loro amminístrati furono considerati come appartenenti all'impero, tanto che nel 952 la grande marca del Friuli fu staccata dall'Italia e unita dapprima al ducato di Baviera, e più tardi a quello di Carinzia.

In questa manicra Trieste assieme al suo retroterra montuoso, Carso, Alpe Giulia e Istria grigia (calcarea). furono staccate politicamente dall'Italia civile di Augusto 
(ossia da quel territorio che aveva lingua, leggi e costumi romani). A riprova però della sua italianità, nella lotta dei comuni italiani contro gl'imperatori tedeschi della casa di Svevia, partecipò, come fu già detto, alla Lega Lombarda.

Intanto Venezia estendeva la sua potenza per mare ed anche per terra e non tollerava rivali. Trieste invece che si riteneva l'erede morale e commerciale di Aquileja non voleva piegare il capo. Così nacque la rivalità commerciale, per la quale i Triestini dovettero cercare appoggio nei patriarchi di Aquileja, feudatari e principi immediati dell'impero. La conseguenza di quest'antagonismo cconomico fu che verso la fine del XIV secolo e precisamente nell'anno suaccennato nel mentre Venezia consolidava definitivamente la sua posizione sulla costa orientale adriatica, Trieste, che non poteva più sperare aiuto dai patriarchi di Aquileja, perchè erano essi stessi all'orlo del tramonto per causa appunto di Venezia, si indusse ad accettare il dominio degli Asburgo. Questi principi, che erano già da qualchie anno padroni di Duino, del Carso e della contea di Pisino, confinavano ormai col territorio di Trieste.

La famiglia principesca degli Asburgo proveniva dai paesi alpini dell'odierna Svizzera tedesca e alla sua comparsa nella storia nel XIII secolo aveva possedimenti anche nell'Alsazia e nella Svevia. Con tutti quei mezzi coi quali gl'individui si fanno ricchi ed i principi accrescono i loro possedimenti, ma specialmente con accorti maritaggi a scopo di successione ereditaria, gli Asburghesi riuscirono a fondare un impero di prin'ordine, che gravitò ben presto anche sull'Adriatico.

Nel secolo precedente i possedimenti degli Asburghesi avevano, avuto un ingrandimento capitale in seguito all'estinzione dell'altra famiglia principesca germanica doi Babemberghesi. Possedevano questi i paesi alpini nordorientali (odierna Austria e Stiria) come derivazione dall'antica marca orientale, fondata da Carlomagno contro i nemici del germanesimo. Coll'aiuto di Rodollo d'Asburgo, che si trovava allora sul trono di Germania, la fami-

Ingrandimento degli Asburgo. 
glia ottenue non solo l'Austria e la Stiria, vacanti, ma anche la Carniola e la marca Vendica (1282).

AI principio del XIV secolo (1303) gli Svizzeri con una ribellione riuscirono a rendersi indipendenti dagli Asburghesi: e questi che invece cercavano di ingrandire i loro possedimenti, riuscirono a risarcirsi col guadagnare altri territori alpini, adiacenti ai loro, prima di tutto la Carinzia (1335). - Un duca d'Austria (Ostarrichì) Rodolfo IV, detto per i suoi acquisti il fondatore, volle spingersi ancora più al mezzogiorno, e incominciò nel 1361 una guerra contro il patriarca di Aquileja, più debole di lui, e che terminò con alcuni acquisti da parte degli Asburghesi. Con ciò erano giunti a porre un piede nella pianura friulana, ma non ancora al mare e raddoppiarono gli sforzi. Nel 1363 si fece cedere dall'ultima erede del Tirolo, una vecchia donna, anche questa provincia e così gli Asburghesi divennero d'ora in poi gli unici padroni degli odierni paesi alpini austriaci.

Nel 1374 un altro duca d'Asburgo in seguito alla morte del ramo istriano dei conti di Gorizia ebbe in forza ad anteriori trattati d'eredità alcune parti della Carniola con Duino quale sbocco al mare e dell'Istria montana.

A questo punto l'espansione tedesca degli Asburgo urtò contro quella italiana di Venezia, ma non si venne ancora ad una lotta aperta. Soldati del duca d'Austria si batterono non lungi da Trieste coi Veneziani, ma solamente quali partigiani dei Triestini. Venezia era in quell'epoca assalita da tutte le parti, da Genova, dai patriarchi d'Aquilcja, dai duchi d'Austria, dal re d'Ungheria, senza contare i signorotti d'Italia. Venezia possedera l'Istria marittima e doveva lottare sopratutto cogli Ungheresi per contendere il possesso della Dalmazia. Venezia era la più debole e non poteva arrestare con una guerra l'avanzata dei duchi d'Austria. Per ciò dovette contentarsi di ostacolarli coll'abilità diplomatica. Ma gli Asburghesi, servendosi dello stesso mezzo ed aiutati dall'appoggio morale dei grandi ecclesiastici e laici, che in quei paesi di popolazione prevalentemente romanica s'erano conservati tedeschi, riuscirono a presentarsi sulla scena come, gli 
unici protettori possibili del commercio di Trieste. E i Triestini con quella dedizione volontaria ne riconobbero la supremazia, ma a patto della concessione di franchigie amministrative e commerciali, compreso il diritto di pace e di guerra.

I duchi d'Austria erano riusciti a toccare un porto al mare. Oggi ognuno vede l'importanza di tale passo, piccolo allora nell'apparenza, ma del quale la maggior parte dell'Italia contemporanea forse nemmeno si sarì accorta.

Intanto per comprendere bene il valore effettivo di queste estensioni di potere degli Asburgo bisogna aver presente che il loro dominio non era che parziale e ch'essi alla lor volta dipendevano pure in parte dalla volontà suprema dell'imperatore di Germania. Così p. e. la contea di Pisino fu adoperata costantemente per procacciarsi danaro, cedendone il dominio utile a signori affituari e fu riscattata del tutto appena nel 1848. Trieste dopo la dedizione conservò immutati i suoi statuti fino al 1550 e anche dopo fino alla proclamazione del punto franco (1771) potera considerarsi un piccolo Stato tributario dell'Austria (a somiglianza di quanto arvenne più tardi col Montenegro). - Però il passo più importante era fallo. L'ulteriore ingrandimento del più forte e la sottomissione finale del più debole al momento opportuno, non sarebbero stati che (fuistioni di tempo.

L'ingrandimento era stato infatti preparato tacitamente da trattati scambicvoli d'eredità, conchiusi dagli Asburgo verso questo tempo con varie famiglie dominanli. Di speciale importanza furono i patti colle faniglie d'Ungheria e del Tirolo, nel qual modo venne preclisposta l'unione dei paesi austriaci, boemi ed ungheresi, ossia fu preparata la grande Austria asburgica.

Quest'ingrandimento della lamiglia ducate asburgica merita di essere segualato come un caso tipico della storia. Nel mentre in gencrale i popoli, gli Stati e pere essi i loro capi per estendere il dominio doveltero ricorrere a lolte cruente, gli Asburgo senzia guerer, mat 
colla sola sagacia di saper approfittare delle contingenze della politica, con matrimoni e con trattati d'eredità, riuscirono esattamente in un secolo a riunire nelle loro mani una potenza territoriale con cui cinsero l'Italia al settentrione e all'oriente dalle Alpi all'Adriatico. Già in quest'epoca era chiara la tendenza di fondare nella parte orientale di Europa un regno potente.

Il cammino percorso dalla famiglia principesca degli Asburgo coi possessi della Svizzera lungo le Alpi fino al golfo di Trieste per diventare poi quali sovrani d'Austria i continuatori in forma ampliata della missione nazionale assegnata da Carlomagno alla sua marca orientale, può servire a simboleggiare il movimento dell'espansione germanica.

Infatti essi ebbero la loro culla tra la Svizzera e l'Alsazia, in quel cuneo da cui sembrò che i Teutoni avrebbero infranto la compagine romana. Ma stettero all'ombra fino a tanto che lo spirito del germanesimo, tenuto alto dai suoi imperatori, esercitò il primo periodo della sua pressione verso l'occidente sulla Gallia e verso il mezzogiorno sull'Italia. Tacitamente dopo sei secoli lo spirito romanico aveva ripreso il sopravvento e il germanesimo aveva ceduto nel XII e XIII secolo allo spirito di rivendicazione dei Francesi latinizzati nell'occidente e degl'Italiani, rappresentati dal papato, nel mezzogiorno.

Nel XIV secolo la potenza degl' imperatori tedeschi e del germanesimo era impallidita; ma si riebbe presto per aver cambiato direzione alla sua espansione. Si rivolse ad esercitare la sua pressione verso l'oriente a danno dei suoi prossimi vicini più deboli, gli Slavi. In tale opera appare provvidenziale per il germanesimo la comparsa degli Asburgo, che, riunenclo nelle loro mani territori, popoli e potenza, furono i più fedeli ed attivi continuatori dello spirito di Carlomagno.

Fino al secolo XIII la pressione germanica s'era fatta sentire sull'Italia e sull'Adriatico dal nord-orest. Nei due secoli XIV e XV c'è una sosta in cui tale pressione, sempre esistente, fu meno sensibile. Dal XVI in poi riprende forza, ma dal lato nord-est, non più sotto la mano de- 
gl'imperatori di Germania, ma della potenza particolare degli Asburgo, da quella stessa parte dalla quale i Cimbri avevano molestato le aquile di Roma.

E stato detto che il XV è un secolo nel quale rinac- srcoro xv. quero tutte le energie d'Italia e d'immediato riverbero anche quelle dell'Adriatico.

La prima manifestazione in questo senso fu la restrizione di quella stragrande dispersione di poteri sovrani e il riassorbimento di molte di quelle piccole seni-indipendenze, ch'erano state la conseguenza della mancanza di un'autorità direttiva unica in Italia, nei Balcani e sull'Adriatico e la causa di quel pullulare di organismi statali piccoli e anemici.

t:

In questo studio che tratta solamente delle due coste dell'Adriatico, gli avvenimenti della Lombardia, come parte della Padania, non poterono che venir sfiorati, giacchè essi si seguivano del tulto indipendentemente da questo mare: però colla loro azione influivano indirettamente sugli Stati che avevano la loro vita alla costa e particolarmente su Venezia. Per questo motivo noi, che di fronte al secolo $\mathrm{XV}$ possiamo vantarei della sapienza di posteri, dobbiamo spingere lo sguardo ancora un poco più in là della valle padana, fino proprio alle radici delle Alpi piemontesi, ove come contrapposto, quasi casuale, all'espansione degli Asburgo sulla parte orientale dell'Adriatico erano sorti un piccolo Stato e una dinastia, destinati a far molto per l'Italia e per l'Adriatico in antagonismo agli Asburgo e all'Austria.

A cavaliere delle Alpi, tra il bacino del Rodano e quello del Po, s'era formata in seguito alle trasmigrate Savoia. zioni germaniche e secondo il suo spirito feudale mal contea (Sapaudia), che in principio gravito rerso il liodano e i paesi franchi. Già verso il 1000 i conti di Sitvoia (Maurienne) s'erano trapiantati al di qua defle $N_{\text {pi }}$ 
nel bacino del Po. Quando poi alla fine del XIV secolo il loro dominio, favorito da una legge sull'indivisibilità del paese, si estese dal lago di Ginevra fino a Nizza, comprendendo anche Pinerolo, Chieri, Savigliano e parti della diocesi di Ivrea e Vercelli in Italia, l'imperatore Sigismondo innalzò la Savoia (prima vicariato imperiale) al grado di ducato (1416). Malgrado dunque la presenza di una catena montuosa robusta vediamo formarsi dopo la caduta della romanità anche da questa parte un organismo statale a cavallo del confine d'Italia, che fu poi attratto per legge geografica sempre più nella Padania.

Le condizioni politiche della Francia non permettevano più un'espansione nella valle del Rodano: quelle dell'Italia invece sì. D'ora in poi l'espansione dei duchi di Savoia e Piemonte seguì il corso del Po, finchè dopo tre secoli di vicende passate ora bene e ora male nell'antagonismo tra i re di Francia e gl'imperatori di Germania al principio del XVIII entrarono direttamente a far parte dell'ulteriore storia della Padania.

Lombardia. Un consimile risveglio politico sotto forma d'innalzamento dinastico di una famiglia dominante si riscontra anche nel rimanente dell'alta Italia.

A Milano, estintasi nel 1447 la casa Visconti, il regime aristocratico repubblicano continuò colla casa Sforza. A Mantova i Gonzaga nel 1432 ed a Modena gli Estensi nel 1452 elevarono sè e le loro città al grado ducale.

Venezia e l'Istria.

In questo secolo anche Venezia toccò il colmo della sua potenza.

Giì negli anni 1404 e 1405 aveva esteso i suoi possessi su Vicenza, Verona, Bassano, Feltre, Belluno, Padova coi rispettivi territori; nel 1417 comperò da Federico "dalla tasca vuota》 Rovereto; nel 1420 e 1421 pose fine al patriarcato di Aquileja e si consolidò nel Friuli e nell'Istria marittima.

E venuto il momento di dire qualche cosa in particolare dell'Istria. - Quest'estrema regione d'Italia, risparmiata, come si disse, nella trasmigrazione dei barbari, divenuta nei secoli successivi il terreno di confine tra l'influenza politico-nazionale latina dei Papi e di Vene- 
zia e quella germanica degl'imperatori tedeschi e dei duchi d'Austria, fu divisa e sofferse per le guerriglic e le incursioni dei popoli di terra e per la pirateria dalla parte di mare. La sua condizione migliorò sotto la dominazione veneziana che fu favorevole alla collura civile. Tra l'Istria e Venezia si stabilirono i rapporti più intimi e cordiali. Non meno di novantuna faniglie patrizie di Venezia provennero dalle città dell'Istria. Capodistria sola diede cinque dogi alla Serenissima. Dall' Istria Venezia tracva a preferenza le querce per le sue galere e per le fondamenta degli edifizi, la pietra per le costruzioni, le ciurme più ardite, i piloti più sicuri, quei battaglieri uomini di mare, piranesi, che si distinsero nella guerra di Chioggia e in tante altre.

Contemporancamente all'Istria Vcnezia assicurò il suo possesso anche in Dalmazia; indi nel 1428 ebloc Brescia e Bergamo; nel 1440 Ravema, nel 1448 Crema e nel 1484 Rovigo.

I suoi possedimenti nel solo Adriatico arrivavano nella Padania dal lago di Como e dall'Adda ad Aquileja e comprendeva a mezzogiorno tutto l'estuario del Po. Sulle coste oricntali poi possedeva tutta l'Istria, dal vallone di Muggia in poi, indi si estendeva lungo le isole del litorale croato fino alla Dalmazia (terraferma ed isole) giù giù, meno la minuscola repubblica di Ragusa, fino ad Antivari. Da qui a Vallona il possesso di Venczia fu breve e limitato alle città costiere, Dulcigno, Scutari, Alessio, Durazzo, Vallona.

Ricca, potente, bene amministrata, Venezia era dovunque temuta e contava come una potenza mondiale, anzi era la prima potenza commerciale del mondo. Sotto di lei fiorirono le scienze e le arti, il commercio e l'industria. Il. suo governo era mite, gli aggravi crano lenui. Il governo aristocratico condamava è vero il popolo all'inazione, ma acuiva tanto maggiormente l'amer di pattria e la saggezza politica della casta dominante.

Venezia era nata e cresciuta sopratutlo scinume le regole e gl'insegnamenti della geografiat. Il fallo storico) della distruzione di Aquileja da solo non avedbe dato 
vita alla Venezia che noi studiamo e ammiriamo, se la nalura, ossia la posizione geografica, non avesse protetto i primi aliti della nuova cittì. Ai primi Veneziani non era concesso altro che muoversi sul mare, ed essi fecero i primi passi lasciandosi portare dalle correnti del mare e del vento (il maistro) e giunsero in Istria, poi lungo le isole in Dalmazia. Il loro salto sulla costa orientale, anzichè una lenta discesa lungo l'occidentale, dopo la descrizione geografica, apparisce la cosa più naturale. Pcrò con ciò solo i Veneziani non sarebbero diventati ancora i dominatori dell'Adriatico. Essi progredirono perchè poterono immettere radici sulla loro terraferma, ch'era fortunatamente piana, aperta e libera e permise loro di attingere forza in tutto quel tratto che ra dal mare ai contrafiorti delle Alpi. Se non avesse aruto questa terra e le Alpi e non fosse stata lo sbocco cconomico della Padania Venezia non sarebbe andata molto più in là della pirateria, come i Narentani, che anche averano esteso il loro dominio sulle isole circostanti, ma inaridirono. Per attenuare il danno dell'insediamento di montanari a Trieste ed a Fiume, Venezia dovette inevitabilmente pretendere per sè il dominio esclusivo del mare. E questa è la ragione della sua politica inesorabile, ma logica e fatale. Altrimenti la geografia, che con una mano l'aveva tratta sul mare, coll'altra ve l'arrebbe annegata.

La stessa ragione condusse Venezia fino al Narenta. Forse Venezia si sarebbe contentata di questo limite per la sua posizione sull'Adriatico, giacchè il resto della cosha orientale ha poco valore sociale, economico e nautico. Però l'allettancnto dei commerci fuori di questo golfo impose ai Veneziani la necessità di averne in mano la porta. Per ciò Venezia in battaglie navali a Durazzo e a Butrinto (nel cauale di Corfù) attaccò i Normanni che s'erano messi a contenderle il dominio dell'Adriatico meridionale. Le Bocche di Cattaro, i porti dell'Albania e Corfì dalla parte esterna essa li volle solo per maggior sicurezza dell'Adriatico. Così si spiega anche perchè Venezia in questi ultimi paesi lasciò sussistere molta li- 
bertà, tanto che il di lei dominio fuori della Dalmazia apparisce meno effettivo.

All'osservatore attento non sfugge nemmeno che questi possedimenti di Venezia sbocconcellati sull'Adriatico mancavano di consistenza e per ciò si sareblse potuto pronosticare sin d'allora che il suo dominio non cra destinato a durare eternamente.

Giacchè il racconto dei fatti di Venezia ci ha portato sulla costa orientale, restiamoci ancora un poco per dire qualche cosa della repubblica di Ragusa, che può dirsi una leggiadra miniatura, fatta sul modello di Venezia.

Anche per Ragusa il XV e il XVI secolo (quest'ultimo almeno nella sua prima parte) sono l'epoca del fiore.

In primo luogo estese il suo dominio in terraferma comperando dai signori serbi piccoli tratti di territorio e villaggi. Poi i Ragusei estesero i loro commerci, per terra fondando agenzie in Bosnia, in Macedonia, in Bulgaria, in Rumenia, ebbero fattorie fin nel centro dell'Asia, e per mare solcandolo fino alle coste non solo d'Italia, ma anche di Spagna. Ragusa teneva orunque colonie rette colle patrie leggi. La sua flotta ascendera a quatiocento navigli e con questi trafficava nel Jonio e nol mar Nero, in Asia e in Africa. - Ragusa, dopo essere stata una stazione di crociati, divemne un emporio di prim'ordine pei commerci di tutto l'oriente, ricetto ospitale di principi, culla delle lettere, splendore delle scienze. Ragusa, più che umo Stato, era una gran casa di commercio. La cittì aveva raggiunto l'apice di abitanti (40000) e di ricchezze davvero colossali. In questo tempo furono chiamati molti dotti d'Italia quali istrultori e professori. E quando la lingua slava, introdotta dalla nuova popolazione, incominciò ad infiltrarsi nei giudizi, tali er:mo le tradizioni del senato, che questi, proprio nel 1472, emanò una legge, secondo la quale nelle arringhe c nclle sentenze non si dovera usare altra lingua che litaliana (nisi lingua latint ragusea). E l'italiano rimase la lingua ufficiale della repubblica e dei commercianti.

Le relazioni diplomatiche di Ragusa con Venezia in quest'epoca, come fu accennato alliove, non potevano 
dirsi fraterne; erano però in buoni termini. - Venezia, che dapprima aveva tentato di soffocare la crescente floridezza di Ragusa, accortasi che indirettamente giovava anche a lei di avere un adito sempre aperto al traffico dei Balcani, lasciò sussistere la repubblica di San Biagio per proprio tornaconto.

A quest'interpretazione dei fatti proveniente da fonte veneziana, scrittori ragusei oppongono che Venezia non si azzardò di toccare Ragusa, perch'era spalleggiata dalla Serbia, la cui potenza per terra era per i Veneziani un'incognita. Per ciò, dicono i Ragusei, Venezia non stimò opporluno di incappare nello Stato serbo, che avrebbe potuto divenire per lei un brutto ginepraio.

Comunque siasi, sta il fatto che Venezia e Ragusa fiorirono contemporaneamente sul mare e nei commerci.

Bassa Italia. L'assorbimento per parte di Venezia e di Ragusa del commercio dell'Adriatico coll'oriente danneggiò, com'era naturale, le città costiere della bassa Italia. Tra queste Bari e Brindisi, come le principali, rialzatesi dopo l'occupazione saracena, avevano raggiunto qualche importanza durante le crociate, ma decaddero.

In quest'epoca il paese era dominato dagli Angioini, che avevano la sede a Napoli. Il loro dominio datava dalla fine del secolo XIII, ma il governo era funestato quasi ininterrottamente da dissidi intestini e da guerre (cogli Aragonesi di Sicilia, col Papa, cogli Angioini d'Ungheria) alle quali cose contribuì in gran parte la malvagilti dei singoli reggitori. Quando nel 1435 si estinse la discendenza diretta della casa d'Angiò, gli Aragonesi, che già occupavano la vicina Sicilia, un po' per questo fatto, in po' per diritti matrimoniali, affacciarono pretese su Napoli e le mandarono ad effetto dopo anni di guerra $(1442)$.

Scanderbeg. Per le cose dell'Adriatico interessa inoltre di rilevare che alle lotte che continuarono tra i partigiani degli Angioini ed i nuovi signori, gli Aragonesi, presero parte dall'altra costa dell'Adriatico Scanderbeg coi suoi Albanesi.

Scanderbeg areva fondato in opposizione alla rapida e terribile avanzata degli Osmanli, quell'effimera Albania 
indipendente, basata solamente sul valore delle armi, che non durò clie circa quattro lustri. Dopochè nel 1461 nella pace col sultano l'Albania era stata abbandonata formalmente in suo potere, Scanderbeg, che avera bisogno della guerra per vivere, passò nel 1462 coi suoi guclrieri nelle Puglic e contribuì alla vittoria degli Aragonesi. Per ciò re Ferdinando gli concedette il dominio di Tr’ani, Manfredonia, il monte Gargano ed altre terre. Dopo la sua morte un altro buon numero di Albanesi immigrarono in Italia ed altri ancora sopravvennero via via fino alla metà del secolo XVIII per sfuggire alle stragi turche.

Gli Aragonesi s'insediarono nella bassa Italia; ma il Gli Arag nesi. loro governo, che durò poco più di mezzo secolo, fu tormentato da continue guerre.

Oltre alle lotte colla nobiltà, vi furono guerre per quistioni ereditarie famigliari coi re di Francia ed anche con quelli di Spagna. Anzi in un'alleanza fra questi due era stato pattuito di dividere lo Stato e precisamente le Puglie e le Calabric dovevano toccare agli Spagnuoli, il rimanente ai Francesi. Ma contrasti sulla spartizione della preda mandarono a monte il disegno, finchè battuti i Francesi in tutte le regioni (nelle Puglie presso Cerignola) il regno delle Due Sicilic passò direttamente in possesso della corona di Spagna.

Del governo degli Aragonesi, che non fu migliore di quello degli Angioini, si dirà più tardi parlando in generale del dominio spagnolo nelle regioni dell'Adriatico.

Per completare non solo l'enumerazione degli Stati Cesare Borgis. maggiori cle si affacciavano sull'Adriatico, ma rilevare anche fatti importanti per la storia, occorre dire nuovamente qualche cosa dello Stato della Chiesa.

Lo Stato pontificio, devastato al principio di questo secolo (XV) dai Napoletani (angioini) fu ricostituito, dopo terminato lo scisma, da Martino V (un Colomna) nel 14:20. Ma non riacquistò più l'antico lustro, un po' perchè le famiglie aristocratiche avevano acquistato tanta indipendenza da rendere lo Stato pontilicio un organismo statale 
più di nome che di fatto, un po' per la corruzione dei Pontefici stessi che della loro dignità fecero una fonte di commercio. Incominciò allora quella piaga del nepotismo, accompagnata da ribellioni e da lotte, che tanto danneggiò l'autorità morale del Papa, le condizioni materiali di Roma e dello Stato della Chiesa, finchè gli eccessi dei Borgia non vi misero involontariamente una fine.

Precisamente alla fine del secolo di cui trattiamo salì sulla sedia pontificia col nome di Alessandro VI (14921503) il cardinale Borgia, uomo di straordinaria capacità e di eloquenza meravigliosa, ma rinomato per la sua cupidità e crudeltà e per la mancanza di ogni sentimento di pudore.

Alessandro VI si adoperò a sottomettere nello Stato della Chiesa la nobiltà feudale. Ma nel far ciò aveva di mira non l'interesse della Chiesa, nıa quello della sua famiglia, specialmente di suo figlio Cesare, al quale diede ducati e principati. - In tali avvenimenti Cesare Borgia entrò in scena proprio nell'ultimo anno di questo secolo, cioè nel 1499, e il campo delle sue imprese fu precisamente la costa dell'Adriatico. Ritornato in quest'amno dalla Francia con un corpo d'armati s'impossessò nelle Romagne di tutte quelle signorie, che s'erano ribellate al Papa, Imola, Forlì, Pesaro, Rimini, Faenza, Perugia, ecc., e poi si fece da suo padre nominare duca di Romagna. Nel 1502 s'impossessò medianie un tradimento del ducato d'Urbino e della marca di Camerino. Stava già all'apice della sua potenza quando la morte di suo padre, il pontefice Alessandro VI, lo privò improvvisamente della base, su cui poggiara il suo edifizio di violenze. Fu per ciò che il successore di Alessandro VI, il papa Giulio II della Rovere (1503-1513) potè incamerare a sè quei possedimenti e ricostituire lo Stato della Chiesa in quella forma ed estensione geografica, che conservò sino agli ultimi tempi. Lo Stato temporale della Chicsa occupava ora la costa dell'Adriatico per il lungo tratto dalle foci del Po al Tronto.

Le considerazioni sul valore morale di questi fatti saranno esposte nel trattare del secolo venturo, al quale essi veramente appartengono. 
Per l'Adriatico però bisogna subito notare che da que- Condizioni p? sto momento la sua costa occidentale ha acruistato una litiche e civili sto momento la sua costa occilentale ha acrilistato mina delliatico divisione politica non mutevole, non già perchè tutti gli alla fine dèStati che vi erano formati avessero i requisiti di stabilità duratura, ma perchè lo Stato pontificio, come euneo nel mezzo, impedì che si alterasse la delinitazione politica.

Tre erano gli Stati d'Italia che si bagnavano ora nell'Adriatico, Venezia, lo Stato pontificio e il regno delle Due Sicilic (sotto gli Spagnoli). Questi tre con Milano e Firenze formavano i cinque Stati che tenevano l'equilibrio politico dell'Italia e dell'Adriatico. Però ad una maggiore fusione dell'Italia non si giunse a causa di cucl particolarismo e di quello spirito d'indipendenza, che se erano stati favorevoli per opporre resistenza alla supremazia germanica, impedirono la sottomissione di tanti poteri locali ad un capo comune.

Malgrado questo miserando frazionamento politico, congiunto a tremende lotte fratricide, l'Italia alla fine del medio evo superava tutti gli altri paesi d'Emopa per lo splendido sviluppo della sua coltura.

Sotto la bandiera di San Marco ci si presenta in quest'epoca una nuova espansione della maggiore vilatiti della parte occidentale adriatica verso la meno fortumata orientale. Quest'espansione differisce dalla precedente dei Romani, perchè era scomposta e mancava di una forte direttiva politica, ma forse per ciò si presenta sollo una veste più simpatica. Era una trasmigrazione più della coltura che del dominio. Ciò nulla meno fu efficace e benefica perchè proveniva da esuberanza di vila di un popolo, che, se anche era diviso politicamente, intellettualmente progrediva su una sola via.

Nel medio evo la letteratura italiana fu la più importante di tutte le letterature d'Europa. I centri più importanti del rinascimento umanistico erano Firenze e Romil, ma anche Venezia e Milano vi presero parte notevole. Si aggiunga a ciò le universita di Bologna e Salemo, delle quali la prima s'illustrò per la collura della giurisprudenza e la seconda per la medicina. Era quindi impossi- 
bilc che la costa orientale adriatica, così vicina a queste ciltà, non ne risentisse un influsso benefico.

Talvolta i dotti, professori, giurisperiti, medici, teologi d'Italia venivano chiamati a prestar la loro opera e il consiglio nelle cittadelle della costa orientale. Tal'altra vi si recavano come nel rifugio più adatto in seguito alle fazioni di parte (Guelfi e Ghibellini).

Contemporaneamente al rinascimento d'Italia e nella proporzione in cui si trovano press'a poco la luce del sole con quella della luna, abbiamo un rinascimento delle Icttere anche sulla costa orientale adriatica, cui contribuirono non solo i latini, ma anche gli Slavi. La letteratura nazionale dei Croati e dei Serbi in questi paesi dai primordi del secolo XV a tutto il XVII porta una marcata impronta dell'influenza umanistica italiana. I componimenti in lingua slava non sono che copie o rifacimenti dei maggiori e migliori componimenti dei poeti d'Italia ed hamno oggi importanza più come documenti storici, che quali composizioni letterarie.

Ma il soffio della coltura italiana nelle forme letterarie e scientifiche dell'umanesimo e del rinascimento superò la costa e si spinse molto al di là fino nei pacsi alpini dell'Austria e in Ungheria. In questo paese particolarmente sotto l'influenza degli Angioini di Napoli ebbe luogo per le relazioni coll'Italia un potente rivolgimento nella coltura e nelle costumanze della popolazione.

Politicamente la costa occidentale dell'Adriatico aveva raggiunto, come fu rilevato, un assetto stabile. Ma anche l'oricntale si era avviata ad un migliore orientamento. I particolarismi locali dei germogli non vitali e delle speranze indigene dei Croati, rappresentate dai conti di Bribir c dalla pirateria degli Almissani, dei Serbi, degli Albanesi erano stati tolti da Venezia. Le incursioni dei Saraceni erano diminuite; i Bizantini, che avrebbero potuto considerarsi anche quali intrusi dell'Adriatico, si face- 
vano poco sentire; gli Ungheresi avevano un tratto di mare sul litorale croato, perfino i duchi d'Austria, quale esponente del germanesimo, avevano raggiunto lo sbocco al mare con Trieste. Sembrava che fosse sorta per l'Adriatico una nuova era di tregua politica e di floridezza commerciale, per la seconda volta sotto il benefico influsso della civiltà d'Italia.

Ma la stabilità è contraria alle regole del progresso umano.

Nuovi, se non tutti gravi, cambiamenti s'erano delineati già nel secolo ora ricordato per cause interne ed esterne all'Adriatico. Fra le prime va ricordata la discordia degli Stati dell'alta Italia, fra le seconde la comparsa dei Turchi in oriente d'Europa ed i tentativi d'ingrandimento della famiglia d'Asburgo.

Già nel secolo XV incominciò in Italia e precisamente dai confini occidentali ed orientali delle Alpi - quindi da regioni che interessano l'Adriatico - quella perniciosa intromissione di potentati stranieri, purtroppo invano temuta da patriotti previdenti.

Il timore dei piani di conquista del re di Napoli fecero sì che uno Sforza di Milano, Lodovico il Moro, si rivolgesse nell'ultimo decennio del ' 400 al re di Francia per aiuto. Questa chiamata fu fatale, perchè l'Italia e con essa l'Adriatico, che per secoli erano riusciti a tenersi liberi dalla supremazia degl'imperatori di Germania, ricaddero in schiavitù politica degli stranicri e divennero il teatro delle lolle, epiche ma sanguinose, tra i Francesi e gli Asburgo per il predominio in Europa. La lega di Cambrai segnerà o suggellerà ben presto la dipendenza d'Italia e la decadenza anche dell'Adriatico.

Dei Turchi, che sull'Adriatico non ebbero che un cam- I Turchi al. po d'azione del tutto secondario, saramno ricordati solamente quei singoli falti, che interessano principalmente le sue coste.

Presa Costantinopoli nel 1453 e posta fine così delinitivancute alla signoria greca-bizantina, si preparavano a soltentrare anche nei di lei possedimenti. La penisola balcanica e la strada verso la pianura mongrical crane 
loro aperte da parecchi decenni. Si diressero di qua, giacchè per fortuna dell'Adriatico anche questi barbari non erano marittimi. Il lettore avrà già osservato che veramente barbari non sono generalmente che popoli di regioni montanare. I popoli che vivono sul mare possono essere predoni e pirati, ma barbari di costumi no, perchè il mare ingentilisce.

I Turchi dunque fecero la loro prima comparsa sull'Adriatico meridionale verso il 1467 , dopo aver posto fine in quest'anno al regno di Scanderbeg. Nel 1470 compirono la conquista dell'Albania e nel 1480 fecero un'incursione sulla costa occidentale, ove conquistarono e devastarono Otranto. Siccome però l'Albania, militarmente importante, per la storia e per la civiltà, meno che all'epoca romana, ebbe scmpre poco valore, anche l'occupazione turca non segnò per ciò solo la rovina dell'Adriatico.

Vinta la resistenza anche degli Albanesi, i Turchi si spinsero subito avanti con scorrerie, che forse avevano lo scopo di provare gli ostacoli da vincere. E così comparvero lungo la costa dalmata, ma per terra, dietro le prime linee di monti, depredando e ammazzando, fino nelle regioni montanc dell'Istria e del Carso triestino e più in là ancora oltre l'Isonzo nella pianura friulana fino ad Udine, accolti, come si può pensare, tutt'altro che gradevolmente dalle popolazioni e dalle milizie della repubblica veneta. Contro le loro incursioni fu anzi eretta (1479-1481) la fortezza di Gradisca.

A dire il vero fra quelle orde c'erano in gran parte anche Bosniaci e Croati, poco meno barbari dei Turchi, incalzati da questi. Di tali scorrerie ve ne furono parecchic, incominciando dal 1470 fino al 1501. Grandi furono i danni sofierti dalle popolazioni dell'interno della Dalmazia e dell'Istria, che, decimate, impoverite, perirono riducendo in miseria intere regioni. I vuoti prodotti in Dalmazia furono più tardi e lentamente riempiti da altri Slavi. In Istria invece la Serenissima vi rimediò improvvidamente trasportando i Morlacchi dalla Dalmazia. Queste infiltrazioni demografiche e le ulteriori immigrazioni 
di Morlacchi, ivi condotti dalla repubblica per colmare $\mathrm{i}$ vuoti delle pestilenze dei secoli XV, XVI e XVII furono fatali, perchè alterarono il carattere nazionale delle campagne dell'Istria, prima esclusivamente e prodigiosamente italiane, malgrado le stragi della trasmigrazione delle genti.

I Turchi in questo frattempo fecero anche delle conquiste durature. Nel 1463 conquistarono gran parte della Bosnia e resero tributario il paese del Narenta alle spalle di Ragusa, territorio che sottomisero completamente solo nel 1483. Questo territorio, che gli Slavi chiamarono Zachumia e Travun, verso la metà del secolo precedente era stato innalzato dall'imperatore di Germania al grado ducale, col titolo di "Ducato di San Sabba". In seguito a ciò, per una corruzione di termini (duca in tedesec si dice Herzog), prese il nome di Herzegovina, col quale ì conosciuto anche oggigiorno.

Ottenuta la signoria di Trieste i duchi d'Austria contunuarono la politica d'ingrandimenti mediante maritaggi

Gli Asbur gliesi. e trattati d'eredità in caso di morte.

Siccome l'Adriatico non interessava loro gran che, in primo luogo perchè essendo oriundi da paesi alpini anch'essi non avevano domestichezza col mare, in secondo perchè con Venezia, retla a repubblica, non era il caso d'imparentarsi proficuamente, si rivolsero altrove. A noi che studiamo l'Adriatico non interesserebbe nemmeno di sapere che cosa abbiano fatto al di là dello spartiacque. Siccome però da quei maneggi uscì più tardi quell'Austria, che tulti conosciamo, che pesa e vorrebbe pesare sempre più sull'Adriatico, è necessario di occuparcene, almeno sommariamente.

La risoluta energia di uno dei duchi d'Austria, Alberto II, riuscì a procurare alla sua famiglia le corone d'Ungheria e di Boemia. Egli fondò in tal maniera la prima unione personale fra l'Austria, l'Ungheria e la Boemia, che fu ricca di grandi conseguenze. Quando poi fil imnalzato anche sul trono terlesco (1438) fu compiula quella riunione di paesi, che diede nei secoli successivi la sua impronta speciale all'Austria. Da questo duca ed 
imperatore la corona tedesca rimase sino al 1806, quindi per tre secoli e mezzo, meno una sola interruzione nella famiglia degli Asburgo.

Suo figlio e successore Federico III fu un uomo debole. Il primo sfregio che si lasciò fare nella sua qualità di erede dei diritti degl'imperatori tedeschi partì dall'Italia e precisamente da Milano. Estintasi la valorosa famiglia dei Visconti (1447) il condottiere Francesco Sforza si appropriò la dignilà ducale senza curarsi affatto dell'imperatore e dell'impero. E questo fu il periodo in cui l'influenza della corona germanica in Italia $\mathrm{fu}$ ridotta al minimo.

Ma già alla fine di questo secolo, anzi proprio nell'anno 1500 , la politica ereditaria degli Asburgo registra un altro piccolo acquisto sull'Adriático, ${ }^{1}$ ) la contea di Gorizia con Gradisca e Pisino, in seguito all'estinzione della linea dei conti di Gorizia. Questo avvenimento ridondò a danno della repubblica di Venezia.

Ma sulla portata politica di questi fatti che si connettono cogli avvenimenti del secolo successivo si dirà nel prossimo capitolo che tratta del secolo XVI.

Intanto le due forze, ultime ricordate, dei Turchi e degli Asburgo, per quanto siano state in lotta, concorsero da due parti differenti ad aggredire ed indebolire Venezia, che forse avrebbe resistito, se un nuovo fatto imprevisto, ma di importanza storica mondiale, la scoperta della via marittima alle Indie occidentali, essiccando le fonti della sua ricchezza, non l'avesse indebolita nelle sue radici.

1) Tappe dell'esp.nsione austriaca sull'ddriatico. - Dalla parte orientale: Duino (1374), Trieste (1382), Gorizia, Gradisca, Pisino (1500), Ungheria (1526), Dalmazia (1797), Bosnia-Erzegorina (1878). - Dalla parte occidentale: Rorereto (1516), Lombardia (1535 e 1714), Parma (1735), Venezia (1797), Jodena (1814), Napoli (1516 e 1711). 


\section{EVO MODERNO.}

Il passaggio dall'evo medio al moderno, che, quando. si studia la storia, apparisce unicamente per ragioni tecniche come un cambiamento di scena, si compì invece anche per l'Adriatico senza scosse o mutamenti rumorosi.

La Serenissima continuò a dominare sulle sue coste; gli altri Stati e popoli continuarono a vivere ed a muoversi subordimatamente. I cambiamenti più noteroli avvennero gradatamente e più tardi, ancora una volta in particolar modo sulla costa orientale per opera, come si disse, degli Asburghesi e dei Turchi.

La vita locale dell'Adriatico portò d'ora in poi per tre secoli (XVI, XVII e XVIII) l'impronla principale di Venezia. Il dominio della Serenissima non fu però senza pericoli e contrasti: ebbe da lottare contro gli Asburghesi, i Turchi, i Papi e gli Spagnoli di Napoli. Siccome però il teatro diplomatico e guerresco di queste lotte giacque fuori dell'Adriatico, si può dire che questo mare, ridivenuto anche politicamente italiano per opera di Venezia, ebbe nuovamente un periodo di monotonia storica e, pur godendo di un benessere economico, rientrò per tre secoli nella penombra. Premetteremo quindi alcune rapide considerazioni sulla politica generale.

Ancora una volta, a riprova della sua umile missione storica, l'Ádriatico, tanto nei suoi piccoli quanto nei suoi grandi cambiamenti, subì l'eco dei maggiori avvenimenti del mondo.

L'epica lotta medievale fra gl'imperatori tedeschi e il papato, rappresentanti il primo l'idea germanica, il secondo della latinita e che avera reso l'alla Italia per secoli teatro di vicende guerresche, era terminata colla viltoria, non rumorosa, ma effettiva del papato. Nellitalia meridionale l'influchea pontilicia aveva chiamato in opposizione agl'imperatori di Germania prima gli Angioini francesi, poi gli Aragonesi spagnoli. Nell'Italia di mezzo lo 
Stato del Pontefice e la Toscana non avevano nulla da fare cogl'imperatori tedeschi. Venezia che vigilava sulla costa orientale pensava a tener in scacco il germanesimo, rappresentato dagli Asburghesi, alla porta orientale d'Italia. La Savoia col Piemonte s'era emancipata coll'elevazione a ducato. Quando anche nella Lombardia il regime repubblicano raggiunse piede fermo a Milano, a Mantova, a Modena, le relazioni dell'alta Italia coll'impero divennero assai tenui, e si potè quindi dire che la penisola, e con lei l'Adriatico, rispetto alla Germania erano davvero indipendenti.

Nel secolo XVI lo splendido risveglio intellettuale dell'Italia, favorito sotto certi aspetti dal disordine politico (non c'era in Europa un paese disordinato politicamente come l'Italia) attrasse nella sua orbita il papato, che veramente fu sempre più o meno influenzato dalle condizioni morali e civili d'Italia. I pontefici Alessandro VI Borgia, Giulio II Della Rovere, Leone $\mathrm{X}$ dei Medici si occuparono troppo dei beni terreni, del loro potere temporale e delle cose d'Italia, trascurando gli interessi religiosi del papato in Europa e nel mondo. Questa loro sbagliata attività ebbe per conseguenza la riforma religiosa e l'emancipazione protestantica di una gran parte dei popoli anglo-germanici. L'Adriatico era troppo saturo di cattolicismo perchè il protestantesimo riuscisse a porvi piede saldo.

Il mecenatismo dei Papi, tanto benemeriti a quest'epoca in Italia per lo sviluppo delle belle arti e della poesia, non portò loro quel giovamento che avevano avuto invece i loro predecessori nei secoli III e IV, indi nel VII e da ultimo all'epoca delle crociate col favorire la coltura e gli studi e coi ripetuti tentativi riusciti a porsi a capo del progresso intellettuale. Allora 'la Chiesa reagiva come tendenza morale perfezionata contro lo sfacelo del mondo romano pagano del III e IV secolo, contro la barbarie pagana nordica del VII o contro l'inferiorità brutale maomettana nelle crociate. Essa 'allora attraeva gli uomini mighiori e di maggior ingegno, che divenivano di conseguenza i suoi più validi sostegni. 
Nel Cinquecento invece il papato non rappresentava prù lo specchio della moralità. D'altra parte l'umanesimo del rinascimento letterario traeva la sua origine proprio dal paganesimo greco-romano, ispirandosi al culto non d'idee trascendentali, ma della natura. I dotti umanisti non erano più, come una volta i più celebrati luminari delle università, altrettante colonne della Fede; invece di essere devoti alla Chiesa, seguendo il nuovo indirizzo scientifico, le si mostravano indifferenti e spesso ostili.

Malgrado l'ostilità dell'umanesimo latino ed i rovesci della riforma germanica, il papato, la Chiesa e l'autocrazia degl'imperatori avevano ancor'a forza sufficiente per affrontare la situazione e provocare la controriforma col Concilio di Trento e colla guerra dei trent'anni. Fu però unn successo effimero.

La rottura, religiosa e politica nelle forme, verteva in sostanza tra il dogma e la libertà di pensiero. Quci paesi e quegli Stati che, mantenendosi cattolici, caddero sotto l'influenza dei Gesuiti e dell'istruzione impartita esclusivamente da loro, come l'Italia, la Spagna, l'Austria e la Polonia, rimascro molto indietro in confronto dello sviluppo scientifico iniziato in Inghilterra ed in Germania nel sccolo XVIII. Questi due paesi in grazia della libertà di studio e d'indagine si svilupparono fino a raggiungere l'egemonia industriale e politica odierna. L'Adriatico fu necessariamente e fatalmente compreso nella slera della reazione cattolica. I Gesuili si sparsero in tulli i luoghi maggiori anche della costa orientale fino all'Albania.

Il bavaglio messo dai Gesuiti al libero progresso degli studi ne determinò la stagnazione e colla decadenza civile delle nazioni latine incominciò anche pel papato quella curva discendente della sua parabola che lo doveva condurre alla breccia di Porla Pia.

Dell'arresto della potenza papale, manifestatosi già alla fine del XY secolo, approfittarono i secolari competitori, gl'imperalori di Germania. Siccome questa dignitit dal 1433 cra passata nella casa d'Asburgo e continuó ad essere una specie di loro prerogativa, l'influenza di questa casa giunse addiritlura alla supremazia sull' Europa 
occidentale. L'ascensione incominciò sotto Massimiliano I. Era stata preparata con patti accorti e fu poi favorita da matrimoni fortunati e dalla scoperta dell'America. Questo fatlo, in parte accidentale, ridondò per lungo tempo ad esclusivo favore della casa asburgica, al cui impero mondiale spagnolo per parecchi decenni non potè pareggiarsi nessun'altra potenza. Quest'egemonia toccò l'apice sotto Carlo V e Filippo II. Il mondo latino le preparò un fatale avversario, non più nei Papi, ma nei re di Francia che per secoli, incominciando da Carlo VIII (1483-1498), avversarono il dominio degli Asburghesi, sia da soli, sia alla testa di alleati, che trovarono ora nei Turchi, ora nei protestanti, finchè non riuscirono ad abbatterlo durante la guerra dei trent'anni (1618-1648).

D'allora in poi il predominio politico passò alla Francia, che lo mantenne attraverso le vicende della rivoIuzione e dell'epoca napoleonica per perderlo a Sedan.

All'influenza direttrice dei tre attori principali - Asburgo, papato, re di Francia - ora ricordati, si deve aggiungere quella relativamente più prossima dei Turchi e degli Spagnoli. Questi due popoli divenuti marittimi e affermatisi nel Mediterraneo (gli Spagnoli col possesso della Sicilia e di Napoli, i Turchi colla conquista dell'Africa setlentrionale) giunsero in parte per via di terra ed anche per via di mare in contatto immediato coll'Adriatico, che fu per conseguenza ancora una volta la linea di confine dei tanti antagonismi.

Da questo abbozzo delle potenze d'Europa nell'evo moderno cercheremo ora di sgomitolare i fili principali e più notevoli degli avvenimenti del mare che c'interessa.

srcoro xir. Sul mare, come tale, dominava tra la fine del XV e Venezin. il principio del XVI incontrastato il leone di San Marco.

Però la fioritura di Venezia non era sbocciata nel momento migliore per la maturazione di frutti duraturi, giacchè anche altri Stati e popoli raggiunsero contemporaneamente l'apogeo della loro forza. Nel secolo XVI furono all'apice della potenza politica gli Asburghesi, quali rappresentanti del germanesimo, gli Spagnoli, i Turchi; le repubbliche e gli staterelli d'Italia brillavano per la col- 
tura e la Francia progrediva risoluta nella via ascensionale. Da tale esuberanza di energie potera prodursi o l'equilibrio o un gran cozzo colla caduta dei piè piccoli e deboli. Avrenne il secondo.

Erano tempi di turbolenze civili per quistioni religiose e di smania generale di conquiste guerresche. Il progresso della coltura e del benessere economico, favorito in gran parte dalla scoperta dell'America, fece nascere nei vari ceti sociali delle popolazioni il desiderio di conservare ed aumentare le loro prerogative contro le tendenze dei principi di consolidare nelle loro mani ogni potere. Questa lotta fra libertà municipali e centralizzazione imperiale diede vita, inquietudine e risalto a tutto il secolo XVI e fu visibile meglio che altrove nell'alta Italia.

Avvennero per ciò nuove lolte e guerre per le quali l'organismo di Venezia, non bene assicurato territorialmente, anzi insidiato nell'Adriatico e colpito nelle sue altre fonti mariltime, diveme appunto più debole e declinante.

I Veneziani avevano compreso molto bene che per conservare il loro Stato occorreva ampliare il territorio di terraferma, ossia crescere per poter resistere alla concorrenza degli altri competilori più grandi territorialmente. Per ciò li vedemmo spingersi nel secolo precedente fino all'Adda ad occidente, all'Isonzo ad oriente, al vescovato di Trento a sellentrione el a Cervia a mezzogiorno. Inoltre avevano occupato alcune cilti della costa pugliese: Monopoli, Polignano, Trani, Mola, Otranto, Barletta, ecc.

Con ciò si urtarono con tulli i ricini, che credettero di scoprire in loro l'intenzione di estendere la signoria su tutta l'Italia.

In questo lrattempo avennero inoltre in tulti gli Stali che contornavano Venezia degli avenimenti, per i quali la Serenissima fu presa in tale un ingranaggio d'inimicizie, che corse il pericolo di essere stritolala.

Quando gli Asburghesi nel 1500 raccolsero l'eredità dei conti di Gorizia, la repubblica di Venezia non mancò 
di sorgere a reclamarla anch'essa, dicendo che l'accordo ereditario non era valido perchè il Goriziano apparteneva alla chiesa di Aquileja, nei cui diritti essa era sottentrata. Sorsero così delle contese armate, durante le quali Venezia, per rappresaglia, impedì all'imperatore di passarc per i suoi territori quando volle recarsi a Roma per l'incoronazione. Ne nacque una guerra.

Poco diversamente andarono le cose al confine meridionale. Venezia potè conservare Ravenna e Cervia per quasi tre quarti di secolo. Ma quando dopo la morte di Alessandro VI Borgia, papa Giulio II volle incamerare il ducato della Romagna fondato da Cesare Borgia, si vemne ad ostilità anche da questa parte.

Per iniziativa di Giulio II fu conchiusa il 10.decembre 1508 la celebre lega di Cambrai tra lui, il re di Aragona Ferdinando I il Cattolico, interessato per i possessi della bassa Italia, e l'imperatore Massimiliano d'Austria, cui accedette anche il re di Francia Luigi XII, interessato per le città della Lombardia, coll'intendimento nientemeno che di distruggere la repubblica di San Marco.

Ma i Veneziani, conservando la riputazione della loro sagace politica, sventarono quella lega innaturale, cedendo subito al Papa Ravenna e Cervia e a Ferdinando I i porti della Puglia. Nè queste furono le sole perdite: nella pace conchiusa nel 1517 Venezia riebbe Verona, ma perdette Cremona e la riva dell'Adda. Rovereto, Riva c Gradisca rimasero nelle mani degli Asburghesi, che non se le lasciarono sfuggire mai più. - Nelle paci relative fatte per forza e in fretta non si designarono esattamente tutti i confini. Sotto il titolo di possesso di Gradisca gli Austriaci tennero anche Aquileja e Marano lagunare. Vi furono contestazioni posteriori fra Veneti ed Austriaci ed anche oggi precisamente per questa stessa causa la quistione dei confini orientali tra l'Italia e l'Austria (pare incredibile che possa essere una conseguenza di quell'epoca) non è ben chiara.

Venezia s'era dumque salvili, na aveva fatto il primo passo sulla via del declivio.

Con tutto ciò essa contiuuò a prosperare. Tranquilla 
nei suoi possessi, che godevano di un governo mite e di tenui aggravi e le erano perciò fedeli, fortunata ancora nei commerci, fu turbata soltanto dai Turchi, delle cui molestie si dirà nel parlare dei progressi dell'islamismo.

Ai suoi sudditi essa concedeva molte libertì, purchè non si occupassero di politica. A questa cura aveva da provvedere il senato o consiglio dei Pregadi, al cquale erano demandati gli affari di politica estera, e gli ambasciatori, che la repubblica, prevenendo in quest'uso tutti gli altri Stati d'Europa, teneva presso le corti straniere. Che il sistema fosse buono, almeno per quei tempi, è provato dai successi e dagli espedienti coi quali la repubblica seppe cavarsi da brutti imbarazzi. L'acume politico dell'aristocrazia veneziana era anzi tanto sviluppato che Ia storia di molti avvenimenti generali di quest'epoca non ha altra o miglior fonte che i rapporti degli ambasciatori veneziani al loro senato. Tanto erano divenuli acuti osservatori e coscienziosi informatori.

Come prova del suo benessere e del suo senso artistico basti ricordare tutti gli edifizi pubblici e privati costruiti nei suoi possessi a Udine, a Palmanora, a Capodistria, a Pirano, a Zara, a Sebenico, a Traú, a Lesina, a Curzola, ecc. che datano quasi tutti da questo e dal secolo precedente.

Quest'opulenza però è un'ostentazione forzala per abbagliare i propri cittadini e gli stranieri. Venezia avera sulle sponde adriatiche due rivali, l'Austria e la Spagna, affermatesi di fresco, ma bramose di espansione. Per ora specialmente gli Spagnoli della bassa Italia, essendo un popolo marinaro per eccellenza, esercitarono una terribile concorrenza ai commerci di Venezia col levante. Lo sforzo d'impossessarsi di tutto l'Adriatico le era stato fatale. Dall'impari lotta la repubblica di Sall Marco era uscita stremata di forze ed esausta di danaro. La concorrenza anche degl'Inglesi e degli Olandesi nel commercio del mondo doveva darle l'ultimo tracollo.

Papa Giulio II della Rovere (1503-1513) può essere to stato pou. chiamato l'ultimo Pondatore dello Stato della Chiesa. Eggli tificio. voleva non solo ricostituire il polere temporale, mat fare 
dello Stato pontificio lo Stato dominante in Italia e liberare infine la penisola dagli stranieri (detti ancora i barbari).

Il primo intento gli riuscì giacchè riebbe tutte le Romagne e incorporò allo Stato della Chiesa Modena, Reggio, Parma e Piaccnza. Se anche queste ultime ciltà riandarono presto perdute, si può dire ch'egli ridusse da ultimo lo Stato della Chiesa a quella estensione, che conservò fino agli ultimi tempi.

Giulio II, uno di quei papi del '500, prettamente italiani, che, a torto o a ragione non conta, s'interessarono più delle cose della loro patria che dell'eredità di San Pietro, s'era assunto un còmpito non facile. Per doninare in Italia doveva fiaccare Venezia e per liberare l'Italia non poteva far a meno dell'aiuto di questa. Imprudentemente incominciò col rivolgersi contro Venezia, e non riuscì. Allora strinse con Venezia la lega santa per cacciare dall'Italia i Francesi e attrasse dalla sua parte anche l'imperatore di Germania. Ma la sua morte (1513) troncò anche i progetti. I Francesi invece continuarono ad immischiarsi tanto nella Lombardia che nel Napoletano.

A dispetto degli sforzi di Venezia e di Giulio II per redimere la penisola politicamente, i Francesi s'insediarono nell'Italia alta e gli Spagnoli (ramo asburghese) nella bassa. Questo risultato fu l' effetto di lunghe guerre, combattute per lo più in Italia ed ebbe la conseguenza che la parte settentrionale d'Italia incominciò a dipendere dalla Francia e la meridionale dalla Spagna, mentre gli Stati di mezzo non ebbero più vita indipendente, ma dovettero cercare la loro salvezza accostandosi ora all'una e ora all'altra potenza straniera. In altre parole l'Italia fu ridotta in quello stato in cui si trovò press'a poco ai nostri tempi la penisola balcanica.

Arl ogni modo sono notevoli questi due tentativi pratici, antagonistici, ma contemporanei, di liberazione ed unificazione d'Italia e dell'Adriatico. A loro si deve accompagnare l'altro, pure contemporaneo, ma teoretico, di Machiavelli. Essi dimostrano se non altro che lo svi- 
luppo intellettuale del rinascimento italiano non era scompagnato da sentimenti d'indipendenza politica.

Varie sono le cause per le quali questi sforzi non poterono conseguire il fine desiderato. Innanzi tutto nessun grande interesse, nessun bene permanente dei popoli moveva tutta questa attività politica, la cui molla era il solo momentaneo interesse dei dominanti. Per ciò mancava la costanza. Secondariamente se Venezia e il Pontefice, che si potevano in certo modo considerare di forze pari, non erano riusciti a scavalcarsi, non sarebbe stato mai possibile che uno di loro vincesse i Francesi e gli Spagnoli, malauguratamente chiamati ad intromettersi negli affari della penisola, o gli Asburghesi, assorti proprio in quel tempo al primo posto della politica mondiale. In queste condizioni anche se la sorte avesse fatto nascere il principe invocato da Machiavelli, si può dubitare del successo della sua opera patriottica.

Malgrado ciò non sarà privo d'interesse considerare un po' più da vicino il tentativo di Venezia.

Venezia possedeva sulla costa orientale tutto quello che la geografia e la politica contemporanea le permettevano. I Veneziani però comprendevano il lato debole del loro possesso. Essi vederano che i loro territori della Dalmazia e dell' Albania, interrotti in terraferma, non potevano essere conservati che coli'assoluto dominio sul mare; vedevano crescere i loro nemici tanto dietro l'Istria, dietro la Dalmazia, nell'Albania, quanto al di là del Po e dell'Adda; si sentivano nel pieno vigor della forza e del senno amministrativo, e per prevenire assalti nemici, tentarono d'ingrandire i loro posisessi. Lo Stato pontificio nell'Italia media $\mathrm{e}$ il regno di Napoli nella bassa erano sconvolti politicamente. Ne approfittarono per metter piede nella Romagna e nelle Puglie. Questo passo dimostrerebbe ch'essi non tendevano tanto al dominio dell'Italia, quanto piuttosto ad assicurar meglio nelle loro mani il dominio del mare Adriatico con un ampliamento di possessi anche sulla costa occidentale. Nè d'altronde è ammissibile che i Veneziani, tanto sagaci, abbiano con- 
cepito il piano di dominare l'Italia intera, impresa alla quale non avevano un interesse diretto e per la quale sarebbero loro mancati i mezzi.

Tanto più interessante è quindi quel loro tentativo per questo studio sull'Adriatico. Qualora i Veneziani fossero. riusciti in quell'intento, avrebbero rinnovato in senso inverso quello che i Bizantini avevano già fatto nei secoli VII, VIII, IX, ossia il dominio incontrastato sul mare mediante l'occupazione di tutti i punti importanti delle due coste. L'insuccesso dei Veneziani si può spiegare con ragioni storiche del momento; però in esso, come nella breve durata dei possessi solidi dei Bizantini, hanno operato sopratutto cause geografiche.

I Veneziani nell'espansione al di là del Po urtarono contro i possedimenti temporali del Papa. Se però Pipino non avesse fatto le donazioni i Veneziani non vi avrebbero trovato che forse una repubblica di Bologna e una continuazione dell'esarcato ed avrebbero magari potuto sottometterli, come avevano vinto la resistenza dei patriarchi di Aquileja nel Friuli. E probabile che questa espansione avrebbe allarmato la Toscana e Roma (che, del resto avrebbe potuto continuar ad esistere e prosperare anche se la costa adriatica fosse caduta tutta in mano dei Veneziani), indi che ne sarebbero derivate delle guerre e che magari i Veneziani avrebbero oltrepassato anche gli Apemnini. Tutto ciò però non sarebbe stato inevitabile geograficamente, perchè I'Apennino umbro-toscano è alto sufficientemente da permettere ai suoi due versanti di vivere indipendentemente. Dal lato puramente geografico Venezia avrebbe potuto quindi impossessarsi anche d'Ancona e continuare eziandio la sua marcia lungo il versante adriatico dell'Apennino. Dove però la geografia si sarebbe opposta ad un duraturo possesso della sola costa è nelle Puglie. Abbiamo veduto che perfino i Normanni, sbarcati su questa costa, quando vollero consolidarvisi, furono attratti all'interno e finirono col calare dall'altra parte nel Napoletano, appunto perchè nella parte meridionale dell'Italia l'Apennino non ha una catena determinata di divisione e di confine 
Queste considerazioni accompagnate dalla visione dell'Adriatico quale fu ai tempi dei Romani, dei Bizantini e dei Veneziani c'induce alla affermazione geografica, che questo mare, il quale può dirsi il golfo più simmetrico che la natura abbia creato nell'Europa e forse su tutta la terra, non è adatto a formare uno Stato. Ciò avrebbe forse potuto avvenire qualora tutti gli Apemini avessero la forma e l'altezza che hamo nell'Italia di mezzo e tutto il Carso avesse la forma e la robustezza della catena del Bebio (Velebit). Così però com'è fatto esso fu e sarà sempre diviso e dipenderà per due terzi dall'Italia e per un terzo dai Balcani.

Dal lato puramente geografico il tentativo di Giulio II quale signore dello Stato pontificio, arrebbe avuto maggiore probabilità di riuscita per l'Italia, se anche non per l'Adriatico. L'idea era però sorta in tempi non adatti e anche se fosse riuscita non avrebbe avuti effetti duraturi. Il peso della signoria spagnolo-asburgica la fece tramontare subito e per sempre anche da questa parte. Intanto le idee dei Pontefici e gl'interessi del papato si modificarono talmente che questo Stato, il quale per un attimo era sembrato l'animatore dell'ideá italiana, ritornò ad essere invece il più forte e il più lungo ostacolo alla rigenerazione d'Italia.

Nello studiare l'ulteriore sviluppo dell'Austria e della Gli Asburga. famiglia asburgica occorre distinguere tra i possessi territoriali dello Stato e della famiglia congiunti come tali, e quelli della famiglia sola, (fuali imperatori di Germania.

Daremo la preferenza allo studio dei paesi austriaci, perchè questi almeno in principio interessano più da vicino l'Adriatico.

Abbiamo veduto come l'Austria nel 1500 sia venuta in possesso del Goriziano e ne sia derivata una guerra con Venezia. Aggiungeremo clıe già allora l'Austria per combattere i Veneziani incominciò ad adoperare truppe croate.

Quando poi nel 1516 gli Austriaci s'impossessarono anche di Rovereto e di altri luoghi a mezzogionno, li incorporarono al Tirolo come arrotondamento. In tal modo 
l'Austria era venuta ad insediarsi solidamente nella parte montuosa tutt'all'intorno dell'Italia al suo confine nordorientale.

Stava allora a capo della famiglia d'Asburgo Massimiliano I, uomo di pronta intelligenza e di grande attività, che avendo ottenuto anche il posto d'imperatore germanico, cercò di favorire in ogni modo la potenza della sua casa. Tentò, ma senza risultato, di elevar l'Austria a regno o a Stato elettorale della Germania. Allora la sua mente, sempre inquieta per l'avvenire della sua casa, rivolse l'attenzione all'ingrandimento dei possedimenti territoriali. E combinò due matrimoni, quanto mai accorti, uno colla famiglia regnante di Spagna, l'altro con quella d'Ungheria, che dovevano portare in breve agli Asburgo un Iustro strepitoso. Egli preparò e facilitò alla sua famiglia l'acquisto non solo dell'Ungheria, ma contemporaneamente della Boemia, e pose con ciò la base della preponderanza politica dell'Austria nel sistema degli Stati curopei.

Per effetto del primo matrimonio un ramo degli Asburgo sali il trono di Spagna, da cui dipendeva ora anche il regno di Napoli e quando nel 1516 si estinse in questo la famiglia regnante la corona passò per eredità appunto ad un asburghese (che fu Carlo V). - Questa signoria sul Napoletano è però da considerarsi ancora come dominio spagnolo, perchè di fatto veniva esercitata da vicerè, venuti dalla Spagna. In ogni caso quest'estenş,ione del potere degli Asburgo sull'Adriatico occidentale, per quanto saltuaria e politicamente spagnola, è già un fatto. di non poca importanza. E realmente gli Spagnoli, popolo navigatore e guerresco, all'apice della potenza proprio in questo secolo, esercitarono grande influenza su questo mare. Essi aiutarono in primo luogo i Veneziani nelle guerre contro i Turchi nell'Adriatico e fuori di esso (a Lepanto). A Castelnuovo nelle Bocche di Cattaro esiste ancora un forte detto spagnolo in memoria del concorso dato da questi ai Veneziani nel 1538 alla conquista delle Bocche. Anche sull'isola di Lesina esiste un forte detto spagnolo. Le spedizioni maritlime degli Spagnoli nel Me- 
diterraneo ebbero poi, come si vedrà, una grande influenza indiretta sulla repubblica di Ragusa.

Ma ben più notevole fu il successivo accpuisto degli Asburgo. Quardo nel 1526 (in seguito alla battaglia di Mohacs combattuta contro i Turchi) si estinse anche la famiglia regnante d'Ungheria, questo paese e la Bocmia vennero per successione ereditaria a Ferdinando I d'Austria, il quale per ciò può essere considerato come l'effettivo fondatore dell'odierna monarchia austriaca.

Con ciò la casa d'Asburgo acquistò nel litorale croato e nel golfo di Fiume un nuovo tratto di costa dell'Adriatico e venne a gravitarvi di conseguenza con un'ampiezza di territorio, mai prima avveratasi. Nemmeno i Carolingi avevano ammassato politicamente all'Adriatico verso il confine orientale d'Italia un retroterra così vasto.

L'avanzarsi e l'ingrandirsi della potenza asburghese cambiò aspetto ai paesi nord-orientali dell'Adriatico. Nel mentre prima per la mancanza di un grande potentato vicino vi si erano formati molti gruppetti di enti politici, indipendenti amministrativamente e governati da principi ecclesiastici o laici sotto l'egida della sorranità feudale dell'impero germanico, ora la dipendenza di questi enti dalla casa d'Asburgo e la loro appartenenza allo Stato austriaco li privò gradatamente delle prerogative autonome per cementarli nella compagine eterogenea del nuovo Stato.

Lo sviluppo di questo Stato era in parte il prodolto del lavorio della casa regnante, ma in parte anche un portato dei tempi. Nei secoli XV e XVI avvenne la fusione di alcuni Stati cone la Spagna e la Russia, o l'ingrandimento di altri, come la Francia, la Prussia, la Polonia. Eguali tendenze s'erano manilestate anche in Italia con scopi regionali. Altrettanto accadera ora in Austria con interesse dinastico. Si sarebbe quasi tentali di esclamare che quel principe che Machiavelli aveva invano invocato per l'Italia, era nato... in Austria.

Morto Massimiliano I nel 1519 gli suceesse suo nipote Carlo V, che eletto contemporancamente anche at trono di Germania, quale imperatore tedesco ed erede dei troni 
(li Spagna, d'Austria e di Borgogna, dominò su di un impero mondiale, di cui l'Austria veramente non rappresentava che una piccola parte e l'Adriatico una ancora più piccola.

Sebbene dunque l'Austria e gli Asburghesi avessero raggiunto l'Adriatico su una zona abbastanza vasta, non pensavano di affermarvisi ancora. Varie sono le cause. In primo luogo Venezia non si sarebbe lasciata portar via il suo commercio marittimo. In secondo luogo l'Adriatico andava perdendo d'importanza tanto per l'Austria quanto per gli Asburgluesi. La comparsa dei Turchi a Costantinopoli e il loro avanzarsi nella penisola balcanica aveva inaridito il commercio coll'oriente. I due sbocchi poi dei paesi austriaci, che non conoscevano ancora la grande industria, su Trieste e Fiume, erano sufficienti per quei tempi. Nè d'altronde gli Stati continentali pensavano allora di contendere le vie del mare ai popoli marittimi per posizione geografica. La scoperta dell'America da ultimo areva attratto i popoli navigatori del Mediterraneo, come gl'Italiani del versante tirrenico, gli Spagnoli, i Francesi, verso l'Atlantico, non solo, ma aveva dato vita per la prima rolta nclla storia ad un'attività marinara e commorciale dai mari settentrionali d'Europa per opera degli Olandesi, Francesi, Inglesi. Il commercio mondiale d'Europa, che prima era concentrato nel Mediterraneo e nei suoi golfi adiacenti si spostò verso l'Atlantico, e l'Adriatico, che appena da poco era risorto a nuova vita, illanguidì nuovamente. La casa d'Asburgo infine, in grazia dei suoi possedimenti spagnoli e olandesi d'oltre mare, ebbe campo di estrinsecare la sua attività ed ambizione su mari e regioni, divenuti molto più importanti dell'Adriatico. Per queste ragioni e forse ancora per cause politiche interne l'Austria per tutti i secoli in cui durò il dominio di Venezia non esercitò un'influenza decisiva sui destini dell'Adriatico.

Carlo $\mathrm{V}$ aveva invece rivolto tutta la sua attenzione ai re di Francia, ch'egli considerava rivali nel dominio del mondo. Francesco I di Francia nel 1515 aveva ritolto Milano agli Sforza. Carlo $\mathrm{V}$ si rivolse per ciò contro di 
lui ed ebbe la fortuna di abbatterlo subito al principio della lotta. Così ebbe fine il predominio francese nell'alta Italia durato circa quattro lustri del principio di questo secolo e la signoria spagnola non fu più contrastata nella bassa Italia.

Sorse allora nel pontefice Clemente VII l'idea di fare un nuovo tentativo per liberare l'Italia dagli stranieri, di cui non restava più che l'imperatore Carlo $\mathrm{V}$, e fece una nuova lega santa col re di Francia, collo Sforza di Milano e con Venezia. Ma l'intento fallì. In conseguenza di quest'insuccesso Clemente VII dovette riconoscere la signoria di Carlo $\mathrm{V}$ in Italia e quando nel 1535 si estinse la famiglia degli Sforza, Milano fu occupata da un Asburgo. Il sopravvento della famiglia spagnolo-asburgica in Italia cra ormai assicurato; ed i re di Francia invano tentarono di contrastarla ancora con una serie di guerre, finchè nella pace di Catcau-Cambrésis (1559) dovettero riconoscerla definitivamente.

L'influsso spagnolo dominava ora anche nei più piccoli staterelli d'Italia. Col favore degli Spagnoli i Gonzaga di Mantova ottennero il margraviato del Monferrato, resosi vacante. Parma e Piacenza furono fuse in un ducato e date ad un Farnese. Il Papa dovette contentarsi di uno Stato conforme agl'interessi spagnoli. Da ultimo anche la Savoia, che nella pace di Cateau-Cambrésis, aveva riavuto il Piemonte, dovette appoggiarsi al sistema politico della Spagna. Così dalla metì del secolo XVI la penisola italiana, e particolarmente quella sua metì che spetta all'Adriatico, cadde in una totale dipendenza degli Spagnoli.

La grandezza della Spagna e la suil signoria sull'Italia, durata nella bassa due secoli e mezzo e nell'alla mo e mezzo, fu la rovina della penisola e dell'Adriatico.

Non le facciamo carico di aver sviato il commercio dal Mediterraneo e di aver privato l'Italia della sua posizione privilegiata come centro del commercio mondiale, e consideriamo soltanto la sua influenza demoralizzatrice sulla terralerma.

Gli Spagnoli inebbriali delle loro virtù militari e delle

Conseguenze del dominio spagnolo. 
Loro conquiste portavano ovunque con sè un'albagia irragionevole e un cieco fervore religioso, che li rese schiavi della più materiale superstizione. Nell'idea di sostenere la fede cattolica come il còmpito più nobile demandato dalla storia alla loro nazione, essi si lasciavano ridure ad un docile strumento dell'inquisizione e della reazione clericalc.

I loro vicerè nella bassa Italia represscro ogni libertà di pensicro e abrogarono un po' alla volta l'antica costituzione. Il clero e la nobiltà accumularono tutte le ricchezze nelle loro mani e si formarono i latifondi e le manimorte. I funzionari non avevano limite nella loro corruzione; il popolo tenuto a bella posta nell'ignoranza e oppresso da balzelli impoverì e si abbrutì.

Nel rimanente dell'Italia e durante la prima metà del secolo, le arti belle avevano raggiunto il massimo fiore, specialmente a merito dei Pontefici. Papi, cardinali e principi, in ispecie della casa Medici, andavano a gara nel proteggere le arti e i loro cultori. Il rinascimento era all'apice del suo splendore. A ragione il cinquecento (secolo XVI) viene detto il secolo di Leone $\mathrm{X}$ o quello d'oro dell'arte e della pocsia in Italia.

Eppure il dispotismo gerarchico del cattolicismo, sostenuto dal governo spagnolo, soffocò tutta quella splendida fioritura. Per far valere la loro influenza nei paesi dominati direttamente, nonchè sugli altri Stati indipendenti territorialmente, ma non politicamente, come lo Stato pontificio, Venezia, la Toscana, gli Spagnoli mantenevano numerosi escreiti (s'era già introdotto l'uso delle milizie regolari) e un esteso servizio di polizia. Del resto si curarano poco delle quistioni giuridiche e municipali, specialmente nell'alta Italia. Ma coll'aver soffocato ogni libertà di pensiero, nell'interesse apparente della Chiesa romana, gli Spagnoli non fecero un buon servizio nemmeno ai Pontefici, nè li compensarono con ciò della perdita d'autorità politica, per la quale avevano lottato tanti secoli. Prescindendo dalle contese locali italiane nessuno dei Papi della metà di questo secolo potè innalzare la woce in un'importante quistione europea. Il concilio di 
Trento, tenuto per caso in una città, che non è priva dinteresse indiretto per l'Adriatico, fu un insuccesso. Perfino il più energico dei pontefici del secolo XVI, Sisto V, non riuscì ad ottencre dei risultati che nell'assetto interno dello Stato della Chiesa col dar la caccia ai banditi e coll'ordinare le finanze. Paolo V poi, che non voleva tollerare intromissioni di sorta del potere temporale nelle faccende ecclesiastiche, dovette veder fallire tulti i suoi mezzi spirituali di fronte alla vicina e piccola repubblica di Venezia, che aveva dato ricetto a fra Paolo Sarpi, l'implacabile storico del concilio di Trento, e l'aveva nominato suo teologo e consulente nel senso delle teoric protestanti. Per vincere, l'inquisizione dovette farlo pugnalare.

Tutto ormai declinava in Italia e nella metì dell'Adriatico che la bagna.

Ma anche sull'altra costa le cose non anclavano meglio; I Turchi. da questa parte principalmente per opera dei Turchi.

Al principio del secolo XVI, proprio quando per una strana coincidenza l'occidente d'Europa (Italia, Spagna, Asburghesi) era in fiore, anche i Turchi ragginmsero l'apice della loro potenza sotto Solimano II (1520-1566).

Siccome noi non abbiamo da occuparci che dei paesi situati in immediata vicinanza dell'Adriatico, basterà ricordare che i Turchi, raggiunta la costa dell'Adriatico nell'Albania e conosciuti i paesi che stavano innanzi a loro colle scorrerie che abbiamo accennato, si accinsero ad affrontare i popoli più agguerriti. Nel 1526 vinsero la famosa baltaglia di Mohacs, in seguito alla quale gli Ungheresi dovettero celere anche un'ultima parte della Bosnia, e Ragusa si vide indotta ad umiliarsi al Turco e porsi sotto la sua protezione.

Venezia, che da più di mezzo secolo aveva avuto la necessità di affrontare per mare e l'occasione di sperimentare la violenza dei Turehi, che le avevano tolto la maggior parte dei suoi possedimenti in oriente, se li vide ora avvicinare sempre più minacciosamente dalla parte di terra e occupare anche alcmi paesi interni della Dalmazia, confinanti colla Bosnia. Dovette per ciò sostenere con loro continue guerre in Dalmazia e fuori. 
Abbiamo detto che i Turchi non erano un popolo marittimo. E non lo furono mai. Però colla conquista di tanti paesi ai mari Egeo e Jonio e nel Mediterraneo orientale e sopratutto colla sottomissione di quegli Stati barbareschi nell'Africa settentrionale, da cui già una volta rano usciti i Saraceni, essi riuscirono a mettere insieme una flotta di terribili pirati, che incominciarono a molestare inıpunemente tutto il resto del Mediterraneo, le coste dcll'Italia e l'Adriatico. Nel 1521 il vicerè d'Algeri, Uzuz-Ali, veme colle sue galee e con una turba di barbari a invadere l'Adriatico orientale. Vi furono scontri a Catlaro, a Culzola, a Lissa, alla Brazza (Bol). Nel 1566 in un'incursione turco-saracena fu distrutta la città di Ortona. - A ricordo p. e. delle lotte sostenute in questo mare contro le aggressioni dei Turchi i popolani di Curzola ballano ancora la moresca, in cui figurano i cristiani che tolgono al re dei Mori la sua «bula» ossia la sposa ad essi rapita.

Per porre un argine a queste terribili incursioni le potenze interessate, sebbene fossero anche allora rose dalla gelosia e dall'invidia - ossia lo Stato pontificio con Sisto V, Venezia, Genova e Spagna coll'asburghese Filippo II, - strinsero, sia pur per breve tempo, una lega che fruttò la vittoria navale di Lepanto (1571), cui presero parte, qual più qual meno, con proprie navi, tutte le città marine dell'Adriatico. La flotta papale veniva subito al secondo posto dopo quella veneziana. Dopo questa vittoria delle armi cristiane, da cui per discordia e gelosie personali non si seppe trarre il debito frutto, la potenza dei Turehi incominciò a declinare un po'alla volta fino ai fatti dei giorni nostri.

I Turchi non erano nati che per la guerra. Non ávendo e non potendo più conquistare altro, dovettero adatlarsi a vivere, come si vive in pace. E infrollirono, anzi marcirono.

La finc del secolo XVI, incominciato con albori di gloria, segna anche per i Turehi, come per i contemporanei loro avversari, il principio di una parabola disecndente 
Abbiamo parlato del benessere di Ragusa. Tale stalo Ragusa. invidiabile durò anche per tutto il secolo XVI. Vedemmo anche come fu indotta a mettersi sotto la protezione dei Sultani. Tale cambiamento, dalle apparenze sgradite, permise invece ai Ragusei di godere di una sufficiente indipendenza, anzi li favorì, perchè li mise in grado di commerciare liberamente fra l'occidente e l'oriente anche quando a Venczia ciò non era concesso a causa delle suc periodiche guerre col Turco. Così p. e. i calzolai di Ragusa divemnero ora i fornitori di babbuce (papuzze) per la Turchia.

Però a causa di queste relazioni coi Sultani i Ragusei ebbero delle molestie da parte delle potenze cristiane. E allora siccome a causa delle guerre in oriente il commercio era spesso inceppato davvero, essi offrirono i loro navigli in nolo e i loro capitani in sérvizio agli Spagnoli. Le flotte di Spagna ebbero navi e condottieri ragusei nclle grandi spedizioni di Tunisi (1535), Algeri (1541) sotto Carlo $\mathrm{V}$ e in quella dell'invincibile armata (1588). Ma di queste la prima soltanto, come si sa, cbbe esito felice, ed i Ragusei ritornarono in patria carichi di boltino. Nella seconda e nella terza invece gli elementi distrussero la maggior parte della flotta ed i Ragusei ne furono damneggiati sensibilmente. Così incominciarono i rovesci anche per loro.

L'avanzata dei Turchi portò all'Adriatico oricntale in- I pirati duldirettamente ancora due faslidi: i pirati dulcignotti e gli eignotti. Uscocchi.

Approfittando della guerra dei Veneziani coi Turchi, gli abitanti di Dulcigno (albanesi) si fecero corsari per conto proprio. La loro flotta raggiunse il numero di mille navi. Le barche dulcignotte, di una forma speciale, furono terribili nell'Adriatico e devastarono le coste dell'Albania, della Grecia c dell'Italia meridionale. Un pascià, Solimano, rinscì a sorprenderle e incendiarle d'un colpo nel loro porto.

Questa parola di origine slava e che vorreblbe dire i Gli tseoenhi. fuggiaschi o i banditi, era adoperata nel secolo XVl per indicare gunegli abitanli slavi rella Bosnia e della Serhia, 
che al principio di esso avevano dovuto abbandonare le loro terre e ricoverarsi altrove. Ne abbiamo già veduto una parte precedere e accompagnarsi ai Turchi nelle devastazioni dell'Istria, del. Carso e del Friuli.

Vi furono però di loro altri due gruppi principali, uno dei quali operava in terraferma e aveva il centro d'azione a Clissa e poi a Sign (in Dalmazia, sopra Spalato) e l'altro sul mare e aveva il nido a Segna e sui monti che la coronano. Erano predoni feroci nel vero senso della parola. Stavano sotto la protezione dell'Austria, che li sosteneva e stipendiara, e molestavano i Veneziani, i Turchi ed anche i Ragusei (questi ultimi col pretesto che erano alleati dei Turchi). Avevano barche velocissime e la loro speciale bravura consisteva nelle sorprese notturne durante $\mathrm{i}$ tempi burrascosi. Formavano in tutto circa seicento uomini, ma crano rinforzati da avventurieri e galeotti di ogni provenienza dall'Adriatico ed anche dal Mediterraneo. Quelli di terraferma devastavano le campagne dei territori soggetti a Venezia lungo tutta la costa fino a Zara. Più di tutti ebbe da combatterli Venezia durante ottant'anni interi, per mantenere di fatto quella supremazia dell'Adriatico, ch'essa vantava di diritto. Quando alfine i Ragusei, stanchi di essere aggrediti dagli Uscocchi in casa propria, nella penisola di Sabbioncello, si rivolsero al Papa, che fece le rimostranze alla corte d'Austria, i Turchi mossero per causa loro una guerra all'impero e più tardi i Veneziani fecero la stessa cosa, anche l'Austria si persuase di doverli finire. E infatti al principio del secolo XVII dopo sanguinose repressioni e feroci rivolte furono sbandati e dispersi nell'altipiano croato.

La pace fra l'Austria e Venezia fu conchiusa nel 1617 a Fiume, o più precisamente in un vicino convento di cappuccini.

Dopo la guerra degli Uscocchi, Venezia e l'Austria munirono i loro confini dell'Istria, devastati da quei predoni, di nuove tribù slave; Venezia le prese dalla Dalmazia, l'Austria dal litorale croato.

secolo xir. Questo secolo differisce essenzialmente dal precedente. 
Si può dire un secolo di sosta e di stagnazione per litalia e per l'Adriatico e quindi di poca importanza. Non vi furono notevoli cambiamenti, anzi dalla parte occidentale territorialmente nessumo, dall'orientale pochi. Noralmente è un secolo di decadenza su tutta la linea nel senso di continuazione della china precedente.

Malgrado la pace relativa, l'Italia giaceva oppressat sotto il peso dell'assolutismo politico e religioso della Spagna.

La scienza, che nei paesi protestanti era rinata e procedeva libera e trionfante sulla via dell'esperimento, nei paesi cattolici era soffocata dall'intransigenza della fecle. Basta citare un fatto per tutti, la persecuzione di Galileo Galilei, costretto ad abiurare per rispetto alla fede quello che l'indagine scientifica gli avera insegnato essere positivamente vero.

Nella bassa Italia la costa adriatica non poteva essere fiorente, se poco lontano, a Napoli, scoppiava l'insurrezione di Masamiello.

Nello Stato pontificio il nepotismo e la caltiva amministrazione portarono più volte la nave di San Pietro all'orlo del fallimento. Urbano VIII spese somme lavolose in fortificazioni e per l'esercito; lentò, ma inutilmente, di sottrarsi alla potenza degli Asburghesi durante la guerra dei trent'anni. Al contrario collaiuto francese la santa sede riuscì ad impossessarsi clel ducato d'Urbino dopo l'estinzione dei Della Rovere. Innocenzo XI chbe da fare per riordinare le linanze.

La letteratura e l'arte, che furono sempre il baromedro d'Italia, erano degenerate nelle sdolcinature del secentismo e uclle ampollositì del barocco.

Però verso la fine di questo secolo, essendosi la Francia a merito di Luigi XIV innalzata al predominio in Europa, l'influenza francese incominciò a soppiantare la spagnola. Si prepararano grandi e gravi arrenimenti pel secolo venturo.

Venezia ed i Turchi non deposero le armi per lullo Guerra di questo secolo.

Siccome però i Turchi lotlavano contemporancamente. Veneziat $(0,1$ T'ureo. coll'Austria e cogli altri Stali delloriente d'Europla dai 
Balcani in su, Venezia si regolava secondo l'andamento delle cose da quella parte di terra.

Una guerra violenta scoppiò nel 1645 , che durò fino al 1669 , nel qual anno nella pace di Candia, Venezia dovette adattarsi a perdere quest'isola importante e accontentarsi di una demarcazione, a lei sfavorevole, dei confini in Dalmazia, sulla base dell'uti possidetis. A Venezia rimasero cioè soltanto i territori alla costa, occupati durante la guerra, che poi vennero indicati coll'appellativo di "vecchio acquisto». - La linea fissata dal commissario reneto Nani, fu detta per ciò "linea Nani».

Però, appena i Turchi furono sconfitti sotto Vienna, Venezia riprese coraggio e in alleanza cogli Stati orientali (lEuropa riprese la guerra che terminò colla pace di Karlowitz (1699), ${ }^{1}$ ) pure sulla base dell'uti possidetis.

In forza di questa i Veneziani riebbero solo una parte del retroterra dalmata (fra cui sei piazzeforti) e precisamente quella che si trova tra la prima catena di monti alla costa e le monlagne successive, inoltre la foce del Narenta fino a Gabella, Castelnuovo nelle Bocche di Cattaro. Questo fu detto il "nuovo acquisto».

In tale incontro fu stabilita anche la neutralità di tutto il territorio di Ragusa. Caposaldo della politica di questa repubblica era il principio di non urtarsi con nessuno (lei suoi vicini più potenti e che avrebbero potuto danneggiarla. Ragusa si trovava libera nei suoi commerci sotto la protezione nominale della Porta e per ciò, siccome in questa guerra i Veneziani incalzando i Turchi nella regione del Narenta s'erano strelti un po' troppo addosso al territorio dei Ragusei, questi acconsentirono volentieri che il porto di Klek col villaggio di Neum fossero ceduti ai Turchi, desiderando prudentemente di evitare il conLatto territoriale coi Veneziani in Dalmazia. Così la Turchia fino al 1878 ebbe con una striscia di pochi chilometri anche lo sbocco all'Adriatico di mezzo nel canale del Narenta di fronte a Sabbioncello.

1) Ora Karlovei in croato, Karlícza in ungherrse, città sul Danubio, nel comitato dell'antico Sirmio. 
In complesso Venezia e la Turchia chiudevano il secolo con un patto transitorio, spossate e declinanti tuttc due. Per Venezia però si veleva chiara la tendenza a riconsolidarsi almeno sulla costa dalmatit.

La sorte di Ragusa non poteva sottrarsi al destino Ragusa generale; e quindi questo secolo segna anche per lei un. regresso, e non indifferente.

In principio del secolo era ancora fiorentissinı. Ma per la morte di molte centinaia di giovani affogati sotto Tunisi, Algeri, Tripoli, per la perdita in settant'ami di circa duecento navigli, per le pestilenze degli anni 1548 e 1562, ma sopratutto in conseguenza del terribile terremoto del 1667, rimase mezza rovinata, diminuita di popolazione, scoraggiata.

Vi successe una quicta decadenza politica, corruzione nell' aristocrazia dominante (effetto inevitabile dell'ozio nella ricchezza), lotte di partito tra la vecchia nobiltà e quella mova di borghesi ammessi dopo il terremoto. Anche l'istruzione non fu più attinta direltamente dall'Italia, ma anche qui s'insinuarono i Gesuili, che la avocarono a sè.

In questo sccolo, principalmente per opera del Gondola (Gundulić) e del Palmolta (Palmotić) anche la lingua serba fu usata in forma letteralia meno imitalrice dell'arte italiana, che nel secolo precedente.

In questo sccolo l'Austria non feece alcun passo aranti sull'Adriatico, ma si consolidio al confine dell'Isonzo. 1)opo un secolo dachè possedera Gradisca, si accese tra l'Austria e Venezia una nuova guerra, alla fine del $\mathbf{1 6} 15$, a causa del possesso di questa cittì. La guerra, che si concentrò attorno a Gradisca, porla il nome di guncra gradiscana. Fu una guerra minula e durò due amni. La fortezza rimase però all'Austria.

All'interno dell'Austria avvennero invece degli arvenimenti importanti, che devono venir ricordati, onde romprendere di poi quello che succederà al suo estemo.

In quanto riguarda particolarmente la dinastia degli Asburgo basta dire che la sua stella continuò a hrillarre sul mondo da Carlo V proprio fino all'ullim'ora del se- 
colo (1700). Fu però appannata dall'antagonismo della Francia, impersonata prima in Richelieu, poi in Luigi XIV.

L'Austria, come tale, continuò il suo duello colla Francia, ma ne uscì con danno.

Oltre alle guerre in cui fu implicata per questo motivo, l'Austria, come Venezia, ebbe da lottare tutto il secolo coi Turchi. Pel valore di Eugenio di Savoia l'Austria ritolse alla Turchia la maggior parte dei territori perduti. La pace di Karlowitz non fu definitiva nemmeno per lei. Era però evidente anche da questa parte che la Turchia era ormai in perdita e non si sarebbe rialzata più.

A leggere le vicende più spesso caltive che buone e le gucree da cui fu sconvolta l'Austria in questo secolo, si sarebbe tentati di dedurre che anche la sua compagine statale ne abbia risentito dei prégiudizi. Invece per quella tendenza di re e principi, generale in quest'epoca in Europa e ch'era stata incominciata dalla Spagna, di restringere cioè le costituzioni e le libertà delle classi alte, anche in Austria il dominio assoluto della dinastia regnante d'Asburgo aveva preso un deciso sopravvento sulle velleità particolariste degli elementi che la componevano. $\mathrm{Per}$ effetto di quest'azione incominciò anzi a farsi sentire anche nei paesi austriaci una unione sempre più intima fra loro, nella quale fu compresa ben presto anche l'Ungheria.

Si sa che prima, sia per ragioni interne di famiglia, sia per opportunità di amministrazione, gli stessi arciduchi ed imperatori d'Austria avevano spartito i loro territori fra i figli. Tale era appunto il caso nell'epoca di cui si tratta. Questo sistema pericoloso fu ora abrogato, ed estintasi nel 1665 una linea laterale i paesi furono riuniti in una mano e da quel tempo in poi non si fecero più spartizioni nel territorio di casa d'Ásburgo.

L'idea di uno Stato indivisibile, in germe già al tempo di Ferclinando I (morto nel 1564) e già chiaramente manilesta solto Ferdinando II (morto nel 1637) ebbe la sua più viva espressione sotto Leopoldo $\mathrm{I}$, il quale ottenne che nella dieta di Presburgo (1687) l'Ungheria venisse trasformata in un regno ereditario nella linea 
maschile austriaca e fosse abrogato il diritto degli Stati di opporsi a mano armata al re nel caso di violazione da parte sua delle leggi regnicolari. - Le mire di Massimiliano I di creare uno stato burocratico furono ora raggiunte in grazia dell'assolutismo che, vincendo la resistenza degli Stati, potè produrre una rapida fusione dei paesi. Per l'azione dei principi in quest'epoca si svilupiò pure la coscienza d'un'Austria indivisibile e già al tempo di Leopoldo I (1657-1705) il nome "Austria» appare quale contrassegno ufficiale di tulti i paesi ereditari della casa d'Asburgo.

Mentre però nei paesi erceditari austriaci l'assolutismo progrediva sempre più, gli Ungheresi seppero conservarsi la loro costituzione privilegiata, cosicchè già in cquesto periodo nella forma di gorerno si manifesta come una specie di dualismo.

Ma quanto più potente diveniva l'Austria, tanto più intenta era la Francia ad umiliaria.

Il secolo XVIII è anche per le regioni dell'Adriatico un secoro xrm secolo di rinnovamento. Esso incominciò e si cliunse con eventi memorabili. Gli arvenimenti, che successero al suo principio, presentano però un nodo di fatti difficili ad esporsi in ordine cronologico.

Per non guastare il filo della logica occorre ricordare dapprima l'ultima guerra contro i Turchi, sostemula ciopo tre soli lustri, dai Vencziani e dall'Austria nello stesso tempo. Venezia e l'Austria cioè, in altrilo ai loro confini e sull'Adriatico, avevano interessi comuni nei Balcani, e si trovarono d'accordo da questa parte. (Anche allora proprio come oggi!)

Nel 1714 la Porta avera ritollo la Morea ai Veneziani. Fine della La repubblica trovandosi alle strelle si rivolse all'impe- suerre di veratore d'Austria, Carlo VI, che fece delle rimostrinze a chi.

Costantinopoli; ma essendo stata respinta la mediazione mosse subito guerra alla Turchia. Il genio mililare del principe Eugenio di Savoia costrinse i Turchi dopo splendide vittorie alla pace di Passarowit\% (esaltamente l'oyalrevac in Serbia, 1718), cui aceedettero, triculo prolitlo sull' Adriatico anche i Veneziani. Anche questa arrenne? 
sulla base dell'uti possidetis. Venezia ebbe oltre ad alcune piazze nell'Albania (venela), in Dalmazia tutti i territori situati al nord della linea Knin, Sign, Vergoraz, ottenuti nella pace di Karlowitz, sino ai confini naturali delle Alpi dinariche. Fu fatta per ciò una nuova demarcazione dei confini, che dal nome del commissario vencto Alvise Mocenigo prese il nome di linea Mocenigo. Questo fu detto l' "acquisto nuovissimo". - Con ciò ebbero fine le lotte durate due secoli e mezzo fra la Serenissima e la Porta.

Venezia nel- Per quanto Venezia fosse riuscita a conservare la sua l'ult mosecolo della sua esistenza. posizione sull'Adriatico di fronte ai Turchi, aveva riportato delle ferite, ch'erano irrimarginabili.

Privata dei commerci coll'oriente a causa dei Turchi e di quelli del Mediterraneo per le nuove vie marittime del mondo, illanguidì nella sua forza economica e decadde anche nel resto della sua potenza.

Venezia non poteva sapere quello che sappiamo noi, che cioc̀ la pace di Passarowitz avrebbe segnato l'ultima lotta sanguinosa col Turco. Per ciò in una ragionevole previsione di altri conflitti, posta com'era tra due rivali potentissimi, la Turchia e l'Austria, cercò di conservare nelle popolazioni dei confini alla costa orientale e specialmente nei Dalmati quello spirito bellicoso, che le areva giovato tanto nelle guerre sostenute col Turco, e le lasciò a bella posta nello stato di ignoranza in cui si trovavano, anzichè elevarli, perchè facendo di loro dei cittadini dotti, li avrebbe alienati dall'uso delle armi. Venezia aveva dato ricetto entro i suoi confini a gruppi di popolazioni dai Balcani, per lo più Slavi, ma anche Albanesi e Greci. Ciò però non bastava. Un proverbio orientale dice che dove è passato lo zoccolo d'un cavallo turco l'erba noin cresce più. I Turchi nelle loro infinite escursioni (la storia ne ricorda più di dieci maggiori) avevano distrutto e desolato il paese. Le popolazioni (slave) dell'interno, taglieggiate dai Turchi ed esauste dalle guerre combattute con condotticri veneziani, non avevano più forza da rialzarsi. Così quest'infelice costa orientale, che avera tanto sofferto nella trasmigrazione dei popoli, che 
non aveva potuto rimettersi nemmeno nei secoli di mezzo per l'inferiorità civile iniziale degli Slavi che l'arevano occupata, ebbe per colpa dei Turchi l'ultima rovina.

La debolezza di Venezia era già palese in quest'epoca. Dalla pace di Karlowitz essa non prese parte più a nessun avvenimento mondiale e adottò il sistema pericoloso, perchè negativo, della neutralità disarmata. Essa si astenne da tutte le guerre combattute in Europa, ma ciò non impedì che già nella guerra di successione spagnola, soldatesche austriache e francesi scorrazzassero pel suo territorio devastandolo. Si direble che si sentiva vecchia e per ciò desiderava la pace ad ogni costo. E tale si mostrò appunto nel 1728, quando venuto l'imperatore d'Austria a Trieste, mandò un'ambasciata solenne a complimentarlo.

L'aristocrazia veneziana, ch'era quella che aveva aruto il merito di tener su lo Stato nei secoli precedenti, immiserì nel facile godimento delle ridchezze, nell'ozio e in frivole contese.

La pace di quasi un secolo fu fatale alla Serenissima, perchè preparò la sua caduta. L'ora del tramonto non era Iontana.

L'astuzia ragusea, che non era venuta meno nella sven- Ragusa. tura, trovò il modo di insinuarsi anche nella pace di Passarowitz. In questa fu stabilito, a somiglianza di quanto era stato fatto per Kilek in quella di Karlowitz, che la Turchia avrebbe avuto un secondo sbocco nell'Adriatico, e precisamente nelle Bocche di Cattaro, col territorio detto della Sutorina, ch'è una piccola striscia di terra fra la Punta d'Ostro e Castelnuovo. Questa soltile distinzione, di pochissimo valore territoriale, ma di molta importanza politica, sussiste tutt'ora. Klek e Sutorina dipendono dall'Erzegovina.

Al principio di questo secolo la magnifica llotta ragusea era ridotta a venti trabàccoli e l'esercito a un centinaio di mercenari, comandati da un governatore delle armi, "cletto dal re di Napoli".

Nel suo particolare anche la storia di Ragusa seguc in questo secolo una linea concava. Nella prima meti 
Lulio exa decaduto, anche la letteratura. Nella seconda invece commercio, ricchezza e letteratura si rialzano per l'instancabile attività dei Ragusei. Nei secoli XVI-XVIII liagusa fu considerata l'Atene della letteratura serba.

Ma Ragusa risente un nuovo dammo dalla guerra russoturca (1768-1774). Nel 1768 l'ammiraglio russo Orlow comparve nell'Adriatico (nuovo, inatteso, per quanto breve, attore su (juesto mare) con una flotta poderosa e fece quello che si usa fare anche oggigiorno: fermò come navi nemiche quelle di Ragusa, che trasportavano granaglie e vetlovaglic per i Turchi a Costantinopoli. Venezia che in altri tempi aveva sostenuto inflessibilmente il principio che nari stranierc non dovevano veleggiare nell'Adriatico, non ebbc la forza nemmeno di far sentire la sua voce.

I! resto del secolo, ossia gli ultimi anni di Ragusa, si comnettono, come quelli di Venezia, alle vicende napoleoniche.

Gli Asburghesi.

Distingueremo per un po' le lendenze e gli avrenimenti dinastici degli Asburgo rispetto all'Italia da quelli dell'Austria, quale anteriore loro paese ereditario. Tutti due ebbero grande influenza sull'Adriatico.

Nel 1700 morì l'ultimo rampollo maschile della linea spagnola della casa d'Asburgo, che in forza a quel titolo godeva il Milanese e la bassa Italia. La vacanza di seggi cosi ambiti doveva provocare la guerra; scoppiò inlatti col grande rivale Luigi XIV di Francia.

Qui $110 n$ ì il luogo di esporre il corso degli avvenimenti svoltisi fatalmente ancora una volta nella Padania (battaglic di Carpi e Chiari, 1701, Torino 1706) e nell'Adriatico (assedio e bombardamento di Pescara), che si sostenne a lungo contro le armi austriache in grazia delle fortificazioni erettevi ancora dall'epoca di Carlo V.

Dopo da due a tre lustri di guerre la corona di Spagna passavi sulla testa di un Borbone francese e gli Asburgo in compenso di questa diminuzione di potenza e di lustro s'insediavano come linea austriaca a Mantova, incamerata cuale feudo vacante dell'impero, indi nel $\mathrm{Mi}$ lanese, nel Napoletano. Malgrado ciò la potenza della l'amiglia d'Asburgo, per quanto fosse discesa dalla som- 
mità in cui s'era trovala solto Ciarlo $V$, era ancora ragguardevole. Fu questa l'epoca in cui la monarchia au. striaca raggiunse la massima sua estensione e divenne la potenza davvero dominante nella penisola italica.

Una parte importantissima in queste guerre fu eserci- Savoia. tata dalla Savoia, che seppe curare anche molto bene i suoi interessi dinastici.

I duchi di Savoia dipendenti geograficamente dalla Francia avevano parteggiato finora per lei anche nella politica. Avvenne però che Vittorio Amedeo II nel fervore della guerra per la successione di Spagna si schierasse dalla parte degl'imperatori di Germania, il cui esercito in Italia era appminto capilamato dal celebre Eugenio di Savoia. Alla fine della guerra il duca di Savoia ebbe dalla Francia tutte le fortezze e le vallate alla parte orientale delle Alpi Cozie e Marittime, dall'imperatore di Germania e dall'eredità di Spagna tutto il Monferrato, parti considerevoli del ducato di Milano (fra altro Alessandria, la Lcmellina, Val Sesia) inoltre la Sicilia col titolo di Re. Fu questo il secondo passo dei Savoia sulla strada delI'Italia e dell'Adriatico. In seguito però Vittorio Amedeo II ripassò dalla parte dei nemici dell'Austria e per ciò nel 1720 fu costretto a scambiar la Sicilia colla Sardegna. In quesl'occasione prese il titolo di re di Sardegna, che i suoi successori non lasciarono finchè venne il momento di assumere quello d'Italia.

Nell'amno 1748, in seguito alla guerra di successione austriaca, il regno di Sardegna ebbe dall'Austria ancora Tortona, Novara ed altri territori confinanti sul Ticino.

La nuova dinastia borbonica in Spagna mise in opera tutti i mezzi per riacquistare l'antica posizione degli Spagnoli in Italia. Ciò non le riusci che in parte coll'acquisło Cambiamenti dinastici in Italia. dei ducati di Parma e Piacenza dopo l'estinzione dei Farnesi nel 1731.

Poco dopo durante i rivolgimenti per la sanzione prammatica della casa d'Austria li cambiò con quesla per Nilpoli e la Sicilia, nel qual regno fu creala ma secondogenitura borbonica, che durò fino al 1861 .

Gli Asburghesi coll'acquisto di Parma e Piacenza in- 
grandirono i loro possedimenti in Lombardia e misero un piede al di là del Po.

Contemporaneamente fu stabilito che il duca Francesco di Lorena, genero presuntivo dell'imperatore l'Austria, avrebbe ceduto la Lorena e ne sarebbe stato 1.isarcito colla Toscana quando il trono mediceo fosse rimasto vacante per l'estinzione di cuella casa. La morte dell'ultimo granduca della famiglia dei Medici avvenne nel 1737 e Francesco di Lorena entrò allora in possesso del suo nuovo Stato, che fu dichiarato una secondogenitura dalla casa d'Asburgo-Lorena. Nel 1741 Massa e Carrara passarono agli Estensi di Modena e nel 1748 Parma e Piacenza furono restituite ai Borboni di Spagna, pei quali fu creata quivi una nuova secondogenitura.

Da tutti questi rimaneggiamenti di Stati subentrò finalmente all'esierno della casa d'Asburgo una specie di equilibrio tra lei e la Francia, dopo due secoli e mezzo di antagonismi, che avevano tenuto in sussulto la politica d'Europa. Questo equilibrio fu ancora meglio assicurato dal sorgere della monarchia prussiana (il principe elettore di Brandeburgo assunse nel 1701 il titolo di re di Prussia) e dal rafforzarsi della Russia (Pietro il Grande, 1689 al 1725).

Considerazioni politico-sociali.

Pur comprendere la ragione intima dell'arbitrio col quale i principi si toglievano o si assegnavano troni, signorie, territori, e delle guerre spesso d'usurpazione combattute a questo scopo nei secoli XVII e XVIII, bisogna astrarr: dalle idee sociologiche moderne, basate sulla compartecipazione dei popoli al governo. Il periodo che va dalla metà del seicento alla metà del settecento è caratterizzato quale periodo del potere illimitato dei principi. Questo concetto non ha da essere inteso nel senso di contrasto a qualche limitazione anteriore. Il tentativo dell'aristocrazia c del clero, detti altrove gli «stati», nel secolo XVII, di ottenere una voce nell'amministrazione del governo era stato vinto. I principi (siano essi stati principi veri, oppure imperatori, re o altrimenti titolati nelle loro signoric) fino all'epoca di Carlomagno avevano governato a loro talento. C'è però una differenza tra il modo 
nel quale il loro impero era considerato nel medio evo e in questo periodo dell'evo moderno. Allora i principi regnavano come emanazione del potere divino ed i popoli, vinti e vincitori, si ritenevano tanto inferiori e lontani dal potere, che gli si inchinavano volonterosamente fino quasi ad adorarlo.

Alla potenza materiale dell'imperatore non poteva osare di contrapporsi che il Papa, rappresentante in terra del potere divino; e questi concetti erano posti tanto in alto tutti due, che solo tra loro poteva sorgere un antagonismo. Se Arrigo IV andò a Canossa (1077), solo un Ottone I potè deporre papa Giovanni XII (963).

Ora invece $\mathrm{i}$ due strati di vincitori e vinti erano scomparsi nella fusione operata dai secoli ed i popoli evoIuti da una maggior generalizzazione della coltura incominciavano a divenir consapevoli della loro forza, ed avrcbbero volentieri incamminato trattative coi principi per ottenere qualche debole libertà. Un vago sentimento di sviluppo nazionale aveva già rotto le tenebre del medio evo. Ma i principi volendo prevenire il pericolo di simili velleità, concentrarono nelle loro mani ogni potere e lo esercitarono non solo a loro tornaconto e capriccio, ma perfino illimitatamente contro le aspirazioni ed i desideri dei popoli. Si direbbe che in quell'epoca i principi, presentendo qualche cambiamento, avessero avuto fretta di rintuzzare ogni velleità di condominio, giacchè specialmente nei paesi caltolici si afirettarono a diminuirc l'influenza anche della Chicsa sullo Stato. Il Papa non era più una grande potenza colla quale ognumo doveva assolutamente far $\mathrm{i}$ conti: Canossa non avreble potuto più ripetersi.

Tale evoluzione ebbe origine dai cambiamenti nella vita economica, sociale, intellettuale dei popoli e fu sforzata d'un tratto dalle nuove correnti d'idee, che venivano dalla Francia e per le quali si prepararono radicali innovazioni.

Alcuni mutamenti economici furono accenuati dopo le crociate e l'argomento di questo studio non concede di parlarne più a lungo. 
La coltnra finora presso tutti i popoli - se si eccettuino forse i Greci - era stata un privilegio delle classi alte e ricclıe ed aveva progredito per lo più sotto l'impulso di volontà singole. - La prima quasi-generalizzazione della coltura si cbbe in Italia nel rinascimento cinquecentesco in grazia delle libertà popolari. Siccome però queste libertà non erano regolate, la decadenza politica che ne derivò danneggiò anche la coltura.

L'umanesimo italiano passò fortunatamente in Francia e lì trovò terreno propizio anche dal lato politico; giacchè questo paese sin dalla fine dell'evo medio era stato riunito in uno Stato nazionale. Si ebbe qui per conseguenza una nuova rifioritura nei secoli successivi XVII e XVIII, per la quale la Francia si pose alla testa della civiltà d'Europa.

Gli Enciclo-

All' umanesimo già abbastanza antireligioso del seperlisti. colo XVI, tennero dietro nel XVII gli studi filosofici e nel XVIII la letteratura dell'illuminismo, che occupandosi di riforme nella vita religiosa, amministrativa ed economica, ne stimolò il desiderio fra il popolo ed esercitò una straordinaria influenza sulle moltitudini. Basti infalti nominare i rappresentanti principali di questa letteratura, Voltaire, Montesquieu, Rousseau e gli Enciclopedisti. Per influsso di queste idee avvenne in Francia che l'opinione pubblica provocò ed ottenne l'allontanamento dei Gesuiti.

Era quella la prima volta clopo i tempi classici di Grecia, di Roma e delle repubbliche italiane, che il popolo sotto la veste di opinione pubblica s'imponeva in atti di governo. La coltura e la libertà mercè le quali la Francia marciava già risolutamente sulla via del progresso, portarono ben presto ancora molti altri frutti maggiori, che ridondarono a benefizio non dei Francesi soli, ma di tutta l'umanità.

Le idee di progresso, propagate dalla Francia, abbracciavano la filosofia, la religione, la posizione giuridica dei cittadini nello Stato, l'economia politica: erano state sviluppate dagli studi filosofici dei secoli XVII e XVIII ed cbbero nuovo vigoroso alimento dagli studi di scienze 
matematiche e naturali, coltivati teoricamente ed applicati in pratica negli ultimi tre secoli.

Il fervore religioso che aveva dato vita alle crociate era scomparso e al suo posto fioriva la letteratura antireligiosa degli illuministi, dei materialisti, degli enciclopedisti.

Nel mentre i principi credevano di arer debellato le brame di libertà degli Stati (clero, nobiltà, militari) solgevano voci (Montesquicu, Rousseau) a reclamare la libertà legale del singolo, anche infimo membro del popolo. I principi per paralizzare la resistenza degli Stati avevano inaugurato il sistema mercantile, che favoriva esclusivamente l'industria e il commercio. Ma già l'errore cra stato scoperto ed era sorto a combatterlo il sistemal fisiocratico, il quale insegnava che l'unico patrimonio di un popolo erano i beni guadagnati col lavoro della terra e voleva per ciò che si favorisse e proteggesse sopratutto il ceto dei contadini. Questo principio fu poi corretto dallo scozzese Adamo Smith, il quale pose giustamente i fondamenti dell'agiatezza nazionale nel suolo, nel lavoro e nel capitale.

Di pari passo col progresso delle scienze teoretiche avanzarono le naturali.

Per effetto di questi studi e delle nuove idee sviluppate da essi si cambiò il modo di pensare di quasi tutte le persone colte d'Europa.

La prima a risentire il contraccolpo di questa rivoluzione intellettuale fu la Chicsa. Stati cattolicissimi e che nei secoli precedenti avevano coltivato la superstizione religiosa come mezzo di governo, incominciarono a voler diminuire l'influenza della Chiesa sullo Stato.

Papa Paolo III nel 1540 aveva confermato la compa- 1 Gesuiti. gnia di Gesù per soddisfare al bisogno d'una nuova milizia spirituale, che servisse non solo di scudo, ma auche di spada contro la riforma. Invece i Gesuili non solo non ottemnero successi contro i protestanti, ma si altirarono colla loro spinta intransigenza religiosa e colla tendenza di limitare il potere regio anche l'odio di coloro che prima per fervore religioso avevano dato loro 
ampia protezione e ricetto. Federico II di Prussia era volteriano.

Perfino in Austria Maria Teresa ed i suoi successori, tra cui specialmente Giuseppe II, introdussero nei rapporti colla Chiesa tanti e tali cambiamenti da ridurla ad una completa subordinazione dallo Stato. Maria Teresa proibì ai vescovi ed ai capi degli ordini la corrispondenza immediata con Roma, abolì le penitenze pubbliche e riscrvò all'approvazione dello Stato la scomunica. Cessò la libertà d'imposta fruita da parte del clero e fu limitato il numero dei conventi. Giuseppe II s'ingerì perGiuseppe II. sino nelle cose spettanti al culto. Stabilì il numero delle messe, proibì i pellegrinaggi ed emise delle prescrizioni riguardo alla tumulazione dei cadaveri. Colla patente di tolleranza (1781) eguagliò ai cattolici i protestanti ed i Greci non uniti, conferì diritti civili agli ebrei, sicchè alla dominante Chiesa cattolica non rimase che il privilegio del pubblico esercizio religioso.

A scongiurare colpi sì terribili il papa Pio VI si recò personalmente a Vienna nel 1782, ma nemmeno tale atto di umiltà riuscì a distogliere l'imperatore dalle riforme ecclesiastiche, chè anzi l'anno successivo (1783) Giuseppe II colla patente sul matrimonio tolse a quest'ultimo il suo carattere strettamente ecclesiastico tramutandolo in un contratto civile.

Basterebbero questi cenni dell'arbitrio con cui i principi trattavano il Pontefice in contrapposto alle scomuniche con cui Gregorio VII, Innocenzo III, Innocenzo IV riducevano ai loro piedi e al loro volere un Arrigo IV, Filippo II Augusto di Francia, Federico II Huhenstaufen per comprendere quale rivoluzione d'idee s'era già effettuata in Europa.

Queste considerazioni non parranno inutili, quando si ricordi che il papato, assunto a potenza mondiale appunto per aver tratto i suoi succhi dal suolo immortale di Roma e d'Italia, divenne in seguito m'istituzione eminentemente italiana; lo prova il fatto che fu influenzata tanto nel bene quanto nel male dall'ambiente locale. Però, sia che il papato avesse esercitato un'influenza uni- 
versale, europea o solamente italiana, l'effetto delle sue azioni si ripercosse sempre in prima linea altraverso il suo Stato temporale sull' Italia, sulla costa occidentale dell'Adriatico, sull'Adriatico tutto. E appunto sulla costa orientale dell'Adriatico tra la catena delle Alpi dinariche e il Narenta che si potrebbe tracciare la linea di demarcazione storica tra i raggi d'azione del cattolicismo da una parte e dell'ortodossia e dell'islamismo dall'altra, ossia tra Roma e Costantinopoli, o meglio ancora tra l'Italia e i Balcani. E per esprimere l'energia morale del papato fu scelta appunto ia storia delle sue vicende politiche, perchè è nella politica che si manifesta più chiaramente l'altività esteriore dei popoli.

A questa evoluzione politica del papato l'Italia non contribuì consciamente che al primo e all'ultimo atto della scena.

Nell'evo medio e nei primi tre secoli dell'evo moderno il papato era subordinato alla politica italiana, ma anche gl'Italiani erano schiavi delle idee del papato.

Coll' umanesimo l'Italia diede la spinta alla coltura e alle prime ribellioni del pensicro con Gerolamo Savonarola (nato a Ferrara), fra Paolo Sarpi, veneziano - altre due città situate casualmente nel bacino adriatico Giordano Bruno. In questi secoli l'Italia ebbe anche i suoi primi storici e Machiavelli, il primo scrittore unicamente politico. - Ma non poteva dare di più nel campo politico-sociale, perchè il suo sminuzzamento in tauti staterelli non offriva il modo che di apprendere a reggere Stati piccoli con un governo repubblicano oligarchico, per conseguenza aristocratico. Mancava in tutta l'Italia, se si eccettui il Napoletano, che però era dominato da stranieri, il campo materiale di esperimento per far prove di amministrazione civile in grande e quindi di far studi economici e sociali colla partecipazione di altre classi della popolazione, che non fossero state quelle privilegiate e che si ritenevano le sole capaci di dirigere la cosa pubblica. Il servaggio straniero impedi all'talia di contribuire in questo secolo al grande lavoro intellettuale preparatorio degli altri popoli. Per ciò essa durante la 
rivoluzione francese e l'epoca napoleonica apparisce sitibonda importatrice delle idee d'oltr'alpe. - Ma quando la giustizia e la libertà, applicate al governo dei popoli, portarono da quel contrasto tra coercizione e libertà di pensiero, l'idea della nazionalità, l'Italia, svincolatasi felicemente dai ceppi medievali, divenne d'un balzo l'antesignana del principio dell'indipendenza nazionale ed anche l'Adriatico ne subì di riverbero una ricostituzione su basi del tutto nuove.

Per tutto ciò e per quei fatti che ancora hanno da venire parve opportuno di far presente al lettore quelle considerazioni.

Condizioni dell'Italia ossia della costa occidentale dell'Adriatico.
Da quanto ̀̀ stato esposto si rileva che in sostanza nemmeno nel secolo XVIII non vi furono sulle coste dell'Adriatico grandi cambiamenti politici; anzi si può dire che, dopo superati i mutamenti dinastici del suo principio, già ricordati, e prescindendo dalla bufera napoleonica che si connette al successivo, regnò la quiete fin dopo la rivoluzione francese.

Però di quei mutamenti dinastici l'Italia fu la vittima.

La teria fiorita dell'umanesimo era divenuta in un solo secolo il pascolo dell'Arcadia. Di quest'avvilimento una parte della colpa cadeva sugl'Italiani e un'altra sugli stranieri. Gl'Italiani, godendo nel '500 di un'invidiabile libertà individuale, pensarono più a combattersi fra di loro che a premunirsi contro l'invidia degli altri. Gli stranicri attratti dalla luce irradiata dall'Italia furono invogliati a toccarla e ci riuscirono, perchè la resistenza politica degl'Italiani non era forte. Avvenne del rinascimento italiano quello ch' era successo alla civiltà greca in condizioni consimili di discordia e di debolezza politica: attrasse gli assetati, che non sapendo attingere alla sua fonte, l'essiccarono.

Ma l'Italia è terra benedetta e fertile.

Nella prima metà di questo secolo sembrava perduta irremissibilmente. Bastò che nella sua seconda la pressione politica diminuisse d'un poco e un soffio caldo le venisse d'oltr'alpe, perchè nuovi germogli mostrassero d'un tratto verdi foglioline. 
La corrente dominante anche in Italia in questo secolo è la reazione contro la strapotenza del clero. Nel secolo XVIII, ossia nell'epoca dell'illuminismo, l'autorità dei Papi in riguardo politico decadde sempre più. Gli Stati cattolici vicini intrapresero più volte atti di violenza contro quello della Chiesa per costringere il Papa a fare delle concessioni. Ciò non di meno dopo i rimaneggiamenti dinastici sembrò raggiunto un certo equilibrio anche da questa parte.

Gli Asburghesi avevano bensì la prevalenza nell'alta e nella media Italia, ma erano tenuti in iscacco dai Borboni della bassa, da Venezia, che ancora contava qualche cosa, e dalla Sardegna. Lo Stato del Papa pareva messo apposta in mezzo ai contendenti per impedire che si azzuffassero.

Il rallentamento generale dell'oppressione religiosa permise che germogliassero in più luoghi idee nuove.

Nella bassa il dominio borbonico in principio fu molto meno opprimente dello spagnolo-asburghese. Il ministro liberale Tanucci potè introdurre molte utili riforme.

Milano godette anche di maggiore indipendenza e approfittì nelle quistioni religiose e amministrative delle ardite innovazioni introdolte dallo spirito modernizzatore di alcuni governanti. Siccome infine le nuove dinastie - fecero tutto il possibile per adattarsi all'ambiente della nazionalità italiana, la divisione politica d'ftalia non era più sentita come una grande sciagura.

Per completare l'esposizione dello stato dell'Adriatico L'A ustria. in quel secolo di sordo fermento, che è il XVIII, occorre parlare separatamente dell'Austria, anche perchè di poi si possano comprendere meglio i fatti del secolo suceessivo, nel quale l'Austria conquistò sull'Adriatico una posizione di prim'ordine.

Distingueremo l'espansione territoriale, i riordinamenti interni e il suo orientamento verso mezzogiorno e all'Adriatico, dividendo la materia per argomenti a scopo di maggior chiarezza logica, anclie se la cronologia ne risulterì alquanto alterata. 
a) Espansione territoriale.

Degli acquisti in origine dinastici, che poi divennero austriaci, nella penisola italica, fu già parlato.

L'ingrandimento territoriale rappresenta il programma della casa d'Asburgo in questo ed anche nel secolo successivo. Con questa tendenza s'attirò le gelosic oltrechè della Francia e della Spagna anche di principi tedeschi, che incominciarono a farle opposizione ed a porre un argine alla sua intromettenza politica. - Essa inviluppò per conseguenza sè e lo Stato in molte guerre, da alcune delle quali uscì anche con danno. Così p. e. la perdita della Slesia portò la Prussia vincitrice nel numero delle grandi potenze europee e la fece divenire necessariamente sua tremenda rivalc. Però le perdite e gli acquisti transitori di paesi che non riflettorro l'Adriatico non hanno interesse pel nostro studio, nel quale sarà limitato l'accenno a quei soli paesi lontani, che essendovi stati aggregati definitivamente, hanno un valore ed un peso nella complessiva conformazione organica dell'Austria e nella sua posizione sull'Adriatico. Faremo una sola cccezione per ricordare che nella pace di Passarowitz l'Austria aveva avuto al di là del Danubio e dei monti transilvanici, che formano anche oggi il suo confine verso la penisola balcanica, la Serbia e la piccola Valacchia, ma le perdette nel 1739. Con ciò ebbero fine anche da questa parte le guerre coi Turchi e incominciò quella stagnazione dell'islamismo in Europa, ch'era preludio di tramonto.

Nella prima spartizione della Polonia, avvenuta nel 1772, l'imperatrice Maria Teresa ottenne la Galizia orientale e la Lodomiria (paesi al di là dei Carpazi) con due milioni e mezzo d'abitanti e nella terza, avvenuta nel 1795, l'Austria ebbe il ducato di Cracovia. Nel 1775 poi mediante semplici negoziati e per denaro acquistò dalla Turchia la Bucovina. Alla fine del secolo l'Austria fece ancora degli altri importantissimi acquisti e precisamente sull'Adriatico; ma di questi si dirà più tardi separatamente.

b) Riordina- Gli anteriori paesi ereditari austriaci (titolo corrisponmenti interni dente appunto alla formazione dinastica dello Stato) non erano ancora bene amalgamati fra loro, come si vide, che ne capitarono degli altri. 
Per consolidare tutti questi possedimenti all'interno, l'imperatore Carlo VI, che temeva di non avere eredi maschi, procurò di far valere una nuova legge ereditaria, la cosidetta sanzione prammatica, allo scopo di mantenerc l'unità della monarchia e di assicurarla alla sua famiglia. La sanzione prammatica stabiliva che i paesi ereditari austriaci non dovessero essere mai divisi e che all'estinzione del ramo maschile degli Asburghesi avesse diritto alla successione il ramo femminile.

La prammatica sanzione fu pubblicata nel 1713 e negli anni successivi fu accettata da tutti gli Stati provinciali dei paesi austriaci. L'mperatore si adoperò allora perchè fosse riconosciuta generalmente in Europa e a tale scopo fece grandissimi sacrifizi. Però, invece di seguire il consiglio del vecchio ed esperto principe Eugenio di Savoia, che gli diceva di colmare il tesoro e di aumentare l'esercito, preferì di battere la via delle trattative. S'impigliò per questo in nuove guerre, ma da ultimo, malgrado alcuni sacrifizi, otteme il suo intento.

Per effetto appunto di questa legge di famiglia quando nel 1740 Carlo VI morì, gli succedette sua figlia Maria Teresa, maritata con Francesco di Lorena, dal che la casa prese d'allora in poi il nome d'Asburgo-Lorena. Un impero fresco ed eterogeneo, tenuto insieme solo dalla persona del sovrano, caluto in mano d'una domna, nentmeno preparata ad un còmpilo così arduo, doveva necessariamente andar soggelto a scosse. Ai nemici individuali interni ed esterni, si aggimnsero le nuove idee di progresso, propagate dalla Francia e che già piechiavano impazienti ai confini dell'Austria.

L'Austria, come tale, si sa che non ha esercitalo mai - e non aviebbe nemmeno poluto farlo - un'inlluenza direttiva sulle correnti intellettuali d'Europa. Le sue condizioni poi erano tali che i primi a comprendere il vatlore delle nuove tcorie e ad altuarle nel proprio interesse dovevano essere i circoli del governo. In tale opera di modernizazione primeggiarono Maria Teresa e suo figlio Giuseppe II, ai cuali storici cortigiani attribuiscono troppo merito nellopera riformalrice. Essi fecero, è ve- 
ro, molli cambiamenti sulla via del progresso e particolarmente Giuseppe II, imbevuto d'idee filosofiche moderne, andò molto in là nei piani di riorganizzazione. Però il vero motivo di tale amore di progresso proveniva dall'interesse dinastico, perchè l'ultimo scopo delle riforme era il reprimere le ultime libertà feudali e centralizzare, ossia concentrare il potere assoluto nelle proprie mani, regolando e unificando l'amministrazione e la legislazione della monarchia.

Delle innovazioni particolari nell'amministrazione della giustizia, delle finanze, dell'economia politica, degli affari militari non è qui il caso di parlare dettagliatamente. Alcuni cambiamenti nel campo religioso furono già citati. Basterà rilevare, che molto di ciò che all'osservatore superficiale apparisce merito dei sovrani, non avveniva per loro iniziativa spontanca, ma per una pressione ormai sentita dell'opinione pubblica. Così p. e. la tortura come mezzo d'inquisizione era stata ben riconosciuta in una "giurisdizione criminale» introdotta provvisoriamente, ma dovette venir abolita nel 1776. E qui non bisogna dimenticare che il milanese Cesare Beccaria già nel $\mathbf{1 7 6 4}$ aveva dato alla luce quel gioiello, ch'è la sua opera $D c i$ delitti $e$ delle pene, la quale se anche necessariamente fu stampata fuori dei confini austriaci, aveva suscitato grande scalpore.

Il fervore centralistico di Giuseppe II, che del resto non faceva altro che imitare Federico II di Prussia, andò tant'oltre da concepire il piano di introdurre ovunque la lingna tedesca come unica lingua di governo e da iniziare così a poco a poco la fusione, o più esattamente la snazionalizzazione dei diversi popoli non germanici dell'Austria. Questi tentativi rappresentano però già ai nostri occhi il culmine dell'opera della famiglia degli Asburgo per consolidare lo Stato da loro formato tra le Alpi, il bassopiano ungarico, il Danubio e l'Adriatico.

L'effetto fu invece precisamente opposto a quello voluto, perchè in questo modo si fece divampare l'odio contro l'elemento tedesco anche là, dove prima non esi- 
steva. La reazione manifestatasi contro queste tendenze segna il principio di rivolgimenti e sgretolamenti centrifughi. Questi tentativi abortirono in primo luogo perclıè i tempi crano ormai troppo progrediti per una simile violentazione della coscienza nazionale dei popoli più evoIuti, almeno, e poi perchè furono fatti senza tatto e con precipitazione. D'altronde a mandarli ad effetto anche in altri momenti sarebbe occorsa qualche cosa più del potere autocratico d'un solo regnante ed uno spazio di tempo più lungo che non sia la vita d'un nomo.

La tendenza a dar maggiore coesione fra loro ai paesi posseduti non riuscì che riguardo ai paesi tedeschi austriaci e boemi. Le riforme nel campo ecclesiastico e la centralizzazione germanizzatrice in quello politico provocarono un fermento considerevole in Ungheria, la quale ancora nel secolo precedente aveva dato filo da torcere alla dinastia austriaca. Per allontanare pericoli maggiori si dovettero ristabilire i primieri ordinamenti.

Accanto all'Ungheria, la Lombardia fu l'altro paese che interessa l'Adriatico, ove l'opposizione del popolo riuscì a mantenere una posizione privilegiata e hove la centralizzazione dovette venir rallentata, concedendosi perfino l'esenzione dalla coscrizione militare.

Questo ducato, che aveva tenuto testa a Federico Barbarossa, era un po' troppo lontano da Vienna e vicino invece alla Francia. Le idee cosidette sovvertitrici vi si erano insinuate più facilmente che altrove. La coltura letteraria e di scienze naturali (unico esercizio intellettuale concesso in tempi di schiavitù) aveva ripreso vigore nell'alta Italia col classicismo. Per ciò la corte di Vienna pensò di attirare questo paese a sè per ora colle blandizic. Ebbe una speciale riorganizzazione militare e fu istituito per lei un apposito consiglio italiano a Vicmua. Nel ducato di Milano fu promosso il benessere economico, si favorirono le scienze, fu fondata l'accademia di belle arti detta di Brera, il poeta Metastasio fu accolto con grandi onori alla corte di Vienna, e il Parini chbe protezione a Milano dal governatore. Parecchi membri delln famiglia imperiale prescelsero a loro domicilio i L'Adriatico. 
paesi in Italia e perfino gl'imperatori si misero a studiare e ad apprendere la lingua italiana come obbligo di regno.

Dopo tante vicende la famiglia d'Asburgo poteva dire di essersi finalmente assicurata un possesso se non omogeneo e compatto, almeno unito territorialmente.

Erano però questi gli ultimi bagliori del feudalismo medievale, anzi piuttosto gli ultimi tentativi dell'assolutismo dei principi per salvare il loro patrimonio, tentativi che nella loro frettolosità tradivano perfino un vago senso di paura, dinanzi ai prodromi sordi, ma minacciosi, di una nuova rivoluzione d'idee e di principii sociali.

c) Orientamento verso mezzogiorno e all'Adriatico.
La dinastia degli Asburgo nella sua parabola ascensionale aveva tentato d'insediarsi in tutti i migliori troni d'Europa. C'era riuscita in Germania, in Ispagna ed in Italia. L'opposizione della Francia l'aveva respinta un po' per volta dalla Spagna, dal Belgio e dalla bassa Italia. Aveva potuto mantenersi nell'alta Italia.

Da un secolo aveva cambiato tattica, ossia aveva ripreso quella più modesta dei primi tempi : di cercar cioè nuovi acquisti di territori, anche piccoli, attorno al suō nuclen principale. Verso la Germania fece cattiva prova perchè la Prussia le tolse la Slesia e mise un inesorabile alto là. Guadagnò abbastanza nello smembramento della Polonia, ma così venne a trovarsi a contatto colla Russia, dalla quale non c'era nulla da sperare. Il momento storico, forse più che la geografia, le mostrò ora aperta la strada soleggiata del mezzogiorno, ove poteva avanzare in tre direzioni, nell'Italia, nei Balcani e sull'Adriatico.

Fra la penisola italica e la balcanica ognuno avrebbe preferito la prima: in primo luogo perchè senza confronto migliorc e poi perchè da questa parte la resistenza militare e politica era minima, mentre al di là del Danubio stavano ancora i Turchi, che per quanto battuti avevano ceduto solo al genio di Eugenio di Savoia.

L'Austria, non curandosi allora dei Balcani, conchiuse un altro di quei matrimoni bene calcolati coll'ereditiera di Modena, che, come vedremo, doveva condurre al principio del secolo venturo alla fondazione di una terzogenitura asburgica. 
Giá coll'acquisto di territori nelle penisole balcanica e italica cra connesso per l'Austria anche il problema di un'affermazione ed eventualmente d'un'espansione sul mare che le congiunge. La ricchezza raggiunta in breve tempo dagli Spagnoli, dai Portoghesi, dagli Olandesi, dagli Inglesi, dai Francesi sulle vie del mare avera insegnato a tutti i popoli il grande valore di questo mezzo quale strada commerciale. Ed anche Carlo VI, valendosi dei consigli di quel grande nomo che fu il principe Eugenio di Savoia, si accinse di proposito a promovere le industrie ed i commerci per terra e per mare. Per mare egli voleva raggiungere le Indie occidentali dall'Olanda (porto di Ostenda) e le Indic orientali attraverso l'Adriatico. Noi naturalmente non ci occuperemo che di questo.

Finora Venezia avera sempre dichiarato e sostenuto che questo mare appartenevá a lei. Considerato però l'indebolimento della Serenissima, Carlo VI feee un tentativo. Nel 1717 dichiarò libera la navigazione per tutto l'Adriatico e libera alle navi l'entrata e l'uscita dai porti adriatici. Da questo momento Trieste e Fiume furono considerati porti franchi. Venezia taccue. Il primo passo, impunemente a suo danno, era stato fatto. Era questa la prima volta nella storia, che uno Stato continentale dell'Europa di mezzo ed avente soltanto uno sbocco relativamente piccolo sull'Adriatico, tentava di affermarvisi. La cosa era possibile unicamente a causa della schiavitù politica in cui erano cadute le popolazioni indigene marinare delle duc penisole italica e illirica e delle deplorevoli condizioni di Venezia, che sentiva mancarsi sempre più le forze per opporsi al nuovo intruso.

Apriremo una piecola parentesi per dire fualehe cosa del passato di Fiume, ch'è la citta posta proprio sulla soglia dell'Italia geografica.

All'epoca romana (dalla quale bisogna prendere le mosse ogni volta che si voglia studiare qualche istituzione civile sulle coste dell'Adriatico) nel litorale, ora dello croato, primeggiava Tarsatica, da cui deriva l'odierna Tersatto. La regione apparteme quindi ai Franchi e nel $\mathrm{X}$ secolo ru retta dai re croati. Nel XIII secolo esistera

Trieste e Fiume porti franchi. 
già una borgatía, da cui si sviluppò la città di Fiume, che passò in feudo dei patriarchi d'Aquileja, indi dei signori di Duino, dei Frangipani, e nel 1471 fu acquistata per danaro dall'imperatore Arrigo III, che la innalzò al grado di città immediata dell'impero.

Gli anni immediatamente successivi al 1717 furono impiegati per preparare le opere portuarie. Vari porti aspiravano alla fortuna di divenire emporio commerciale, come Aquileja, San Giovanni di Duino, Fiume, Buccari, Portoré. Trieste stessa non aveva allora che circa tremila abitanti. Siccome però il principe Eugenio di Savoia properdeva per Trieste in primo luogo, nel 1723 questa città con Fiume furono dichiarate formalmente porti iranchi. Quindi si pensò di allacciarle alla capitale Vienna. Una strada naturale non esiste; per ciò occorreva costruirne una artificiale attraverso il Semmering. La strada fu terminata nel 1728 e in quest'anno Carlo VI scese a 'Trieste per la solenne inaugurazione. Venezia, che una volta vi avrebbe partecipato con galere e cannoni, mandò, come vedemmo, un'ambasciata a complimentare.

Il commercio di transito per l'Italia era diminuito ancora dall'epoca della riforma, perchè l'antagonismo politico-religioso e sopratutto la scoperta delle nuove vie commerciali l'aveva instradato al di sopra delle Alpi.

Carlo VI, che già nella pace di Passarowitz aveva saputo procurarsi libertà di commercio per i suoi paesi sul Danubio, pensò ora di incanalare tutto il commercio attraverso i suoi Stati ed i suoi porti. Già sotto Leopoldo I era stata istituita una compagnia commerciale levantina. A Vienna fu formata ora una compagnia orientale con grandi capitali e con grandi privilegi, che doveva esserc l'anima del commercio austriaco.

Ma i piani navali di Carlo VI, o forse dei suoi consiglieri, andarono tant'oltre che s'incominciò a costruire anche una flotta da guerra per la protezione dei bastimenti mercantili austriaci. Al comando di quest'armata fu scelto un Parravicini, genovese, per sfruttar meglio l'antagonismo contro Venezia. Però per la poco buona prova fatta dalla compagnia orientale e per la gelosia 
delle potenze marittime settentrionali, Inghilterra e Olanda, dovette sacrificare da lì a pochi anni la compagnia commerciale e l'armata navale. Abbandonato quindi il pensiero di convertire Trieste in un arsenale da guerra, prevalse l'altro di farne una piazza importante puramente per il commercio. Il tentativo, per quanto abortito, era in ogni caso abbastanza significante.

Maria Teresa, figlia di Carlo VI, che gli successe al trono (nel 1740) continuò l'opera paterna e diede ai due porti franchi i più ampi privilegi. Fra altro protesse le colonie di nazioni estere, concesse libertà di culto ai Greci e agli ebrei, ecc. Nelle innovazioni politico-amministrative, per cui va noto il governo di Maria Teresa e di Giuseppe II, anche Trieste ebbe a subire l'imposizione del tedesco.

In quanto riguarda Fiume particolarmente c'è da notare che questa città nel 1776 fu incorporata alla Croazia, ma subito tre amni dopo ne venne escorporata e come corpus scparatum fu annessa direttamente alla corona di Santo Stefano, ossia all'Ungheria. Tutti samno che tra Maria Teresa c gli Ungheresi passava una grande simpatia.

In complesso durante questo secolo le due cittadine di Trieste e Fiume, prima insignificanti, crebbero sempre più e divennero porti notevoli nei traffici dell'Adrialico.

\section{IA RIVOLUZIONE FRANCESE.}

Tutto al di là delle Alpi, in Francia, in Germania, in 'Austria era in fermento di rinnovazione. Solo l'Adriatico, tolti questi piccoli falti appena visibili, ora accennati, stagnava; nclla Padania l'oppressione stranicra, alla costa occidentale la superstizione, la miseria, il dolce far niente, elevati a sistema di governo; alla parte orienlale sotto Venezia e Ragnsa vecchiaia e indolenza; al di lit nei Balcani la barbarie turea.

A turbare quel fatale torpore dell'Adriatico venne la grande rivoluzione politica e sociale di Francia nel 1789. 
Alimentata da molti secoli di angherie e di oppressioni di ogni genere, preparata da un paio di secoli di studi filosofici e provocata dal disagio economico scoppiò con una veemenza mai ancora veduta nella storia e imprevista per chi considerava il mondo colle idee tramandate dall'evo medio. Per trovare un avvenimento consimile bisogna risalire ai tempi classici di Atene e di Roma e pensare alle rivoluzioni, che precedettero la costituzione di Solone e alle riforme dei Gracchi, colla differenza maggiore che, nel mentre allora gli effetti, pur grandiosi, degli sconvolgimenti sociali erano rimasti limitati agli organismi civilizzati di Atene e di Roma, ora essi varcarowo i confini della Francia, ove erano stati prodotti, e si dilatarono immediatamente su due terzi dell'Europa c nell'America.

I disordini, gli sconvolgimenti e le guerre, causati dalla rivoluzione di Parigi, durarono precisamente un quarto di secolo e rimasero limitati alla Francia solamente nei primi tre anni, indi si propagarono ai paesi adiacenti del continente, in Germania, in Italia, in Austria, in Spagna. L'Adriatico fu anche preso in quest'orbita e messo totalmente sossopra.

Durante questo periodo avvennero mutamenti colossali nella vita dei popoli e nei confini dei singoli Stati.

I. bencfizi portati innegabilmente dalla rivoluzione francese non devono far dimenticare gli orrori da cui fu macchiata. Se si considera però che il popolo francese era stato oppresso per lunghi secoli dal potere regio e dai ceti elevati dẻlla società coi mezzi più atroci, si deve conchiudere che la reazione sanguinosa della feccia popolare entrava nel corso inevitabile e naturale delle cose, nel momento in cui doveva ristabilirsi violentemente l'equilibrio. Un ragionamento consimile dev'essere fatto a proposito degli eccessi che, in proporzioni minori, scoppiarono in altri paesi, in Italia e nelle regioni dell'Adriatico.

La proclamazione dei "diritti degli uomini", l'abolizione dei privilegi della nobiltà e del clero, il supplizio del re e della regina per atto di una frenetica volontà 
popolare, divenuta improvvisamente sovrana, la proclamazione della repubblica, dovevano inebbriare la plebe di Parigi e della Francia, ma spaventare nello stesso momento i troni degli altri Stati d'Europa.

Una reazione coalizzata per contrastare i progressi della propaganda rivoluzionaria e repubblicana francese era quindi naturale e prevedibile. Era del pari naturale che l'Austria, come lo Stato più grande e più assolutista, stesse alla testa dei conservatori.

Quello però che portò a questo periodo di fortunose vicende la nota imprevista ed imponderabile, fu la comparsa di Napoleone, dell'uomo fatale che per oltre tre lustri, dal 1796 al 1814 temne la Francia e l'Europa soggiogate alla sua capricciosa, autocratica ed ambiziosa volontìt.

Napoleone forse pensò e potè illudersi di essere stato il fattore principale della storia del suo tempo. I posteri, senza menomare la parte avuta dalla sua influenza personale, possono asserire che anche lui fu uno strumento della maturità dei tempi. La sua opera gigantesca si sfasciò colla sua caduta, e ciò dimostra che la volontà d'un uomo, anche se si chiami Napoleone il Grande, non è gran cosa nella ruota della storia. Nel solco segnato dal suo passaggio germogliò invece il seme di nuove idee, abbastanza differenti da quelle da lui propugnate $\mathrm{e}$ preferite, e ciò prova che i bisogni collettivi dànno la vera spinta al progresso dell'umanità.

L'animo irrequieto del Bonaparte mosse le turbe sonnolente e capovolse troni tarlati.

Il suo primo passo tenue una direzione geograficamente naturale e fatalmente storica: scese in Italia e bastò la sua presenza nel bacino padano per sconvolgere tutto l'Adriatico. 


\section{CONDIZIONI DELL' ITALIA E DELL'ADRIATICO.}

L'Italia era in quest'epoca un corpo smembrato ed inerte. Il prestigio e il potere dei governi principeschi assoluti, di Napoli nella bassa Italia e del Papa nella media, erano offuscati dalla tirannia e dalla superstizione. La repubblica nazionale di Venezia (e altrettanto si può dire incidentalmente di Genova) decadeva per la concorrenza nei commerci e per la corruzione interna dei costumi dei patrizi, dai quali poi anche la plebe traeva cattivo esempio.

Il solo regno di Sardegna conservava un'apparente lihrstà; ma era troppo piccolo, appartato e dipendente dall'influenza francese per esercitare un'azione salvatrice. Nella Lombardia spadroneggiavano gli stranieri tedeschi; ovunque il feudalismo della nobiltà e del clero si sgretolava per la propria corruzione.

Queste condizioni avrebbero potuto trascinarsi ancora per molto tempo, perchè avendo la servitù politica e la povertà economica impedito la formazione di un ceto operaio e industriale (il quarto Stato) mancava una classe numerosa di cittadini, desiderosa e capace di tendere ad un miglioramento sociale e di produrre qualche cambiamento, sia pur con mezzi violenti. I soli letterati ed i dotti tenevano viva la fiammella della coltura, segno della vigoria della stirpe, che nemmeno la schiavitù aveva potuto spegnere. Però anche l'attività letteraria era rivolta piuttosto a magnificare il passato, che ad affrontare l'avvenire, che si presentava troppo tetro. Le condizioni politiche e civili d'Italia erano allora tanto misere da permettere agli stranieri i più atroci insulti. Metternich potè rispondere, poco dopo, a chi parlava d'Italia, che l'Italia non era altro che un'espressione geografica e Lamartine vi aggiunse non molto più tardi (1825) il giudizio ancora più caustico, che l'Italia cra null'altro che la terra dei morti.

E sc non proprio realmente morta, certo sprofondata 
in letargo, era allora la penisola italica. E siccome gli Italiani, che anche nei periodi più dolorosi della loro storia averano contato qualche cosa, se ne stavino inerti senza coscienza, e gli altri due popoli minori dell'Adriatico, gli Slavi e gli Albanesi, quasi nemmeno sapevano di esistere, anche il mare che a noi tanto interessa, seguiva ora come nei tempi passati la medesima sorte delle due penisole che lo racchindono.

L'alta Italia padana e le due coste dell'Adrialico erano come un vasto campo lasciato a maggese in altesa di venir coltivato. I Francesi non furono i pacilici e benefici dissodatori: bisogna per altro riconosecre loro il merito di avervi scarato alcuni solchi profondi, che poi vivificarono tulto il bacino.

La prima comparsa di un escrcito francese in Italia nel 1796, mandatori dal direttorio al comando del giovane generale Bonaparte, mostrò alle attonite popolazioni i seguenti strabilianti mulamenti: ${ }^{1}$

la sconfilla del vecchio ed agguerrito esercito austriaco per parte di giovani truppe meschinamente vestite ed approvrigionate;

l'annessione alla Francia della Savoia e di Nizza (che appartenerano al re di Sar(legna);

la creazione di una repubblica cispadana colla Romagna, tolta al Papa, e col ducalo di Modena;

la caduta della secolare repubblica di Venczia e l'abolizione della sua antica costituzione oligarchica, sostituita da un governo democratico;

la successiva rapida conversione della repubblica cispadana in cisalpina coll'aggiunta della Lombardia;

lat conversione della repubblica genorese in ligure coll'introduzione anche qui di una costituzione democratica ;

l'ingrandimento dell'Austria coi territori reneti di terralerma fino all'Adige e con quelli dell'Istria e della - Dalmazia e il suo conseguente insediamento su ma buona parte della costa orientale dell'Adriatico.

Poco dopo, nel 1799, la propaganda rivoluzionaria pose fine a Roma alla signoria pontilicia. Invano un esercito

L'Adriatico. 
austro-russo cercò di venirc in soccorso al Pontefice, operando intanto uno sbarco e l'occupazione di Ancona. Il Papa trascinato a Valenza vi fu tenuto prigioniero fino alla sua morte. Il re di Sardegna dovette cedere alla Francia il Piemonte. Il re di Napoli, che temendo a ragione per l'esistenza del suo regno s'era unito alla coalizione contro i Francesi, vide malgrado i suoi sforzi convertirsi il suo regno in una nuova repubblica chiamata partenopea. D'un tratto dunçue tutto l'Adriatico, meno alcuni tratti - austriaco, raguseo e turco - aveva camsbiato governo. Essendo poi stato cacciato dai Francesi anche il granduca di Toscana, quasi tutta la penisola e metà dell'Adriatico era in loro potere ed arbitrio.

Però la sconfilta delle armi francesi in Italia durante l'assenza del Bonaparte in Egilto fece sparire d'un tratto tutte le nuove repubbliche italiane e ristabilire l'antico ordinc di cose a Napoli, in Toscana, nello Stato pontificio e in Lombarlia; solo a Genova sostenevasi ancora il generale Massena.

Ma il Bonaparte, fattosi frattanto primo console, colla battaglia di Narengo ristabilì in parte il dominio francese in Italia. La repubblica cisalpina divenne repubblica italiana ed egli ne fu il primo presidente. Il duca di Parma, invece del suo regno, aggregato alla repubblica ilaliana, ottemne la 'Toscana col titolo di regno d'Etruria. Il re di Napoli rimase in possesso dei suoi territori, il Papa riebbe lo Stato pontificio, il Piemonte fu unito alla Francia. Ottenuta nel 1804 la corona imperiale creditaria in Francia, Napoleone l'ammo appresso s'incoronò a Milano anche come re d'Italia. Tutti questi fatti, l'annessione della repubblica ligure alla Francia, ed altre istituzioni a favore della famiglia Bonaparte palesavano troppo chiaramente le aspirazioni di Napoleone alla signoria di tulla la penisola.

Si formò anche per questa ragione la terza coalizione; ma Napoleone colla battaglia detta dei tre imperatori ad Austerlitz riuscì ancora vittorioso. Nella pace di Presburgo (1805) si fece cedere dall'Austria, al regno d'Italia, tutti i territori già acquistati dalla cessata repubblica ve- 
neta, cioè l'Istria e la Dalmazia. In Italia ancora Napoleone s'impossessò di Napoli, perchè il re delle Due Sicilie s'era associato alla coalizione, e fece dono di qucslo regno a suo fratello Giuseppe Bonaparte.

In seguito l'attenzione di Napoleone, divenuto il potente imperatore dei Francesi, fu occupata coi pacsi della Germania e della Spagna. Si rivolse nuovanente alle cose d'Italia quando il Papa non voleva accelere al blocco continentale e allearsi con lui contro l'Inghilterra. Napoleone risolse allora di abolire il potere temporale dei Pontefici (1809). Amnettè il resto dei possedimenti pontifici alla Francia, come già prima aveva rimnito l'altra parte al regno d'Italia. Il Papa, che a causa di queste violenze aveva scagliato l'anatema contro Napoleone, fu improvvisamente callurato e condotto a Sarona.

Napoleone ebbe da occuparsi ancora una volta delle cose d'Italia, anzi precisamente di quelle dell'Adriatico. Onde uniliare per sempre la sua più grande rivale, l'Austria, e toglierla dal numero delle grandi potenze, pensò di respingerla dall'Adriatico. Per ciò nella pace di Vienna (1809) si fece cedere una parte della Carinzia, la Carniola, Trieste, Gorizia, Gradisca, l'Istria austriaca, il litorale ungarico e la Croazia dalla sponda destra della Sava, e Fiume. Da queste province, dalla Dalmazia, staccala dal regno d'Italia, dal territorio di Ragusa, - ove anche truppe francesi averano posto fine all'indipendenza della secolare repubblica - dall'Istria e dalla Pusteria formò il nuovo Stato delle province illiriche, che fece amministrare da un governatore generale, il maresciallo Marmont. Il Trentino (Tirolo meridionale) fu unito atI'Ilalial.

Napoleone aveva loccalo l'apice della sua potenza. Quando nel 1811 ebbe un figlio da Maria Luigia d'Auslria gli diede il titolo, che doveva essere fatidico, di he di Roma.

L'ascensione del Bonaparte era slata rapicla: molto più rapida, anzi fulminca, fu la sua cadula. Alla Beresina (1812) il suo astro si oscurò; alla grande batlaglia dei popoli presso Lipsia (1813) si eclissò per sempre. 
Già colla seconda di queste due sconlitte si dissolvettero da sè le istituzioni di Napolcone in Italia e le province illiriche coll'Adriatico vennero di nuovo in mano dell'Austria.

\section{CONSEGUENZE E CAMBIAMENTI SULL'ADRIATICO.}

Falti come quelli ch'erano avvenuti in Italia negli ultimi cuattro lustri e che erano di natura tale da alterare e distruggere idee inveterate da secoli, non potevano venir cancellati d'un tratto.

Le inprese gueresche del Bonaparte, che accompagnarono e seguirono la rivoluzione sociale e politica di Francial, furono per l'Italia e per l'Adriatico quello ch'è lo straripamento d'un fimme per la vallata sottostante. Durante l'inondazione avvengono devastazioni, schianti, rovine; quando poi l'acqua si ritira, lascia dietro di sè un terriceio, il limo benefico, che ricompensa i danni col rigoglio di una vegetazione spontanca lussureggiante e con uri abbondante raccolto. Così la rivoluzione francese lasciò in Italia il fermento di nuove idee e di move aspirazioni.

Che il trono di Napoli avesse subìto dei cambiamenti, non era cos'a insolita nè per il passato nè per il presente.

Nemmeno il rimpiccolimento transitorio del regno di Sardegna alla sola isola di questo nome e il suo ingrandimento finale coll'acquisto di Genova potevano ancora attrarre l'attenzione del mondo e degl'Italiani sulla dinastia di Savoia. Nessuno allora avrebbe indovinato che dal piccolo Piemonte sarebbe uscita la virtù civile e bellica, rinnovatrice d'Italia e dell'Adriatico.

Nuovi invece negli annali d'Italia erano l'abolizione di quel potere temporale dei Pontefici, che gl'Italiani s'erano abituati a considerare da secoli come una necessità, e lo scempio che i Francesi avevano fatto impunemente della persona del Papa, che pure gl'Italiani consideravano come una loro speciale istituzione. Le vicende toccate al Pontefice derono aver meravigliato anche il 
resto del mondo. Però siccome erano decretate da Napoleone, e le sue azioni non tolleravano critiche nè intrcmissioni, nessuno si mosse.

Certo è che gl'Italiani e gli stranieri devono essersi accorti che il potere temporale dei Papi avera terminato la parabola della sua missione storica c che la sua esistenza non era più una mecessità nella politica.

Grave ed irreparabile per l'Adriatico fu la caduta di Venezia.

La decadenza della Serenissima era palese da mo o due secoli; però senza la violenza di Napoleone, la sua ultima ora non sareble ancora suonata. Il senato di Tenezia nella sua debolezza avera cercato di salvare sè e lo Stato mantenendosi neutrale nella guerra fra Napoleone e l'Austria. Errore senile, che una rolta non sarebbe stato certamente commesso. Il Bonaparte invece aveva fatto già il suo piano di sacrificare la Serenissima, offendola come oggetto di compensazione alla corte di Viemna. Per ciò favorì dei moti rivoluzionari in terraferma alle sue spalle, oncle trovare un pretesto d'intervenire. I Veneziani invece, che dopo la concuista della Lombardia da parte dei Francesi, avevano preveduto bensi le loro intenzioni, ma non escogitato il mezzo per sventarle, caddero nel trancllo: e approfittando del linto imbarazzo di Napoleone vollero dislurbare quella spolendida marcia da Mantova a Leoben, in Stiria, con cui il Bonaparte, sfatando l'uso invalso da ben quindici secoli. che per ruella strada gli esereiti poterano solamente discendere in Italia, avera ristabilito le tradizioni gloriose dell'epoca di Mario e di Marc'Aurelio. Ma la vendetta di Napoleone non si fece attendere. Col pretesto di castigarla d'aver favorito l'insurrezione dei suoi popoli, le dichiarò la guerra.

Il senato, risto l'estremo pericolo, pensò di salvare almeno lo Stato, sacrificando sè stesso, e pose mano ald una troppo repentina modificazione costituzionale in senso democratico. Ma non era fuesto lo scopo cui tendera il Bonaparte, che non se ne teme pago, e il 16 misggio 1797 vi fece entrare tremila soldati francesi. Emano 
queste le prime milizie straniere che contaminavano la piazza di San Marco.

Napoleone la depredò delle sue navi, delle provvigioni di guerra e di un gran numero di oggetti d'arte, e la consegnò di poi nella pace di Campoformio (17 ottobre 1797) in braccio al suo più terribile nemico, all'Austria, insieme ai suoi più importanti possedimenti, l'Istria, la Dalmazia e Caltaro.

La sorte di Venezia era dolorosa per sè stessa e divemne ancora più triste per la sua vergognosa rassegnazione. Sebbene disponesse ancora di milizie, particolarmente dalmate, le Cernide, anelanti di difenderla, si lasciò sopraffare senza opporre resistenza. Dodicimila dalmati, fatti venire per proteggerla dall'invasione francese e ch'erano decisi d'impedire che la Francia infliggesse l'estremo oltraggio alla repubblica, dal senato furono colla forza ma vilmente fatti imbarcare e trasportare altrove. Invano un conte Viscovich di Perasto condusse la sua nave coi suoi Bocchesi all'assalto e al massacro della fregata francese denominata per ironia Il liberatore d'Italia, che aveva sforzato il porto interno di Venezia. L'antica dominante decrepita cadde ingloriosamente, ma superbamente, come un aristocratico che preferisca essere calpestato e sopraffatto, piutlosto che degradarsi a scambiare dei pugni coll'aggressore plebeo.

Ciò la rese ludibrio dei vincitori, che a detta del Cantü utentarano, come trafficanti, di usurparle fin l'ultimo diritto alla sventura, la pietà, diffamandola a guisa del giovinastro, che espone alle risate la donna ch'egli contaminò».

Malgrado i suoi difetti e il regime antiquato, rigidamente oligarchico, il governo di Venezia non era nè cattivo, nè immorale da meritare quella sorte e quella riputazione, che Napoleone e certi scriltori francesi sparsero maliziosamente nel mondo e nella letteratura sentimentale dell'epoca. Che l'amministrazione della Serenissima abbia avuto ancora un lato buono, è provato dal dolore spontaneo e sincero con cui fu appresa la notizia della sua caduta nelle città istriane e dalmate e dalle lagrime ir- 
resistibili con cui le popolazioni della costa orientale adriatica accompagnarono la consegna del vessillo di San Marco agli Austriaci. A Zara, portatolo in Duomo, dopo intonato il De profundis, fu baciato con entusiasmo dai cittadini addolorati e sepolto. A Perasto, la piccola ciltadina delle Bocche di Cattaro, gli celebrarono le ultime esequie nella caltedrale e poi lo deposero sotto l'altar maggiore come reliquia nazionale.

Venezia ch'era vissuta tredici secoli (423-1797) con pochissime sommosse e neppur una guerra civile aveva beneficato la costa orientale adriatica, per la seconda volta nella storia dopo di Roma, con una lunga pace durata quattro sccoli (dal 1409 in poi). Senza della Serenissima anche l'ostacolo naturale della Montagna Nera e il valore dei suoi montanari sarebbero stati insufficienti a frenare l'irruzione dei Turchi e la Dalmazia certamente e l'Istria fors'anche, seguendo la sorte dell'Allania, dell'Erzegovina e della Bosnia, invece di un proveditore europeo avrebbero subito un pascià asiatico.

Prima, dalla caduta della romanitì, la costa orientale adriatica era stata traragliata da contimue guerre e soperchierie. Ora erano le ciltì dalmate che si dilaniavano tra loro; ora erano gli stranicri (ungheresi) che dispotizzavano a loro bell'agio. Venezia vi portò quella pace, che da lei potreble dirsi pace veneziana, ehe se da un lato fu benefica, perchè tolse di mezzo ogni molivo di contesa, dall'altro fu purtroppo fatale, perchè infiacchì gli animi. - Nella Dalmazia, nazionalmente mista, ove il dominio fu pienamente effettivo, il governo vencto si appoggiava all'clemento latino, non tanto forse per un preconcetto etnico, chè l'idea nazionale in quell'epoca non era aneora formulata, ma per lue ragioni pratiche, una d'affinita naturale tra la propria composizione sociale e quella dell'elemento romanico delle citti della costa e l'altra della superioriti civile di fuest'elemento e della sua esclusiva capacita a compartecipare al governo detla cosa pubblica. Però a Venezia mai renne in mente di opprimere le popolazioni slave dell'interno, anzi diede loro appoggio morale e ricelto, più volte perfino a damno del- 
l'elemento latino, e se ne servì nella lolta contro il 'Turco, comune nemico.

Sia però che si tratti di dominio effettivo o titolare il governo di Venezia per il suo rispetto alle istituzioni locali, per la tutela della religione, per la mitezza dei balzelli e sopralutto per aver condotto la sicurezza e la pace, ful amato e lasciò un affettuoso clesiderio in tutti quelli ch'erano compianti come suoi servi.

Allo sfasciamento del governo della Serenissima si ripetè sull'Adriatico lo spettacolo offerto dalla latinità di Roma tredici secoli addietro. Quella costa orientale adriatica, clle in principio s'cra opposta qua c lì all'occupazione veneziana, dopo che conobbe i vantaggi materiali e inorali del suo governo, l'amò d'intenso e sincero afietto e quando cadde perchè in sostanza i tempi esigevano un'evoluzione, fu l'ultima ad abbassare il glorioso ressillo e continuò anche sotto nuovi dominatori a lottare per conservare l'idioma e le istituzioni ricerute da Venezia.

Politicamente il giorno della caduta di Venezia (16 maggio 1797) segna per l'Adriatico una data fatale. Finora i'Adriatico non avera conosciuto che clominatori marittimi, o indigeni come i Romani ed i Veneziani, o affini come i Greci ed i Bizantini. Col passaggio di Venezia, delI'Istria, della Dalmazia, di Ragusa, di Cattaro agli Asburgo, in aggiunta agli sbocchi di Trieste e di Fiume, che già si trovarano nelle loro mani, la parte più importante dell'Adriatico fu spostata di botto verso l'Europa centrale e si trovò per la prima volta nella storia alle dipendenze di un potente Stato continentale.

Il Regno Ben comprese l'errore Napoleone stesso, che tentò di ripararvi colla creazione del regno d'Illiria. L'idea non era sbagliala per sè stessa, giacchè Napolcone, divinando la gravità degli attriti nazionali, voleva opporre questa barriera alla discesa dell'elemento germanico. Comprendendo che soltanto la coltura italiana potera tener viva questa sua nuova creazione, Napoleone vi anneltè anche l'Istria e il V'encto. Questa necessitì momentanca tolse valore all'istituzione, che soffriva già di un difetto originario di precipitazione e di prematurità. Le province 
illiriche non solo mancavano di quell'omogencità nazionale, che doveva essere l'essenza delle riorganizzazioni politiche, ma ledevano il concetto geografico e nazionale di un'Italia in formazione, ch'egli stesso vagheggiava. Per ciò la creazione mostruosa scomparve colla stessa facilità, con cui cra stata prodotta.

Il governo napoleonico sulla costa orientale dell'Adriatico va però notato per rilevanti vantaggi economici e morali. Ovunque fu favorita l'istruzione pubblica, italiana beninteso, e furono tolte le pastoie clerico-scolastiche alla coltura. Il paese fu amministrato bene e furono promossi i lavori stradali. Il merito di tutto ciò, particolarmente in quanto riguarda la Dalmazia, appartiene at maresciallo Marmont e al proveditore Vincenzo Dandolo.

L'altività commerciale e navigatrice di Ragusa continuc̀ La caduta di malgrado la decadenza generale dell'Adrialico e quella Ragusa. interna della piccola repubblica fino al principio del secolo XIX, ma dirella specialmente al Mediterraneo e all'Allantico.

I rovesci politici che avevano travolto Venezia erano stati causa di una scossa fatale anche per la altrettanto vecchia compagine di Ragusa, che infatli non le sopravvisse che di due lustri. Malgrado i due cuscinetli di territorio turco a Klek e Sutorina, Ragusa cra incuneata tra i possedimenti veneli di Dalmazia e d'Albania (Bocche di Cattaro e costa del Montenegro fino Antivari). La piccola repubblica si trovì ora esposta alle brame insidiose dei due principali liquidalori della Serenissima, gli Austriaci ed i Francesi, nonchè alle molestic dei Montenegrini e dei Russi, nemici dei Francesi. Erano tempi troppo turbotenli e i governi capricciosi, perchè i Ragusei polessero negoziare con efficacia la loro neutralità.

Il senato di Ragusa tentò ad imitazione di quello di Venezia di non immischiarsi nelle laccende politiclse che turbinavano nell'Adriatico, ma la neutralití imbelle nom giovò nemmeno ad esso.

Nel 1797 milizic austriache oceuparono la Dalmazia e le Bocche di Cattaro, il cui territorio era slato invaso 
anche dai Montenegrini, speranti di poter giungere finalmente allo sbocco del mare. Il nuovo governo austriaco ne approfittò subito per rinchiudere nelle fortezze di Cattaro quei cosidetti giacobini lombardi, patriotti della repubblica cisalpina, che sono ricordati nella Bassvilliana del Monti.

Nel 1805 il generale francese Lauriston (più tardi governalore di Venezia) incaricato di occupare Venezia e la Dalmazia, col pretesto di moti interni tra nobili e democratici, ruppe la neutralità di Ragusa, la occupò militarmente e vi si sostenne contro forze preponderanti dei Russi e dei Montenegrini.

L'anno seguente in seguito alla pace di Presburgo il generale francese Nolitor, mandato in Dalmazia come governatore, occupò nuovamente la città ed i forti. Ragusa, assediata dai Montenegrini per terra e bloccata per mare dalla squadra russa dell'ammiraglio Sinjavin, che avera occupato anche le Bocche di Cattaro, patì nuovi guasti.

Battuti i Montenegrini e costretti i Russi a levare il blocce di Ragusa, Molitor dopo di aver occupato Cattaro, per porre un argine all'influenza che la Russia andava acquistando fra le popolazioni slave, soppresse anche Kagusa. Un decreto napoleonico abolì nel 31 gennaio 1808 definitivamente la repubblica, che da allora in poi segù le sorti della Dalmazia. Ragusa, secondo i progetti di Napolcone, dovera diventare la sua base d'operazione per il Mediterraneo orientale. Ma la realizzazione non andò più in là della nomina del maresciallo Marmont a duca di Ragusa.

La battaglia di Lissa del 1811.
In omaggio alla cronologia storica e per non intralciare altrove la narrazione logica con un fatto staccato, ricorderemo in questo punto la memorabile battaglia franco-italiana ed inglese nelle acque di Lissa del 1811.

I Francesi per conservare la loro posizione dominante nel regno italico (costa occidentale adriatica) e nelle pro- 
vince illiriche (costa orientale) averano bisogno del pieno possesso del mare Adriatico. - Gl'Inglesi che li combattevano appunto per mare s'erano annidati a Lissa, donde inceppavano non solo i movimenti della flotta da guerra, ma anche delle navi mercantili di Venezia e di Trieste. Si è per ciò che in quest'epoca i Triestini cercarono una via commerciale terrestre fino in Turchia ed a Salonicco.

In quell'epoca una marina italiana era appena in formazione e quella francese era disseminata in tulti i mari. Nemmeno le forze navali inglesi erano considerevoli, anzi numericamente erano inferiori, ma erano comandate dal valente ammiraglio Hoste, che appunto a Lissa si coperse di gloria e che di poi aggiunse nuove foglie alla corona di lauro negli assedi di Cattaro e di Ragusa. La vittoria arrise agli Inglesi; dunque anche questa volta ebbe esito sfavorevole al possessore della costa. Essi di conseguenza poterono estendere la loro occupazione ad un gruppo maggiore di isole dalmate (tra cui Curzola) nonchè alle Bocche di Cattaro (1813) meno la città, che fu tenuta dai Montenegrini.

Quella giornata di Lissa segnò la fine della signoria francese nell'Adriatico. Al rapido tramonto dell'astro napoleonico tenne dietro la scomparsa del dominio francese anche delle coste.

II 1814 segna l'entrata delle armi austriache in Dalmazia, a Ragusa e nelle Bocche di Caltaro, che d'ora in poi formarono un unico complesso amministrativo, meno le tre isole di Veglia, Cherso ed Ossero, che furono staccate dalla giurisdizione della Dalmazia e aggregate all'Istria.

Passate le vicende della rivoluzione francese e del Bonaparte l'Austria per azione indirella riuscì ad eliminare le influenze indigene di Venezia e di Ragusa, e l'intrusione esterna dei Russi, degli Inglesi, a relegare nuova- 
mente in montagna i Montenegrini e s'insediò padrona sulla parte migliore della costa e del mare Adriatico.

Non fu accolta con simpatia in nessuna parte e meno che altrove a Ragusa. I nobili ragusei, per non dare il loro sangue agli usurpatori, fecero il proponimento di non maritarsi e di lasciar piuttosto estinguere i loro blasoni secolari; e mantennero la parola.

Il Montenegro.

E venuto il momento di aprire un capitolo apposito pel Montenegro, onde ricordare alcune cose che si riferiscono unicamente a questo paese.

L'esistenza politica del Montenegro è la conseguenza del fenomeno geologico-geogralico, già più volte accennato. Quei monti aridi, a quanto sembra disabitati fino a tempi relativamente recenti e per di più inaccessibili, non attrassero le brame nè dei Veneziani, nè dei Turchi, che non ci trovarono un tornaconto ad incontrare sacrifizi sproporzionati per averli e stimarono più opportuno di limitarsi al possesso, gli uni della costa e delle Bocche di Cattaro, gli altri delle pianure ad oriente ed a settentrione. Così quest' altipiano montuoso rimase semilibero in mano dei pastori introdottisi dai vicini territori balcanici. Quando poi i Turchi ebbero ridotto in loro potere effettivo tutta la penisola balcanica, sentirono in parte lo stimolo dell'ambizione e in parte il bisogno di piantare la loro bandiera anche sulla Montagna Nera; ma malgrado ripetute poderose spedizioni militari non riuscirono a tenerlo soggetto militarmente che nell'ultimo decennio del secolo XVII. Nella seconda metà del XVIII i Montenegrini riuscirono a liberarsi anche dal pagamento del tributo al sultano.

Di una vita civile sull'altipiano del Montenegro nelle condizioni geografiche e storiche del secolo XVIII non è il caso di parlare. Ma anche per non perire politicamente c'era bisogno di molta accortezza e di molto eroismo. Un continuo stato di guerra coi Turchi, più che dannoso, era fino ad un certo punto giovevole e consono alla natura forzatamente adottata dalla popolazione. Economicamente non si poteva far a meno di appoggiarsi alla più evoluta vicina del mare, Venezia, e per ciò i signori 
del Montenegro nei secoli passati avevano accettato stipendio e l'alto dominio della Serenissimn. Però per tenersi in bilico tra una superiorita militare ed una economica faceva di bisogno una protezione ancora maggiore e i Montenegrini per simpatia religiosa e nazionale la cercarono nella Russia, colla quale s'erano strelli in un legame di clientela politica fino dai tempi di Pietro il Grande.

Caduta Venczia i Montenegrini sperarono, come fu accennato, di allargarsi verso il mare. Ma si trovarono di fronte i Francesi. Il maresciallo di Napoleone, Marmont, non riuscì nè a vincerli colle cattive e nemmeno ad avvincerseli colle buone, perchè i Montenegrini rimasero, nel loro evidente interesse, fedeli alla Russia.

Venuta l'Austria a succederc definitivamente a Venezia, i Montenegrini si trovarono ancora a peggior partito che nel secolo precedente, perchè l'Austria, oltre a possedere anche Ragusa, era una potenza terrestre e avrebbe potuto, come infatti avvenne, stringere il Montenegro anche dalla parte di terra. Intanto l'Austria non trascurò, come vedemmo, di ricacciare subito i Montenegrini lontano dal mare e di rintuzzare anche in seguito ogni loro tentativo di raggiungere uno scalo al mare. Appunto con questa mira l'Austria si fece cedere dal Montenegro verso un compenso in danaro nel 1841 il territorio di Pastrovicchio, un piccolissimo tratto di costa in continuazione ad oriente di Budua.

\section{IL CONGRESSO DI VIINNA.}

Il congresso di Vienna (1814-15) sancì da solo la restaurazione degli antichi monarchi, senza che i popoli (e specialmente gl'Italiani, che furono i maggiomente colpiti) potessero far sentire la loro voce.

L'Austria oltre alle province illiriche (in cui c'erano per lei i movi acequisti dell'Istria veneta, della Dalmazia, di Ragusa e delle Bocche di Cattaro) ebbe la Lombardia, il Vencto col Friuli, quale compenso per i Paesi Bassi 
perduti nel 1797, il Tirolo (Trentino), ecc. Nel granducato di Toscana e nel ducato di Modena ritornarono membri della famiglia d'Austria. L'imperatrice Maria Luigia ottenne Parma, Piacenza e Guastalla. Il Papa ritornò sul trono pontificio; il re di Sardegna ricbbe i suoi possedimenti in Italia ed ottemue anche Genova. Solamente a Napoli rimase per alquanto tempo Murat. Per l'ulteriore storia locale dell'Adriatico ricorderemo due episodi della fuga di Murat: Ia sua sconfilta a Tolentino $(3 \mathrm{mag}-$ gio 1815) e il bombardamento di Pescara da parte degli Austriaci.

Dopo il fallimento della tentata riscossa di Napolcone durante i cento giorni dal ritorno dall'Elba c la fuga di Murat da Napoli, che ritornò sotto il dominio dell'antica dinastia borbonica spagnola, i monarchi sopravvissuti alla bufera napolconica, credettero di aver seppellito colla Santa Allcanza anche in Italia e nel bacino dell'Adriatico lo spirito rivoluzionario. Ma s'ingannarono.

\section{L'A USTRIA.}

La restaurazione della Santa Alleanza condusse sotto le ali dell'Austria non solo le province illiriche, ma anche la Lombardia. Più che Venezia e la sua credità, l'Austria aveva acquistato la porzione migliore della Padania e dell'Adrialico, per valore storico, civile, geografico e marillimo, ed era divenuta la prima potenza su questo mare.

L'Austria comprese molto bene il nuovo orientamento politico, c s'avvide che l'Adriatico rappresentava ora la sua nuova base e un nuovo grande polmone, e ne approfittò per sanare le ferite che le erano state inflitte da altre parti.

Le guerre napolconiche portarono non pochi cambiamenti allo stato territoriale dell'Austria. Prescindendo da quelli in Italia c sull'Adriatico, la lega colla Russia le fruttò nella terza spartizione della Polonia (1795) la Ga- 
lizia occidentale e Cracovia, ma nel 1797 perdette per sempre i Paesi Bassi. Quando poi Napoleone nel 1804 si proclamò imperatore dei Francesi e nel 1805 s’incoronò a Milano come re d'Italia, Francesco II assunse il titolo d'imperatore ereditario dell'Austria in seguito alla legge prammatica dell'11 agosto 1804 ed elevò con ciò i paesi da lui governati, e che in forza alla sanzione prammatica erano uniti inseparabilmente, ma senza titolo comune, ad impero creditario.

E cvidente l'importanza di questi fatti. L'Austria che finora aveva avuto la forma di uno Stato sopralutto dinastico, avendo perduto ormai i paesi staceati, che rappresentavano un possesso personale della corona, si restrinse nell'estensione territoriale, ma acquistò nome, forma e consistenza. Restara però alla famiglia degli Asburgo-Lorena ancora un titolo fuori dei conlini austriaci ed era quello d'imperatori tedeschi. Napoleone prese di mira anche questo e glielo tolse.

La lotta che il genio superbo del Bonaparle sostenne contro tutta l'Europa cbbe come perno la continuazione del ducllo secolare tra i due grandi imperi dei Franchi occidentali (gallici batinizzati) e dei Franchi orientali (germanici), rappresentati in quest'ultimo stadio dalla grandezza degli Asburgo. Per abbatterla Napoleone si giovò delle gelosie degli stessi altri principi tedeschi, desiderosi di scuotere la supremazia austriaca e promosse la formazione della conlederazione renana (1806, sollo il suo proteltorato. Ciò inclusse Francesco II (come imperatore d'Austria Francesco I) a rinumziare alla dignità d'imperatore tedesco-romano, altro falto importantissimo, se si ricorda che gli Aslumgo la coprivano, meno un'interruzione insignilicante, dal 1438.

Il congresso di Vienna offrì il destro all'Austria di ingerirsi nuovamente negli affari di Germania. Quando dopo lunglue sedute fu risollo che invece dell'antico impero tedeseo dovesse sorgere la Conlederazione Germanica, l'Austria entrò pure a farne parte, però solo colle sue province tedesche. E interessante poi di notare che lira rueste pretese province terlesche furono comprese abu- 
sivamente e senza chiedere il parere degl'interessati, anche il cosidetto litorale austriaco (Trieste, Gorizia, l'Istria settentrionale) nonchè il Trentino, quale parte del Tirolo, tanto che anch'essi dovettero mandare i loro delegati alla dieta federale di Francoforte, ch'era presieduta dagli ambasciatori d'Austria. Uno dei patti della confederazione ch'ebbe, e non ha perduto ancora valore anche per l'Adriatico, è questo: che i confederati si obbligarono di assicurare e difenderne l'integrità.

Grandi e gravi erano state le umiliazioni subite dall'Austria per parte di Napoleone, a placare la cui smodata ambizione non valse nemmeno il sacrifizio della figlia di Francesco I, Maria Luigia, che per opera di Metternich dovette divenire sua seconda moglie.

Il rapido tramonto dell'astro napolconico, le violenze dei Francesi, apparse maggiori per l'immaturità dei popoli a comprendere il lato buono della loro rivoluzione, permisero all'Austria non solo di riaversi delle sconfitte patile, ma di riprendere per qualche tempo la posizione più notevole fra le grandi potenze.

La Santa Al- Le gravi vicende toccate a tanti principi e a tanti poleanza. poli pei l'ambizione di un sol nomo, non rimasero senza inlluenza sul sentimento religioso, ancora abbastanza vivo, di coloro che sopravvissero alla fine di quella grande catastrofe. Per ciò l'Austria era riuscita a stringere d'accordo coi monarchi di Russia e di Prussia la cosidetta Santa Alleanza, a partecipare alla quale invitarono tutti i principi cristiani d'Europa. Però questa lega non ebbe nè la durata voluta dai principi, nè i frutti sperati dai popoli per la contrarictà d'interessi dei singoli Stati e per l'opposizione dei governi ai desideri di riforme politiche ed amministrative, manifestati dai popoli.

Limitandoci a dire dei paesi dell'Adriatico occorre rilevare che le dinastic rimesse sui troni crano state accolte dappertutto con malfidenza e antipatia. Per ciò non scntendosi sicure cercarono tutte un appoggio nell'Austria, che in tal maniera ridivenne la potenza dominante anche sull'Italia. Dall'Austria furono tese le fila di una vasta sorveglianza poliziesca e di quel sistema di spionaggio, 
applicato a lutta la penisola, che ancora non è scomparso dalla memoria degl'Italiani.

Scffocate momentaneamente le tendenze troppo rivoluzionarie, il desiderio di libertà dei popoli prese necessariamente forme più modeste e si contentò di domandare riforme costiluzionali. Il congresso di Vienna anzi volendo mostrare la gratitudine clei principi per i sacrilizi falli con tanto entusiasmo dal popolo tedesco nella lotta contro Napolsone, aveva promesso il ristabilimento delle costiluzioni provinciali in tulli i paesi della Conlederazione Germanica. Questa promessa non lu osservala invece proprio dalla maggiore delle polemze ledesche, dall'Austria.

Dope secoli di vicende e di lotle per ampliare la propria potenza ed ingrandire i lerritori la rasa d'Asburgo era riuscila a mettere insieme uno stato pur sempre bue rocratico, mancinte di consistenza geografica, storica, economica e nazionale, tenuto su amministrativamente, e nel quale solo l'inlluenza personale dinastica, ossia l'assolutismo monarchico, poleval agire come cemento per tenerlo unilo. Questo Stato arava subito scossc violenti per lotte religiose, politiche, sociali ed economiche, ma aveva potuto superarle perchè religione, politica, anti-leudalismo, industrialismo erano applicabili senzat distinzione a tulli i popoli temuti insieme dalla volonlat dinaslica. Anzi la previdenź e la sagacia politica averano suggerito ad alcuni regnanti (Massimpliano I, Ferdinando I, Calrlo VI, Maria Teresa, Cinseppe II) l'espediente di larsi modernizzatori e di meltersi a capo delle riforme per dominare la situazione delle nuove esigenze piuttosto ch" esserne colti alla sprovvista c forse travolti. L'Auslria aveva mostrato quindi di poter sopportare molle burrasehe. Però il buon senso insegnava ch'essa difficilmentc sarebbe stata in grado di affrontarne una: quella della concessione di libertà individuali costituzionali, dictro alle quali facevano capolino liberti colletlive nazionali in antagonismo col concetto di sovranita dinastirát degli Asburgo. Era evidente che per le nuove tendenze politico-sociali

\section{L'Adriatico.}


o l'Austria sarebbe riuscita a soffocare ogni moto popolare, oppure le rivendicazioni nazionali avrebbero finito col produre il suo sgretolamento.

L'Austria si trovò quindi costretta fatalmente a schierarsi contro ogni concessione di libertà costituzionali. E come il progresso tumultuoso della rivoluzione francese aveva favorito la creazione del suo campione nel genio militare ed amministrativo del Bonaparte, la reazione Metternich. austriaca fu imperniata e personificala dalla mente gretta del prineipe di Metternich.

Il conte (più lardi principe) Clemente - ironia dei nomi simbolici - di Metlernich, che escreitò un'influenza politica assai grande per mezzo secolo, era entrato ai servizi dell'Austria nei primi anni del secolo XIX. Nominato nel 1809 ministro di Stato, diresse gli affari esteri e condusse a line nell'anno decisivo 1813 l'alleanza dell'Austria cogli avversari di Napolcone. Ebbe parte imporlantissima nol congresso di Vienna e si deve ascrivere a sua opera anche la slipulazione della Santa Alleanza. Ebbe dei successi nella diplomazia e molti meriti nella politica estera dell'Austria, però essendo di poca levatura nell'amministrazione interna dello Stato fu la causa principale del malcontento dei popoli. Fu lui ad imprimere all'Austria quel carallere spiccalo di arversaria di ogni movimento proveniente dal popolo e di più grande baluarlo della monarchia legiltima.

Nel primo fiorire della Santa Alleanza Metternich potè reprimere i moli nazionali colla forza della polizia e dello baionette. Incominciarono quei moli in Germania, ove per la delusione delle promesse costituzionali si manifestò, specialmente nelle università, un forte malumore. Melternich naturalmente fu pronto a convocare un'adunanza di ministri e di uomini di Stato per trattare sui provvedimenti da prendersi in questo riguardo. Fu soppressa la libertì di stampa, istituita una commissione centrale d'inchiesta per le cosidette mene demagogiche, fu ordinata una più rigorosa sorveglianza delle università e furono sciolte le societì accademiche e gl'istituti di ginnastica. 
Il movimento fu segnito subito dagl'taliani. Cardulo L'Italıa at. Napoleone il romanticismo nella lelteratura el i Carbo-torno al 1820. nari nella politica cercarono di promuovere da una parte la coltura del popolo e dall'allra di prepararlo allidea delle libertà costituzionali e dell'unità d'Italia. Erano questi i primi passi da farsi necessariamente sulla via della redenzione, e la direzione fu presa dalle classi colte.

Mancando l'appoggio di una vasla opinione pubblica i patriotti italiani procedevano a tentoni, talvolta con alli sbagliati, ora in una parte d'Italia ed ora nell'altria, approfittando di tulte te cause di malcontento per sobillare e far propaganda contro le dominazioni stranicre. Nell'alta Italia le popolazioni erano irritate a cansa delle irragionevoli el esagerate persecuzioni poliziesche dell'Austria, nella media contro la reazione religiosa e nella bassa per il malgoveruo amministrativo che aveva fatlo nascere banditi da tulte le parti. In quest'epoca il centro delle agitazioni stava appunto vicino all'Adriatico nelle Romagne, a Parma, a Modena, ove il carbonarismo aveva messo le più salde radici. E anzi interessante di far conoscere la bizzarea iclea dei più avanzali lra $\mathrm{i}$ Carbonari, di sostiluire ai governi stranieri la repubblica ausonica. Questal doveva essere formalat dal continente italiano e dalle isole e coste dell'Adriatico, appartencente alla repubblica veneta, fino alle Bocehe di Callaro.

Malgridlo l'insistente sorveglianza della polizia scoptpiarono insurezioni nel 1820, come riverbero della rivoluzione spagnolat, a Napoli ed a Torino per le (fuali il re delle Dne Sicilie e quello di Sardegna furono costrelli a concedere la costituzione. Nella Lombardia scoppiarono i moti insurergionali del '21.

Lat rivoluzione di Napoli, capitanala dal gencrale Prpe, ebbe a teatro particolarmente il versante lirrenico dengli Apennini, sebbene l'insurrezione si losse estesal anche alle Puglie e nel Molise, e venne repressa senza speriali conseguenze per la sloria dell'Adriatico. P'er ciò a pere hrevila ei liniteremo a dire gualde dellaglio solamente dei falti del Piemonte, che si mostrarono più importanti anche per le loro conseguenze. 
La reazione aveva ristabilito anche nel regno di Sardegna (Savoia) l'inquisizione, la disparià giuridica del popolo e l'antica gimrisdizione. Ma le classi colte della popolazione avevano qui ricevuto e conservalo dalla rivoluzione francese delle idee liberali, che s'insinuarono nella nobiltà e nei militari. Per ciò il fermento diveme generalc e i Carbonari poterono inscenare quella conginra e rivoluzione militare che provocò l'abdicazione del re. Fu creala una Giunta che agì in nome del "regno d'Italia» e il principe Carlo Alberto proclamò col tricolore alla mano di acceltare lo statuto costituzionale.

Ma Melternich ebbe ancora facile viltoria. Dopo i congressi di Lubiana (1821) in cui fu deciso l'intervento austriaco nel Napoletano e di Verona (1822) truppe austriache ristabilirono ovunque il potere assoluto che non lu più lurbato fino alla fine del decennio. Era questa l'epoca del Conciliatore, del Pellico e dello Spielberg.

Frallanto in Germania si preparava un nuovo scacco per l'Austria. La formazione della lega doganale (Zollverein) avvinse sempre più gl'interessi economici dei piccoli Slati tedeschi alla Prussia, mentre l'Austria divenne sempre più estranea non solo allo sviluppo maleriale, ma anche al progresso scientifico della Germania.

\section{NUOVE IILEE POLITICHE, SOCIALI E NAZIONALI.}

Quando nell'assemblea nazionale di Parigi (1789) i rappresentanti borghesi (terzo stato) proclamarono soprat tulto «i diritti degli uomini» non tulli i contemporanei avranno compreso subito la portata pratica di quesla cnunciazione. Essa fu il germe di quella poderosa e mai prima veduta rivoluzione d'idee per la quale gradatancule in Francia e negli altri paesi e popoli all'abolizione dei privilegi della nobiltà e del clero tenne dietro il sentimento di sempre maggiori liberlà e della conseguente partecipazione di tutti al governo.

Questo sentimento che prese nella pratica il concetto e la forma di democrazia portò i popoli alla coscienza 
che non i principi nè l'aristocrazia soli averano da comandare, ma che il popolo era il rero sovrano di sè stesso.

Dal concetto di una libertà individuale non poleva andar disgiunto quello di una liberta collelliva. La ricerca dell'ente più piccolo capace di esprimere e di lar valere queste libertà condusse alla proclamazione del principio di nazionalità.

Questo cosidetto principio si dimostrò, è vero, più lardi conluso nella teoria e non sempre applicabile nella pratlica. Il sentimento però che gli diede origine fu considerato per tutto il secolo XIX come la scoperta dell'anello prima mancante nella scala evoluliva della libertà dall'individuo attraverso la lamiglia, nella patria, verso l'umanità. Esso servì di piattaforma per i postulati di libertà popolarc.

La coscienza della libertà individuale spinse i popoli a lottare per abolire la schiavitù polilica e per ollenere le costituzioni: quella della liberla colletlira a loltare per formar Stati nazionali; l'una e l'altra insieme, ossia guella della sovranità del popolo, ad abolire le monarcinie ed a crcarc repubbliche.

La coscienza civile delle popolazioni delle due coste dell'Adriatico era ancora troppo irrigidita, apalica, imbevula di preconcelli feudali aristocratici per comprendere ed abbracciare spontaneamenle queste move idee. Esse vi furono per ciò importate in gram parte per lorza. Venczia che avera dominato negli ullimi secoli sull'Adriatico colla politica e coi commerci vi avera consolidato la sua distinzione marcata la nobilta e plebe, lat prima delle quali aveva in mano lutta la cosa pubblica e la seconda non poteval lag allo che obbedire e diverLirsi. D'un nazionalismo, nel senso moderno, non c'era ncmmeno l'ombra; anzi la politica esclusivamonte cennomica di Venezia, lrascurò e perlino damneggiò la quistionc nazionale.

Dalle ricerche, per quanto ancola imperpelte, falle dis storici e studiosi, si sa che le radici dell'idea matronale italiana, che fu la prima a lormarsi nei paesi dell'Adria- 
tico, sono letterarie. Gli albori del nazionalismo italiano datano dal principio del XIX secolo ed ebbero origine in parte per imitazione, in parte per reazione delle idec venute di Francia. Le prime opere ispirate o imbevute di sentimento nazionale sono letterarie: il Misogallo dell'Alfieri, le Ultime leltere di Jacopo Ortis del Foscolo, Il primalo d'Italia del Gioberti. Dalla letteratura l'idea nazionale passò nella vita privala e nella politica.

I Francesi, e specialmente Napoleone, fecero e dislecero sull'Adriatico a capriccio e con violenza senza punto curarsi della volontà del popolo o d'interessi nazionali. Napoleone anzi avrebbe volentieri gallicizzalo; ma dovette suo malgrado inchinarsi alla grandezza intellettuale degli Italiani; e nel mentre proclama il toscano lingua ufficiale accanto al francese nei paesi ad occidente dell'Adriatico, in quelli della costa orientale favorì energicamente la lingua e la coltura italiana a Trieste, in Istria, in Dalmazia (aveva progetlato di istituire a Zara un'università degli studi).

Malgrado le violenze la dominazione francese fu benelica, perchè spazzò via abusi e pregiudizi medievali. I popoli dell'Adriatico a contatto dei Francesi e delle altre nazioni, cui si avvicinarono per le vicende guerresche di Napoleone, appresero l'uso delle armi, purtroppo dimenlicato; si abiluarono a muoversi in un ambilo sociale ed economico più libero, e se anche le speranze che alcuni patriolli avevano riposto nell'aiuto di Napoleone per ristabilire l'antica milà nazionale andarono disillase, lu una cosa benelica anche il risveglio dopo un lelargo secolare.

Gli studi lelterari, che avevano servito almeno a tener deste le menti degl'Italiani, giovarono ora a lar conoscere lo splendore del passalo e furono lo sprone che colla rampogna di un amaro confronto eccitò gli animi ad osare la rigenerazione della patria. - Gl'Italiani intuirono il benelizic della rivoluzione franeese e della differenza tra il loro governo e quello austriaco. Dopo hulto dovendo per l'una ragione o per l'altra far sacrilizio clella vili, gl'Italiani preferirono morire comballendo sotto le glo- 
riose bandiere di Francia, che marcire nei sotterranei delle prigioni e fortezze austriache. Così si spiega perchè essi presero poea parle alle guerre l'Europa per liberarsi dal violento dominio di Napoleone. Al contrario gl'Italiani (e fra loro c'erano moltissimi adriatici dell'una e dell'alta costa) resero segnalali servizi a $\mathrm{Na}$ poleone nel 1812 nella campagna di Russia e net 1813 contro la confederazione europea, astenendosi anche in palria loro da moti rivoluzionari antilrancesi.

Le idee di Francia cambiarono faceia a quasi lutla l'Europa. Ma nel mentre altrove e nella Francia stessa dalla rivoluzione si svilupparono o idee sociali, oppure tendenze mazionali, per lo piò staccate, l'ra gl'ttaliani gli sforzi per l'emancipazione sociale furono condotli di pari passo e in perfelta unione coi tentalivi di liberazione politica, tanlo che oggi sarebhe diflicile di sceverare e stabilire quanta parte abbia avuto nella rigencrazione dell'Italia, e di riverbero di tulli i popoli dell'Adriatico, l'idea economica e cuanta l'idea nazionale. Così l'opera rivoluzionaria nel volger d'un (fuarto di secolo raggiunse nelle due coste dell'Adriatico una meta, (he pochi in principio avianno intravedula.

\section{AVVENIMENTI NELIA MET' BALCANICA DELL ADRIATIC().}

Le idee innovatrici, o se vogliamo pure rivoluzionarie, ora accennate serpeggiarono in tulte le regioni dell Adriatico, dell'una e dell'altra costa; ma dalla pate orientale non allecehirono direllamente che nei luoghi freondali dalla collura ilaliana. Ora, siccome quest'influchzal s'elal fermala alla costa, al di là di questa linea regnava ancora il mondo orientale.

Le idee rivoluzionarie francesi non entrarono mat nelle teste dei Turchi. Nemmeno i popoli balcanici ("rano maturi per comprenderle; però questi, oppressi dal lul giogo inumano, sentivano il bisogno di rischiare fualumgune 
cosa per scuoterlo. E quindi della massa d'idee liberali, seminate dai Francesi, essi non si assimilarono per or:a che quelle riguardanti la libertà individuale, religiosa e indirettamente solo nazionale.

La Turchia dopo le aggressioni russe del secolo XVIII si trovò al principio del XIX nella necessità di riordinare il suo stato interno, di dover sanare le finanze, ristabilire la disciplina e l'autorità del governo e lottare contro i tentativi d'insubordinazione di diversi Pascià e dei popoli soggiogati. - A riverbero debole, ma sincrono di quanto accadeva alle coste dell'Adriatico e in Italia, anche la penisola balcanica nei primi tre decenni del secolo XIX fu teatro di lotte per l'indipendenza.

11 Montenegro è l'unico territorio che i Turchi per difficoltà geografiche non erano riusciti a soltomettere del lutto. I Montenegrini, eccitati dai manifesti di Giuseppe II e di Caterina II nelle guerre contro i Turchi, approfittarono di tutte le guerre della Russia contro i Francesi e contro i Turchi per rompere l'anello politico che li soffocava. Vedemmo i loro sforzi, riusciti vani, per divenire padroni del mare che lambe i piedi delle loro montagne.

Ai Montenegrini tennero subito dietro i Serbi, che incominciarono un'insurezione nel 1804 terminata nel 1816 abbastanza felicemente col raggimgimento intanto dell'autonomia sotto $\mathrm{m}$ principe indigeno.

Contemporaneamente ai Serbi si sollevarono gli Albanesi, capitanati dal prode e generoso Ali di Tepeleni, Pascià di Giannina, che verso il 1820 potè dirsi indipendente di fatto nel regno epiroto da lui raccolto intorno allo splendore della sua reggia di Giannina. La sua azione non ebbe che una durata effimera, ma servì di aiuto ai Greci, che, insorti nel 1822, colle simpatie del mondo civile per i benefizi della loro coltura nel passato e coll'aiuto della Russia, dell'Inghilterra e della Francia, che a Navarino nel 1827 distrussero la flolta turca, potè

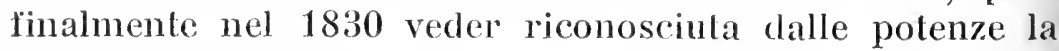
sua indipendenza.

Nel decennio 1820-1830 anche i Valacchi (Rumeni) 
della penisola balcanica scossero il giogo turco conquistando intanto l'autonomia. Sicchè c'è da concludere, che la riscossa dei popoli balcanici contro la Turchia aveva avuto maggior successo che quella degl'Italiani.

Un consimile periodo, ricco di guerre e di battaglie, terrestri e navali, non era certamente adatto a sollevare le sorti dell'Adriatico, che per prosperare ha avuto sempre hisogno di pace per i suoi commerci marittimi.

L'Austria, che come governo, aveva preso il posto di Venezia, era ancora depressa e disorientata. Eliminata, se non ancora del tutto espulsa, dalla Germania, andava perdendo il suo antico carattere, prevalentemente tedesco. S'era procurata un forte incremento d'Italiani; ma l'Italia non poteva esscre il perno della sua politica. Slava non voleva divenire e per ciò era perplessa e nella perplessitì faceva nell'Adriatico per forza e timidamente una politica italiana, lasciando sussistere, ove non le davano ancora ombra, usi, legislazione e statuti Iocali.

Ma ad aumentare la depiessione dell'Adriatico, alla causa politica s'era aggimnta un'altra di natura tecnica, la decadenza della marina a vela per la comparsa della macchina a vapore applicata alle navi.

Quel congegno ideato dit Watt nel 1784 e applicato da Fulton (1803) ad una barcaccia sulla Senna, che non aveva avuto la fortuna d'interessare la mente pur geniale di Napoleone, all'atto pratico aveva dato risultali stupefacenti; e i primi decenni del secolo XIX bastarono a farne comprendere ovunque l'immensa utilità riservalagli nell'avvenire.

La marina a vela dell'Adriatico in quest'epoca avrobbe avuto bisogno di un rimnovamento radicale. Finora per essere buoni marittimi occorreva prima un grande corredo di esperienza e di cognizioni nautiche e poi un gruzzolo di capitale in danaro. La navigazione a vapore invertì le parti; esigette forti capilali pecuniari e cognizioni tecniche e solo secondariamente l'esperienza nautica che, nel mentre una volta richiedeva anni ed anni di vita sul mare, ora potè venir acquistala collo studio

L'Adriatico.

Decadenza della marina a vela. - Origini della navirazione a vapore. 
in un periodo molto più breve. Le popolazioni litoranee dell'Adriatico, impoverite per la decadenza generale dei commerci, mancando dell'clemento capitale e non potendo procurarsi da solc facilmente le nozioni tecniche, dovettero lasciar inaridire scoraggiate il loro immenso tesoro di esperienza nautica, accumulata da secoli e ch'era divenuta una cosa superflua. Per ciò tutte le plaghe eminentemente nautiche (legli ultimi secoli, come la laguna di Venezia, le isole dall'Istria alla Dalmazia, Ragusa, le Bocche di Cattaro, i porti delle Marche e delle Puglie, intisichirono scmpre più.

La navigazione a vapore richiedente enormi capitali per l'acquisto dei battelli, per opere portuarie e per una migliore organizzazione commerciale, andò ad annidarsi nei porti maggiori, capaci di fornirle tutto ciò, oltrechè dall'intraprendenza privata collettiva più forte, anche dagli appoggi morali ed economici di un organismo solido, quale lo Stato.

L'Austria, che già nei secoli precedenti aveva mostrato di aver compreso il valore economico delle vie del mare, appena potè, rivolse l'attenzione all'Adriatico e, in aggiunta a quanto aveva fatto prima per i porti di Trieste e di Fiume, ora (1830) concesse il diritto di porto franco anche a Venezia, la decaduta regina dell'Adriatico.

Altre cure ancora stavano per essere rivolte dal governo austriaco al mare Adriatico. Ma il 1830 è un anno critico c ci costringe ad interrompere la narrazione dei fatti economici per dar posto a quella dei subbugli politici.

\section{LA RIVOLUZIONE DI LUGLIO DEL 1830 E SUE CON- SEGUENZE SULLE DUE PAR'TI DELLAADRIATICO.}

Nel luglio del 1830 scoppiò a Parigi una nuova rivoluzione, ch'ebbe per conseguenza la caduta dei Borboni in Francia e l'eco d'insurrezioni in altri paesi, Belgio, Spagna, Svizzera, Polonia ed Italia.

In Italia dopo la rivoluzione di Luglio i liberali spe- 
rarono di ricevere aiuto dalla Francia e inscenarono una rivoluzione - questa volta nello Stato pontificio, dove il fermento contro il rigore di papa Gregorio XVI nei rapporti spirituali e temporali avera raggiunto il colmo.

In quanto a circostanze locali, la rivoluzione ebbe origine e sfogo anche questa volta nella parte adriatica dello Stato pontificio, in Romagna, ove i Carbonari stavano sempre in testa, perchè oltre ad essere più lontana da Ronı: era anche più accessibile alle idee innovatrici. - In pochi mesi le autorità pontifice erano scaceiate dalle Legazioni, dall'Umbria, da Parma, da Modena ed i deputati dei paesi liberali formarono la costituzione delle "province confederate d'Italia». Ma anche quest'insurrezione fu subito ed egualmente repressa dall'Austria. Gl'Italiani confederati furono battuti a Rimini e gli Austriaci, dopo di aver occupato Ferrara, Parma, Bologna, ricordussero sui troni di Parma e di Modena i regnanti scacciati dai moli popolari. Gl'Italiani compromessi volevano dopo la capitolazione di Ancona cercare la fuga oltremare, ma le navi austriache in crocera s'impadronirono dei disgraziati Carbonari, che furono cacciati nelle prigioni di Palmanova e di Dalmazia.

L'insana severiti del duca di Modena e del Papa provocarono una seeonda rivolta, parimenti repressa nel 1832. La Francia stette come le altre potenze a guardare, c si limitò ad occupare Ancona dalla parte di mare.

Tutti questi centativi convulsi di riseossa e le reazioni furibonde di alcmi governanti, che riuscirono a rimettersi a posto solo mercè l'intervento delle baionette austriache, produssero un orientamento nell'opinione pubblica d'Italia e d'Europa, nel senso che tutti si persuasero come l'Austria era la principale, anzi l'unica, sostenitriee dei tanti dominatori stranieri d'Italia e che senzal di lei gli staterelli polizieschi d'Italia non avrebbero potuto più reggersi. Questo stato di cose diveniva di vionno in giorno più insostenibile e se ne accorsero anchle alcuni principi come Carlo Alberto di Sardegna, il granduca di Toscana e la granduchessa di Parma, che cor- 
carono di acquistarsi la simpatia del popolo colla mitezza di governo e coll'introduzione di riforme, ormai richieste insistentemente dalle esigenze dei tempi.

In conseguenza di ciò anche tra i patriotti italiani presero consistenza due correnti, del resto elementari. Gli uni, i più ferventi e radicali, volevano spazzar via tutti gli stranieri; gli altri, moderati e conservatori, pensavano che forse si sarebbero potuti ottenere dei sensibili miglioramenti politici anche con un federalismo costituzionale.

In tale subbuglio di tendenze compariscono in Italia, come poco tempo prima Napoleone e Metternich, due nuove figure d'uomini, che nel loro nome riassumono due programmi opposti: Mazzini e Radetzky, venuti nell'alta Italia (Padania) uno dall'occidente, l'altro dall'oriente. Nel 1831 fu trasferito a Milano il già noto capo dello stato maggiore generale Radetzky, non più giovane (era nato nel 1766) che rimase d'allora in poi in Italia fino alla sua morte, avvenuta a Milano nel 1858, e può essere qualificato il rappresentante obbediente, ma inesorabile, dell'oppressione militare.

Mazzini, il solitario, ascetico, infaticabile apostolo e patriotta repubblicano, esule in Svizzera, aveva fondato nel 1832 l'associazione della "Giovine Italia» e diretto al re Carlo Alberto di Sardegna quella famosa lettera, che lo cccitava a mettersi a capo dei liberatori d'Italia.

Per comprendere bene quest'invocazione alla futura "spada d'Italia»: bisogna tenere presenti le condizioni generali d'Italia e quelle particolari del regno di Sardegna.

Il malcontento era generale in tutte le tre parti d'Italia, ma da esso solo non sarebbe uscita ancora la libertà. Occorreva trovare il nocciolo di una organizzazione civile c militarc, segnatamente, capace di raggruppare attorno a sè le forze vive e turbolente degl'Italiani per disciplinarli e condurli alle guerre di liberazione. La bassa Italia non si prestava a questo scopo per la sua corruzione civile, cui seguiva fatalmente l'impreparazione e deficenza militare, come s'erano potuti persuadere i patriotti dall'inliclicissimo esito dell'insurrezione del Pepe nel 1820. E poi nel Napoletano regnavano principi stranieri. 
Restavano duc sole altre istituzioni nazionali, il papato nella media e la Sardegna nell'alta Italia.

Il papato per la sua storia e per la sua costituzione non poteva ispirare fiducia.

In Sardegna era morto nel 1831 il reazionario Carlo Felice. Essendosi estinta con lui la linea diretta della famiglia gli successe, sccondo le decisioni del congresso di Vienna, Carlo Alberto, principe di Savoia-Carignano, cle sebbene avesse piegato all'assolutismo, cercò di introdurre utili riforme nella giustizia, nell'istruzione, nei commerci e sopratutto nell'aumento ed efficenza dell'esercito. Ora la Sardegna meglio amministrata, libera anche dalle pastoic religiose, collocata in alto nclla Padania e spalleggiata dalla Francia, donde venivano tutte le speranze di liberalismo, governata da un principe in fama di tacito liberale, attirava gli sguardi ed i cuori di molti patriolti.

L'idea unitaria italiana si propagò dal settentrione verso il mezzogiorno. Giunta agli Apcunini fece gorgo nelle Romagne e da lì proseguì in due fiolti: tmo maggiore penetrò oltre i monti in Toscana c s'insinuò negli Stati del Papa; l'altro minore corse giù lungo la costa occidentale adriatica. Fino al 1830 la direzione del movimento nazionale era tenuta dai Carbonari, nomini di alta intelligenza e di gran cuore, diramatisi un po' dappertutto a spargere il lievito delle nuove idec e tra i quali primeggiò, a proposito dell'Adriatico, quel patriotta insigne che fu Terenzio Mamiani della Rovere, nalivo di Pesaro.

Ma dopo il 1830 la popolarità e il prestigio del carbonarismo incominciò a declinarc. La loro azione era troppo teoretica e avvolta nel mistero. Appunto verso il 1830 si fece rimarcare tra la gioventù genovese un giurista, Mazzini. Nato cospiratore, egli si mise subito a capo di coloro che volevano l'azione rivoluzionaria immediata. Fu Mazzini che, proscritto, concepì il progetto di trasportare in terra straniera la direzione del movinento nazionale.

Per effetto della sua propaganda avennero tra il $18: 30$ 
e il 1848 parecchi tentativi insurrezionali a mano armata, organizzati dal nord verso il sud d'Italia, collo scopo di far insorgere tutto il paese da Ferrara alle Pinglie.

Questi tentativi tennero due vie. I principali ed anche più fortunati furono quelli ch'ebbero per campo d'azione il versante occidentale dell'Apennino. L'Adriatico fu per lo più evitato per una doppia ragione, geografica e politica - la vicinanza e vigilanza dell'Austria. Anzi si può dire che nelle lotte 'per il risorgimento d'Italia all'Adriatico toccarono le note più dolorose. Com'è naturale il concorso più numeroso dei combattenti per la causa nazionale fu fornito dai cittadini della costa occidentale adriatica. Però anche dalle regioni della parte orientale non mancarono gli entusiasti ed $\mathrm{i}$ generosi. Fra $\mathrm{i}$ volontari lombardi c'erano molti trentini e fra i veneti erano commisti istriani e dalmati.

Mazzini, impaziente e deciso a sforzare la mano al tempo, organizzò nel 1834 dalla Svizzera col concorso di fuggiaschi italiani, polacchi e tedeschi, quell'infelice spedizione di Savoia, che se anche fallì, mantenne vivo il fermento nel popolo e accrebbe l'odio contro l'ingerenza straniera, che privava gl'Italiani di ogni libertà.

I fratelli Ban-

Di un'influenza diretta sull'Adriatico è stata poi l'altra spedizione, pure infelice, dei fratelli Bandiera. Attilio ed Emilio Bandiera, due giovani reneziani, figli del barone Francesco, pure veneziano, ma ammiraglio austria$(\mathrm{C}$, noto all'Italia per la cattura sul mare nel 1831 dei Carbonari fuggiaschi da Ancona, e di una Pizziolato da Spalato, erano ambiduc ufficiali della marina da guerra anstriaca. Entusiasmatisi per l'idea nazionale e per le teorie mazziniane disertarono nel 1844 , Attilio dalla fregata Bellona in rada di Smirne ed Emilio durante una licenza da Trieste. Essi speravano che il loro esempio arrebbe prodotto una diserzione in massa dalla flotta e (Jall'esercito; ma s'ingannarono. Raccoltisi a Corfù decisi ad un tentativo rumoroso ad ogni costo c illusi sullo stato d'animo delle popolazioni della bassa Italia intrapresero in venti compagni, quattordici dei (fuali appartenevano 
regionalmente all'Adriatico, una spedizione in Calabria per suscitare un'insurrezione in questa provincia e nelle Puglie. Sbarcarono nel golfo di Taranto, ma traditi, incompresi, furono arrestati presto e fucilati.

La giovinezza, i cospicui natali, l'abnegazione eroica dei fratelli Bandiera fecero echeggiare questo lugubre dramma in tutto l'Adriatico e in tutta l'Italia. Mazzini per scagionarsi di (quell'azione inconsulta afíermò che $\mathrm{i}$ fratelli Bandiera avevano agito di proprio impulso e contro il suo parere. Questa dichiarazione però non impedì che nell'anno seguente 1845 scoppiasse un altro molo insurrezionale mazziniano a Rimini, che costò nuovo sangue all'Italia.

Simili a quelle sopra descritte per gl'Italiani della $P a$ dania e della bassa Italia, erano le condizioni dei popoli dell'Adriatico.

L'Austria, che lo dominava, l'opprimeva col peso della sua stagnazione. L'assolutismo, le guerre e le innovazioni introdotte col solo scopo di consolidare l'milà della monarchia avevano provocato un ristagno di tulta la vila dello Stato. Le finanze, i commerci e le industrie dell'Austria erano rovinati. Metternich stesso incominciava ad accorgersi di aver fatto ma politica sbagliata e che um progresso moderato non potera che giovine? allat conservazione dello Stato.

Colla morte di Alessandro di Russia (1825) l'influenza di Metternich, che avera subito lo scaceo della liberalzione della Grecia, ${ }^{1}$ ) areva incomincialo a venir meno. $E$ anche nella politica interna si lacevis sentire una eorrente a lui contraria, per cui avrobbe ceduto alquanto e promosso il benessere economico, ma trovò opposizione nell'ostinatezza di francesco $I$, che non voleva sitperne assolutamente d'imnovazioni. Morto Franceseo I nel 1835 gli successe l'inctto Ferdinando I, sotto il cui regno le tendenze antonomistiche-nazomali dei popoli si fecero sempre più lorti.

1) L'Austria areva sostenuto anche allora l'integritit dell 'Turchia di fronte agli altri. 
Malgrado ciò i tre lustri di pace relativa goduti dall'Austria dal 1832 al 1848 le servirono per cercar di rialzare le condizioni economiche dell'Adriatico (che è quella parte dell'Austria, che sola c'interessa direttamente).

\section{LA MACGHINA A VAPORE.}

Un capitolo importante nella storia del vapore applicato alla navigazione è costituito dal tentativo di GiuRessel. seppe Ressel, fatto a Trieste nel 1829, di usare l'elica come motore dei battelli in luogo delle ruote laterali (támbure). Un accidente di poco conto nella prima prova indusse la polizia austriaca a proibire ulteriori tentativi, e così non si udì più parlare dell'clica fino al 1836 quando l'inglese Smith suscitò grande scalpore con un vapore ad elica.

Frattanto nel 1833, sebbene nel 1830 fosse stato dichiarato punto franco il porto di Venezia, era stato fon-

Il Lloyd. dato a Tricste il "Lloyd austro-ungarico", come società di assicurazione, e nel 1836 fu allargato per la navigazione del Levante e dotato di una sovvenzione da tutti i due governi di Vienna e di Budapest. Era evidente che l'Austria per ragioni di opportunità, che non scaturivano dalla nautica, dava la preferenza a Trieste.

In questa maniera Trieste divenne la prima piazza commerciale dell'Adriatico e andò a sostituirsi nel Levante, ncll'Egeo, nel Mediterraneo orientale e più in là ancora, a Venezia, che dopo il colpo fatale del 1797 e l'occupazione austriaca del 1815 non riuscì a rialzare il capo. Per l'esattczza bisogna rilevare che il governo austriaco non obliò del tutto Venezia, anzi cercò di unirla per la via del mare con Trieste e per la via fluviale colla Lombardia. Battelli del Lloyd (nove piroseafi e cento barcacce) esercitarono dal 1853 al 1859 la navigazione sul Po e sui suoi affluenti. Però non si deve dimenticare che queste curc partivano da criteri di utilità per la navigazione militare ed avevano lo scopo di avvicinare meglio le province italiane al resto della monarchia. 
Di pari passo coi progressi della macchina a vapore applicata alla navigazione andavano quelli della locomotiva nella trazione ferroviaria. Nel 1838 fischiò in Austria la prima locomotiva per un píccolo tratto vicino a Vienna e nel $1839 \mathrm{fu}$ aperto un piccolo tratto di ferrovia di soli otto chilometri anche su territorio italiano.

Per iniziativa d'un italiano, il veneziano Carlo Gliega (nato nel 1802), s'intraprose in Austria verso il 1848 a convertire la strada carrozzabile aperta da Carlo VI attraverso il Semmering in una strada ferrata. I lavori furono incominciati appunto nel turbolentissimo 1848 c terminati nel 1853. Nell'anno seguente 1854 la ferrovia da Vienna arrivava fino a Lubiana; ma già nel 1851 il centro della monarchia austriaca era stalo congiunto coi porti del mare del Nord. Trieste ne soffriva nei suoi traffici e dopo molte suppliche si costruì nel 1857 il tronco da Trieste a Lubiana. Ma era tardi, perchè Amburgo aveva già vinta la concorrenza commerciale con Trieste. A maggior danno di Trieste la società francese che aveva costruita la ferrovia detta meridionale, impose tariffe alte che la misero in inferiorita nella concorrenza coi porti del mare del Nord.

Ma dobbiamo abbandonare le considerazioni economiche per ritornare indietro col tempo, ai fatti nazionali particolari della costa orientale dell'Adriatico.

\section{L'ILLIRIS M O.}

La costa orientale adriatica, abitata da popolazione italiana cittadina alla sponda e da Slavi contadini nell'interno, presentava fra il ' 30 e il ' 48 un aspelto singolare.

Gli Slavi, per la loro inferiorili iniziale sino dal secolo VII, per la corrispondente superiorità dei Latini e poi per i falti storici di Venezia e dei 'Turchi, erano rimasti in letargo sino alla fine del secolo XVIII, e non avevano una marcala coscienza individuale. Furono de- 
stati dalla rivoluzione francese, le cui teorie di libertà, eguaglianza e fraternità pervennero loro per due strade, dagl'Italiani al mare direttamente e dai Magiari attraverso $\mathrm{i}$ Tedeschi indirettamente in Croazia.

Sorse allora, tra il 1830 e il 1840 , come suole accadere in tutte le rivoluzioni, un uomo, personificatore della nuova idea, che bandì il concetto nazionale dell'illirismo. Fu questi Lodovico Gaj, nato nel 1809 a Krapina, luogo a settentrione di Zagabria in territorio montuoso, su un affluente di sinistra della Sava. Gaj era animato da sentimenti patriottici e vedendo che la discordia era la causa principale della debolezza politica degli Slavi suoi connazionali, cercò un'idea capace di unirli e affratellarli tutti in una sola aspirazione di progresso e di libertà, materiale e civile. Attratta la mente dalla creazione napoleonica delle province illiriche e nella credenza erronea che gli Slavi fossero infatti i discendenti degli antichi Illiri, perchè ne abitavano i territori, si fece il banditore dell'illirismo, che doveva essere l'idea magica, unificatrice, di tutti gli Slavi (Illiri) dispersi dal Danubio all'Adriatico.

L'idea, formulata in maniera da non urtare le suscettibilità particolari di nessuno dei maggiori gruppi slavi, invaghì le menti colte di molti, ma non potè resistere alla verilà storica, che gli Slavi non sono affatto i discendenti degli Illiri. Per ciò decadde e scomparve presto, anche per la ragione che fu sbancata da un'altra, più seducente ancora, quella del panslavismo, importata appunto verso il 1848 dalla Boemia, ove anche era apparso il banditore di un nuovo verbo, nella persona del patriotta slovacco Jan (Giovanni) Kollar.

Per quanto sia stato sepolto presto, l'illirismo non poteva venir dimenticato, perchè rappresenta il primo risveglio veramente nazionale degli Slavi alla parte orientale dell'Adriatico. 


\section{LA RIVOLUZIONE DEL 1848.}

La terza grande rivoluzione europea del febbraio 1848 non avvenne nè improvvisa e localizzala, come quella del 1789, nè impreparata, come quella del 1830. Sintomi evidenti facevano comprendere dappertutto che qualche cosa di grave stava per scoppiare.

Ci limiteremo, secondo l'argomento, ai prodromi manifestatisi in Italia e in Austria.

Morto nel 1846 il papa Gregorio XVI, che aveva ristabilito il regime poliziesco coll'aiuto delle baionette austriache e francesi, il partito dei cardinali riformatori elesse in opposizione all'influenza anstriaca il cardinale Mastai-Ferretti, appartenente ad una famiglia di Sinigaglia e in fama di propenso alla causa nazionale, che assunse il pontificato col nome di Pio IX. Il nuovo Papa pronunziò apertamente che le condizioni d'Italia avevano bisogno di una riforma, temperò l'asprezza del regime poliziesco e nel principio del 1847 concesse la formazione di un embrione di costituzione colla Consulta di Stato. Queste riforme, ancorchè deboli, ebbero immediatamente due conseguenze, prima quella di rendere il Papa popolare in tutta l'Italia, tanto da far convergere sulla sua persona le speranze di molti patriotti, e seconda quella di provocare ovunque domande di consimili concessioni politiche.

Il contagio dell'esempio scoppiò prinla di tutto in Sardegna. Carlo Alberto, che in principio del suo regno per necessità politica aveva mostrato di appoggiarsi ai governi antichi, incominciò dal 1846 a seguire, specialmente di fronte all'Austria, maggior indipendenza d'azione. Dopo l'elezione di Pio IX fu trascinato apertamente dalla corrente nazionale, tanto che alla fine del ' 47 fece concessioni liberali, prometlendone delle maggiori, ehe infatti accordò nel febbraio del 1818.

L'esempio di Pio IX e di Carlo Alberto si ripereosse subito nel Napoletano, dove nel gennaio del 1848 seop- 
piò da parte dei Siciliani uma rivoluzione, che si confuse coi fatti maggiori del ' 48 .

Questo fermento delle regioni italiane, esenti dall'influenza diretta dell'Austria, produsse un'agitazione viva e palese negli animi degli altri Italiani dell'Adriatico, soggetti all'Austria.

Molto meno evidente era invece il movimento politico negli altri popoli dell'Austria. C'erano qua e là delle manifestazioni singole con desideri di riforme. Per esempio i Magiari, capitanati dal conte Stefano Széchényi, voIevano una riforma liberale e nazionale della loro costituzione; i Boemi, destati dalla propaganda panslavista del Kollar, già ricordato, volevano l'autonomia della Boemia sotto la sovranità austriaca; i Croati e gli Sloveni venivano destati al sentimento nazionale dall'illirismo del Gaj; ma tutte queste manifestazioni non arrivavano all'importanza del movimento politico disegnatosi in Italia c anche in Germania dal 1840 in poi.

Malgrado però questa calma apparente il isistema della repressione, impersonato da Metternich, aveva fallito. Se n'era accorto lui stesso. Ormai non c'era che la cecità della corte di Vienna a voler perpetuare uno stato di cose, condannato dal tempo.

In tali condizioni la rivoluzione di Febbraio doveva scoppiare in Austria come un fulmine, apportatore d'incendio.

L'impulso rivaluzionario venme anche questa volta dalla Francia. Nel mentre i popoli nei paesi ora nominati lottavano per avere appena un barlume di costituzione, le idee sociali avevano preso in Francia tale sviluppo fra le masse da formare già un quarto ceto, l'operaio, così forte ed organizzato, da mettersi in lotta col ceto possidente e da dare i primi segni di quella quistione sociale del proletariato, che divenne acuta in tutto il mondo nella seconda metà del secolo passato.

Il risorgere della repubblica in Francia, alla cui presidenza già nel decembre 1848 veniva portato un Bonaparte (Luigi Napoleone, futuro imperatore, Napoleone III dal 1852), esercitò grandissima influenza su quasi tutti 
gli Stati europei, nella maggior parte dei quali incominciarono nuovi moti rivoluzionari.

Immenso fu l'effetto prodotto dalla rivoluzione di Febbraio in Germania, ove si giunse a formare una conlederazione, meglio compaginata, coll'esclusione dell'Austria.

Più profondamente della Germania fu sconvolta l'Austria dagli avvenimenti del marzo 1848, perchè in mezzo alla bufera di insurrezioni, cui parteciparono i popoli malcontenti, a lei soggetti, sembrò realmente che fossé venuto il momento della sua dissoluzione.

L'agitazione era diretta apparentemente contro il principe di Metternich, che col suo rigore s'era attirato l'odio generalc. Per salvarsi dal furore del popolo di Vienma Metternich dovette darsi alla fuga. Però dopo la sua caduta dal potere apparve manifesto che i popoli dell'Austria aspiravano ad altri scopi.

I Tedeschi volevano aver condizioni costituzionali moderne, nı accompagnate in loro favore dal più rigido accutramento politico-amministrativo all'interno e da una più intima unione colla Germania all'estero.

Gli Slavi crano meno precisi e risoluti nelle loro aspirazioni, ma davano a vedere chiaramente di tendere anch'essi all'indipendenza. I Cechi (Boemi) a Praga aspiravano al federalisno, come ad un passo necessario sulla via di maggiori libertà costituzionali; i Polacchi a Leopoli (Lemberg) ed a Cracovia tentavano d'iniziare il ristabilimento della Polonia.

I più turbolenti furono gli Ungheresi e gl'Italiani; anz; la loro azione, concorde in buona parte per nesso morale, non , può venir esposta separatamente, perchè è connessa più che nell'andamento cronologico nel nesso caulsale. Merita d'altronde di rilevare con particolar studio questo movimento, in primo luogo perchè si svolse dall'una all'altra parte dell'Adriatico, in seconclo luogo perchè fu sincrono e consono sebbene gli attori fossero due popoli niente affatto affini. C'è però tra la rivoluzione: italiana e quella ungherese una differenza intima, che non può venir sorpassata.

Considerate nelle linee generali averano tufle dur ramse: 
e tendenze nazionali e sociali; considerate però megtio nella sostanza presentano delle forti differenze nello spirito d'idee che agitavano i due popoli.

In Italia l'idea nazionale spianava le barriere regionali; i principii democratici restringendo sempre più i privilegi della nobiltà e completando la caduta di questa casta , entusiasmavano tutte le masse; la libertà era predicata ed applicata come un bene generale, sicchè le tre grandi idee di nazionalità, democrazia e libertà fecero fremere tutti gli animi e la sconvolsero tutta dall'Alpi alla Sicilia.

In Ungheria invece le idee non potevano svolgersi in questo modo per la ragione ch'essa non era, nè è oggigiorno, uno Stato nazionalmente omogeneo. Le idee suaccennate vennero accolte e bandite principalmente dai Magiari, nazionalità dominante, ma come mezzo per estendere ancora più la loro egemonia. E siccome quest'egenınia era in parte pesante, in parte pericolosa per le laltre nazionalità, queste interpretando anche le correnti moderne a loro favore, si mostrarono decisamente avverse ai postulati dei Magiari. Ne seguì che in Ungheria l'idea nazionale acuì invece gli odii e le barriere fra popoli che di buono o malgrado convivevano da secoli; che la libertà per mancanza di concordia minacciò di venir soffocata; e che anche quella che fu raggiunta degenerò in tirannia a danno delle nazionalità minori, non magiare, che furono oppresse. Nemmeno l'idea di democrazia potè raccogliere in Ungheria quei successi ch'ebbe invece in Italia. Il feudalismo aveva in Ungheria' radici troppo profonde per venir soppiantato d'un tratto e poi era necessario per la conservazione dell'egemonia sociale e nazionale magiara; per ciò l'attuazione" di riforme in questo senso rimase riservata a tempi futuri. Ciò non di meno siccome la parte ricca, colta ed intelligente del popolo magiaro abbracciò con entusiasmo. l'idea d'indipendenza nazionale, la rivoluzion? che vi scoppiò di riverbero agli sconvolgimenti polit. i di Europa e particolarmente a quelli d'Italia fu di una gravità eccezionale per le sorti dell'Austria. 
Gli Ungheresi videro giunto il momento favorevole per ottenere maggiori libertà ed indipendenza amministrativa e desideravano per ciò in principio un'unione soltanto personale coll'Austria.

Allorchè alla notizia della rivoluzione di Parigi il popolo, persuaso che la bancarotta dello Stato era ormai inevitabile, prese d'assalto le casse dello Stato, il discorso infuocato - detto il discorso battesimale della rivoluzione austriaca - che Kossuth tenme il 3 marzo 1848 a Budapest fu il segnale di una valanga, a Vienna e nelle altre province, di petizioni contro il governo per avere istituzioni più liberali, che la censura e la polizia dovettero guardare impotenti. Non v'ha cittadina dell'Adriatico orientale, che nei suoi ricordi del '48 non abbia da registrare una straordinaria effervescenza popolare con istituzione di una guardia nazionale, sfoggio di coccarde e nastri dai tre colori italiani, eclissamento della polizia.

Gli altri Italiani, specialmente quelli del LombardoVeneto, che mai avevano potuto adattarsi al giogo del governo austro-tedesco, tentarono di staccarsi dallo Stato austriaco con insurrezioni (le cinque giornate di Milano, la sommossa di Venezia, ecc.). Immedialamente furono mandate in Italia tutte le milizie disponibili. Fra i reggimenti mobilizzati c'erano i Croati (lei cosidetti confini militari (regioni lungo la Sava), orde feroci rimaste celebri per la loro avidità depredatrice.

Ma i rigori polizieschi coi quali il sistema di Metternich aveva creduto di reprimere nel Lombardo-Veneto e negli altri Stati italiani (eccettuato il Piemonte), ligi all'Austria o per paura, le nuove idee di libertì civile si mostrarono inutili, anzi non fecero che gettar esca sul fuoco.

Di fronte alle rivoluzioni irresistibili concessero costituzioni Carlo Alberto, Pio IX, Ferdinando II di Napoli, Leopoldo II di Toscana. Di questi solo Carlo Alberto e in principio Pio IX averano abbracciato sinceramente la causa nazionale e per ciò il giubilo e le speranze degli Italiani furono concentrate su loro due.

Il tornaconto politico moveva certamente l'mo e l'al- 
tro. Il Papa aveva molte ragioni per credere che opponendosi alla corrente nazionale, la sua nave ne sarebbe stata travolta. Per ciò stimò opportuno di far rivivere l'idea guelfa medievale e arginare la fiumana ponendosene a capo.

Ma contemporaneamente l'idea ghibellina aveva trovato un inatteso sostegno nel re di Sardegna: e Pio IX prese la mezza misura di mandare truppe papaline con volontari nell'alta Italia a combattere contro quelle dell'Austria. Questa rispose con un lungo bombardamento di Ancona, che fu costretta alla fine alla capitolazione dal generale Wimpffen.

Il Piemonte, situato geograficamente alla punta estrema di quel bacino che anche nella sua parte di terraferma chiamammo dell'Adriatico, sembrava essere formato meno di nessun'altra regione per influire sulle sorti di questo mare. Invece nel mentre appena singoli preveggenti avevano incominciato a sperare nelle virtù civili e militari dei Piemontesi, era già destinato che a loro sarebbe toccata la fortma e l'onore di capitanare il risorgimento politico d'Italia, di cementare il nuovo Stato e indirettamente di rendere italiani non solo il Tirreno, ma anche l'Adriatico.

Il Piemonte, paese fecondato più degli altri dalle nuove idee rivoluzionarie provenienti dalla Francia, ma minacciato dalla reazione austriaca imperversante in Lombardia, oscillava fra le due correnti opposte. Intanto era divenuto il rifugio di tutti i proscritti d'Italia, la fucina ove si preparavano le armi per la riscossa, la sentinella che vegliava ansiosamente sugli avvenimenti della penisola. La notizia che il re di Sardegna colle riforme costituzionali e col liberalismo s'era messo a capo del movimento nazionale italiano suscitò ovunque, anche lungo l'Adriatico, un giubilo indescrivibile. Gl'Italiani nella vivacità del loro temperamento meridionale inneggiarono da un capo all'altro della penisola al re, detto per iperbole la "spada d'Italia», ripetendo un po' troppo affrettatamente la frase ormai storica "che l'Italia farà da sè».

Carlo Alberto, cui si presentava la possibilità di in- 
grandire il suo regno, approfittò dell'occasione ed entrò con un esercito in Lombardia per sorreggervi l'insurrezione. L'esercito piemontese, composto in gran parte di volontari raccogliticci, non era adeguato alle truppe allenate di Radetzky. Ciononpertanto in principio la sorte dell'armi volse favorevole agl'Italiani, che presero Milano e costrinsero Radetzky a retrocedere fino a Verona e rinchiudersi nel quadrilatero (Verona, Legnago, Mantova, Peschiera). Questi successi insperati portarono l'entusiasmo c l'imprudenza degl'Italiani oltre i limiti, in cui invece avrebbero dovuto tenersi. I patriotti più ardenti volevano spingersi avanti, togliere al nemico tutte le parti "del suolo sacro", conquistare l'Istria, la Dalmazia. Altri proponevano addirittura di occupare l'Illiria e spingersi su Vienna.

Ma il disinteressamento del Papa e del granduca di Toscana dalla causa nazionale, a malgrado dei tentativi d'intesa di Gioberti, e l'indisciplinatezza dell'esercito sardo, produssero un cambiamento nella prossima sorte delle armi. Carlo Albcrto sconfitto a Custoza (26 luglio 1848) fu costretto a ritirarsi. La guerra però non fu ancora terminata.

Quest'aggressione dall'esterno per rinforzare la rivoluzione interna del Lombardo-Veneto era senza dubbio pericolosa per l'Austria, ma se anche il maresciallo Radetzky non l'avesse respinta, l'Austria avrcbbe perduto due province, che già le si stacchcranno più tardi, senza per ciò vedere compromessa la sua esistenza. Molto più grave fu invece il rischio corso a causa della rivoluzione in Ungheria, perchè se fosse riuscita, l'Austria avrebbe subìto una scossa tale nelle suc fondamenta, da slasciarla; forse, certamente da modificarne la compagine.

L'accertare se e quali intese secrete di governi o di uomini politici abbiano corso dall'una parte all'altra dell'Adriatico fra Italiani e Ungheresi è un còmpito degrli storici. In questo rapido quadro basta l'accenno che le rivoluzioni in Italia e in Ungheria scoppiarono quasi contemporancamente, colla precedenza però del LombarL'Adriatico. 
do-Veneto, tanto che l'esercito austriaco dovette venir diviso per fronteggiare il duplice pericolo. $\mathrm{Fu}$ appunto il contegno ostile dei Magiari in quest'epoca (seconda metà del 1848) che impedì all'Austria di trarre vantaggi notevoli dalla vittoria di Custoza.

Per reprimere le insurrezioni nel Lombardo-Veneto l'Austria ci teneva fin dal 1831 il maresciallo Radetzky. Riguardo al trattamento dei rivoluzionari ungheresi l'imperatore era invece indeciso. Le popolazioni minori non magiare dell'Ungheria, ossia i Croati, i Serbi ed i Rumeni, ostili naturahnente al nuovo Stato unitario magiaro, s'erano rivolte all'Imperatore perchè le proteggesse. Ma questi, curante sopratutto dei propri interessi, esitava. Dapprincipio vedeva un tornaconto nel tenere dalle sue i Magiari, ma poi che questi gli si mostrarono davvero pericolosi, favorì i non Magiari, tra cui emergeva il ba110 croato Jellacich. L'imperatore credette di poter rivolgere una buona parte delle truppe contro i Magiari e ne affidò la direzione al Jellacich. Ma la rivoluzione scoppiata a Vienna stessa il 6 ottobre 1848 e gli altri sconvolgimenti indussero l'imperatore Ferdinando I ad abdicare in favore di suo nipote, l'arciduca Francesco Giuseppe.

Questo giovane arciduca di soli diciotto anni, che si vide sollevato al trono in condizioni tutt'altro che rosee, ebbe da pensare prima di tutto a reprimere i moti rivoluzionari, e facendo di necessità virtù pubblicò in marzo del 1849 una costituzione valevole per tutto l'impero e cuindi anche per l'Ungheria.

Però approfittando di questi imbarazzi del governo anche gl'Italiani avevano ripreso coraggio e si ebbero l'insurrezione di Venezia con Manin e col dalmata Tommaseo e il nuovo attacco dei Piemontesi (che del resto erano inferiori agli Austriaci) respinto da Radetzky a Novara (23 marzo 1849).

In Ungheria invece gli eserciti degli Honved (insorti) capitanati dal generale Görgey respinsero le truppe austriache mandate contro di loro. La dieta ungherese proclamò allora (14 aprile 1849) la deposizione della casa 
d'Asburgo e la nomina di Kossuth a governatore dell'Ungheria.

Assicuratosi in Italia il governo austriaco potè concentrare le truppe contro l'Ungheria. Malgrado ciò si salebbe trovato ancora in brutti frangenti se in quel momento critico non fosse stato soccorso dalla Russia, che per la partecipazione dei Polacchi alla lotta degli Ungheresi temeva dei pericoli per sè e per la sua parte della Polonia. Colla sconfitta del generale ungherese Görgey a Vilagos (13 agosto 1849) per parte dei Russi, ebbe fine anche la guerra in Ungheria.

L'Austria era salva e l'Adriatico risoggiogato

La vittoria di Radetzky a Novara aveva avuto un contraccolpo su tutta l'Italia. Il granduca di Toscana ed i duchi di Modena e Parma poterono ritornare nei loro Stati. La Francia spedì un corpo d'esercito a Roma per rimettervi il Pontefice. Indarno Garibaldi difese la città; essa cadde in mano dei Francesi. Gli Austriaci ristabilirono quindi il potere dei Papi nella Romagna e nelle Marche. Anche il re delle Due Sicilic abrogò la costituzione. Solo il re di Sardegna, Vittorio Emanuele II, mantenne lo statuto costituzionale concesso da suo padre Carlo Alberto. Il Lombardo-Veneto venne organizzato ed amministrato a norma delle altre province austriache. Trieste e l'Istria dovettero subire l'imposizione della burocrazia tedesca. La Dalmazia non fu curata, perchè contava poco. $\mathrm{Fu}$ abrogata l'antica costituzione ungherese e in tutta l'Austria fu ripristinato l'assolutismo, concentrando ogni potere nelle mani dell'imperatore (ministero Bach, ai 31 dicembre 1851).

Questi provvedimenti del governo avevano ancora lo scopo di fare dell'Austria uno Stato unitario. 


\section{ALCUNE CONSIDERAZIONI SULLA RIVOLUZIONE ITALIANA DEL 1848 CON SPECIALE RIGUARDO ALL'ADRIATICO.}

Ancora una volta armi austriache dalla parte dell'Adriatico e per di più, cosa strana, francesi da quella del Tirreno ristabilirono in Italia l'antico regime di reazione ed oppressione politica e clericale. Per indicare con una parola sola la sintesi di tutti i patimenti da cui furono colpiti gl'Italiani dopo il ' 48 non c'è che il termine terrorismo, e, cosa dolorosa, questo terrorismo gravò maggiormente sulle province dell'Adriatico.

Però il ritorno dell'assolutismo non poteva formare l'epilogo d'una rivoluzione così profonda come quella che aveva sconvolto l'Europa in tre grandi periodi, 1789, 1830 e 1848. Non fu che un'altra perentesi intermedia in quel grandioso rivolgimento che tra la fine del XVIII e nel corso del XIX secolo trasformò l'assolutismo feudale del medio evo nel moderno industrialismo liberale e nazionale.

Fino al 1848 le idee politiche degl'Italiani erano abbastanza confuse.

Ma la dura scuola del '48 e '49 ebbe l'effetto di far sparire i sogni informi e di togliere i dibattiti e gli attriti fra i partiti repubblicano e monarchico sulla formazione federale o unitaria, che prima avevano agito così perniciosamente nell'azione pratica per ottenere la liberazione politica.

Fino al 1848 il pensiero rigeneratore d'Italia non aveva che un solo apostolo, Mazzini, che nel suo ascetismo diritto e radicale, non vedeva che l'ultima meta dei suoi sogni, la repubblica. Per ragioni di politica e di temperamento l'idea repubblicana aveva fatto il maggior numero di proseliti nella bassa e nella media Italia.

In quest'ultima parte un numero non esiguo di pa- 
triotti, oscillanti tra il conservatorismo e la rivoluzione, avrebbero voluto concordare le duc tendenze e propendevano per una confederazione, che in pratica non avrebbe avuto fortuna.

Nel Piemonte, ove la monarchia era profondamente radicata negli animi di tutte le classi del popolo, l'idea repubblicana stentava ad attecchire ed i patriotti avevano abbracciato il programma di vaste riforme liberali e costituzionali, ma sempre sotto l'egida monarchica.

Nel Lombardo-Veneto il pensiero dominante era la cacciata degli Austriaci; e per ciò tutti i moti, anche se singoli e staccati e provocati ora da mazziniani, ora da liberali, ora da aderenti del Piemonte, erano sempre ed inevitabilmente diretti ad espellere la forza armata dell'Austria o almeno a provocare imbarazzi al governo.

Nella Venezia Giulia, e particolarmente nella città di Trieste, tolti i patriotti più chiaroveggenti, preoccupazioni d'indole economica fecero restar perplessa buona parte della popolazione sull'opportunità di estendere anche a questo paese il movimento separatista italiano antiaustriaco. Più vive erano le ansic e le aspettative delle cittadelle dell'Istria e della Dalmazia, sempre memori di aver appartenuto per secoli a Venezia; ma cui per la esiguità del numero dei cittadini non si giunse a movimenti esterni di speciale entitì.

1 rovesci del '48 schiariromo l'atmosfera politica e dic- Garibaldi. dero campo agl'Italiani di apprezzare le gesta di un nuovo eroe provvidenziale, Garibaldi.

Compromessosi nel 1834 (a 27 anni d'età) in una congiura mazziniana e condannato a morte, era riuscito a ricoverarsi in Francia, donde era passato in Americi. Alla notizia dello scoppio della rivoluzione nazionale in Italia del' 48 era accorso in patria, ma vi giunse quando il primo periodo fortunato della guerra nell'alta Italiat era passato. Si offerse di prestare servizio sotto Carlo Alberto, ma fu respinto dall'austerita gerarchica dei Picmontesi per diffidenza a causa della sua aderenza al programma ultra rivoluzionario di Mazzini. Il comitalo di difesa to diresse allora a Milano ove formo un corpo 
di volontari, coi quali oppose accanita resistenza alle truppe austriache di gran lunga più numerose e meglio a'gguerrite. Quest'ardita resistenza gli procurò immensa popolarità.

La accrebbe ancora la difesa di Roma tanto contro le truppe francesi, fatte sbarcare da Napoleone, allora principe pretendente, che aveva bisogno dell'appoggio del clero, a sostegno del potere temporale del Papa, quanto contro quelle borboniche di Napoli. Perduta però la miglior parte delle milizie per combattimenti, marce forzatc, malattie, Garibaldi è costretto a cercar salvezza nella fuga e si getta sulla parte adriatica dell'Apennino. Parecchie volte in pericolo di essere fatto prigioniero dalle truppe croate dell'Austria dopo mille peripezie da romanzo riuscì a prendere il mare a Cesenatico in una barca di chioggiotti ed a rifugiarsi nelle lagune di Comacchio, donde si ricoverò in Toscana. - Sopraffatto dall'andamento generale della politica di reazione dovette ricoverarsi in Piemonte, ma subito esulare anche da qui fuori del territorio d'Italia. Attorno al suo nome s'erano pcrò già formati il fascino e un'aureola di gloria.

San Marino, Nella memorabile ritirata da Roma Garibaldi si ricoverò per brevissimo tempo nella repubblica di San Marino. Il nome di questa repubblichetta, oltrechè riportare il pensiero dei fatti generali dell'epopea italiana alle regioni particolari dell'Adriatico, riallaccia idealmente le due coste, perchè secondo la leggenda San Marino che fondò la città nel IV secolo d. C. sarebbe venuto dalla Dalmazia, e precisamente da Arbe. - San Marino, la cui indipendenza municipale era stata riconosciuta dai Pontefici (Urbano VIII nel 1631) ed anche da Napoleone, divenne il rifugio di compromessi politicamente, tanto che nel 1851, siccome il Papa chiedeva l'estradizione di diversi rifugiati, ottocento austriaci e duecento gendarmi pontifici vi fecero irruzione. In seguito si manlenne strettamente neutrale, perfino negli sconvolgimenti italici degli anni ' 59 e ' 60 , e fu per ciò rispettata anche dal governo italiano.

Tra i rovesci patiti dagl'Italiani nel ' 48 è necessario 
ricordare altri episodi, che si riferiscono specialmente all'Adriatico.

Mentrechè -sul Mincio ferveva ancora la guerra fra La flotta sarda Piemontesi e Austriaci, la flotta sarda, comandata dall'ame Trieste. miraglio Albini e rinforzata dalla squadra napoletana e veneta, veniva sull'Adriatico in cerca dell'austriaca. Ma siccome questa non accettò battaglia e si rifugiò a Pola, la flotta sarda gettò le àncore nella rada di Trieste. Le truppe austriache si misero subito in posizione di difesa, ma dopo tre giorni con meraviglia generale la flotta sarda, anzichè procedere al bombardamento, salpò per prendere il largo. Si seppe di poi ch'era stata richiamata per ordine del governo di Torino, giacchè la Germania aveva protestato contro l'eventuale occupazione ci Tricste. Questa intromissione era una conseguenza , I trattato di Vienna del 1815, secondo il quale l'imperatore Francesco I areva aggregato alla confederazione germanica, come fu già rilevato a suo posto, anche il cosidetto litorale austriaco, che i confederati s' crano impegnati a difendere e ad assicurarne l'integritì.

$\mathrm{Ma}$ contro questa nuova ingerenza germanica a Trieste e nel Trentino, si ebbero nel ' 48 delle proteste. Sempre in forza del trattato di Viema, quanclo in quell'anno sí raccolse la costituente germanica a Francoforte, Trieste, l'Istria e il Trentino dovettero anche mandarvi i loro delegati. Una giunta triestina, che di triestino non aveva che il nome e che era presicduta da un carinziano e da un prussiano clomiciliato da linghi anni a Trieste, s'era affrettata ad inviare il suo presidente ed il vicepresidente, ossia i due indicati stranieri, a Francoforte, che furono così i rappresentanti di sè stessi o tutt'al più della colonia tedesca, ma non di Trieste. Bisogna tuttavia riconoscere, ad onore di cuei due delegati, ch'essi portarono onestamente a conoscenza della dieta le proteste dei Triestini contro l'inclusione arbitraria nella confederazione germanica. Più encrgiche furono invece le protestc dei delegati, veramente italiani dell'Istria (Facchinetti e Madonizza) e del Trentino (il poeta Gazzoletti e Marsilli) che chiesero la separazione clell'Istria e del 
Trentino dalla confederazione. Un altro strenuo propugnatore dei diritti nazionali del Trentino fu il barone A. Prato.

L'avversione incorreggibile di Ferdinando di Napoli e di Leopoldo di Toscana ad ogni concessione costituzionale e nazionale e il voltafaccia di Pio IX dopo $\mathrm{Cu}-$ stoza, che non volle più saperne della causa nazionale, apersero definitivamente gli occhi degl' Italiani. La rivoluzione del '48 aveva costato un grande sforzo e immense perdite, ma fu come una prova generale, che mostrò gli errori degl'Italiani e il modo nel quale essi avrebbero potuto far breccia contro i molteplici loro avversari.

Le cinque giornate di Milano e la sommossa di Venezia coll'eroica difesa del forte di Malghera insegnarono che la furia popolare può valere a qualche cosa, a patto che sia appoggiata dal di fuori. Un urto felice contro l'Austria avrebbe prodotto immediatamente lo sfacelo dei troni di Toscana e di Napoli, l'ultimo dei quali aveva il suo lato debole nella Sicilia, e avrebbe reso l'Italia signora dell'Adriatico. Garibaldi aveva messo arditamente la mano nella quistione di Roma e insegnato che per giungervi bisognava andare nelle Marche dalla parte dell'Adriatico. Allora tutti compresero che per raggiungerc lo scopo finale dell'indipendenza e la libertà nazionale occorreva smettere le fazioni e agire concordi con un piano prestabilito sotto la guida di uno Stato; e questo non poteva essere che la Sardegna.

La quistione italiana aveva varcato i confini della penisola ed era entrata nel dominio della grande politica europea. Infatti dopo Custoza Francia e Inghilterra, le due potenze che allora dirigevano la politica europea, cercarono di regolare il futuro assetto d'Italia in via pacifica, ma i loro sforzi arenarono di fronte all'irritazione scoppiata nell'Italia stessa contro Carlo Alberto per l'esito infelice della campagna. Dopo Novara l'Austria voleva annientare addirittura la Sardegna, ma ne fu impedita dalle potenze occidentali, che s'intromisero. Carlo Alberto salvò lo Stato rinunziando subito dopo l'infau- 
sta battaglia di Novara alla corona a favore di suo figlio Vittorio Emanuele II.

La condizione della Sardegna era oltremodo difficile perchè circondata tutt'intorno da vicini male intenzionati. Suo còmpito principale era guadagnare la protezione delle altre potenze contro l'Austria. E infatti riuscì a riavere dall' Austria tutti i suoi territori pagando una forte indennità di guerra. Tutti questi fatti e la conservazione dello statuto costituzionale destarono negl'Italiani la speranza che il giovine re Vittorio Emanuele II sarebbe stato il primo e il solo a levare nel momento opportuno il vessillo dell'indipendenza d'Italia.

Durante questo storico duello fra gl'Italiani e l'Anstria, combattuto nella metà superiore dell'Adriatico, la parte orientale non italiana di questo mare dormiva. Però le attuali condizioni politiche dei Balcani presentano molte analogie e contingenze, assai simili a quelle quarantottesche d'Italia di tre quarti di secolo fa.

L'Italia aveva un cuore: Mazzini; un braccio: Gari- L'Italia tra baldi; e una spada: Vittorio Emanuele II. Le occorreva il' 48 e il ' 69. una mente e la ebbe fortunatamente nel conte Camillo di Cavour. Tutti quattro gli eroi provenivano dall'alta Italia.

L'entrata di Cavour (11 ottobre 1850) nel ministero di agricoltura e commercio segna la rigenerazione della Sardegna. Dotato di una preparazione economica egli si diede a tutt'uomo a ordinare l'istruzione, il regime daziario, a costruir strade, ponti e ferrovie. Furono regolate le finanze, fu pagato il debito di guerra all'Austria, fu consolidato l'esercito. Le speranze degl'Italiani furono realizzate per merito principale del conte di Cavour, che resse per un decennio la politica estera del regno di Sardegna.

Durante questo tempo anche l'Austria, godendo di pace interna, cercò di sanare le molte ferite. Ma non ebbe speciale fortuna; e ancora meno ne trovò nella politica estera. Era riuscita a tenersi in piedi, ma la sua debolezza ormai era stata palese c di ciò approfittarono i

L'Adriatico. 
vinti del momento, preparandosi tacitamente a quel migliore avvenire, cui tendevano da parecchi decenni.

Uno degli atti più geniali e fortunati di Cavour fu, com'è noto, la partecipazione di un corpo di esercito sardo, coll a capo il generale Lamarmora, alla spedizione di Crimea. La Sardegna a nome dell'Italia non aveva alcun interesse da far valere nel Mar Nero e quindi la spedizione sarda non era destinata che a provocare sacrifizi di danaro e di vite umane. Ma Cavour volle parteciparvi allo scopo - dicono gli storici - di tener desta l'attenzionc dell'Europa alle encrgie italiane e di metter poi una voce della penisola italiana nel capitolo degl'interessi politici europei. - Oggi però noi possiamo scorgere in quell'atto una portata ben maggiore. Se anche l'Italia era in subbuglio ed i Balcani stavano quieti, tutte le due penisole che racchiudono e formano l'Adriatico andavano rigenerandosi. - La metà del secolo passato ¿̀ la prima volta in cui l'Europa assistette ad uno scoppio di panslavismo e ne fu perplessa.

L'imperatore russo Nicolò, la cui attività era cresciuta enormemente per il soccorso che, essendo il suo paese rimasto illeso dai rivolgimenti del ' 48 , aveva potuto prestare all'Austria contro gli Ungheresi, pieno di fiducia nclla sua potenza e approfittando delle condizioni europee, ancora sconvolte, aveva voluto dare il colpo mortale alla Turchia. Il miraggio di Costantinopoli aveva attirato la politica russa ancora nei secoli precedenti, ma come tendenza burocratica. Questa volta invece l'inupresa fu illuminata da uno sprazzo di panslavismo che impressionò: molti temettero di vedere la Russia non solo sul Bosforo, ma anche sull'Adriatico, e in atteggiamento più pericoloso che all'epoca napoleonica.

La reazione della gelosia europea non poteva mancare. Inghilterra e Francia, cui si unì più tardi anche l'Austria, fecero una lega per opporsi allo smembramento della Turchia. Cavour trovò il modo d'insinuarvi il corpo l'esercito sardo. Si vemue così alla guerra detta di Crimea $(1853-56)$ in cui gli alleati soffersero grandi perdite a causa dell'inverno e del coléra, perdite che furono rime- 
diate dal corpo d'esercito sardo. - La Turchia conservò momentaneamente la sua integrità; il panslavismo e la Russia dovettero cedere davanti all'Europa.

L'Austria, immediata vicina nei Balcani e interessata per l'Adriatico, sprecò rilevanti somme di danaro per gli armamenti, perdette a causa di malattie numerose truppe durante l'occupazione della Valachia, ma dovette adattarsi a veder sorgere dai principati danubiani lo Stato nazionale indipendente di Rumenia.

Intanto gl'Italiani non davano tregua.

Appena si preparavano in Europa gli armamenti guerreschi per la guerra di Crimea, Mazzini, impaziente e che non tralasciava di approfittare di ogni complicazione, organizzò nel febbraio del 1853 un moto rivoluzionario a Milano, ehe abortì, ma che merita di essere notato perchè vi presero parte anche gli ungheresi ivi di guarnigione.

Il governo piemontese poi istigava continnamente in segreto le popolazioni del Lombardo-Veneto contro l'Austria. Nel 1857 il maresciallo Radelzky s'era ritirato a riposo definitivo ed era passato da Verona a Milamo, ove morì un anno dopo. Cavour aveva alzato la voce al congresso di Parigi (1856) per farvi conoscere i lagni d'Italia. Russia e Inghilterra s'erano mostrate disposte di concedere un aiuto, ma esilavano a porgerlo coi fatti.

\section{LA GUERRA RRANGO-I'TALIANA DEL 1859 CONTRO L'AUSTTRIA.}

Si trovò allora in Francia Napoleone III, che da una guerra lira l'Austria e la Sardegna sperava di provocare una revisione dei trattati del 1815 e di trare quindi dei vantaggi per sè. Cosi gl'llaliani risollevarono il capo certi che l'Austria non avrebbe trovato alleali. Nellormai famoso convegno di Plombières (1858) Napoleone III e Cavour si accordarono su 111 progello di liorgamizzazione dell'Italia. L'alla Italia doverat essere librebla fino 
all'Adriatico e appartenere alla Sardegna: nel rimanente della penisola doveva formarsi una confederazione di Stati sotto la presidenza onoraria del Papa. Cavour finse di accettare.

Già il $10^{\circ}$ gennaio 1859 la Francia ruppe le relazioni diplomatiche coll'Austria; e dappoichè la Sardegna s'armava e riceveva rinforzi dalla Francia, l'Austria tenne verso di lei quel linguaggio che $i$ più forti usano sempre in casi simili coi più deboli: le impose l'ultimatum di disarmare entro tre giorni oppure di aspettarsi l'inizio delle ostilità di gucrra. La Sardegna non cedette e il maresciallo Gyulai, succeduto a Radetzky, aprì le opcrazioni. Ma fu sconfitto a Magenta (4 giugno) e Solferino (24 giugno 1859). Mai le speranze degl'Italiani furono così rosce come in questo momento. Invece Napoleone, che temeva l'intromissione della Prussia qualora avesse proseguito le operazioni di guerra, s'arrestò inaspettatamente a mezza strada del suo programma di liberare l'Italia fino all'Adriatico. Nell'armistizio di Villafranca (novembre 1859), conchiuso da lui con amaca delusione degl'Italiani, l'Austria cedette a favore del re di Sardegna la Lombardia fino al Mincio.

I principi di Toscana e di Modena dovevano rientrare nei loro possessi, donde erano stati espulsi, però senza l'aiuto di armi. In conseguenza a queste deliberazioni il presidio austriaco che teneva occupata Ancona ancora dal 1848 dopo la capitolazione ottenuta da Wimpffen, se ne andò finalmente. L'Austria promise inoltre di entrare col Veneto in una confederazione italiana da costituirsi secondo il progetto di Napoleone III. - Queste erano le decisioni della diplomazia. Però ad eccezione del Veneto, che doveva restar ancora in possesso dell'Austria, tutte le altre deliberazioni erano destinate a non divenir effettive ancora prima della loro sottoscrizione.

Come conseguenza di quest'impotenza della diplomazia, anche i territori della Toscana, di Modena e di Parma, appartenenti alla seconda e terzogenitura della casa d'Asburgo, nonchè della Romagna, dove con plebisciti era già stata decisa la deposizione delle anteriori dina- 
stie e l'annessione alla Sardegna, venuero in mano all Italia (1860) il cui nuovo governo raggiunse così la costa dell'Adriatico.

Il patriottismo e le tendenze unitarie degl' Italiani crebbero sempre più. Buona parte dell'alta e della media Italia crano già rimnite. Contro l'Austria, ancora troppo forte, e contro il Papa, protetto da Napoleone III, non si potega agire. Per ciò il cosideńto partito d'azione rivolse l'attenzione alla bassa Italia. Scoppiata l'insurrezione in Sicilia (primi d'aprile del 1860) Garibaldi ri sbarcò coi suoi Mille senza grandi difficoltì e sovvenuto segretamente dal Piemonte riuscì col concorso delle popolazioni ad impadronirsi dell'isola. Presa anche Napoli e costrelto il giovane re Francesco II alla resa della fortezza di Gaeta, Garibaldi, dopo di aver istituito dappertutto governi provvisori, si avvicinò colle sue schicre ai confini meridionali dello Stato pontificio.

La prospettiva che anche il territorio di Roma avesse da ricadere in mano di un governo repubblicano spaventò Napoleone III, il quale si mise per ciò d'accordo con Cavour affinchè truppe sarde occupassero le Marche e l'Umbria pontificia e da lì passassero nel Napoletano, onde sostituire alla diltatura di Garibaldi un gorerno monarchico più regolato. La cittì di Ronı e il cosidelto patrimonio di San Pietro non doverano venir toceati. Lo truppe papaline osarono affrontare il corpo d'esercito sardo comandato da Cialdini a Castelfidardo, ma furono disfatte. Anche Ancona, dove s'era rilugiato il generale Lamoricière, dovette capitolare pochi giorni dopo. Dopo di ciò anche l'occupazione di Napoli fu impresa di breve tempo. I plebisciti proclamarono l'annessione del regno delle Due Sicilie e della maggior parte dello Stato pontificio.

I combattimenti di Castellidardo e l'Ancona, ol'a l'icordati, sono per la storia d'Italia episodi secondari; per quella dell'Arliatico hanno invece importanza ritpitale, perchè proprio duel piccolo tratto di mezzo della costa occidentale dell'Adriatico, love aveva av'ulo origine il potere temporale dei Papi, servi da ponte di conginn- 
zione per la ricostituzione politica della penisola italica c per la rigenerazione dell'Adriatico. - Fatta eccezione di Roma e del suo territorio, di Venezia e dell'Istria, del Trentino e del Canton Ticino, di Nizza voluta da Napoleone e per ciò ceduta alla Francia, della Corsica, di Malta e di Pelagosa, della repubblica di San Marino e del principato di Monaco, quasi tutta l'Italia geografica era ormai in possesso del re di Sardegna, il quale, per volere del parlamento italiano, assunse il titolo di re d'Italia (14 marzo 1861).

Superato appena, se anche solo in parte, felicemente un problema, quello dell'unità, i patriotti italiani si trovarono di fronte ad un altro scabrosissimo, quello della capitale. Secondo l'opinione di tutta la nazione questa non poteva essere che Roma. Ma l'opinione pubblica francese, che vedeva di mal'occhio sorgere uno Stato italiano vigoroso, impediva di toccar Roma e la teneva sempre presidiata da milizie francesi. D'altronde anche l'ostilità delle potenze che, eccettuata l'Inghilterra, non avevauo riconosciuto il regno d'Italia, consigliavano di agire con prudenza. - Cavour cercò di venire ad un accomodamento pacifico col Papa colla formula di una "Chiesa libera in libero Stato", ma le sue speranze riuscirono vane di fronte all'irreconciliabilità della curia romana. In mezzo a tali maneggi il grande statista morì improvvisamente il 6 giugno 1861 e la sua perdita parve, e fu in parte, irreparabile.

I suoi successori, senza riguardo al partito cui appartennero, ebbero il merito di non lasciarsi strappare la di"czione degli avvenimenti dal partito d'azione e di aver. conservato le simpatie personali e l'aiuto di Napoleone III. Non potendo aver Roma, rivolsero le mire al resto dell'alta Italia. Nel 1865 Lamarmora quale presidente dei ministri incanminò trattative coll'Austria per la cessione del Veneto verso un alto compenso pecuniario, ma Viemna le respinse. 


\section{L'AUSTRIA TRA IL '59 E IL'66.}

In Austria l'assolutismo applicato con eccessiva durezza non aveva fatto altro che inacerbire maggiormente gli animi. Di ciò si avevano prove abbastanza evidenti dopo la guerra del ' 59 . I ministri reazionari dovettero dare le dimissioni e furono eletti dei nuovi, che apersero trattative coi rappresentanti popolari. Ma non si potè venire ad un accordo tra governo c popoli. Per ciò l'imperatore Francesco Giuseppe I si risolse di accordare ai suoi dominii un governo costituzionale, il cosidetlo diploma d'ottobre del 1860.

Bastò questo embrione di statuto, perehè gli appetiti nazionali dei popoli dell'Austria, a lungo repressi, si scatenassero con straordinaria veemenza. Delle tendenze nazionali degl'Italiani, dei Magiari, dei Tedeschi, abbiamo avuto oceasione di parlare. La costituzione del "60 sciolse le brame anche degli Slavi, e non solo di quelli ilel settentrione, Boemi e Polacchi, ma anche di quelli dicl mezzogiorno, Croati, Sloveni e Serbi. - Si domandò la ricostiluzione del preesistito triregno di Croazia, Slavonia e Dalmazia, al quate doveva essere annessa anche la città di Fiume. Il panslavismo seese giù come min folata e travolse gli animi degli Slavi dell'Arliatico, già riscaldati dal contatto cogl'Italiani e speciahmente dagli esempi contagiosi dell'università di Padova, che molti studenti avevano disertato per seguire le orme di Garribaldi.

Tale statuto fu accollo di conseguenza slavorevolmente dagli. Ungheresi e dai Tedeschi, che da una riorganizzazione federativa con prevalenzal deghi Slavi nello Stalo temevano per la loro egenronia. - Per ciò il diploma lu sostituito dalla cosidetla patente di febmraio del 1861, con cui anche l'Austria cutrò delinilivamente nel novero degli Stati costituzionali.

Ma neppur questa chbe l'approvarione generale. I partigiani dell'assolulismo, ch'erano numerosi nell'esercito e 
nella burocrazia, e i sostenitori delle idee feudali e clericali pronosticavano addirittura la fine della vecchia Austria. Gli Ungheresi, che ambivano l'autonomia, l'osteggiarono senz'altro. Venezia, l'Istria e la Croazia non mandarono i loro rappresentanti. Per risolvere il contrasto l'imperatore sospese ncl 1865 la costituzione.

Ma oltre ai travagli costituzionali e nazionali interni e alla questione italiana sempre aperta, l'Austria aveva da salvaguardare la sua posizione a settentrione nella Germania. Nel 1863 credette venuto il momento per riguadagnare una posizione direttiva nella confederazione germanica; ma i tentativi andarono falliti per l'opposizione della Prussia. Da qui nacquero gravi attriti; e la discordia andò tant'oltre che nel 1865 la Prussia sentendosi ormai forte della mano di Bismarck e del suo esercito dichiarò sciolta la confederazione e presentò un nuovo disegno con esclusione dell'Austria. Indi la guerra tra la Prussia e l'Austria.

\section{LA GUERRA ITALIANA DEL '66.}

L'Italia, che spiava il momento favorevole per allontanare gli Austriaci dal Veneto, si associò quale alleata della Prussia.

In questa guerra l'Italia era numericamente superiore all'Austria tanto per terra quanto per mare, anzi specialmente per mare.

Sebbene l'Austria possedesse da quasi cinque secoli Trieste, il cui porto mette in comunicazione il centro della monarchia col mare Adriatico, pure, a causa di Venezia, questa città non aveva potuto raggiungere importanza come porto e per ciò anche non si era sviluppata una vera marina da guerra (flotta) austriaca. Appena nel 1798 e poi nel 1814 col possesso di Venezia l'Austria potè promuovere la sua attività sul mare, ma nel 1848 con Venezia perdette nuovamente quasi tutta la sua flotta.

Nel 1856 dopo molte incertezze sul luogo più conveniente per formare un porto moderno di guerra (essen- 
dovi buone ragioni anche per Muggia, Pirano, Sebenico e Cattaro) fu scelta Pola e nel 1861 fu posta la prima pietra dell'arsenale. Nei tre lustri, dal '50 al '65, l'Austria aveva dato notevole e regolare sviluppo alla sua marina da guerra, che infatti fece buona prova a Lissa, specialmente per il valore personale di Tegethoff.

Da parte italiana invece, se era stata fatta l'Italia e se era stata messa insieme una flotta di gran lunga più numerosa e tecnicamente piì forte dell'Austria, non erano purtroppo fatti ancora gl'Italiani. La flotta italiana mancava di omogencità ed era composta di squadre scisse da pregiudizi regionali e da gare personali. Così, male preparata e peggio condotta dal Persano, la flotta italiana si presentò nella infausta giornata del 20 luglio 1866.

Una guerra per il possesso di Venezia non poteva essere combattuta lontano dall' Adriatico. L' ambasciatore germanico a Roma conte Usedom suggerì al Lamarmora il noto piano "di colpire l'Austria nel cuore»: con un'ardita offensiva fino nell'Ungheria e nell'interno dell'Austria. Il Lamarmora preferì di avanzare con due corpi d'armata alla destra e alla sinistra del Po e di passare forzando il quadrilatero. Ma a Custoza (24 giugno 1866) fu battuto dall'arciduca Alberto che aveva saputo scegliere posizioni migliori. Lamarmora dovette abbandonare il piano dell'ofiensiva.

Frattanto i Prussiani riportavano il 3 luglio la vittoria decisiva di Königgrätz in Boemia. L'imperatore Francesco Giuseppe I cercò la mediazione dell'impesatore francese, che ancora godeva una posizione dominante in Europa, al quale offerse il Veureto, e Napoleone III, geloso dei successi delle armi prussiane, accettò. - Ma il ministero italiano Ricasoli per non venir meno ai patti colla Prussia continuò le operazioni militari. Cialdini passò il Po e veme senza colpo lerire fino alle fortificazioni di Venezia. Garibaldi avanzava sulle Alpi e gì̀ gl'Italiani sperando di conquistare anche il Trentino e l'Istria chtrarono in questi due paesi. Per risolvere la guerra occorreva un'azione decisiva sul mare, e per ciò fu dato 
ordine al Persano, che finora era stato inoperoso nelle acque d'Ancona, di attaccare e distruggere la flotta austriaca internata a Pola. Ed egli andò.... a Lissa. La vergognosa perdita di questa battaglia provocò un violento malcontento in tutti gl'Italiani, producendo anche una depressione degli animi. Per consiglio di Bismarck il governo italiano conchiuse l'11 agosto un armistizio e ritirò le truppe dal Trentino e dall'Istria.

Nella pace conchiusa poco dopo a Praga l'Austria si obbligò verso la Germania, oltre al pagamento dell'indennitì di guerra, ad uscire dalla Confederazione germanica. La pace coll'Italia fu conchiusa a Viemna e in essa fu riconosciuto all'Italia il possesso di tutto il regno Lombardo-Veneto, che Napoleone aveva già ceduto a re Vitlorio Emanuele, però entro i suoi attuali confini c verso l'assumzione da parte dell'Italia di 35 milioni di fiorini del debito dello Stato austriaco.

Così l'Austria perdette per sempre quell'egemonia in Italia e in Germania che aveva conquistato nel 1815 e riconquistato nel 1849.

Ma anche l'Italia non aveva potuto coronare col successo tutti i suoi sforzi. Le conseguenze della sconfitta di Lissa furono e sono gravissime per l'Italia e per la sua posizione nell'Adriatico. Una vittoria l'avrebbe portata di botto in possesso delle regioni geograficamente e storicamente italianc più contrastate, non molto grandi, ma strategicamente importantissime, il Trentino e l'Istria, e anche di tulta l'eredità materiale e morale di Venezia sull'Adriatico. Con una vittoria a Lissa sarebbe stato tagliato corto a tutte le velleità di espansione sull'Adriatico del germanesimo, dello slavismo, degli Ungheresi. La rientrata dell'Italia nell'eredità della Serenissima sarebbe apparsa allorá la cosa più naturale del mondo e si sarebbero evitati attriti e lotte per il futuro. L'imperizia del Persano ripiombò l'Italia nello scoraggiamento e nella dipendenza strategica dell'Austria, che dai due speroni avanzati del Trentino e dell'Istria minaccia e domina collo spauracchio del suo esercito, rinforzato là dal germanesimo, qua dallo slavismo, quel bacino adriatico 
che per legge geografica non può avere che un solo dominatore, gl'Italiani.

La lotta epica e secolare tra il germanesino e la latinità, che nella lunga linea di contatto s'era spinta in giù fino all'Adriatico, avrebbe potuto essere chinsa a Lissa; e invece rimase malauguratamente aperta. Giacrchè il movente recondito delle lotte che ancora dall'epoca del primo Napoleone l'Austria sosteneva contro i Francesi e gl'Italiani era la tendenza della prima ad allargarsi sull'Adriatico $e$ dei secondi a respingerla. Fino dal secolo XVIII il germanesimo da questa parte cra vincitore. $\mathrm{Fu}$ battuto per breve tempo da Napoleone col regno d'Illiria, indi fu respinto fino all'Isonzo. La lotta naggiore e forse definitiva fu ancora una volta rimandata ad un'epoca futura.

\section{CONDIZIONI INTERNE \\ DELL'ITALIA E DELL'AUSTRIA DOPO IL '66.}

Siccome colla guerra del '66 si chiude rispelto a noi il periodo delle guerre reciproche tra l'Austria e l'Italia e si potrebbe dire anche delle conseguenti complicazioni guerresche di questi due Stati coll'estero per l'Adriatico durate mezzo secolo, sarà opportuno di dire alcune parole sulle loro condizioni interne e sulla loro nuova posizione rispetto all'Adriatico.

Coll'acquisto del Veneto gl'Italiani potevano dire di aver raccolto le parti vitali delle loro "membra sparte». Restava sempre da risolvere la quistione di Ronna capitale. Il partito d'azione, impaziente, insisteva per una pronta soluzione. In conformitì alla convenzione di settembre appena si formo un esercito papalino, il presidio francese abbandonò Roma e lo Stato pontificio. Per la prima volta dopo molti secoli il stolo dell'Italia antical poteva dirsi quasi libero da milizie straniere. Il governo italiano si sarebbe contentalo di questo successo e inlatvolò nuovamente trattalive colla curia romana per un componimento pacifico; ma i tentalivi lurono acculti con 
ruvida repulsione. Allora il partito d'azione ricorse ai latti e Garibaldi coi suoi volontari entrò nello Stato del Papa. L'Austria questa volta non si moss'e, ma dalla Francia un corpo d'esercito rioccupò militarmente lo Stato del Papa. La sconfitta delle armi garibaldine a Mentana e l'appoggio poco velato con cui il ministero Rattazzi aveva favorito l'impresa garibaldina, peggiorarono la posizione dell'Italia di fronte alla Francia, che poteva lagnarsi della rottura della convenzione. La conseguenza di tutto ciò fu che l'Italia cadde ancora più alla dipendenza della buona volontà di Napoleone.

Il pessimo stato delle finanze, il deficit enorme, la geIosia dei capipartito, l'antagonismo fra mezzogiorno e settentrione, l'indolenza di una parte della popolazione, rendendo difficile un pronto consolidamento del nuovo Stato, depressero l'entusiasmo degl'Italiani, che accorgendosi di dover intraprendere un lavoro colossale di rinnovamento interno vi si accinsero, ma trascurarono per ciò la politica estera. In quest'opera di riorganizzazione si distinsero ancora una volta i piemontesi (il vecchio partito della consorteria) che si dimostrarono i più capaci a continuare la redenzione morale d'Italia.

Tra gli organismi che avevano bisogno di una riorgaLa marina uizzazione c'era la flotta. L'infelice battaglia di Lissa aveitaliana. va mostrato la necessità di rinnovare la marina italiana dalle fondamenta. L'Italia aveva messo in mare alcune navi corazzate, ${ }^{1}$ ). ma la maggior parte erano ancora in legno. Per ciò ne furono vendute trentadue e costruite delle nuove. Di pari passo colla rinnovazione della flotta andarono i lavori portuari in generale e quelli della costruzione di basi navali militari in particolare. Per ragioni geografiche e storiche gl'Italiani rivolsero l'attenzione a quello dei loro tre mari ch'è il più importante, il Tirreno, e mediante il quale essi devono curare la posizione di grande potenza mediterranea. Già nel 1861 posero le basi del massimo porto di guerra alla Spezia.

1) Le prime navi corazzate elano state usate in Francia per cura di Napoleone III dopo la guerra di Crimea (1854) e di poi con nuovi progetti verso il 1858 . 
Nell'Adriatico invece, quasi volessero distogliere gli sguardi dalla ferita sanguinante di Lissa, non solo non fecero quasi nulla per fortificarsi militarmente nei tre unici porti naturali di Venezia, Ancona e Brindisi, ma non si curarono nemmeno di rialzare le sorti economiche di Venezia e di migliorare le condizioni nautiche dei molti porti secondari. La bandiera da guerra italiana si mostrò poco sull'Adriatico e osservò sempre un'astensione misteriosa riguardo ai porti dell'altra sponda, che furono visitati solo da navi a vela e da qualche vapore della defunta società Florio-Rubattino.

Quest'astensione degl'Italiani dall'Adriatico - se non fu un crrore - fu una fatalità, perchè permise all'Austria di conservarvi il primo posto.

Il cattivo stato delle finanze anche dell'Austria persuase i suoi reggitori dopo la guerra del '66 a mutar rotta e a concedere le tanto sospirate riforme costituzionali, sołddisfacendo in pari tempo le domande nazionali in senso federalistico.

Il ministro Belcredi aveva concepito il piano di frazionare tutti i territori dell'Austria in cinque regni, retti a sistema feudale e uniti solo dalla persona dellimperatore. Contro questo progetto che avrebbe dato il predominio agli Slavi (Boemi, Polacchi e Croati) insarsero i Tedeschi e i Magiari, che avevano ancora in mano il potere e non volevano rinunziarvi.

L'imperatore Francesco Giuseppe I comprese che gli conveniva risolvere quello stato di cose e contentare gli Il dualismo in Austria. Ungheresi. Così per salvare lo Stato da ulteriori scosse a causa delle piccole nazionalità si formò il compromesso tra l'Austria e l'Ungheria e si ricorse al cosidetto dualismo. Secondo questo fu assicurata ai Tedeschi l'egemonia in Austria e ai Magiari in Ungheria. Allo scopo di frantumare le nascenti velleità nazionali dei Croati, quale avanguardia dello slavismo serbo-balcanico e del panslavismo, la Dalmazia fu assegnata al governo di Vienni, Fiume a quello di Budapest. Invano i Croati elevarono la voce per la ricostituzione del loro antico triregno. II governo tollerò che la prima rappresentanza della Dahına- 
zia, riuscita italiana ed animata dal desiderio di conservare l'autonomia amministrativa per tutelar meglio la propria nazionalità, che incominciava ad essere minacciata, si astenesse dal mandare delegati alla dieta di Zagabria. Ma quello che nel momento parve un appoggio del governo in favore degl'Italiani non era che una manovra politica, perchè il contegno del governo, come si dirà tra breve, mutò di lì a poco in senso diametralmente opposto.

intanto la legislazione e l'amministrazione interne furono divise e condotte indipendentemente per l'Austria, come "paesi rappresentati al consiglio dell'impero", per l'Ungheria come paesi della corona ungarica. Al contrario si volle mantenere intatta la rappresentanza all'estero e l'unità nell'esercito, nella marina, nelle finanze e provvisoriamente anche nelle relazioni commerciali. D'allora in poi la monarchia, divisa nell'amministrazione interna in Cisleitania e Transleitania, viene chiamata Austria-Ungheria. La legislazione vigente in Austria si basa sulla costituzione di febbraio del 1861, completata dalle leggi del '67; in Ungheria invece è in vigore l'antica costituzione di quel paese, modificata dalle leggi del ' 48 .

Il costituzionalismo concesso dopo tanta attesa in dosi minute e con pregiudizio di molte aspirazioni nazionali doveva necessariamente produrre nuovi malcontenti. E infatti i ministeri austriaci ebbero da lottare per parecchi

Gli Slavi. anni coll'opposizione degli Slavi. I Boemi pretendevano una costituzione speciale per i paesi della «corona boema» (Boemia, Moravia e Slesia); i Polacchi domandavano la completa autonomia della Galizia, gli Sloveni la creazione d'un regno sloveno. I deputati dell'Istria o si astenevano di presentarsi al parlamento oppure se vi comparivano stavano dalla parte dell'opposizione. In Dalmazia scoppiò nel 1869 la sollevazione dei Bocchesi nel Crivosie (montagne al di sopra di Risano) che esentati in origine dal servizio militare nell'esercito, non volevano assoggettarsi alla nuova legge, che areva creato ic milizie della Landwehr, in cui per una finzione burocratica, avrebbero dovuto ora servire anch'essi. 
In mezzo a tanti trambusti l'Austria-Ungheria (che per brevità continueremo a chiamar talvolta scmplicemente Austria) s'accorse che la sua posizione nell'Emopa dal '66 in poi s'era cambiata. Senza rinunziare del tutto alla speranza di riacquistare eventualmente col favore della sorte le posizioni perdute in Germania e in Italia essa comprese molto bene che gli avvenimenti del futuro l'avrebbero orientata verso i Balcani. La Dalmazia, e con essa il confine più delicato dello Stato, penzolava al mezzogiorno come una funicella attaccata alla monarchia da un solo capo. Tra i popoli dell'Austria c'era un numero considerevole di Slavi: Slavi erano nella maggioranza anche i popoli della penisola balcanica. Dopo il duello contro la latinità combattuto colla Francia e cogl'Italiani, l'Austria doveva prepararsi a sostencrne un altro cogli Slavi e colla Russia.

Malgrado Ia viltoria di Lissa l'Austria cble il presentimento che il somo degl'Italiani non sarebbe stato di lunga durata; c mutò politica la questa parte. Senza divenire slavofila, incominciò a fare politica slava. Fino al ' 66 per non alienarsi maggiormente gl'Italiani favoriva il loro elemento sull'Adriatico, anzi se ne servì per reprimere i primi moti di panslavismo, dietro il quale temeva la Russia. Dopo Lissa, per impedire che anche la costa orientale dell'Adriatico divenisse un giorno italiana, decise che doresse diventare per ora slava, ma in contrapposto alla politica slava balcanica della Russia. E incomineiò dalla Dalmazia dove la minoranza numerica degl'Ttaliani, danneggiali socialmente dal costituzionalismo, rendera più facile l'esecuzione rapida del capitombolo della politica. Che questo piegamento verso il croatisino non fosse altro che una manovra lo prova il fatto che a Fiume, dove dal ' 18 al '67 i Croati averano guadagnato tereno, fu invece lavorito l'elemento italiano.

Lissa nel '66 e il dualismo austro-ungarico col compro-

Importanza dell'Adriatico per l'Austria. messo ungaro-croato nel '67 dovevano aver tagliato le ali nello stesso tempo all'iredentismo italiano ed a quello croato.

D'ora in poi nella coscienza dei governanti dell'Au- 
stria-Ungheria l'Adriatico divenne il pezzo più geloso di confine :

a) perchè essendo l'unico tratto di mare, la sua conservazione era una condizione indispensabile per l'esistenza di grande potenza;

b) perchè questa via del mare era la migliore per partecipare coi prodotti dell'industria al commercio del mondo:

c) perchè l'Adriatico è la via geografica più breve traa il centro dell'Europa e l'Oriente;

d) perchè proprio la sua costa orientale, connessa alla penisola balcanica, veniva a costituire una parte capitale nel nuovo orientamento della politica austro-ungarica.

E infatti l'Austria-Ungheria vi rivolse cure sempre maggiori, sviluppando la marina tanto da guerra che quella mercantile. Per diversi lustri la bandiera austriaca fu la dominante incontrastata sull'Adriatico, compresi i porti della costa occidentale.

\section{LA GUERRA FRANCO-PRUSSIANA DEL 1870. ROMA CAPITALE D'ITALIA.}

Per cause estranee all'Adriatico avvenne nel 1870-71 il duello tra Napoleone III e Bismarck, ossia la guerra franco-germanica coll' esito che tutti sanno. In conseguenza delle vittorie della Prussia la confederazione germanica si estese anche alla Germania del sud. La confederazione assunse quindi il titolo d'impero tedesco e il re di Prussia ne divenne l'imperatore (18 gennaio 1871). Così compivasi l'unità della Germania a base nazionale ed era posto il coronamento ad un'orientazione del tutto nuova nella politica europea. La Germania era ora più grande, più solida e più forte dell'Austria e venne ad occupare il di lei posto nel settentrione d'Europa.

Eppure anche quest'avvenimento ebbe una duplice ripercussione sulla sorte dell'Italia e dell'Adriatico.

In primo luogo con esso fu sepolto per sempre il so- 
gno del sacro romano impero della nazione tedesca, sostenuto negli ultimi secoli dalla famiglia d'Asburgo e col cui pretesto questa s'era ingerita anche troppo negli affari dell' alta Italia, ch'è contemporaneamente il bacino superiore dell'Adriatico.

In secondo luogo la guerra del 1870 portò un'insperata fortuna al coronamento dell'unità italiana. Là Francia fu costretta a richiamare da Roma le milizic destinate alla sicurezza del Papa, e il governo italiano occupò Roma (20 settembre 1870) ponondo fine al dominio temporale dei Pontefici.

L'epilogo era tanto logico, naturale, inevitabile, che si effettuò quasi come una quistione interna italiana e il mondo non se ne commosse che a parole.

In quanto all'Adriatico, siccome le Romagne e le Marche erano già incorporate al regno d'Italia, la caduta del potere temporale del Papa non vi produsse alcuna ripercussione diretta. Il fatto però ha un valore indiretto non indifferente anche per il nostro mare, perchè per la prima volta dalla caduta della romanità l'Italia e la costa occidentale erano reintegrate in un corpo solo e l'energia che non poteva mancare di svilupparsi era destinata ad espandersi, come era già avvenuto una volta nella sua storia, anche al di là dell'Apennino tra le Alpi e i paesi carsici lungo il terzo mare d'Italia.

L'Europa e la civilta colla costituzione dei regni d'Italia e di Germania ebbero ordine e pace al mezzogiorno e al settentrione. Restava da regolare ancora un vasto paese ad oriente, la penisola balcanica.

\section{SULL'ADRIATICO ORIENTALE.}

L'assetto politico di questa penisola la per l'Adriatico La penisola anche un'importanza capitale, perchè sebbene sia bagnahalcanica. ta nella sua parte maggiore da due altri mari, l'Egeo e il Jonio, nella parte minore tocca l'Adriatico. Anzi, come fu giả dimostrato, il disordine politico dei Balcani inllui sinistramente sull'Arlriatico.

L'Adriatico. 
Solo mezzo secolo fa la penisola balcanica era ancora dominata dai Turchi. Però la loro decadenza, che cresceva rapidamente, invogliò lo zar Alessandro II di Russia a tentare un'altra volta di mandar ad effetto quello che si dice fosse il testamento politico di Pietro il Grande: aprirsi un varco al mezzogiorno, cacciando i Turchi dall' Europa e conquistando Costantinopoli con buona parte della penisola balcanica.

I Turchi stessi prestavansi, a così dire, all'esecuzione di tali disegni. Il loro odio contro i cristiani rendeva vana l'emancipazione pacifica di questi ultimi; onde i cristiani insorsero più volte, ma le loro ribellioni furono punite col rigore più crudele.

Naturalmente i popoli più ostinati nelle ribellioni erano quelli che stavano alla periferia dell'impero turco, come più lontani da Costantinopoli, e fra questi i Bosniaci, che essendo a contatto e spalleggiati da un paese civile come l'Austria-Ungheria, potevano all'occorrenza rilornirsi e rifugiarsi oltre i confini turchi. Inoltre le popolazioni della Bosnia, in prevalenza serbe, ricevevano soccorsi dai loro connazionali dei principati di Serbia e Montenegro.

Così, approfittandosi dei torbidi dinastici di Costantinopoli, avvenne nel 1876 un'insurrezione bosniaca, cui si unirono Serbia e Montenegro. Ma i Turchi forti ancora militarmente riportarono la vittoria.

La guerra rus- Sorse allora in difesa dei cristiani e Slavi lo zar di Russo-turca del $1877 \cdot 78$. sia, che nell'aprile del '77 dichiarò la guerra alla Turchia. Associatasi alla Russia anche la Rumenia i Turchi soggiacquero, malgrado la valorosa difesa, al numero superiore di milizie di cui disponevano gli avversari. Questi dopo la capitolazione di Plevna valicarono i Balcani, conquistarono Adrianopoli e minacciarono Costantinopoli. - L'Europa fu presa dallo spavento di vedere l'oriente russificato, e la gelosia spinse tosto l'Inghilterra spalleggiata dall'Austria e dalla Germania, press'a poco come nella guerra di Crimea, a prendere le parti dei Turchi per non lasciar cader Costantinopoli nelle mani dei Russi. - Ma la Russia evitò la lotta concludendo 
col sultano la pace di Santo Stefano (17 marzo 1878) nella quale fu riconosciuta l'indipendenza della Rumenia, della Serbia, del Montenegro, che ottennero pure degl'inglandimenti territoriali. La Bulgaria doveva godere l'autonomia pagando un tributo.

Anche questo terzo sforzo della Russia di imporre sè e lo slavismo all'Europa, era stato vinto dall'Europa coalizzata. Se la Russia fosse riuscita nel suo intento in questo o nei secoli precedenti, forse avrebbe russificato i molti popoli slavi minori della penisola balcanica, privi allora di una marcata coscienza nazionale per mancanza di coltura e quindi il panslavismo russo sarebbe stato davvero imponente e pericoloso per gli altri popoli d'Europa. Siccome invece la geografia aveva frazionato durante i tempi di mezzo la compattezza slava costringendo ogni frazione di quel popolo a vivere da solo ed a differenziarsi per conseguenza dagli altri, e la reazione degli altri popoli non slavi aveva respinto le prime velleità d'ingrandimento smisurato della Russia, il pericolo maggiore del panslavismo poteva dirsi superato.

Però se era scartato il pericolo russo, non si poteva cancellare lo slavismo. Era ormai evidente che la spartizione della Turchia fra le potenze maggiori era "impossibile per la reciproca gelosia. Quindi sotto il pretesto della emancipazione graduale dei popoli con delimitazione geografico-nazionale, fu trovato il ripiego di creare quegli staterelli nazionali bisognosi dell'appoggio esterno. La gelosia dei grandi pensò a seminare il seme cattivo della zizzania sotto forma di protezione, più o meno interessata, dei piccoli. Si può però ritenere che con ciò sia stata rotta la fibra al panslavismo. Gli Slavi polranno civilizzarsi, evolversi, divenire forti e potenti quanto si voglia. Ormai ogni sehiatta laa ma propria caratieristica ed è costretta a cercare salvezza sopratutto nelle propric forze. Quanto maggiore sarà lo sviluppo civile degli Slavi, tanto maggiore sarà la differenziazione e quindi lostacolo a tentativi di nuova fusione. Gli Slavi furono avviati sulla slessa strada dei Francesi, degl'Italiani, de- 
gli Spagnoli, ecc., degl'Inglesi, dei Tedeschi, ecc. memori gli uni dell'origine latina, gli altri germanica, ma divisi in molte nazionalità geografiche, piuttosto antagoniste che alleate.

Il Congresso di Berlino.
La pace di Santo Stefano fu però assoggettata ad una ratificazione e questa avvenne a Berlino nel 1878, ove si riunirono a congresso per iniziativa dell'Austria i rappresentanti delle principali potenze europee sotto la presidenza di Bismarck.

Il congresso di Berlino, oltrechè importanza in sè medesimo per le deliberazioni che vi furono prese, ha un valore speciale essendo una piattaforma sulla quale la diplomazia aveva regolato le sorti politiche della penisola balcanica.

I principi di Serbia e Rumenia assunsero il titolo regale. La Rumelia orientale, quale provincia autonoma, fu staccata dalla Turchia e assegnata alla Bulgaria. Alla Grecia fu promesso un ingrandimento al confine settentrionale, che infatti le fu concesso l'anno seguente colla cessione di alcuni distretti dell'Albania e della Tessaglia. L'Austria poi ebbe il mandato di occupare la BosniaErzegovina - ma di ciò diremo più in dettaglio. Il congresso di Berlino corresse in sostanza la pace di Santo Stefano col restringere alquanto le perdite della Turchia e col limitare l'influenza russa nei Balcani a vantaggio dell'Austria-Ungheria. Colle decisioni del congresso di Berlino stanno poi in nesso anche alcuni minori acquisti territoriali, proprio sulla costa adriatica, generalmente meno noti e che per ciò devono venir segnalati.

Il Montenegro, sul cui piccolo trono sedeva l'accorto e valoroso principe Nikita, s'era impigliato nella guerra colla Turchia per ingrandirsi dalla parte d'oriente, giacchè non poteva da quella opposta. I pacsi al suo oriente spettano veramente alla nazionalità albanese, ma quando si tratta della lotta per l'esistenza non si bada ai sentimentalismi. I Montenegrini avevano così conquistato tre paesi al mare e precisamente Spizza, Antivari e Dulcigno. Ma l'Austria che, come al principio del secolo ed anche in seguito ha l'interesse politico di tenere lo sla- 
vismo lontano dall'Adriatico, vi si oppose e volle per sè Spizza, che le fu anche assegnata. Dopo molte discussioni Antivari e Dulcigno finirono col passare al Montenegro, che così ebbe raggiunto finalmente l'Adriatico.

La Bosnia sta non solo conficcata come un cunco L'occupazione tra la Slavonia, pertinente politicamente all'Ungheria, e la Dalmazia formante parte dei paesi austriaci, ma come fu già rilevato nella storia dell'epoca veneziana, pcnetra fin dentro nel corpo della Dalmazia, quale fu ricostituita dall'Austria al congresso di Vienna, colle due appendici di Klek e Sutorina e la sminuzza in tre parti. Essa è abitata da popoli linguisticamente e nazionalmente affini a quclli dei paesi finitimi austro-ungarici.

La propaganda serba in Bosnia e montenegrina in Erzegovina aveva già dato segni di vita altiva e ciò non poteva garbare all'Austria che incominciò a vedere in quei paesi una polvericra, dalla quale l'incendio nazionale slavo avrebbe potuto troppo facilmente dilatarsi nei suoi territori. D'altronde il possesso della Dalmazia, posta com'era attaccata geograficamente non all'Austria ma all'Ungheria e accessibile senza toccarc suolo ungherese, solo per mare, era troppo labile. Per togliere di mezzo il golfo troppo slavo, cine s'insimuara nei suoi territori, tagliare la testa alla propaganda serba, assicurarsi il possesso duraturo della Dalmazia coll'attaccarla al suo rectroterra e, quello che più importava ai nuovi fini della sua politica, consolidarsi meglio sull'Adriatico, l'AustriaUngheria fece quello che avrebbe tentalo qualunque altro Stato, che si fosse sentito in forze sufficenti - prevenne il pericolo e volle incatenarc a sè le due province che mostravano tanta voglia di sottrarsi ai Turchi. A tale scopo alimentö secretamente l'insurrezione e lor\%ò ghli avvenimenti. Ma il congresso di Berlino, che non fu capace di prendere che mezze misure, contento l'Austria solo in parte concedendole l'occupazione ma colla salvaguardia dei diritti di sovranità del sultano. L'Austria, che badara più alla sostanza che alle apparenze, entrò nelle due prorvince, agguerrita militarmente, e vinta la resistenza dei mussulmani, le occupò nel termine di tre mesi, aspellan- 
do che venisse l'occasione propizia per annettersele definitivamente.

Questo successo estero poco rumoroso, ma rilevante dopo gli scacchi subìti negli ultimi anni nella politica germanica e italiana, rialzò il prestigio e il morale della monarchia, offrendole un compenso sufficente, se non equivalente, di due altre province in luogo del LombardoVeneto e valse particolarmente a confortare il suo amor proprio, mostrandole una nuova via alla politica imperialista.

Orientamento dell' Austria verso i Balcani.

Ogni ingrandimento degli Asburgo, più che l'effetto di un'espansione per esuberanza di vita, fu uu accorto impicgo della propria forza a danno di vicini pericolosi, ma più deboli, onde prevenire la formazione di Stati nemici e rivali. Questo era stato il movente che li aveva allettati ad immischiarsi dal secolo XVI in Germania e dal XVIII in Italia. Chiusa ora in un cerchio insormontabile a settentrione dalla Germania e ad occidente dall'Italia, l'Austria non ebbe aperta che la via dell'oriente verso i Balcani e vi si avventurò colla meta verso Salonicco per controbilanciare e contrastare la marcia della Russia su Costantinopoli.

Alla concezione di tale piano contribuirono certamente lo sfacelo del dominio turco in Europa e lo stato d'inferiorità politica in cui si trovarono fino a qualche anno fa gli Slavi. La dimostrazione di tale inferiorità e delle sue cause sarebbe lunga e fuori di posto. Però è un fatto innegabile che dei tre gruppi etnici principali dell'Europa, Latini, Germanici e Slavi, questi ultimi diedero il minor contributo al progresso della civiltà. I Tedeschi tanto in Austria quanto in Germania ed i Magiari in Ungheria tennero e tengono in parte ancora da secoli sottomessi gli Slavi Ioro vicini.

Nella marcia verso i Balcani si trovarono d'accordo tanto l'Austria, ossia i Tedeschi, quanto l'Ungheria, ossia i Magiari, giacchè tutti due avrebbero molto da temere da un ingrandimento della potenza slava in Europa. Ma i più fervidi nell'impresa si mostrarono i Tedeschi, che, spalleggiati dai loro connazionali della Germania, 
inaugurarono quella politica di espansione, per il momento di forma piuttosto economica ed industriale, ch'è conosciuta coll'espressione di "Drang nach Osten" ossia la spinta verso l'oriente.

Per tener aperta la strada di Salonicco l'Austria l'anno seguente a cuello della sua entrata in Bosnia, ossia nel 1879, occupò con presidi militari il Sangiaccato di Novibazar, regione lungo il corso del fiume Lim (confluente della Drina) clie separava la Serbia dal Montenegro.

Nello stesso anmo fu conchiuso per opera di Bismarck un traltato di alleanza fra la Germania e l'Austria, che nei suoi efletti innalzò ancora più l'egennonia militare tedesca in Europa, assicurò l'Austria, sempre in subbuglio all'interno, da pericoli esterni, ma la legò a rimorchio della Germania, cosa che merita attenzione come segno dell'inversione delle parti tra questi due Stati più potenti dell'Europa centrale c della decadenza politica dell'Austria-Ungheria.

\section{SLAVOFILIA AUSTRIACA SULLADRIATICO.}

L'annessione della Bosnia-Erzegovina confermò l'Austria a proseguire sull' Idriatico nell'orientamento slavofilo inaugurato dopo Lissa e ad accentuare di conseguenza la sua politica antitaliana.

L'esecuzione di questo piano fu facilitata dalle inutate condizioni della politica interna austriaca. Gli Slavi in generale in tutta la monarchia e nelle regioni dell'Adriatico particolarmente costituivano l'elenento campagnolo della popolazione, più numeroso, ma povero ed incolto. Le città della costa, abitate a prol'erenza dagl'Italiani, rappresentavano invece la superiorita della coltura e del censo. Fino a tanto che coll'assolutisino continuavano a sussistere per un rimasuglio anacronistico in parte condizioni sociali di tipo feudale, gl'flaliani cittadini, se anche inferiori di numero, conscrvando il potere, predominavano sugli Slavi campagnoli, anche dal lato nazionale. 
La costituzione diede l'ultimo colpo ai privilegi feudali degli aristocratici e dei ricchi e spostò il potere a favore delle masse. Questa livellazione, benefica ed equa dal lato sociale, fu dannosa all'elemento italiano dal lato nazionale, perchè privandolo del potere, lo portò di botto alla mercè di un elemento inferiore e avversario nazionale. Malgrado questa evoluzione dei poteri civili, che accompagnata dal risveglio politico degli Slavi avrebbe prodotto inevitabilmente anche in caso di passività del governo una lotta con caratteri nazionali piuttosto che sociali, l'elemento italiano, abbandonato anche a sè stesso, avrebbe trovato nella sua superiorità culturale la forza ed i mezzi da conservare l'esistenza nazionale. Ma qui subentrò l'azione del governo, che col favorire ogni e qualsiası aspirazione degli Slavi e col negare c reprimere ogni domanda o reazione degl'Italiani, mutò violentemente la bilancia in favore dei primi. Furono abolite le scuole italiane e sostituite con croate; fu favorita in tulti i modi l'immigrazione slava nelle città italiane; nelle elezioni quello che non potevano fare le manipolazioni secrete ed i soprusi palesi ammantati di legalità fu affidato alle baionette. La difesa degl'Italiani fu necessaria e strenua, ma non giovò ad arrestare la marea slava, mossa appositamente dal governo austriaco lungo la costa orientale dell'Adriatico in direzione da sud verso nord. L'onda del croatismo governativo travolse così rapidamente i municipi italiani dalle Bocche di Cattaro in su e sỉ arrestò al comune di Zara, che gl'Italiani riuscirono a conservare. La Dalmazia, che anche dopo la caduta di Venezia aveva mantenuto nel suo esterno un carattere evidente ed innegabile d'italianità, fu slavizzata in pochi lustri. L'Istria, Trieste, Gorizia si sentirono seriamente minacciate di dover subire a breve scadenza una sorte eguale.

Però a tale contegno l'Austria poteva sembrare spinta dalla politica italiana di quegli anni. 


\section{LA POLITICA ITALIANA DAL 1871 AL 1887.}

La non celata antipatia della Francia verso il giovine regno d'Italia indusse i ministri di questa subito dopo il 1871 ad appoggiarsi nella politica estera alla Germania e indirettamente all'Austria. Però l'azione dell'Italia in mezzo agli altri Stati d'Europa non poteva aver che debole effetto fino a tanto che non fossero state regolate le condizioni interne e specialmente le finanze.

Malgrado contrasti di partito ed errori di ministri le cose procedevano bene. Nell' anmo 1877 il parlamento italiano potè votare quella memorabile legge che stabilì il carattere offensivo della flotta italiana. Così sorsero le possenti corazzale Italia, Lepanto, Duilio, che per anni furono le maggiori del mondo.

Ma le vedute dottrinario-radicali dei ministeri Cairoli e Depretis impressero alla politica estera italiana un altro indirizzo. Essi speravano di poter approfittare degli antagonismi nazionali d'Europa per completare la formazione anche geografica d'Italia e ritenevano di riuscirvi più facilmente qualora si fossero tenuti intanto in disparte quali spettatori senza essere legati con nessun Stato. Essi inaugurarono per ciò quella politica detta delle mani nette, che fidando nell'amicizia della Francia, divenuta repubblicana e radicale, dovera invece farli restare a mani vuote tra anare delusioni.

Quando nel 1878 l'Austria occupò la Bosnia-Erzegovina e' l'Italia uscì dal congresso di Berlino colle mani vuote, l'opinione pubblica italiana non potè nascondere la sua invidia e, non avendo da segnare un altro successo corrispondente, incominciarono da parte della lega per l'Italia irredenta, capitanata da Garibaldi, pubbliche manifestazioni per il passaggio all'llalia del Trentino e dell'Istria con 'Trieste. Queste dimostrazioni rimasero in 
un limite platonico fino alla congiura del triestino Guglielmo Oberdank contro l'imperatore Francesco Giuseppe I (1882). Anche nella quistione d'Oriente l'Italia mostrò l'intenzione di occupare l'Albania. Sembrava da tutto ciò che l'Italia avrebbe intrapreso una politica attiva anche nell'Adriatico, come l'aveva già inaugurata nel Mediterraneo coll'aver posto gli occhi su Tunisi, che per la sua posizione e per la numerosa immigrazione d'Italiani era venuta a cadere nella sfera d'interessi dell'Italia.

Invece un altro fatto inatteso venne a ripiombare gli animi degl'Italiani nella costernazione. L'interessamento del governo di Roma per le regioni dell'antica Cartagine mosse la Francia a fare un colpo ardito, ad occupare cioè la Tunisia (1881) con un futile pretesto e tanto rapidamente che il governo italiano non avrebbe potuto impedirlo altrimenti che con una guerra. Sotto l'impressione di questo fatto che oltre ad umiliare l'amor proprio del giovine regno, sventava la sua naturale e necessaria espansione demografica è minacciava il suo progresso con un pericolo prima non osservato dall'occidente, avvenne nella politica italiana un mutamento radicale e sorprendente anche nella direzione d'oriente; si dimenticò il secolare, storico e ancora vivissimo odio per l'Austria e l'Italia aderì nel 1883 all'alleanza austrogermanica, dando così origine a quel trattato della triplice, che aveva di mira - e difatti corrispose finora allo scopo - di assicurare il godimento della pace ai tre Stati contraenti in prima e all'Europa in seconda linea. Però con esso fu posto un suggello d'immutabilitì all'aspetto politico dell'Adriatico.

Gl'Italiani, che avevano regolato intanto l'amministrazione interna e riordinato le finanze, erano ora impazienti di rifarsi degli scacchi subìti nell'Adriatico (1878) e nel Mediterraneo (1882). Vedendo che tutti gli altri popoli avevano inaugurato con successo una politica estera coloniale furono presi dalla smania di fare altrettanto. Essi si lasciarono così indurre a quell'impresa nel Mar Rosso e in Abissinia (1885) che, lungi dal portar rose, fu per decenni un ginepraio, da cui non colsero che ama- 
rezze e che fece loro dimenticare ancora più i veri e reali interessi nel vicino Adriatico. Appunto in quest'anno e come indice della nuova orientazione politica fu creato in Italia un nuovo porto di guerra, ma a Taranto.

\section{LA TRIPLICE ALLEANZA.}

Gl'Italiani nel loro ardore giovanile di far molto e presto credevano di agire il più delle volte di proprio impulso. Invece la mente fredda e calcolatrice di Bismarck aveva saputo predisporre gli avvenimenti in modo che riuscissero vantaggiosi in primo luogo al popolo germanico.

Per ripicco all'occupazione di Tunisi da parte della Francia, l'Italia un anno dopo aveva acceduto all'alleanza austro-germanica. Nessuno si mascondeva l'ibridezza di questa alleanza coll'Austria, che non poteva aver sinpatie nei popoli e ch'era stata accettata dagl'Italiani come una necessità dura el inevitabile e sperabilmente passeggera. Invece il contegno sempre ostile della Francia la ribadì ancora più. La Francia aveva assmonto verso l'Italia un contegno sempre più minaccioso coll'intenzione evidente di intimorirla e di legarla al suo caro. Le rinfacciava l'ingratitudine, la dameggiava commercialmente e le suscilava contro il clericalismo colle riverdicazioni del potere temporale del Papa. In tali condizioni della politica generale il ministro degli esteri Robilant conchiuse nel 1887 il trattato formale della triplice alIeanza, il cui scopo era di mantenere la pace curopea. Il fine éra certamente lodevole in sè stesso; ma equivaleva alla conservazione dello stulu quo nell'Adriatico, ossia alla negazione delle tradizioni e delle necessiti della politica estera italiana.

Ciò non per tanto la politica triplicista fu continuata anche da Crispi, che cercò di rinsaldare la posizione dell'Italia, appoggiandosi particolarmente alla Germania. Nol mentre duncpue nei primi moti per la ricostituzione d'Ita-

L'Adriatico. 
lia, fino al 1882 , l'inimicizia fra l'Italia e l'Austria lungo tutto il bacino dell'Adriatico era apparsa una fatalità inevitabile, contingenze della politica generale europea mutarono improvvisamente le cose e arrestarono per alquanto tempo quell'evoluzione che, mossa dagli uomini, era stata determinata invece dalla natura intima del progresso civile ed economico dei popoli.

I'Italia doveva essere preparata piuttosto ad un conflitto colla Francia e per ciò furono spese somme ingenti per la riorganizzazione dell'esercito e della flotta. Le manifestazioni irredentistiche furono sconfessate e represse dal governo ed anche fra l'opinione pubblica italiana le velleitì di rivendicazione territoriale sull'Adriatico furono poste a dormire.

Tuttavia i patti della diplomazia furono ancora una volla impotenti a fremare l'evoluzione fatale della storia dei popoli, e lo statu quo dell'Adriatico, conservato a stento e per forza, fu minacciato dalla dissoluzione del regime turco nei Balcani.

\section{ALCUNI FATTI DELLA PENISOLA BALCANICA.}

Abbiamo veduto il lento, ma irresistibile risveglio dei popoli balcanici, accompagnato dal desiderio di liberazione religiosa-nazionale e le tendenze di instaurarvi in un modo o nell'altro l'egemonia dello slavismo ortodosso, suscitate dalla Russia. Però la penisola balcanica non è abitata solamente da Slavi: ci sono i Greci, i Rumeni, gli Albanesi i quali, come gli Slavi, al contatto della civiltà occidentale latino-germanica incominciarono qua e là a solidificarsi.

I consolidamenti avvenuti in quest' epoca riguardano paesi distanti dall'Adriatico e quindi non lo toccano che indirettamente in quanto sarebbero stati capaci di influire sull' assetto complessivo della penisola balcanica. Però non possono venir sorpassati in silenzio. Nel 1881 la Rumenia, nel 1882 la Serbia assunsero il titolo di re- 
gno, nel 1885 la Rumelia orientale fu unita maggiormente quale provincia al principato di Bulgaria. Malgrado le convulsioni che allora tenevano in sussulto la penisola balcanica e facevano temere imminenti complicazioni, passarono precisamente tre lustri senza che in tutti i Balcani si avesse da registrare un cambiamento o un avvenimento notevole politico.

I tre lustri di molto benevola attesa accordati dai uristiani soggetti ai Turchi e dall'Europa al governo ollomano per effettuare un miglioramento alle popolazioni non mussulmane trascorsero invano, anzi dimostrarono l'inettitudine incorreggibile della razza turca ad adattarsi ai sistemi civili europei. Avvennero allora nuove ribellioni in più parti dell'impero turco e particolarmente a Creta. I Greci, impazienti di continuare la liberazione dal giogo della mezzaluma e frettolosi di prevenire nella penisola balcanica rivendicazioni di altri popoli, prendendo appiglio dai fatti di Creta, presero imprudentemente le armi provocando la Turchia a battaglie campali (1897). Guerra greco. Ma l'atto temerario cbbe per loro effetto disastrosn: chbero la peggio e furono costretti a rodere il freno, guardando ed attendendo che anche gli altri si movessero per azzardarsi di attaccare un'altra volta il nemico, che da quattro secoli e mezzo sta insediato al loro posto a Costantinopoli.

Grande era il desiderio dei popoli Slavi balcanici e dei Greci di mettere un fine al dominio turco, almeno in Europa. Ciò non per tanto passarono ancora due lustri interi di ribellioni e di massacri di cristiani durante i quali la diplomazia enropea fece rani e ridicoli tentativi di rigenerazione del governo turco.

Nel 1908 avvenne quella rivoluzione dei giovani tur- La rivoluziochi, che scmbrò apportatrice di nuovo vigore nell'ormai iradicio governo turco. Ci furono allora coloro che ci re dei givvani turchi. credettero; oggi anche ruesta illusione ì svanita.

Il sorgere di un nazionalismo imperialistico tureo indusse l'Austria-Ungheria a provedere alla conservarione delle due provincie di Bosnia e d'Erzegovina, nelle quali aveva profuso milioni in spese militari e civili e 
che evidentemente nè essa aveva l'intenzione di restituire più, nè altri dei vicini avrebbero avuto la forza di strapparle. Avvenne allora la proclamazione dell'annessione (settembre 1908) accompagnata da quella dell'indipendenza totale della Bulgaria, che assunse essa pure il titolo di regno.

I'annessione della BosniaErzegovina.
Tutti ricordano ancora l'irritazione che questo atto provocò in tutti i Serbi, l'esasperazione con cui fu appresa particolarmente da quelli indipendenti del regno di Serbia e del principato del Montenegro e la tensione diplomatica che ne derivò colla Serbia e colla Russia; accompagnata da una mobilitazione militare effettiva nei paesi annessi.

Se ne discusse molto anche dall'opinione pubblica italiana, purtroppo passionatamente e senza oggettività, per quel rimasuglio atavico di malavoglia contro l'Austria, e s'incolpò il governo italiano di essere rimasto passivo. Però già oggi si è fatto strada il convincimento che il governo italiano non poteva contenersi altrimenti. Per calmare gli avversari dell'annessione basta rammemorare alcune circostanze. Essa costò all'Austria parecchie centinaia di milioni e aggravò il suo bilancio rendendolo sempre meno elastico. La sua annessione, semplificando i rapporti internazionali, complicò invece le condizioni interne già abbastanza intricate, dando nuova esca alla gara fra Tedeschi e Magiari per la preminenza nelle nitove regioni, aumentando il numero degli Slavi nella polictnica monarchia e dando forma più precisa alla tendenza nazionálistica degli Slavi meridionali, che domandano la trasformazione dell'Austria in monarchia trialista. Per giustificare lo strappo arbitrario del trattato di Berlino e dare una prova apparente di rinunzia ai piani di espansione balcanica, l'Austria ritirò da una parte i suoi presidi militari dal Sangiaccato di Novibazar e rinunziò dall'altra alla polizia marittima della costa del Montenegro, che per tal modo divemne libero nel suo porto di Antivari.

La restrizione mentale dell'Austria di rimettere piede nel Noribazar alla buona occasione s'è dimostrata un 
sogno svanito, e il Montenegro, divenuto maggiorenne, potè nel 1910 assumere anch'esso la corona regale. È vero che quest'innalzamento di grado avrenne anche coll'appoggio e favore dell'Austria, che cerea di seminare la discordia fra i due Stati serbi ai suoi confini meridionali, però per ogni eventualitì ormai tutti i sovrani dei cinque Stati balcanici sono diplomaticamente alla pari.

Tulti questi atti non crano certo adatti a formar la maggior fortma dell'Austria, che, davvero, si è con essi chiusa per sempre la strada di Salonicco.

Ormai siamo portati a discorrere di fatli tanto recenti, L'occupazione che, se anche storici, non permettono di formulare giu- italiana della dizi apodittici. Per ciò ricorderemo semplicemente la seconda conseguenza della rivoluzione giovane turca, l'occupazione italiana della Libia. Senza indagare le cause che inclussero il governo italiano ad impossessarsi della Tripolitania e della Cirenaica, basterà accennare all'avvenimento, perchè, sebbenc il suo teatro sia stato lontano dall'Adriatico, pure si può prevedere che prima o dopo in alcune delle sue ulteriori conseguenze anch'esso avrà un contraccolpo sul mare che resta sempre l'oggetlo principale di cuesto studio.

La guerra italo-lurca ha messo a nudo diverse verità:

1) Il rimovato spirito bellicoso e l'organizzazione militare dell'Italia, segni indubbi di maturilà politica.

2) L'incvitabile scomparsa della potenza lurea, che cacciala dall'ultimo suo possesso di quell'Africa settentrionale, fino a mezzo secolo fa sua sino all'Algeria, non poteva più resistere a lungo nemmeno alle scosse dei popoli balcanici.

3) L'esistenza di una mal dissimulata acredine tra l'opinione pubblica austriaca e quella ilaliana, sintomo di una grave causa latente di antagonismo. Basto infatti un'impresa cosi lontana dai confini italo-austriaci, perchè l'opinione pubblica di certi circoli dell'Austria ne approfittasse per restituire agl'Italiani quella malavoglia che tre anni prima questi averano dimostrato per l'annessione della Bosnia. 
4) La lenta formazione di una comune coscienza nazionale albanese. La coscienza individuale di razza ì stata negli Albanesi viva ognora e fu anzi la molla che spinse questo popolo a lottare ed a resistere a tutte le imposizioni esterne, compresa l'ultima e più forte dei Turchi. Però fino a pochi anni fa gli Albanesi crano discordi tra loro anche nelle minime frazioni di tribù a tribù, e nella massima parte fautori e sostenitori del governo turco, sotto il quale avevano potuto espandersi e ingrassarsi a carico dei Serbi.

L'Austria da settentrione e l'Italia da mezzogiono avevano cercato di acquistare le loro simpatie. Nuove idee penetrarono dal di fuori tra quelle selvagge popolazioni montanare e si formò così, almeno nelle poche persone educate all'europea, il sostrato di una vera coscienza nazionale comune, influenzata per ora da due correnti superficiali esterne, una di italofilia, l'altra di austrofilia. Per effetto di questo progresso gli Albanesi approfittando degl'imbarazzi momentanei del governo turco pretesero l'autonomia, la delimitazione dei loro confini, scuole, lingua, mezzi pecuniari propri. Questa inattesa trasformazione mise in campo un nuovo erede dei Turchi e aiutò la diplomazia a creare l'Albania autonoma, quale tentativo di risolvere pacificamente il problema dell'equilibrio o dello statu quo dell'Adriatico.

La liquidazio. ne della Turchia europea.
Abbenchè secondo il trattato preliminare della pace di Londra (17-30 maggio 1913) la Turchia sia rimasta in possesso di Costantinopoli colla penisola di Gallipoli e con quel tratto di territorio europeo che va dal golfo di Enos nell'Egeo al porto di Midia sul Mar Nero, si può dire senza tema di smentite che l'influenza turca in Europa è ormai liquidata.

Il Mar di Marmara coi due stretti del Bosforo e dei Dardanelli più che un segno di demarcazione geografica tra l'Europa e l'Asia o, secondo i casi, il ponte di passaggio tra questi due continenti, è stato dal medio evo in poi uno dei punti strategici della diplomazia internazionale, attorno al quale si sono logorate le energie di più di un popolo (Greci, Turchi, Russi, Veneziani, Geno- 
vesi). Molti deplorano che nè la diplomazia, nè le armi alleate dei Balcanici abbiano risolto anche il problema di Costantinopoli. Lo storico, osservatore posato e privo di sentimentalismi, dirà che la quistione di Costantinopoli nel 1913 non era ancora matura. La sua risoluzione arverrà, si può star sicuri, e per parte dell'Europa, non dell'Asia. É anzi certo che l'eredità di Costantinopoli, sia che clecada a favore di uno dei primi iscritti (Bulgari, Greci, Russi, Inglesi) o di qualche altro Stato che all'oc:casione non rifiutcrebbe di adirla, avrà un contraccolpo nel Mediterraneo e indirettamente nell'Adriatico. Trattandosi però di influenze lontane e mediate non ci diffonderemo ad esaminarle ed osserveremo piuttosto le conseguenzc che la scomparsa dei Turchi dall'interno dei Balcani potrebbe avere per l'Adriatico.

Nei giudizi con cui ognuno di noi accompagnò l'inizio, le varie fasi e la fine della recente gucrea turco-balcanica, buona parte fu presa dal sentimento. In generale ai cristiani toccò maggiore ammirazione e meritata, perchè vinsero: ai Turchi toccò invece l'esecrazione. Da qualcuno si volle qualificare la gucrea come una crociata, ma il segno di Cristo non fu che un pretesto o una finzione accampata contro la mezzaluna, quale emblema dei seguaci di Naometto. In sostanza lottarono due tipi di civiltà, l'europea contro l'asiatica. Anche i Turchi, dei quali oggi si dice tanto male, hanno qualche lato buono, ed anche a loro bisogna concedere qualche briciolo di ragione, almeno soggettiva. Con mezzi non mollo differenti da quelli pei quali oggi perdettero, cinque secoli la i Turchi vincevano, e come! Quale elemento fece passare la superiorità da ma civiltà all'altra? La forza, la sola violenza biutale.

Per effetto più dello studio che per conoscenza personale nella maggior parte dei casi, noi siamo abituati a considerare i Turchi fin dalla loro comparsa in Europa quale un flagello. Il nostro orgoglio di razza privilegiata ci fece considerare loro superiori anche quando essi fitcevano tremare meza Europa. Certo i Turchi sempre eguali nelle virtù militari, nella moderazione per le esi- 
genze della vita, nel loro speciale cnlusiasmo religioso fatalistico, mancano della più elementare facoltà di adattamento ad altri regimi di vita e di quella irrequicta attività organizzatrice, che sono in contrapposto le caratteristiche degli Europei. La cui superiorità consiste oggi nell'aver sviluppato ad un alto grado la capacità di servirsi di tutti i mezzi offerti dall'intelligenza per aumentare quel benessere, cui li spinge la loro stessa natura.

Coll'invasione arabo-turca in Europa cessò il movimento dei popoli da oriente verso occidente. I Turchi furono proprio gli ultimi ad arrivarvi, mossi da un'idea primordiale: il trionfo di una religione. E sono anche $5^{1: i}$ ultimi a partire, cacciati da un'idea più evoluta, quella della libertà nazionale come espressione di una migliore civiltà.

La sorte aveva offerto ai Turchi la possibilità d'immortalarsi, qualora avessero saputo europeizzarsi come i Magiari, i Bulgari, i Finlandesi, ed assimilare nella nazionalità, anzichè soltanto nella religione, la spruzzaglia di popolazioni di cui è cosparsa la penisola balcanica. Ma non furono capaci di adempiere una missione consimile forse per inettitudine congenita e forse anche in parte perchè vi si oppose l'eterna nemica dei Balcani, la geografia.

Essi se ne sono andati e stanno ormai Iontani dall'Adriatico senza rimpianto, ma non senza conseguenze. Non è possibile che un dominio durato un mezzo millennio non abbia lasciato dei solchi profondi nella psiche dei popoli dominati. Sarebbe quindi vana cosa il pretendere che per essere cessato il governo turco scompaiano d'un tralto le tracce dei suoi usi e costumi.

Ora l'Adriatico non sarà più colla sua parte orientale la linea di contatto tra l'islamismo e la cristianità cattolica. Scomparso lo Stato scomparirà anche la religione mussulmana. Fra gli Slavi non sarà difficile la conversione all'ortodossia; ed anche gli Albanesi per forza di ambiente, sia che vivano autonomi sia che vengano sottomessi da altre potenze, per europcizzarsi dovranno cristirnizzarsi. 
La cessazione dello Stato turco per poco non portò di botto lo slavismo strapotente all'Adriatico. Per ora fu sventato il progetto serbo-greco di dividersi l'Albania. Ma è certo che l'Austria e l'Italia avranno ancora da far i conti collo slavismo e un po' anche coll'ellenismo per la divisione delle influenze nell'Adriatico. 



\section{L'ADRIATICO NELLA POLITICA.}

La nostra mente che seguiva con una presunta sicurezza di discernimento gli avvenimenti del passato remoto, ma che aveva già incominciato ad essere guardinga nel giudicare il più prossima ieri, si arresta involontariamente quando arriva al giorno d'oggi.

Per miopia innata, per stanchezza individuale, per l'inesperienza generale, i nostri giudizi sui fatti contemporanei sono lenti e labili. Difficili, se non addirittura impossibili, sono le mostre previsioni riguardo a quelli dell'avvenire.

La ragione sostanziale della differenza nella nostra facoltà discernitrice starebbe in ciò, che per i fatti del passato abbiamo non solo la conoscenza dell'operato delle forze imprevedibili e imponderabili, che ci sfugge per il presente e ci manca per il futuro, ma anche la conferma e il paragone degli avvenimenti postcriori. Eppure malgrado la sosta difettosa della nostra mente, la vita collettiva dell'umanità continua, come quella dell'individuo, indisturbata il suo corso collo stesso ritmo. Sitpendo dunque che il mondo cammina mosso da leggi inmutabili, parzialmente a noi note dall'esperienza del passato, possiamo avventurarci a guardare il clomani, che forse noi non vedremo, aiutandoci con delle deduzioni generali, che scbbene saranno forse distrutte da forze ignole, perchè la logica non agisce nel mondo ma solo nclla nostra mente, avranno pure qualche probabilità di az- 
zeccare nel vero. E poi se lo studio del passato ci conforta pei suoi ammaestramenti e quello del presente ci allieta o accora secondo che in esso vediamo avvenimenti a noi buoni o cattivi, l'indagine del futuro ci attrae col fascino dell'ignoto.

\section{IL PROBLEMA DELL'ADRIATICO.}

Qualcuno potrebbe non essere d'accordo nell'ammettere l'esistenza d'un problema dell'Adriatico. Noi invece, dopo avere studiato varie cause d'indole genorale e circostanze particolari, lo ammettiamo senz'altro per i nostri tempi.

Il problema è complesso per ragioni economiche, nazionali, politiche. Cercheremo di esaminarle onde poter poi fare più comodamente le considerazioni opportune.

\section{L'IDEA DI NAZIONALITÀ.}

L'idea che nel secolo passato ha fatto maggior cammino e che continuerà a correre anche nel presente è quella di nazionalità.

Nata dallo spirito di libertà e di democrazia dei tempi recenti essa entusiasmò molte menti, di cui alcune la vollero elevare all'onore di un principio. Dimostratasi insufficente a risolvere tutti i problemi in cui avrebbe dovuto trovare applicazione nel vecchio e nel nuovo mondo, si ridusse alla sua vera essenza, di un s'entimento, comnaturale a quello di libertà, che spinge gli uomini a cercare nella patria il gradino per salire dall'individualismo all'umanità.

Sarebbe esagerato il ritenere che il solo sentimento di nazionalità abbia fatto cambiar faccia politica all'Europa e in particolare abbia aiutato gl'Italiani, i Tedeschi, i popoli dei Balcani a riordinare la loro patria; che per ́esso solo la Turchia si sia sgretolata e che esso solo forse 
farà suonare un giorno l'ora fatale anche per l'AustriaUngheria.

Esso è però oggi una formula, un'etichetta, che si adatta benissimo a ricoprire tutte le tendenze civili, culturali, economiche, di benessere sociale, di progresso, d'indipendenza e per ciò tutti i popoli che vogliono raggiungere questa meta agitano in prima linea la bandiera nazionale.

L'idea nazionale fa ancora il suo corso e rappresenta alla mente dei pensatori un ciclo, in cui noi viviamo $e$ ci agitiamo quasi inconsciamente, come le genti del medio evo vissero e pensarono in conformità all'idea religiosa, che produsse quel graude movimento demografico che furono le crociate.

E come l'ilea religiosa, accanto all'innegabile impulso dato al bene e al progresso, fu accompagnata da fatti che riuscirono damnosi come il monachismo col numero stragrande dei conventi, l'impinguamento del clero, la formazione della manomorta, la stagnazione intellettuale, così anche l'idea nazionale, che pur agisce come una molla in avanti, dà segni di degenerazione nel militarismo, nella pace armata, che già rallentano il progresso della civiltà e la opprimeranno sempre piò̀ fino a clanneggiare lo sviluppo economico dei popoli. Allora gli uomini si accorgeranno, come noi vediamo per il medio evo, che i mali concomitanti e derivati dal nazionalismo esagerato furono maggiori del bene e lo disprezzeranno per ricorrere alla riforma dellat società su basi comuniste o sindacaliste, che oggi sono ancora allo stadio teorico.

Intanto come nel medio evo la coltura e il clero si accompagnavano e chi voleva raggiungere un perfezionamento intellettuale assumeva l'abito del prete, così noi oggi vediamo i militari elevati al primo posto nella classe sociale e quasi tutti i patriotti adorare il dio Marte. Ora siccome si prevede che questo ciclo del nazionalismo debba giungere al suo compimento, almeno per l'Europa, se ne deduce che non solo la Turchia, ma anche l'Austria-Ungheria dovamno subire inevitabilmente modificazioni politico-nazionali. 
La diplomazja, si sa, è ostile per principio ad ogni mutamento politico. I beati possidentes non rinunziano che per forza al loro possesso e alla loro beatitudine. Però anche la forza della díplomazia ha un limite, che l'arresta quando scoppiano avvenimenti decisivi nella storia dei popoli. Essa si avvantaggia spesso dell'azione personale, senza dubbio influente e alle volte decisiva di ministri o sovrani, attivi e geniali. Ma quest'azione, che pur fa sentire il suo valore nella storia, è di troppo breve durata, perchè non abbraccia che una parte, talvolta minima, della vita d'un uomo, già insignificante a paragone dei secoli che sono concessi alla vita delle nazioni.

Se volessimo cercare degli esempi dalla storia vicina dell'Adriatico, potremmo citarne tanto a favore della diplomazia, che dei popoli. Dante e Machiavelli, due menti geniali, intuendo fatti che si avverarono appena dopo molti e molti secoli, fecero ai loro tempi politica attivissima, ma senza risultato. Napoleone il Grande inutilmente mise a soqquadro l'Adriatico per allontanare l'Austria; l'austriacissimo principe di Metternich, fornito pure di un'immensa influenza politica, invano credette di poter soffocare il movimento italiano sull'Adriatico; e Bismarck con Andrassy se risorgessero vedrebbero con rammarico il fallimento dello statu quo nei Balcani.

Quello invece che il genio e la potenza smisurata non poterono ottenere, fu dato di raggiungere ad uomini originariamente oscuri, quali Mazzini e Garibaldi, oppure a principi animosi, ma con poteri limitati, come Vittorio Emanuele II di Sardegna ed oggi Nicola del Montenegro. La ragione del successo starebbe in ciò che tutti questi ebbero la fortuna di agire nel momento e nel senso favorevole agl'interessi del loro popolo.

Si vede quindi che in tutte le epoche della storia le correnti anonime, ma grandi e vitali dei popoli, dopo giri tortuosi e viziosi finiscono sempre col trionfare. Così sarà anche della riorganizzazione nazionale dell'Europa, come del resto possiamo già dedurre dagli aggruppamenti statali avvenuti finora.

La liberazione dei popoli balcanici dal giogo ottomano 
ha risollevato la quistione degli Slavi meridionali (jugoslavi) e fatto riavvampare indirettamente tutte le brame del panslavismo. Piccoli attriti locali stridono in più parti dell'Europa, ovunque popoli diversi vengono a contatto. Però se osserviamo l'assetto subìto dall'Europa nell'ultimo tempo per l'idea dominante di nazionalitì, vediamo che ormai l'occidente, il settentrione, il mezzogiorno ed anche l'oriente sono placati. Non rimarrebbe da regolare che il centro occupato appunto dall'Austria-Ungheria, unico Stato formato da u'n'accozzaglia di popoli malcontenti e discordi. - Dopo la liquidazione della Turchia europea la posizione dell'AustriarUngheria non è punto invidiabile. Ed è interessante, particolarmente per questo studio, di notare che le maggiori probabilitì di mutamenti nazionali nella compagine dell'Austria si famno sentire precisamente su quelle delle sue regioni che sono confinaric sull'Adriatico.

In parte fu dettol e in parte ognuno lo sa, che l'odicrna configurazione politica dell'Adriatico non coincide colle divisioni geografiche e nazionali. Nel momento attuale le maggiori velleità di mutamenti partono dai Balcani.

La lega balcanica aveva deliberato, e senza l'interwento della diplomazia l'avrebbe anche mandato ad effetto, di spartirsi l'Albania, nel qual caso la Serbia e la Grecia sarebbero divenuti Stati politicamente adrialici.

Il Montenegro che ha bisogno del mare per non morire soffocato nei monti e lo vede ai suoi piedi nelle Bocche di Cattaro, per scendervi dovette andare a sghenbo prima nei porti albanesi di Antivari e Dulcigno, ora nella malarica Bojana. Si dice di conseguenza che esso avrebbe grande bisogno di ingrandirsi non solo colle Bocche di Cattaro, ma anche coll'Er\%egovina e col territorio dell'antica repubblica di Ragusa fino al Narenta, giacchè altrimenti corre pericolo o di essere soflocalo dall'Austria o di essere assorbito dalla Serbia.

Per nessumo è mistero che la Serbia non ha rinunziato ai suoi diritti storici e mazionali sulla bosnia e che la tutto il possibile per provare anche coi fatti di essere divenuta il Piemonte dei Balrani. 
Si dice poi che gli Slavi meridionali (Serbi, Croati e Sloveni) intendano di rivendicare tutti i territori dove essi formano anclic una parte soltanto dell'elemento campagnolo. I Croati poi, almeno fino a pochissimo tempo fa, speravano di ottenere una specie di egemonia sugli Sloveni a nord e su alcuni Serbi a mezzogiorno (ricostituzione del triregno croato e reimpasto dell'Austria nel trialismo).

A Fiume, città italiana con retroterra slavo, comandano i Magiari del bassopiano pannonico. Il fiumicello Judrio è un segno troppo insignificante per formare il confine divisorio fra due grandi potenze in antagonismo. Inoltre esso taglia in due una regione che è e resterà uniforme in natura e in popolazione. Si dice quindi che gl'Italiani non abbiano rinumziato al loro programma di completare volentieri il loro Stato portandolo al confine naturale col Trentino e colla regione Giulia, basandosi su buone ragioni geografiche, storiche ed etnografiche.

Si dice che i Tedeschi per il grande progresso della loro patria hammo bisogno di estendersi economicamente fino all'Adriatico e che per ciò considerano Trieste come una loro Amburgo meridionale dell'avvenire.

Si dice - e questa è senza dubbio la cosa più certa che l'Austria non intende di cedere nemmeno un palmo del suo territorio.

Qualcuno potrebbe smentire tutti questi si dice. La storia continuerebbe il suo cammino egualmente. L'assetto odierno dell'Adriatico mostra una pausa, un limite accidentale eschusivamente a noi, che non lo possiamo osservare altrimenti. Ma già fra cent'amni gli storici sorvoleranno sul periodo dei primi lustri del secolo $\mathrm{XX}$, che non avranno segnato altro che la continuazione di quei preparativi, che l'idea nazionale convertirà in fatti della storia.

Ma poi se anche non si volesse tener alcun calcolo di quegli indizi di fermento che pullulano sotto i nostri occhi, bisogua pensare che nell'immutabilità dell'armonia dell'universo c'è ma legge che vuole mutabili contimamente nella forma tutte le istituzioni uname. Ed anche 
la formazione degli Stati e la vita dei popoli, come quella degl'individui, cresce, si sviluppa e muove per dar luogo ad altri organismi, che si moveranno in un modo conforme.

Tutti potramno quindi essere d'accordo che o prima o dopo cambiamenti politici nell'Adriatico saranno inevitabili. Ognuno può vedere lo slavismo che si matura nell'oriente dei Balcani. E come un secolo fa circa si preparavano dei cambiamenti per la costa occidentale dell'Adriatico dalla regione del suo bacino ad esso meno vicina, il Piemonte, così oggi possiamo prevedere che anche la costa orientale sarà sconvolta per contraccolpo di ciò che è avvenuto nei Balcani e di ciò che sta maturandosi ancora a Costantinopoli.

Se questo studio avesse veduto la luce avanti la guerra balcanica contro la Turchia, in questo punto sarebbero state inevitabili delle considerazioni preventive sull'atteggiamento dell'Austria rispetto a quella crisi che la toccò forse più vitalmente di quella italiana.

\section{LA SORTE DELLAUSTRIA.}

Ormai si sa che la liquidazione della Turchia sulla cui eredità anche l'Austria vantava un ipoteca e di prima iscrizione, avvenne senza la di lei partecipazione e con tutto suo damno, perchè se fu liquidata la Turchia fu liquidata anche la pretesa marcia dell'Austria, culale rappresentante del germanesimo, per il Novibazar, Mitroviza avanti fino a Salonicco. L'Austria ha cessato di essere la printa creditrice politica dei Balcani. Ma la scomparsa della 'Turchia porterà all'Austria ancora un altro trentendo contraccolpo. Ormai l'Austria non solo è chiusa in quel fatale anello di ostilita nazionali anche da oriente, ma è l'unico Stato contro il quale si appunteranno d'ora in poi tulte le mire tanto di rivendicazioni giustificabili, quanto di espansioni ambiziose. Colla liberazione degli Slavi balcanici cessò anche quella parvenza di missione dell'sustria, di portare la civilta a popoli soggiogali dalla barbaric. 
Cause economiche, nazionali e civili hanno prodotto lo sgretolamento della Turchia e secondo alcume previsioni cause eguali dovrebbero produrre un effetto consimile anche nell'Austria-Ungheria. Tolta però la causa remota, ossia la tendenza dei popoli al miglioramento, eguale in tutti i due casi, le forme di tale evoluzione saranno differenti. In Turchia, ch'era la negazione della civiltà, appena i popoli soggetti raggiunsero la coscienza dello stato avviliente in cui erano tenuti, non ebbero un campo su cui tentare in via pacifica o costituzionale di ottenere dei miglioramenti. Ogni mezzo ragionevole era escluso coi Turchi e per ciò ai popoli oppressi non restò altra via da battere che quella di contrapporre violenze alle violenze turche, con insurrezioni, con stragi, colla guerra.

Nell'Austria invece, ch'è uno Stato eminentemente civile e bene organizzato, il malcontento non ha la sua origine in una lotta disperata per l'esistenza, che o bene o malc è assicurata a tutti i popoli, quanto piuttosto nell'allettamento ad uno sviluppo sempre maggiore, suscitato dal progresso raggiunto dagli altri Stati, con un'evoluzione più libera. Il rapido progresso mediante la libertà nazionale non è solo una fantasia di animi patriottici, ma una forma perfettamente concreta dell'evoluzione dei popoli. L'irritazione in Austria è diretta quindi sopratutto contro l'imperfetto sistema di governo.

Per ciò anche gli effetti nelle probabili alterazioni dovrebbero essere differenti. Se ormai anche i popoli balcanici avevano compreso l'inutilità e il danmo di dilaniarsi a vicenda per far godere i terzi, non sarà forse lontano nemmeno il tempo in cui anche $i$ popoli dell'Austria-Ungheria con un'intesa metteranno fine al medievale divide et impera, ch'è la divisa di governo a Vienna ed a Budapest.

L'Austria-Ungheria è oggi nella sua costituzione copiata dal modello di Stati nazionalmente omogenei e amministrata in base al sistema della rappresentanza proporzionale del numero degli abitanti. Questo sistema che negli altri Stati ha anche i suoi inconvenienti e gli op- 
positori per lo squilibrio che ne deriva lra i vari strati sociali, in Austria è addirittura maleviso e cuasi insostenibile, perchè opprime le nazionalità minori numericamente. Da questo sistema ci sono quindi gli avrantaggiati ed i danneggiati e, perchè tutti i popoli dell'Austria-Ungheria si decidessero a rischiose rivoluzioni, occorrerebbe che si presentasse, almeno per i più, la prospettiva di migliorare il loro sviluppo civile senza perdere il bene che già godono. Prima di ricorrere dunque a mezzi estremi le nazionalità dell'Austria cercheranno, come del resto hanno falto sinora, col tendere e reclamare un'amministrazione più equanime basata su un'eguale e non pregiudizierole distribuzione dei diritti politici, di raggiungere qualche cosa di simile al federalismo. L'Austria dovrà per forza cessare dal copiare le istituzioni costituzionali dell'Inghilterra e della Germania e cercar di cavare qualche buona idea dagli esempi della Svizzera, del Belgio, degli Stati Uniti d'America. Essa sarà dunque verosimilmente messa al bivio o di evolversi federalisticamente o di sgretolarsi. Dalla strada in cui la porteranno la saggezza dei suoi reggitori e la fatalita degli avvenimenti dipenderà la sua sorte.

Il pronostico pessimista potrebbe del resto fallire del tutto o in parte, come ogni previsione d'astrologo, molto più che l'Austria ha ancora for'za bastante per tenere in rispetto i presunti suoi eredi.

Nè tulti d'altronde sono di quellopinione pessimista. Una mente geniale sopratulto per esperienza politica, quella del principe Ottone di Bismarck, si espresse che l'Austria è una necessitì e che se non ci fosse, bisognerebbe crearla.

La sentenza, con tutto il rispetto per la sua provenienza, deve aver riflesso principalmente gl'interessi della Germania alla fine del secolo passato, giacchè non è ben chiaro l'utile derivato al benessere generale d'Europa da uno Stato poco omogeneo perfino geograficamente, esteso dalle $\Lambda_{1}$ i ai Balcani e dalla Sarmazia all'Adriatico, di cui si sa che negli anni 1830, 1848, 1859, 
1866, 1878, 1908 e 1912 fu causa e spettacolo di trambusti che pregiudicarono invece la pace d'Europa.

La sentenza poi dovrebb'essere intesa in senso relativo anche in ragione di durata, giacchè si deve pur concedere la supposizione che questo Stato "necessario" possa diventare un giorno teatro di aspre competizioni nazionali. Forse nemmeno il principe di Bismarck ha previsto che un ulteriore ingrandimento dell'Austria nei Balcani o il crescere della coscienza e forza nazionale degli Slavi entro i confini austriaci potrebbe invertire le parti dell'egemonia finora goduta dai Tedeschi e far desiderare anche a loro che cessi la "necessità" sentenziata.

A sostegno di coloro che si ostinano a pensare al finis Austriae sta il fatto che l'Austria già nel 1848 non fu lontana dal suo dissolvimento e che le tendenze centrifughe di alcune nazionalità continuano innegabilmente a farsi sentire ancora. Malgrado l'intervento esterno della Russia i Ma'giari riuscirono in tre o quattro lustri ad ottenere mano libera negli affari interni. Il dualismo accettato dal trono nel 1867 come una necessità inevitabile per salvare almeno il prestigio della dinastia, non fece col tempo che accrescere le screpolature lungo la Leitha (fiumicello di confine tra l'Austria e l'Ungheria). Sono noti i secolari e ancor oggi continui dissidi tra la "nazione ungherese» e "la corona" (la dinastia degli Asburgo) per ottenere la prima e non dare la seconda quelle concessioni che dovrebbero produrre l'indipendenza nazionale completa dell'Ungheria.

Sono note del pari le simpatie intellettuali dei Tedeschi, degl'Italiani, dei Rumeni, dei Serbi per i loro connazionali dei rispettivi Stati liberi e degli altri Slavi per tutto ciò che è prodotto dal mondo slavo. Si odono spesso accuse reciproche contro le nazionalità perchè vammo troppo oltre con queste loro simpatie e contro i governi austriaco e ungherese, perchè impediscono le manifestazioni che trovano eco al di là dei confini. E chiaro che questi due governi, a meno di prołlure il caos in casa loro e suicidarsi, non possono dare alle troppe loro nazionalità tutto quello che godono le altre frazioni mag- 
giori indipendenti éd è pure spiegabilissimo il motivo pel quale le nazionalità soggette all'Austria e all'Ungheria, vedendo i vantaggi goduti dai loro connazionali, sentano gravosamente il peso della soggezione. Il malessere generale che ne nasce è fatale e non dà speranza di soluzione pacifica.

Di contro però a queste forze centrifughe esistono anche delle tendenze centripete, di cui si deve tener anche conto per non errare nel giudizio. Lasciamo stare i Tedeschi, che hanno tutto il tornaconto a continuare nell'andazzo politico attuale, che procura loro l'egemonia. Ma i Magiari stessi che fecero il colpo del '48 e continuano a lotlare per l'indipendenza, oggi sono in parte perplessi e divisi in due partiti se convenga loro staccarsi del tutto dall'Austria. Anch'essi devono andar cauti nelle loro pretese, perchè l'Ungheria non è uno Stato nazionalmente omogeneo. Dei suoi venti milioni di abilanti solo olto o nove sóno Magiari. Vengono poi gli Slavi con tre milioni di Serbo-Croati, due di Slovacchi e mezzo circa tra Ruteni e Sloveni, indi i Latini con tre milioni di Rumeni e con circa 27000 Italiani di Fimme e da ultimo i Tedeschi con due milioni abbondanti. -- Unita all'Austria l'Ungheria non solo conta per grande potenza, ma meno che ai confini colla Serbia e colla Rumenia è protelta - si passi il paragone troppo materiale - come un tuorlo d'uovo dall'albume e può intanto esplicare maggior energia all'assimilazione forzata delle nizionalità minori. i: un fenomeno abbastanza strano questo della nazione magiara che da una parte lotta a roce alta per la propria libertà e dall'altra opprime nazioni più deboli. Essa si giustifica dichiarando che l'intransigenza è per lei quistione vitale, perchè altrimenti verrebbe oppressa lei. La verità è che più della giustizia vale la forza e l'egoismo più dell'altruismo.

Ci sono poi i Bocmi (Cehi) che, memori di avere avulo dal $\mathrm{X}$ a tutto il XIII secolo $11 \mathrm{n}$ grande Stato, lottano ancora contro i loro secolari nemici, i Tedeschi, per conservare almeno la loro nazionalità. Negli ultimi decemni hanno anzi ottenuto dei suceessi di rivendicazione e per 
ciò comprendono di aver dell'interesse alla continuazione dell'attuale stato di cose ed a non lasciar che l'Austria vada ancora in sfacelo, finchè non saranno meglio preparati, giacchè altrimenti toccherebbe loro la sorte dei Polacchi in Germania.

In condizioni consimili si trovano i Polacchi in Galizia. Dopo la tragica spartizione, chi li malmena meno è l'Austria, e pur sognando sempre la ricostituzione del loro regno, vedono che se c'è il caso di sperare un aiuto csterno, questo non potrebbe venire che, sia pure per ragioni indirette, da un'Austria slava, e per ciò stanno col governo e sono per la conservazione della monarchia.

L'Austria conta ancora due nazionalità slave minori, gli 'Sloveni ed i Croati, che entusiasmati ormai anch'essi per tutto ciò che sa di panslavismo, nella politica pratica lavorano per la ricostituzione dell'antico regno di Croazia. Da soli sarebbero impotenti ad agire e non si nascondono che in Austria non stanno poi tanto male e sotto un altro padrone starebbero peggio. Per ciò anch'essi stanno col governo e sperano intanto nel trialismo austriaco.

In Ungheria l'altra parte dei Croati, gli Slovacchi ed i Rumeni, bistrattati dai Magiari, impotenti ad aiutarsi da soli e privi di aiuti esterni, non hanno altra speranza che nell'equanimità del sovrano e guardano quindi all'Austria.

Strano fenomeno anche questo dell'Austria, dove tutti sono malcontenti, nessuno ama lo stato per sè stesso, anzi ognuno lavora pro domo sua, eppure ci sono tanti interessati a non lasciarlo dissolversi.

E sembrerà più strano ancora e addirittura ozioso che a proposito dell'Adriatico si siano tirati in ballo Boemi, Polacchi e Rumeni, che stanno le milla miglia lontano. Eppure finchè dura uno Stato che abbracciando tutte le regioni dall'Elba e dai Carpazi all'Adriatico, può rivolgere contro di questo braccia e redditi pecuniari di quei paesi, nel fare previsioni su eventuali modificazioni per la base marina di quello Stato, bisogna tener conto di tutta la sua potenzialità.

Nè quelle indicate sono le sole sue cause, sia pur 
involontarie, di sostegno. C'è per gili Stati sconnessi, come per gl'individui malandati in salute, una certa inesplicabile forza di gravità, che li conserva in vita al di tà di ogni previsione.

E un fatto ormai sperimentato che un organismo complicato, com'c̀ uno Stato ai giorni nostri, non può risolversi nel nulla. Perchè si disfaccia occorre che ci sia già pronto un altro organismo in cui possa continuare, magari in frazioni, a svolgersi la vita civile. Così p. e. la Germania e l'Italia non avrebbero potuto prosperare e sarebbero piombate nell'anarchia se in luogo dei governi là spodestati, qua espulsi, la Prussia e il Piemonte non avessero portati pronti tutti gli ordinamenti necessari al funzionamento dell' or'ganismo statale. Lo stesso fatto si ripetè di recente colla Turchia, ove limmaturitì dei popoli balcanici a reggersi da soli fu una delle cause che prolungarono la vita del governo ottomano. Per questa ragione in parte l'Austria fu salva nel '48. Tutti i popoli erano malcontenti, ma meno gl'Italiani e gli Ungheresi, tutti gli altri non sapevano che cosa sostituire al governo di Vienna. S'ebbe molta confusione, ma passata questa l'unico, o il miglior governo, fu quello di prima.

Fra coloro che giudicano pessimisticamente l'avenire dell'Austria ci sono alcuni che pensano ch'essa forse prima che non si creda potrebbe scindersi in duc, Austria e Ungheria, ognuna per sè.

Questa soluzione non potrebbe essere definitiva per ragioni sopratulto geografiche. L'Ungheria nellat suat configurazione geografica e lasciando a parte lutli gli altri riguardi, potrebbe benissimo stare da sè: l'Austria odlerna invece no.

Una provincia oggi dipendente da Viemna, la Dalmazia, è staccala territorialmente dalle altre. Dove andrebbero in quel caso questa e la Bosnia-Erzegovina? Si pensi alle difficoltà tecniche d'm'amministrazione che doresse tenere uniti i paesi alpini del Tirolo colla Bucovina, che guarda nel Mar Nero. Oppure si verebbe all'ulloriore inevitabile conseguenza che l'Austria si riducesse alle pro- 
vince alpine, alta e bassa Austria, Stiria, Salisburgo, Tirolo, Carinzia e Carniola, quale primo gruppo, e alle province nordiche di Boemia, Moravia e Slesia quale secondo gruppo, che formano la vera, antica Austria tedesco-boema, come ai tempi di Rodolfo d'Asburgo nel XIV secolo.

Allora forse l'idea di Bismarck che nei punto di contatto di quattro popoli, Slavi del nord, Tedeschi, Magiari e Slavi del sud, sia necessario uno Stato neutro per smussare gli attriti apparirebbe più ragionevole, sebbene in contrapposto si potrebbe osservare che ormai nessuno ritiene pir̀ indispensabile a questo scopo nemmeno la Svizzera o il Belgio.

Altri di coloro che attendono, ma sono più pazienti, ritengono che l'Austria subirà prima un rimedio palliativo, il federalismo. Chi lo invoca dimentica però che per eseguirlo sinceramente bisognerebbe mutare le basi della polietnica monarchia, spostando i confini di quasi tulte le province e alterando per conseguenza ragioni storiche inveterate (cosa difficile anche solo tecnicamente) e che la prima conseguenza di questo cambiamento sarebbe la tanto temuta prevalenza degli Slavi. Se ora che duc popoli soltanto hanno voce in capitolo la discordia è endemica, figurarsi la babilonia che nascerebbe quando a parlare fossero almeno cinque popoli maggiori e alcuni altri di numero inferiore. L'esperimento è ritenuto arrischiato e d'altronde le nazionalità da sole non potrebbero nemmeno tentarlo. L'unico ente capace e che forse potrebbe vederci del tornaconto a tentarlo sarebbe la Corona; però a ciò sarebbe prima di tutto necessaria una mente geniale e una mano di ferro.

Una similitudine - con licenza del lettore per uno sfogo poetico anche ora che la ragione dovrebbe funzionare da sola - spiegherà meglio che cento frasi la sorte politica che l'idea nazionale potrebbe preparare ai paesi dell'Austria.

Quando un grande fiume straripa e allaga i territori che gli stanno intorno egli ne diviene il padrone e impone alle sinuosità della terra l'uniformità della super- 
fice piana della sua acqua. Quando poi l'acqua cala e il lago ritorna fiume, spuntano un po' alla volta i vertici di monticelli, indi tutte le falde e da ultimo si asciugano anche le bassure.

Lo stesso procedimento accompagnò in forma astratta gli Stati creati da grandi capitani, da dinastie, da popoli conquistatori, da Alessandro il Macedone, ai Romani, a Carlo Magno, agli Osmanli, a Napoleone e, cosa naturale ma che pur molti stentano a riconoscere, si ripeterà anche riguardo all'Austria. Questo Stato che si estese per impulso dinastico e raggiumse la massima estensione nel secolo XVII è già in deflusso evidente dall'epoca napoleonica. Ha appena terminato, e non vittoriosamente, il ducllo secolare colla Francia e le lotte cogl'Italiani e colla Germania, e già s'è impigliato in un nuovo duello, non meno grave, cogli Slavi e colla Russia. Le previsioni anche da questo lato non gli si presentano favorevoli. La Russia, meno nella Polonia, è quasi invulnerabile per l'Austria; mentre invece l'Austria rispetto alla Russia ha piì di un tallone d'Achille. L'Austria-Ungheria oggi ostenta ricchezza e forza, come Venezia dopo Cambrai, ma la sua decadenza è evidente a chiunque la studi con un po' d'attenzione.

Bisogna attendere perchè il tempo e il sole prostiughino il resto.

Nelle considerazioni sugli effetti che l'idea nazionalistica potrebbe produrre negli Stati polietnici d'Europa l'Austria fu paragonata alla Turchia. Però non si deve dimenticare una enorme differenza che le distingue. L'Austria è uno Stato civile, modernamente amministrato, che se non avesse appunto il tarlo delle competizioni nazionali, potrebbe fiorire ancora. Per ciò malgrado l'eguaglianza apparente della composizione polietniea, le loro condizioni di vita sono del tulto differonti.

La scomparsa della Turchia sarì semza dubbio un guadagno per la civiltà e per persuadersene basta pensare ai rapidi progressi raggimuli da tutli gli Stati balcanici dopo la liberazione. I popoli dell'Austria invece possono dare abbastanza liberamente il loro contributo 
alla civiltà. Se non che l'Austria, come Stato, non è nè può mettersi più alla testa del progresso, per la ragione che lo spirito animatore dello sviluppo moderno è la libertà nelle sue varie forme e molteplici applicazioni, e l'Austria, che non può concedere certe rivendicazioni, deve necessariamente astenersi da esperimenti di cui non si conosce la portata oppure si sa che sono riusciti pericolosi. Altri Stati d'Europa dove il principio dei diritti dell'uomo fu sviluppato nelle sue conseguenze sono oggi retti a repubblica con spiccato carattere antireligioso.

Il perno dell'Austria invece è, e non può essere altrimenti, la dinastia. Questa, che s'è ingrandita per la fortunata sostituzione ad altre estinte, per continuare ad esistere ha bisogno di appoggiarsi al legittimismo: l'imperatore d'Austria ancor oggi non può essere tale che per grazia di Dio. Per ciò l'Austria che poteva prosperare nei secoli in cui trionfava il culto gerarchico, oggi è paralizzata e deve andar cauta nel concedere, perchè il troppo liberalismo non potrebbe che nuocerle, e bisogna che si rassegni a seguire talvolta a malincuore le esigenze dei tempi in cui tutti aspirano sopratutto alla libertà.

Non è quindi da meravigliarsi affatto che l'Austria cerchi ancora il suo sostegno principale nel militarismo e nel clericalismo. Il primo oltre a rappresentare la forza reale porta con sè il vanto trasmessogli dai secoli più antichi di essere l'emanazione diretta del potere sovrano, che si compiace di figurare come suo capo. Il secondo dispone di un'organizzazione vastissima e docile per ignoranza e per la debolezza umana nel sentimento religioso. La croce e le baionette sono mezzi potenti quando si tratta di far passi avanti, ma nella difesa di uno Stato costituiscono le ultime trincee.

Per questo suo fatale irrigidimento, derivante da difetto d'origine e dalle peculiari sue condizioni nei secoli delle libertà, potrebbero derivare åll'Austria - come già avvenne nel '48 - delle scosse anche per cause estranee alla nazionalità, da cui invece fu risparmiata la Turchia.

Questa scomparve tra l'esecrazione dei popoli civili per non aver saputo adempiere la sua missione. L'Austria 
potrebbe dissolversi o rimpiccolirsi, anche con qualche rimpianto, per aver adempiuto la sua.

Esaurite le speculazioni sulla sorte che potrebbe toccare al secondo dei due Stati d'Europa, ecco l'elenco e l'indicazione della forza numerica approssimativa in abitanti dei popoli che, secondo l'idea di nazionalitì, potrebbero formare degli Stati tra il Danubio, l'Egeo e l'Adriatico :

Magiari 8-9 milioni; Rumeni 9-10 milioni; Bulgari 6-7 milioni; Jugoslavi (Serbi, Croati, Sloveni) 8-10 milioni; Greci (compreso l'Arcipelago) 5-6 milioni; Albanesi 1-2 milioni.

\section{L'ASSETTO PRESENTE DELL'ADRIATICO.}

L'assetto politico presente dell'Adriatico dala nelle sue linee generali stabili da undici lustıi, poco più di mezzo secolo, ovverossia dal 1866, anmo nel quale coll'acquisto della Venezia, il regno d'Italia si costituì nella sua forma conservata fino al giorno d'oggi.

Osservandolo però nei dettagli si dovrebbe fare un'ulteriore distinzione. A sette lustri di distanza da noi, cioè nel 1878, il congresso di Berlino assegnò all'Austria il territorio di Spizza e al Montenegro Antivari e Dulcigno (occupate veramente appena nel 1880). La recentissima pace di Londra assegnò al Montenegro ancora un piecolo tratto di territorio, le foci della Bojana. In questincontro la diplomazia partorì quell'ibrido feto, ehe non si sa ancora se sia nato vivo o morto, ch'è lo Stato d'Albania, autonomo, ma senza altre leggi che quelle della gelosia altrui.

La rapida corsa da noi fatla attraverso i secoli dovrebbe aver dimostrato che le relazioni tra le due coste adriatiche non furono reciprocamente sincrone. Ciò non 
per tanto un osservatore inesperto potrebbe essere indotto a ritenere che nell'attuale divisione delle due coste, dell'occidentale nazionalmente italiana nel regno d'Italia, e dell'orientale, nazionalmente mista italiana e slava, nella polietnica monarchia austro-mngarica, sia raggiunto uno stato di cose ideale e duraturo.

Cesseremo ormai di fare induzioni su ciò che potrebbe accadere nell'Adriatico in linea politica, in primo luogo perchè simili pronostici sono eccessivamente labili e in secondo perchè la loro realizzazione dipende da circostanze estrance al presente studio; ci occuperemo invece a preferenza delle condizioni nazionali ed economiche, più facili ad essere esaminate e.più forti nelle loro conseguenze, essendlo più volte la causa determinante di apparenti effetti politici.

L'Italia. Abbiamo veduto le ragioni di politica interna ed estera, per le quali gl'Italiani dopo Lissa stornarono gli sguardi dall'Adriatico.

La crisi finanziaria e lo scetticismo degli animi che tennero dietro allo sforzo del risorgimento politico li avevano indotti ad occuparsi principalmente di assettare le loro cose interne $\mathrm{e}$ in conseguenza dei tre mari che bagnano la penisola si diede la preferenza al Tirreno, ch'è senza dubbio il più importante non solo, ma che alla costituzione del regno d'Italia si mostrò di essere il più vitale, e poi al Jonio. Solo di quando in quando si ricordarono dell'Adriatico perchè la loro attenzione vi fu attratta dall'attività navale dell'Austria. Fu per ciò che il governo italiano intraprese delle opere onde ampliare ed assicurare il porto di Venezia e creare una seconda base navale militare a Brindisi.

All'intraprendenza privata italiana non si ha da ascrivere che l'attività commereiale nella bassa Italia e particolarmente del porto di Bari. Bari è stata nell'antichità cá è anche oggi la città più attiva ed intraprendente del mezzogiorno d'Italia sull'Adriatico. Bari è oggi il terzo emporio dell'Adriatico con una popolazione di oltre centomila abitanti. E quello che è più notevole il suo rinnovamento è dovuto interamente alle energie locali. 
Più di recente si ebbe il risveglio marittimo di Venezia, che vedendo il suo porto frequentato da molte navi con bandiera austriaca, provvide a mostrare anche l'italiana sull'altra sponda.

Tutto ciò è poca cosa per una nazione marinara come l'italiana e sarebbe sconfortante se non fossero già palesi i segni di un miglioramento.

La crescente prosperità economica, la gara dell'Austria, il fermento nei Balcani, in una parola l'avvenire dell'Italia come grande potenza marittima hanno reso consci gl'Italiani anche dei loro doveri verso l'Adriatico. Da un lustro a questa parte l'Italia ha dato mano alla costruzione del grandioso acquedotto delle Puglie, all'adattamento di tutti i suoi porti adriatici, anche minori, alla sistemazione equa e razionale della navigazione a mezzo dei nuovi servizi marittimi. L'energia del leone di San Marco s'è finalmente desta. Ma l'Italia arrì sciolto il suo còmpito verso l'Adriatico e verso la civiltà appena il giorno in cui, dopo di aver attuato varie opere minori, avrà reso realtà il progetto del grande Leonardo da Vinci di congiungere Milano col mare mediante un canale navigabile.

Dopo l'Italia verrebbe veramente la volta dell'AustriaUngheria. Però con riguardo alla importante sua posizione, da cui si sprigiona l'altro problema dell'antagonismo coll'Italia, per necessità di esposizione sarà tratlata da ultimo.

1 maggiori progressi - proporzionalmente parlando - Il Montzfurono raggiunti invece, embrerebbe quasi incredibile, negro. dal Montenegro.

Questo paese noto fin sa per le sue montagne, ha dalla parte di mezzogiorno 'a vista aperta sul mare e su un porto di primo rangr: solamente che questo mare e il porto - le Bocelie d' Caltaro - appartengono all'Austria.

F. oltremodo in'eressante di considerare un po' l’influenza che ebbe nella storia la posizione geografiea di 
questo nodo scomposto di rocce, gettato dalla natura, quasi per rompere la monotonia, nel mezzo di quelle poco rilevanti catene di monti, che a guisa di fascie d'un nastro, corróno Iungo la costa orientale adriatica dal Carso della regione Giulia fino alla punta Menders. Questo mucchio di monti erti, aguzzi, fitti, difficilmente accessibili fu nell'antichità il rifugio e il cuore del popolo illirico. Decaduto questo ed infiltratisi gli Slavi esso aiutò una parte dei vinti di Cossovo a porre un duro inciampo alla marcia dei Turchi, che dovettero scansarlo e battere un'altra strada. Obliato per secoli ridivenne ai nostri tempi la rocca di un altro popolo, il Serbo. Ai suoi piedi si arrestarono Turchi, Albanesi, Veneziani, Francesi ed Austriaci.

Durante la decadenza dei Turchi i Montenegrini scherzarono a fucilate alla loro fronticra orientale e calarono alla prima buona occasione nella valle di Scutari. Fino a poco tempo fa dovevano stare in sussiego molto serio verso il loro confinante di ponente. Ma da quando la tecnica militare diede occhi, voce e fulmini alla fata dell'invitto Lovcen, essi non temono più il nemico nemmeno da questa parte. Chi fosse preso dal desiderio di animare le cose immote vedrebbe disegnarsi alle Bocche di Cattaro la linea di una lotta tra la violenza degli nomini e la resistenza della natura. Da una parte, al basso e al mare, l'Austria ricca e potente per tutte le conquiste della mente, ma ferma suo malgrado e irritata; dall'altra il Montenegro povero, ma fiero non per tanto c contento solo perchè la natura lo aiutò a salire su in alto. Nel mezzo di sì epica contesa sembra di veder tesa dalla vetta del Lovcen al porto d'Antivari una grande tabella colla scritta: "Per di qua non si passa". Forse nel momento di una visione consimile un alto funzionario militare austriaco avrebbe pronunciato, a quanto si dice, la sentenza che "alle Bocche di Cattaro si decideranno un giomo i destini dell'Austria».

Coll'aiuto italiano la rada di Antivari ha acquistato un porto discreto e la bandiera montenegrina può ora sventolare liberamente sull'Adriatico. Quante persone non 
hanno forse ignorato la sua presenza? Eppure essa ̀̀ là, e non può essere dimenticata. Essa è piccola e modesta e non sventola che su navi minime; ma esiste e soslituisce per l'amor proprio degli Slavi quella rinfoderata di San Biagio della repubblica di Ragusa. Sono in errore coloro che ritengono essere il popolo montenegrino esclusivamente montanaro. Una frazione di esso lungo la costa, diviso da un confine politico, possiede ottime attitudini marinare, di cui diede prova in varic epoche. E se un giorno i due elenenti della marina e del monte venissero sposati con ma stessa bandiera, si può star certi che una marina serba sarebbe presto formata. Senza le gole della montagna nera gli Slavi non avrebbero ancora una bandicra sull'Adriatico.

E consigliabile di azzardare oggi un gitudizio sull'av- L'Albania. venire dell'Albania?

Le condizioni dell'Albania sono state sempre molto confuse, il carattere delle sue popolazioni si è mostrato sempre tale da giustificare i timori che si nutrono sulla scarsa maturila politica e sulla poca loro attitudine a formare un organismo di stato indipendente. L'Albania fu creata come punto monentaneo di equilibrio negativo nei Baleani e nell'Adriatico per interesse e tornaconto di altre grandi potenze. Eppure tali maneggi furono ammantati col pretesto dell'ecpuità nazionale. La diplomazia ha voluto imitare i cavalieri medievali che nel prendere le armi per spirito di aventura o di conquista dicevano di farlo in difesa della fede.

Comuncue siasi, in mo studio come il presente, che spera di riuscire coscionzioso e nell'imprevedibilití di quello ch'è riservato dal fuhro, l'Alhania non può nè deve venir trascurata.

Gli Albanesi del resto, come i Montenegrini, abitano in prevalenza sui monti. Quelli pero di loro (he stanno alla costa sono navigatori. Nelle condlizioni create all'Albania dai Turchi non si può parlare proprianente di una marina albanese. Sta il falto pero che nella navigazione a vela hanno posto anche gli Albancsi. come marinaj al soldo di allri, oppure como proprialari spe- 
cialmente gli Scutarini) di velieri. La maggior parte delle navi a vela che fino a poco tempo fa percorrevano l'Adriatico sotto bandiera turca, appartenevano ad Albanesi. Ed oggi che l'Albania ha assunto una propria bandiera, non sarebbe la cosa più inverosimile se la vedessimo un giorno sventolare oltrechè nei porti dell'Adriatico, anche su navi nel mare.

L'Austria-Un- L'Austria-Ungheria è un organismo statale particolare gheria.

che richiede in ogni circostanza uno studio speciale. Ed anche in quest'incontro per esaminare le sue tendenze economiche e nazionali sull'Adriatico occorre far distinzione fra le esigenze dello stato ed i bisogni e i desideri dei vari popoli, che non collimano, anzi sono troppo spesso in contrasto. Esporremo prima le aspirazioni dei popoli, che sotto la sudditanza austriaca vivono o vogliono espandersi nell'Adriatico, onde di poi siano più chiare le contingenze del governo.

Come tutti sanno, anche nei territori dell'Adriatico, inchiusi nei confini austro-ungarici, l'elemento italiano è il più ragguardevole per indigenato, capacità nautiche, civiltà, censo e numero. Di esso però parleremo a lungo più avanti.

Accenneremo per ora brevemente agli altri.

I Croati e il La storia dei Croati è lunga, ma assai modesta. Ebbero trialismo. qualche notorietà quando furono aiutati dopo il mille dagli Ungari e nel XVI secolo (gli Uscocchi) quando erano favoriti dagli arciduchi d'Austria. Del resto stettero in letargo. Una fortuna consimile capitò loro dopo Lissa a causa del nuovo orientamento politico dell'Austria. Elevati d'improvviso all'onore e ai favori di partito di governo approfittarono per affermarsi; più che ingrandirsi, perchè ciò non era possibile, a spalle degl'Italiani. Il governo, pur di reprimere questi ultimi, tollerò che i Croati avanzassero le più ardite pretese, tra cui fece subito capolino quella della ricostituzione del loro antico triregno di Croazia, Slavonia e Dalmazia sepolto, come abbiamo veduto al principio del XII secolo per opera degli Ungari. Concessa nel 1860 la prima costituzione a tutto l'impero i Croati approfittarono per 
far valere le loro domande nazionali, contro le quali non ci sarebbe stato nulla a ridire dal lato dell'equità, se non avessero implicato l'oppressione degl' Italiani. Allora apparvero essi pericolosi al governo che li perseguilò. Fu anzi l'opposizione degl'Italiani che fece in apparenza naufragare momentaneamente il progetto.

Intanto gl'Italiani da maggioranza divennero minoranza in Dalmazia; e allora i Croati poterono dar libero sfogo alle loro richieste, di fronte alle quali il governo si mantenne passivo fino a tanto che si trattava di aspirazioni platoniche; ma in quanto alla loro effettuazione pratica fece per lungo tempo le orecchie da mercante. Quando poi l'Austria occupò la Bosnia-Erzegovina, l'idea della ricostituzione del triregno croato fu ampiata nel senso che a questo regno dovrebbero essere annessi anche tutti gli altri territori, ora austriaci, abitati da Slavi meridionali, quindi oltre a tutti quelli ora nominati anche il litorale (in bloceo, Istria, Trieste, Goriziano) e i paesi degli Sloveni (Carniola, parte della Carinzia e della Stiria). Per procurare poi un valore a fuesto nuovo regno si propone dai Croati, che guidano il movimento, di trasformare l'Austria da Stato dualislico Austria-Ungheria in un trialismo austro-nngarico-croato.

Il progetto errò nelle menti dei patriotti e dei politicanti, senza trovare nè ascolto nè opposizione, fino alla recente crisi balcanica, quando sembrò che alli personaggi dell'Austria volessero farlo oggelto di studio e forse di attuazione. Forse si ventila l'idlea di farne nel peggiore dei casi un vice-reame sul lipo del LombardoVeneto: Sarebbe difficile di indovinare ora fino a dove arrivino le intenzioni sincere di appoggio al trialismo. $\mathrm{Si}$ comprende però che in fuesto movimento nazionale degli Slavi sono in ginoco tanto interessi di popolo, quanto manove di governo. Che però il trialismo salvo errore e prova in contrario - non sia destinato ad aver altro che simpatic platoniche nelle eccelse sfere, lo si deve dedure da queste due considerazioni d'ordine reale. Il trialismo scompiglierebbe più che la costituzione dell'Austria quella dell'Lngheria e quindi avrà 
sempre ostili i Magiari. Poi, se oggi che le deliberazioni in Austria devono esser prese in due, gli affari dello Stato s'incagliano, figurarsi le difficoltà che nascerebbero quando a deliberare fossero in tre, reciprocamente ostili. Qualora poi da ultimo gli Slavi arrivassero ad aver tanto potere, anche alla loro mente si affaccerebbero piani migliori, e il sogno del trialismo, sorto nelle tenebre di una notte politica, dileguerebbe cogli albori del primo giorno di libertà.

In quanto poi all'importanza dei Croati come elemento marinaro bisogna riconoscere che quella parte di loro che abita alla costa e sulle isole, mescolatasi ai latini, dai cuali apprese l'arte del navigare, possiede oggigiorno buone qualità nautiche. In particolare il governo di Zagabria s'interessa molto e lodevolmente del progresso nautico dei porti di Buccari e Portorè, ove vi sono cantieri (da qui uscì la prima nave corazzata dell'Austria), scuole, navi d'istruzione marittima, ecc.

Individualmente però i Croati non riuscirono ad affermarsi durevolmente sul mare per le ragioni geografiche esposte nella prima parte di questo studio. Però sotto le bandiere prima di San Marco ed ora dell'AustriaUngheria essi prestarono sempre buoni servizi. Sono consci e superbi di queste loro attitudini e prestazioni e non è raro il caso di udir proclamare in discorsi e sui giornali, un po' prematuramente è vero, l'avvenire infallibile della marineria croata.

I Tedesshi I tedeschi nelle regioni dell'Adriatico non hanno che piccole colonie sporadiche e non raggiungono numericamente che una frazione minima. Però anche questi pochi, consci di appartenere ad una grande nazione, tenaci per l'istinto della loro razza, organizzati maestrevolmente dal centro della Germania, favoriti dalla burocrazia del governo, non vogliono cedere un palmo della posizione privilegiata che deriva loro dagli ultimi rimasugli del feudalismo medievale. Per loro, il Tirolo settentrionale e il meridionale (Trentino) deve restare indiviso per sempre, e Trieste coll'Istria appartengono a quella marca meridionale, che finora non esiste che sulla 
carta, ma di cui la confederazione germanica non intende dimenticarsi. Essi si erano spinti verso l'Adriatico per due vie, quella dell'Adige e quella del Carso. Vedendo di non poter più raggiungere colle loro propaggini nazionali il bacino dell'Adriatico per la prima, pensano che oggi forse l'impresa di arrivare ad un porto al sud potrebbe loro arridere attraverso il Carso e concentrano i loro sforzi più che mai su Trieste. Il governo mantiene per loro scuole tedesche a Trento, a Rovereto, a Gorizia, a Trieste, a Pola senza contare i luoghi minori sovvenzionati dalle loro socielà private.

Il Lloyd una volta tutto italiano oggi ì germanizzato anche nelle apparenze esterne. Col denaro, colla burocrazia, col militare i Tedeschi vorrebbero essere i regolatori dall'alto della vita pubblica. Politicamente però sono privi d'importanza e per ciò a Trieste la loro colonia deve contentarsi di trar partito dall'opportunilì del momento della lotta ben più aspra, ch'è combaltuta oggi tra gl'Italiani e gli Slavi. Quando il pericolo italiano sembrava minaccioso per la monarchia essi inclinavano a sostenere gli Slavi. Oggi che lo slavismo costituisce un principio di minaccia per la loro egemonia in Austria e in Europa sembrano invece disposti ad accostarsi agli Italiani. Senza il governo austriaco e la grande Germania non sarebbero calcolabili.

Da quando il mare si rielevò nell'importanza economica dei popoli anche nazioni che non lo hanno per confine tentano di utilizzarlo come via commerciale. Fra queste sull'Adriatico sono i Magiari.

Essi rammemorano sempre con orgoglio l'ammonimento fatidico di Kossuth: "Maggiari, al mare» (a tengere Magyar!).

Le cure che il governo di Budapest dedica alla navigazione marittima, guerresca e commerciale non possone essere valutate che da chi abbia osservato da vicino il porto di Fimme. Il governo ungherese non solo sovernziona laulamente le due societi di navigazione ungarocroata per l'Adriatico e Adria per il Mediterrameo, coestruì cantieri, la fabbrica di torpeclini, ece, ma rorrebbe

L'Adriatico.

I Jagriar: 
accrescere il valore del proprio elemento con una propria bandiera (quistione che fu oggetto di non facili trattative col governo di Vienna) e avvia giovani magiari, tolti dalla loro puszta, alla carriera nautica, per lo più nella marina da guerra.

Gli Sloveni. Un po' l'attrazione ed il valore reale del mare, un po' il cattivo esempio, e un po' di megalomania fecero venire delle velleità navali anche ad un popolo che non è stato, non è e non sarà mai altro che montanaro, agli Sloveni. Essi abitano il Carso carniolino a settentrione di Trieste e non raggiungono il mare che con deboli propaggini a Duino, perchè la popolazione di tutta quella costa è italiana. Essi noll ebbero mai domestichezza col mare e non potrebbero avventurarsi nemmeno se un cataclisma dovesse seppellire l'Austria-Ungheria, l'Italia e la Germania, e metter su, come un'arca di Noè, il regno di Croazia, perchè in tal caso sarebbero i Croati che se li assorbirebbero.

Eppure bastò che le promesse del governo mettessero loro in vista il miraggio della conquista di Trieste, perchè qualcuno di loro vedesse già a Trieste.... il porto di Lubiana e proclamasse quel golfo nientemeno che il.... sinus carniolinus.

Gli Sloveni sarebbero destinati a minare l'italianità dal basso, e come mezzo di lotta si servono del danaro profuso loro dagli altri Slavi che si estendono fino alla Boemia. Gl'Italiani presi in una morsa tra i Tedeschi e gli Sloveni si difendono opponendo a tutti scuole e la loro brillante coltura.

\section{L'AUSTRIA NELL'ADRIATICO.}

Passiamo ora ad esaminare le tendenze e l'opera dell'Austria-Ungheria quale Stato amalgama di questi e di altri popoli.

Dalla caduta della Serenissima e dal congresso di Vienna, che consegnò definitivamente all'Austria Venezia c la costa orientale adriatica, l'Austria potè, e non a torto, 
dirsi l'erede di Venezia; anzi avrebbe potuto chiamarsene anche la padrona. Tale eredità del resto non si limitò alla presa di possesso dei beni materiali, ma fu estesa anche ai beni ideali, perchè l'Austria si arrogò perfino la protezione dei Cattolici nei Balcani occidentali e fuori d'Europa, prima esercitata dalla Serenissima quale un privilegio.

In quanto alla successione nell'Adriatico l'Austria coll'aver posto a Trieste il capo-linea della navigazione lungo l'Istria e la Dalmazia e coll'aver diramato da Trieste importanti linee di navigazione per il Levante, ritenne di aver fatto, nel suo interesse, di Trieste la regina dell'Adriatico e l'erede della Serenissima nelle tradizioni Ievantine.

Una simile asserzione d'eredità, giustificabile relativamente finchè l'Austria possedeva il Lombardo-Veneto o almeno il solo Veneto con Venezia, non calza più oggigiorno. $\mathrm{E}$ vero che in assenza e durante la depressione di Venezia, Trieste assorbì la massima parte del commercio di navigazione lungo la costa orientale adriatica e nei mari del Levante, ma da ciò non si può trarre ancora la conclusione assoluta che Trieste abbia sostituito Venezia nella missione storica dei commerci adriatici. L'asserzione zoppica innanzi tutto dal lato dialettico, perchè -Venezia non può essere sostituita da alcun'altra citlì.

In primo luogo Venezia era oltre che un porto raro uno Stato indipendente. Trieste non è invece che uno scalo come ce ne sono tanti altri. Venezia per la sua posizione geografica, come fu dimostrato nella prima parte di questo studio, può sola essere la mediatrice degli scambi commerciali tra le regioni adriatiche ed esclusivamente esportatrice di prodotti della Padania. Trieste, appartata in un angolo, non rappresenta che il porto di transito del commercio dai paesi alpini fino al Danubio. Venezia, decaduta momentaneamente, non è scomparsa, anzi rigenerata nel regno d'Italia, intende di riguadignare l'influenza perduta. L'Austria non rappresenta quindi più l'eredità di Venezia, se non che nel senso che nell'Austria sta oggi raccolta l'eredita di tutte quelle inimicizic dei 
Tedeschi, degli Ungheri, degli Schiavoni, che Venezia Ira. dovuto combattere nel lungo corso di tredici secoli della sua esistenza.

Per effetto dell'eredità di Venezia l'Austria conta tra i suoi sudditi gl'Italiani dell'Istria e della Dalmazia. In generale poi gl'Italiani soggetti ancora all'Austria vivono, tutti nel bacino geografico dell'Adriatico. Nel mentre per ragioni geografiche e storiche indistruttibili ed inoppugnabili essi vi dovrebbero occupare ancora, anche nell'ambito. dei confini austro-ungarici, il primo posto, a causa della politica austriaca essi sono costretti a lottare febbrilmente soltanto per vivere.

Quando fra il trattato di Campoformio e il congresso di Vienna, l'Austria succedette a Venezia sull'Adriatico, le acque di questo mare non erano ancora mosse dalla corrente nazionale. Però linguisticamente e civilmente l'Adriatico continuò ad essere italiano, come l'aveva lasciato la sua secolare dominatrice. Questo stato di cose, malgrado il pronunziato movimento nazionale italiano durato mezzo secolo e gli sconvolgimenti del '48 e del ' 59 , continuc̀ per quell'andazzo naturale fino al '66, ossia alla battaglia di Lissa. Perduta. Venezia e il Veneto, l'Austria ebbe paura di perdere l'Istria e forse qualche cosa altro ancora. Per ciò mutato il suo obbiettivo politico dalla penisola italica a quella dei Balcani, cambiò sistema e (trattandosi dell'Adriatico, si passi una frase marinaresca) virò di bordo, dandosi a perseguitare gl'Italiani ed a favorire gli Slavi, come fu dimostrato.

Dopo il '66 avvemnero per le due coste dell'Adriatico due fatti opposti. Sull'occidentale cessarono i moti nazionali e l'attività civile fu rivolta ai miglioramenti sociali ed economici: Sull'orientale invece, prima relativamente tranquilla, si scatenò una bufera di aspre lotte, rinfocolate dalla mescolanza della popolazione e tenute vive dal governo, il cui programma ormai troppo evidente è di distruggere l'elemento italiano dal Judrio alle. Bocche di Cattaro, stritolandolo in una morsa slavizzatrice dal basso, germanizzatrice dall'alto. Altro che eredità dị Nenezia! 


\section{GARA COMMERCIALE FRA TRIESTE E VENEZIA.}

Fra Venezia e Trieste, insegna la storia e lo dice il presente, esiste senza dubbio sotto alcuni aspetti una rivalitì d'interessi economici.

Ambedue derivano, si può dire, la loro esistenza da Aquileja, Venezia in linea materiale, Trieste in linea quasi locale e morale. Venezia in posizione di gran lunga più favorevole che Trieste, crebbe prima e ad un'altezza, che non fu concessa a Trieste.

Venezia non permetteva che altri venissero a disturbarla nei suoi traffici. Trieste che si riteneva erede dello spirito commerciale di Aquileja non voleva essere dipendente da Venezia e cercò di emularla. Da questa gelosia d'interessi sorsero attorno il mille delle lotte che si protrassero per molti secoli successivi. Finchè poterono, i Triestini si sostennero da soli; poi siccome i Veneziani avevano occupato l'Istria inclinarono verso i patriarchi di Aguileja o i conti di Gorizia. Quando anche yuesti perdettero influenza si rivolsero ai duchi d'Austria, come fu detto. La rivalitì commerciale fra Trieste e Venezia fu dunque per una metà, aceanto alla debolezza del confine geografico, la causa dell'insediamento di Tedeschi 'sull'Adriatico. Fintantochè Venezia era potente Trieste dovelte contentarsi di vivacehiare. Col tramonto di Venezia Trieste incominciò ad attivare scambi commerciali colla sponda occidentale adriatica, con Bari, Brindisi, Otranto e con Napoli. Caduta Venczia, l'Austria le sostituì quasi in tulto Trieste, e si servì lino al '48 del tornaconto commerciale per tener Trieste lontana e contraria alla mova corrente dell'idea nazionale.

Considerando ora la quistione dal lato locale si deve ritenere che tale rivalita è determinala più forse che dalla loro vicinanza dall'identiciti di mezri, di eni devono servirsi ognuma per mantenere la propria prosperitì.

$\therefore$ Tutte due hamo, al loro settentrione lat copona dei 
paesi alpini; tutte due respirano sull'Adriatico e tutte due volendo avvantaggiarsi del commercio di mediazione tra il continente europeo e i paesi d'oltremare fuori d'Europa, sono condotte dalla direzione geografica dell'Adriatico a trafficare nei mari del Levante. $E$ naturale quindi che ognuna cerchi di attrarre a sè quant'è più possibile del commercio del retroterra, di affermare la propria influenza commerciale sull'Adriatico stesso e di trasportare la maggior quantità di mercanzie nella parte orientale del Mediterraneo.

Però se Venezia, quando aveva da pensare alla propria esistenza quale Stato indipendente, doveva necessariamente procurare con tutti i mezzi il massimo assorbimento del commercio dell'Adriatico e soffocare gelosamente ogni rivalità e specialmente quella di Trieste, dietro la quale stavano appiattati i duchi d'Austria, pericolosi per il suo possesso dell'Istria, ora che può avvantaggiarsi delle risorse morali e materiali del regno d'Italia non ha più bisogno di essere tanto intransigente. Trieste non potrà mai strapparle il commercio della Padania, come viceversa Venezia non potrà più impedire che a Trieste affluiscano e passino le merci per i paesi carsici ed alpini che le stanno alle spalle.

In quanto all'Adriatico ci sarebbe posto per più d'uno, purchè il progresso civile rendesse più attivi gli scambi commerciali, sopratutto tra le due sponde. E siccome l'Adriatico va rialzandosi da questo lato, è sperabile che anche questa causa di concorrenza per scarsezza di commercio scompaia.

Per i commerci del Levante si può dire la stessa cosa. Anche qui sono cresciuti, ed i concorrenti, sebbene siano molti, vivono tutti. Anzi si potrebbe calcolare con probabilità, che i meno fortunati di questi concorrenti trasportano oggi più mercanzie che Venezia quand'era sola.

Il timore che la prosperità di una implichi la decadenza dell'altra è tanto meno giustificato oggi che evidentemente progrediscono entrambe.

Venezia e Trieste nel tempo presente e nel futuro potranno agire come scali e centri di attrazione di territori 
distinti nel transito locale e marittimo tanto in senso della latitudine che della longitudine geografica.

Anzi la rivalità è tenuta viva ancora dalla loro pertinenzal a due Stati differenti, che devono necessariamente trovarsi spesso in antagonismo e sarebbe attenuata nel giorno in cui un solo governo aresse da provvedere al benessere di ambedue queste importanti città adriatiche.

Quel poco di rivalità derivante dalla vicinanza che resterebbe sempre, servirebbe di sprone alla gara nel far meglio.

Qualche cosa di simile, ma in proporzioni assai ridotte, della gara fra Venezia e Trieste, si presenta nella rivaliti di Fiume con Trieste. Qui però si tratta di una maggiore vicinanza locale e di una minore differenza di retroterra.

Malgrado gli sforzi del governo ungherese dal ' 47 in poi, Fiume non progredì nella misura che forse esso sj aspettava. Ciò significherebbe da una parte che la posizione di Trieste è migliore come scalo del medio Danubio e dall'altra che Fiume col solo commercio proveniente dall'Ungheria non può divenire un emporio di primo rango. Fiume dunque non dovrebbe essere destinata sotto nessunat costellazione politica arl assunere importanza capitale. Dal che si deve conchindere che se il nesso politico coll'Ungheria le riesci di qualche giovamento economico nou è una condizione capace di correggere l'imperfezione geografica della sua posizione rispetto ai traffici dell'Adriatico.

\section{LA MISSIONE DI TRIESTE.}

All'epoca romana quando la pianura veneto-friulana era tutta coperta da boschi e intralciata da torrenti verso i monti e da lagune verso il mare, Artuileja, situata sulla via Emilia e difesa dalla natura fu seelta come stazione del confine italico orientale. Le furono affidati due compiti, uno militare di sbarrare la strada ai barbari invasori, 
l'altro civile di irradiare la luce della coltura romana al di là dei confini e di reclutare e romanizzare le milizie semi-barbare. Ai Romani provenienti dalla Padania mai sarebbe venuto in mente di scegliere a tale scopo il punto esposto strategicamente ove ora si distende Trieste.

Distrutta Aq̣uileja e decaduta la romanità sull'Adriatico nessun luogo ebbe più prosperità, eccettuato Venezia, che risorse in grazia alla sua eccellente posizione. geografica e s'impose a tutte le regioni circonvicine soffocando i rivali, tra cui nel secolo XIII compariscono anche. i Triestini. Più tardi lo stato poco sviluppato di civiltà dei paesi carsici della regione Giulia e dei paesi alpini orientali, le lotte locali, il disgregamento politico, la scarsezza dei commerci, assorbiti quasi totalmente da Venezia, impedirono a Trieste la formazione anche di un mediocre scalo commerciale.

Trieste incominciò a crescere dal principio del XVIII secolo, apparentemente per merito delle cure che le rivolsero gl'imperatori d'Austria, da Carlo VI in poi, ma in sostanza perchè s'erano fatti vivi ed avevano bisogno di un'espansione l'industria ed il commercio dei paesi austriaci, che avevano dato la spinta all'accrescimento anche di altre città, come Vienna, Praga, Graz, ecc.

Che il solo fatto di essere un porto austriaco non produsse la prosperità di Trieste è provato dalla circostanza che Trieste apparteneva all'Austria da oltre tre secoli ed era rimasta nell'abbandono per il motivo che i paesi del suo retroterra non avevano raggiunto ancora quel grado di progresso che dà vita ai commerci.

Per quel primo motivo e non per questo Trieste continuò a prosperare durante i secoli XVIII e XIX fino a raggiungere presentemente quell'estensione e quel benessere che la fanmo somigliare ad una seconda Milano e che nella sua storia fino al giorno d'oggi rappresentano l'apice. Ed essa crescerà ancora anche senza troppe cure del governo, perchè lo sviluppo straordinario delle città ¿̀ un fenomeno dei tempi moderni, in cui le industrie pel bisogno d'intelligenza é di mano d'opera cercano di cólocarsi a preferenza nella vicinanza di città e contribui- 
scono a produrre quell'agglomeramento di popolazione, indicato comunemente col nome di urbanismo.

A coloro i quali pretendono che Trieste debba la sua fortuna e prosperità all'Austria si potrebbe far osservare che la stessa cosa era stata detta per un po' di tempo. riguardo a Venezia, ma che i fatti poi hamo dimostrato che l'asserzione non era esatta. Venezia che dopo la sua cadula ebbe un periodo di depressione per questo fatto e per la trasformazione della marina dalla vela al vapore, rifiorì alquanto sotto l'Austria e molti credettero che staccata dall'Austria e privata del commercio austriaco d'oltr'alpe, sarebbe ricaduta. Invece, superata una nuova crisi inevitabile, ma passeggera, ridiveme lo scalo naturale della Padania e lo sbocco delle regioni alpine e potrà riacquistare importanza anche nell'Adriatico e nel Levante appena gli Italiani lo vorranmo passando dalle parole ai fatti.

Consimili previsioni lugubri si ripetono oggi per Trieste pel caso che il confine politico venisse portato dall'occidente al suo oriente. Certo clıe se ciò avenisse, \{rieste subirebbe una scossa ed ma crisi economica che la danneggerebbero, ma solo momentaneanente, giacchè Trieste verrebbe ad occupare di falli (fuel posto che all'epoca romana era tenuto da Aquileja.

Uno Stato formato entro i limili geografici della penisola italica ha bisogno di grandi centri di atlivazione e di irradiazione ai punti estremi e cone all'occiclente corrispose a questo compito sempre Genova, alloriente si ebbero Aquileja, indi Venezia ed oggi Trieste. Anzi tanto è profonda la veritì di questo falto che Trieste compie questa funzione anche oggi ch'è divisa dal confine politico e che la sua italiantiti c̀ combattuta con accanimento pari alla sna baldanzal. Trieste alssorbì già colonie francesi e tedesche importatevi artilicialmente a

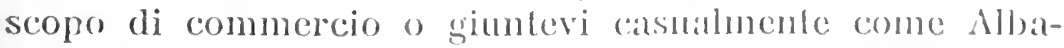
nesi, Armeni, Greci. Ed anche gli Sloveni a Trieste non potranno essere mati altro che mon colonia destinalia ad essere assorloila prima o dopo come le allee.

iTrieste sia clue appartenga politicamente all'Austria o L'Adriatico. 
all'Italia avrà sempre un'importanza speciale economica; civile e militare. Il transito del commercio non potrebbe mai venirle a mancare del tutto. Sotto qualunque bandiera si trovi, rimarrà sempre uno dei due capi della via più breve dal Danubio all'Adriatico e nessuno potrà portarle via il suo raggio economico naturale, che va fino a Vienna. D'altronde essa è il centro naturale di tre regioni vicine, il Friuli (orientale), la Carniola e l'Istria. Essa non andrebbe dunque incontro alla rovina collo spostamento del confine politico, purchè beninteso improvvide barricre doganali non facessero sviare il commercio ad un altro porto estero.

Qualcuno dirà che se l'Au'stria perdesse Trieste certo. per ripicco incanalerebbe altrove il suo commercio dell'Adriatico. Riconosciamo però a questo proposito che il porto di Trieste ha per l'Austria tanto valore ch'essa non lo perderà se non quando sia fiaccata troppo fortemente. Essa potrà perdere altre parti della costa ed anche Fiume potrà divenire per lei un porto quasi straniero, ma dorrà fare ogni sforzo possibile per conservare Pola o almeno Trieste. Appunto perchè riteniamo che Trieste non sarà strappata all'Austria che nel giorno in cui guesta sia ridotta nelle condizioni in cui la mise Napoleone nel 1809 colla costituzione del regno d'Illiria, riteniamo pure che se si avrerasse il passaggio di Trieste all'Italia, l'Austria non avendo un proprio porto sull'Adriatico, di buon o di cattivo grado, il commercio dei paesi alpini fino a Vienna, a meno di non volervi rinunziare affatto o incorrere in spese sproporzionate per la via di Amburgo, cosa poco probabile, non avà ancora altra strada migliore di quella di Trieste. Non vediamo forse che il commercio della Svizzera meridionale, in quanto trova il suo tornaconto negli scali del Mediterraneo, si scarica nel porto di Genova? 


\section{PRETESA PRECARIETA}

\section{DELLA FLORIDEZZA DI TRIESTE.}

Considerando il rapido e recente accrescimento di Trieste qualcuno ritiene che la sua floridezza abbia da essere precaria. Certamente non è così solida come quella di Nenezia, provata da tutte le vicende della storia. Trieste non è situata in un punto di valore commerciale stabile, come Venezia che dalla sua fondazione ad oggi ebbe importanza, per lo meno locale.

Trieste fino al secolo XVIII era un porlo secondario, ma bisogna riconoscere anche che tale slato inferiore al valore della posizione geografica fu delerminato in parte da ragioni storichic, in parte dalle condizioni infelici del suo retrolerra. - Trieste è inleriore a Venezia perchè ad enorme differenza di questa ha un retroterra povero delle risorse naturali linora maggiormente sfruttate e perchè deve di conseguenza cercare lontano le materie da commerciare. Però la civiltà melle in valore forze naturali che per la barbarie sono improduttive. Per dimostrarlo non occorre spendere molte parole e basterà invece citare ad esempio la Padania, incli, in senso inverso, tutta la costa sellentrionale dell'srica oppure anche la Bocmia, che nell'antichita era ma regione tutta foreste ed accute ed oggi è uno dei paesi più industri d'Europa. E come il progresso generale della civilta moderna produsse la vita industriale anche là dove, come nei paesi delle alpi orientali, non esisteva, è̀ motivo da sperare che ulteriori applicazioni praliche di indagini e invenzioni scientifiche per l'atmentato benessere della vita materiale, provocheranno qualche sfrutamento utile anche di quelle regioni carsiche finota poco curate per la loro conformazione sassosa, brulla, ventosa.

Una di queste potrebbe essere l'ulilizazione industriale della pietra calcare del Carso. Questo allipiano non è che un immenso ammasso calcareo c da esso oggri non si 
ritrae, con mezzi non ancora perfetti, altro che la calce viva. Se però si riuscisse a procurar energia elettrica a buon mercato, vi sarebbe la possibilità di estrarre dai numerosi suoi banchi calcarei il carbonio puro, il carburo di calcio, il calcio metallico, la calcio-cianamide, la calce idraulica ed altri prodotti pei quali la calce serve di materia prima. Di grande importanza per tutta quella regione potrebbe divenire l'impiego del calcare frantumato per il cemento armato. Oggi poi che l'uomo, oltre all'aver tentato di utilizzare le onde marine, procede alla conquista dell'aria, è lecito sognare almeno che si riesca a trarre qualche utile sia pure intermittente, da quell'immensa e tremenda forza naturale ch'è la bora.

Ma se anche queste speranze ardite dovessero dimostrarsi fallaci, la prosperità di Trieste non sarà limitata alla sua dipendenza dall'Austria, ma durer⿳亠口冋 per lo meno fino a tanto che dureranno le condizioni industriali e commerciali che hanno fatto fiorire il centro dell'Europa nei tempi a noi più vicini.

Trieste inoltre non conta oggi che come punto di transito del commercio da nord a sud e manca di buone comunicazioni nella direzione dall'occidente verșo oriente. Trieste, quando fosse liberata dall'incubo della strategia militare, potrebbe divenire la stazione importantissima di una ferrovia commerciale costiera dal bassopiano veneto-friulano per Aquileja, Monfalcone, Duino, diretta a raggiungere lungo il vallone di Muggia nella via più breve il fiume Culpa e quindi lungo la Sava il Danubio e le due pianure ungarica e valacca. Il progresso industriale e commerciale, che ha ormai quasi saturato l'occidente e il centro dell'Europa, cerca di espandersi per forza di circostanze nell'oriente balcanico. E siccome l'avvenire commerciale anche di queste regioni è ormai certo, non sono del tutto vane nemmeno le speranze che Trieste diventi. la stazione intermediaria meglio situata per gli scambi commerciali terrestri tra le due penisole italica e balcanica. Ed anche in tal caso l'irresistibilità del fattore economico sarebbe superiore alle barriere e alle bizze politiche. 


\section{PERSECUZIONE DELL'ELEMENTO ITALIANO.}

In conseguenza del programma di distruggere l'italianità della costa orientale adriatica onde poter fare di questa costa un territorio austriaco, la prima ad essere sacrificata fu, come si disse, la Dalmazia.

A completare poi la presunta croatizzazione di questa provincia fu emanata nel 1909 un'ordinanza speciale sull'uso delle lingue negli uffici dello Stato, che sostituendo al secolare uso dell'italiano intempestivamente il croato, suggerì ad un giornale tedesco la frase di commento, che con quell'ordinanza il governo austriaco seppellì San Marco. (Forse a titolo di eredita?).

In questo modo fu compiuta a spron ballulo quell'artiñiciosa metamorlosi esterna, che dorreble dare alla Dalmazia un carattere pretlamente slavo. Ma non si riuscì a forzare menomamente la nalura, peschè ognuno che viaggi la costa orientale adriatica s'ateorge che le citti conservarono la loro impronta veneziana, cosi come i cuori conservarono la coscienza italiana e le bocche abituate a parlare il dialetto delle lagune continnano e continueranno a parlarlo ancora.

Quando la slavizzazione della Dalmazia, intrapresa per opportunita balcanica, era già progredila, il governo fece scoccare l'ora per l'Istria, essendogli parso di trovarvi per di più una necessità stralegica. L'Istria ha la fortuna - la disgrazia, come si voglia, di posserlere Pola. La germanizzazione della marina da guerra alustriaca trova un ostácolo nell'ambiente italiano di Pola. Inoltre non è facile, nè comodo. affilare le armi rontro gl'tlaliani sotto i loro stessi occhi. Il governo rede quindi di avere diverse ragioni per ritenere questedemento, olleche apoditticamente inficlo, anche molto male piazalo. Tullo cio è più che bastante per determinarlo a sbatrazarsene a sostituire al suo posto altri ritenuti migliori, per escmpio i Croati. Come sapete l'Istria odierna amministralivanente 
austriaca è composta della provincia veneta, mariltima, ilaliana, e della provincia liburnica austriaca, montuosa, slavizzata. All'Istria appartengono inoltre le tre isole di Veglia, Cherso e Lussin, clie sotto Venezia ed anche prima facevano parte della Dalmazia e che le furono assegnate dopo la caduta napoleonica. Oggi le forze degl'Italiani e degli Slavi in Istria si tengono quasi in bilico, con maggioranza italiana; ma colla tendenza del governo a mettere continuamente pesi nel piatto degli Slavi, si deve necessariamente temere che la bilancia tracolli dalla parte loro.

Trieste, il centro dell'italianità in Austria, e sulla parte orientale dell'Adriatico, cittì fiorente che si arroga la pretesa di divenir sede di una università italiana, non poteva non subire la sorte clelle altre terre italiane. E siccome i pochi Slavi del suo suburbio nulla possono tentare contro la compagine cittadina, il governo prima mobilizzò il socialismo intermazionale a parole, slavofilo nei fatti, e poi le scaricò addosso gli Slavi di tutta la Carniola e perfino della Boemia.

Apparentemente per non fare un'cccezione alla parola d'ordine, ma certo per strategia e per fiaccar prima la resistenza degl'Italiani, non furono risparmiati nemmeno i bonari e pacifici abitatori del Friuli orientale, rei di essere troppo vicini al Judrio. Ed ora anche quelle popolazioni hamno da subire il gusto di incursioni degli Slavi che, accortisi dopo secoli di non star più bene lin montagna, vorrebbero scendere a riscaldarsi nelle basse triulane. Gorizia deve sostenere l'urto più forte.

C'era fino a pochi lustri fa ancora un angolo dell'Adriatico dove gl'Italiani erano tollerati in pace, vale a dire a Fiume. Ma dacchè il governo di Budapest adottò nell'amministrazione interna i sistemi di. Viemna e si mise in testa di magiarizzare, tutti gl'Italiani soggetti alla monarchia austro-ungarica sono trattati egualmente male.

Ma il governo austriaco ha fretta, molta fretta. E siccome gli Slavi, malgrado tutte le concessioni ed i favori, potrebbero venir tenuti in rispetto dagl'Italiani, anche soli, ancora per molto tempo, il goremo si compiace di 
ricorrere ad altri mezzi per affrettare la pulizia nazionale della sua costa. La polizia di Stato, gli agenti provocatori, le spic, con tutta la fioritura di amenita da loro prodigate, come le proibizioni, gli scioglimenti, le espulsioni, i sospetti, le delazioni, i processi, a Gorizia, a Trieste, a Pola, a Fiume, a Zara e nelle altre città minori, hanno prodotto negl'Italiani un'esasperazione generale e tanto più giustificata in cquanto che gl'Italiani comprendono di essere le vittime innocenti di rancori impotenti contro fatti inclultabili della storia e contro il suo fatale cammino e samo di venir trattati come un capro espiatorio da un governo che crecte di propiziarsi col loro sacrifizio un migliore arrenire.

\section{ERRORE POLITICO DELLAUSTRIA.}

L'esperienza del secolo passato dovrebbe insegnare ai governanti dell'Austria che i sistemi (puarantolteschi, ora rimessi in onore, abortiscono il loro efletlo. Le persecuzioni, oltre che esasperare gli ardenti, hamno avuto l'effetto di destare i sonnolenti e di anmentare per conseguenza il numero dei malcontenti. In cincuanl'anni di repressione l'italianiti della costa adriatica, ristretla in apparenza, si consolidò nella sostanza. Trieste nel 1818 eral molto meno italiana che oggidi. Fino a quell'mno il governo la chiamava la "fedelissima» e nel 18.51 l'invilava a farsi rappresentare alla dieta di finncoforte. Nell'Istria la minaccia slava produsse $n$ risreglio benefico di energia e di concordia. E se il Frinli oriculale ne segura fra breve l'esempio piủ che la cosidella propaganda irredentistica, Lanto leggermente tirala in ballo, si avrì da ringraziare il sistema di governo. Lo stesso vale gia per Fiume, dove bastò un tentativo di ostracismo alla lingua italiana per produre la piò viva reazione del sentimento. In Dalmazia la lingua italiana topo mezo secolo di persecuzioni i ancora sulla bocea di tutti coloro che ha parlavino prima.

L'oppressione degl'Italiani in Austria lua ragggiunto or- 
mai il massimo limite e ad onta di questa essi hanno edificato tre belle città, Trieste, Pola e Fiume, dove una volta non c'erano che borgate, hanno strappato al governo scuole nella loro lingua e se anche non sono progrediti politicamente, come avrebbero fatto in condizioni meno critiche, la loro posizione in quanto a numero, lingua e coltura non è peggiorata.

Il governo dovrebbe quindi persuadersi che le persecuzioni, oltrechè essere odiose, sono inutili. Del resto poi un po' di persecuzione giova praticamente. Senza il martirio politico non si sarebbero purificati nemmeno gl'Italiani dell'altra sponda.

L'oppressione di quella frazione italiana, ch'è soggetta all'Austria, non potrà essere eterna e se anche quest'oppressione dovesse essere intensificata, cosa quasi impossibile e ormai anche poco probabile, non potrà produrre la distruzione, perchè vi si oppone quell'assioma che un popolo o frazione di popolo superiore per civiltà, appoggiata al suo tronco, sia direttamente come i Trentini, sia indirettanente come gli Adriatici, che sono congiunti dal mare, non può soccombere di fronte a masse, più numerose, ma meno colte.

Gl'Italiani dell'Austria dal canto loro conoscono la loro posizione nella gcografia e il loro còmpito nella storia: sanno che la lotta per l'esistenza nazionale è divenuta ormai per loro un'oceupazione alla quale si sono abituati, come altri popoli si dànno alla caccia, alla navigazione, alla guerra, anche ai divertimenti, per. passatempo, e non cederanno. Nè il secolo ventesimo è stato scelto bene per sradicare gl'Italiani dall'Adriatico.

Ma ammettendo pure per una inconcessa ipotesi, che il piano del governo anstriaco dovesse riuscire, esso non ne ritrarrebbe egualmente alcun vantaggio. Perchè l'Austria perseguita in ultima analisi gl'Italiani? Non certo per procurarsi il gusto di convertire i tre quarti di milione d'Italiani suoi sudditi in Slavi, Tedeschi e Magiari e aumentare, specialmente sulla costa adriatica, il numero dei Croati. Essa lo fa per impedire - diciamo l'ostica parola - rivendicazioni irredentistiche, o forse per lo 
scopo più pratico di sbarazzarsi di un elemento che le è stato sempre infido - sarebbe puerile voler falsare la storia - e cle simpatizzando col probabile nemico di domani potrebbe divenire pericoloso.

Lasciamo stare questo scopo pratico dell'Austria e vediamo che cosa voglia l'irredentismo. Nelle declamazioni si ode dire che l'Austria detiene paesi che spetterebbero all'Italia per diritto geografico, storico ed etmografico. Ora siccome le parole, anche se reboanti, non sono cannonate, tutti samo che l'Austria mon corre alcun pericolo di perdere le sue province abitate da Italiani, fino a tanto che l'Italia non abbia la possibilità e la volontà, ossia la forza, di strappargliele. Per l'Austria quindi da questo lato ¿̀ affatto inconcludente di conservarle italiane o snazionalizzarle. Che se viceversa renisse il momento nel quale l'Austria fosse condannata a perdere il litorale adriatico, la slavizzazione non ne impedirebbe il passaggio all'Italia, che nella forza della vittoria troverebbe ragioni sufficenti per gitustilicare il suo atlo, anche se l'Austria le avesse distrulto il tilolo della nazionalità in quel modo che tutti samo.

E possibile però anche che l'Austria persegua uno scopo recondito e remolo, quello di tener vivo l'astio fra Italiani e Slavi per vederli un giorno accapigliarsi per bene tra loro. Certo è liallanto che per un osservalore il quale abbia a propria disposizione soltanto il buon senso e la logica il procelere dell'Austria apparisce strano, inutile e damoso ad essa stessa. Ricorderemo il noto adagio greco: "Conosci te stesso", per dire che proprio l'Austria polietnica (lovreble nel suo interesse nostrarsi assai tollerante delle incvitabili simpatic nazionali tra popoli aflini divisi, com'è il caso sull'Adriatico, da un confine politico non bene perectlibile.

L'inficrire ingiustificalo dell'Austria contro gl'Italiani, suoi sudditi, Lurba evidentemente le relazioni tra i due Stati vicini ed ora alleati e se anche non si possa dedurre che ne deriveramno perturbazioni internazionali - schbene alle volte l'incendio nasce dat una lavilla - servira a documentare le tendenze degli Stati e delle nazioni su L'Adriatico. 
questo mare nel tempo in cui viviamo. Sotto l'egida dell'Austria Tedeschi, Magiari, Sloveni e Croati dovrebbero poter svilupparsi sull'Adriatico e gl'Italiani no. L'ingiusto traltanento se fa sofirire gli oppressi, procura loro certamente le simpatie degl'imparziali e ragionevoli, e, provocando in loro la coscienza di essere dalla parte della ragione, procura loro il conforto morale, non disprezzabile, della resistenza per una buona causa.

La repressione del sentimento italiano era stata. elerata ad assioma d'una necessità di Stato anche per il Lombardo-Vencto. La storia dimostrò poi che il preteso alto sistema di governo non fu altro che un basso ed. inutile sfogo di polizia.

\section{RECENTE POLITICA AUSTRIACA SULL'ADRIATICO.}

Per quanto possa apparire noiosa la ripetizione, è un fatto, di cui si sarà persuaso ogmuno che conosca o abbia studiato le condizioni interne dell'Austria, che in ogni quistione, sia governativa, amnuinistrativa, scolastica, religiosa, economica e da qualche tempo anche militare, prima o dopo si vede far capolino inevitabilmente l'aspirazione nazionale. Si chiami questo stato di cose una piaga, lo si paragoni ad un labirinto interminabile, esso esiste e nessumo potrebbe negarlo.

Non sarebbe però giusto dar all'Austria, com'è governata oggi, tutta la coipa di queste lotte. Esse esisterebbero anche sotto un'altra costellazione amministrativa. L'Austria ha invece il torto, anzichè di averle attenuate, come ne avrebbe avuto oltre il dovere anche la possibiliłà ed i mezzi, accordando di fatto a tulti i suoi popoli quel rispelto e quella sicurezza che sono sanciti nelle sue leggi, di averle rinfocolate fino al parossismo, con danno della tranquillità generale dei popoli e forse dei suoi stessi interessi.

L'Adriatico ed i paesi da esso bagnati, per rialzarsi dalla prostrazione in cui li lasciò la decadenza della marina a rela avrebbero bisogno di speciali cure in opere portua- 
rie, in congiunzioni ferroviaric, in sovvenzioni alla marina mercantile. L'Austria invece da parte sua non fece che poco e anche questo con obbiettivi puramente strategici. Imperatori come Carlo VI, Maria Teresa, avevano rivolto attenzione speciale al porto di Trieste e alla socicti del Lloyd. Per l'incuria del governo in tempi più l'ecenti l'una e l'altro erano andati deperendo, e se alla citla fu data di recente una seconda congimnzione ferroviaria, la transalpina attraverso i Tauri e le Caravanche e se ne sta ampliando il porto, ciò fu fatto non collo scopo precipuo di favorire il commercio, ma di riversare più facilmente soldati al conline orientale verso il Friuli.

Perdite d'afíari, diminuzioni di merci e l'opposizione del governo di Budapest ad aumentare la sovvenzione al Lloyd, divenuto una socictà bmocralizzata c germanizzatrice, indussero il governo di Vienna ad assumere questa società per proprio conto nel 1892, dandole il titolo di Lloyd austriaco.

Non l'intraprendenza commerciale, ma gelosic tra Vienna e Budapest diedero poscia origine alla socictì di navigazione ungaro-croata, con sede a Fimme, in concolrenza al Lloyd di Trieste.

L'intraprendenza di singoli armatori privati dell'Istria e della Dalmazia ha dato vita a socictà di navigazione con sede a Trieste, a Lussimpiccolo, a Fiume, a liagusa, ma di queste il governo si cura poco o solo quel lanto che gli serve per avere una flotta di navi da trasporto e introdmre nelle direzioni commerciali anmiragli a riposo.

L'Adriatico ed i paesi della sua costa orienlale si crede di poterli tener avrinti colla burocrazia, con seuole tedesche, con banche slave, con ospizi marini per conto di municipi alpini, coll'inviarvi i turisti, con qualche esposizione a Vienna. Ma con lutte queste precanzioni il governo austriaco deve sentirsi ancora poco sicuro sult'Adriatico, perchè vi ammassa dappertutto e sempre più militari. L'elemento che nel frasario austriaco viene qualificato "dall'interno della monarchias ma che con parola più chiara dovrebhe venir detto montanaro, abbonda per- 
fino nella marina da guerra, tanto che non è raro il caso di udire che marinai, caduti in mare, annegarono per non aver saputo nuotare. Viceversa l'elemento italiano, marinaro della costa, viene relegato lontano nell'interno.

L' Austria dal 1866 al 1908 si cra ostinata a non vedere sull'Adriatico altro pericolo che da parte degl'Italiani. Per ciò aveva allevato artificialmente e rinfocolato il sentimento nazionale dei Croati e degli Sloveni. La recente guerra balcanica le insegnò che se gl'Italiani non si lasciarono prendere nè colle blandizie nè colla violenza, anche gli Slavi da lei favoriti si preparano a darle filo da torcere. Dopo tante benemerenze spese dall'Austria per istillare agli Sloveni ed ai Croati il sentimento di una individualità nazionale e religiosa differente dai Serbi, bastò il fragore delle armi balcaniche, perchè il loro nazionalismo assumesse d'un tratto quel grado d'intensità, che a proposito degl'Italiani si usa indicare colla parola irredentismo.

Dunque disorientazione politica e militarismo sono i due fenomeni di cui l'Austria dà spettacolo di fronte ai Balcani e all'Adriatico. Ma chi ne sofire di più momentaneamente è il secondo.

La maggior parte delle opere portuali costruite lungo la costa orientale austriaca deve la sua origine alle esigenze della marina da guerra. Lo stesso dicasi per l'attività nelle costruzioni navali e pei cantieri di Trieste, Pola, Fiume, Monfalcone. Tutti gli altri porti che non hanno la sorte di servire direttamente agli scopi della marina da guerra vengono trascurati. Unicamente ai bisogni militari vanno debitori i luoghi della costa alla preferenza nelle modernissime costruzioni radiotelegrafiche.

Trascurata poi del tutto è la terraferma. L'Istria e la Dalmazia non avrebbero ancora nemmeno quei piccoli tratti di ferrovia, nè un telefono senza le esigenze strategiche. L'Austria tratta la costa adriatica coi criteri e coi metodi che altri Stati adoperano nelle colonie di recente acquisto. Il Carso è sempre povero; i montanari dell'Istria e clelka Dalmazia dopo un secolo di dominio 
austriaco sono ancora in miseria. Le popolazioni languiscono e chi ha cervello comprende e perde sempre più la fiducia nelle promesse governative.

Questa è brevemente l'opera compiula dall Austria sulla costa adriatica negli ultimi cincuant'anni.

\section{L'EQUILIBRIO DELL'ADRHATICO.}

La parola equilibrio - un neologismo diplomalico ha un concetto vago e labile ed è usata il più delle volte per esprimere la tendenza alla conservazione di uno stato di cose. Essa ̀̀ pari quindi a cuell'altra frase creata dalla diplomazia dello statu quo.

Considerato dunque bene, l'equilibrio non è nella politica nè nella vila dei popoli uno stato elernamente duraturo, perchè non rappresenta che la stasi momentanea quando le forze del progresso, insito nella nalura umana, riposano o si tengono temporancanente in bilico. II moto è l'essenza della vila e la quiete non rappresenta che stadi di transizione, precisamente come nella bilancia che per sua natura e per corrispondere al suo scopo deve oscillare, e sta ferma sollanto quando un peso le impedisce di muoversi. L'equilibrio poi non è una forma politica precisa, giacchè può sussistere sollo le condizioni più disparate e perfino opposte.

Qual equilibrio più bello e sicuro sull Adriatico di quello che vi regnava all'epoca romana? l'oi si chbe l'equilibrio solto i Bizantini che dell Adrialico non occuparano che i punti più importanti della costa e solto i Vencziani che possedevano solamente la costit opientale. Anche nella prima metà del secolo passato vi fu un equilibrio, tulto a favore dell'Austria, che pure non possedeval che una parte della costa dal Po a Callaro.

L'Adrialico per la sua ristrettezza non si presta a che due grandi potenze vi si sviluppino simultancamente senza pericolo di urtarsi. LiAlriatico differisce per cio grandemente dagli altri mari, come il Mediteraneo o il Mar 
Nero, che hanno una circonferenza ampia, rotondeggiante. Esso dovrebbe venir confrontato piuttosto al Baltico. Di conseguenza la miglior forma di equilibrio per tali mari è quella di cui un solo Stato gode la supremazia incontrastata.

Se però noi vogliamo cercare una forma d'equilibrio per l'Adriatico con due potenze non potremo trovarla altrimenti che dividendo le due coste in modo che lo Stato. che possiede l'occidentale inferiore geograficamente, abbia degli altri vantaggi che lo compensino militarmente (non bisogna dimenticare lo spirito dei tempi in cui viviamo) in confronto del maggior valore dell'orientale. $\mathrm{E}$ questo è appunto il caso ora tra l'Austria e l'Italia ove se la prima possiecle i migliori porti, la seconda clispone della testa (la Padania) e di tutta la metà del mare compresa l'imboccatura. La giustezza di questo concetto sembra essere stata riconosciuta anche dalla diplomazia dei due Stati a proposito della recente spartizione delle sfere d'influenza sulla costa albanese, giacchè l'occupazione dell'Albania (Durazzo e Vallona particolarmente) per parte di uno solo avrebbe inevitabilmente per conseguenza la superiorità di questo sull'altro.

Ma quanto stento ci volle per produrre questa forma di equilibrio a due. Occorsero cincuant'anni di aspre lotte per la creazione del regno d'Italia e altri cincquant'anni spesi per la formazione degl'Italiani, trent'anni di alleanza un po' forzata tra i duc secolari e mortali nemici, la liquidazione della Turchia europea. Eppoi appena stabilito questo difficile equilibrio ecco sorgere una nuova minaccia di perturbamento, il canale di Corfù e lo stato della Grecia.

Ma prescindendo anche da tutte le possibili fonti di alterazione che potrebbero avvicinarsi dai Balcani, esistono all'interno dell'Adriatico altre cause che lo rendono estremamente labile. 


\section{A NTAGONISIIO.}

L'Austria s'era abituata fino al 1866 a godere il predominio incontrastato sull'Adriatico. Si rassegnò à dividerselo territorialmente malgrado la vittoria di Lissa, ma continuò a conservarvi il primo posto nella navigazione.

La marina da guerra austro-ungariea - ancle per ragioni di spazio in contrapposto all'italiana spesso asscnte - vi era sempre presente e vigile e così pure nel movimento commerciale tanto fra le sue sponde quanto tra i suoi scali e quelli di altri mari primeggiavano navi dei porti dalla parte orientale Trieste, Fimme, Lussino, Ragusa, battenli bandicra austro-mungrica.

L'Italia intanto avendo raggiunto anche la maturiti economica volle pareggiare la posizione anche nel terzo dei suoi mari, ma bastò questo sviluppo naturale e giustificalo per provocare gelosic.

L'opposizione dell'Austria è però anche una manifestazione spiegabile, che si potrebbe dire storica. E destino che ogni volta che l'elemento italico fu portato ad espandersi sull'Adriatico abbia avulo da incontrare resistenza negli abitatori dell'altra costa. I Romani trovarono gli Illiri, i Veneziani Iottarono coi Narentani, coi Croati, cogli Uscocchi, coi Turchi, e gl'Jaliani hamno avuto da urtarsi coll'Austria. E una fataliti che sia così e si potrebbe quasi dire che la lotta anzichè fra popoli, che non furono sempre gli stessi, sia proprio fra gli elementi delle due coste, la penisola italica contro la balcanicil.

L'Austria ha bisogno dell'Adrialico come di ma condizione vitale per la sua conservazione di grande potenza. Anzi il tratto di costa adriatica posseduto dall'Austria rappresenta il minimo necessario fanto relativanente alle altre grandi potenze, quanto assolutamente riguanklo alla sua potenzialitit. Esiste rioe nno squilibrio trat la forza terrestre e il possesso marillimo dell'Austria, e per ciò ora che tulti gli Stati ambiscono al grado di grameli 
potenze estendono la loro forza proporzionalmente sui mari, l'Austria deve ritrarre dall'Adriatico tutte le sue risorse. Per non restar indietro agli altri vuole anch'essa avere una flotta potente, superiore alle esigenze locali. della sua costa, capace non solo di difenderle ma di cercare all' occorrenza il nemico altrove. Per ciò essa deve mettere in opera tutti i mezzi onde conservarsi aperta l'uscita dall'Adriatico e poter portare la voce della sua potenza in tulte le quistioni marittime del Mediterraneo. La privazione dell'Adriatico la menomerebbe ipso facto e privandola della forza navale la ridurebbe a potenza di. secondo grado.

'Per I'Italia l'Adriatico, se anche fu talvolta trascurato, non è meno importante. Per essa, invero, questo mare non è assolutamente necessario onde conservare la potenza marittima. Però al bacino dell'Adriatico appartengono le due regioni della Lombardia e del Veneto, che sono per l'Italia una condizione vitale non solo quale grande potenza terrestre, ma come fu provato dalla storia, per la sua esistenza. Ora da questa parte l'Italia deve, tollerare dei gravosi diritti di servitù a vantaggio dell'Austria. Lo sperone del Trentino sporgente dalle Alpi nel piano tra la Lombardia e il Veneto domina queste regioni. Il confine orientale fissato al Judrio nel basso Friuli lascia aperta una grande porta a tutto vantaggio dell'Austria. Fino a tanto dunque che questa potenza tiene non solo il Trentino e l'alto Friuli, ma anche l'Istria con Pola, l'Italia non può dirsi sicura in casa sua. Contro questa minaccia da parte di uno Stato superiore per forza terrestre l'Italia non può difendersi altrimenti che opponendo una superiorita navale. L'invasione di un esercito nemico dalle Alpi nel Friuli e nel Veneto non può venir trattenuta che con contrattacco dal mare nell'Adriatico. Anche l'Italia deve quindi a tutti i costi tener aperta l'entrata in cuesto mare per non vedersi compromessa ancora più seriamente nella sua esistenza. Per ciò se l'Italia fece dei tentativi per portare i suoi confini settentrionali e orientali alla linea naturale dello spartiacque dei monti sulle Alpi e sul Carso non si può rimprove- 
rarle di aver perseguito sogni megalomani. Tali sforzi derivano dalla coscienza della propria conservazione.

Qui sta il perno di tutta la quistione del problema dell'Adriatico, del suo equilibrio, dell'antagonismo italoaustriaco: uno non può muoversi e progredire senza urtare o pregiudicare l'altro. Per ciò fu detto con molta ragione che l'Italia e l'Austria non possono essere che alleate o nemiche.

Malgrado però l'attuale alleanza ogni movimento dell'una provoca la reazione dell'altra. Alla fortificazione di Pola l'Italia non potendo far di più oppose il riordinamento strategico delle piazze di Venezia e di Ancona. Quando l'Austria nell'ultimo decennio del secolo scorso imprese a fortificare le Bocche di Cattaro, con Teodo quale stazione della marina da guerra, l'Italia fece poco dopo altrettanto a Brindisi. Alle stazioni semaforiche, di torpediniere e di siluranti poste dall'Italia lungo la sua costa adriatica, l'Austria contrappose da Trieste a Cattaro un'infinità di consimili stazioni, con apparati radiotelegrafici e con linec telefoniche militari.

Ma la prova più palese di un sordo rancore specialmente da parte dell'Austria è data dall'ingiusto trattamento, più volte ripetuto e dibattuto, degli Italiani, suoi sudditi.

Se dunque malgrado un equilibrio che geogralicamente dovrebbe essere ritenuto ideale e un'alleanza trentenne ogni mossa di una potenza suscita le gelosie dell'altra, vuol dire che su questo Adriatico esiste anche nelle condizioni attuali un antagonismo fatalmente inevitabile, che la brama generale di pace non può nemmeno far tacere.

\section{PREDONINIO.}

Se l'equilibrio illusorio è turbato da $11 n$ antagonismo insanabile, il passo ulteriore ad una lotla per il predominio è breve.

Se anche in questo incontro vogliamo ricorrere agli ammaestramenti della storia, vedremo che l'Adriatico per 
la sua forma geografica ristretta e prolungata, come fu ripetuto più volte, non ebbe periodi lunghi di equilibrio stabile, che quando un solo popolo o Stato ebbe in esso la supremazia incontrastata. Ed a conferma basta ricordare la pace romana, durata sette secoli, il dominio bizantino di cinque secoli, e quello di Venezia di otto secoli (in questo senso dal XII a tutto il XVII secolo). Tutti gli altri periodi in cui si ebbe sulle due sponde il fiorire di popoli o Stati differenti, furono brevi e forieri di lotte, che si esplicarono per lo più in guerre. Tali furono quelli del I e II secolo a. C., quando la marineria illirica si trovò in antagonismo colla romana, indi nei secoli IX e X si ebbero le lotte tra Bizantini, Veneziani, Narentani, Saraceni, nei secoli XI-XIII tra Veneziani, Ungari, Croati, Normanni e infine allo svolto del secolo XVIII-XIX tra Napoleone e l'Austria. Sull'antagonismo recente dell'ultimo mezzo secolo tra l'Austria e. l'Italia, che ha raggiunto nell'ultimo lustro il punto culminante, suscettibile di tenere ancora in bilico le forze, non si può dare un giudizio, perchè gli manca la soluzione. - Si può rilevare solamente che se l'Austria ebbe il sopravvento a Lissa e il predominio nella navigazione, l'italiana è stata sempre sull'Adriatico la nazione di gran lunga superiore a tutte le altre.

Se poi vogliamo indagare quale fu l'esito di questi vari antagonismi secolari per l'Adriatico vedremo un fatto strano e apparentemente in contraddizione colla geografia locale, che la vittoria finale rimase alla costa occidentale, meno favorita dalla natura. I Ronıni ed i Veneziani furono i più fortunali e più duraturi trionfatori sull'Adriatico. I Greci prima ed i Bizantini poi nel porre le basi del loro predominio sull'Adriatico scelsero punti della costa occidentale i primi nella bassa Italia, Magna Grecia, i secondi alle foci del Po, esarcato di Ravenna.

Sulla costa orientale si decisero le sorti guerresche per la supremazia dell'Adriatico.

Tutte le cinque battaglie navali più importanti, combattute a questo scopo e già ricordate, a Zara nel 1202, a Curzola nel 1298, a Pola nel 1379, a Lissa nel 1811 e 
di nuovo a Lissa nel 1866 ebbero per scena un punto della costa orientale. Eppure solo una, quella più recente di Lissa, arrise al possessore della costa rispettiva. In quanto però alla seconda Lissa noi sappiamo che con essa non fu dimostrata la superiorità nautica nè dei popoli, nè degli Stati in rivalità sull'Adriatico, ma solo quella individuale dell'ardimento e dell'avvedutezza di Tegethof sulla pusillanimità e imprevidenza di Persano, che si lasciò dopo lunga attesa attaccare in condizione d'inferiorità nautica e in stato di stanchezza per i bombardamenti del giorno precedente. Del resto a proposito della Lissa del '66 si potrebbe permettersi di usare il bisticcio che in quella battaglia combatterono italici contro italiani.

In sostanza tutte quelle cinque battaglie non dimostrarono altro che la vittoria arrise ai rispettivi ammiragli vincitori pel semplice effetto della preparazione $\mathrm{e}$ della tattica usata e non per vantaggio di acque o di coste. La spiegazione di questo costante predominio storico della costa occidentale è offerto dalla geografia. La costa occidentale ricevette un decisivo aiuto materiale e morale dalla Padania e dall'altro versante apenninico, aiuto che invece in senso inverso e corrispondente mancò sempre alla costa orientale. La geografia poi corresse sempre anche gli errori degli ammiragli; c come malgrado le sconfitte di un Dandolo a Curzola e di un Vettor Pisani a Pola i Veneziani si risollevarono a Chioggia e rimasero padroni dell' Adriatico invece degli intrusi Genovesi, così le sconfitte di Dubourdieu e di Pasqualigo alla prima e di Persano alla seconda Lissa non impedirono alla vinta Italia di continuare a rielevarsi e svilupparsi brillantemente sul mare. 


\section{DOMINIO ASSOLUTO.}

Il concetto di predominio presuppone la coesistenza di più rivali, uno dei quali ottenne la superiorità sugli altri.

Quali sarcbbero i limiti di questo predominio sull'Adriatico e potrebbe esso convertirsi in dominio assoluto?

La storia c'insegna che al solo impero mondiale dei Romani era riuscito di ottenere un dominio assoluto terrestre e navale sull'Adriatico con esclusione di rivali. Però anch'essi avevano dovuto tollerare e servirsi per la loro marina dei preziosi elementi nautiçi offerti dagli Illiri e dai Greci dell'altra costa. E nemmeno questa loro opera colossale resistette, perchè fu turbata proprio sull'Adriatico, prima clie negli altri mari, ancora dal III e IV secolo dalle trasmigrazioni dei popoli, che fecero poi della romanità sulla costa orientale quella strage che fu illustrata nel capitolo relativo.

I Bizantini dominarono per mare, ma non ovunque per terra e quindi la loro dominazione, sebbene sia stata poderosa e secolare, non può dirsi esattamente un dominio assoluto, ma piuttosto una supremazia, perchè anch'essi ebbero da lottare con Goti, Longobardi e Franchi.

I Veneziani, che rimnovarono in parte le tradizioni romane, e in parte seguirono l'esempio dei Bizantini, per le mutate condizioni politiche dell'Europa, dovettero contentarsi anch'essi di un predominio sul mare e di un possesso abbastanza contrastato su parti singole della costa, lasciando Trieste agli arciduchi d'Austria, Fiume agli Ungheresi, il litorale croato ai parenti degli Uscocchi, Ragusa a libera repubblica e ai Turchi due spiragli a Neum Klek ed a Sutorina e la massima parte della costa dell'Albania.

Oggigiorno le condizioni nazionali e politiche dell'Europa sono talmente cambiate noll solo in confronto ai tempi anttichi, ma anche a quelli juì recenti dell'età di 
mezzo, che una conquista territoriale di tutto il bacino adriatico per parte di uno dei due maggiori possessori di costa odierni è inconcepibile. - Non ci rỉuscì l'Austria, che mostrò di volerlo tentare un secolo fa, ma lu respinta, e non vi riuscirebbe nemmeno l'Italia, che se anche per aver ricostituito lo Stato nazionale con Roma capitale dovesse essere presa dai più ambiziosi sogni imperialistici, non potrebbe ritentare le imprese dei Romani, perchè la sua posizione nel mondo non è più, nè potrebbe divenire, quella di Roma antica. Ammettiamo per un istante che l'Austria s'indebolisca col tempo e che l'Italia si fortifichi fino al punto di essere superiore alla prima non solo per mare, ma anche per terra. L'Italia qualora tentasse la conquista di tulta quanta la costa orientale dell'Adriatico avrebbe contro di sè non solo Tedeschi, Ungheresi, Slavi, Greci, Albanesi, ma tre quarti dell'Europa. Ad una simile impresa si opporrebbero anche insormontabili ragioni geograliche. Il possesiso duraturo di quel tratto di costa che sla alle falde del monte Velebit e poi di quell'altro che va dal Narenta al Drino è condizionato all'accuisto del suo retroter'ra. Per mandar ad effetto tale dominio l'Italia dovrebbe rinnovare come i Romani la conquista della massima parte della penisola balcanica fino forse al Damubio e più in là ancora, cosa che si deve ritenere a priori inammissibile. Anche i Veneziani avevano dovuto contentarsi delle isole del Quarnero, della Dalmazia lino al Narenta e dell'Albania.

Dunque la geografia e la storia di tutti i tempi c'insegnano che nemmeno l'Italia, la nazione marinara pel eccellenza e che più delle altre domino l'Adriatico, potrebbe aspirare ad un predominio assoluto, esclusivo su questo mare. 


\section{PREDOMINIO DELL'TALIA.}

All' Italia più che a nessun altro Stato è riservato di riprendere una posizione predominante sull'Adriatico. Ch'essa finora sia stata più volte assente da questo mare noll conta per ciò che potrebbe essere nell'avvenire. E che anche di recente, come i Romani nel III secolo a. $\mathbb{C}$., abbia rivolto la sua attenzione e cura piuttosto al Mediterraneo, non può essere che di buon augurio. Nessun contemporaneo, che conosce i motivi pei quali l'Italia si decise ed accinse alla conquista della Libia, malgrado le rettoriche riesumazioni di storia degl'Italiani stessi, oserebbe asserire che l'Italia andò in Africa per seguire pedissequamente le orme delle gesta di Roma. Ci andò per la forza maggiore degli avvenimenti; e se un giorno al posto del delenda Carthago dovesse sorgere un delenda Biserta, la colpa non sarebbe degl'Italiani, ma della storia, che spesso ama ripetersi.

Il fatto appunto che l'Italia risorta abbia sentito il bisogno di affermarsi prima sul Mediterraneo e sulle coste dell'Africa che nell'Adriatico potrebbe servire a dimostrare come certe espansioni debbano seguire leggi geografiche e climatiche immutabili, per cui si ripetono anche a grandi distanze di tempo in forme quasi eguali. Anche i Romani seguirono dapprima la via naturale dell'espansione a mezzogiorno e solo più tardi furono indotti a spingersi nell'Adriatico incominciando dal bacino inferiore. Il consolidamento ai loro confini settentrionali e nella parte superiore dell'Adriatico fu l'ultima parte delle conquiste e il coronamento della loro grand'opera.

Gl'Italiani intanto hanno registrato in Africa un visibile successo. A farli aver cura sempre maggiore dell'Adriatico penseramo parimenti gli altri.

La supremazia morale sull'Adriatico gl'Italiani potranno ormai conquistarla quando vorrammo, perchè è la natura che li favorisce. Oggi l'Austria sembra avere la pre- 
cedenza ed essere la più agguerrita. E che per ciò? Anche gl'tlliri scorrazzavano l'Adriatico da padroni prima dei Romani ed i pirati Narentani prima dei Veneziani. E poi? L'elemento italiano lottò e facendo passi più lunghi e più rapidi vinse già tutte le due volte.

\section{COZZO TRA L'ITALIANITÁ, LO SLAVISHO E IL GERMANESIMO.}

Nell'Adriatico stanno di fronte tre grandi elementi storici: l'italianità, lo slavismo e il germanesimo. Accanto o poco lontano vi sono altri tre popoli minori, gli Albanesi, i Magiari, i Greci.

Come in tutto il mondo e in tutta l'Europa c'è anche attorno all'Adriatico un risveglio generale di energia nazionale, economica, intellettuale, che non permelte a nessuno di riposare. Chi volesse oggi - come sempre del resto nel mondo - rinunziare alla lotta per vivere in pace, sarebbe soffocato. E per ciò, malgrado che si odlano spesso lamenti per lo spreco di energie che viene fatto nell'attrito e che animi mossi dall'amore della giustizia e dal desiderio di pace invochino almeno un armistizio, la lotta continua fatalmente, inesorabilmente; perchè è superiore alla volontà umana e dipende da una legge di natura, che nessum ripiego della diplomazia potrà frenare. Piuttosto che una gara di Stati s'è scatenata sull'Adriatico dalla fine dell'altro secolo una lotta di popoli. Sempre più si avvicina il giorno in cui tutti gli elementi dell'Adriatico, ma specialmente i tre maggiori, potrebbero diare di cozzo.

La parte settentrionale alta, asciulta, dell'Adriatico è Il germane stata per oltre dieci secoli, dall'evo medio fino a einquant'anni fa, l'agone in cui furono combattute le maggiori battaglie dell'epica lotta sostenuta dalla latinitì per respingere l'invasione germanica. Le vittorie furomo conquistate per lo più a prezzo di un'intesa tra i due magggiori popoli latini, i Francesi e gl'Italiani. Nel tragico 1870 
quest'alleanza mancò e d'allora in poi il germanesimo trionfa sul Reno, ma anche sull'Adriatico colla triplice alleanza, che non è altro se non una conseguenza della preminenza germanica. Infatti datano dall'anno 1871 j sogni grandiosi del pangermanesimo, che vuole espandersi in tutto il mondo, ed invadere l'oriente e il mezzogiorno d'Europa (Drang nach dem Osten, Drang nach dem Süden).

L'Austria, in cui predoninano i Tedeschi, anche per l'influenza della famiglia regnante, tedesca, viene sempre più foggiata ad imagine e similitudine della Germania nell'amministrazione pubblica, nel sistema militare, negli usi. Dall'Austria fu tedeschizzata negli ultimi decenni la marina da guerra ed ora si tenta di fare altrettanto della mercantile, degli uffici dello Stato e dove fu possibile anche delle scuole in tutte le regioni dell'Adriatico. Attraverso l'Austria i Tedeschi comandano a Trieste, a Pola, in Dalmazia, in Bosnia, sull'Adriatico dunque e ncll'interno dei Balcani. Ora poi la politica estera imperialista della Germania che avrebbe voluto tener in piedi il cadavere turco, lavora a tutt'uomo per acquistarsi influenza economica nei Balcani e nell'Asia minore e tenta tutti i mezzi per procurarsi un porto di sbocco nel Mediterraneo. Che Trieste debba apparire loro come lo scalo ideale e il coronamento dei loro sogni di egemonia, si capisce. Bismarck disse un giorno che chi tocca Trieste, urta la punta della spada della Germania. Rettorica da soldato, inelegante, ma eloquente.

Il possesso di Trieste però non rappresenta per il Germanesimo che una comodità commerciale. Tutti i paesi tedeschi più vicini all'Adriatico, come il Tirolo, il Salisburgo, le due Austrie, le parti alpine della Stiria e della Carinzia appartengono gcograficamente al bacino del Danubio. Ora al Germancsimo che tiene avvinto a sè il primo tratto del Danubio dalla sorgente a Passavia (confine austriaco) tornerà sempre più conto di attirare a sè anche il resto di quello che spingerlo a gravitare al mezzogiorno col pericolo di vederselo staccare. I Tedeschi poi per chi considera le cose dal punto astratto dell'e- 
quità teorica avrebbero assai magri titoli per affacciarsi sull'Adriatico. Tutti sanno però che questo popolo tenace e valoroso, in continua ascensione da parecchi secoli, non ha raggiunto ancora il vertice della parabola ed è per ciò un concorrente non disprezzabile.

Alla minaccia del Germanesimo che grava sull'Adria- Lo slarismo. tico dal di là delle Alpi si aggiunge e contrappone ormaj il nembo dello slavismo, avanzantesi dall'oriente.

Lo slavismo s'è destato alla vita civile dell'Europa più tardi, appena in conseguenza della grande rivoluzione francese e diede segni del suo risveglio per la prima volta nel 1848. La prima vampata d'idee panslaviste partì dai Bocmi educatisi al contatto dei Tedeschi. Dai Boemi furono comunicate agli altri Slavi dell'Austria, ai Russi, ai confratelli balcanici. Per gli Slavi dell'Austria e dei Balcani l'idea panslavista rappresentò sempre la tendenza al progresso, alla libertà, mercè la solidarietà di ìazza.

In Russia invece le peculiari condizioni della mentalità di quel popolo vi aggiunsero un nuovo coefficente, la religione, e facendo dell'ortodossia un caposaldo del loro panslavismo, lo modificarono nel panrussismo. Allettata dall'eguaglianza di religione della maggior parte degli Slavi dei Balcani e con buon numero di quelli dell'Austria, la Russia sognò nella seconda metì del secolo passato di divenire la gran madre di tutti gli Slavi e di riunirli a sè dal Baltico all'Adriatico, all'Egeo, al Mar di Marmara.

Ma differenze linguistiche, culturali, psichiche, religiose, climatiche determinarono una reazione liberale, capitanata dai Polacchi, che se accederebbero all'idea di un panslavismo, si ribellano alla schiavitù del panrussismo. La gelosia delle potenze e dei popoli non slavi favorì questa reazione e per ciò nel recente trionfo dello slavismo nei Balcani noi abbiamo veduto realizzarsi non l'idea russa, ma quella del panslavismo liberale, individualistico.

Delle due correnti in eui si suddivise lo slavismo la più pericolosa sarebbe stata cjuella del panrussismo. Fortunatamente essa è tenuta lontana dall'Adriatico dall'argine dei Rumeni, dei Magiari e dei Tedeschi, che tagliano 
in due lo sterminato mondo slavo dell'oriente d'Europa. Contro l'Adriatico va dunque addensandosi veramente la marea degli jugoslavi, anzi siccome i Bulgari non oltrepassano l'Egeo, soltanto dei Serbo-Croati-Sloveni.

Lo slavismo, arretrato in progresso e scisso da una divisione territoriale, voluta non dagli uomini ma dalla storia, non presenta un pericolo, cui tutti devollo pensare con trepidazione. Se ne parla spesso, perchè la fantasia del sentimento si pasce volentieri di frasi iperboliche. Ma il panslavismo è l'indice d'uno stato d'animo, non d'uno stato politico e per ciò nella realtà non ha maggior valore di quelle altre nebulose che si dicono il pangermanismo, il panellenismo, il panislamismo, la latinità e così via.

Ciò non per tanto gli Slavi che si estendono ad arco di cerchio dal Baltico all'Adriatico, picchiano alle porte della Germania, in casa dell'Austria e alla costa orientale dell'Adriatico. Rispetto allo slavismo la sorte dell'Austria e quella dell'Adriatico sono connesse e non saranno risolte senza una lotta cogli Slavi.

Bisogna riconoscere ad ogni modo che finora nè il panslavismo in generale, nè lo slavismo balcanico in particolare hanno elevato una formale e grave minaccia contro l'Adriatico. Non mancarono invece pretese di rivendicazione $o$ meglio di usurpazione da parte dei più piccoli gruppi di Slavi, i Croati e gli Sloveni. Chi s'interessa della lotta nazionale che si svolge sulla costa orientale dell'Adriatico avrà avuto certamente occasione di udire proclamare da parte slovena che Trieste ha da diventare il golfo della Carniola e il porto della Boemia, oppure da parte Croata che una metà dell'Adriatico da Trieste a Spizza è già croata; che l'Adriatico fin oltre Durazzo ha da essere un mare slavo. Non sarà raro nemmeno il caso di udir esaltare una marineria croata ed altre simili sentimentalità.

In realtà le cose stanno altrimenti. Gli Slavi vengono, sì, secondi sull'Adrialico per posizione geografica e per numero. Dal Judrio a Pola non hamno che insignificanti punte avanzate sul mare. Da Pola in giù, eccettuate le 
città della costa, ove per ragioni storiche si conserva fin da un'epoca anteriore alla loro immigrazione un elemento italico, essi occupano tutto il territorio dal Quarnero all'Albania, compreso il retroterra per un tratlo interno abbastanza profondo. Il loro numero odicrno complessivo può essere indicato in cifra rotonda con sette milioni all'incirca. Di questi però non gravita veramente sull'Adriatice nemmeno un milione. Essi potrebbero tenere un posto più importante di quello che hanno raggiunto finora, se non fossero stati divisi politicamente, religiosamente, nazionalmente. Il loro basso stadio di civillà alla comparsa nella storia, la mitezza d'animo innata e un po' d'indolenza, li resero meno agguerriti nella lotta per l'esistenza: furono sopraffatti da nemici più forti e portano ancora oggi le conseguenze di quei difetti iniziali. Gli Slavi quindi che per numero e posizione geografica dovrebbero essere i secondi sull'Adriatico, per cfuesto loro frazionamento passano in terza linea e la gara nel cozzo decisivo sarà risolta tra gl'Italiani e i Tedeschi.

Del resto l'Adriatico non è nemmeno per lo slavismo un'arteria vitale. Lo slavismo anzi, sia che lo consideriamo nel suo complesso abbracciante tutti gli Slavi d'Europa, oppure ristretto a quelli dei Balcani, o sia infine solamente a quelle tre schiatte che gli stamno vicino (Croati, Serbi e Sloveni) non è stato nè sarà mai un elemento marinaro. Abbiamo delto e dimostrato più volte che Serbi, Croati e Sloveni gravitano geograficamente ed economicamente verso il Dambio. Tolla quindi quella piccola parte dei Croati, che vive sulle coste della Dalmazia e - se vogliamo assegnare esclusivamente ai Serbi quelle poco numerose popolazioni slave che vivono attorno a Ragusa e alle Bocche di Caltaro - tolla anche questa minima frazione serba, gli Slavi dell'Adriatico non hanno nemmeno domestichezza col mare. $\Lambda$ i Serbi, ed anche ai Croati - qualora riuscissero a formarsi nuo stato solo e indipendente - basterebbe mo sboceo commerciale.

Trieste, che non ha importimza cippilale per il Germanesimo, ne ha ancora meno per lo slavismo. Siluala 
ad un'estremità periferica del non compatto e contrastato possesso nazionale, essa attira oggi gli Slavi soltanto come un centro di coltura, di cui hanno grande bisogno. Trieste è per gli Slavi troppo fuori di mano e per conquistarla lo slavismo tutto dovrebbe fare uno sforzo ancora più grande che i Tedeschi.

Trattandosi dunque di rivendicazioni platoniche si potrebbe non curarle soverchiamente, se gli Jugoslavi non fossero divenuti i più inquieti dei popoli d'Europa e se le loro pretese non rischiassero di trovar appoggio nelle esagerazioni dell'idea panslavista.

L'italianità. L'italianità sull'Adriatico soffre ancora le conseguenze fatali di Lissa.

Nei primi anni che seguirono a quel lutto, lo scoraggiamento potè far credere ad una perdita irreparabile. Oggi noi possiamo giudicare quella giornata più serenamente e paragonarla ad una delle battaglie perdute dai Romani, p. e. a quella di Drepano (Trapani) nell'anno 249 a. C., durante la prima guerra punica. Anche allora i Romani avevano esaurilo tutti i mezzi per terra e per mare onde vincere il nemico e dopo una lotta di quindici anni si vedevano in una condizione più pericolosa della primitiva, nè sapevano più a qual partito appigliarsi. I Romani del resto furono più fortunati perchè riafferrarono la vittoria in un decennio, mentre la sconfitta di Lissa arrestò l'italianità sull'Adriatico per quasi mezzo secolo.

Ormai anche gl'Italiani hanno superato lo scoraggiamento.

Intanto, anche senza affrontare battaglie, anch'essi devono tutelare i loro interessi per non lasciarsi sgravare dagli altri concorrenti. I cinquanta anni d'esistenza del regno d'Italia dimostrano che l'Italia può esistere e prosperare anche senza il predominio sull'Adriatico. Occorre però che nessun pericolo la minacci da questa parte, giacchè la sicurezza dell'Adriatico è per lei, a differenza degli altri popoli, una necessità per le ragioni addotte nel capilolo sull'antagonismo italo-austriaco. Qualora quindi dovessero minacciare dei cambiamenti, che non potreb- 
bero essere che in suo damo, essa avrebbe il dovere di correre ai ripari.

Intanto il còmpito dell'Italia sull'Adriatico è di tutclare tutte le propaggini del suo elemento nazionale lungo la costa orientale dell'Adriatico. La conservazione dell'italianità del Trentino, della Venezia Giulia, dell'Istria e delle oasi italiane a Fiume, in Dalmazia, in Albania, nelle attuali condizioni, è questione oltre che d'interesse, anche di dignità nazionale.

Nel presumibile urto fra i tre elementi maggiori non mancheranno di esercitare una qualche azione anche i tre popoli minori.

Più lontani dei Tedeschi dall'Adriatico sono i Magiari. I Magiari. Il loro campo d'azione naturale è il medio Danubio col Tibisco. Fiume è per loro un lusso. Però siccome hanno posto il piede politicamente sull'Adriatico già da otto secoli, sono un popolo impetuoso, prepotente, poco scrupoloso, ma vitale, e prima di perdere il tratto che li congiunge al mare sarebbero indotti a mettere sossopra tutti i Balcani, occorre tener conto anche della loro presenza come attori secondari, per lo meno.

Fuori dell'Adriatico, ma in posizione da far da cu- I Greci. stode alla sua entrata dalla parte esterna, sta m altro popolo, il Greco, il quale col possesso dell'isola di Corfù, ch'è l'unica in prossimita dello stretto di Otranto e di una parte, almeno, del suo ciulale, può esercitare anche un'influenza sulla sorte del nostro mare.

I Greci avrebbero volentieri esteso le loro conquiste fino a Vallona e in tal caso avrebbero aruto voce in capitolo anche nell'interno dell'Adriatico. Non ci sono riusciti e probabilmente non ci riusciramno nemmeno in un avvenire prevedibile.

Per i Greei l'Adriatico non ì altro che un buon campo d'affari.

Però la posizione dei Greci a Corfù potrebbe essere 
fonte di un'amicizia assai ricercata per gli altri possessori dell'Adriatico.

Gli Albanesi. Quale sarà l'avvenire degli Albanesi, mancanti di tutte quelle cose anche più elementari che occorrono per formare uno stato civile, nel guazzabuglio di passioni religiose e politiche, che non permettono ancora di prevedere in che direzione si metterà la nascente coscienza nazionale e il desiderio di progresso? Se arriveranno a capire i loro veri interessi, sia da soli, sia per suggerimento altrui, cercheranno di vivere in buona armonia coi loro più forti vicini i Serbi e i Greci in terra e gl'Italiani sul mare e troveranno forse nella gelosia e nell'antagonismo dei popoli maggiori il modo di conservare la loro prosperità. - Sia però ch'e ottengano l'autonomia e magari un'indipendenza o ritornino ad essere soggetti a dominatori stranieri, la loro non sradicata presenza sul cardine orientale della porta dell'Adriatico agirà sempre come freno alle espansioni di tutti i popoli circonvicini, in qualsivoglia direzione, e per ciò anch'essi non mancheranno di aver una qualche parte nell'altalena degli squilibri dell'Adriatico.

\section{IL FUTURO.}

L'Adriatico, ch'è un'importantissima via di comunicazione per il commercio internazionale, per nessuno dei popoli che vi si affacciano è l'arteria principale ed esclusiva della loro vitalità.

Non è per gl'Italiani, che possono prosperare negli altri due mari migliori che li attorniano e che sotto certe premesse possono tollerare che rimanga qual è. Non è per la grande Serbia, che ha per bacino principale i fiumi Morava e Drina e per sbocco naturale economico l'Egeo, a Salonicco. Non è per gli Albanesi, che hanno sempre preferito il monte al mare. Per le due nazioni minori i Croati e gli Sloveni l'Adriatico sarebbe incontestabilmente molto utile; però è altrettanto certo che essi non potranno mai affermarvisi individualmente 
ed energicamente e che dipenderamno più dei Serbi dalle concessioni, che saranno fatte loro dai popoli maggiori. Per i Tedeschi ed i Magiari uno sbocco nell'Adriatico, come fu già delto, non è che un lusso. Per i Greci infine l'Adriatico non può essere altro che un campo d'attività commerciale, come qualunque altro mare.

Il segreto dell'equilibrio dell'Adriatico sta dunque nell'egemonia di un popolo, che non può essere che l'italiano, e nella libertà d'esistenza nazionale o di attività commerciale, secondo i casi, per tutti gli altri, ossia mella formula "vivere e lasciar vivere» precisamente come nel commercio.

L'assetto presente dell'Adriatico rimarrà quindi presumibilmente inalterato per qualche lustro o decemnio, finchè nessuno si muoverà, giacchè la grande c tanto temuta guerra mondiale non scoppierà molto probabilmente proprio a causa solamente delle compctizioni sull'Adriatico. Ma se per altre cause dovesse scatenarsi nel mezzo o nell'oriente dell'Europa una grave bufera politica ¿̀ certo che l'Adriatico vi sarcbbe coinvolto.

Del resto perchè in un bacino della grandezza dell'Adriatico si compiano dei mulamenti non occorre nemmeno che succedano alterazioni violente. Il tempo fa maturare le cose anche quando stanno in pace. Il tempo è come un orologio, la cui lancelta imperceltibilmente cammina. Se, distratti, non vi poniamo atlenzione continua, ci accorgiamo ad un tratto che non è più l'ora di prima. Così cammina anche l'indice del progresso dei popoli. In dieci, venti, cincuant'anni, l'esponente dell'energia dei popoli che stamno sull'Adriatico sarà certamente cambiato e allora per logica e naturale conseguenza si compirebbe anche senza scosse il livellamento dei valori reciproci. Così si è compiuto nell'ultimo mezzo secolo il progresso dell'italianità.

Abbiamo già accennalo altrove ed ora i necessarion La risurrezio di ripetere, che ai nostri occhi le maggiori prohaloiliti di nedeibalcau. 
alterazioni violente sull'Adriatico stanno venendo dalla partc dei Balcani.

Inaspettatamente e contro tutte le profezie degl'iniziati e della diplomazia è toccata proprio a noi la fortuna di assistere alla cacciata del Turco dai Balcani, dopo cinque secoli che vi si era insediato. La penisola balcanica, la più orientale e la più infelice d'Europa, che nel corso della storia aveva cambiato più volte nome e nazionalità, illirica, greca, slava e turca, fu riportata nel volgere di un secolo nelle condizioni in cui si trovava press'a poco nei tempi remotissimi dell'antichità. Dopo più di venti secoli abbiamo veduto risorgere la Dacia (Romania), la Moesia e la Tracia (Bulgaria colla Rumelia), la Grecia, l'Illirio greco (Albania) e in parte anche il resto geografico dell'Illirio barbaro (Montenegro e Serbia). La Macedonia, paese e popolazione incerti nell'antichità, non ha perduto neppur ora il suo ibridismo.

I Balcani ${ }^{1}$ ) per volere della geografia, che può venir violentata, ma non tollera modificazioni innaturali, sono ritornati in quello stato che Greci, Persiani, Macedoni, Romani, Germani, Slavi e Turchi invano avevano alterato.

Bisogna però anche aggiungere che la resurrezione dei Balcani segna il primo, vero trionfo dello slavismo nel mondo, proprio là dove era sembrato ch'esso avesse trovato la sua tomba (Adrianopoli 1363, Cóssovo 1389 e 1448, Nicopoli 1396, Varna 1444). - La grandezza della Russia, ossia del panrussismo avvenne per opera di una sola schiatta slava, la russa, e di una dinastia, mosse nelle loro imprese da fini imperialisti burocratici, piuttosto che essere spinte da un sentito bisogno di espansione per esuberanza di vitalità. Nel recente trionfo dei quattro Stati balcanici, tre dei quali sono slavi, cooperarono invece tulte le forze dello slavismo: quelle dei popoli slavi balcanici o jugoslavi, Bulgari e Serbi in prima

1) La penisola balcanica ha preso questo nome d'indicazione puramente geografica dalla parola turca Balkán (monte) colla quale fu chiamato per antonomasia l'antico Haemos dei Greci, ch'è la più alta catena della penisola e dovrebbe essere il suo confine settentrionale. 
linea, quella della Russia sotto forma di appoggio diplomatico e di aiuto materiale e quella dello slavismo rimanente (Slavi dell'Austria) colla simpatia morale in seconda linea, che anche pesò nella bilancia degli avvenimenti internazionali. Questa volta dunque si tratta di un risveglio notevole per la sua origine, ossia la coscienza di aver raggiunto la via della civiltà e per la sua finalità ossia la tendenza alla libertà, al progresso illimitato, perfino ad un predominio nel mondo. Giacchè la cacciata dei Turchi che riempì l'Emopa di ammirazione per il valore delle armi slave, inebbriò gli animi degli Slavi d'un entusiasmo indescrivibile, che fece loro intravvedere la realizzazione d'un grande sogno. Sapendo di non aver nè un grande passato, nè un presente brillante, gli Slavi hanno concentrato le loro speranze nel futuro e concepito press'a poco l'idea che, come l'antichità è stata dei Latini e il presente è dei Germanici, il futuro sarà loro.

Noi non li seguiremo in questi voli lirici, giacchè sappiamo che per pretendere all'egemonia nel mondo è necessario per lo meno di aver prima superato tulti nella civiltà ; studieremo invece quali potrebbero essere le conseguenze immediate dell'ultima vittoria, dello slavismo nei Balcani.

É certo che Bulgari e Serbi progrediramo rapidamente. Ma nessuno potrebbe pronosticare con fondatezza come e fin dove. Per formulare un giudizio sulla portala del progresso nei Balcani, occorre aspeltare almeno qualche decemnio. Giaechè la storia che non va mai disgiunta dalla geografia c'insegna a difficlare di quella penisola.

Delle regioni balcaniche a noi non interessano da vicino che quelle che stamno verso l'Adriatico.

Sarà un miracolo se l'Albania froverà in sè stessa tante risorse della terra e degli uomini da bastare a sè stessa.

Del Montenegro e dell'Erzegovina si sa che sono paresi classicamente sterili. Buoni frutti al contrario potrebbero dare le regioni della Serbia (vecchia e nuova), della Bosnia, della Croazia, della Slavonia. Ma ciò non basta ad assicurare una vita libera ed indipendente ai rispettivi abilatori. Prese complessivamente queste regioni formano 
un altipiano esteso, ma informe, rotto da valli scoscese e burroni intransitabili, digradante verso le regioni del Danubio, senza un confine isolatore da nessuma parte, non verso settentrione, nè verso oriente. Solo verso occidente vi sarebbero le catenelle di monti, che seguono l'altro versante adriatico della Dalmazia. Per quest'impossibilità di condurre una vita propria questi paesi furono la disgrazia degli Illiri e dei Romani e contribuíscono senza dubbio alla poca fortuna dei Serbo-croati, che le hanno ereditate.

Comunque, è certo che i Serbi, spinti dal bisogno di progresso e di maggiore indipendenza politica, da esuberanza di giovani forze, dagli ardimenti del risvegliato sentimento nazionale, tenteranno di ingrandirsi e fortificarsi coll'unione di quanti più potranno confratelli, ora. soggetti all'Austria-Ungheria. L'idea nazionale ha fatto grandi progressi fra questo popolo. Già le differenze fra Serbia e Montenegro, i due Stati nazionalmente del tutto omogenei, sono ridotte alla soluzione del problema dinastico e gli attriti in parte artificiali, fino a qualche lustro fa violenti tra Serbi e Croati sono, per effetto dell'entusiasmo guerresco, quasi scomparsi. La maggior coltura ha fatto vedere anche ai Serbi l'avvenire da un punto di vista più elevato ed anche fra loro l'idea religiosa ha ceduto il posto all'idea del bene della nazione.

Anche senza che il giornalismo serbo e russo l'avessero proclamato apertamente, si sarebbe potuto prevedere che il trionfo degli Slavi nei Balcani, che rappresenta la seconda tappa vittoriosa del panslavismo, avrebbe avuto per logica conseguenza il desiderio di completare la redenzione della razza col liberare gli Slavi dell'Austria-Ungheria, soggetti politicamente ai Magiari e ai Tedeschi. Anzi le prossime rivendicazioni nazionali dei Serbi saranno dirette particolarmente contro i Magiari.

Gli Slavi del- Noi crediamo che gli Jugoslavi s'illudano un po' nella l'Austria. facilità di questa nuova impresa. La rapida ed improvvisa soluzione del problema balcanico (non ancora interamente di quella che la diplomazia chiamava la quistione d'Oriente) è stata possibile perchè l'impero turco 
era nell'ultimo sfacelo, perchè di fronte ad esso i Balcanici erano divenuti lentamente i più forti e perchè le maggiori simpatie dell'Europa erano per la cansa profondamente giusta degli oppressi.

Simpatie i Serbi ne troveranno ancora senza dubbio e sopratutto tra gli altri Slavi. Anzi è perciò che nel problema dell'Adriatico, sebbene per la sua soluzione in quanto allo slavismo non si presentino nell'agone che i soli Serbi ed i Croati, non si deve mai perdere di vista tutta la mole del panslavismo. Se dunque nel terzo grande sforzo dello slavismo per la liberazione degli Slavi dell'Austria-Ungheria il lato sentimentale della quistione sarà lo stesso che nel secondo, perchè vi dovranno concorrere tutte le forze slave, il lato pratico sarà molto differente per la maggior resistenza che gli verrà opposta.

Nella recente guerra balcanica la reclenzione degli Slavi non doveva teoricamente toccare altri popoli, anzi, se mai, era destinata a portare anche a quelli la tanto sospirata libertà. Eppure non mancarono segni evidenti di reazione contro l'appetito slavo da parte di tutti i toccati Greci, Albanesi, Rumeni.

Il tentativo di liberazione degli Slavi dell'Austria provocherà resistenze d'indole locale e generale. Gli Slavi costituiscono in Austria un elemento rispettabile per numero e per collura. Ma non sono compatti territorialmente e per conseguenza nemmeno concordi nei bisogni economici e nelle aspirazioni politiche. Se essi s'insinuano tra gli altri popoli e ne minacciano l'egemonia, anche questi li sminuzzano e li dominano colle loro maggiori energie di civiltà. Un'aggressione isolata e parziale contro l'integrità della monarchia Austro-Ungarica provochcrebbe dunque la reazione di almeno due dei suoi popoli, cosa di cui si deve tener conto.

Se poi lo slavismo si levasse tutto quanto in armi per abbattere dalle fondanenta la compagine della duplice monarchia asburgica e minacciasse di rompere l'argine divisorio di Tedeschi-Magiari-Rumeni, che la storia ha gettato nella metà dell'oriente emropeo, per allargare e russificare più di un terzo d'Europa, la reazione sirchbe 
pari all'aggressione. Non solo tulti i popoli minacciati Tedeschi, Magiari, Rumeni, Greci, Italiani e diciamo anche gli Albanesi, dovrebbero fare uno sforzo altrettanto energico per salvarsi, ma anche altri popoli d'Europa li aiuterebbero per tutelare i loro interessi indiretti. Non fu proprio la Russia quella che nel 1848, quando l'Austria minacciava di sgretolarsi per decomposizione interma, ci trovò il proprio utile a tenerla in piedi? Se la Turchia trovò per secoli dei sostenitori, non foss'altro nei suoi creditori, come non prevedere che anche l'Austria avrebbe degli aiuti provvidenziali da interessi radicati, cui nessuno rinuncia se non per forza e all'ultimo momento? - Giugurta pronosticò alla fine del II secolo a. C., la caduta di Roma per corruzione. Eppure durò ancora quasi sei secoli, perfino con sprazzi di progresso.

Se dunque da mma parte possiamo prevedere alcuni dei combiamenti che saranno prodotti dalla marcia dello slavismo, dall'altra ci manca un fondamento per intuire quale potrebbe essere l'esito finale della grande lotta, che sta per essere ingaggiata tra lo slavismo e l'Europa. Dobbiamo attendere che il nostro secolo ci porti qualche nuovo indice per poter appena pronosticare se il panslavismo sarà il vincilore $o$ il vinto.

A chi studia l'Adriatico interessa sin d'ora di considerare il problema dello slavismo. Gli Jugoslavi minacciano egualmente e contemporaneamente l'Adriatico e l'Austria-Ungheria. Le due quistioni sono, fu già detto, connesse intimamente.

Più che all'Ttalia s'impone pel momento all'Austria l'obbligo di affrontare il problema, arduo davvero. Iu principio l'Austria mostrò di volerlo risolvere avauzando energicamente e legando a sè gli Slavi balcanici. S'impossessò della Bosnia-Erzegovina, occupò il Novibazar, mise solto tutela Serbia e Montenegro, ipotecò la linea di Salonicco. Poi d'improvviso le cose cambiarono. I due pupilli vollero diventare maggiorenni e l'Austria presto mise il catenaccio alla Bosnia-Erzegovina. Credette di aver vinto, ma fu una vittoria di Pirro. I due pupilli divennero maggiorenni di fatto. si allearono e chiusero 
anch'essi la strada dei Balcani. Ora l'Austria ha nellit Bosnia una nuova Lombardia, nell'Erzegovina, quasi quasi, un secondo Veneto. Serbia e Montenegro tengono il posto del Piemonte. E l'Austria dev'essersi accorta anche lei di essere incappata in un ginepraio dal quale non potrì uscire che colle vesti lacerate. Non potendo più inveire militarmente contro i due Stati serbi, perchè nè le tornerebbe conto di annetterseli, nè gli altri gliclo permetterebbero, l'Austria per controbilanciare l'attrazione centrifuga da loro esercitata sui suoi paesi slavi meridionali non ha che due mezzi : reprimere e far aumentare il malcontento e l'odio, oppure concedere e far crescere gli appetiti. Tutte queste due vie non possono condurre che ad un solo risultato: la perdita dei paesi, da poco acquistali.

Stando alle enunciazioni serbe i conlini della fulura grande Serbia dovrebbero coincidere con quelli della Bosnia-Erzegovina e raggiungere l'Adriatico dal Drino al Narenta. Però anche i Croati cogli Sloveni hamo notilicato le loro pretese. Secondo i loro sogni i confini della grande Croazia dovrebbero toccare a settentrione la Mur e la Drava, ad occidente l'Isonzo e lorse il Tagliamento, ed a mezzogiorno l'Arlriatico giù giò lino al Narenta e magari più basso ancora fino al Drino.

Sarebbe premaluro esternare oggi um giudizio se gli Slavi meridionali dell'Adriatico riusciramno a formare una grande Serbia o una Jugoslavia assorbente Croati e Sloveni, oppure due Stati, la grande Serbia e la grande Croazia, oppure la grande Serbia solamente. Conoscendosi la recente al\%ata di scudi dello slavismo e la veemenza irresistibile della corrente nazionale, queste enumciazioni, se anche intempestive, devono essere tenute a memoria.

Una simile eventualità toccherebbe in prima linea gli interessi dell'Austria-Ungheria, ossia nazionalmente pallando, dei Magiari e dei Tedeschi, ma non può lasciano indifferenti nemmeno gl'Italiani, per due ragioni, che poi si riducono ad una sola. Per gl'Italiani si tratterebbe della comparsa di un muovo concorrente sul mare e della 
tutela della loro integrità territoriale, culminanti tutte le due quistioni in un pregiudizio del loro naturale predominio sull'Adriatico.

Ora se l'Italia ha, come l'Austria e come ognuno, da pensare prima di tutto ai casi propri, nei riguardi dei Balcani l'interesse italiano non è identico con quello austriaco. L'Austria-Ungheria, Stato polietnico, ha da preoccuparsi di ogni organismo nazionale maggiore formantesi in vicinanza dei suoi confini. L'Italia, Stato eminentemente nazionale, no.

L'attuale assetto politico dei Balcani non è opera di-. retta dell'Italia. Quando il panrussismo minacciava d'invadere i Balcani la gelosia delle potenze occidentali diede vita un po' alla volta a staterelli indipendenti. Quando poi sorse il pericolo che l'Austria s'insediasse nella penisola balcanica, la Russia le contrappose lo stesso giuoco favorendo l'ingrandimento e il consolidamento di quegli staterelli, creati proprio contro di lei. Che quest'assetto dei Balcani corrisponda oltrechè all'equità anche all'interesse dell'Italia, si comprende. La sua posizione geografica la chiama ora a far verso gli Slavi balcanici tuna funzione storica simile a quella che la Francia e l'Inghilterra esercitarono nel suo risorgimento. Ma non per gli Slavi balcanici soltanto l'Italia ha dell'interesse a divenire fautrice di organismi nazionali, invece di Stati ingenti per espansione dinastica o ambizione di dominio. Certo che un'Ungheria graverebbe sull'Adriatico meno dell' Austria-Ungheria, come è ora costituita, e il risorgimento degli antichi regni di Boemia e di Polonia verrebbe anche salutato con simpatia sincera, perchè oltre ad allontanare buona parte di quel peso artificiale che l'Austria fa ora gravitare sull'Adriatico, servirebbe da argine contro le possibili espansioni al mezzogiorno di quei due colossali organismi statali nazionali, che sono la Germania e la Russia. L'Italia può dunque rallegrarsi dì aver la fortuna rara che il suo interesse nazionale coincida con quello degli altri popoli e di poter quindi farorire quello e questi nei limiti dell'equità.

Per conseguenza anche la formazione di una grande 
Serbia, di una Croazia (per necessità non tanto grande quanto la sognano i suoi patriotti) o di una maggiore Jugoslavia, con possesso territoriale sull'Adriatico, non dovrebbe incontrare l'opposizione incondizionata dell'Italia, come non la trovò di recente la richiesta serba di uno sbocco, per ora commerciale, su questo mare. Da questo lato le simpatie e le alleanze degl'Italiani.saramo per gli Slavi.

Altra sarebbe invece la cosa se i Serbi si affacciassero all'Adriatico come avanguardia del panslavismo invasore, immemore dei diritti altrui. Allora potrebbe succedere quello che certuni in Austria si aspettano meno, che cioè gl'Italiani trovassero nella necessità della difesa identitì d'interessi coi Magiari e coi Tedeschi, e che l'Italia, come Stato, si stringesse ancora più all'Austria per sostenerla. Secondo la minaccia, ossia l'aziome, si determinerì la.difesa, ossia la reazione. La minaccia italiana tra Lissa e la triplice provocó un'intesa fra Tedeschi e Slavi. La marcia progettata dall'Austria nei Balcani avvicinò gli Slavi agl'Italiani; una minaccia slava rinsalderebbe l'alleanza austro-italiana. In questo stesso ordine d'idlee dovrebbero muoversi i Magiari: minacciati dagli Slavi (altro pericolo non hanno oggigiono da temere) non potrebbero che appoggiarsi ai Tedeschi e agl'Italiani e se per caso maggiori attriti fra Budapest e Vienna dividessero quei due popoli, unili dalla storia e dal pericolo, vedremmo rimnovarsi gli entusiasmi italo-ungheresi dell'epoca garibaldina.

Tutto questo, è vero, non è altro che ginnastica cerebrale. Ma per considerare le possibili azioni e reazioni tra i popoli non c'è misura migliore della forza nazionale, noll tanto perchè questa è la forma più sintelica ed evidente della vitalità dei popoli, quanto perchè essa ha più o meno agito sempre, anche in epoche di altre tendenze civili e sociali, e può venir per conseguenza considerata come una forza costante e duratura nell'edifizio della storia. Per ciò si può prescindere fino all un certo punto dal tener calcolo dell'inlluenza che nello srolgimento ulteriore delle vicende storiche sull'Adriatico po- 
trebbero avere i rappresentanti della politica, come sovrani, ministri, diplomatici, che non sono il più delle volte che fattori occasionali e secondari. Proprio noi contemporanei abbiamo veduto un classico esempio dell'impotenza della diplomazia a violentare la geografia e la storia.

Nell'attuale equilibrio forzato dell'Adriatico anche le correnti nazionali sono indecise nelle alleanze e tutti i popoli devono condurre una politica di opportunità a due facce. Involontariamente sono tutti presi nell'ingranaggio di una sola ruota. Esistono però alcune quistioni locali in cui sono in ballo gl'interessi di due soli competitori. Tali sono p. e. quella italo-tedesca pel Trentino, quella ungaro-serba per la Bosnia e regioni slave finitime, e una lotta di tal genere potrebbe nascere un giorno fra Italiani e Serbi per l'Albania o per la Dalmazia. C'è però una regione in cui sono in ginoco gl'interessi degl'Italiani, dei Tedeschi, degli Slavi ed anche dei Magiari ed è quella dell'Istria colle due città di Trieste e di Fiume.

Nell'Alpe Giulia e nel Carso che sovrasta a questa penisola Latini, Germani, Slavi, Ungheri stanno combattendosi ancora dal settimo secolo per il possesso territoriale. Ma in grembo alla civiltà la vita di Trieste, dell'istria, di Fiume, spesso combattute, fu sempre italiana.

L'Istria con Trieste sarì dunque il pomo della discordia e il premio della lotta secolare fra Italiani, Tedeschi e Slavi per l'Adriatico.

Un solo sguardo alla posizione geografica e politica, alla proporzione numerica, al valore della coltura, mostra evidentemente la preponderanza degl'Italiani. L'italianità è la sola che può dire di avervi solide radici e per ciò a lei deve arridere il trionfo. Gli altri non hanno che tenui abbarbichi. La minaccia tedesca, l'abbiamo già detto, è grave. Però non è escluso che le pretese germaniche su Trieste per incorporarla alla loro finora ipotetica Marca meridionale (Südmark) si risolvano in una bolla di sapone, come la marcia austriaca su Salonicco. La minaccia slava è anche seria presentemente, ma soltanto perchè è gonfiala artilicialmıente dalla politica au- 
striaca che non permette agli Sloveni di avere un centro proprio, nè di altingere ad altri loro affini, e li dirige su Trieste per sollevare i Tedeschi e premere la mano sugl'Italiani. Il valore dell'Istria e di Trieste per lo slavismo ¿̀ presso che nullo. Giacciono fuori della sua orbita e non hamno nemmeno $u$ valore di scalo e di transito, come Salonicco, o altri porti dell'Ariatico e dell'Egeo. La regione a mezzogiono dell'Alpe Giulia ha importanza locale soltanto per gli Sloveni. Chi giudica spassionatamente non può dar torto agli Sloveni se anch'essi per vivere sperano e s'illuctono di aprirsi un varco nel mondo per la via di Trieste. Altra è invece la sorte che prepara loro il destino, malgrado i più nobili sentimenti di giustizia. Relegati nell'ariclo Carso e riclotti a poro più di un milione di anime, questi superstiti di una schiatta più numerosa, come gli anlichi Celli in quelle regioni, non possono aspetlarsi che due alternative: nell'ipotesi migliore di vivere all'oseuro sui loro monti, nella peggiore di venir stritolati nel cozzo di organismi più grancli.

E per ciò che se Trieste dovesse ressare di essere jlaliana, prima che slava, potreble divenire germanica.

Altra importanza puramente locale è quella costituila dall' Istria per i Croati. Prima di tullo il foro nucleo principale è in Croazia e gravita verso il Damubio. L'Istria adriatica non ha nessun legame geografico (e non ne ebbe per ciò nemmeno nella storia) colla Croazia. In sccondo luogo i Croati oltre ad occupare (come del resto anche gli Sloveni ed i Serbi) regioni indecise e prive di confini naturali hanno la disgrazia che i loro territori sono contestati da altri popoli più lorti. I Serbi furono più fortunati. Dopo di aver salclato il loro conto coi Thurchi possono dire che la terra su cui dormono è loro. Nessuno la prelende più. Dei paesi popolati dai Croali al contrario la Croazia è ungherese da olto secoli, la T):almazia fu vencziana e croata solo ipoteticamente, l'Istria non fu croata mai. La storia dei Croati ì meschina. " la loro civilta raccogliticeia. La ricostituzione di una gramde Croazia è un'impresa assai problematica e per (o)par, L'Adriatico. 
bisogna dire il vero, più della geografia che della storia; non è escluso il pericolo che in reazione ai tentativi di liberazione tocchi loro invece la sorte dei predecessori Illiri, ridotti oggi sì e no, alle poche tribù albanesi.

Se e quando le divergenze che ancora tengono discordi Bulgari, Serbi, Croati e Sloveni scompariranno sarebbe difficile far ora delle previsioni. Si può però ammettere che per effetto del progresso, della civiltà e dell'idea nazionale in uno o due secoli forse gli attriti tra Slavi meridionali non saranno maggiori e più gravi di quelli che talvolta fanno capolino in Italia fra Veneti e Liguri o fra Milanesi e Napoletani. Ma se anche questa fusione dovesse avverarsi e se queste schiatte slave riuscissero a vincere l'opposizione inmmancabile dei Magiari e dei Tedeschi e formassero un solo grande Stato dal Danubio all'Egeo con un'ampia diramazione laterale sull'Adriatico, è certo che questo Stato non potrebbe in nessun caso avere territorialmente quella estensione massima che vorrebbero assegnargli i panslavisti, nè potrebbe divenire una potenza di prim'ordine pari a quelle che oggi si qualificano col titolo di grandi. Nella miglior ipotesi di rivendicazione completa territoriale e di un aumento di popolazione non arriverebbero tutti insieme Bulgari, Serbi, Croati e Sloveni a venti milioni. Gli Slavi saranno i primi a dover rinunziare a certe pretese troppo avanzate verso l'occidente e in quanto all'Adriatico non potranno mai divenire il fattore preponderante. Essi, come gl'Illiri antichi, hanno un grande nemico, la geografia.

Il miglior avvenire sull'Adriatico, ce lo insegnano la geografia e la storia e ce ne affida il presente, è riservato all'italianità. Molti sono abituati a considerare l'italianità come repressa sull'Adriatico - ma non importa. Solo dieci anni fa le condizioni dei Croati sottomessi ai Magiari e degli Sloveni compressi dai Tedeschi, apparivano di gran lunga migliori in confronto a quelle dei Serbi della Macedonia soggiogati dai Turchi. Da qui a un decennio saranno i Croati e gli Sloveni che dovranno invidiare i Serbi della Macedonia. Sono i Balcanici che hanno avuto la fortuna di Cenerentola. 
Qualche cosa di simile potrebbe toccare agl'Italiani, ora angariati entro i confini austriaci.

I Latini stabilitisi sull'Adriatico come in casa loro non poterono essere respinti dai Goti, dai Longobardi, dai Franchi e nemmeno dagli Slavi. Se la latinità deperì nella parte balcanica dell'Adriatico ciò arvenne più per colpa intrinseca della corruzione romana negli ultimi tempi c per causa della geografia, che per merito direlto di Germani o di Sarmati.

Malgrado la repressione nei pacsi soggetti all'Austria l'italianità nel grande bacino dell'Adriatico progredirà. Essa progredisce già dagli ultimi secoli del medio evo, dalla qual epoca gl'Italiani hanno incominciato a penetrare nell'alta valle dell'Adige e dove malgrado la secolare unione politica di quei paesi alla grande Germania e l'odicrna lotta del pangermanismo solto l'cgida anstriaca, l'italianità guadagna anzichè perdere terreno. Essa ha già assorbito diverse frazioni minori di Tecteschi nel Veneto e di Slavi immigrati nel Frinli; e se la politica doll'Austria non le avesse messo un freno sulla costa oricntale, parecchi di quegli Slavi cui la imprevidenza della Serenissima diede ricetto contro i Turchi nelle canpagne prima italiane dell'Istria e in Dalmazia, non le sarebbero più nemici.

Se l'Italia continuerà a fare quei progressi, che già rallegrano gli animi dei suoi cittadini, nè i Tedeschi delle Alpi, nè gli Sloveni del Carso, nè i Magiari della Puszta, nè i sedicenti Croati dell'Istria e della Dalmazia e nemmeno dei maometlani camufrati da Albanesi, potranno arrestare il suo sviluppo.

Confortati da questo sostegno della natura gl'Italiani, e specialmente quelli che oggi sono repressi in AustriaUngheria, possono fiduciosi guardare in faccia l'avvenire. Nè il pericolo tedesco e nemmeno quello sloreno " croato faranno suonare l'ultima ora dell'italianitì di Gorizia, di Trieste, di Pola, di Fiume, ora più deste che mai. Basterebbe che in queste ciltà cessasse l'imposizione esotica di un governo nemico e in meno di un lustro diverebbero tulte italiane pure, come Venezia, come V'e- 
rona, come Udine, Così anche gl'Italiani della Dalmazia non devono lasciarsi intimorire dalla minaccia del nembo slavo balcanico.

La politica estera degli Stati oggigiorno forma una rete che abbraccia non solo l'Europa, ma quattro dei cinque continenti almeno, ed uno Stato non può muoversi o regolare un conto col suo vicino senza tener calcolo degli umori c degl'interessi di tutti gli altri. - Lo spirito dei nostri lempi, come associò gli nomini e li spinse a formarc sindalcati, monopoli, trust, così produsse fra i popoli gli aggruppamenti diplomatici, le intese, le alleanzc. Prima di venire quindi ad una soluzione definitiva delle varie quistioni che sono oggi pendenti sull'Adriatico, occorreramo molte conlerenze o convegni, più o meno socreti. Ma se anche si dovesse venire ad una conflagrazione violenta in cui l'ilalianità avesse da sostencre da sola l'urto del pangermanesimo e del panslavismo coalizzati, non si arrebbe la dispcrare della vittoria. Tedeschi e Slavi tendono all'Adrialico per ambizione o per tornaconto; gl'Italiani invece vogliono rimanervi per difendere l'integrità e l'esistenza di tutta la loro patria. II Carso istriano, l'Alpe Giulia, le Dolomiti trentine sono le porte della Padania e d'Italia. Il solo bassopiano lombardo-veneto vale un terzo dell'Italia intera. Altrove e lontano da qui sono invece le regioni madri dei Germani e degli Jugoslavi. Se anche gl'Italiani sono inferiori numericamente bisogna considerare che Tedesnchi e Slavi non potrebbero procedere all'assalto che con forze limitate. Gl'Italiani invece combattendo pro aris et focis opporrebbero tutle le loro forze per terra e per mare. $E$ il trionfo finale arride sempre al buon diritto.

Tulli i popoli oppressi che vogliono farsi strada nel mondo devono ricorrere in principio alle combriccole, alle congiure, agli attentati, alle ribellioni, alla guerra ad ogni costo e disperata per la vita o per la morte. Così hanno fatto gl'Italiani c così hanno dovuto fare anche gli Slavi balcanici. Ma appena raggiunto il primo gradino di liberlà, per quanto possa riuscire doloroso rinumziare ad epiche tradizioni, bisogna cambiar tattica. Alla rivolta 
convulsa occorre sostituire il lavoro serio ed ordinato, che segna la via vera e lunga del progresso. Compenetrati dalla logica ferrea di questi ragionamenti i palriotti ilaliani fervidi ed impazienti per gioventù dell'nna e dell'altra parte dell'Adrialico smelteranno le illusioni d'imprese mazziniane e garibaldine, che per liflalia hamo fatto il loro tempo e sono oggi inconsulte e si asterranno da concioni o dimostrazioni irredentistiche, le quali anzichè giovare, danneggiano chi le intraprende e la calısa. Nessuma persona ragionevole potrebbe oggi ammetlere o pretendere che l'Italia metla a repentaglio lopera invidiabile di mezzo secolo per formare imprese che non le riuscirebbero oggi colla prepotenza, ma che forse sortiramno qualche effetto quando un palcato ragionasnento avrà fatto dissipare dei malintesi. Tulto quello che l'Ilalia può ora dare agl'Italiani dell'Austria-U'ngheria è l'aiuto d'una simpatia morale, tenuta nei limiti della corretlersa diplomatica.

Nelle attuali condizioni politiche la parola dordine degl'Italiani sull'Adriatico dovrebbe essere: aspettare. E gl'Italiani che hamo saputo allendere altri avenimenti epocali, senza di cui non sarebbero ancora liberi, altenderanno in omaggio a quel buon senso imalo alla loro razza, che attese e sopportò anche la sehiavilì quando non sareble stalo possibile liberaseme. Quate pii bella prova di semo politico lu ofterta dallat nazione italianal, risorta in una vampa di sentimento, che cuclla di aver fatto tacere per mezzo secolo limpulso del sentimento che la spingeva a socerrere i l'ratelli irredenti? Quante volte non si elevarono grida disperate di dolore da Trento, da Trieste, dall Istria, dalla Dalmazia imploranti soccorso! Eppure la nazione ilaliana, che gli stranieri ritengono ancora focosa r lrivola, oflésa nei suof piò rari affetti e nella sua dignilid. seppe lremarsi "lavolane in

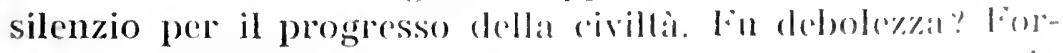

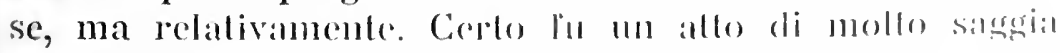
opportunita. - Il callio trallamento dell linstria verse

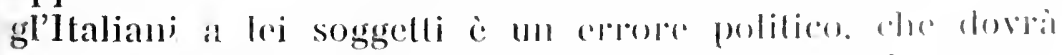

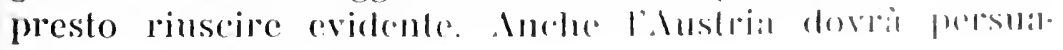


dersi dell'inulilitì teorica e pratica dei suoi sforzi per estirpare l'elemento italiano dall'Adriatico e allora come ha trattato bene, anche troppo, gli Slavi per acquistarsi simpatie tra i popoli balcanici, tanto più dovrà trattare equamente anclic gl'Italiani, nel suo stesso tornaconto, onde non affrettare la conversione di un popolo, che sa farsi rispettare come l'Italiano, da tacilo alleato in aperto inimico.

Aspettare significa dar al tempo la possibilità di far indebolire gli odlerni avversari e rinforzare i possibili futuri alleati. Ogni italiano deve quindi persuadersi che il coronamento dell'impero d'Italia col riacquisto di quelle terre che furono parte della X regione italica di Augusto e col predominio sull'Adriatico non potrà avvenire, come per Roma, che quando gl'Italiani saranno più forti di tutti i loro vicini. E bisogna che si rasseguino a pazientare ed a lavorare intanto nel campo della coltura.

Del resto, si creda, che il frutto non è mai tanto dolce come quando è maturo e sta per cadere da solo 'dall'albero.

\section{L'ULTIMA RATIO.}

Le considerazioni degli ultimi capitoli, che non hanno altro intendimento che quello di essere freddamente logiche, potranno apparire a qualcuno machiavellistiche.

Fra i sentimenti, quello dell'equità, è senza dubbio uno che stimola maggiormente l'umanità nel suo cammino ascensionale. Ma se grande è lo stimolo, piccolo è il successo, perchè la giustizia, considerata come una meta ideale, trascende al di là delle forze umane.

Chi non desidera pentirsi delle proprie azioni non dovrebbc perdere mai di vista questo faro ideale. Chi però vuole cogliere dei successi, deve pensare praticamente ai mezzi per raggiungerli, e purtroppo reprimere il sentimento, anche se nobile, per non guastare la realtà coll'utopia.

Al progresso umano, che nelle sue grandi linee tende 
innegabilmente alla giustizia, succede quello che tocea alle piante nel crescere, che tendono verso la luce. Se non la possono raggiungere direttamente, si piegano e vi si rivolgono per vie contorte. Le povere piante crescono deformi. E un peccato per l'estetica, ma così vuole un inesorabile legge di natura.

Uno strazio consimile colpisee la giustizia quando la politica le proietta la sua ombra. I due canoni fondamentali della diplomazia sono la furberia e la forza: la giustizia non vale che come un miraggio.

Lo stato prevalente del mondo è il moto. Tutto in esso si cambia, si modifica, si trasforma, dal suo regno mincrale sconvolto a malgrado della sua rigidità, al regno vegetale che cresce, liorisce e muore per subilo rinascere, al regno animale, che si sposta e sovrappone continuamente e tra cui primeggia quell'essere debole e forte allo stesso tempo, ma irrequieto, ch'è l'nomo, sempre in subbuglio a causa delle sue passioni mai sazie.

Il mondo è mosso e dominato dalla for\%a. Dacchè mondo è mondo, il pesce grande ha mangiato il piccolo, e così continuerà ad onta di tulti gl'idealismi contrari.

L'umanità cammina verso la libertà tortuosamente, come un fiume verso il mare deve deviare ogni qual volla incontra l'intoppo di una rupe. Limmanilit stancal alle volte si ferma, come il finme che s'allaga, a contemplare la bellezza serena della pace. Ma questa contemplazione, come quella della dolceza nel sentimento, della grazia nell'arte, del bello assoluto nell'universo, è mo statdio passeggero che sari sciupato da un rude soffio di forza, appena che l'umaniti dovrì ripendere il suo faticoso e fatale movimento, senza del quale morrebbe.

Gl'ideali della pace, della giustizia, dell'eguaglianza perletta, esisteramo forse nell'altro mondo, ma allora l'nmanità, come il fiume che raggiungendo il mare cessal di esistere, non sarà più quella che noi vediamo c dol)biamo studiare.

Ormai venti e più secoli di storia positiva e la conoscenza di tutlo il nostro globo ei hammo insogralo abbastanza bene quali siano le correnti dellmmaniti. 
Glideali continucranno ad allettare le sue brame e l'imponderabile a farsene giuoco. Ma gli uomini finchè saranno tali e non angeli, daranno ancora sempre spettacoli di egoismo, di prepotenza, di sopraffazioni. Il mondo è stato sempre dei forti ed i piccoli od i deboli furono e saranno schiacciati. Mors lua, vita mea. Purtroppo!

Questo speltacolo nauseante quando abbiamo sott'occhio l'atto della violenza, sublime culando invece non vediamo che la grandiosità dei trionfi compiuti, durerà quanto l'nomo.

Intanto tutti i popoli, consci dell'incluttabilità di questo ingranaggio, vi si acconciano e non potendo ottenere trionfi definitivi da una parte si contentano di successi parziali dall'altra. La legge dell'adattamento vale per i popoli come per gl'individui. - Quante sopraffazioni non iurono intraprese come ripiego per compensare la cattiva riuscita di altri tentativi!

La storia di tutti i popoli, ma specialmente di quei due classici che hanno chiuso il loro ciclo, dei Greci e dei Romani, ¿̀ un esempio parlante di questa verità. Ma anche senza sforzare la mente nell'investigare ed abbracciare periodi lontani, ci sono esempi a noi vicinissimi e visibili a tutti di questa tendenza generale anche nei popoli, come negl'individui, a cogliere il massimo successo col minimo sforzo e col minimo rischio.

La Francia baltuta nel 1870 morde il freno della rivincita e, non potendo ingaggiarsi in una nuova lotta per riprendersi l'Alsazia-Lorena, s'è rifatta in Africa fondando quel vasto impero coloniale che va dalla Sirte minore alla costa dell'Atlantico. La Germania, che sogna di divenire ancora più grande espandendosi territorialmente verso l'oriente e il mezzogiorno, non reputando giunto ancora il momento di dar di piglio alle armi per questo scopo, s'è messa in una lotta économica coll'Inghilterra per aprirsi un varco commerciale nei grandi mari del mondo. La Russia, impedita dalla gelosia coalizzata dell'Europa di estendersi nei Balcani e battuta dal Giappone anche nella sua avanzata suppletoria nell'Asia, vorrebbe ora allargarsi sull'Armenia o sulla Persia, che non han- 
no la forza di difendersi. L'Inghilterra s'è fatta mondiale approfittando astutamente delle debolezze e delle discordie altrui. L'Austria quando fu respinta dall'Italia si rifece nei Balcani, ed ora che le fu chiusa questa via proclama di voler conservarsi aperta quella del mare ed arma la sua flotta. L'Italia, che per completare la sua indipendenza politica arrebbe bisogno di fortificarsi geograficamente sulle Alpi, trovò più conveniente per ora di espandersi dall'altra parte del Mediterraneo e s'impossessò della Libia. I piccoli Stati balcanici, che arevano connazionali da redimere in Austria e in Turchia, diedero addosso piuttosto a quest'ultima per una ragione assai ovvia a comprendersi. E la Turchia che non può rifarsi delle perdite su altri più deboli all'estero massacra i cristiani che le sono soggetti e che secondo la sua mentalità sono i colpevoli delle disgrazic che le capitano addosso.

Questa catena di soprusi ticne avvinta la storia di tutto il mondo ed è tanto ferrea, che ormai non vale quasi nemmeno arrossire, perchè nessuno ha più le mani perfettamente pure del sangue dei più deboli.

Eppure l'umanità progredisce verso una mela, cui è attratta per forza e ch'essa nemmeno bene distingue. Oggi la prossima tappa o il faro più vicino apparisce l'indipendenza dei popoli. E siccome il ciclo della nizionalità non è ancora completo e chiuso, tutti i popoli e gli Stati, grandi e piccoli, che hamno o non hamo partite aperte, non potendo fare altrimenti si armano militarmente per ricorrere all'ultima ratio della guerra appena si offra loro la possibilità propizia.

Guai del resto se il legittimismo losse inviolabile e la decrepitezza elerna. Il rinnovamento è la vita del mondo e della società umana. Senza errori e senza cadute non v'ha progresso. Il mondo senza la benelica legge del rinnovamento, se oggi apparisee imperletto, sarebbe forse cento volte peggiore. E nel rinnovamento i bene che abbiano la preferenza i lorti sui deboli.

Se tutto ciò da qualcuno sarì chiamalo colla parola L'A driatico. 
poco simpatica di machiavellismo, sia pure. Il metodo di osservazione, basato logicamente sull'esperienza, avrà almeno il merito di aver guardato in faccia l'avvenire scnza il velo di pericolose illusioni.

\section{RIA SSUN'T 0 .}

Riassumiamo brevemente gl'insegnamenti della geografia e di venti secoli di storia positiva.

Il bacino dell' Adriatico racchiuso dalle Alpi, dall'Apennino e dal Carso, protetto poderosamente dalle prime, meno dal secondo e poco dal terzo, ricco più che ogni altro mare, perchè è asciutto nella parte superiore, stretto nella lunghezza, ma congiunto da un'estremità all'altra dalla via acquea marina, fluviale, lacustre, forma un complesso geograficamente caratteristico, singolare, indivisibile.

Non c'è in Europa, e nemmeno nel mondo, un insenamento di mare così regolare, così grande, così importante nell'economia dei popoli. La Padania è il più irrigato, il più fertile, il più bel bassopiano d'Europa. La gran valle dell'Ebro in Spagna, quella del Rodano in Francia, e le due valli del medio e basso Danubio, l'ungarica e la valacca, le somigliano in parte, ma sono inferiori. Il Tirreno e l'Egeo hanno maggior valore dell'Adriatico nella storia della civiltà; ma l'Adriatico considerato nella sua forma e lunghezza complessiva dal Piemonte ad Otranto li supera per importanza economica. Il Tirreno servì agli scambi commerciali tra l'Italia apenninica, le tre maggiori sue isole e la punta dell'Africa. L'Egeo fece nascerc la civiltà mediterranea e congiunse l'Asia minore ai Balcani, il Mar Nero al Mediterraneo. L'Adriatico servì a tenere in comunicazione le parti più vitali di tre continenti: il centro d'Europa, l'Egitto dell'Africa, la Mesopotamia e l'India dell'Asia.

Solamente l'Asia e l'America possiedono un'unione di terra e di mare paragonabile a quella dell'Adriatico, la prima nella Mesopotamia col golfo Persico, la seconda 
nel Mississipì col golfo del Messico. Senza voler menomare affatto la grandiositi e il valore economico della Mesopotamia e delle prateric delle Saranne, possiamo dire che nessuno eguaglia l'Adriatico nell'armonia. Prima di tutto il contorno dell'Adriatico è definito chiaramente. Poi, tolto quel tratto di costa melmosa che ra dal Savio all'Isonzo, il passaggio rapido dal mare al colle e al monte forma una delle attrattive più pittoresche. Lal terra si sacrificò in più punti al mare, ma il mare benclica coi suoi meandri ogni più remoto angolo d'insenatura.

L'Adriatico ha, è vero, un gran clifetto, la streltezza delle due coste nei due lati longitudinali. Però anche questo difetto non è privo di un vantaggio. La povertà della terra spinse i suoi abitatori a cercar lavoro sul mare, li spronò all'attività e li preservò dallo snervamento della ricchezza. La parabola di quasi tutli gli Stali è la decadenza interna per corruzione dopo di aver accumulato tesori col lavoro. Venezia e Ragusa, raggimuta l'opulenza, decaddero e perirono per forze maggiori esterne: la scoperta dell'America, le stragi dell'islamismo, la bufera sociale di Francia, la violenza militare di Napoleone. Malgrado tutto, la fibra sana di Venezia e di Ragusa sarebbe forse sopravissuta se non fossero state troncate la prima dalla lega di Cambrai, la seconda dal terremolo del 1667.

L'Adriatico per i monti che lo cingono può essere considerato come un bacino chiuso. Eppure lia tante aperture naturali in tutte le clirezioni, che non inperli nè immigrazioni, nè emigrazioni. La sua forma allungala dal settentrione al mezzogiono costituisee unottima via di comunicazione tra l'Europa e l'Oriente, e nello stesso tempo la vicinamza delle sue coste favorisec gli scamb, reciproci fra l'Italia e i Balcani.

Per l'equilibrio della temperatura non solo nel suo bacino, ma anche tra $\mathrm{i}$ due continenti enropeo ed alpicano, l'Adriatico è attraversato periodiramente da Ire colrenti aeree principali, costanti e note. In direzions Iongitudinale da settentrione a mezzogiorno soffia il vento di maistro, proveniente dalle Alpi e dalla Panlania ascint- 
to, fresco, rigeneratore delle energie. In direzione opposta da mezzogiorno verso settentrione imperversa il sirocco, proveniente dall'Africa e dal Mediterraneo, umido, piovoso, uggioso, caldo, snervante. In direzione della latitudine, però solo da oriente verso ponente, infuria la bora, vento freddo, violento, vorticoso, distruttore.

Con una somiglianza perfetta e non solo reltoricamente accidentale si mossero sull'Adriatico anche le correnti umane. Nè è da meravigliarsi di questa coincidenza perchè il vento e gli uomini per muoversi devono cercare le vie basse e piane, mari, pianure, vallate e valichi che spesso sono gli stessi per tutti due.

Per le vie della bora vennero gl'Illiri, gli Unni, i Cimbri, gli Avari, gli Slavi, i Magiari, i Tartari, i Turchi, gli Austriaci; -- per quelle dello scirocco i Greci, i Romani in principio, i Bizantini, i Normanni, gli Arabi; - per quelle del maistro i Galli, di nuovo i Romani, i Franchi, i Veneziani, i Francesi di Napoleone, gl'Ttaliani. La similitudine potrebbe essere continuata istituendo un paragone fra la mancanza di vento e il passaggio di popoli direttamente dalla metà della costa occidentale all'orientale, nonchè tra gli effetti della temperatura dei venti sulla superfice terrestre e quelli dell'indole e della civiltà dei vari popoli sul progresso dell'umanità. I popoli che seguirono la strada della bora portarono per lo più distruzione o per lo meno sono stati negativi nel senso che non eressero nulla di grande e duraturo; quelli che venzero collo scirocco recarono un temperamento caldo, ma usi snervanti, che procurarono la decadenza rapida. Solo coloro che partirono dalla Padania portarono seco uno spirito rigeneratore e lasciarono benefici effetti duraturi. La parte buona del dominio romano sulla parte orientale dell'Adriatico provicne dall'avanzata di questo popolo per la via del Po e delle Alpi e le sue tracce si conservano ancora in ragione decrescente con questa linea. I Franchi ristabilirono il bene inapprezzabile della giustizia, guastata dalla corruzione orientale.

La signoria secolare di Venezia, prescindendo da quegli appunti che si possono fare ad ogni manifestazione 
umana, è stata proporzionalmente ai mezzi di cui disponeva la Serenissima, una delle più benefiche. I Francesi portarono la rivoluzione di nuove idee, il cui licvito rigeneratore fermenta ancora.

Singolare questa coincidenza di effetti fra la natura, l'origine e la direzione dei venti e quella dlei popoli!

Nelle relazioni coll'esterno l'Adriatico partecipa alla storia dell'umanità: nelle relazioni interne fra le sue due coste l'Adriatico ha invece mina sua storia lutta speciale. L'Adriatico può essere diviso in tre bacini; di questi, clue, il settentrionale e il meridionale, stamo sotto l'influenza della costa italiana e il medio soltanto appartiene per la ricchezza d'isole all'àmbito preponderante della costa orientale.

Con queste distinzioni geografiche si comprenclono meglio le relazioni reciproche tra le due coste. Le correnti migratorie dalla costa occidentale verso l'orientale furono sempre - e si possono osservare anche ai giorni nostri duplici e per ciò più forti. La corrente migraloria ned scnso inverso fu al contrario sempre debolissimil. La costa orientale, più fortunata dal lato della navigazione, ma infelice per la sua posizione, attaccata al Balcani. fu la più bersagliata, ma esercitò appunto per questo per l'Adriatico e per la sponda opposta un azione benefica, arrestando la fimmana dei popoli barbari dell Oriente e dell' Asia.

Chiusa nella sua parte centrale dal predominio italico nell'alto e nel basso Adriatico, la costa orientale subì in tutti i tempi quasi un dirillo di servitù da parte dell'occidentale. Sebbene appartenga geograficamente ai Balcani, la sua storia dev'essere compresa in quella delloccidentale. La sua civilti anche oggi è loggiata sul tipo italico.

Scorriamo a conferma l'insegnamento dei secoli. Nol principio del secondo secolo avanti Cristo i Romani posero piede sulla costa orientale per non abbandonarta che alla fine del quinto d. C. - A ribadire ancoral pii l'indivisibilità dell'Adriatico nella spartizione dellimpero in occidentale ed orientale solto Teodosio find del $11 \mathrm{sec}$ 
d. C.), l'Illirio fu diviso ancli'esso in due parti e l'occidentale colla Dalmazia fu assegnata all'Italia. Spentosi l'ultimo riflesso della dignità imperiale romana coll'imperatore Giulio Nepote nel 480, subito nel 481 Odoacre occupò tutte le due coste dell'Adriatico e poi per meglio governarle trasportò la sua sede da Roma a Ravenna. Ai Goti si sostituirono nel VI secolo i Bizantini. Dalla metà di esso l'Italia divenne provincia dell'impero d'Oriente e la corte di Costantinopoli affidò il governo dell'Adriatico agli èsarchi di Ravemna. Il dominio bizantino unico ed effettivo sul mare e sulle coste adriatiche, alquanto contrastato nel retroterra, durò di fatto fino a che fir sostituito da Venezia (XI secolo).

L'Adriatico fu dunque per questi dodici secoli ininterrotti un tutto politico. Durante l'epoca bizantina avvennero è vero dei fatti che scossero alquanto la compagine, prima unita, dell'Adriatico. Nella seconda metà del secolo VI i Longobardi vi affermarono dei diritti, nia appena nominali. Al principio del VII vi s'insediarono sulla costa orientale gli Slavi; la dominazione carolingia nell'VIII e IX secolo fu più effettiva, ma anche limitata. Durevole, ma di carattere effimero, fu la signoria del regno di Croazia su parte della costa orientale nel $\mathrm{X}$ ed XI secolo.

Nel X secolo compariscono i Veneziani. Dall'XI al XIII l'Adriatico per opera delle crociate ritorna sotto l'influenza marittima d'Italia e di Venezia particolarmente. Dal XII a tutto il XIV s'ebbero tre secoli di lotte fra Veneziani e Ungheresi per il predominio sull'Adriatico a mezzo della conquista della sua costa orientale. Venezia trionfò al principio del $\mathrm{XV}$ e vi si mantenne signora fino a tutto il XVIII. Il contrasto oppostole dai Turchi per due secoli (XVI e XVII) non fece che sfiorare l'Adriatico.

Il dispotismo napoleonico donette anche rispettare la geografia e la storia e assegnò la costa orientale dell'Adriatico al regno d'Italia. L'Austria, se la corrente nazionale non l'avesse arrestata, avrebbe instaurato sull'Adriatico le tradizioni occidentali, italiche, dei Franchi. Respinta dalla costa occidentale fu lei ad aizzarle contro 
l'orientale ed a far rivivere quegli odii e quei rancori che le avevano travagliate fra l'XI e XV secolo e ch'erano scomparsi per merito della moderazione e della saggezza politica di Venezia.

Un effettivo dominio balcanico sull'Adriatico non ebbe mai luogo. E sia che su questo mare abbia pesato la voce italica di Roma, dei Papi, di Venezia, oppure quella esotica dei Goti, dei Longobardi, dei Franchi, degli Ottoni di Germania, dei Bizantini di Costantinopoli, degli Ungheri, degli Austriaci, la vita civile di tutte le due coste adriatiche fu costantemente di origine e di tipo italico.

Ma volendo anche sottrarre dal nesso statale dell'occidente italico il tempo delle dominazioni puramente politiche (bizantina, carolingia, croata, unglerese e austriaca, contrastate e interrolte, che non potrebbero sommare tutt'al più che a sette secoli di fromle a quattordici di indiscusso dominio dell'Italia), non ne verrebbe alterata la conclusione, che la costa orientale dell'Adriatico subì maggiormente l'influenza della penisola italica per mare, che quella della penisola balcanica per terra, e che essa non esercitò mai alcun influsso storico, nè di dominio, nè di civiltà sulla sua sorella opposta.

In dipendenza da questa situazione geografica e storica si svolsero sempre anche i passaggi minori dei popoli tra le due coste.

Ai tempi antichi, popoli illirici o loro affini si sparsero dalla penisola balcanica nell'Italia, ma furono assorbiti dai Romani. Al principio del medio evo e più tardi, Slavi calarono per terra nel Friuli orientale, o per mare a Venezia, ma anche di questi non rimane che un debole ricordo. Solto l'infuriare delle atrociti turche, numerosi Albanesi furono scaraventati oltre l'Adriatico nella bassa Italia e in Sicilia. Ne sopravvivono diversi ancora, ma politicamente si deve dire che si mantennero soltomessi. Nel Molisano vivono pochi Slavi, cipjoilati non samno essi stessi da dove, ma molto probabilmente dalla costat dialmata e per soltrarsi a qualche massacro fureo. . Ia anche della loro esistenza si sa più per le ricerehe di alle'i, che per estrinsecazione della lo:o individualili natrionale. 
Singoli Slavi scalmanati vorrebbero che anche alle foci del Po abbiano vissuto colonie slave e intendono di comprovarlo col nome della città di Ravenna, che secondo loro sarebbe una corruzione italianizzata della parola ravna (la piana) formatosi per la conformazione della terra in quella regione.

L'ipotesi è senza dubbio azzardata, come lo è l'asserzione di certi pangermanisti che Ravenna debba essere stata tedesca all' epoca dei Goti, perchè le loro leggende ricordano una Rabenschlacht o battaglia di Rabern = Ravenna, fra Teodorico da Verona (Dietrich von Bern) ed Ermanrico. Si direbbe da qualche umorista che per purificarla da simili profanazioni, proprio a Ravemna toccò l'onore di accoglieré le ossa di Dante, il padre della lingua e della rimovata nazione italiana e di conservare la fiamma ideale eterna dell'amor patrio degl'Italiani.

Per esaurire l'enumerazione delle tracce di popoli della penisola balcanica dalla parte italica dell'Adriatico, diremo che nelle Puglie e nella terra di Otranto esistono ancora dei rimasugli di parlata greca e che negli Abbruzzi c'è il villaggio di Scanno, sperduto nei monti, i cui abitatori conservano ancora tipo ed usi turchi.

Non ripeteremo la storia dell'espansione mondiale romana dall'Adriatico al Danubio. Però anche dopo la sua rovina gl'Italiani tennero occupate per tutti i secoli le regioni situate entro il loro confine naturale fino al Quarnero "che Italia chiude e i suoi termini bagna". - Le poche infiltrazioni al di qua di questa linea, riassorbite tacilamente o da riassorbirsi alla prossima buona occasione, non hanno importanza e sono ad ogni modo compensate dall'esistenza ininterrotta di un elemento nazionale italiano, colonie od oasi come si vogliano chiamare, Iungo tutta la costa orientale fino allo stretto di Vallona.

Per esaurire anche da questa parte le tracce provenienti dall'altra costa diremo che, prescindendo dai monumenti romani imponenti, ma muti, per tutto l'interno balcanico della costa orientale esistono ricordi vivi di Roma, nei nomi di località, negli usi e costumi, nel tipo 
dei popoli che seguirono, nei numerosi Curzovalacchi dell'Albania, nei pochi Rumeni alle falde orientali del Monte Maggiore in Istria.

Gli abitatori dell'Adriatico appartengono per quattro quinti all'elemento italico. Vengono poi con un numero ed estensione notevole (quasi un milione) gli Slavi e quindi gli Albanesi (mezzo milione da Scutari a Vallona). Gli altri Tedeschi, Greci, Magiari sono oggigiorno affatto secondari. Prevale dunque potentemente e sotto tutti gli aspetti l'elemento italico. E se un giorno dovesse nascere la fusione e formarsi un nuovo tipo nazionale, l'adriatico, esso non potrebbe essere che man derivazione dell'italiano.

Questo nesso costante fra l'Adriatico e l'Italia non è frutto della storia, ma opera della geografia. Le $M_{p i}$ e gli Apennini che hanno creato I'Adriatico, hamno falto anche I'Italia. L'Adriatico non esisterebbe senza I'Ilalia, come l'Italia non potrebbe vivere se l'Adriatico fosse di altri.

L'Adriatico e l'Italia sono tanto intimamente connessi, che la strada verso l'Italia porta sull'Adriatico e chi viaggia per l'Adriatico finisce in Italia. Questa veriti balza agli occhi dalle storie di Roma, di Venezia, di Francia, di Savoia da una parte, dei Barbari, di Bisanzio, dei Nolmanni, dell'Austria dall'altra.

I Romani non dominarono tutto l'Adriatico che dopo di aver conquistata la Padania e per conquistarla mossero attraverso l'Adriatico. I Veneziani che lenevano giat la costa orientale dell'Adriatico pensarono di oceupare a mezzo di esso anche l'oceidentale. Napoleone irruppe tra le Alpi e gli Apennini per scendere in Italia e le sue lluppe: ed i stro generali di passo in passo arrivarono fino alle Puglic da un lato ed a Cattaro dall'altro. Quando la dinastia di Savoia seese dai suoi monti al piano d'Italia non aveva certamente di mira l'Adriatico. Eppure ogsi regna su quasi due ter\%i di esso. Tulli i Barbari da $\mathrm{Ml}$ tila a Solimano tendevano all'Italia. Soltanto per nom aver saputo navigare inciamparono nell'Adrialico o con tutto ciò scorrendo la sua costa orientale vennero a nno-

L'Adriatico. 
rire alle porte della Padania. Bisanzio attraverso l'Adriatico ebbe le sue due coste, indi regioni notevoli d'Italia e da ultimo l'impero su tutta la penisola. Perfino i Normanni ebbero la bassa Italia involontariamente scorrazzando l'Adriatico. I duchi d'Austria dal Danubio tesero al mare più vicino l'Adriatico e riuscirono ad insediarsi a Trieste. I loro successori furono attratti in Italia e la occuparono fino agli Apennini. La Padania sola costituisce un terzo dell'Italia.

La storia d'Italia influì sempre sull'Adriatico. Le loro sorti sono congiunte indissolubilmente.

\section{GONGLUSIONE.}

Per giudicare convenientemente l'Adriatico bisogna considerarlo oltrechè solo o congiunto all'Italia, anche nel nesso dell'Europa e del mondo.

Tendenza del L'umanità, dopo secoli di cammino che noi non riuprogresso nella civiltà mo derna. sciamo più nemmeno a calcolare, si trova ora in un momento di risveglio, di attività e di slancio universali.

Questo progresso veramente non è nuovo, perchè data dal secolo XV, da quando cioè per l'impulso della navigazione il mondo civile, ristretto all'Europa e ad una parte dell'Asia e dell'Africa, si allargò fino a raggiungere gli estremi confini del globo terrestre.

Le scoperte scientifiche e pratiche che accompagnarono quel rinnovamento dell'attività umana contribuirono a far mutar rotta al cammino ideale dell'umanità. Gli uomini, quando si videro aperta la via dell'America, si accorsero che il misticismo religioso che li aveva fatti combattere inutilmente tante crociate per il lustro dei Papi e degl' Imperatori li aveva incatenati in un campo troppo ristretto d'idee e di azione materiale. Rivolgendo le prore delle navi e le menti verso paesi non ancora dominati, assaporarono tutta la voluttà dell'essere liberi.

Da allora la società dell'Europa cambiò aspetto. Nel mentre prima immensi tesori di energia venivano consumati nel combattersi a vicenda, ora gli uomini liberati 
dalle pastoie religiose e politiche si rivolsero con entusiasmo allo studio, al lavoro proficuo. Per ciò noi vediamo da prima impallidire $\mathrm{i}$ due grandi astri sorti per frazionamento dalle rovine dell'impero mondiale romano, il papato e il sacro romano impero della nazione germanica, e tramontare sempre più l'aureola semi-divina dei Pontefici ed Imperatori, che per accrescere il proprio lustro avevano combattuto la libertà dei singoli.

Il principio trascendentale del potere sovrano ed assoluto aveva terminato il suo còmpito e la socictà divenne matura per altri destini.

Così fu preparata la via della grancle rivoluzione sociale, ch'ebbe principio dalla Francia nel 1789, e dalla quale furono aperte le porte a tutte le libertì singole e collettive, diritti degli uomini, costituzionalismo, indipendenza nazionale.

Forse non per puro caso anche questo nuovo fiotto di sangue nella vita dell'umanità ebbe per compagni importanti invenzioni. Prima di tutto ancora al principio del secolo a noi precedente comparve la macchina a vapore che rese l'uomo ben presto padrone della superfice del mare e della terra. Il secolo nostro colla macchina per volare, coi perfezionamenti elettrici della telegrafiia e della telefonia senza fili, coi sollomarini, colla scoperta dei due poli, renderà l'uomo sempre più dominatore degli elementi della terra.

Il nostro secolo non cammina dunque su una strada nuova, ma segue quella traceiatagli dai precedenti e particolarmente dal XIX. Notevole è però che il progresso della civiltà, per usare una frase conlemporanea, procede a tutto vapore.

Il mondo è ormai conosciuto nella slla estensione; non rimane quindi altro che da rifare passi gia latli per studiarlo e sfruttarlo nelle parti trascurate.

L'industrialismo, quale applicazione pratica di invenzioni teoriche, trasforma sempre piu il tenore di vita e le idee degli uomini. Per esso è aumentato il benessere materiale, è facilitato il guadagno, è cecilala la cupicligia della ricchezza. Quando gli uomini avevano poco da la- 
vorare o anche lavorando cose semplici avevano la possibilità di fantasticare, era facile che sui loro cervelli facessero presa le idee religiose e che di conseguenza fosscro sottomessi alle istruzioni di una superiorità gerarchica. Oggi invece che la pigrizia e il dolce far niente sono in discredito e che tutti per vivere devono lavorare febbrilmente e nolti anzi" finiscono col subire la forza dell'abitudine e l'ossessione del lavoro, resta assai poco tempo da fantasticare alle cose che non ci riguardano immediatamente. Chi sa di dover lavorare, sa non solo voler riposare e divertirsi per ritemprare le forze di tanto in tanto, ma anche organizzarsi per rendere meno gravoso il proprio lavoro.

L'ambiente moderno non impone più fede e rispetto religioso come nell'evo medio; anzi insegna ad aver fede in una direzione opposta, nella più completa libertà individuale, politica, nazionale. In un periodo di studi tecnici devono necessariamente prevalere il positivismo sul sentimentalismo, lo scetticismo sulla fede, l'energia individuale e collettiva sulla rassegnazione passiva, propria a tutte le religioni. Così si spiegano non solo la comparsa delle tendenze irreligiose, antidinastiche, antistatali (massoneria, repubblica, socialismo, anarchia), peculiari alla nostra società, ma anche l'impossibilità di cambiarle e I'inutilità degli sforzi di tutti coloro che vorrebbero alterarle. La febbre attiva della nostra società segue nell'altalena del progresso umano al raccoglimento mistico del medio evo, come questo tenne dietro in naturale riposo alla vita intensa della civiltà greco-romana da una parte e allo sciupamento di forze della trasmigrazione dei popoli dall'altra. Noi che presi dalla stessa febbre vediamo il lavoro intenso dell'umanità dei nostri giorni ce ne compiacciamo e lo riteniamo buono, se non proprio oltimo.

Potrebbe succedere che a questo nuovo sforzo sia per seguire un indebolimento e una nuova decadenza. Alcuni vogliono anzi già vedere in questo nostro ritorno al paganesimo i germi della sfrenatezza e della dissoluzionc. Può darsi che abbiano ragione; ma le loro preoc- 
cupazioni sono perfettamente inutili ed innocue. L'umanità cammina oggi a tutto vapore non tanto perchè ognuno di noi vi mette un atomo di energia, quanto perchè così deve avvenire per altre cause maggiori; e l'umanití, che nel suo lungo viaggio ha saputo scegliere la via migliore guidata dal suo misterioso e per noi imponderabile destino, la troverà anche durante e dopo questo titanico sforzo industriale, tecnico, realista, anche senza o malgrado le nostre filosofiche previsioni.

Tra le forme di libertà portate dalle rivoluzioni del L'indipendensecolo passato primeggia quale specialità del nostro temza nazionale. po l'idea dell'indipendenza nazionale. Essa è quasi la sintesi di tutte le altre libertà parziali dell'individuo, della patria, della società e il mezzo migliore per raggiungere mediante l'impiego non limitato della lingua materna e delle rimanenti facoltà intellettuali il massimo grado clello sviluppo civile.

Questa tendenza che incominciò a farsi sentire al principio del secolo scorso come la prima conseguenza collettiva dei diritti dell'uomo, proclamati di fresco, e che domina le idee e la politica anche del nostro, ha aperto nella storia dell'umanità un ciclo, che se non sarà dei più grandiosi, abbraccerà certo alcuni secoli.

Potrebbe apparir strano che l'idea dell'indipendenza nazionale, così semplice, intuitiva, giusta e pratica, abbia tardato tanto a farsi strada nel mondo. Gli è che l'idea di patria nazionale è un concelto evoluto ben differente da quello elementare di patria, quale attaccamento al luogo nativo. Per comprendere la patria in senso nazionale occorre che un popolo abbia raggiunto uno stato di coltura avanzato, altrimenti, come lo provano oggi all'evidenza gli Albanesi, essa può produrre degli eroismi singoli, non mai un organismo vitale socialmente.

Poi bisogna considerare parechie circostanze. Per l'umanità i secoli equivalgono ai giorni della vita dell'individuo. La distribuzione e la formazione degli Stati aviulti la rivoluzione del 1789 datava ancora in gran parte nelle sue fondamenta dalla trasmigrazione dei popoli. ed era aggravata dalle posteriori incursioni di asiatici. Per dare 
a quella nuova idea un contenuto pratico erano necessarie due cose: occorreva da una parte che i popoli oppressi si preparassero con l'acquisto di altre piccole libertà allo sforzo della riscossa, e dall'altra che l'opinione generale d'Europa fosse un po' intenerita al sentimento di giustizia, come correttivo allo stato di violenza, tramandato fii dai primi secoli dell'era volgare. A conferma di ciò basta citare il risorgimento politico degl'Italiani, dei Greci ed ora degli Slavi balcanici. L'indipendenza di. questi popoli fu oltenuta in prima linea col sacrifizio di vite dei popoli interessati, ma è dovuta in buona parte anche alla simpatia degli altri popoli per una causa giusta, che corresse gli errori della diplomazia, quale espressione della prepotenza, dell'egoismo e dell'ingordigia collettiva dei più forti.

I Balcani e il Prima di fare pronostici sui Balcani sarà bene consiloro avvenire.

derare questa penisola da tre punti di vista: dal suo valore geografico intrinseco, dal suo nesso nel mondo, dall'indole dei popoli che la abitano.

La natura è stata veramente matrigna coi Balcani. La prima disgrazia geografica, che balza agli occhi anche dell'osservatore più inesperto, è la mancanza di confini a settentrione. La catena del Balcan, antico Haemos, non la chiude e non la protegge che debolmente e imperfettamente verso le foci del Danubio. Il resto ad occidente è aperto. Per ciò questa penisola a differenza dell'Italica e dell'Iberica non ebbe, e non potrà avere mai un carattere proprio.

I Balcani sono divisi in parecchie regioni (bacini fluviali) principali, nessuna delle quali può dirsi veramente florida. I venti freddi del settentrione le rendono inferiori ai paesi di eguale latitudine dell'Italia e della Spagna: La sola Grecia e il versante dell'Egeo godono di un clima mediterraneo. I Balcani potrebbero ridivenire ubertosi, come furono ai tempi della civiltà greco-romana, ma occorrerebbero fatiche ingenti per rigenerarli e poi per la loro conservazione.

La natura, oltre ad aver creato questa penisola montuosa, arida, frastagliata, sconnessa, la collocò a contatto 
dell'Asia, che le portò più male che bene. I Balcani non potranno sottrarsi mai al loro amaro destino di formare la porta per i passaggi tra l'Europa e l'Asia. I Balcani, paese complessivamente povero, sono stati la terra classica della discordia interna e dellinsidia esterna: essi furono sempre e resteranno il tallone d'Achille dell'Europa.

Per non divagare troppo - sebbene sarebbe utile nello studio dell'Adriatico di figgere lo sguardo bene addentro in questa equiroca penisola - non ci occuperemo delle attitudini o dell'indole dei Rumeni, dei Greci, degli Albanesi e nemmeno dei Bulgari, che a quanto si può prevedere non sono destinati ad esercitare un'influcnza diretta sull'Adriatico e ci limiteremo ad osservare la natura dei Serbi, che conservano in maggior copia le caratteristiche della razza slava.

Si sa che la caratteristica principale dei popoli slavi è la resistenza alle imposizioni esteriori, accompagnata da una mite rassegnazione, povera di energiil attiva e priva spesso della più elementare organizzazione. Per effetto di questa speciale, ineffabile encrgia passiva gli Slavi sopravvissero probabilmente nei Balcani a tanti disastri, che forse avrebbero fiaccato temperamenli più impulsivi, ma d'altra parte non raggimsero quello sviluppo civile che sarebbe stato possibile di ottenere. A questa debolezza intrinseca bisogna aggiungere l'azione deleteria di cinque secoli di dominio turco.

Per raggiungere il livello degli altri popoli e poter fare senza pericoli quel salto vertiginoso dalla barbaric nella civilta bisognerebbe che i popoli balcanici, oltre al risveglio innegabile delle loro energie interne, godessero anche dell'aiuto esterno dei loro vicini. Purtroppo la terra dei Balcani ha dato sempre ricea messe di zi\%zania, e quasi non bastasse la produzione sponlaneal, lat diplomazia europea ve ne semina dell'altra a piene mani. Una rigenerazione rapida e rigogliosa dei popoli balcalnici per effelto soltanto della liberazione polilica, simile agli esempi offerti dagl'Italiani e dai Tedeschi, salrebhe una vana speranza, almeno per ora. 
E appena cessata la lotta cruenta dei popoli balcanici oppressi contro il secolare oppressore, il Turco, e già si delineano gli attriti futuri fra gli alleati di ieri. Lo spettacolo è doloroso e desolante. Però a conforto dell'umanità offesa e travagliata bisogna convenire che senza errori non si è maturato nessun popolo, anche di quelli situati in condizioni migliori dei Balcani. Dal momento che l'Europa non sa, non può, o non vuole, aiutar a risolvere l'intricata spartizione della penisola balcanica, bisogna che i popoli che la abitano facciano da soli. E quistioni simili non furono risolte mai colla penna o colle forbici, ma sempre unicamente dalla spada e dal cannone.

Il merito prossimo della reiezione dei Turchi dai Balcani spetta ai popoli dei rispettivi paesi; ma c'è anche un merito remoto, quello della civiltà nel più ampio senso concepibile.

L'industrialismo moderno ha bisogno di sempre nuove fonti per trarre materie prime da lavorare e di maggiori campi di consumo per i suoi prodotti artificiali. Furono già messe in azione le parti anticamente trascurate del settentrione d'Europa; solo nella sua parte sud-est giacevano per causa dei Turchi inoperose regioni, che una rolta furono floride per civiltà. I Turchi non vogliono lavorare, non vogliono permettere che altri lavorino con loro e nemmeno cedere colle buone un palmo delle loro antiche conquiste. Essi avranno le loro buone ragioni, ma anche il progresso civile, in senso emropeo, dell'umanità ha i suoi bisogni imperiosi e le sue leggi fatali. Non essendo possibile intendersi colle buone nè patteggiare, l'urto brutale diviene inevitabile e tutti quei popoli ch'ebbero la mala ventura di essere vicini ai Turchi dovettero ricorrere alle violenze guerresche per adempiere la missione loro affidata dalla storia della civiltà.

Il giorno in cui l'ultimo turco porterà politicamente le calcagna al di là del Bosforo sarà una data storica memorabile. Intanto in pochi decenni dal $1912 \mathrm{i}$ popoli balcanici, già avviati sulla strada della libertà, del lavoro, del progresso, daranno - gicva almeno sperarlo - 
al mondo un nuovo esempio dell'immenso valore morale e materiale dell'indipendenza nazionale e politica.

Col tramonto della mezzaluna si può considerare scomparso anche il sogno di uno Stato unico nei Balcani. Si può ammettere l'ipotesi d'un'evoluzione civile per opera della quale Serbi e Bulgari si considerino in un giono di là da venire sinceramente fratelli; ma una fusione nazionale completa dei popoli dal Danubio e dalle Alpi transilvane all'Egeo, al Jonio e all'Adriatico non avverrì mai. Al sognatore ottimista non resta quindi altra visione che un equilibrio di Stati e popoli minori tenuto su per forza ad immagine di quanto avviene oggi nel resto d'Europa tra le sue grandi potenze, oppure nel migliore dei casi una confederazione forte di nome, ma debole di fatto per screpolature insanabili del suo edificio.

Malgrado ciò non bisogna ancora farsi illusioni troppo rosee. La civiltà europea ha invaso e vi cammina a passi tenaci; ma questi passi sono piccoli e lenti per quello sterminato continente. L'Asia poi ha in Persia, in India, in Cina, al Giappone tante pericolose energie dormenti, che forse l'Europa potrebbe pentirsi un giorno di averle svegliate. E se dovesse succedere unáa reazione dell'Asia contro l'invasione europea, simile a quella che gia avvenne contro l'espansione romana, la prima vittima sarebbero ancora una volta i Balcani.

Poi c'è da pensare che appena dopo espulso il 'Turco, sarì da temersi che incomincino dei seri attriti internazionali per la quistione di Costantinopoli. Indi potrebbe acuirsi la quistione nazionale in Austria-Ungheria.

Ma ammettendo pure che tutti questi problemi vengano risolti secondo l'equità, in modo da garantire finalmente a questa vecchia e tribolata Europa (e all'Adriatico indirettamente) pace e tranquillità anche nelle regioni di mezzogiorno e d'oriente, rimarebbe ancora da tener gli occhi rivolti più a settentrione, verso la Russial. Il vasto impero del grande Zaj è ancora un po' sempre la gelida Sarmazia, semi-incognita al resto di Enropia, ove oggi oltre 120 milioni di popolo, tult'altro che omogeneo, si agitano convulsamente con una tragica passio- 
nalità per rigenerarsi civilmente e per raggiungere terre soleggiate, da cui furono finora ostinatamente respinti.

Tutti si augurano di veder sorgere dopo il tramonto della mezzaluna, l'alba d'un idillio pacifico sul sanguigno cielo d'Europa. Chi sa che invece il sasso di Sisifo di altre guerre e forse di altre trasmigrazioni di popoli non sia per insegnare anche ai posteri, che l'uomo non ha a cercare salvezza che nella forza e nella lotta.

L'avvenire dell'Adriatico dipende da quello delle sue due coste, ossia dalla sorte d'Italia, dei Balcani' e dell'Austria.

Per l'Italia l'avvenire non potrebbe presentarsi più lusinghiero di quello che è. La cacciata dei Turchi segnerà anche per i Balcani una nuova era, migliore della passata, e influirà beneficamente anche sull'Adriatico. Non bisogna dimenticare che la comparsa dei Turchi segnò il principio della decadenza di Venezia.

Per quanto è dato di leggere nel futuro il programma nazionale, contenuto nella formula «i Balcani ai popoli balcanici", verrà ad avere forse il suo naturale e logico completamento coll'altro "l'Austria-Ungheria ai suoi popoli». - Il programma di completare il riordinamento d'Europa secondo la grande corrente nazionale è il principio della resurrezione anche dell'Adriatico. Esso farebbe scomparire in primo luogo il pericolo slavo, che s'era fatto sentire al principio e durante il secolo passato di un'occupazione russa dal Baltico all'Egeo, - arresterebbe l'espanisione germanica, - diminuirebbe l'oppressione antinazionale della duplice monarchia danubiana. Mettendo al posto di colossi dell'epoca prenazionale, tanti popoli minori irrequieti e in antagonismo, ma liberi e costretti a cercare il proprio benessere negli scambi reciproci, si darebbe un impulso mai prima visto e oggi forse appena immaginabile al progresso intellettuale e materiale di quelle regioni d'Europa, che furono anticamente le prime ed ora sono invece le ultime a sentire l'influsso benefico della civiltà.

Per il benessere dell'Adriatico è necessario che una nazione, la più grande e la più forte, abbia la supremazia 
politica e militare e le altre minori godano piena libertà commerciale. L'odierno antagonismo militare italo-austriaco non è foriero di pace duratura. La nazione predominante sull'Adriatico non s'è trovala mai sulla costa oggi occupata dall'Austria. L'Austria d'altronde non è nemmeno all'altezza d'una tale missione. Per il benessere avvenire dell'Adriatico è desiderabile che cessi l'oppressione di uno Stato antimarinaro per natura ed antinazionale per costituzione organica, quale è l'austriaco. Questo Stato vi ammassa oggi artificialmente e forzatamente popoli e regioni che non hanno alcuna domestichezza col mare e coll'Adriatico in particolare, perchè sono attratti geograficamente altrove. Chi violenta la geografia provoca la storia.

L'Austria ha riconosciuto l'importanza del suo possesso adriatico, eppure per conservarsclo non sal lar altro di meglio che tenerselo avvinto colla for\%al. Tulto quello ch'essa ha falto per la costa orientale ebbe scopi militari. Da Fiume ad Antivari non costrui che una sola linea ferroviaria tra la costa e l'interno, a scartamento ridotto, ed anche questa per ragioni strategiche attraverso le regioni più aride dell'Erzegovina. Non è con sistemi militari coloniali che si amministra nel secolo $\mathrm{XX}$ una regione d'Europa che vuole ritornare atla luce della civiltà e del progresso. L'arer anteposto an sogno dinastico ambizioso all'interesse dei popoli, del progresso. della civilti, ¿̀ un indice del faltimento dellat missionc dell'Austria e le procurerì le più acerbe e giustilicale rampogne degli storici futuri.

Anche la sorte dell'Italia sull'Adriatico è già traccialla. Oltre alle cause del suo interessancenlo per questo mare. gia ricordate, ne esiste un'altra, anche importantissina. la quistione del suo mezogiorno. - Nei primi studi sulto squilibrio eivile tra il settentrione e il mezogiormo d'Italia si eredette di aver seoperto la causal in un non ben precisabile difetlo etnico di razze. Oggi invere ogntume può accorgersi che l'inferioriti civile del mo\%grionnto ir una conseguenza del disagio economico dorivante a liflesso dalla barbaric dei paesi circonvicini, i lalcani (l'E- 
ropa, l'Asia minore, l'Africa settentrionale. Nell'ammirare il benessere economico della Padania si dimenticava di vedere che oggi al di là delle Alpi fiorisce la civiltà e di ricordare che quando nei tempi antichi i Balcani, l'Asia minor: e l'Africa rappresentavano la luce del mondo e l'Europa di mezzo era immersa nelle tenebre, il mezzogiorno d'Italia superava il suo settentrione. - Fortumatamente il nefasto regime turco è scomparso da tutta la lunga costa settentrionale dell'Africa, dai Balcani, e sparirà sperabilmente presto anche dall'Asia minore. I segni di rigenerazione del mezzogiorno d'Italia sono già spuntati numerosi; e noi ne citeremo per l'Adriatico uno solo, il più eloquente, lo sviluppo del porto di Bari.

E bastato che l'unità italiana e il risveglio economico. dell'Adriatico offrissero qualche condizione favorevole di sviluppo, perchè il popolo pugliese uscisse dal letargo secolare e prendesse nella vita della nazione il posto che la sua intelligenza e la sua operosità gli assegnano. E se i Balcani si apriranno presto all'operosità e alla coltura occidentale, Bari sarà capace di un ulteriore sviIuppo meglio di qualunque altra città adriatica della sponda occidentale e forse anche dell'orientale.

Anche a Brindisi il commercio si sollevò subito dopo il taglio di Suez.

Un altro sintomo favorevole per l'Adriatico è che il Mediterraneo dopo cinque secoli va ridiventando un centro notevole - se anche non più l'unico come nell'antichità - di civiltà e di commerci. La gravitazione politica internazionale va nuovamente avvicinandosi al Mediterraneo, che è il mare d'unione fra tre continenti.

Il canale di Suez, sognato, si dice, dai Veneziani nel secolo XV, aperto dal 1869, lotta da quasi mezzo secolo per riavere dalle colonne d'Ercole quel transito commerciale che l'America gli aveva strappato.

L'asse dell'Europa, che nell'evo medio e nei primi secoli del cosidetto evo moderno peregrinò tra Parigi, Madrid, Londra, Vienna, Berlino, sta per ritornare al suo posto naturale, a Roma. L'Italia non è assurta ancora al grado di potenza regolatrice del vasto mondo: 
Però nella moderna tendenza degli Stati a non rimaner isolati, ma di formar aggruppamenti di potenze, l'Italia può dire già di essere il pendolo della bilancia politica d'Europa e quindi la regolatrice e l'arbitra della pace e della guerra. L'Italia ha già ripreso la sua missione di naturale superiorità geografica e storica, quale centro d'irradiazione sui Balcani attraverso l'Adriatico e la sua costa orientale. Come nei tempi passati, i paesi ad oriente dell'Adriatico non risorgeranno veramente alla civiltì che coll'aiuto morale proveniente dall'Italia. Questo còmpito dell'Italia sarà maggiormente facilitato, in quanto che un altro popolo, figlio di Roma, il Rumeno, è destinato per la sua posizione e per le sue attitudini a divenire l'arbitro delle sorti future dei Balcani.

\section{AUGURI0.}

Se questo sogno di libertà e di equitì nazionale, che liddriatico fortunatamente coincide colla floridezza dell'Adriatico, doin fiore. vesse avverarsi, noi o i nostri posteri vedremo assurgere questo mare in grazia ai progressi della tecnica moderna ad una prosperità superiore a quella di Roma e della Serenissima.

La sua bellezza naturale è rimasla inalterata. L'azzurro carico dell'onda, il celeste terso del firmamento, il roseo dell'aurora e il rosso infuocato dei suoi tramonti hanno conservato negli abitatori delle sue rive vivacita di sentimento e un senso innato del bello estetico.

Le sue coste, qui ampic e distese, lì frastagliate, offrono la più ricca varietà ed originalità di panorami. Solo l'Adriatico poleva dar vita ad una Venezia.

I benefizi del sole torrido, dell'acqua jodata e della brezza vivificante chiamano le genti dalle regioni nelbiose a ritemprarsi sulle sue sabbic. Mancano ancora in maggior numero quei perlezionamenti che possono a devono venir fatli dalla mano dell'uomo.

Quando mediante un imboschimento razionale sarì ripristinata l'ombra, e cillà, ville, bagni, vapori, moderni 
e numerosi, offriranno a tutti un soggiorno comodo e gradito, le coste dcll'Adriatico, oggi ancora in più punti selvagge, ridivenute gentili come ai tempi romani, potranno gareggiare coi giardini delle Esperidi.

Il vero risorgimento dell'Adriatico sta però ai nostri tempi nello sviluppo commerciale. L'Adriatico serve oggi al transito delle merei dal settentrione verso l'oriente e il mezzogiorno; e per ciò non vi sono che due sole grandi citta, Venezia e Trieste. Certo che la funzione commerciale precipua dell'Adriatico sta nclla mediazione in linea longitudinale. Però per la poca distanza tra le due coste sono facili anche gli scambi nel senso della latitudine, c questi invece oggi sono purtroppo ancora scarsi.

La storia c'insegna che quando Venezia non esisteva c Trieste era forse un villaggio di pescatori, la maggiore, anzi l'unica metropoli dell'Adriatico era Salona, grande come mezza Roma. Però allora la penisola illirica, fecondata dai Romani, era tutta orti e giardini, mentre oggi l'erba stenta ancora a crescere dopo il passaggio dei zoccoli dei cavalli turchi. Si pensi che mentre i Romani vi avevano costruito una fittissima rete di strade, che oltre ad essere militari erano anche commerciali, in partenza dalla costa, ora sulla sponda orientale da Fiume a Vallona non ci sono ferrovie, perchè quei piccoli tratti mozzi, fatti dall'Austria per scopi strategici, sembrano messi là solo per ironia.

La risurrezione anche dei Balcani è finalmente assicurata. E noi possiamo già vedere cogli occhi della fantasia risorgere Spalato al posto di Salona, Gravosa-Ragusa a quello di Epidauro, Durazzo e Vallona all'antico sito, crescere e raggiungere l'antica floridezza Pola, Brindisi, Bari, Ancona, Ravenna. Non una ma dieci ferrovie dall'Adriatico al Danubio, venti linee di vapori tra le due sponde, transatlantici da Venczia e da Trieste per Suez, Gibilterra, i Dardanelli, libertà nazionale e politica per i popoli, industrie locali ovunque sia possibile attivarne nei luoghi della costa, trasformeranno l'Adriatico da un lago morlo nel mare più vivo, interessante c ricercato d'Europa. 
L'Adriatico, che per la sua vicinanza al Mediterraneo, L'Adriaticn fu la regione ove la civilti mosse i prini passi, quando e la terza bambina uscì dalla culla di Creta, se la vede ritornare Italia. fatta grande dal giro d'Europa, dopochè crebbe fecondando gradalamente tutti gli altri mari il Tirreno, l'Atlantico, il mare del Nord, il Baltico, il mar Nero.

Le rive dell'Adriatico, troppo magre per elaborare una civiltà propria, vissero di luce riflessa. Ebbero però la fortuna di non essere lontane da un gran faro luminoso, quello di Roma e d'Italia. Su queste sponde, batlute non si sa più se dalle onde del mare o dalla marea umana, si ripercossero le vicende di fuasi tulti i popoli che dall'Asia venmero in Europa e di fuelli che seguirono la strada inversa. Bufere politiche trasportarono i popoli da una sponda all'altra. Però in questo scambio reciproco di energia umana tra le due coste non bisogua dimenticare la differenza essenziale. I popoli che dalla costa orientale passarono sull'occidentale vi vennero poveri, disperati, imploranti ricetto. Quelli invece che dall'oceidentale si recarono sull'orientale furono ricchi, se non altro di beni intellettuali, e per ciò, esscndo superiori, si mantennero anche da dominatori. E tutto ciò non avenne per un privilegio prepotente degl'Italici a (lispelto ed a danno prima degli Illiri, poi degli Slavi. Arvenne così e non altrimenti perchè anche l'espansione dell'energia umana è soggelta alle leggi solari.

Nella conca occidentale degli Apemnini è il grande serbatoio e il segreto della inestinguibile vitaliti italical, che sia come conquista militare, sia come preminenza religiosa, sia come superiorita artistica, disgiunte o riunite, s'irradiò e bencficò tante regioni a lei circostanti e specialmente la costa orientale dell'Amrialico. Su questat passarono e si fermarono popoli e Stati senza mumero, ma nessuno lasciò traccia duratura e incancellabile di una civiltà superiore che Roma e Vencoia. IE non pere solo caso la resurezione dell'Anrialico incomincio e si compì prima dalla parte italica.

Anche volenclolo questa costa non polrohte sollrarsi alla missione imperitura di sorella maggiore dellorien- 
tale. L'Italia, che coll'impero romano, accogliendo per qualche tempo liberamente nel suo seno tutte le religioni, aveva fondato l'unità del mondo antico evi diffuse la propria coltura, chie nell'evo medio fondò la Chiesa cattolica pure universale e nel rinascimento mercè la fusione della coltura pagana colla cristiana iniziò la società moderna, sta ora fortunatamente per riavere la sua missione storica.

La grande idea di nazionalità sconvolse e riformò l'Europa. L'Italia più che nessun altro popolo è nella posizione fortunata di conciliare il proprio avvenire con quello di tutte le nazionalità, maggiori e minori. Essa può e deve quindi diffondere, anche con sacrifizio di qualche piccola ambizione, il tipo statale di nazionalità.

Come l'individuo deve sacrificare la sua esistenza per il bene della patria, così una nazione deve saper contribuire al progresso di una più vasta civiltà umana. In ciò sta l'ultima ragione del valore, della sua dignità nella storia del mondo.

Non l'Austria-Ungheria dunque, ma l'Italia riprenderà la missione di naturale superiorità geografica e storica sull'Adriatico. Nella lotta fra geografia e storia prevale la prima; giusta prevalenza, quella della madre sulla figlia.

La civiltà incominciò ad albeggiare sui Balcani, ma splende già alta sull'orizzonte d'Italia. Ed è bene che Italiani e non Italiani rammentino che per legge naturale, geografica e storica, anche il prossimo risorgimento dell'Adriatico sarà prodotto e segnato dal genio della terza Italia! 
Avvertenz. Dell'Edtore . . . . . . Pag. vil PARTE PRIMA.

\section{L'ADRIATICO NELLA GEOGRAFIA.}

L'Adriatico e la Padania formano un bacino solo. Pag. 1 Collegamenti delle Prealpi e delle isole.

I tre bacini dell'Adriatico

Il settentrionale. - Il medio. - L'inferiore.

La costa.

L'Apenninica. - La Padanica. - La Carsica. - La valle del Narenta. Il Montenegro. - Suddivisioni e tratti. - Bocche di Cattaro.

Le due coste. . . . . . . . . . . . . . 12

Il confine orientale d'Italia. - Venezia.

Valore economico e sociale delle due coste. . . . 14 L'Apenninica. - La Puglia. - La Capitanata. - Il litorale molisano e abbruzzese. - Il Marchigiano. - L'Emiliano. - La Padania - La Carsica. L'Albanese. - Raccoldo tra i versanti omonimi.

I valichi . . . . . . . . . . . . 30

Sull'Apennino. - Tra le Alpi. - Nel Carso.

Le regioni . . . . . . . . . . . . . . 36

La Puglia. - Il Molise. - Gli Abbruzzi. - Le Marche. - Li Emilia. - La Padania. - Il Carso. - L'Istria. - 11 litorale croato. - La lialmazia. L'Erzegovina e il Montenegro. - Influenza delle regioni sul carattere degli abitatori.

Confronto fra le due coste . . . . . . . . . . 47

Funzione sociale dell'Adriatico . . . . . . . 55 
Parte Seconda.

\section{L'ADRIATICO NELLA STORIA.}

Popoli antichi

Pag.

62

I Greci. - I Bizantini. - 1 Romani. - Conquista romana dell'Illiria.

La pace romana di sette secoli. Periodo d'oro dell'Adriatico.

Evo medio

L'Adriatico durante le incursioni dei barbari. - I Longobardi. - Gli Àvari.

- Gli Slavi. - Cambiamento dell'Adriatico per la trasmigrazione dei popoli. - Tramonto della latinità sulla costa orientale dell'Adriatico.

Dalla caduta della romanità (VII secolo) alla comparsa

di Venezia (X secolo) . . . . . . . . . . . . . 96

Gli eredi politici di Roma durante il V-VIİ secolo. - Albori di vita nuova dall'VIII al X secolo. - I Francbi. - I Pontefici. - Venezia. - I Croati. - I Narentani. - I Saraceni. - Gli Ungheri. - Antagonismo fra il Papato e l'Impero.

Quattro secoli (XI-XIV) di lotte per l'Adriatico tra Veneziani, Slavi ed Ungheri .

Secolo XI: Fra Veneziani e Slavi. - Breve storia dei Re Croati. - I MIagiari dell'Adriatico. - L'Adriatico occidentale. - I Normanni. - Paragone fra i Narentani e i Normanni. - I Pontefici. - Considerazioni riassuntive.

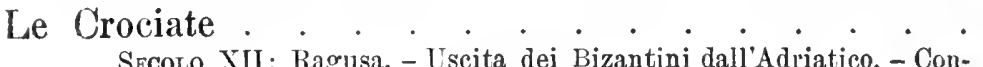
121 Secolo XII: Ragusa. - Uscita dei Bizantini dall'Adriatico. - Con-
segıenze delle crociate. - Stato Pontificio • 121

Secolo XII! : La bassa Italia. - Lo stato della Chiesa. - I Municipi italiani. Albori del trecento . . . . . . . 130

Secolo XIV: Venezia. - Lo Stato Pontificio. - Gli Angioini di Napoli. - Il ililanese. - Nell'Adriatico settentrionale. - 1 pirati almissani. - I Conti di Bıibir. - Il regno di Serbia. - Trartko, re di Bo >nia. - Ragusa. - I Mongoli. - Gli Asburghesi (l'Austria). - Trieste. - Ingrandimento degli Asburgo . . . . . . . . . . 135

Secolo XV: Piemonte e Savoia. - Lombardia. - Venezia e l'Istria. Ragusa. - Bassa Italia. - Scanderbeg. - Gli Araronesi. - Cesare Borgia. - Condizioni politiche e civili dell'Adriatico alla fine dell'evo medio. - I Turchi all'Adriatico. - Gli Asburghesi . . 151

Evo moderno. . . . . . . . . . . • . .

Secolo XVI: Venezia. - Lo Stato pontificio. - Gli Asburgo. Conseguenze del dominio spagnolo. - I Turchi. - Ragusa. - I pirati dulcignotti. - Gli Uscocchi . . . . . . . . . 168

Secolo XVII: Guerra col Turco. - Ragusa. - L'Austria. . 184

Secolo XVIII: Fine delle guerre di Venezia coi Turchi. - Venezia nell'ultimo secolo della sua esistenza. - Ragusa. - Gli Asburghesi. - Savoia. - Cambiamenti dinastici in Italia. - Considerazioni politico-sociali. - Gli Enciclopedisti. - I Gesuiti. - Giuseppe II. - Condizioni dell'Italia ossia della costa occidentale dell'Adriatico. L'Austria. - a) Espansione territoriale. - b) Riordinamenti interni. c) Orientamento verso mezzogiorno e all'Adriatico. - Trieste e Fiume porti franchi. . . . . . . . . . . . . 189 
La rivoluzione francese e Napoleone . . . . Pag. 209

Condizioni dell' Italia e dell'Adriatico . . . . . . 212 Conseguenze e cambiamenti sull'Adriatico . . . . 216

La caduta di Venezia. - Il Regno d'Illiria. - La caduta di Raysusa. La battaglia di Lissa del 1811. - Il Montenegro.

Il Congresso di Vienna . . . . . . . . . 225

L'Austria . . . . . . . . . . . . 226

La Santa Alleanza. - Metternich. - L'Italii attorno al 18:20.

Nuove idee politiche, sociali e nazionali . . . . 232

Avvenimenti nella metà balcanica dell'Adriatico . . 235

Decadenza della marina a vela. Origini della navigazione a vapore.

La rivoluzione di luglio del 1830 e sue eonseguenze sulle due parti dell'Adriatico. . . . . . . . 238

I fratelli Bandiera

La macchina a vapore. . . . . . . . . . 2 t4

Ressel. - Il Lloyd.

L'illirismo . . . . . . . . . . . . . . 245

La rivoluzione del 1848 . . . . . . . . 247

Alcune considerazioni sulla rivoluzione italiana del 1848

con speciale riguardo all'Adriatico . . . . . . 256

Garibaldi. - San Marino. - Ia flotta sarda a Trieste. - Costituente Germanica e Trieste. - L' Italia tra il '48 e il '59.

La guerra francoitaliana del 1859 contro l'Austria . 263 L'Anstria tra il ' 59 e il '66 . . . . . . . . 267

La guerra italiana del ' 66 . . . . . . . . . . 268

Condizioni interne dell' Italia e dell'Austria dopo il '66. 271

La marina italiana. - Il dualismo in Austria. - Gli slari. - Importanza dell'Adriatico per l'Austria.

La guerra franeo-prussiana del 1870. Roma capitale d'Italia . . . . . . . . . . . . . . . 276

Sull'Adriatico orientale. . . . . . . . . . . . 277

La penisola balcanica. - La guerra russo-turea del 1877-78. - 11 Congresso di Berlino. - L'occupazione della Bosnia-Erzegovina. - Orientamento dell'Austria verso i Balcani.

Slavofilia austriaca sull'Adriatico . . . . . . . 28:3

La politica italiana dal 1871 al 1887 . . . . 255

La Triplice Alleanza . . . . . . . . . 287

Alcuni fatti della penisola balcanica . . . . . 2si

Guerra greco-turca. - La rivoluzione dei giovani turchi. - Lannessione della Bosnia-Erzegovina. - L'occupazione ifaliana della Libia. - La liquidazione della Turchia europea. 
Parte Terza.

\section{L'ADRIATICO NELLA POLITICA.}

Il problema dell'Adriatico. . . . . . . . Pag. 298 L'idea di nazionalità . . . . . . . . . . . ivi La sorte dell'Austria . . . . . . . . . . . . 303 L'assetto presente dell'Adriatico . . . . . . . 313

L'Italia. - Il Montenegro. - L'Albania. - L'Austria-Ungheria. - I Croati $e$ il trialismo. - I Tedeschi. - I Magiari. - Gli Sloveni.

L'Austria nell'Adriatico . . . . . . . . . . 322 Gara commerciale fra Trieste e Venezia . . . 325 La missione di Trieste . . . . . . . . . . 327 Pretesa precarietà della floridezza di Trieste . . . 331 Il Carso e la sua calce.

Persecuzione dell'elemento italiano. . . . . . . 333 Errore politico dell'Austria . . . . . . . . . 335 Recente politica austriaca sull'Adriatico . . . . . 338 L'equilibrio dell'Adriatico. . . . . . . . . . 341 Antagonismo . . . . . . . . . . . . . . 343 Predominio . . . . . . . . . . . . . . 345 Dominio assoluto . . . . . . . . . . . . 348

Predominio dell'Italia . . . . . . . . . . . 350 Cozzo tra l'italianità, lo slavismo e il germanesimo . 351 Il germanesimo. - Lo slavismo. - L'italianità. - I Magiari. - I Greci. Gli Albanesi.

Il futuro

Gli Slavi dell'Austria.

L'ultima ratio . . . . . . . . . . . . . 374 Riassunto . . . . . . . . . . . . . . . 378 Conclusione . . . . . . . . . . . . . . 386

Tendenza del progresso nella civiltà moderna. - L'indipendenza nazionale. - I Balcani e il loro avvenire.

Augurio

L'Adriatico in fiore. - L'Adriatico e la terza Italia. 


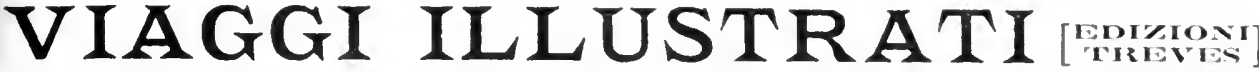

Le origini della civiltà mediterranea. Saygy, er rierche di Angelo sioni nel testo e una tarola a colori. Nuova edizione postuma, preceduta da studi del pro fessor 1I. L. Patrizi e di Enrico Thovez sulla vita e le opere di Angelo 11 osso. L. 12 -

Escursioni nel Mediterraneo e gli scavi di Creta, di Angelo mosso. ln-8, con 187 incisioni a due tarole fuori testo (1.907) .

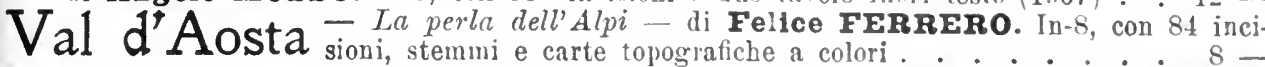

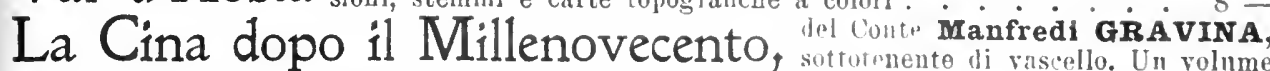
in-8, illustrato da 88 incisioni e 2 carte fuori iestu (1907).

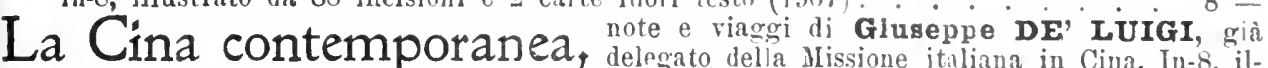
lustrato da 140 incisioni fuori testo e 4 cart gengrafiche.

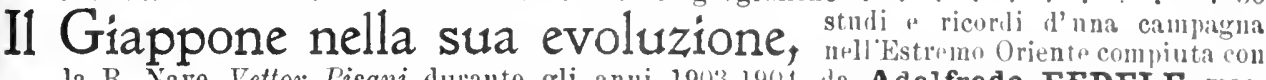
la R. Nave Vettor Pisani durante gli anni 19013-1904. da Adelfredo FEDEE macchinista mavale nellarmata italiana. Un volume in-4 di grau lusso di 216 pagine con 20 incisioni e sei grandi quadri a colori (1905)

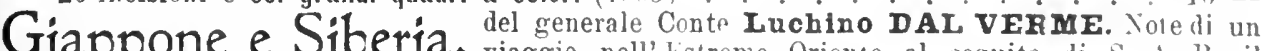
ap viaggio nell' kstremo Oriente al seguito di $S$. A. R. il

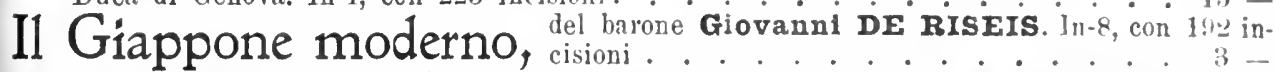

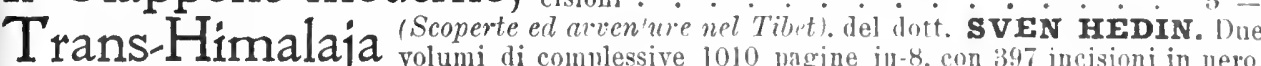
due panorami, otto tavole a

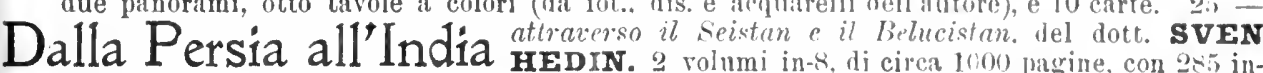
HEDIN. 2 rolumi in- $\$$, di cilca 1000 pacine, con 285 in-

La scoperta del Pol Po Nord,

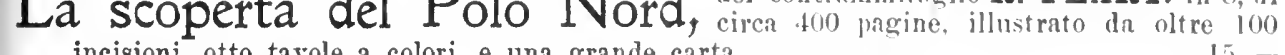

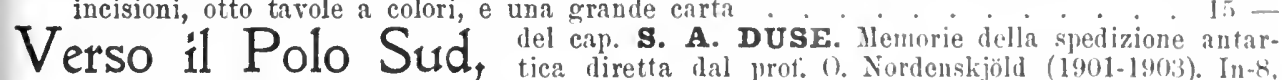
con 148 incisioni e carte

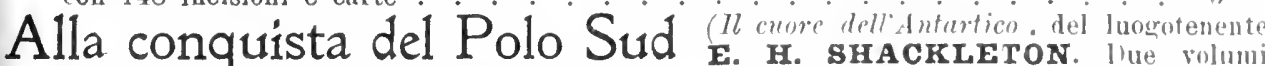
in-8 rrande, di complessive parine 914 con E. H. SHACKLETON. lue volum frontispizi in eliotipia e una grande carta.

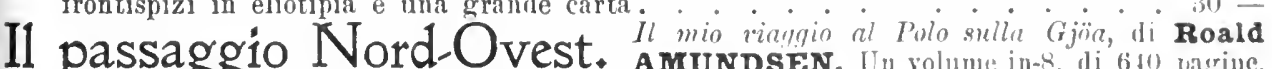

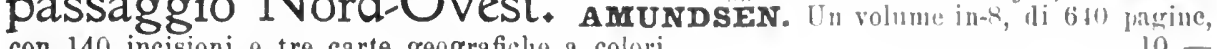

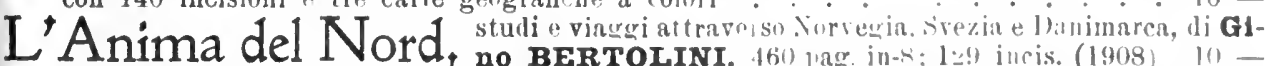

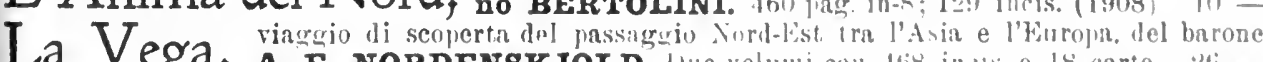

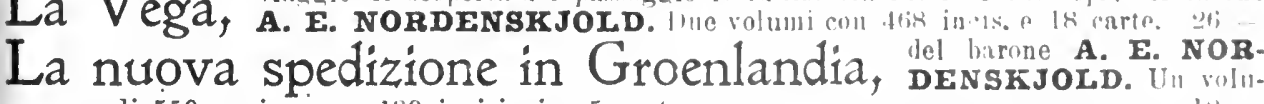

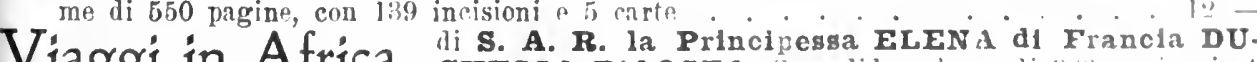

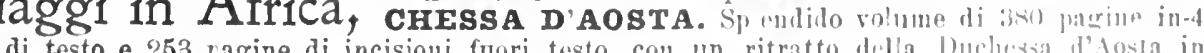
eliotipia colla sua firma autografa e unit carta a culori.

La stessa opera - testo francese -

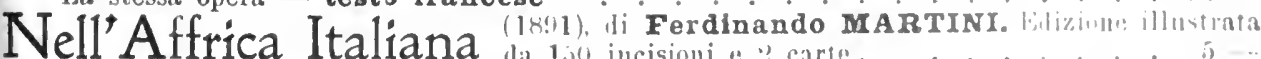

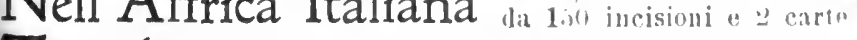

Trípolitania, di Domenioo tumiatr.

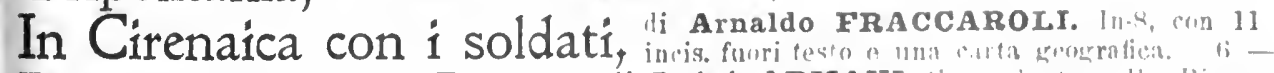
Díciotto mesi al Congo,

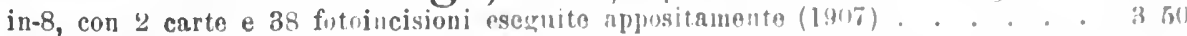




\section{STORIA D'ITALIA}

NARRATA DA

\section{FRANCESCO BERTOLINI}

ILLUSTRATA DA

\section{LODOVICO POGLIAGHY ED FDOARDO MATANIA}

Storia di Roma Legato in tela e oro.............. Eilizione di gran lusso in-folio. . . . . 40 - 4 Legato in tela $\theta$ oro e tagli dorati . . . 50 Vi sono ancora alcune copie dell'edizione in-8. Bellissimo volume di 1060 pagine riccamente illustrato da 230 disegni, legato alla bodoniana . . 20 - $\mid$ Legato in tela e oro..... . . . . $25-$ Queat' opera insigue, sia dal lato letterario sia|narrazione, la dottrina storica su cui essa poggia, dal lato artistico, ebbe già graude successo ed ha ed encomiò pure i disegni che la illustrano, Infatitl ottenuto il premio del Consiglio superiore di istru- pregio singolare dell' opera sono la ricehezza e la zione mubhlica dietro la relazione dettata da Mi- finitezza delle illastrazioni, lavopo originale di Lo chele Amari che l'ilevò la novità e lucidezza della dovico Pogliaghi.

\section{Medio Evo}

Qui il nostro illustre storico espon Q popolo italiano; come, in mezzo all' attrito fira due stilpi e due civiltà, sorgesse la Chiesa fortifieata: e come, in mezzo ad altro grande attrito. sorgesse la creazione piú feconda e più civile del Medio Evo, nei su
il Comune. Col racconto dei fatti politici intreccia

dalle invasioni barbariche fina a tutto il 1300. Illastrato da LODOVICO POGLIAGHY. Un magnifico volume in-folio di 720 pagine con 96 grandi quadri, edizione di gran lusso . . $45-$ Legato in tela 8 ого............... . . 55 quello del fatti morali, dando particolare sviluppo agli eveliti che risguardano la coltura. - Lodovico Pogliaghi, l'artista or"mai celebre per le sue scene romane, ha fatto lunghi e coscienziosi studi artistici per fal rivivere col pellnello il Medio Evo ei suoi costumi, nei suoi personaggi, nei suoi

\section{Il Rinascimento}

e le Signorie Italiane. Illustrato da CoDOVICO PoG I A GIY. Sp!endido volume in-folio di 600 pagine con 73 quadri, edizione di gran lusso . . 36 -

Legato in tela e oro......... $46-$

Il dopnio titolo di questo volume dice l'ampiezza podestà, oggi signoli e sovrani assoluti ; i Visconti dell'argomento e del periodo storico che v'è narl'ato. e gli Sforza, i Gonzaga e gli Estellsi, gli Scaligeri Pigliando le mosse dal primo giubileo romano-papale e i Carrara, i Medici, i Pepoli e i Bentivoglio, ecc.: chiudesi colla fine del secolo XV, che é appunto il è la storia del papato, nell'êra Avignonese, nello secolo detto del Rinascimento. - E, da un lato, la scisma d'Occidente, nella politica del nepotismo storia della coltura classica, che risorge; la storia dinastico.

delle arti, da Giotto a Raffaello, da Branelleschi a L'artista, ormai celebre, che ha avuto di recente Jichelangelo e a Leonardo; la storia della lettera- la massima distinzione all' Esposizione di Ionaeo tura, dall'Allighieri e dal Petrarca, al Poliziano e (Baviera) appunto per i suoi cartoni che servirono al Machiavelli: - e dall'altro lato, è la storia delle ad illustrare le Storie di Roma e del Medio Evo, ha Signorie che sorgono sulle rovine dei Comuni; la qui illnstrato il Rinascimento con la stessa geniastoria dei conquistatori, ieri capitani del popolo o lità artistica e con lo stesso gusto sapiente.

\section{Il Settecento e il primo Regno d'Italia.}

Elizione di gran lus3n, in-folio, illustrata da 25 grandi quadri fuori testo di MODOVIOC POCIICIIr, da 4 incisioni interc late nel testo, fregi, iniziali e testate. . . . . . . . 25 Lerato in tela e oro... . . . . . . . . . . . . . . . . . . . . . . . 35 -

\section{Il Risorgimento Italiano}

$(1815-1870)$. Illustrato da F DOATRO MIATAI A. Nnova ediz. in-folio di 826 pag. illustr. da 103 quadri. 20 -

Legato in tela e oro 40 - I Legato in tela e oro.

Edizione di gran lusso in-folio.

gli permisero di concepire ed eseonire dei quadri

E un'opera che rimarrà, ha detto la rivista Lettere ed Arti (N. 21 del 1889), perchè $\dot{e}$ forse il primo tentativo serio di una vera storia contemporanea. - Per essere fedele alla storia, il pittore fece le più diligenti picerche nei musei, nelle gallerie, nelle collezioni private. Queste ricerche stupendi che colpiscono per la fedele, giusta interpretazione dell' ambiente e dei soggetti, delle persone e delle cose, $\theta$ sono riaseiti $\nabla \theta r e$ opere d'arte, degne di illustrare una storia d'alto valore come questa. 


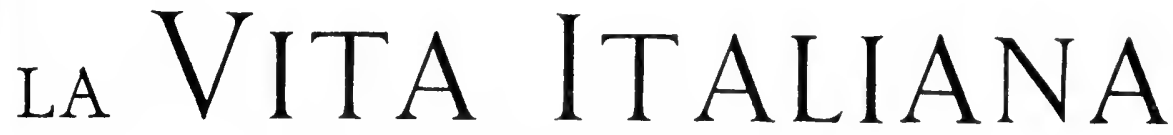

(CONFERENZE FIORENTINE).

GLI A L BOORI DELIA TITA ITATIA NA.

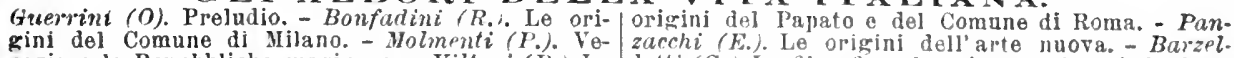
nezia e le Repubbliche marinaje. - Villari (P.). Le losti (G.). Ia filosotla e la scienza nel perjodo delle origini del Comne di Firenze. - Tucco (F.). Gli or- orizini. - Rajue (Pio). Le origini della lingua. dini religiosi e l'eresia. - Bonfadini $(R$.). Le orj- Bartoli (A.), te orjgini della letteratura. - Schupginl della Monapchia in Piemonte. - Bonghi $(R$.$\left.) . le f_{e}\right)^{*}\left(F_{0}\right)$. Le Unirersita o il diritto. - Masi $\left(E_{0}\right)$. orlgini della Monarchia a Napoli. - Graf (A.). Le Epilogo.

Nuova edizione in un sol volume in-16 di 412 pagine, L1re 4. - Legato in telu e oro, Lire 5.

LA VITA ITALIANA NEI, TIE ECEN'TO.

Bonfadili (R.). Le farioni. - Bertolini (F.). Roma Pocma. - Nencioni (E.). La letteratura mistica. o il Papato nel secolo XVI. - Franchetti (A.). lue Barfoli (A.). l'etrarea. - Burtoli (A.). Boccaceio. signorie e le compagnie di ventura. - Tabarini Graf (A.). Il tramonto delle legrende. - Martelli (M.). Le consorterie nella storia fiorentina. - Masi (I). (ili artisti Pisani. - Molmenti (P.). La gran(E.). Svevi e Angioini. - Rnjna (P.). La genesi della dezza di Venezia. - Boito (C.). Santa Maria del Fiore Divina Commetia. - Del Lungo (I.). Dante nel suo e il Duomo di Milano.

Nuora edizione in un sol volume in-16 di 430 pagine, Lire 4 - Leguto in tela p oro, Lire 5. Jiopera è peceduta da una prefazione di Gubo Blati, coi ritratti dei conferenzieri di V. Corcos.

NIET IR I A S C M IINTO.

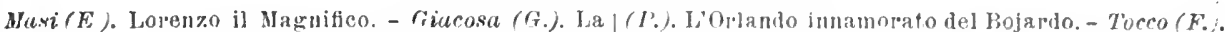
vita privata ne'Castells. - Biagi (G.). La rita pri- Il Savonarola e la profezia. - Martolli(I). L La pí-

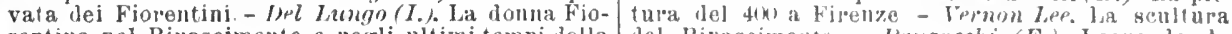
rentina nel Rinascimento e nesli ultimi tempi della del Rinascimento. - I'unzacchi (E.). Lconardo da

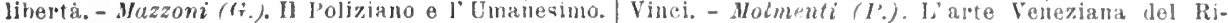
- Nencioni (E.). La jij.ica del Rinascimento. - Rujua nascimento.

Nuova edizione in un sol volume in-16 di 360 pagine, Lire 4. - Legito in tela e oro, L1ro 5.

NET OANQUHCHNO.

Ferrai (L. A.). Franceseo I e Carlo V. - Del Lungo L-Cardncci (G.). L'Ariosto. - Nencioni(F.). Torquato (I.). L Assedio di Fjeenze. - Masi (E.). La Riforma Tasso. - Mazzoni (G.). La lipira dol Cinqueconto. in Italia. - I e Johannis (J.). Condizioni dellecono- P'tnzacchi (E.). Raffiello. - Aid. Symmonds. MI imia pubblica. - Rondoni (G.). Siena nel secolo XVl. chelangelo. - Sillini ('T.). 1I Teat jo del (Jinquecento. - Paoli (C.). Gli serittori politici del Cinquecento. - Biuggi (A.). La musica del Cinquecento.

Nuova edizione in un sol rolume in-16 di 400 pagine, L1re 4. - Legato in tela e oro, L1re 5.

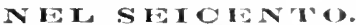

Falorsi (G.). Dalla pace di Castel Cambrese a quella taglia di Lepanto ela poesia politica. - Panzacchi(E.).

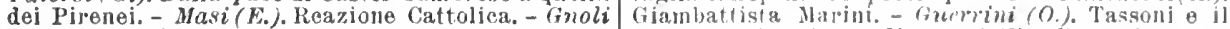
(D.). Roma e i Papi nel secolo XVII - Molmenti(P.). poema eroicomico, - Nenrioni (E..). Barocchismo. La decadenza di Venezia. - Del Lungo (I.). Galileo: Biaggi (A.). La muslea nel secolo XVll. - Venturi la sua vita e il suo pensiero. - Borio (G.). Il pen- (A.). I Caracei e la loro seuola. - Scherillo (M.). siero italiano nel secolo XVH. - Mazzoni (G.). La bat. La commedia dell'arte.

Nuova edizione in un sol volume in-16 di 3r2 pagine, Lire 4. - Legato in trla e oro, L1re 6.

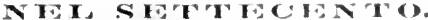

Bonfadini(R.). Da Aquisgrana a Campoformio. - Del doni. - Seruo (M.). (arlo (iozzi. - Mazzoni/fr.). GiusoplLungo(I.). I Medici granduchi. - Masi (F.). Gli avven- pe Papini. - Panzuchi (K.). Viłtorio Altheri - Borio

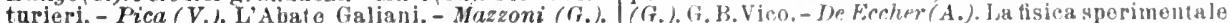
Dal Iletastasio all'Alferi. - Martini (F.). Carlo (iol- lopo Galileo. - Frudeletto (A.). L'arto nel Settocento.

Auova edizione in un sol volume in-16 di 500 pagine, Lire 4. - Legato in tela p oro, Lire 5.

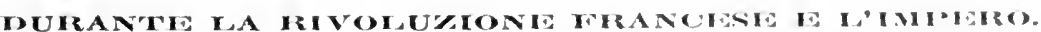

Lombroso (C.). La delinquenza nella Rivoluzione ( ria. - Nitti $(F$.). La trasformuzione socinle. - Mar-

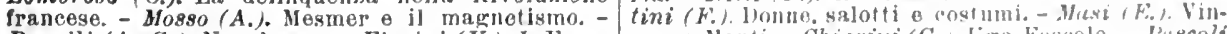
Barrili (A. G.). Napoleone. - Fiorini (V.). I Frnan- cenzo Monti. - Chiarini(G.) l'po Fuscolo. - I'llscoli

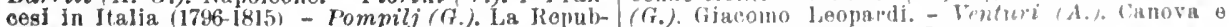

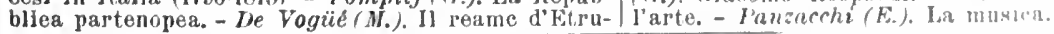

Nuova edizione in un sol volume in-16 di b12 pagine, L1re $6 .-1$ leguto in the uro, Liro 7.

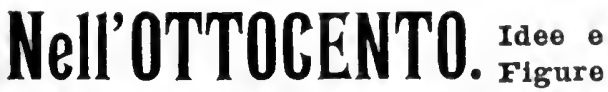
del Secolo XIX, di ERNESTO MASI. Un volume in-16 di 450 pagine. L. 4 Legato in tela $\theta$ oro

\section{Vita moderna degli Italiani, Maghi di A A Q EEL}

DIRIGERE COMMISSIONI E VAGLA AI PRATELLI TREVIS, FDITOR, IN MILANO, VIA PALRIMO, 12. 



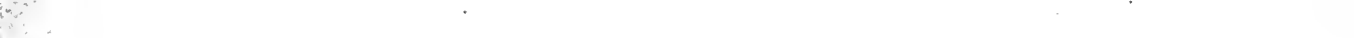





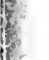

$$
\text { to }
$$





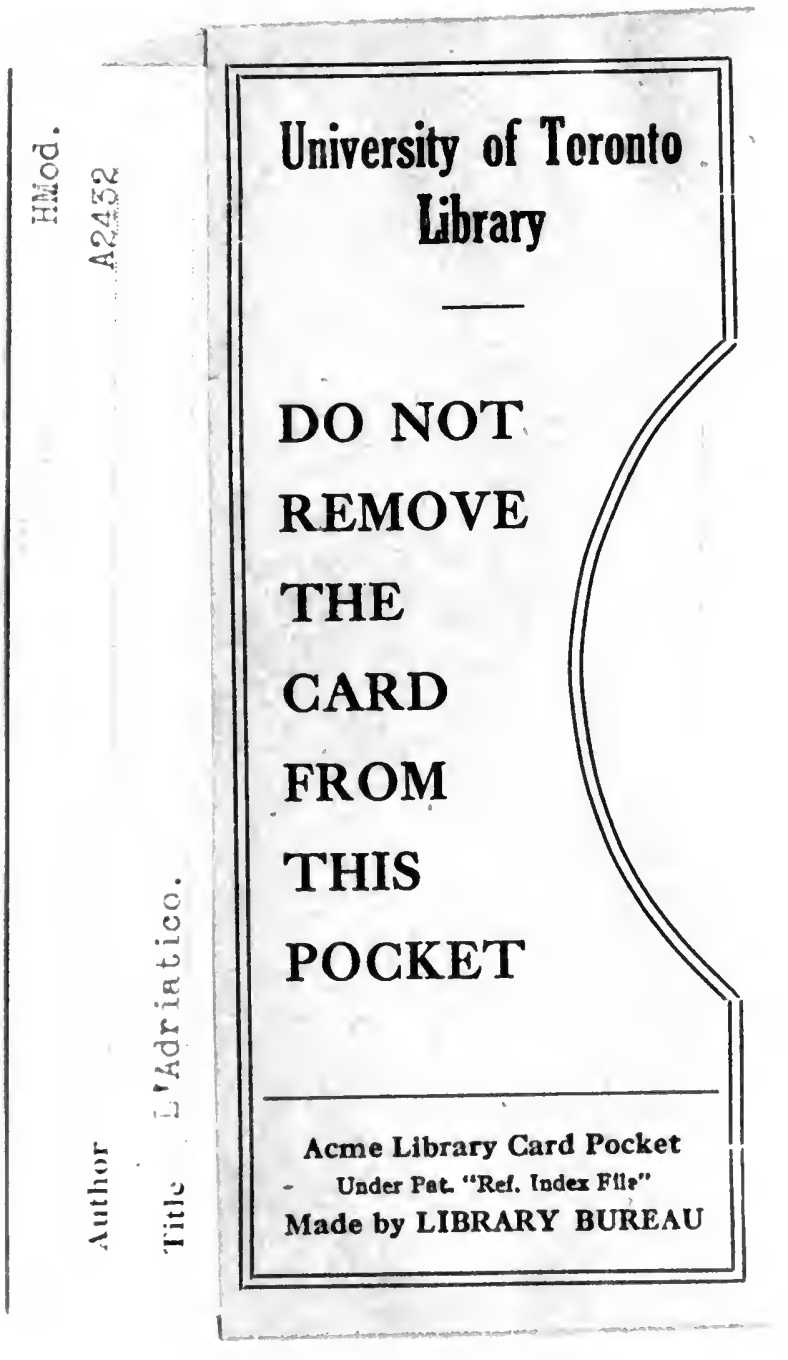


